\title{
Developmental Studies of Appendage Patterning and Formation in Spiders
}

\author{
Dissertation \\ for the award of the degree \\ "Doctor rerum naturalium" \\ of the Georg-August-University Göttingen
}

within the doctoral program Genes and Development of the Göttingen Graduate School for Neurosciences, Biophysics, and Molecular Biosciences Georg-August University Göttingen

\author{
submitted by \\ Natascha Zhang \\ Holzminden
}

Göttingen 2016 


\section{Thesis Committee}

Dr. Nikola-Michael Prpic-Schäper (reviewer and advisor), Department Developmental Biology, Georg-August-University Göttingen

Prof. Dr. Martin Göpfert (reviewer), Department Cellular Neurobiology, Georg-August-University Göttingen

Prof. Dr. Andreas Wodarz

Institute I of Anatomy

University of Cologne 


\author{
Members of the Examination Board \\ Referee: \\ Dr. Nikola-Michael Prpic-Schäper, \\ Department Developmental Biology, \\ Georg-August-University Göttingen \\ $2^{\text {nd }}$ Referee: \\ Prof. Dr. Martin Göpfert, \\ Department Cellular Neurobiology, \\ Georg-August-University Göttingen
}

\title{
Further members of the Examination Board
}

Prof. Dr. Andreas Wodarz

Institute I of Anatomy

University of Cologne

Dr. Gerd Vorbrüggen, Guest Group Molecular Cell Dynamics

Max Planck Institute for Biophysical Chemistry Göttingen

Prof. Dr. Daniel J. Jackson

Evolution of Metazoa

Courant Research Centre Geobiology

Georg-August-University Göttingen

Dr. Sven Bradler

Department Morphology, Systematic, Evolutionary biology

Georg-August-University Göttingen

Date of oral examination: 17.03.2016 


\section{Declaration}

Herewith I ensure, that the thesis "Developmental Studies of Appendage Patterning and Formation in Spiders" has been written independently and with no other sources and aids than quoted. Apart from the publications listed below the thesis has not been published so far. I also declare that I have not previously applied for a doctoral degree at another university.

Göttingen, January 31th, 2016

Natascha Zhang

Publications that are part of this thesis:

Turetzek, N., Pechmann, M., Schomburg, C., Schneider, J., and Prpic, N.-M. (2016). Neofunctionalization of a Duplicate dachshund Gene Underlies the Evolution of a Novel Leg Segment in Arachnids. Mol Biol Evol 33, 109-121.

Schomburg, C., Turetzek, N., Schacht, M.I., Schneider, J., Kirfel, P., Prpic, N.-M., and Posnien, N. (2015). Molecular characterization and embryonic origin of the eyes in the common house spider Parasteatoda tepidariorum. EvoDevo 6, 15.

Janssen, R., Schönauer, A., Weber, M., Turetzek, N., Hogvall, M., Goss, G.E., Patel, N.H., McGregor, A.P., and Hilbrant, M. (2015). The evolution and expression of panarthropod frizzled genes. Frontiers in Ecology and Evolution 3, 96.

Pechmann, M., Khadjeh, S., Turetzek, N., McGregor, A.P., Damen, W.G.M., and Prpic, N.-M. (2011). Novel Function of Distal-less as a Gap Gene during Spider Segmentation. PLoS Genetics 7, e1002342.

Manuscript in preparation:

Turetzek, N., Prpic, N -M. Observations on germ band development in the cellar spider Pholcus phalangioides. In preparation. 
Für meine Tochter

Reya Eliza Zhang 
“Tu die Dinge die du tust, weil du sie liebst und nicht weil ein Anderer dich dafür lieben könnte"

Torben Nehrhoff 2009 


\section{Danksagung}

Als Erstes danke ich meinem Doktorvater Nikola-Michael Prpic-Schäper für die wissenschaftliche Betreuung meiner Doktorarbeit. Ich danke für eine stets harmonische und sehr produktive Zusammenarbeit. Ganz besonders dankbar bin ich für seine Unterstützung, seine Ermutigung mir auch mal was zuzutrauen und sein großes Engagement. Schon während der Anfertigung meiner Masterarbeit hat er mich immer darin unterstützt eigenen Ideen nachzugehen. Vielen Dank für ein offenes Ohr und die vielen interessanten wissenschaftlichen Diskussionen.

Als nächstes möchte ich mich herzlich bei Nico Posnien für die Betreuung des Transkriptomprojektes bedanken. Aber auch für zahlreiche Diskussion über mein Projekt und generelle Fragestellungen der Evolutionsbiologie, welche mich immer wieder dazu gebracht haben meine Arbeit neu zu fokussieren, bin ich sehr dankbar.

Ich danke den Mitgliedern meines Thesis Committes, Andreas Wodarz und Martin Göpfert, für richtungsweisende Kommentare und wissenschaftliche Diskussion meiner Daten.

Ein ganz besonderer Dank geht auch an meine Kollegen aus Labor 2 und mittlerweile sehr guten Freunde Christoph und Montse für ihre nette Aufnahme nach meinem Umzug, die zahlreichen Diskussionen und ihre Unterstützung bei bioinformatischen Fragen. Aber auch Felix danke ich für ein offenes Ohr und sein Engagement mir bei der Optimierung der mikroskopischen Aufnahmen zu helfen.

Desweiteren möchte ich mich bei Ernst Wimmer und Gregor Bucher bedanken. Ihr Rat, Diskussionen und die kritische Hinterfragung der präsentierten Daten in zahlreichen Progress reports hat mich immer wieder dazu angehalten mein Projekt aus anderen Blickwinkeln zu sehen und auch damit dazu beigetragen die Projekte voranzubringen.

Nicht zu vergessen danke ich Sara und Matthias für ihre Einführung in das Arbeiten mit den Spinnen und vielen wissenschaftlichen Diskussionen. Ein ganz besonderer Dank geht auch ein meine lieben Hiwis, Julia Schneider und Julia Golldamm, die mir bei der 
Anfertigung zahlreicher Färbungen helfend zur Seite standen und ohne die, die Arbeit niemals diesen Umfang hätte annehmen können.

Ein großer Dank geht an die ganze Abteilung der Entwicklungsbiologie für ein sehr harmonisches Umfeld und Unterstützung bei jeglichen Dingen, vor allem Birgit, Angelika, Elke und Beate möchte ich herzlich danken. Während der Jahre habe ich in dieser Abteilung viele Menschen kennen gelernt die mir sehr ans Herz gewachsen sind und auch dafür bedanke ich mich. Wie schon genannt grade Niko, Nico, Montse, Christoph und Felix aber auch Dawid, Dippel, Kolja, Peter und Janna haben immer dazu beigetragen den Laboralltag interessant zu gestalten und noch mehr Spaß an der Arbeit zu haben.

Nicht zuletzt danke ich meinen guten Freunden, die mir während der ganzen Zeit den Rücken gestärkt, immer an mich geglaubt und mir Mut gemacht haben: Laura, Conny, Kathi, Marie und vorallem Torben, ich bin euch unfassbar dankbar!

Schlußendlich danke ich meiner Familie für ihre Geduld und ihre Liebe während der ganzen Jahre.

Am meisten danke ich meiner Tochter, dass sie mich immer wieder auf so wundervolle Weise in den Alltag zurück geholt hat. 


\section{TABLE OF CONTENTS}

1. SUMMARY

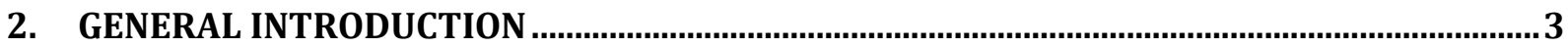

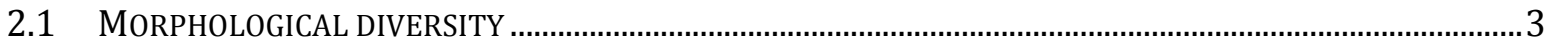

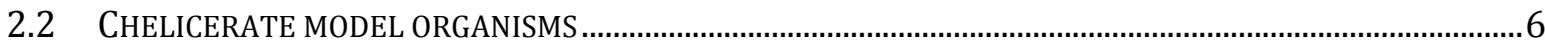

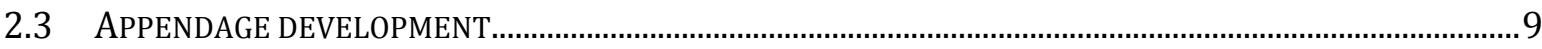

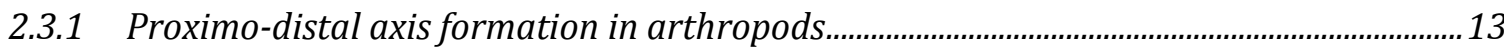

2.3.2 Tarsus segmentation gene network

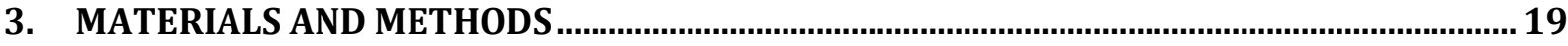

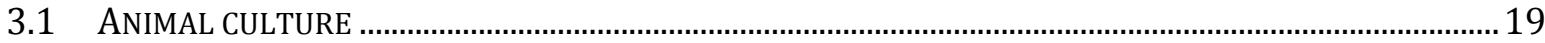

3.2 IDENTIFICATION OF SPIDER ORTHOLOGS AND PHYLOGENETIC ANALYSIS .................................................. 19

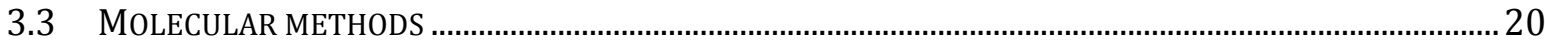

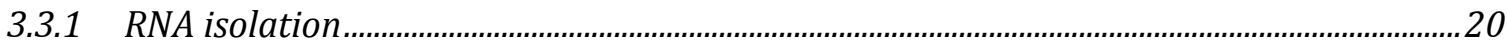

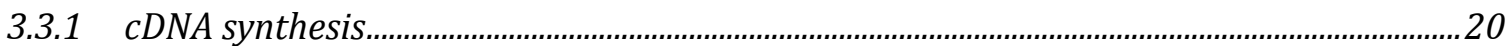

3.3.2 PCR

3.3.3 Cloning, transformation and plasmid preparation ….............................................................24

3.3.1 in vitro transcription of RNA probes and dsRNA for RNAi.................................................25

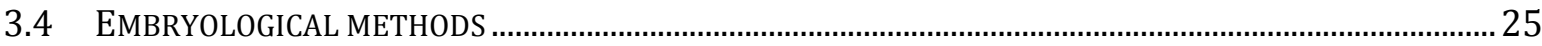

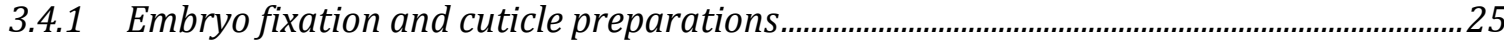

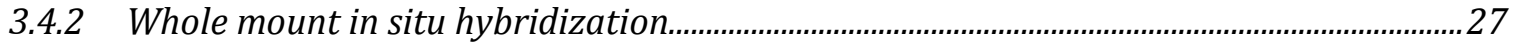

3.4.3 Live imaging of embryonic development of P. phalangoides....................................................28

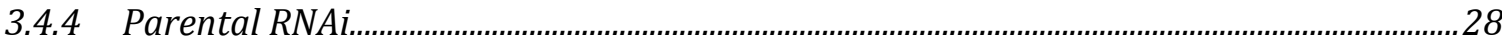

3.5 RNA SEQ, NGS AND DE NOVO TRANSCRIPTOME ASSEMBLY AND ANALYSIS............................................ 32

3.5.1 Read mapping and reduction of transcriptome complexity......................................................32

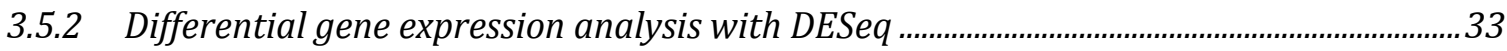

4. PHOLCUS PHALANGIOIDES: A HAPLOGYNE SPIDER MODEL

4.1 OBSERVATIONS ON GERM BAND DEVELOPMENT IN THE CELLAR SPIDER PHOLCUS PHALANGIOIDES ..... 36

4.1.1 Formation of the germ band in P. phalangioides .....................................................................

4.1.1 Segmentation and further differentiation of the germ band ..................................................39

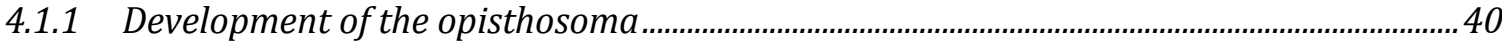

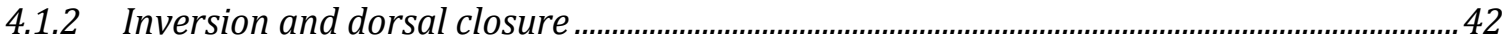

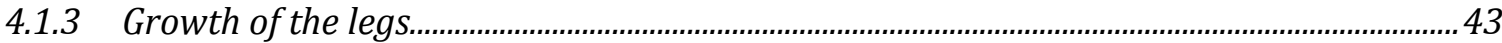


4.2 GENERATING TRANSCRIPTOMIC RESOURCES FOR P. PHALANGIOIDES .................................................. 45

4.2.1 de novo transcriptome assembly and differential gene expression....................................... 45

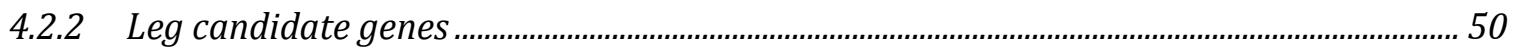

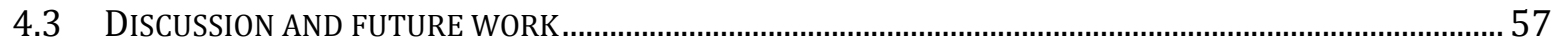

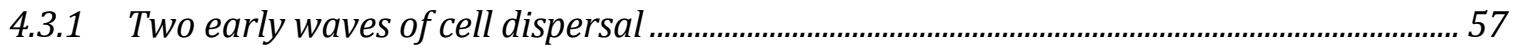

4.3.2 "Preabdomen-postabdomen" subdivision of the opisthosoma ….......................................... 58

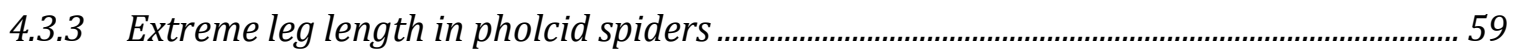

4.3.4 Conserved developmental steps for comparative expressional studies............................... 59

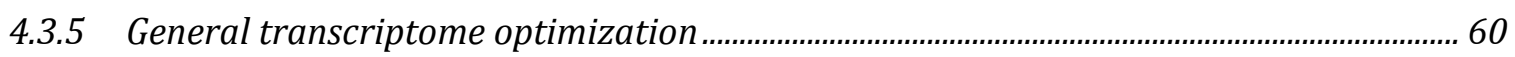

4.3.6 Up-regulated genes in the walking legs of P. phalangioides ................................................ 61

5. LEG GAP GENES IN APPENDAGE DEVELOPMENT OF SPIDERS ….......................................66

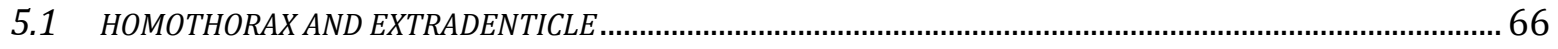

5.1.1 Duplicated hth and genes in P. tepidariorum and P. phalangioides................................... 67

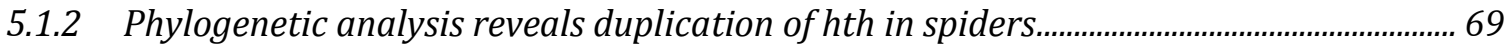

5.1.3 Expression of hth1 and hth2 during P. tepidariorum and P. phalangioides

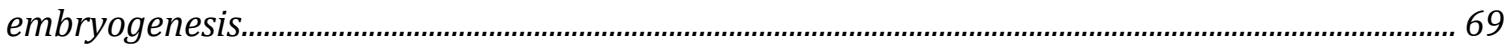

5.1.4 hth expression in the prosomal appendages of spiders.......................................................... 73

5.1.5 Duplication of exd genes in P. tepidariorum and P. phalangioides................................... 78

5.1.6 Phylogenetic analysis of exd homologs in spiders and chelicerates...................................... 79

5.1.7 Expression of exd during embryonic development of P. tepidariorum and P.

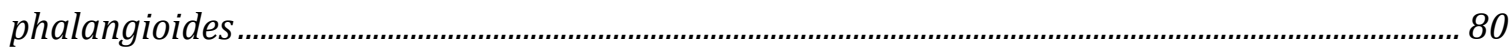

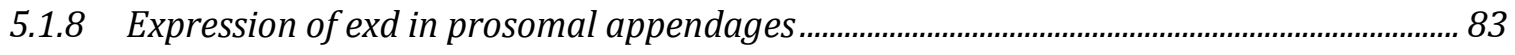

5.2 NEOFUNCTIONALISATION OF A DUPLICATE DACHSHUND GENE UNDERLIES THE EVOLUTION OF A NOVEL

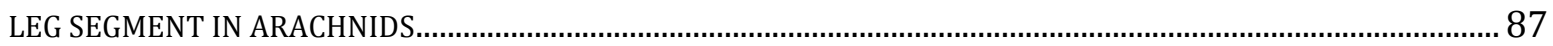

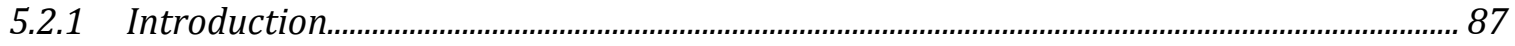

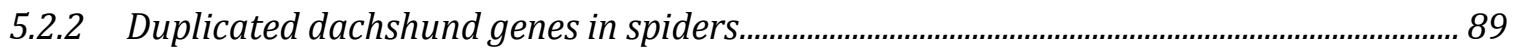

5.2.3 Phylogenetic analysis of dachshund gene evolution and duplication ................................. 91

5.2.4 Expression of dac1 in Parasteatoda tepidariorum and Pholcus phalangioides............... 92

5.2.5 Expression of dac2 in Parasteatoda tepidariorum and Pholcus phalangioides............... 94

5.2.6 Expression profile of dac1 and dac2 in the prosomal appendages..................................... 97

5.2.7 dac2 is required for morphogenesis of the patella leg segment in P. tepidariorum......100

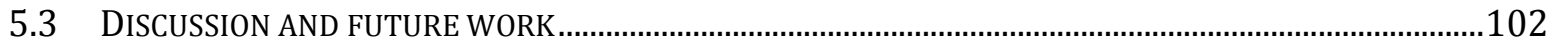

5.3.1 Gene duplication of spider dachshund genes........................................................................102 
5.3.2 Neofunctionalisation of dac2 in the arachnids. 102

5.3.3 Evolution of the arachnid patella: neofunctionalisation of dac2 underlies the evolution

of a morphological novelty. 103

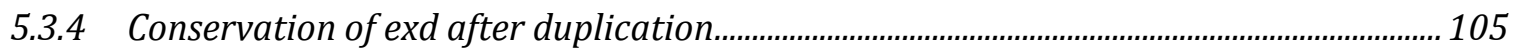

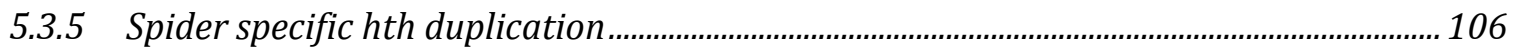

5.3.6 Rapid diversification of hth2 after neofunctionalization .................................................... 107

5.3.7 Leg gap gene conservation and duplication ...................................................................... 109

6. TARSUS SEGMENTATION CANDIDATE GENES IN SPIDERS............................................110

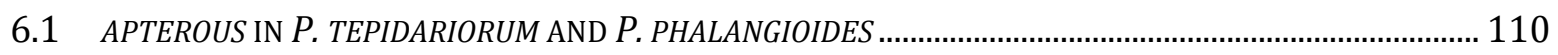

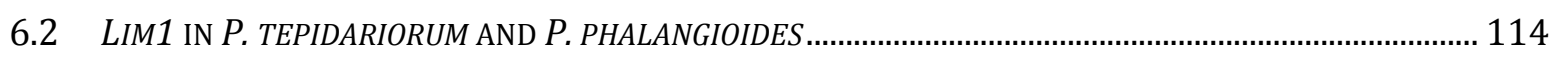

6.3 SPINELESS IN P. TEPIDARIORUM AND P. PHALANGIOIDES …........................................................... 117

6.4 TRACHEALESS IN P. TEPIDARIORUM AND P. PHALANGIOIDES ............................................................. 121

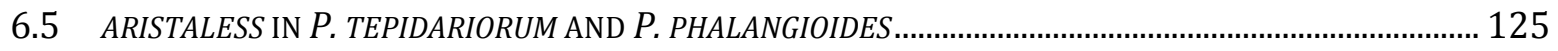

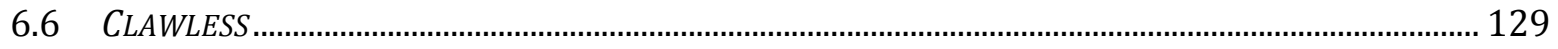

6.6.1 Phylogenetic analysis of clawless duplicates in spider ......................................................129

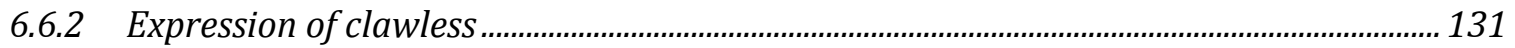

6.6.3 Expression of clawless in prosomal appendages ................................................................135

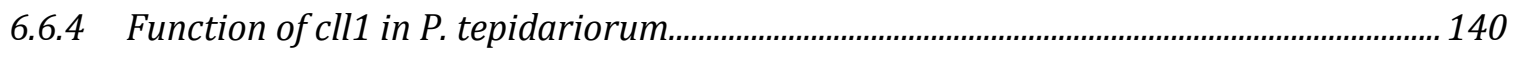

6.7 BARH IN P. TEPIDARIORUM AND P. PHALANGIOIDES ..................................................................... 147

6.7.1 Phylogenetic analysis of Bar in P. tepidariorum and P. phalangioides............................. 147

6.7.2 Expression of BarH homologs in P. tepidariorum ................................................................. 149

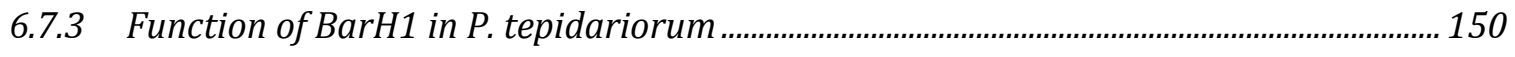

6.7.4 Expression of head and leg developmental genes in Pt-BarH1 RNAi embryos............... 155

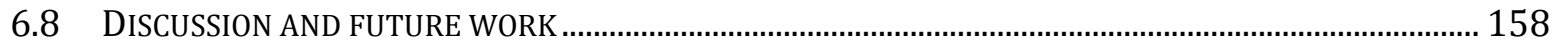

6.8.1 Distal limb development is not conserved for insects and spiders..................................... 158

6.8.2 Cross-regulation of al, cll and Lim1 is not conserved in arthropods ................................. 159

6.8.3 Conservation of distal function of clawless .............................................................................. 161

6.8.4 Other conserved functions of tarsus segmentation genes .................................................162

6.8.5 Possible role for ss and trh in growth regulation during late embryogenesis ................. 164

6.8.6 Novel role for BarH1 in head patterning of P. tepidariorum .............................................. 165

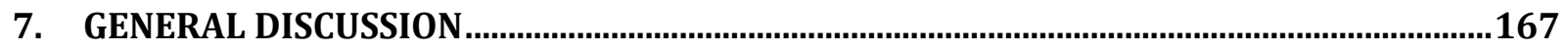

7.1 HOURGLASS MODEL OF WALKING LEG DEVELOPMENT ......................................................................... 167

7.2 DUPLICATION OF GENES FACILITATES DIVERSIFICATION …................................................................. 171 


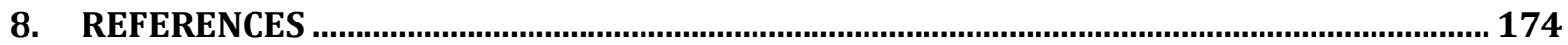

9. REFERENCES OF PUBLICATIONS AND MANUSCRIPTS...................................................... 191

9.1 TURETZEK ET AL. (2016). NEOFUnCTIONALIZATION OF A DUPLICATE DACHSHUND GENE UNDERLIES

THE Evolution of A Novel LEg SEgment in ARACHNids.

9.2 TURETZEK AND PRPIC IN PREPARATION. OBSERVATIONS ON GERM BAND DEVELOPMENT IN THE

CELLAR SPIDER PHOLCUS PHALANGIOIDES ...............................................................................................194

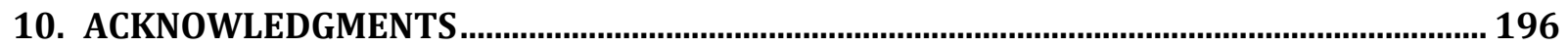

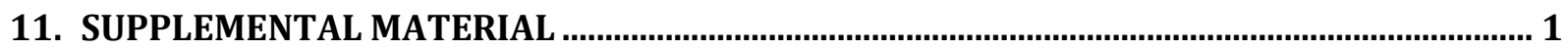

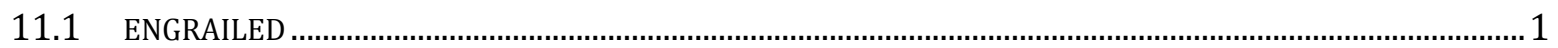

11.2 TIMING OF EMBRYONIC DEVELOPMENT OF P. PHALANGIOIDES …...................................................... 3

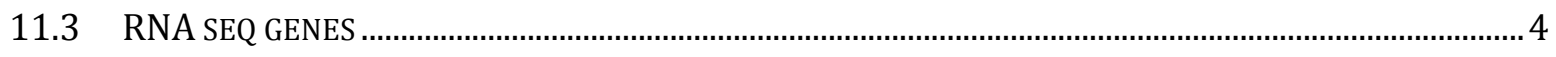

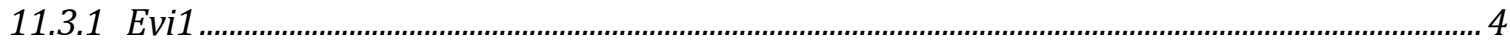

11.3.2 c105514

11.3.3 c95092

11.3.4 c109329

11.3 .5 c109662

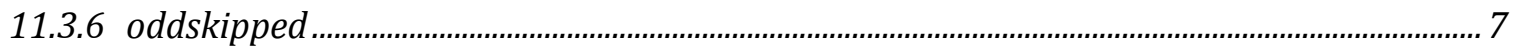

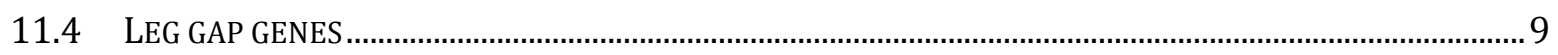

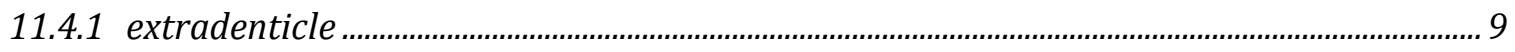

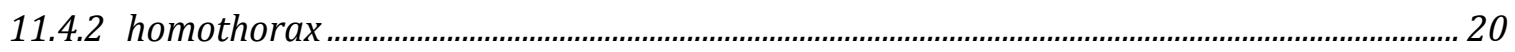

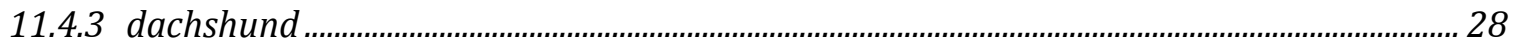

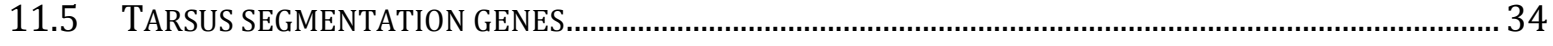

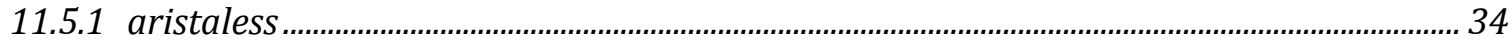

11.5.2 Lim

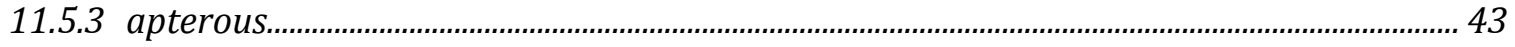

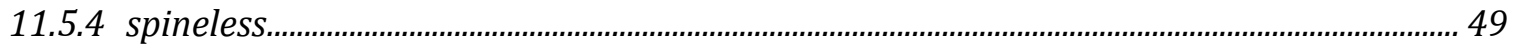

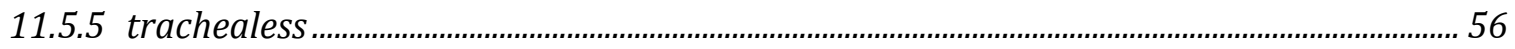

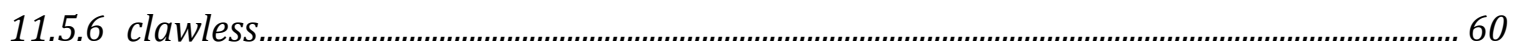

11.5.7 BarH 


\section{List of Figures}

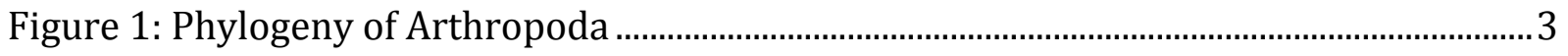

Figure 2: Diversity of arthropod appendages ............................................................................

Figure 3: Spider models used in this study ……………………………………………….....

Figure 4: Simplified leg patterning cascade of D. melanogaster ............................................. 12

Figure 5: Conservation of leg gap genes in panarthropods and spiders................................. 14

Figure 6: Overview of PD leg patterning in D. melanogaster and T. castaneum.................... 17

Figure 7: Consecutive stages of germ band formation and differentiation in $P$.

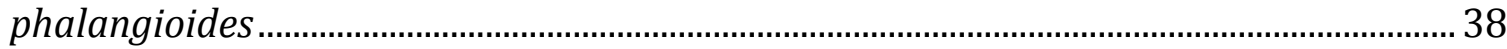

Figure 8: Expression of engrailed (en) in the germ band of P. phalangioides ....................... 41

Figure 9: Formation of the tail...................................................................................................... 42

Figure 10: Formation of the opisthosoma by folding down tail segments on the yolk...... 43

Figure 11: Allometric growth of the legs in P. phalangioides, and ventral closure .............. 44

Figure 12: Overview de novo transcriptome assembly.............................................................. 46

Figure 13: Comparison of gene and transcript numbers after corset...................................... 47

Figure 14: Dependence of transcript number and transcript length ...................................... 48

Figure 15: Principal component analysis of the triplicates for the different sample tissues

Figure 17: Expression of c102331 Pp-odd skipped in P. phalangioides .................................... 53

Figure 18: Expression of $c 109624$ in P. phalangioides ............................................................... 54

Figure 19: Expression of $c 95092$ in P. phalangioides ................................................................ 55

Figure 20: Expression of $c 105514$ and $c 109329$ in P. phalangioides ....................................... 55

Figure 21: Expression of $c 109662$ in P. phalangioides ............................................................ 56

Figure 22: Unrooted 50\% phylogenetic majority rule consensus tree of $h t h$ orthologs ... 70

Figure 23: Expression of $h$ th paralogs in P. tepidariorum ………………………………….... 71

Figure 24: Expression of hth paralogs in P. phalangioides ..................................................... 72

Figure 25: Expression of hth paralogs in prosomal appendages of P. tepidariorum............ 75

Figure 26: Expression of $h$ th paralogs in prosomal appendages of $P$. phalangioides .......... 76

Figure 27: Expression of $h t h 2$ in prosomal appendages of $C$. salei ......................................... 77

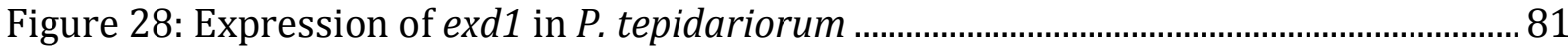


Figure 29: Expression exd1 in P. phalangioides .82

Figure 30: Expression of exd2 in P. tepidariorum 82

Figure 31: Expression exd2 in P. phalangioides 83

Figure 32: exd expression in prosomal appendages of $P$. phalangioides. .85

Figure 33: exd expression in prosomal appendages of $P$. tepidariorum .86

Figure 34: Alignment of the duplicated dac genes from P. tepidariorum (abbreviated PTEP in the figure) and P. phalangioides (abbreviated PPHA in the figure). 90

Figure 35: Unrooted 50\% majority rule consensus tree after Bayesian Markov chain Monte Carlo analysis. .92

Figure 36: Expression of dac1 in P. tepidariorum .94

Figure 37: Expression of dac1 in P. phalangioides. .95

Figure 38: Expression of dac2 in P. tepidariorum .96

Figure 39: Expression of dac2 in P.phalangioides 96

Figure 40: Detection of $d a c 1$ (A-F) and $d a c 2$ (G-L) expression in dissected appendages of

P. tepidariorum. .98

Figure 41: Detection of dacl (A-F) and dac2 (G-L) expression in dissected appendages of P. phalangioides. .99

Figure 42: Malformation of the patella after dac2 RNAi in P. tepidariorum. (A-D) Wild type nymph.

Figure 43: Evolution of a novel leg segment in arachnids linked to dac duplication and neofunctionalisation. 104

Figure 44: Overview spider $h$ th2 walking leg expression..................................................... 108

Figure 45: Expression of ap1 in P. tepidariorum.. 111

Figure 46: Expression of ap1 in P. phalangioides 112

Figure 47: Expression of ap2.2 in P. phalangioides. 114

Figure 48: Expression of Lim1 paralogs during embryonic development of $P$. tepidariorum.... 115

Figure 49: Expression of Lim1.2 in P. phalangioides 116

Figure 50: Expression of spineless paralogs in P. tepidariorum 118

Figure 51: Expression of spineless paralogs in P. phalangioides. 120

Figure 52: Expression of trh1 in P. tepidariorum 122 
Figure 53: Expression of trh1 in P. phalangioides......................................................................122

Figure 54: Expression of trh2 in P. tepidariorum ...................................................................124

Figure 55: Expression of trh2 in P. phalangioides......................................................................125

Figure 56:Expression of aristaless in P. tepidariorum …......................................................127

Figure 57: Expression of aristaless in P. phalangioides...........................................................128

Figure 58: Unrooted 50\% phylogenetic majority rule consensus tree of $c l l$ orthologs ...130

Figure 59: Expression of cll1 in P. tepidariorum........................................................................132

Figure 60: Expression of cll1 in P. phalangioides ......................................................................133

Figure 61: Expression of cll2 in P. tepidariorum....................................................................134

Figure 62: Expression of cll2 in P. phalangioides .....................................................................135

Figure 63: Expression of $c l l 1$ in prosomal appendages of $P$. tepidariorum ..........................136

Figure 64: Expression of cll1 in prosomal appendages of $P$. phalangioides..........................137

Figure 65: Expression of cll2 in prosomal appendages of $P$. tepidariorum ..........................139

Figure 66: Expression of cll2 in prosomal appendages of $P$. phalangioides..........................140

Figure 67: Overview of phenotypes in the different cocoon after cll1 RNAi and control

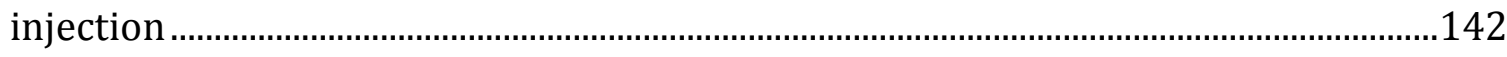

Figure 68: Overview of phenotype in nymphs of P. tepidariorum after cll1 RNAi ............143

Figure 69: Weak Pt-cll1 RNAi phenotype in the walking legs .................................................144

Figure 70: Strong Pt-cll1 RNAi phenotype in the walking legs ..............................................145

Figure 71: Pt-cll1 RNAi effect on nymphal pedipalps....................................................................146

Figure 72: Expression of BarH1 throughout embryonic development of $P$. tepidariorum

Figure 73: Expression of BarH1 in the head of P. tepidariorum ..............................................151

Figure 74: Expression of BarH2 in P. tepidariorum ...............................................................152

Figure 75: Phenotype of Pt-BarH1 knockdown in the different cocoons ............................153

Figure 76: Embryonic and nymphal phenotypes from Pt-BarH1 knockdown ...................154

Figure 77: Overview of embryonic phenotypes in cocoon 2-4 after Pt-BarH1 knockdown

Figure 78: Expression of otd, Dll and Scr in embryos after Pt-BarH1 RNAi........................157

Figure 79: Hourglass model of walking leg diversification development ...........................171 


\section{List of Tables}

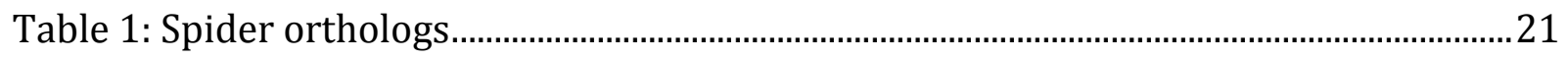

Table 2: Standard oligonucleotides ……………………………………………………...22

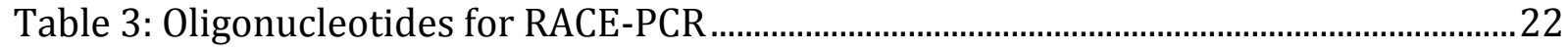

Table 4: Gene specific PCR oligonucleotides ..........................................................................23

Table 5: List of gene clones used in this study ………………………………………..........26

Table 6: dsRNA injection procedure for RNAi ..........................................................................2

Table 7: Differently expressed genes in the different tissues sequenced for $P$.

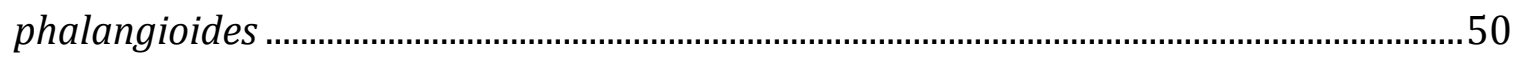

Table 8: Filtering criteria to reduce the number of up-regulated leg gene candidates......50

Table 9: Final candidates and their annotation .........................................................................51 



\section{Summary}

In the animal kingdom arthropods are unparalleled in species number and diversity. Especially their appendages show a great morphological diversity and are adapted for different purposes like feeding, walking, flying, swimming, breathing and courtship. The segmentation of the appendages into podomers is one characteristic trait shared by all extant arthropod groups and, at least in part, might be the key to their evolutionary success. Comparative studies of the genetic mechanisms involved in the formation and patterning of the appendages in different representatives of the four major arthropod clades is important to understand the evolution of this diversity. After the specification of the appendage primordia, appendage patterning in general requires the establishment of an additional axis, the proximo-distal axis. The formation of this axis is highly conserved in the arthropods, including homothorax/extradenticle defining the proximal, dachshund the medial and Distal-less the distal leg area. Further mechanisms of proximodistal patterning involved in tarsus patterning are largely conserved in the insects, but not much is known for arthropods outside the insect clade. To study these processes in non-insect arthropods, I used the main spider model Parasteatoda tepidariorum which has an undivided tarsus and the long-legged cellar spider Pholcus phalangioides, which shows tarsus segmentation that is morphologically similar to the insect tarsus. The conserved leg gap genes were already studied in two other spider species and homothorax and extradenticle were found to be present as duplicates. I studied homologs of all leg gap genes in Parasteatoda tepidariorum and Pholcus phalangioides to clarify the origin of the duplication event and their impact on appendage patterning differences leading to morphological diversification. My data suggest that all proximal and medial leg gap genes were duplicated in the lineage leading to the spiders. Whereas the initial leg gap genes all show the conserved expression as found for other panarthropods and thus might be a synapomorphic trait of the entire phylum, the expression of the paralogs differs indicating that the duplicates have undergone neofunctionalization. However, the origin of duplication as well as the conservation of the new function differs for these 
genes. The duplicate of dachshund is arachnid specific. It is required for patella formation, which is a morphologically novel walking leg segment only present in spiders and allies. The analysis of candidate patterning genes involved in tarsus segmentation of insects shows that these genes have some conserved functions within the phylum of the arthropods, but that their tarsus segmentation function is not conserved between spiders and insects. These data show that the initial steps of proximo-distal axis formation are highly conserved, but that more downstream patterning networks can be altered to drive the development new morphologies. In addition the presence of gene duplications is beneficial for functional gene diversification leading to novel traits whereas the fundamental developmental role of one paralog remains conserved to ensure proper development. 


\section{General Introduction}

\subsection{Morphological diversity}

The animal kingdom impresses by the vast diversity of species and morphologies. This diversification is probably the result of novel traits, which facilitate adaption to ecological niches causing a survival benefit and thus are positively selected over time. Especially the arthropods are unparalleled in species number and the great morphological disparity of the species. The phylogenetic interrelationships of the major arthropods clades (i.e. chelicerates, myriapods, crustaceans and insects) are an intensively debated issue and the great diversity hampers the phylogenetic resolution despite the growing number of available genomic resources (Caravas and Friedrich, 2010; Edgecombe, 2010). The most widely accepted phylogeny (Figure 1) is that the chelicerates, including mites, ticks, scorpions, harvestmen, spiders and the "living fossils" horseshoe crabs, are the most basally branching clade. Insects and crustaceans are grouped together forming a monophyletic group, the pancrustaceans, distally in the arthropod tree. The myriapods form the sister group to the pancrustaceans and together the three clades are referred to as Mandibulata (Regier et al., 2010; Rota-Stabelli et al., 2011).

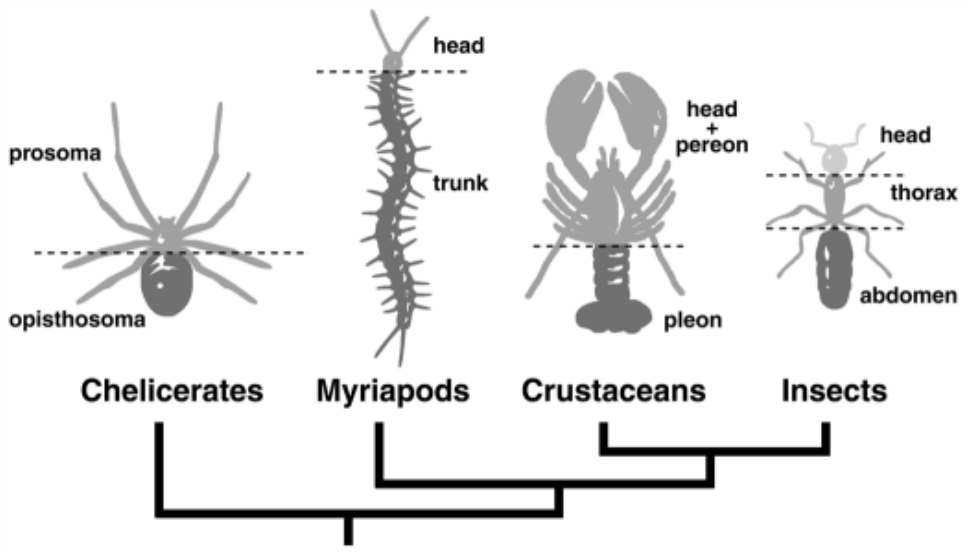

Figure 1: Phylogeny of Arthropoda

Current view of the phylogeny of all four extant arthropod groups. Dark grey denotes the hind body in the different clades. Light grey in contrast labels the more proximal head and thoracic body segments. Picture taken from (Hughes and Kaufman, 2002a). 
The serially homologous appendages of the arthropods are present in a varying number and morphology and have been adapted for special purposes like feeding, sensory function, walking, flying, mating and courtship (Prpic and Damen, 2008)(Figure 2). The appendages are not only diverse between different species, but even in an individual the different appendages on the distinct body segments are morphologically different from each other. One example are isopod crustaceans like the woodlouse, which can have up to 9 different appendage types along their body axis (Kensley and Schotte, 1989). But also in members of the other taxa we find a number of different appendage types (Prpic and Damen, 2008). So the question arises how are the arthropods so successful in terms of evolution, what is the key to their great species number and disparity, especially of the appendages. The disparity probably is the reason for the great species number and this disparity is in all likelihood the result of the fragmentation of their body and appendages into segments (e.g. Angelini and Kaufman, 2005). The subdivision of body and appendages into smaller units facilitates morphological changes of these independent modules, which might be positively selected without disturbing the complete organization of the body. Every multicellular organism arises from one single cell, which divides and gives rise to cells that differentiate during embryonic development and finally form certain organs or tissues.

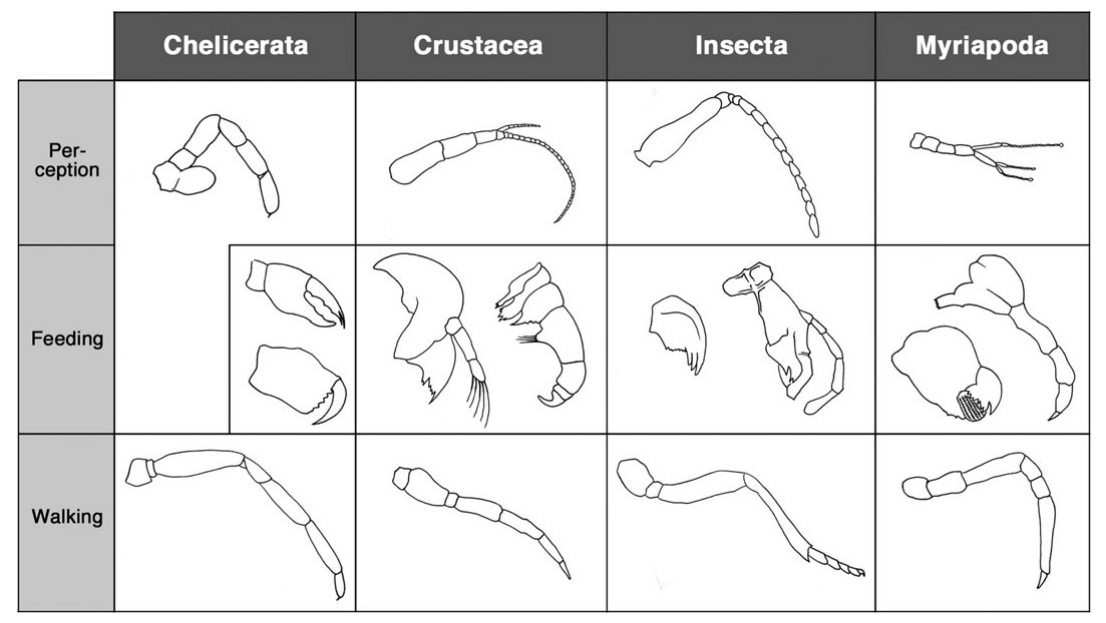

Figure 2: Diversity of arthropod appendages

Examples of different appendage types in the four major arthropod clades serving different purposes. The picture is taken from (Prpic and Damen, 2008) 
Even more interesting is that all cells inherit the same genetic information and different use of this information decides the fate of the cell. To understand the genetic level of this different use and where alterations are possible to develop novel morphological traits, it is necessary to understand general developmental processes involved in body and appendage segmentation. In case of the commonly used arthropod model organism the fruit fly, Drosophila melanogaster (D. melanogaster), many of these processes are well studied. For example, the early gene cascade of body axis patterning involves morphogen gradients setting up the anterior posterior axis, segmentation by the pair-rule genes and positional information provided by the Hox genes (Harding et al., 1986; Johnston and Nüsslein-Volhard, 1992; McGinnis and Krumlauf, 1992). Also the detailed genetic network driving the development of the different fruit fly appendages is extensively studied (reviewed in Estella et al., 2012; Kojima, 2004), but will be described in more detail below. In order to understand how the great morphological disparity was achieved during evolution it is not sufficient to study only one representative of a phylum because this could represent a derived mode (Bolker, 1995; Wilkins, A. S., 2002). Studies of fossil ancestors from the stem-line of the phylum may provide hints how certain morphologies have changed over time (Prpic and Damen, 2008; Whittington and Almond, 1987), but it is not possible to study the gene functions involved in the formation and patterning of these morphologies during embryonic or larval development. The only way to infer the ancestral state and reconstruct the level of conservation and/or diversification through evolution is to compare genetic mechanisms involved in the formation and patterning of morphologies during embryonic development of representatives from different branches of one phylum (Damen, 2007; Diogo, 2015; Peel et al., 2005). Especially the technological advances in sequencing leading to the Next Generation Sequencing (NGS) era facilitate working with a diverse set of non-model organisms of different taxa (Liu et al., 2015).

Diversification of morphological traits during evolution has to be based on diversification at the molecular level. Two possibilities of gene function diversification are changes on the regulatory level or alterations in the coding region of the gene, which are then positively selected over time (Wittkopp et al., 2004). However, most genes fulfil more than one specific function (pleiotropic genes), thus any change in their sequence usually causes multiple effects in different organs or tissues (Rose, 1982). In order to be 
positively selected, the genetic change must have a beneficial or neutral effect on all the different functions of a pleiotropic gene. Thus, genes cannot easily change their function and lead to morphological novelties, because genetic changes bear the risk of being lethal or disturbing finely tuned gene regulatory networks important for proper development or physiology of an individual. Current research has shown that gene duplications can facilitate evolution of novel traits (Innan and Kondrashov, 2010; Kondrashov et al., 2002), by providing genetic material for new functions, but without losing or changing the original gene. In vertebrates duplication of genes or complete genomes is intensively studied (Baskaran et al., 2015; Holland, 2013; Holland et al., 1994; Kasahara, 2013; Marlétaz et al., 2015), but in the arthropods duplication events appear to be less common and intensive research is still pending. Interestingly, recent studies indicate gene or genome duplication events in chelicerates (Di et al., 2015; Nossa et al., 2014; Sharma et al., 2014). Indeed, the pervasive gene duplications have been linked to the evolution of the repertoire of silk and venom genes in spiders, and to specific sensory organs in scorpions (Haney et al., 2016). Thus, extensive gene duplication in arachnids apparently provides the starting material for the evolution of morphological novelties. But not only the extensive duplication events in the lineage leading to spiders makes them good model organisms to study the evolution of the great arthropod disparity, other features of this clade and their importance in evolutionary developmental research are summarized in the following section.

\subsection{Chelicerate model organisms}

Chelicerates have an interesting position in the phylogenetic tree of the arthropods representing the most basally branching group (Caravas and Friedrich, 2010; Friedrich and Tautz, 1995; Giribet et al., 2001). Thus they are the most distantly related relatives to insects including D. melanogaster in the group of the arthropods (McGregor et al., 2008; Regier et al., 2010). In addition they have diverse appendages with different functions on the distinct body segments. Spiders for example have a pair of chelicerae for feeding, the pedipalps, which can be called the Swiss-knife of this taxon full-filling tasks ranging from sensing to sperm transfer, and the walking legs (Foelix, 1996). The 
appendages on the hind body (opisthosoma), give rise to the invaginated book lungs and tubular tracheae as well as the spinnerets for silk production (Foelix, 1996). Spiders also fulfil many requirements important for model organisms (summarized in McGregor et al., 2008). They are easy to keep in the lab, have a relatively short generation time (depending on the species) and the eggs are laid into cocoons, which are easy to access. In contrast to holometabolous insects their embryonic development ends with the generation of nymphs, which largely resemble the adult body morphologies, except for some specialized features used for mating. Thus the genetic background leading to the adult morphology can be studied directly during embryonic development, and not like in D. melanogaster in imaginal discs of the larva.

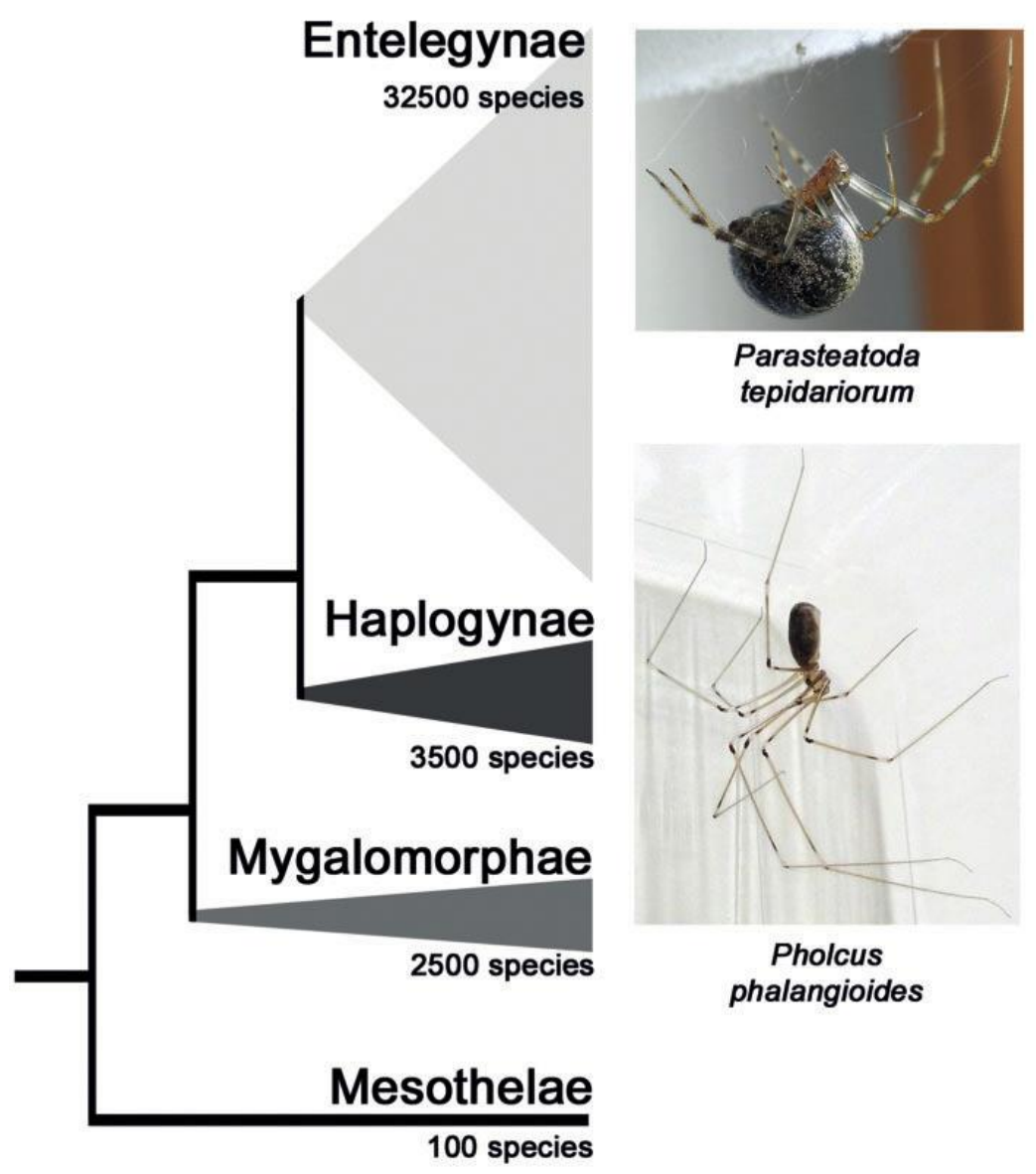

Figure 3: Spider models used in this study

The tree to the left shows a simplified spider phylogeny to show the relationships between the species Parasteatoda tepidariorum (an entelegyne spider) and Pholcus phalangioides (a haplogyne spider). Approximate numbers of described species are given for each clade. Picture taken from Turetzek et al. 2016 
Early genetic studies were performed in the Mexican wandering spider Cupiennius salei (C. salei). C. salei is a member of the most derived and largest spider group (32,500 species, Figure 3), the Entelegynae (Turetzek et al., 2016). These studies already fostered the impact of chelicerate arthropod models for evolutionary developmental biology. Studies of Hox genes in C. salei helped to resolve the homology of the arthropod mouthparts and revealed the presence of duplicated Hox genes (Prpic and Damen, 2004; Schwager et al., 2007). Drawbacks of this species are its large size, its relatively long generation time and a limited toolkit for the study of gene function. Therefore another entelegyne spider species went into the focus of developmental studies, namely Parasteatoda tepidariorum (P. tepidariorum) (Hilbrant et al., 2012; McGregor et al., 2008). This spider species has a shorter life cycle and it is suited for gene function studies by parental RNAi (pRNAi). In pRNAi the double stranded RNA is injected in adult females and possible knockdown phenotypes can be observed in the numerous offspring of the cocoons laid by this female. So far many techniques, like lineage tracing, whole mount in situ hybridization and exogenous protein expression were established in this species (reviewed in Hilbrant 2012). In addition a full transcriptome sequence (Posnien et al., 2014) is available and a genome sequence resource is in preparation (unpublished data, courtesy of Alistair McGregor, coordinator of the P. tepidariorum sequencing consortium). As stated above, one species is not sufficient to understand the changes in evolution leading to novel morphologies. Findings from only one representative always bear the risk being a derived specialty of the used species and must be compared to other members of the same clade. The best option would be to validate the findings from entelegyne spiders with data of a spider species branching off earlier in the lineage leading to the entelegyne spiders (depicted in Figure 3). For that there are three possibilities: (1) Segmented spiders (Mesothelae, approximately 100 Species), which are rare and difficult to keep under laboratory condition. (2) Bird spiders (Mygalomorphae, 2500 Species), which also require a lot of lab space and have long generation times. However, some comparative studies were performed with a member of the bird spiders, Acanthoscurria geniculata (A. geniculata). (3) Haplogyne spiders (Haplogynae, 3,500 species), which are the sister group of the entelegyne spiders and were already in focus of early spider research (Claparède, 1862; Holm, 1940). One of the most widespread and 
best-known member of the haplogyne spider group is the cellar spider Pholcus phalangioides (P. phalangioides, Order: Araneae; Family: Pholcidae). In addition to its phylogenetic position this spider is characterized by special appendage morphologies. This makes it interesting for comparative appendage patterning studies. Other names for this spider species are "daddy long legged" or "vibrating" spider and refer to the development of extremely long walking legs compared to its overall body size. The other characteristic morphological novelty is the segmentation of the tarsus into approximately twenty smaller segments, called tarsomeres (Roberts, 1995). This usually is not found in spiders. These characteristic walking leg features presumably influenced the evolutionary success of this widespread spider species, which is found in nearly every basement around the world. The extremely long legs can be used to catch prey of a much bigger size by throwing the spider silk from a safe distance. The long legs also facilitate vibrating behaviour, which makes the spider blur and thus distracts predators. For these reasons I performed comparative studies of appendage patterning (with a focus on walking leg development) in the two spider species $P$. tepidariorum and $P$. phalangioides and compared them to findings from other arthropod groups studied so far. Thus the following section is a summary about the main mechanisms of appendage development in the fruit fly D. melanogaster and its conservation/ or differentiation in comparison to other species.

\subsection{Appendage development}

D. melanogaster has a very derived indirect appendage development from imaginal discs (Cohen, 1993; Fristrom, and Fristrom, 1993) and thus might not the best representative to study formation and patterning of these outgrows. However, most knowledge about appendage formation is based on intensive studies of this species. The fly leg is subdivided into five distinct segments: coxa, trochanter, femur, tibia and tarsus (Kojima, 2004), which develop from the leg imaginal disc. The tarsus is composed of five tarsal segments (so-called tarsomeres), and the pretarsus, which carries the claw. The common chelicerate leg shows some differences compared to the insect leg. The distal part of the spider leg consists of two segments, the metatarus and tarsus (Foelix, 1996), but usually 
lacks the subdivision of the tarsus (except for P. phalangioides see above). The homology of these distal leg segments between different arthropods is still under debate and requires further genetic studies (Bitsch, 2001). In contrast to all other arthropods the spider leg contains a short segment between the femur and tibia, the patella.

No matter how versatile the different types of appendages are, they all have to undergo the same fundamental processes of development: (1) Specification of appendage primordia. (2) Specification of appendage type and position. (3) Specification of 3D Structure (anterior-posterior axis, dorso-ventral axis and proximo-distal axis). (4) Production of anatomically distinct regions (Segmentation). (5) Allometric growth (Angelini and Kaufman, 2005). These processes are studied in great detail in the fruit fly D. melanogaster. The specification of appendage primordia requires the onset of Dll expression by wingless $(\mathrm{wg}$ ) during embryonic development (Cohen et al., 1993). Dorsal and ventral appendage primordia are then distinguished dorsally by decapentaplegic (dpp) and ventrally by EGF signalling (Goto and Hayashi, 1997; Kubota et al., 2000). Knockout and ectopic expression experiments with the genes buttonhead and Sp1 (homolog of the vertebrate $S p 8$, also involved in leg development) showed complete loss and induction of leg structures, respectively and are thus also required to induce leg identity in the dorsal primordia (Estella and Mann, 2010; Estella et al., 2003). Dll and Sp8 both are known to be conserved factors in early development and onset of appendage development in arthropods and vertebrates (Bell et al., 2003; Kawakami et al., 2004; Panganiban et al., 1997; Treichel et al., 2003). The interactions and hierarchy of these genes are still a matter of debate. In D. melanogaster it was shown that early Dll expression from the Dll304 enhancer does not require $S p 1 / b t d$ function and that $S p 1$ and Dll are initially activated in parallel (Estella and Mann, 2010). However, maintenance of later Dll expression driven by another DLL enhancer DllLT is dependent on the $S p$ gene function. The appendage types along the anterior posterior body axis is largely influenced by the Hox genes (Hughes and Kaufman, 2002a, 2002b). The thoracic Hox gene Antennapedia (Antp) drives the proper development of the thoracic legs in the fruit fly (Schneuwly et al., 1987; Struhl, 1981). The posterior Hox genes Ultrabithorax (Ubx) and abdominal- $A$ ( $a b d-A$ ), on the contrary repress the development of appendages in the abdomen of D. melanogaster and are important for the formation of pleuropodia in the 
beetle Tribolium castaneum (T. castaneum) and the milkweed bug Oncopeltus fasciatus (Angelini et al., 2005; Bennett et al., 1999; Hughes and Kaufman, 2002b). The conservation and exact function of these early mechanisms in appendage specification are, however, still not fully understood for the other arthropod groups. Results from the spider $P$. tepidariorum show that legs develop in the absence of the Antp, but opisthosomal limb buds develop in the presence of Hox gene co-expression of Antp, Ubx and $a b d-A$ (Khadjeh et al., 2012). In addition, $D l l$ is required for the development of the first and second walking leg segment and distal leg development in P. tepidariorum (Pechmann et al., 2011). The mechanisms of appendage allocation and initiation probably do not have a significant impact on diversification of appendage morphology, because these are fundamental processes likely to be virtually identical in all appendages. However, the formation of the proximal distal appendage axis has a significant influence on morphological differences of different appendage types and divergent species. Thus my work focused on the proximo-distal axis (PD axis) patterning and refinement mentioned in steps 3 and 4 above. The genetic networks involved in these processes show, as already observed for body segmentation, a certain level of modularity (simplified in Figure 4). Combined $w g$ and $d p p$ interactions initiates the leg gap genes (hth/exd, dac and Dll), which generally subdivide the leg into proximal, medial and distal regions. Afterwards the EGFR pathway and several downstream targets are activated by Dll, $w g$ and $d p p$ in the distal portion to refine this patterning in the developing fly tarsus, called the secondary PD patterning (summarized in Damen and Prpic 2008; Estella et al., 2012; Kojima, 2004). The joints are finally developed under the control of the Notch pathway (Bishop et al., 1999; Celis et al., 1998; Rauskolb and Irvine, 1999). Data from the spider C. salei, the cricket Gryllus bimaculatus and the beetle $T$. castaneum show that the role of joint formation is a conserved function fulfilled by the Notch pathway (Angelini et al., 2012; Mito et al., 2011; Prpic and Damen, 2009). Preliminary expressional data for Notch pathway genes in the spiders P. tepidariorum and $P$. phalangioides further support the hypothesis that the joint formation itself is not altered between the different species and thus is also not involved in diversification of appendage morphologies ((Pechmann, 2011), and unpublished data). 


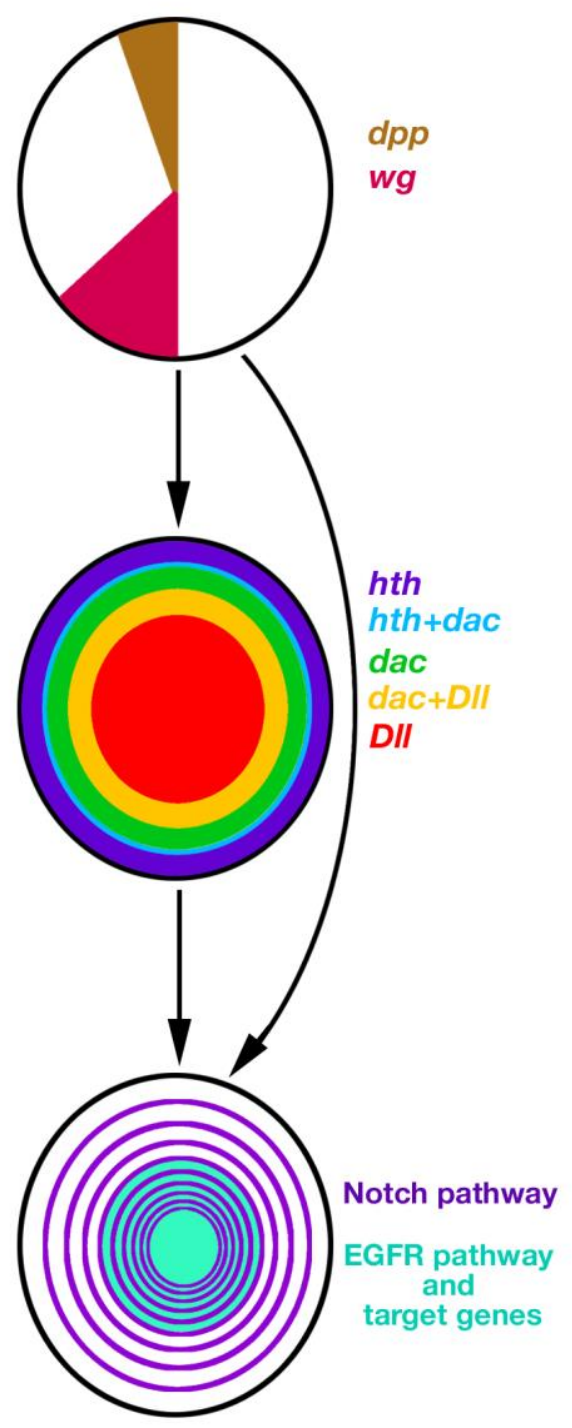

Figure 4: Simplified leg patterning cascade of $D$. melanogaster

Schematic overview of the walking leg patterning cascade in leg imaginal discs of D.melanogaster. The colour code in the disc represents the gene functions given in the same colour on the right. The rings of the Notch pathway represent the joints formed in the leg and tarsus segments. The picture was modified from (Prpic and Damen, 2008) with findings reviewed in (Estella et al., 2012; Kojima, 2004).

To investigate whether the leg gap genes or the genes involved in secondary PD axis patterning are altered and thus may drive differences between spiders and insects as well as the characteristic leg morphology of $P$. phalangioides I studied candidate genes known to be involved in these patterning networks from D. melanogaster. Findings on primary and secondary PD axis patterning in D. melanogaster are summarized and compared to other species in the following. 


\subsubsection{Proximo-distal axis formation in arthropods}

Key players in proximo-distal axis formation are the genes hth, dac and Dll, which are required to pattern the leg into proximal, medial and distal parts, respectively (AbuShaar and Mann, 1998; Lecuit and Cohen, 1997). They were called leg gap genes because the corresponding part of the leg was lost after loss of function experiments (summarized in Kojima, 2004 and Estella, 2012; Cohen and Jürgens, 1989; Cohen et al., 1989; Gonzalez-Crespo and Morata, 1995; Mardon et al., 1994; Rauskolb et al., 1995). Already nearly 20 years ago it is was suggested that different levels of the morphogens $w g$ and $d p p$ are responsible for activation of dachshund and Dll and repression of $h t h$. This hypothesis was challenged by the finding that mutant discs lacking the $d p p$ gradient, can still form a PD axis (Estella and Mann, 2008). This has led to a number of subsequent studies that have further refined our understanding of the role of $w g$ and $d p p$ and their targets like Dll during the formation of the proximo-distal axis of the leg imaginal disc.

Current research on the enhancer elements of Dll showed that co-expression of $w g$ and $d p p$ indeed induces the expression of $\mathrm{Dll}$ in the most distal part in early leg imaginal discs, but that it is not required for the maintenance of Dll (reviewed in Estella et al., 2012). In addition it was shown that $d a c$ is not activated by intermediate $w g$ and $d p p$ levels, but by direct activation of Dll (Giorgianni and Mann, 2011; McKay et al., 2009). wg $d p p$ coexpression thus only play a role in repressing $d a c$ in the most distal part. During leg growth the Dll and dac domains broaden and getting distinct from each other, which is probably maintained by auto regulatory mechanisms, transcriptional memory mechanism (Kim et al., 2008) and other downstream factors involved in secondary PD patterning (see below). The proximal part is defined by the homeobox gene hth distally repressed by wg, dpp and dac (Abu-Shaar and Mann, 1998; Wu and Cohen, 2000). Conservation of PD axis patterning by the leg gap genes was intensively studied in many panarthropod species including onychophorans, chelicerates, myriapods, crustaceans and various insects (Angelini and Kaufmann 2005; Janssen et al., 2010; Prpic and Damen, 2004; Prpic and Telford, 2008; Prpic et al., 2001, 2003; Sharma et al., 2012).

These studies showed that Dll and dac have conserved expression patterns in the developing walking legs of all panarthropods (Figure 5). The expression of $h$ th and exd 
on the other hand differs in myriapods and chelicerates when compared to the pancrustaceans and onychophorans. Studies in the spiders C. salei and A. geniculata, and the myriapod Glomeris marginata showed a reversal of hth and exd expression (Pechmann and Prpic, 2009; Prpic and Damen, 2004; Prpic and Tautz, 2003). In these arthropod species $h t h$ is expressed in the entire walking leg excluding the most distal tip and exd is restricted to the proximal part. In the onychophoran Euperipatoides kanangrensis (E. kanangrensis), the crustacean Parhyale hawaiensis and different insects $h t h$ is restricted to the proximal part and exd expression expands distally (Janssen et al., 2010; Prpic and Telford, 2008). That this reversal of expression could happen and did not lead to morphologically different PD axis is caused by the dependency of $h t h$ on exd. Hth needs exd to be transported to the nucleus (Berthelsen et al., 1999; Jaw et al., 2000; Rieckhof et al., 1997). Thus only in the area of co-expression these genes are functional and can fulfil their proximal patterning function.
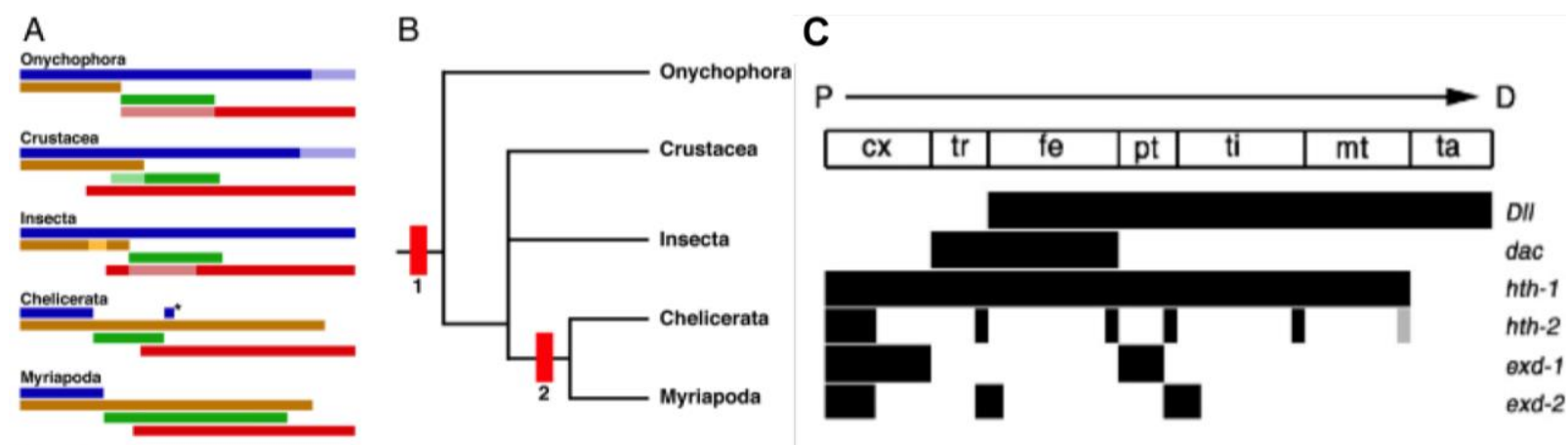

- exd e hth - dac - DII

Figure 5: Conservation of leg gap genes in panarthropods and spiders

A: Expression patterns of hth (orange), exd (blue), dac (green) and Dll (red) in the four major arthropod clades in comparison with onychophorans. B: Phylogenetic relationships of the panarthropods supported by findings from leg gap gene expression. The red box 1 marks the plesiomorphic state of the hth/exd expression pattern typical for onychophorans, crustaceans and insects, and the appearance of the dachshund domain within the DIl domain. The red box 2 denotes the synapomorphic reversal of $h t h$ and exd expression present in chelicerates and the partially non-overlapping appearance of $d a c$ and Dll. A and B are taken from (Janssen et al., 2010). C: Summary of leg gap gene expression including duplicates of $h$ th and exd in spiders. The grey box marks differing expression of $h$ th2 in $C$. salei compared to A. geniculata. modified from Pechmann et al., 2010. cx: coxa, tr: trochanter, fe: femur, pt: patella, ti: tibia, mt: metatarsus and ta: tarsus.

Since the co-expression of hth or exd is at all times restricted to the proximal leg, the divergent expression patterns found in the panarthropods are nevertheless functionally equivalent and the patterning function of $h t h$ and exd in the proximal leg is evolutionarily 
highly conserved. Interestingly studies from the spiders revealed that $h t h$ and exd are duplicated in the entelegyne spider C. salei and the bird spider A. geniculata (Figure 5, C) (Pechmann and Prpic, 2009; Prpic et al., 2003). The expression of these duplicates suggests that they differentiated from their original gene function, and probably underwent neofunctionalisation. The paralog of $h$ th, hth2 even shows different expression patterns in the walking legs of $C$. salei and A. geniculata. Thus studies in more chelicerate species could provide insight in the origin of gene duplication events and subsequent evolution of gene function of the duplicates. Furthermore one ring of expression in the area of the patella was found for the paralogs exd1 and exd2 in C. salei and A. geniculata in addition to the conserved proximal expression. Further studies showed that this ring represents a later function of exd, acting downstream of the Notch pathway after joint formation (Prpic and Damen, 2009). The coincidence of this ring with the chelicerate specific patella suggests that late function of the leg gap genes and their duplicates might have a role in the development of morphological novelties. Studies in the mite Archegozetes longisetosus (A. longisetosus) challenge this hypothesis. Although it is a member of the chelicerates and also has a patella only one exd paralog has been found so far and this gene has no medial expression ring (Barnett and Thomas, 2013). Findings for another chelicerate the harvestman Phalangium opilio (P. opilio), however, revealed the expression of exd1 as a ring in the medial part of the leg (Sharma et al., 2012). To further clarify the evolution of these leg patterning genes in the spiders I have performed additional studies of these genes in P. tepidariorum and P. phalangioides.

\subsubsection{Tarsus segmentation gene network}

The secondary segmentation process that occurs in the tarsus of D. melanogaster requires Dll and the EGFR pathway, which is initiated by wg/dpp (Galindo, 2002; Galindo et al., 2005). The EGFR signaling pathway creates a gradient in the tarsal segment 3 - 5 (t3-5), and the pretarsus. The pretarsus segment is defined by the transcription factors aristaless (al), clawless (cll) and Lim1, which are activated upon high levels of EGFR signaling (Campbell and Tomlinson, 1998; Kojima et al., 2005; Pueyo, 2004; Pueyo et al., 2000; Tsuji et al., 2000). The development of tarsal segment 4 and 5 in contrast requires 
low levels of EGFR signaling activating the homeodomain genes BarH1 and BarH2 and apterous (ap) (Kojima et al., 2000; Pueyo, 2004; Pueyo et al., 2000). The more proximal tarsal segments 1-5 are defined by bric-a-brac (bab), tarsal-less (tal), spineless (ss) and rotund (rn) (summarized in Kojima 2004 and Estella 2012; Baanannou et al., 2013; Emmons et al., 1999; Godt et al., 1993; Kozu et al., 2006).

The exact gene expression patterns that subdivide the tarsus segment of D. melanogaster into several smaller units are established by complex cross-regulatory interactions of these tarsal genes and have been intensively studied in over a decade, but are still not fully understood (Overview in Figure 6). In the pretarsus the proteins $\mathrm{Al}$ and $\mathrm{Cll}$ form a complex, which activates the expression of Lim1 (Campbell, 2005; Miyazono et al., 2010). The Bar homologs are then repressed distally by Lim1 and its cofactor Chip and proximally by ss and $r n$ (Kojima et al., 2005; Pueyo, 2004). Also the genes trachealess (trh) and tango, both predominantly known for the development of the tracheal system during embryonic development (Isaac and Andrew, 1996; Sonnenfeld et al., 1997), were shown to be involved in tarsus patterning by fine tuning the function of BarH in the fifth tarsal and pretarsal segment (Tajiri et al., 2007). For proper development of the more proximal tarsus segments the expression of $d a c$ needs to be restricted to the medial part. It was found that this is performed by the Bar homologs in the early third instar leg imaginal disc (Giorgianni and Mann, 2011). After the onset of $s s$ and $r n$ expression these genes mediate the distal repression of dac (Pueyo and Couso, 2008). bab expression in concentric rings is fine tuned by Dll and $r n$ binding to the leg and antennal enhancer and is then also involved in repression of dac (Baanannou et al., 2013).

The study of the gene battery involved in secondary PD axis formation in the red flour beetle T. castaneum, showed that most genes involved in D. melanogaster tarsus segmentation are also present and have a similar function in the tarsus of the beetle (Overview Figure 6) (Angelini et al., 2009; Angelini et al., 2012; Smith et al., 2014). However, not all genes known from D. melanogaster were included in this study and especially the genes involved in the pretarsus segment development led to contrasting results. Whereas the RNAi of al led to alterations of the claw (Beermann and Schröder, 2004), no obvious leg phenotypes were found after cll RNAi, although expression of this gene is present in the distal tip during embryonic development of T. castaneum (Cande et 
al., 2009; Grossmann and Prpic, 2012). Lim1 RNAi showed the fusion of femur and tibia but in contrast no distal walking leg phenotype. Even simultaneous knockdown of Lim1, $a l$ and cll did only lead to an additive combination of the single RNAi of al and Lim1 (Angelini et al., 2009). Data for Gryllus bimaculatus and Manduca sexta showed that also EGFR signalling and distal expression of $b a b$ and al are similar to D. melanogaster (Miyawaki et al., 2002; Nakamura et al., 2008; Tanaka and Truman, 2007). This further supports the conservation of distal patterning in insects.

A D. melanogaster (leg)

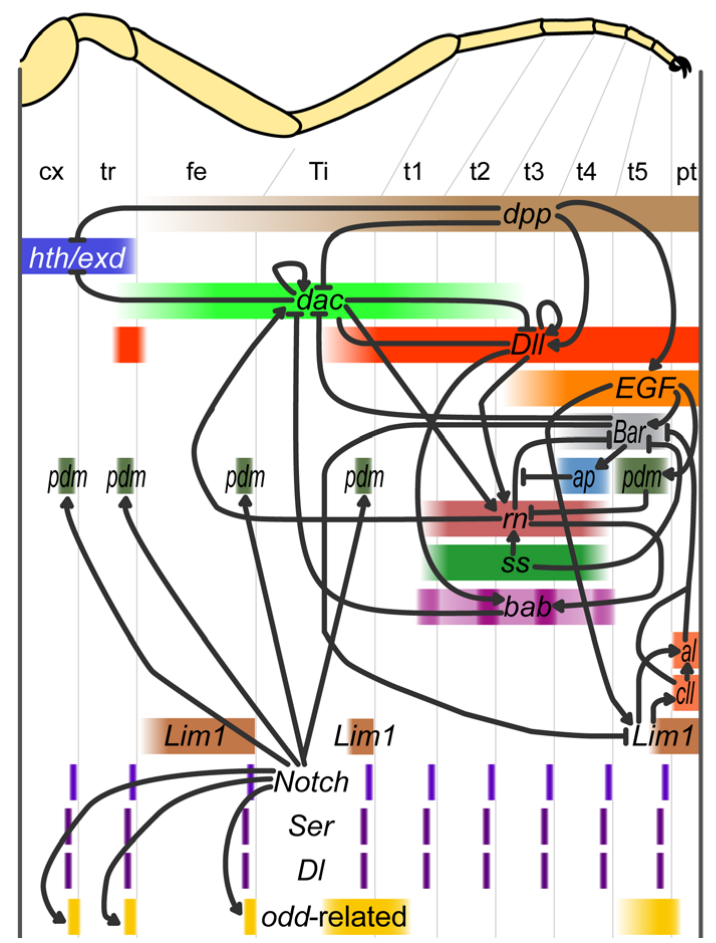

B T. castaneum (leg)

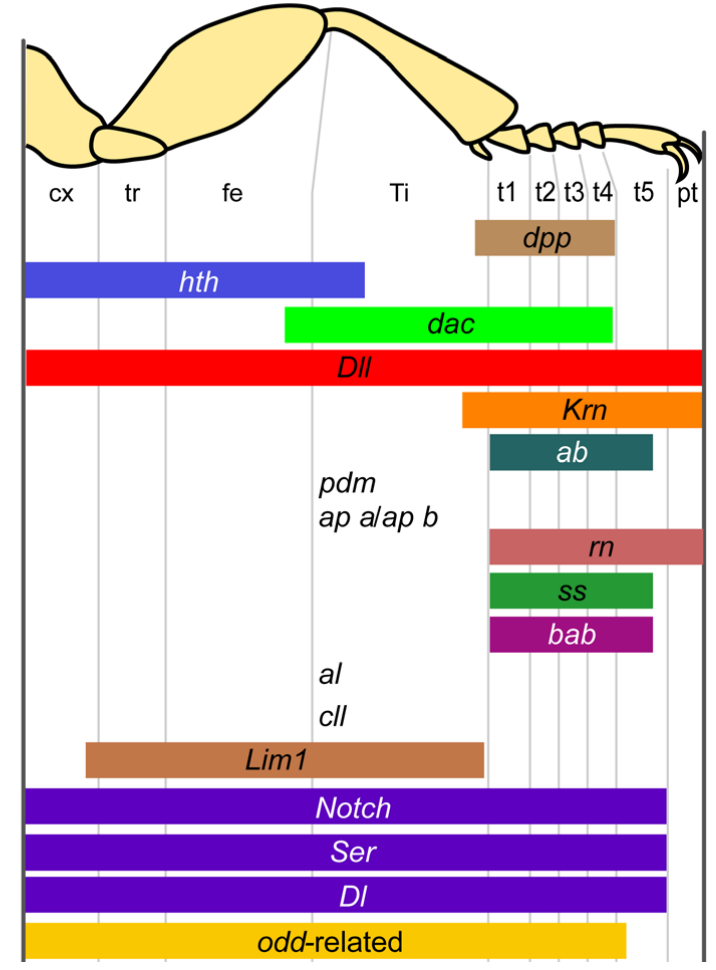

Figure 6: Overview of PD leg patterning in D. melanogaster and T. castaneum

A: Overview of genetic cross-regulation involved in patterning the walking leg and tarsus segmentation of $D$. melanogaster (Summarized from Kojima 2004 and Estella 2012). B: Summary of the patterning cascade in $T$. castaneum. The picture was modified from (Smith et al., 2014).

Contrasting findings from the crustacean Artemia, however, showed that trh is absent from the distal tip of all appendages (Mitchell and Crews, 2002), thus suggesting that conservation of distal patterning in the insects cannot be generalized for all arthropods. Recently six distal limb genes were studied in the onychophoran Euperipatoides 
kanangrensis (Oliveira et al., 2014), which represents the more basal sister group of arthropods (Borner et al., 2014). Comparative studies from insects and the members of the sister group of arthropods could reveal a possible conservation of distal patterning in the last common ancestor even prior to segmentation of the limb. The study shows that the genes cll, al, rn, Lim1 and ss are present in all appendages, but with a different expression in the anterior appendages compared to D. melanogaster. However, it was also shown that at least cll, al, Lim1 and ss are expressed in the distal part of the legs during late embryonic development. This suggests that the distal patterning of the locomotory appendages already involved these genes in the last common ancestor. Another scenario could be that distal limb development independently evolved in onychophorans and higher insects using the same set of genes. The findings for Lim1 of T. castaneum and trh of Artemia would support this scenario. The only possibility to identify the level of conservation of distal limb development and tarsus segmentation and determine whether it was already present in the last common ancestor of all arthropods is to study the distal patterning in more arthropod species. Therefore we studied these genes in the two spider species P. tepidariorum and P. phalangioides. Preliminary data on the expression of EGFR in the spider $P$. tepidariorum (see Supplemental Figure 2) already indicates that the distal organization of the leg is probably independent of an EGFR gradient signalling in the distal part. However, this does not exclude the possibility that the downstream targets were ancestrally used to pattern the distal limb area and are just switched on by another gradient. 


\section{Materials and Methods}

\subsection{Animal culture}

Parasteatoda tepidariorum (P. tepidariorum) and Pholcus phalangioides ( $P$. phalangioides) embryos were obtained from our laboratory stocks in Göttingen, and treated as previously described (Turetzek et al., 2016).

\subsection{Identification of spider orthologs and phylogenetic analysis}

Unless stated otherwise the identification and cloning of candidate genes was based on the transcriptomic resources available for both spiders. To identify genuine spider gene orthologs a blast search was performed using a translated nucleotide query of the gene of interest from Drosophila melanogaster (usually the canonical isoform from uniprot (Consortium, 2015)) in the two spider transcriptomes of either P. phalangioides (Janssen et al., 2015) or P. tepidariorum (Posnien et al., 2014) using Geneious ${ }^{\circledR}$ (version 8.1.5). The best five transcriptome hits were selected as long as their e-value was below e-9. The largest open reading frame was translated into protein sequences. Afterwards a protein blast (http://blast.ncbi.nlm.nih.gov) against the non-redundant protein sequence database including the following organisms and taxa (Drosophila melanogaster (taxid:7227), Mus musculus (taxid:10090), Danio rerio (taxid:7955), Onychophora (taxid:27563), Chelicerata (taxid:6843), Tribolium castaneum (taxid:7070), Apis mellifera (taxid:7460), Anopheles gambiae (taxid:7165)) was performed to identify further homologues. The best fifty hits from the non-redundant protein sequence database with an e-value below e-15 (whereas isoforms of one gene and redundant predicted genes were discarded) were then used to generate a protein alignment with default settings using Clustal Omega (http://www.ebi.ac.uk/Tools/msa/clustalo/ (Sievers et al., 2011)). Based on this Clustal protein alignment a phylogenetic tree was calculated using the parallel version of MRBAYES ((version 3.2.5) (Ronquist and Huelsenbeck, 2003)). After testing mixed amino acid substitution models the correct model was chosen to generate topological convergence using Metropolis coupling for 
maximum 3.000.000 generations or after reaching an average standard deviation of split frequencies below 0.01 . The resulting $50 \%$ majority rule consensus tree was visualized with Geneious ${ }^{\circledR}$ (version 8.1.5). Genuine spider homologs were identified forming a monophyletic group with the candidate gene homologs from other species. Unless stated otherwise the identified $P$. tepidariorum paralogs were named according to their similarity to the D. melanogaster protein, so that the paralog with lower e-value was referred to as first (e.g. clawless1). The P. phalangioides paralogs were named according to their homologs in P. tepidariorum identified with the phylogenetic tree (see Table 1). After this identification of the spider homologs an additional phylogenetic tree was generated for some genes to investigate the phylogenetic relationships between the chelicerate paralogs in more detail. These phylogenetic trees were generated with a subset of homologs for chosen species and calculated in the same way as described above.

\subsection{Molecular methods}

\subsubsection{RNA isolation}

Total RNA for cDNA synthesis was isolated from a combination of different embryonic stages of either P. phalangioides or P. tepidariorum with TRIzol ${ }^{\circledR}$ (Life Technologies, Thermo Fisher Scientific, Carlsbad, CA, USA) according to the manufacturer's instructions. Total RNA extraction for library preparation of Illumina sequencing of $P$. phalangioides was previously described in Janssen et al., 2015.

\subsection{1 cDNA synthesis}

After RNA isolation cDNA was generated with either the SMARTerTM PCR cDNA Synthesis Kit or the SMARTerTM RACE cDNA Synthesis Kit (Clontech, Mountain View, CA, USA). cDNA synthesis of specific stages, organs or tissues, which were needed in lower amounts was performed with the Maxima ${ }^{\circledR}$ First Strand cDNA Synthesis Kit (Life Technologies, Thermo Fisher Scientific, Carlsbad, CA, USA). 
Table 1: Spider orthologs

\begin{tabular}{|c|c|c|c|c|}
\hline Organism & Gene name & Transcript ID & e-value & \\
\hline$P t$ & apterous1 & Locus_4700_Transcript_14/16 & $2.26 \mathrm{e}^{-38}$ & \\
\hline$P p$ & apterous2.1 & comp120245 & $7.35 \mathrm{e}^{-45}$ & not cloned \\
\hline Pt & apterous2.1 & Locus_11195_ & $8.31 e^{-43}$ & not cloned \\
\hline$P t$ & apterous 2.2 & Locus_1_Transcript_14384/166847 & $3.28 \mathrm{e}^{-23}$ & not cloned \\
\hline$P p$ & apterous1 & comp117589 & $4.98 \mathrm{e}^{-40}$ & \\
\hline$P p$ & apterous2.2 & comp118341 & $1.17 e^{-41}$ & \\
\hline$P p$ & aristaless & comp123836 & & \\
\hline$P t$ & aristaless & Locus_18982_Transcript_4/4 & & \\
\hline$P p$ & BarH1 & comp116578_c0_seq1 & $5.00 e^{-44}$ & bad probe \\
\hline$P p$ & BarH2 & comp120407_c0_seq1 & $8.14 \mathrm{e}^{-33}$ & bad probe \\
\hline Pt & $\mathrm{BarH2}$ & Locus_1_Transcript_157093/166847 & $2.31 \mathrm{e}^{-30}$ & \\
\hline$P t$ & BarH1 & Locus_3013_Transcript_10/10 & $6.74 \mathrm{e}^{-43}$ & \\
\hline$P p$ & clawless1 & comp116449 & $9.68 \mathrm{e}^{-49}$ & \\
\hline Pt & clawless1 & Locus_15589_Transcript_1/1 & $4.69 e^{-53}$ & \\
\hline$P p$ & clawless2 & comp121099 & $3.21 \mathrm{e}^{-48}$ & \\
\hline$P t$ & clawless2 & Locus_21582_Transcript_1/1 & $9.04 \mathrm{e}^{-52}$ & \\
\hline$P p$ & $\operatorname{Lim} 1.2$ & comp121897 & $6.45 e^{-58}$ & \\
\hline Pt & $\operatorname{Lim} 1$ & Locus_1_Transcript_73444/166847 & $5.70 e^{-64}$ & \\
\hline$P p$ & $\operatorname{Lim} 1$ & comp125826 & $1.43 e^{-94}$ & \\
\hline Pt & $\operatorname{Lim} 1.2$ & Locus_2322_Transcript_1/1 & $1.23 e^{-47}$ & \\
\hline$P p$ & spineless1 & comp121950 & 0 & \\
\hline Pt & spineless1 & Locus_18705_Transcript_3/6 & 0 & \\
\hline$P p$ & spineless2 & comp125157 & 0 & \\
\hline$P t$ & spineless2 & Locus20228_Transcript_1/2 & $6.80 \mathrm{e}^{-152}$ & \\
\hline$P p$ & trachealess2 & comp119399 & $5.07 e^{-165}$ & \\
\hline$P p$ & trachealess1 & comp128285 & $1.08 \mathrm{e}^{-163}$ & \\
\hline Pt & trachealess2 & Locus_17705_Transcript_7/7 & $4.43 e^{-158}$ & \\
\hline$P t$ & trachealess1 & Locus_1_Transcript_160439/166847 & $1.02 \mathrm{e}^{-168}$ & \\
\hline
\end{tabular}

\subsubsection{PCR}

The strategies to amplify gene fragments from cDNA differed depending on the availability of transcriptomic resources. When no transcriptome was available first small fragments of the gene of interest were amplified with degenerated primers, usually in combination with nested PCR. Subsequently, additional sequence information was obtained by RACE (Rapid Amplification of cDNA Ends) PCR with primers designed depending on these small fragments. All these PCR types were performed with the Advantage ${ }^{\circledR} 2$ Polymerase Mix (Clontech, Mountain View, CA, USA), according to the 
manufacturer's recommendations. For genes identified from the transcriptomes of either P. phalangioides or P. tepidariorum primers were designed with help of Primer3 (Untergasser et al., 2012) and gene fragments were amplified by standard PCR using one of the following thermo stable DNA-polymerases in the given order: Taq DNA Polymerase (New England Biolabs, Ipswich, MA, USA or Life Technologies, Thermo

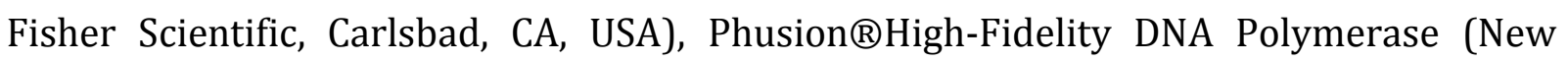
England Biolabs, Ipswich, MA, USA) or Advantage ${ }^{\circledR} 2$ Polymerase Mix (Clontech, Mountain View, CA, USA). Standard PCRs were performed with standard primers and the Phusion ${ }^{\circledR}$ High-Fidelity DNA Polymerase to provide sufficient amounts of template DNA from the cloned gene fragments prior to in vitro transcription of probes or double stranded RNA (dsRNA).

\subsubsection{Standard oligonucleotides}

Table 2: Standard oligonucleotides

\begin{tabular}{|l|l|}
\hline Name & Sequence 5' $^{\prime}$ 3' $^{\prime}$ \\
\hline Topo-1 & TCG GATC CAC TAG TAA CG \\
\hline Topo-2 & GTG TGA TGG ATA TCT GC \\
\hline T7-Sp6 & GTA ATA CGA CTC ACT ATA GGG ATT TAG GTG ACA CTA TAG AAT ACT CAA GC \\
\hline T7 & GTA ATA CGA CTC ACT ATA GGG CGA AT \\
\hline M13 forward & GTA AAA CGA CGG CCA G \\
\hline M13 reverse & CAG GAA ACA GCT ATG AC \\
\hline
\end{tabular}

\subsubsection{RACE-PCR oligonucleotides}

Table 3: Oligonucleotides for RACE-PCR

\begin{tabular}{|l|l|}
\hline Name & Sequence 5' - 3' \\
\hline Pp_exd_1_3'RACE_new_Nt251 & GCG GCC GGT GGC CCC AAT CAG C \\
\hline Pp_exd_1_5'RACE Nt249 & TGT CTG ATT TGG GCC AGT TTC GCC CGG \\
\hline Pp_exd_2_3'RACE_Nt310 & CGA AGG GGT TGC AGG ACC CGA GAA AG \\
\hline Pp_hth1_3'RACE_new_Nt374 & CAG GGA CTG CGA CGG GGG CCT C \\
\hline Pp_hth1_5'RACE_new_Nt 267 & GGT GGT GGC GCC GGT GGT GGA AG \\
\hline Pp-En-3RACE: & CGG CGA CAG GAT TTG GCC CG \\
\hline Pp-En-5RACE & CGG GCC AAA TCC TGT CGC CG \\
\hline UPM & Combination of long[2 $\mu$ M] and short [10 $\mu$ M] \\
\hline UPM long T3 & ATT AAC CCT CAC TAA AGG GAA AGC AGT GGT ATC AAC GCA GAG T \\
\hline UPM short T3 & ATT AAC CCT CAC TAA AGG GA \\
\hline
\end{tabular}




\subsubsection{Gene specific PCR oligonucleotides}

Table 4: Gene specific PCR oligonucleotides

\begin{tabular}{|c|c|}
\hline Name & Sequence $5^{\prime}-3^{\prime}$ \\
\hline Pp_al_for & CCG GTT CTC TCC TTC GAT ATT GTT GTC TC \\
\hline Pp_al_rev & TCA ACT GAC TTC GCC GTT GGA CTT CCT C \\
\hline Pp_apt_2.2_comp118341_f & TCG GAT GAC CAG TGC CAA GAC CTG \\
\hline Pp_apt_2.2_comp118341_r & TCG CTC TTT GTC AGA ACA TCT CGT GGA \\
\hline Pp_apt1_for & ACC GTT ACG TGA CAA CAG TGA GGC GG \\
\hline Pp_apt1_rev & AGC ACC CAT TGT CCC CGA ACC GCT AA \\
\hline Pp_c102331_for & CTT CCT TCG AGC GGC TCT TC \\
\hline Pp_c102331_rev & CTG AAC TCT TTG CCG CAG GA \\
\hline Pp_c105514_g1_i1_for & CGA CAG CCG TCC AAG CTA TC \\
\hline Pp_c105514_g1_i1_rev & CAG GGG TCA TTG TCG TCT CG \\
\hline Pp_c109329_for & GCC ACG ATC TCA ACC ACC AC \\
\hline Pp_c109329_rev & TGC GCT ACC TGT TCG ACC TC \\
\hline Pp_c109624_for & GAC GCT GAG TCC GAT CCA GA \\
\hline Pp_c109624_rev & CTC CGG GTC TTT CGT CGA GT \\
\hline Pp_c109662_for & CGG TGA ACC GTC TTC CAG TG \\
\hline Pp_c109662_rev & GTC TCG GGA CCG TCC TCT GT \\
\hline Pp_c95092_g2_i1_for & CTG GTC TAG TCG CCG CAG TC \\
\hline Pp_c95092_g2_i1_rev & TCC ACG TCC GCA GTC TTT GT \\
\hline Pp_cll_1_comp116449_for & CAT GGA CCA CGG TGA TAC GGA CGA \\
\hline Pp_cll_1_comp116449_rev & CCT CCT CCA TTT CGT CCG CCT GTT \\
\hline Pp_cll_2_comp121099_f & GGA ATC AAT TCC CCG CAA CCG GAC \\
\hline Pp_cll_2_comp121099_r & GTC AGA GAT GTC GGT GGT TGC AGG \\
\hline Pp_hth2_for & CGG TTA TCG GGT GGA CTT CGG \\
\hline Pp_hth2_rev & GGT CCA TGA TGT TCG GAG GCG AA \\
\hline Pp_Lim1_comp121897_f & GGA GGC GTA AGA GGA AGC GGA GG \\
\hline Pp_Lim1_comp121897_r & CAC CGA GGG CAC CGG GGA AC \\
\hline Pp_ss1_for & GGA ACA GCA AAA CCG TCC GTG TCC CG \\
\hline Pp_ss1_rev & TGT AGC GGC GCG TTC TCT GAC ACC TT \\
\hline Pp_ss2_comp125157_f & CGT CGG TGG CTG CCT TTC TAT GTA C \\
\hline Pp_ss2_comp125157_r & CCG ATA TCG ACA GCA GGG GAT TAC C \\
\hline Pp_trh1_comp128285_for & GCG GCA GCA GAA TCG GGC CTA TCC \\
\hline Pp_trh1_comp128285_rev & CCT CAT TAT CAG GCG TTG ATC CAC GC \\
\hline Pp_trh2_comp_119399_for & ATG CAG CCT GAT ACT TAT GGA TCG TTA CC \\
\hline Pp_trh2_comp_119399_rev & GCT CTG TAT GTA ATG GCC GTT ATC CAA GG \\
\hline
\end{tabular}




\begin{tabular}{|c|c|}
\hline Pt_al_for & GCC TCC AAT CCC TAC ACG TAA CAG G \\
\hline Pt_al_rev & GCT TCC CAA AAC ACC ACG CAC \\
\hline Pt_apterous_1_for & GGA GAT ATC GCA GTT GGG TGT CCC \\
\hline Pt_apterous_1_rev & GTG ATG CGA GCC AGA GAA CAC G \\
\hline Pt_Bar_Locus_1_157093_for & GAG ATA GTA GCC CTT CAC TGA AGA ACA \\
\hline Pt_Bar_Locus_1_157093_rev & GAC ACG CAC GAA TGT CCA GTT GC \\
\hline Pt_Bar_Locus_3013_for & CGA TCA TTC TGG TCT GGA AGA AAC AAA C \\
\hline Pt_Bar_Locus_3013_rev & CCT GCG CCA TAG ACT AGT AGT CAG CC \\
\hline Pt_cll_f_L15589 & GCTC TGT GAT CGT ACT GCT CGC GT \\
\hline Pt_cll_r_L15589 & GGT GAA GAT AAA GAG TGT GGA GGT GC \\
\hline Pt_cll2_for & AAC AAT TCG TCG CCG CTA GTT G \\
\hline Pt_cll2_rev & GGT TGT AGA TTT TGA AGT GCG TGC \\
\hline Pt_Lim_1.2_Locus2322_f & CGA CCC AGT CCT CTT GCC TCT TGA \\
\hline Pt_Lim_1.2_Locus2322_r & TCG TGG TGG TCC GTA AGC GTC TGA \\
\hline Pt_Lim1_for & GGT GGC TGT TCC CAA GGC ATA TGT \\
\hline Pt_Lim1_rev & CGA ATG TTC GAG AGT ATT ATT TCC TCC \\
\hline Pt_odds_f_L17047 & GGA AGT TGA GCG GCG ACG TAC C \\
\hline Pt_odds_r_L17047 & CCA GGG TCT TGA TCT CCA CTC TCC \\
\hline Pt_spineless_1_for & GAG AAC TCC GCG CAG TAG CAT GT \\
\hline Pt_spineless_1_rev & ATC TCT TTG TCC TCT GGC AGC GG \\
\hline Pt_ss2_Locus_20228_f & CGT GTC CCT CCA AAA GAT GGC CAA AC \\
\hline Pt_ss2_Locus_20228_r & CCA AGC AAA TCC CTT CCT TCT TCT TCC \\
\hline Pt_trh1_for & CCT GTC AAC TCA TCG GCT GTG TAG \\
\hline Pt_trh1_rev & CCT GTT GAT CTG TCC GGT CAT TAT GC \\
\hline Pt_trh2_for & GC AAC CTG AAG GAT ATG GGT CCA TAC \\
\hline Pt_trh2_rev & GGT TTC TTC TTC GGC ATT CTT GGT GTT \\
\hline
\end{tabular}

\subsubsection{Cloning, transformation and plasmid preparation}

After gel-electrophoresis, fragments of interest were extracted from the gel with the help of the NucleoSpin ${ }^{\circledR} G e l$ and PCR Clean-up (MACHEREY-NAGEL, Düren, Germany) or MinElute Gel Extraction Kit (Qiagen, Hilden, Germany). Gene fragments amplified with either Taq DNA Polymerase or Advantage ${ }^{\circledR} 2$ Polymerase Mix were cloned into the pCR®II vector using the TA Cloning® Kit Dual Promoter (Life Technologies, Thermo Fisher Scientific, Carlsbad, CA, USA). Gene fragments amplified with Phusion ${ }^{\circledR} H i g h-$ Fidelity DNA Polymerase were cloned blunt end into a modified pJet2.1 vector (generated by Stefan Dippel, Department Developmental Biology, Georg-August- 
University Göttingen) using a standard T4 Ligase (5 Weiss units/ $\mu \mathrm{l}$, New England Biolabs, Ipswich, MA, USA or Life Technologies, Thermo Fisher Scientific, Carlsbad, CA, USA). Chemically competent Escherichia coli $\mathrm{dH} 5 \alpha$ or $\mathrm{dH} 10 \mathrm{~b}$ bacteria were transformed with the vectors and positive clones were selected for sequencing and further experiments using blue-white selection after incubation on LB-Agar plates containing $40 \mu \mathrm{l} \mathrm{X-Gal} \mathrm{(4 \%} \mathrm{in} \mathrm{DMF).} \mathrm{Plasmid} \mathrm{DNA} \mathrm{was} \mathrm{isolated} \mathrm{as} \mathrm{with} \mathrm{a} \mathrm{modified} \mathrm{alkaline} \mathrm{cell} \mathrm{lysis}$ protocol (Pechmann, 2011), or with one of the following kits: Zyppy ${ }^{\mathrm{TM}}$ Plasmid Miniprep Kit (Zymo Research Europe, Freiburg, Germany) or NucleoSpin®Plasmid Miniprep Kit (Macharey-Nagel, Düren, Germany). Clones were further tested by restriction digest with either EcoRV or EcoRI (New England Biolabs, Ipswich, MA, USA), in case of pJet2.1 or pCRßII vector, respectively. Clones with fragments of the expected size after digestions were send for sequencing (Macrogen Europe, Amsterdam, Netherlands or LGC Genomics, Berlin, Germany).

\subsection{1 in vitro transcription of RNA probes and dsRNA for RNAi}

After amplification by standard PCR approximately 500ng template DNA were used for in vitro transcription to synthesize dsRNA or Digoxygenin-labeled RNA probes from the cloned gene fragments. Synthesis of the RNA probes was performed according to standard protocols (Prpic et al., 2008a). Kits and precipitation strategy for dsRNA synthesis were previously described in Turetzek et al., 2016.

\subsection{Embryological methods}

\subsubsection{Embryo fixation and cuticle preparations}

Fixation of $P$. tepidariorum embryos after reaching germ band elongation stages was performed according to standard protocols described in pervious publications (Schomburg et al., 2015). Fixation of early embryonic stages of $P$. tepidariorum and $P$. phalangioides embryos were performed according to the fixation protocol previously published for $C$. salei (Prpic et al., 2008b). Cuticle preparations of nymphal stages of $P$. tepidariorum after RNAi experiments were performed with a mixture of lactic acid and Hoyer's medium (1:1) as previously described in Turetzek et. al, 2016. 
Table 5: List of gene clones used in this study

\begin{tabular}{|c|c|c|c|c|c|}
\hline Organism & Gene name & Transcript ID & $\begin{array}{l}\text { Accession } \\
\text { number }\end{array}$ & Clone & Reference \\
\hline $\mathrm{Pp}$ & apterous1 & comp117589 & & own cloning & this work, Appendix \\
\hline $\mathrm{Pp}$ & apterous 2.2 & comp118341 & & $\begin{array}{l}\text { own cloning, } \\
\text { with help of Julia } \\
\text { Schneider }\end{array}$ & this work, Appendix \\
\hline $\mathrm{Pp}$ & aristaless & comp123836 & & own cloning & this work, Appendix \\
\hline $\mathrm{Pp}$ & c102331 & c102331 & & own cloning & this work, Appendix \\
\hline $\mathrm{Pp}$ & c105514 & c105514_g1_i1 & & own cloning & this work, Appendix \\
\hline $\mathrm{Pp}$ & c109329 & c109329 & & own cloning & this work, Appendix \\
\hline $\mathrm{Pp}$ & c109624 & c109624 & & own cloning & this work, Appendix \\
\hline $\mathrm{Pp}$ & $c 109662$ & c109662 & & own cloning & this work, Appendix \\
\hline $\mathrm{Pp}$ & c95092 & c95092_g2_i1 & & own cloning & this work, Appendix \\
\hline $\mathrm{Pp}$ & clawless1 & comp116449 & & $\begin{array}{l}\text { own cloning, } \\
\text { with help of Jana } \\
\text { Holtzheimer }\end{array}$ & this work, Appendix \\
\hline $\mathrm{Pp}$ & clawless2 & comp121099 & & own cloning & this work, Appendix \\
\hline $\mathrm{Pp}$ & dachshund1 & & CUC08949.1 & $\begin{array}{l}\text { Dr. Matthias } \\
\text { Pechmann }\end{array}$ & (Turetzek et al.,2016) \\
\hline $\mathrm{Pp}$ & dachshund2 & & CUC08949.1 & $\begin{array}{l}\text { Dr. Matthias } \\
\text { Pechmann }\end{array}$ & (Turetzek et al., 2016) \\
\hline $\mathrm{Pp}$ & engrailed & & & own cloning & $\begin{array}{l}\text { (Turetzek, } \\
\text { 2011),Appendix }\end{array}$ \\
\hline $\mathrm{Pp}$ & extradenticle1 & comp102492 & & own cloning & this work, Appendix \\
\hline $\mathrm{Pp}$ & extradenticle2 & comp122137 & & own cloning & this work, Appendix \\
\hline $\mathrm{Pp}$ & homothorax1 & comp102262 & & own cloning & this work, Appendix \\
\hline $\mathrm{Pp}$ & homothorax2 & comp120146 & & own cloning & this work, Appendix \\
\hline $\mathrm{Pp}$ & $\operatorname{Lim} 1.2$ & comp121897 & & own cloning & this work, Appendix \\
\hline $\mathrm{Pp}$ & spineless1 & comp121950 & & own cloning & this work, Appendix \\
\hline $\mathrm{Pp}$ & spineless2 & comp125157 & & own cloning & this work, Appendix \\
\hline $\mathrm{Pp}$ & trachealess1 & comp128285 & & own cloning & this work, Appendix \\
\hline $\mathrm{Pp}$ & trachealess2 & comp119399 & & own cloning & this work, Appendix \\
\hline $\mathrm{Pt}$ & apterous1 & $\begin{array}{l}\text { Locus_4700_Trans } \\
\text { cript_14/16 }\end{array}$ & & own cloning & this work, Appendix \\
\hline Pt & aristaless & $\begin{array}{l}\text { Locus_18982_Tran } \\
\text { script_4/4 }\end{array}$ & & own cloning & this work, Appendix \\
\hline $\mathrm{Pt}$ & BarH1 & $\begin{array}{l}\text { Locus_3013_Trans } \\
\text { cript_10/10 }\end{array}$ & & own cloning & this work, Appendix \\
\hline $\mathrm{Pt}$ & $\mathrm{BarH2}$ & $\begin{array}{l}\text { Locus_1_Transcrip } \\
\text { t_157093/166847 }\end{array}$ & & own cloning & this work, Appendix \\
\hline $\mathrm{Pt}$ & clawless1 & $\begin{array}{l}\text { Locus_15589_Tran } \\
\text { script_1/1 }\end{array}$ & & own cloning & this work, Appendix \\
\hline Pt & clawless2 & $\begin{array}{l}\text { Locus_21582_Tran } \\
\text { script_1/1 }\end{array}$ & & $\begin{array}{l}\text { Christoph } \\
\text { Schomburg and } \\
\text { Jana Holtzheimer }\end{array}$ & this work, Appendix \\
\hline $\mathrm{Pt}$ & dachshund1 & & FM945397.1 & $\begin{array}{l}\text { Dr. Matthias } \\
\text { Pechmann }\end{array}$ & $\begin{array}{l}\text { (Pechmann et al. } \\
\text { 2009) }\end{array}$ \\
\hline $\mathrm{Pt}$ & dachshund2 & $\begin{array}{l}\text { Locus_18128_Tran } \\
\text { script_3/3 }\end{array}$ & AKM21240.2 & own cloning & this work, Appendix \\
\hline Pt & Distal-less & & CAS89759.2 & $\begin{array}{l}\text { Dr. Matthias } \\
\text { Pechmann }\end{array}$ & $\begin{array}{l}\text { (Pechmann et al. } \\
\text { 2009) }\end{array}$ \\
\hline
\end{tabular}




\begin{tabular}{|c|c|c|c|c|c|}
\hline $\mathrm{Pt}$ & extradenticle1 & $\begin{array}{l}\text { Locus_1_Transcrip } \\
\text { t_58666/166847 }\end{array}$ & HE608681 & own cloning & (Khadjeh et al., 2012) \\
\hline $\mathrm{Pt}$ & extradenticle 2 & $\begin{array}{l}\text { Locus_1_Transcrip } \\
\text { t_58675/166847 }\end{array}$ & & Dr. Sara Khadjeh & this work, Appendix \\
\hline $\mathrm{Pt}$ & homothorax1 & $\begin{array}{l}\text { Locus_81_Transcri } \\
\text { pt_57/63 }\end{array}$ & HE608682 & own cloning & (Khadjeh et al., 2012) \\
\hline $\mathrm{Pt}$ & homothorax2 & $\begin{array}{l}\text { Locus_10999_Tran } \\
\text { script_20/21 }\end{array}$ & & Dr. Sara Khadjeh & this work, Appendix \\
\hline $\mathrm{Pt}$ & Lim1 & $\begin{array}{l}\text { Locus_1_Transcrip } \\
\text { t_73444/166847 }\end{array}$ & & own cloning & this work, Appendix \\
\hline $\mathrm{Pt}$ & $\operatorname{Lim} 1.2$ & $\begin{array}{l}\text { Locus_2322_Trans } \\
\text { cript_1/1 }\end{array}$ & & $\begin{array}{l}\text { own cloning, } \\
\text { with help of } \\
\text { David Salamanca }\end{array}$ & this work, Appendix \\
\hline Pt & odd-skipped & $\begin{array}{l}\text { Locus_17047_Tran } \\
\text { script_1/1 }\end{array}$ & & own cloning & this work, Appendix \\
\hline Pt & odd-skipped & $\begin{array}{l}\text { Locus_13444_Tran } \\
\text { script_9/13 }\end{array}$ & & own cloning & this work, Appendix \\
\hline $\mathrm{Pt}$ & orthodenticle & & AB096074 & $\begin{array}{l}\text { Dr. Matthias } \\
\text { Pechmann }\end{array}$ & (Akiyama-Oda, 2003) \\
\hline $\mathrm{Pt}$ & $\begin{array}{l}\text { Sex combs } \\
\text { reduced }\end{array}$ & & FM956097 & Dr. Sara Khadjeh & $\begin{array}{l}\text { (Schwager et al., } \\
\text { 2007), sequence } \\
\text { extended by Sara } \\
\text { Khadjeh } \\
\end{array}$ \\
\hline $\mathrm{Pt}$ & spineless1 & $\begin{array}{l}\text { Locus_18705_Tran } \\
\text { script_3/6 }\end{array}$ & & own cloning & this work, Appendix \\
\hline $\mathrm{Pt}$ & spineless2 & $\begin{array}{l}\text { Locus20228_Trans } \\
\text { cript_1/2 }\end{array}$ & & own cloning & this work, Appendix \\
\hline $\mathrm{Pt}$ & trachealess1 & $\begin{array}{l}\text { Locus_1_160439/1 } \\
66847\end{array}$ & & own cloning & this work, Appendix \\
\hline $\mathrm{Pt}$ & trachealess2 & $\begin{array}{l}\text { Locus_17705_Tran } \\
\text { script_7/7 }\end{array}$ & & own cloning & this work, Appendix \\
\hline
\end{tabular}

\subsubsection{Whole mount in situ hybridization}

In situ hybridization of embryos was performed according to standard methods with minor modification (Schomburg et al., 2015). After in situ hybridization and SYTOXGreen (Life Technologies, Thermo Fisher Scientific, Carlsbad, CA, USA) staining whole embryos were imaged with a Leica M205 FA binocular equipped with a QImaging Micropublisher 5.0 RTV camera using combined UV and white light. When necessary appendages were dissected and mounted in $80 \%$ Glycerol in PBST. Images of dissected tissues were taken with a Zeiss Axioplan-2 microscope using an Intas digital camera. For colour and brightness correction of the captured images Adobe Photoshop CS5 Extended or CS6 for Apple Macintosh was used. Staging of embryos for all spiders was chosen on the basis of the staging of P. tepidariorum (Mittmann and Wolff, 2012). 


\subsubsection{Live imaging of embryonic development of $\boldsymbol{P}$. phalangoides}

The time-lapse movies of the embryonic development of P. phalangioides were captured with a Leica M205 FA binocular equipped with a QImaging Micropublisher 5.0 RTV camera. The embryos were covered with Voltalef H10S oil (Arkema) and images were taken in intervals of ten minutes. The single images were combined into a movie using iMovie version 10.0.5 (Apple Macintosh), in a way that every image is shown 0.05 seconds (time-lapse movies are attached as CD-ROM).

\subsubsection{Parental RNAi}

For parental RNAi dsRNA of the cloned gene fragments was injected into sexually mature adult female spiders. The synthesis and precipitation of dsRNA, as well as the injection and statistical analysis of Pt-dachshund1 were performed as described in Turetzek et. al, 2016. For successful RNAi the injection procedure and time point of mating differs for every gene and is described in the following table (Table 6). For all genes at least 3.5$4 \mu \mathrm{g} / \mu \mathrm{l}$ ds RNA were injected into sexually mature female spiders. The first five cocoons produced by injected females were opened and usually half of the embryos were fixed for further experiments. The remaining half was allowed to develop until hatching of the first nymphal stage and screened for any visible phenotypes. Unless stated otherwise only offspring from the females that survived and produced offspring showing a phenotype in at least one cocoon were included in the statistical analysis. That the offspring of some females did not show any phenotype although the females were injected in the same way, with the same batch of dsRNA and treated exactly in the same manner can have multiple reasons. Although the females were fed and mated equally from beginning of the experiment, each individual female might be in a different condition, caused by mite infection, feeding status during the juvenile period and other environmental stress factors. Furthermore it can happen that the injected liquid is pushed out of the injection hole or through the mouth, which cannot be completely avoided even when injected very carefully. Thus the amount of dsRNA might simply not be sufficient to cause an effect. In addition the exact RNAi mechanism in the spider is not yet known and here individual differences and the position of injection might have an 
effect as well. Freshly hatched nymphs showing phenotypes were embedded in Voltalef H10S oil (Arkema, Colombes, France) and boiled for at least one minute. Z-stack images of these nymphs were captured using the confocal laser-scanning microscope (LSM 510, Carl Zeiss, Jena, Germany).

Table 6: dsRNA injection procedure for RNAi

\begin{tabular}{|c|c|c|c|c|c|c|c|c|}
\hline Gene & $\begin{array}{c}\# \\
\text { Females }\end{array}$ & $\begin{array}{c}\# \\
\text { Injections }\end{array}$ & $\begin{array}{l}\text { Day for } \\
\text { recovery }\end{array}$ & $\begin{array}{c}\text { Volume of } \\
\text { dsRNA }\end{array}$ & Mating & $\begin{array}{c}\# \\
\text { Phenotypes }\end{array}$ & $\begin{array}{c}\text { \# Females } \\
\text { died }\end{array}$ & $\begin{array}{l}\text { Included in } \\
\text { statistic }\end{array}$ \\
\hline BarH1 & 10 & 5 & 1 & 2,0 & $\begin{array}{l}\text { after 3rd } \\
\text { injection }\end{array}$ & 4 & 4 & 4 \\
\hline BarH2 & 5 & 5 & 1 & 2,0 & $\begin{array}{l}\text { after } 3 r d \\
\text { injection }\end{array}$ & 0 & 0 & \\
\hline cll1 & 5 & 5 & 1 & 2,5 & $\begin{array}{l}\text { after 3rd } \\
\text { injection }\end{array}$ & 3 & 1 & 3 \\
\hline cll1 & 5 & 3 & 0 & 2,5 & $\begin{array}{l}\text { one day } \\
\text { before } \\
\text { injection }\end{array}$ & 4 & 1 & 4 \\
\hline cll1 & 3 & 5 & 1 & 2,5 & $\begin{array}{l}\text { after 3rd } \\
\text { injection }\end{array}$ & 1 & 0 & $\begin{array}{l}\text { excluded from } \\
\text { statistic }\end{array}$ \\
\hline dac2 & 3 & 5 & 1 & 2,5 & $\begin{array}{l}\text { after 3rd } \\
\text { injection }\end{array}$ & 0 & 0 & \\
\hline exd1 & 3 & 3 & 1 & 2,0 & $\begin{array}{l}\text { after 3rd } \\
\text { injection }\end{array}$ & 0 & 1 & \\
\hline exd1 & 3 & 3 & 1 & 3,5 & $\begin{array}{c}\text { before } \\
\text { injection }\end{array}$ & 0 & 0 & \\
\hline exd2 & 3 & 3 & 1 & 2,0 & $\begin{array}{l}\text { after 3rd } \\
\text { injection }\end{array}$ & 0 & 2 & \\
\hline exd2 & 3 & 3 & 1 & 3,5 & $\begin{array}{c}\text { before } \\
\text { injection }\end{array}$ & 0 & 2 & \\
\hline$h$ th1 & 3 & 3 & 1 & 2,0 & $\begin{array}{l}\text { after 3rd } \\
\text { injection }\end{array}$ & 0 & 0 & \\
\hline$h t h 1$ & 3 & 3 & 1 & 3,5 & $\begin{array}{c}\text { before } \\
\text { injection }\end{array}$ & 0 & 1 & \\
\hline$h$ th2 & 3 & 3 & 1 & 2,0 & $\begin{array}{l}\text { after 3rd } \\
\text { injection }\end{array}$ & 0 & 1 & \\
\hline$h$ th2 & 3 & 3 & 1 & 3,5 & $\begin{array}{c}\text { before } \\
\text { injection }\end{array}$ & 0 & 1 & \\
\hline $\lim 1$ & 3 & 5 & 1 & 2,5 & $\begin{array}{l}\text { after 3rd } \\
\text { injection }\end{array}$ & 0 & 0 & \\
\hline ss1 & 5 & 5 & 1 & 2,0 & $\begin{array}{l}\text { after 3rd } \\
\text { injection }\end{array}$ & 0 & 0 & \\
\hline
\end{tabular}

\subsubsection{Phenotypic analysis of Pt-cll1 RNAi}

Freshly hatched nymphs injected with Pt-cll1 dsRNA were observed and categorized in the following manner: "wild type" no visible effect of the nymphal cuticle can be observed; "not hatched": embryos which either died during embryogenesis or were not able to hatch from the embryonic envelope; "weak" malformation or missing of the joint 
between the metatarsus and tarsus in at least one walking leg or pedipalp; "strong" missing of either the tarsus segment or the entire part of the appendage distal to the metatarsus in at least one walking leg or pedipalp.

In freshly hatched nymphs as well as after the second moulting into second instar nymphs the joints in the prosomal appendages are not fully developed, thus especially the strong phenotype was difficult to distinguish from wild types during these stages. Therefore most of these nymphs were allowed to moult once more into third instar nymphs. These show, in the wild type, fully developed joints and have bristles as well as sensory organs, which were not present in earlier stages. To ensure correct categorization of the phenotypes cuticle preparations of these third instar nymphs were performed in combination or instead of analysing freshly hatched nymphs. The overview shown in Figure 67 (left panel) shows the percentage of these categorized phenotypes from the different cocoons laid by seven injected females.

The cll1 phenotype was observed in two independent experiments and with two different injection procedures. The cll1 phenotype was initially observed in one female (marked in blue Table 6) after injection of three females. The phenotypes from these offspring were not included in the statistical analysis, because the effect was first observed in the forth cocoon and it was not clear if the strong phenotype was already present in previous cocoons and simply was not recognized in first nymphal stages. The statistic thus includes phenotypic observations in cocoon1-5 of seven females injected in two different ways (Figure 67).

As control five sexually mature female spiders were injected with injection buffer in the same way for both different injection procedures as described for Pt-cll1. Two of the control spiders, which were injected three days in a row, only produced cocoons with embryos that died shortly after egg laying. This might be an effect of the injection but is also observed for unfertilized embryos after unsuccessful mating. Because all cocoons showed the effect, which usually tapers of when caused by the injection itself, it is more likely that unsuccessful mating caused the effect. Thus these females and the produced cocoons were excluded from the following statistical analysis (shown in Figure 67, right panel). 


\subsubsection{Phenotypic analysis of Pt-BarH1 RNAi}

The Pt-BarH1 phenotypes were observed in two independent RNAi experiments (5 females in each experiment, from which each time two showed effects in the offspring). Unfortunately all control females injected with injection buffer died, thus the experiment needs to be repeated including a control.

After RNAi of Pt-BarH1 freshly hatched nymphs up to the seventh cocoon from the four females were analyzed (depicted in Figure 76). The category "wild type" is similar as explained for the Pt-cll1 RNAi. The category "not hatched", was further subdivided into "not hatched" and "dead". "not hatched" included all embryos, which finished ventral closure stages indicated by tightening of the embryonic membrane, which makes visible some embryonic structures like the prosomal appendages, but failed to open the egg membrane, which usually bursts above the tooth pic of the pedipalps. "dead" included the embryos, which died during the course of embryonic development, indicated by aggregation of the cells to one spot. In some cases it was needed to distinguish between these categories, then the unhatched embryos were covered with Voltalef H10S oil, which makes the vitelline membrane transparent. The only RNAi phenotype category present after moulting was the missing of the first walking leg segment and was therefore named "L1 missing".

Due to the high number of unhatched but fully developed embryos the fixated embryos for the second, third and fourth cocoon of all four females were analysed and included in the statistical analysis (Figure 77). For a better visualization the embryos were stained with Sytox-Green after fixation. In addition to the categories described above defects involved in segmentation of the embryo were found and described as follows: "head" missing of at least the head segments up to all anterior segments until the first walking leg; "chelicera/Pedipalps/L1": missing or fusion of either chelicera, pedipalps, one of the walking legs or combinations of these effects; "other defects" massive malformation of the embryo, including duplicated axis, duplicated opisthosoma or malformed opisthosoma. 


\subsection{RNA Seq, NGS and de novo Transcriptome assembly and analysis}

RNA isolation, library preparation and sequencing with Illumina HiSeq2000 of $P$. phalangioides samples were previously described in Janssen et al. 2015. In summary, triplicates of 4 different samples (a mixture of all embryonic stages as well as legs (excluding the coxa), prosoma and opisthosoma of freshly hatched first instar nymphs) were sequenced on one lane of an Illumina Hiseq2000 sequencer (Tanskriptom Analyse Labor, TAL, GZMB, Göttingen, Germany). This resulted in 472,770,758 paired-end reads of $100 \mathrm{bp}$ lengths each. The assembled transcriptome that was used in Janssen et al., 2015 represents only a preliminary version. To improve the $P$. phalangioides transcriptome all paired-end reads were de novo assembled with a newer version of the Trinity assembler, using the following settings: --seqType fq --JM 240G -run_as_paired --CPU 6 (version r20140717, (Haas et al., 2013)). Quality trimming and filtering of the raw reads was performed prior to the assembly with trimmomatic (Bolger et al., 2014) as part of the Trinity pipeline. Translated sequences of the entire improved transcriptome version were blasted against the common protein databases SwissProt ((Bairoch and Apweiler, 2000)) and Uniref90 (Suzek et al., 2007). In addition a protein blast search was performed against the gene set of the P. tepidariorum genome (unpublished data, courtesy of Alistair McGregor, co-ordinator of the P. tepidariorum sequencing consortium). The e-value cut-off was set to $5 \mathrm{e}^{-2}$ in all cases. This annotation was performed following the instructions of the freely available annotation pipeline Trinotate (Trinotate Release 2.0.2, https://trinotate.github.io, with kind help of Dr. Nico Posnien).

\subsubsection{Read mapping and reduction of transcriptome complexity}

For differential gene expression analysis it is necessary to map the raw reads of the different tissues against the combined transcriptome. This was performed using bowtie 2 (Version: 2.1.0, (Langmead and Salzberg, 2012)). First of all, the de novo assembled transcriptome was indexed as reference transcriptome. Then the raw reads of the triplicates from the four different samples (embryo, legs, prosoma and opisthosoma) were multi-mapped (100 alignments recorded) end-to-end against this indexed 
reference transcriptome, no mismatches allowed, using the following parameters: - no discordant --no mixed. The generated sam files were converted into bam files using Samtools (Li et al., 2009). Corset (version 1.0.3, (Davidson and Oshlack, 2014)) was used to reduce the number of transcripts prior to differential gene expression calling. Based on the bowtie2 alignments Corset clusters Trinity transcripts into potential gene models (Cluster). Subsequently the reads for each library and cluster were counted and can be used for downstream differential expression analysis. Corset was run in general with default settings and the information, which samples represent replicates, was defined by the command-group.

\subsubsection{Differential gene expression analysis with DESeq}

A principle component analysis was performed for the sample tissues and replicates based on counts from the Corset gene clusters and depicted with the plotMDS command in $\mathrm{R}$ (Version 0.98.1087, including the following packages: limma Version 3.22.1, RColorBrewer, (Neuwirth, 2011)). Afterwards, significantly up- and down regulated (padj < 0.05) corset gene clusters were identified on the basis of the library read counts generating four different comparisons: (1) Leg, prosoma and opisthosoma (all from freshly hatched nymphs) read counts were compared to embryonic read counts (referred to as $1^{\text {st }}$ instar larvae). (2) The leg read counts were compared to the opisthosoma and prosoma read counts (3) The prosoma read counts were compared to the leg and opisthosoma read counts. (4) The opisthosoma read counts were compared to the leg and prosoma read counts. This analysis was performed following the instructions of the R package DESeq2 (Version 1.6.2, (Anders and Huber, 2010)) from bioconductor (Gentleman et al., 2004). The GO-term enrichment analysis of the candidate genes up-regulated in the nymphal legs was performed by Dr. Nico Posnien with Blast2Go (Conesa et al., 2005). To reduce the number of candidate genes we filtered the candidates as follows. First, all candidates with a base mean lower than 10 were discarded, because these usually include gene clusters with very low read counts in every tissue. Then the candidates were sorted for the mean read counts in the three embryonic samples and all below 50 were rejected. This had three reasons: (1) Staining 
of gene expression in nymphal stages of $P$. phalangioides is not yet established. (2) The segmentation of the leg presumably already takes place during embryogenesis and (3) previous data revealed extreme growth of the walking legs during late embryonic development in P. phalangioides. After sorting for the log2Fold change the best 15 candidates were chosen and further studied. Finally all candidates without an annotation in any other organism were excluded. 


\section{Pholcus phalangioides: a haplogyne spider model}

A detailed study of the embryogenesis of a new model organism provides the groundwork to perform comparative studies prior to comparative expressional and functional studies. Comparisons of the general embryonic development of Parasteatoda tepidariorum (P. tepidariorum) and Pholcus phalangioides (P. phalangioides) will clarify if these spiders develop entirely different or if they share comparable tissue organization. Earlier studies already revealed major differences during germ band stages and opisthosomal organization in the embryonic development of the haplogyne spider $P$. phalangioides (Claparède, 1862; Pechmann et al., 2011), which I analyzed in more detail in this study.

The first part of this chapter "Observations on germ band development in the cellar spider Pholcus phalangioides" thus is the detailed description of the embryonic development of the haplogyne spider. It will be submitted as a manuscript to Development, genes and evolution. The data collection was already started during my master thesis and was finalized during this work. My supervisor and I performed the analysis of the data with equal contribution. The text of the manuscript was largely written or rewritten by my direct supervisor Dr. Prpic-Schäper.

In the era of Next generation sequencing (NGS) it is easier to sequence the transcriptome of a new species than facing the problems of fishing for genes in cDNA pools with degenerated primers to clone genes. Thus the second chapter will focus on the de novo transcriptome assembly for the haplogyne cellar spider P. phalangioides. In addition the transcriptomic resources were used for differential expressional analysis to identify genes fulfilling important roles during spider developmental processes. This project was performed in close collaboration and with supervision by Dr. Nico Posnien. 


\subsection{Observations on germ band development in the cellar spider Pholcus phalangioides}

Spider development involves, after cleavage and blastoderm formation, the formation of the so-called germ disc (Andersson 1973). The germ disc represents the anlage of the embryo proper, whereas the remaining cells that are not part of the disc will either degrade or form extra embryonic tissue or yolk. The germ disc is radially symmetric and therefore needs to undergo a transition from radial to bilateral symmetry to form the germ band and ultimately the bilaterally symmetric spider. This process of symmetry break at the transition from germ disc to germ band involves the migration of a group of cells, called the cumulus, from the center to the perimeter of the disc. A few genes that are required for this migration of the cumulus have been identified (Akiyama-Oda and Oda 2003; Akiyama-Oda and Oda 2006, Akiyama-Oda and Oda 2010), but the mechanisms of symmetry break are still only poorly understood.

The majority of recent studies of spider development focused on entelegyne spiders (e.g. Abzhanov and Kaufman 2000, Akiyama-Oda and Oda 2003, McGregor et al. 2008). Although this group of spiders includes the vast majority of all spider species (ca. 33000 species (i.e. over 80\%)) it is unclear whether the processes described on the basis of entelegyne spiders are also representative of the other, smaller spider groups. We have therefore initiated comparative studies in the haplogyne spiders which are phylogenetically the sister group of the entelegyne spiders, but comprise only about 3500 species worldwide. We have chosen the species Pholcus phalangioides as a very common and therefore easily accessible species. The majority of the species of the genus Pholcus are distributed in Asia, but a few species also have a western Palearctic distribution, and P. phalangioides is even a cosmopolitan species (Huber 2011). The species of Pholcus are characterized by their extremely long walking legs that make the animals superficially similar to long-legged species of harvestmen, and that have earned them their English nickname "daddy long leg spiders". So far, we have studied gene expression and function in appendage development in P. phalangioides (Pechmann et al. 2011; Turetzek et al. 2016). Here we focus on the description of germ band formation and further differentiation of the germ band. 


\subsubsection{Formation of the germ band in P. phalangioides}

The first sign of the transformation of the radially symmetric germ disc into the bilaterally symmetric germ band is the aggregation of cells at the center of the germ disc, thus forming the primary thickening (Figure $7, \mathrm{~A}$ ). At $25^{\circ} \mathrm{C}$ the primary thickening is visible approximately 48 hours after egg deposition (see Supplemental table 1). About 10 hours later a smaller cell mass, the so-called cumulus, separates from the primary thickening and migrates towards the rim of the germ disc (Figure 7, B). During migration the cumulus initially remains connected with the primary thickening via a thinner stalk of cells. As soon as the cumulus cell mass separates from the primary thickening, the cells of the primary thickening start dispersing across the entire germ disc. This leads to the dissolution of the primary thickening until it is barely visible anymore. However, at approximately 70 hours after egg deposition, the connection between the dissolving primary thickening and the migrating cumulus ruptures and at the same time the remaining cells of the primary thickening congregate again, thus forming the secondary agglomeration (Figure 7, C). At approximately 72 hours after egg deposition the cumulus cells have reached the rim of the germ disc and the cells of the secondary agglomeration have attained their maximum density (Figure 7, D). Now the secondary agglomeration starts dissolving, the cells segregate and form a posterior lobe, a new and enlarged structure, which will give rise to the posterior growth zone (also referred to as segment addition zone). This event marks the symmetry break that defines the posterior and anterior ends of the germ band and the formation of a bilaterally symmetric germ band is completed at approximately 82 hours after egg deposition, when the germ band shows the first morphological signs of segmentation (Figure 7, E). 

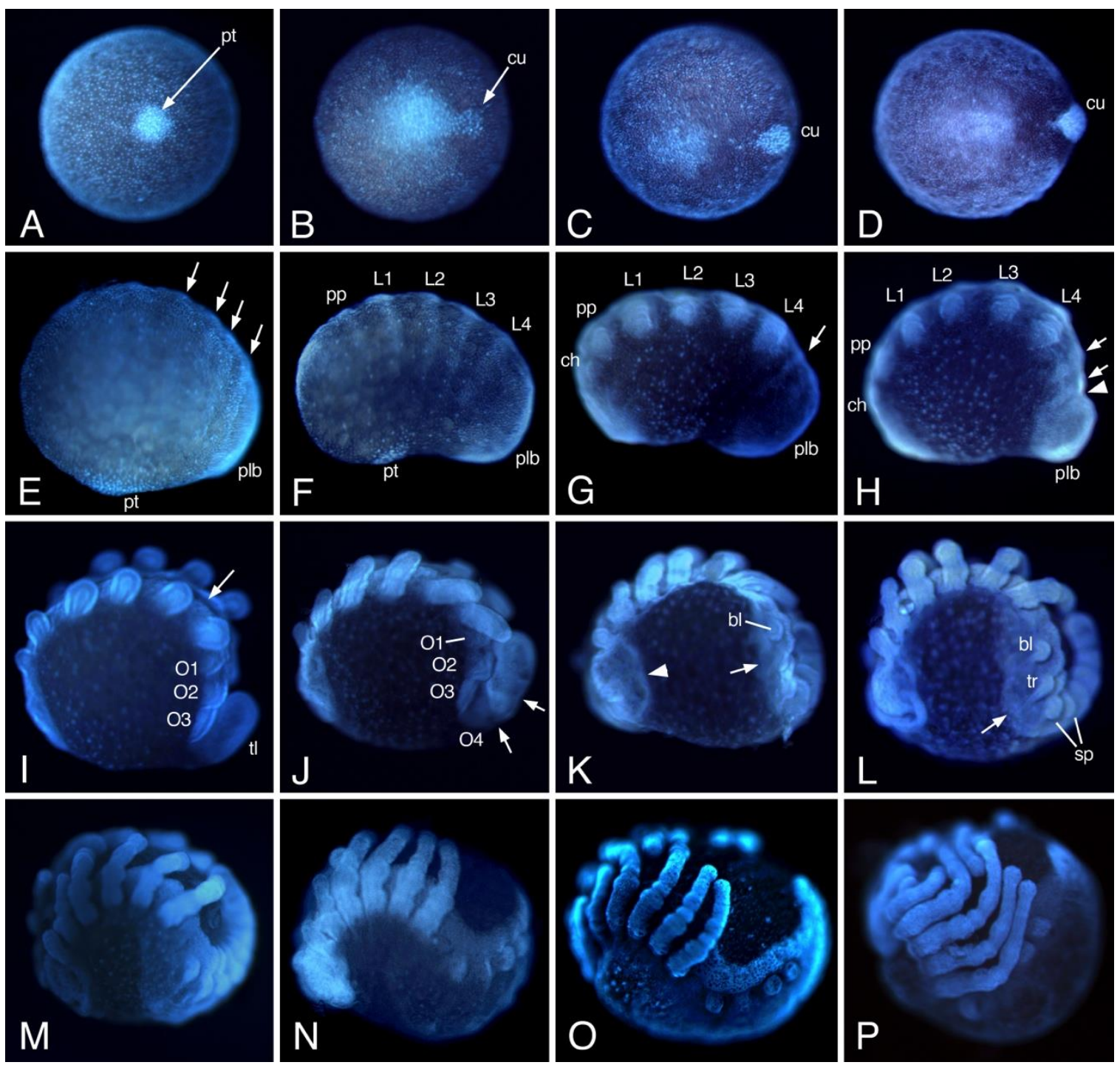

Figure 7: Consecutive stages of germ band formation and differentiation in P. phalangioides

A: Germ disc with the primary thickening of cells in its center. B: Cumulus cells separate from the primary thickening. C: The cumulus is fully separated and secondary aggregation has formed. D: Cumulus has reached the rim of the germ disc. E: Pear-shaped germ band has formed; segments are visible in the posterior part (arrows). F: Anterior segments are visible; embryo attains bean-shape. G: First opisthosomal segment is visible (arrow). H: Tail bud and prosomal limb buds form. More opisthosomal segments are present (arrows) or are in statu nascendi (arrowhead). I: Ventral sulcus forms (arrow); tailbud elongates into tail. J: Opisthosomal limb buds appear; tail segments are visible. K: Brain differentiation begins (arrow); dorsal tissue develops on the opisthosomal segments (arrowhead). L: Dorsal tissue grows further. M: Dorsal tissue overgrows the yolk (arrowhead); tail segments are sequentially folded down on the yolk (arrow). N: Dorsal tissue is almost closed (arrowhead); most opisthosomal segments are now folded down on the yolk (arrow). 0: Dorsal closure complete (arrowhead). P: Prosomal shield forms (arrowhead). For further details for each stage please see text. All embryos shown in E-P are shown in lateral view and oriented with anterior on the left. Abbreviations: bl, book lung bud; ch, cheliceral segment; cu, cumulus; L1L4, leg segments 1 to 4; 01-010, opisthosomal segments 1 to 10; plb: posterior lobe; pp, pedipalpal segment; pt, primary thickening; sp, spinnerets buds; tl, tail; tr, trachea bud. 


\subsubsection{Segmentation and further differentiation of the germ band}

In the early germ band the anterior region is poorly defined, but in the posterior half four segment precursors are already visible (Figure 7, E). The embryo now appears pearshaped because the posterior growth zone slightly protrudes and takes up the entire posterior end of the embryo. The cumulus is still visible on the opposite side of the germ band (the future dorsal side, but no dorsal tissue has developed yet) (Figure 7, E), but will gradually disappear (Figure 7, F) until it has completely vanished at around 96 hours after egg deposition (Figure 7, G). At approximately 92 hours after egg deposition, the germ band is more differentiated also in the anterior portion and the whole embryo is now bent into a bean-shape (Figure 7, F). The segmental anlagen of the cheliceral, pedipalpal and the four walking legs are morphologically visible, and at the molecular level a further segmental anlage is already present in the posterior growth zone as visualized by the expression of the segmental gene engrailed (en) (Figure 8, A). At approximately 96 hours after egg deposition the prosomal segments are fully delineated and the first opisthosomal segment is now also morphologically separated from the posterior growth zone (Figure 7, G). At this stage also the specification of the prosomal limb buds starts and slightly later, at around 106 hours after egg deposition, the buds are formed (later referred to as limb bud development Figure 7, H). This stage also marks the formation of the tailbud (Figure 7, H), which is the posterior growth zone now strongly bulging outward. By generating more opisthosomal segments over the following stages it produces a "tail" that grows away from the embryo and hangs freely in the perivitelline space (Figure 7, I-L) (see also next chapter). All segments, including those in the tube shaped tail, express the segmentation gene engrailed in their posterior portion (Figure 8, B-D). The prosomal appendages continue growing (limb bud elongation Figure 7 , I) and at approximately 130 hours after egg deposition also the first opisthosomal limb

buds, the buds of the book lungs, are specified (Figure 7, J, K). Later also the buds of the tracheal system and the spinnerets are visible (Figure 7, L). At approximately 143 hours after egg deposition the head lobes enlarge indicating the beginning differentiation of the brain (Figure 7, K). The appendages on the prosoma further elongate during these stages. Although the development of the opisthosoma differs compared to the entelegyne 
spiders (described below) general spider tissue rearrangements like inversion (Figure 7, $\mathrm{L}$ and $\mathrm{M}$ ) and dorsal closure (Figure 7, $\mathrm{N}$ and 0 ) are also observed for P. phalangioides. During these rearrangements the prosomal appendages further differentiate and elongate. One of the last major embryonic movements is the closure of the ventral prosomal tissue (ventral closure, Figure 7, P). During this step the podomers of the prosomal appendages differentiate and become visible and the walking legs undergo intensive elongation (discussed below).

\subsubsection{Development of the opisthosoma}

An unusual feature of germ band development of $P$. phalangioides is the mode of opisthosoma formation. The posterior growth zone generates the first three opisthosomal segments while it is still closely attached to the yolk (Figure 9, A and B). These three segments are therefore lying directly on the yolk in the same way as all of the more anterior segments (Figure 9, B). Then the growth zone bulges outward and thus all further segments are formed without connection to the yolk in a tube-shaped tail (Figure 9, C-E). The posterior segments in this tail are closed dorsally, but in fact do not have any true dorsal tissue yet. This is revealed by a detailed sequence of segment formation in the tail (Figure 10). The 04 segment is the first opisthosomal segment that is not formed in direct contact with the yolk, but is included in the tail. However, during further development it opens on its dorsal side and the two halves are then folded down on the yolk (Figure 10, A). Now the 04 segment is very similar to the segments 01 to 03 , and there is no indication anymore that would reveal that it has not been formed in permanent contact with the yolk like 01,02 , and 03. In particular, like the segments 01 to 03 , the 04 segment also has no proper dorsal tissue yet; this tissue forms afterwards during the later phases of inversion (see below). The posterior growth zone continuously adds segments to the tail (Figure 10, B) whereby the tail initially becomes longer. But at the same time more segments are opened "dorsally" and folded down on the yolk (Figure 10, C and D). In this way, the tail becomes shorter after the posterior growth zone has generated the 010 segment and thus terminated its activity (Figure 10, D and E). Finally, all segments are folded down on the yolk and the tail has thus disappeared (Figure 10, F). 
Brightfield
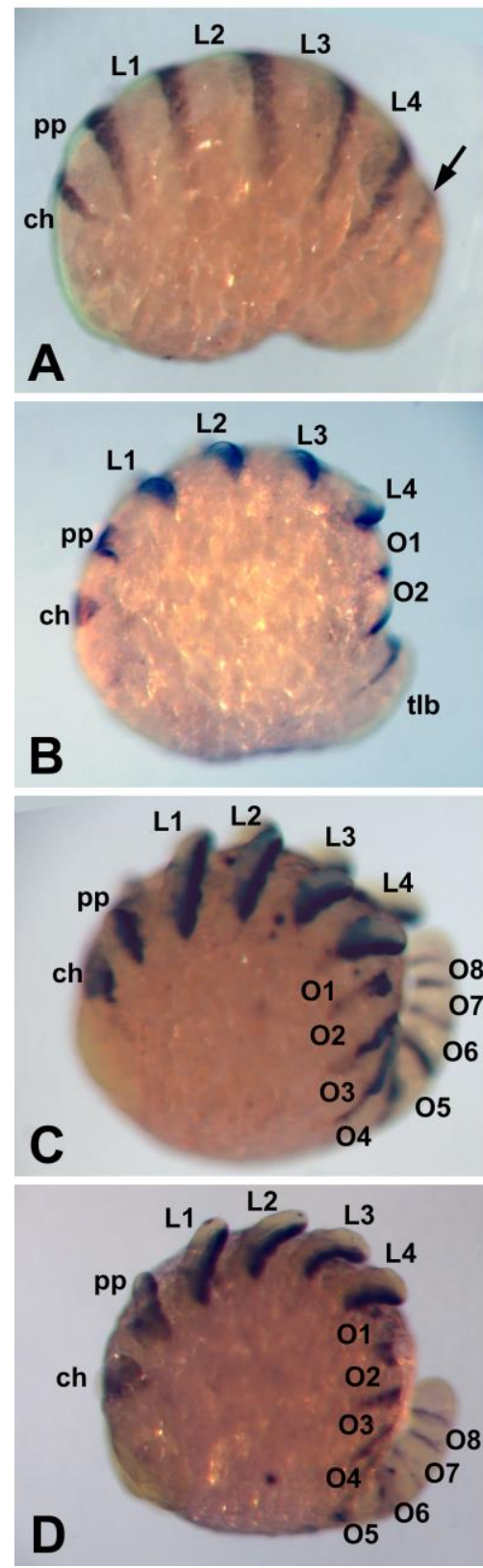

Sytox
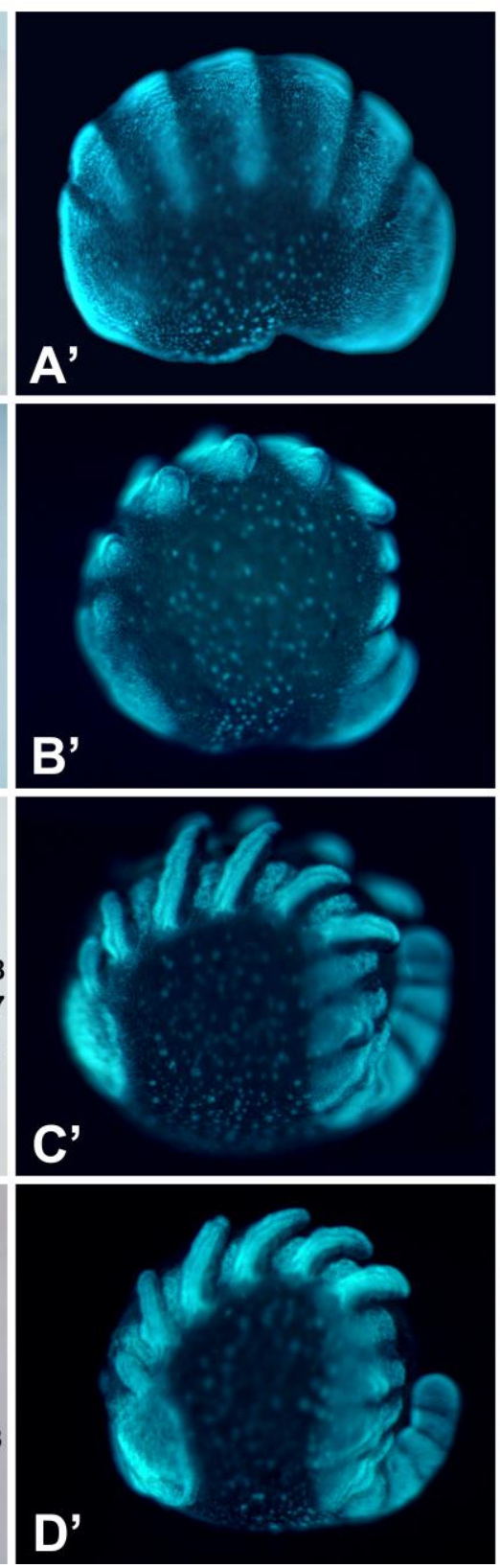

Combined
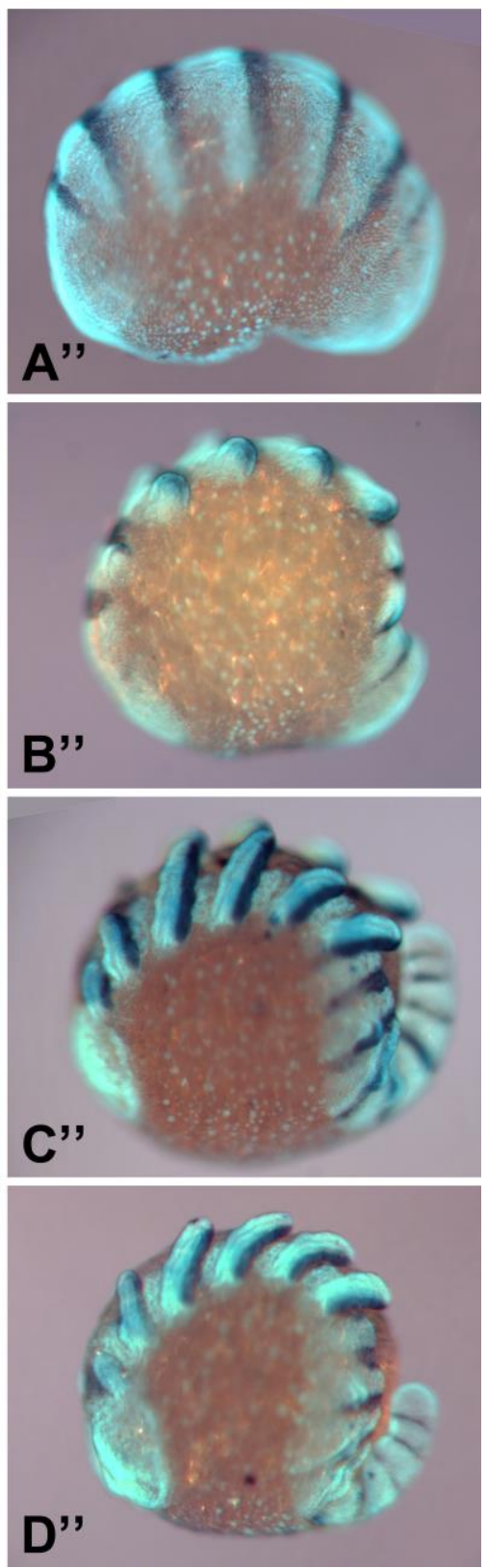

Figure 8: Expression of engrailed (en) in the germ band of $P$. phalangioides

A: Embryo at the bean-shaped stage. All prosomal segments are visible and express en in their posterior portion. Expression of en also prefigures the first opisthosomal segment formed by the posterior growth zone (arrow). B: After tailbud formation, further opisthosomal segments are formed, all denoted by en expression. C-D: Segments in the tube shaped tail also express en. A'-D': The same embryos as in A-D, respectively, cell nuclei have been stained with Sytox Green and visualized with UV light. A"-D": The same embryos as in A-D, respectively. Combined detection of cell nuclei (UV light) and gene expression (white light). All embryos are shown in lateral view with anterior pointing to the left. Abbreviations: see Figure 7. 

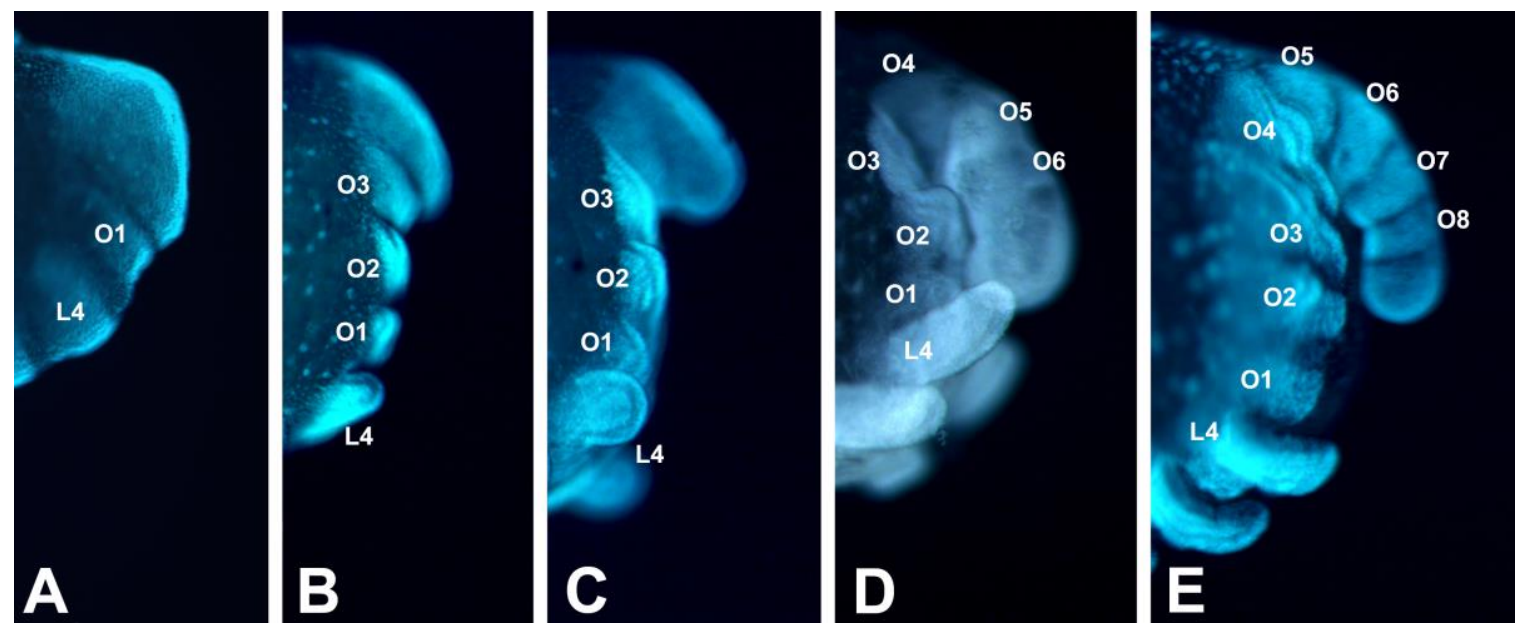

Figure 9: Formation of the tail

A: The posterior growth zone starts bulging outward after it has initiated the formation of the 01 segment. B-C: After the formation of the segments 01 to 03, the growth zone orients away from the body (B) and elongates (C). D-E: All following segments are then formed in the tail (D) that therefore elongates (E) before it shortens again, because the more anterior segments (i.e. more proximal in the tail) are successively folded down on the yolk (see Figure 9). All embryos are shown in lateral view and oriented with the ventral side to the bottom. Abbreviations: see Figure 7.

\subsubsection{Inversion and dorsal closure}

As a preparation for inversion, the germ band starts splitting along the ventral midline at approximately 119 hours after egg deposition (Figure 7, I). This formation of the ventral sulcus initiates the ventral opening necessary for inversion, but on the dorsal side no inversion specific events occur until approximately 143 hours after egg deposition when genuine dorsal tissue develops on the opisthosomal segments (Figure 7, K). This dorsal tissue overgrows the yolk during the following stages (Figure 7, L-N) until the two leading edges meet and fuse on the dorsal side during dorsal closure (Figure 7, 0). After the dorsal side of the embryo has closed over the yolk there is further differentiation of this tissue when it further contracts and forms the uniform prosomal shield (Figure 7, P) at approximately $215 \mathrm{~h}$ after egg deposition. At this stage the tail has vanished and all opisthosomal segments have been folded down on the yolk. Although this process also leads to a partial closure of the ventral side of the embryo (Figure 11, A and B), the ventral side is still open at the time when the dorsal side is fully closed and the prosomal shield forms (Figure 11, C). However, ventral closure proceeds quickly and the prosoma is closed first (Figure 11, D) and shortly after, the ventral side is closed entirely (Figure $11, \mathrm{E})$. 

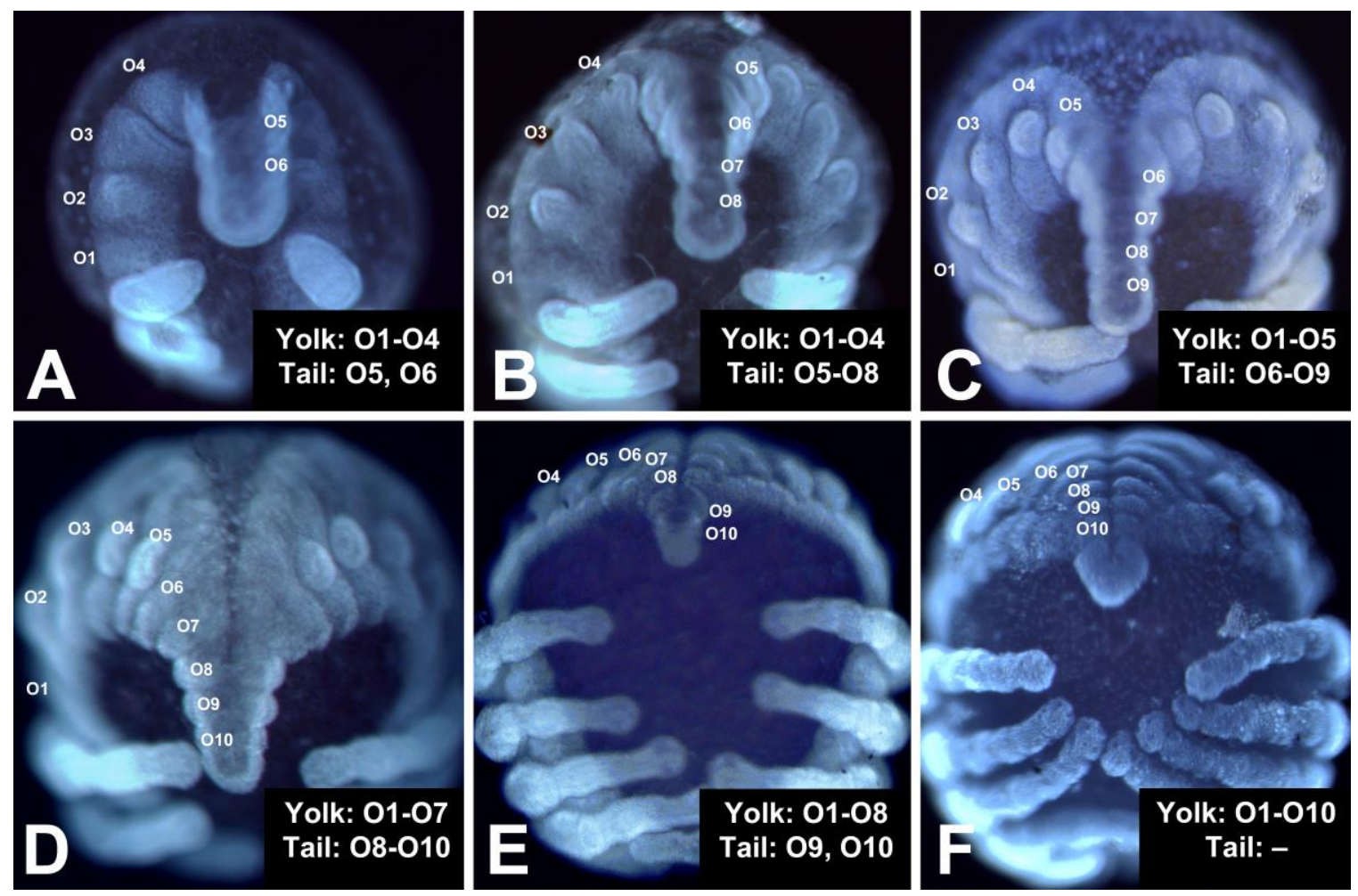

Figure 10: Formation of the opisthosoma by folding down tail segments on the yolk

A-B: The number of segments in the tail grows continuously, but the most proximal tail segment (04) is already at the "hinge" between the segments on the yolk and segments in the tail (A) or fully folded down on the yolk (B). C-E: The growth zone produces more segments, but the speed of folding down proximal tail segments also increases, thus leading to a shortening of the tail. (F) Because the growth zone stops producing segments at one point, but the folding down of segments at the proximal end of the tail continues, the tail is eventually gone. The number of segments already lying on the yolk and those still in the tail is given in each panel at the lower right corner. All embryos are shown in posterior view with the dorsal side pointing upwards. Abbreviations: see Figure 7.

\subsubsection{Growth of the legs}

The legs first appear as small limb buds at approximately 106 hours after egg-deposition, and then continuously grow throughout embryogenesis. The legs are not extraordinarily long before dorsal closure (Figure 11, A). However, around the time of dorsal closure the legs have grown considerably and now especially the distal portion of the first leg pair is overlapping, thus giving the impression that the legs are "too long" to fit next to each other and the embryo looks like "folding its arms" (Figure 11, B). This impression is lost again when the dorsal side compacts during the formation of the prosomal shield (Figure 11, C) and this contraction of the dorsal side also pulls apart the entangled legs (Figure $11, \mathrm{D})$. 
Coinciding with ventral closure, there is a sudden increase in leg growth that leads to the legs overlapping again and the tips are often twisted, obviously because there is not enough space now to accommodate the long legs (Figure 11, E). This length growth continues throughout the rest of embryonic development and in embryos shortly before hatching the legs are so long that they are fully wrapped around the body (time lapse movie provided on CD-Rom, Figure 11, F). In the time lapse movie of embryos just before hatching no rearrangement or development of the remaining embryonic tissue can be observed anymore, but instead only the walking legs are growing further.
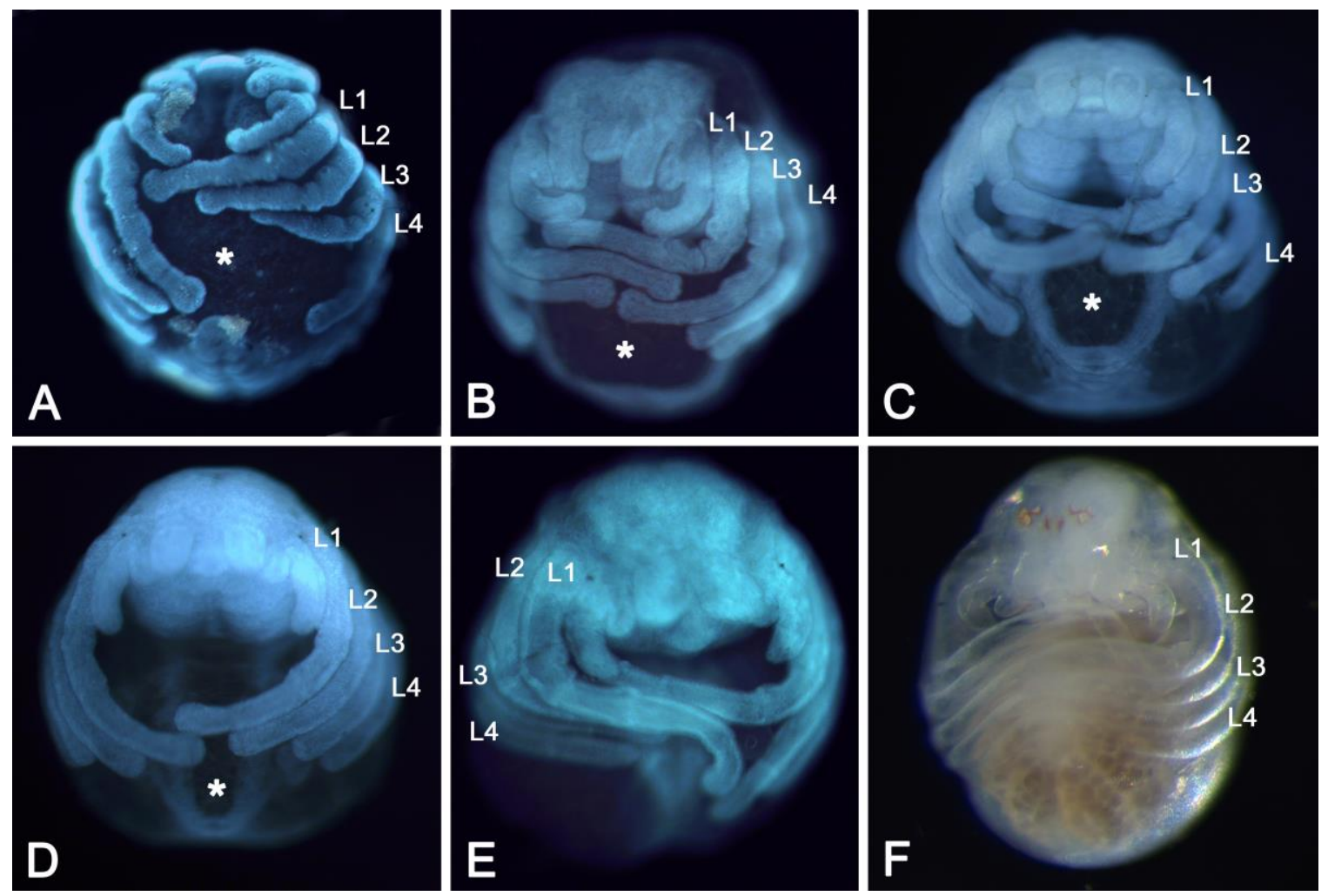

Figure 11: Allometric growth of the legs in P. phalangioides, and ventral closure

A: Shortly before dorsal closure the legs are moderately long and the ventral side is still wide open (asterisk). B: When the dorsal side is closed the legs (especially the first leg pair) interlace. Ventral closure has started, and the gap between left and right half is closing (asterisk). C-D: The formation of the prosomal shield on the dorsal side pulls apart the interlaced legs. Ventral closure proceeds (asterisk) and is first complete in the prosoma (arrow in D). E-F: When ventral closure is complete (arrow in E), a sudden increase in leg growth occurs, and in stages shortly before hatching the legs are fully wrapped around the body (F). All embryos are shown in ventral view with anterior pointing upwards. Abbreviations: see Figure 7. 


\subsection{Generating transcriptomic resources for $P$. phalangioides}

Technical advances giving rise to the next generation sequencing (NGS) era facilitate the fast and relatively cheap generation of transcriptomic and genomic resources for various tissues and species and thus revolutionized biological research (Gilad et al., 2009; Oppenheim et al., 2015; Stapley et al., 2010). This helps to overcome labour-intensive cloning strategies to identify homologous genes in non-model organisms. Whereas genomic information represents the general gene set of an organism, transcriptomes have the ability to provide gene expression data for different tissues or developmental stages of an organism. Thus unbiased transcriptomic approaches are in contrast to the candidate gene approach an auspicious stepping-stone to identify species or lineage specific genes or novel functions of pleiotropic genes driving the evolution of morphological differences between species.

This study was among others performed to uncover the genetic basis causing the special walking leg characteristics of P. phalangioides. Therefore we performed RNAseq, de novo transcriptome assembly and differential gene expression analysis for embryonic and different nymphal tissue. After isolation of a convenient number of leg gene candidates their expression pattern was observed in the haplogyne spider model P. phalangioides.

\subsection{1 de novo transcriptome assembly and differential gene expression}

A preliminary version of the $P$. phalangioides transcriptome was assembled de novo and previously published (Janssen et al., 2015). For the unbiased identification of spider or even P. phalangioides specific leg gene candidates a second assembly was performed using a newer version of the assembler Trinity. With this improvement a full assembly of 284.577 transcripts and 233.353 predicted genes with a GC content of $37.14 \%$ was generated. The N50 of the transcriptome is $1032 \mathrm{bp}$ with a median transcript length of $348 \mathrm{bp}$ and an average transcript length of $659 \mathrm{bp}$. The P. phalangioides transcriptome completeness was assessed using the freely available software BUSCO (Simão et al., 2015). BUSCO uses the comprehensive OrthoDB database (divided into six major phylogenetic clades) of orthologs to assess the completeness of genomes and transcriptomes by comparing the presence or absence of common orthologous genes. 

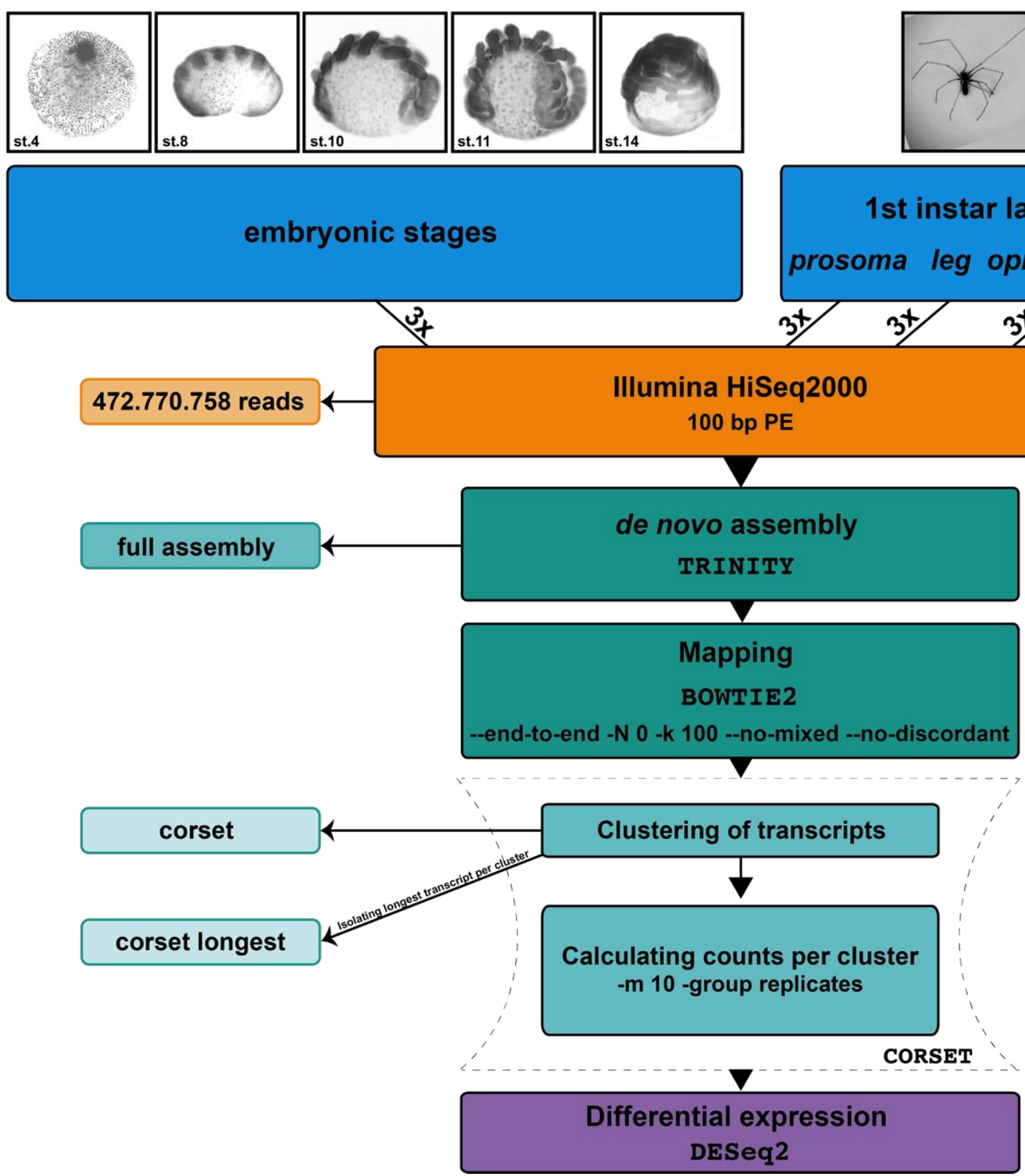

Figure 12: Overview de novo transcriptome assembly

Work flow of RNAseq, de novo transcriptome assembly and quality optimization prior to differential gene expression analysis. Triplicates of four different samples were sequenced with the Illumina HiSeq2000 generating 100 base pair paired end reads. Afterwards assembly and mapping were performed with the software Trinity and bowtie 2 respectively. To reduce the number of overall transcripts the program corset was used to cluster transcripts into gene models and calculating the read counts per cluster. Finally differential gene expression was performed with the $R$ package DESeq2. 
$82 \%$ of the arthropod OrthoDB database orthologs $(n=2.675)$ were found in the de novo assembled transcriptome, and even $89 \%$ in case of the metazoan database $(n=843) .33 \%$ of the arthropod orthologs are duplicated in the P. phalangioides transcriptome. After this quality assessment the program corset was used to cluster transcripts, which might represent isoforms of one gene into gene clusters and counts the reads for these clusters. This clustering reduced the complexity of the reference transcriptome (Figure 13, A) to 142,919 transcripts (Figure 13, panel B) and only 109,640 transcripts (Figure 13, C) if only the longest transcript of each cluster was considered. The reduction of the transcriptome complexity mainly minimized the number of very short transcripts compared to the full transcriptome (Figure 14).

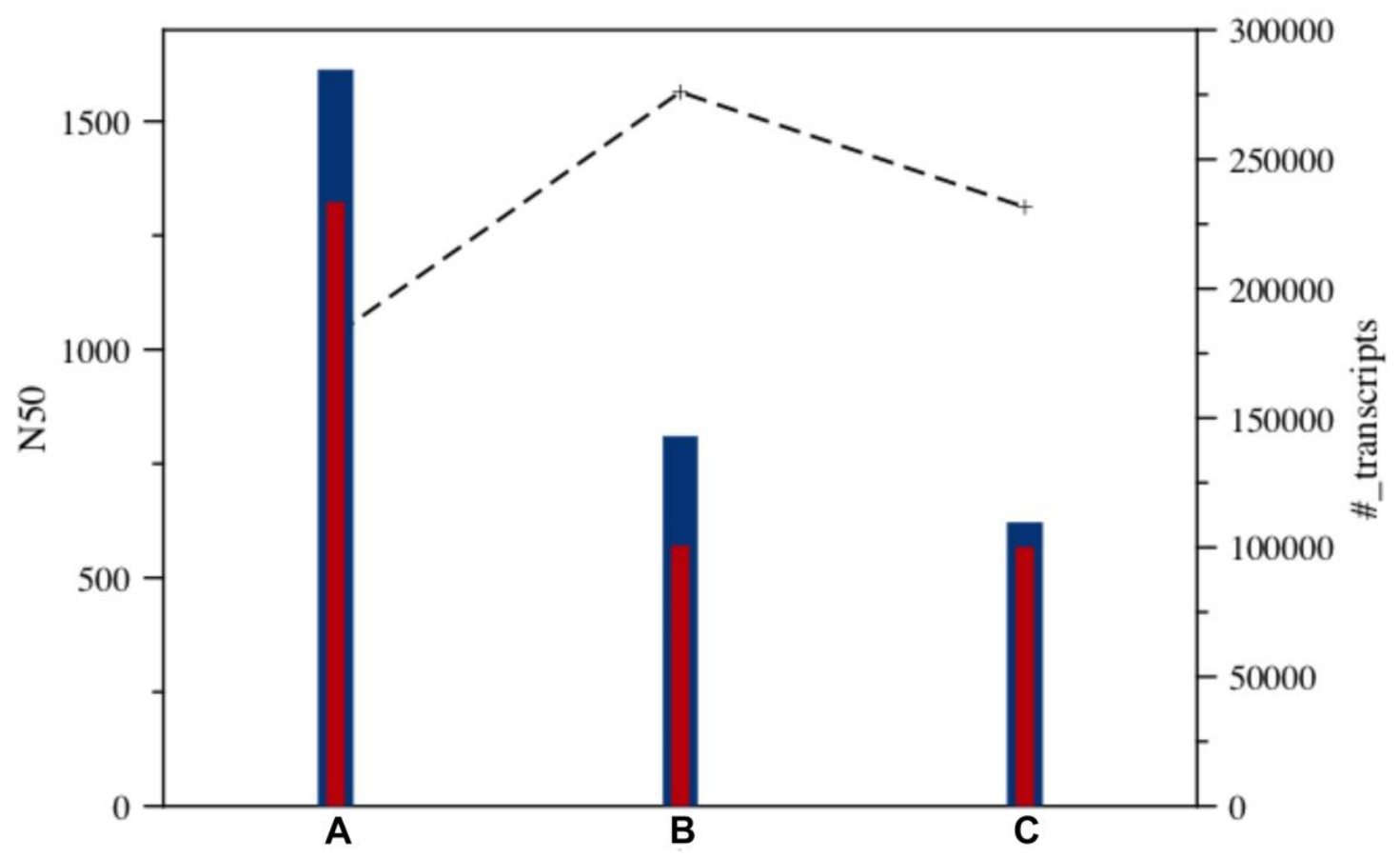

Figure 13: Comparison of gene and transcript numbers after corset

Depicted are the N50 (dashed line) and the number of transcripts (labeled in blue) and genes (red) in the de novo assembled transcriptome before (A) and after optimization with corset (B). C: Total numbers of transcripts and genes when only the longest transcript of the gene cluster was considered. 


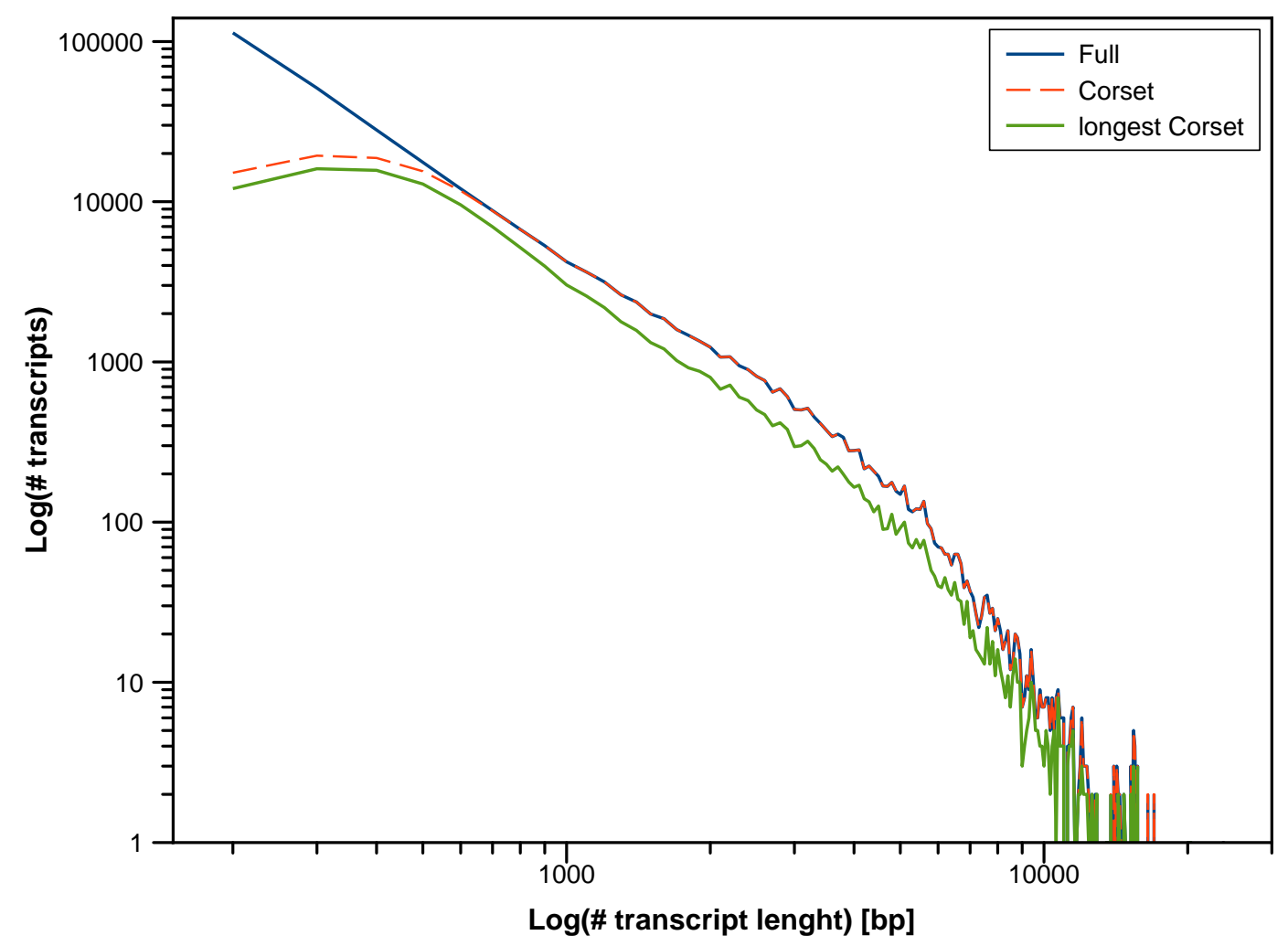

Figure 14: Dependence of transcript number and transcript length

This graph shows the relation of transcript length and number of the de novo assembled transcriptome before and after optimization. The blue line represents the distribution of the length of all transcripts without optimization. The dashed red line shows that after optimization with corset especially short transcripts were discarded what resulted in the overall reduction of transcript number. In contrast the green line shows the length distribution of only the longest transcript of the corset gene cluster. This shows a general reduction of transcript number independent for the length of the transcripts.

Based on the read counts the distribution and relatedness of the triplicates and the different sample tissues was analysed with a principle component analysis. The three biological replicates of the different tissues are always closely grouped together. Also biologically more similar tissues like the samples of the legs (green Figure 15) and the prosoma (magenta, Figure 15 (coxa, pedipalps and chelicera attached)) are grouped more closely, than less related tissues like the opisthosoma (blue, Figure 15). 


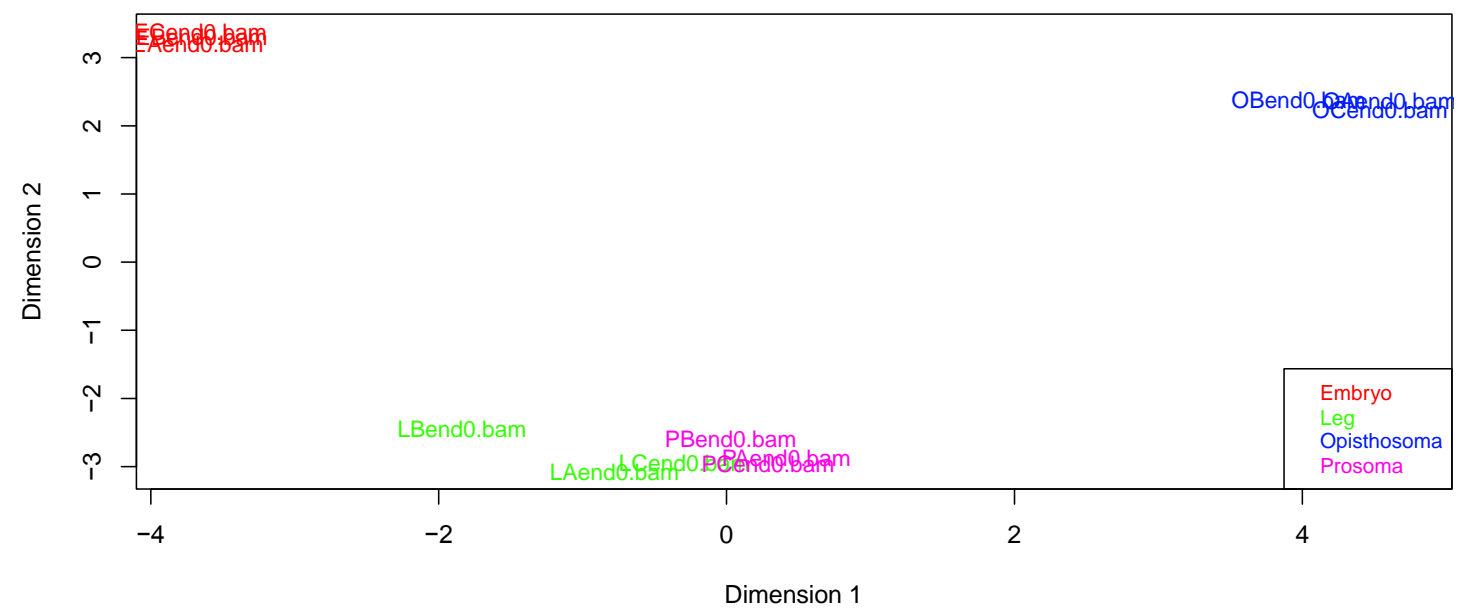

Figure 15: Principal component analysis of the triplicates for the different sample tissues The principle component analysis based on read counts shows that the triplicates of the different sample tissues are always grouped closely together. The triplicates are named as the following: Embryo tissue (red): EAend0.bam, EBend0.bam, ECend0.bam; Prosoma (magenta): PAend0.bam, PBend0.bam, PCend0.bam; Opisthosoma (blue): OAend0.bam, OBend0.bam, OCend0.bam and Walking legs (green): LAend0.bam, LBend0.bam, LCend0.bam. The embryonic samples and the opisthosomal samples are found in upper proximity of principle component 2 but are far away from each other along principle component 1. Walking leg and prosomal samples are grouped more closely together in the most negative part of principle component 2 .

In order to identify genes with different expression levels in the different tissue samples, the read counts of the different stages and tissues were compared using the program DESeq2. The comparison of mixed embryonic tissue samples to all nymphal tissue samples revealed the highest number of up regulated genes (14.212) (Table 7). Comparisons of prosomal tissue samples to walking leg and opisthosomal tissue samples revealed around 7.000 up-regulated genes. A similar amount of up-regulated genes was observed for the opisthosomal tissue samples compared to the other two nymphal tissues. Comparison of the walking legs samples to the prosomal and opisthosomal tissue samples indicated only 1.490 up regulated genes. The number of down-regulated genes ranges between 3.000 and nearly 5.000 for the different comparisons of the nymph tissue samples, and is lower than the identified 9.000 down-regulated genes in the comparison of the embryonic tissue samples to the samples of nymphal tissues. In this study I focused on the 1.490 genes up-regulated in the legs and their possible role in the specialized leg formation of $P$. phalangioides. 
Table 7: Differently expressed genes in the different tissues sequenced for $P$. phalangioides

The list shows the number of genes, which were significantly differentially expressed (padj< 0.05 ) in the different comparisons. i) Comparison of the embryonic samples to all nymphal samples. ii) Comparison of freshly hatched nymph prosoma samples to freshly hatched nymph walking leg and opisthosoma samples. iii) Comparison of the walking leg samples to the samples of the prosoma and opisthosoma. 1.490 genes were identified to be significantly up regulated in the walking legs of freshly hatched nymphs indicated in green. iv) Comparison of the opisthosomal samples to the samples of the walking legs and prosoma.

\begin{tabular}{lcccc}
\hline & i) 1st instar larva & ii) prosoma & iii) leg & iv) opisthosoma \\
Up regulated & 14.212 & 6.934 & 1.490 & 6.976 \\
Down regulated & 9.158 & 3.071 & 3.197 & 4.711 \\
\hline
\end{tabular}

\subsubsection{Leg candidate genes}

In total 1.490 transcripts were identified to be up-regulated in the walking legs of freshly hatched nymphs (Table 7, highlighted in green). Functional annotation (using gene ontologies) of these overexpressed genes was performed with the Blast2Go annotation pipeline in addition to the general transcriptome annotation with SwissProt, Uniref90 and the P. tepidariorum genome gene set. The gene ontology (GO) term analysis (Figure 16) revealed many general processes like transcriptional regulation, cuticle formation metabolic and cellular processes. But also growth related processes like regulation of cell proliferation and negative regulation of apoptosis were found. Based on the GO term analysis developmental genes involved in the Notch signalling pathway and in imaginaldisc derived leg segmentation are especially up-regulated in the walking legs of the $P$. phalangioides.

Table 8: Filtering criteria to reduce the number of up-regulated leg gene candidates

\begin{tabular}{|l|r|}
\hline Criteria & \# candidates \\
\hline leg up padj $<0.05$ & 1.490 \\
\hline basemean $>10$ & 1.215 \\
\hline Embryomean $>50$ & 582 \\
\hline sort log2fold & best 15 \\
\hline Annotation in any other organsim & 9 \\
\hline orf $>300$ & 8 \\
\hline
\end{tabular}


To reduce the number of candidates from 1.490 to a number of genes that can be functionally tested, we applied different criteria (Table 8). The rejection of all candidates with generally low expression (base mean lower than ten) and low expression in the embryo (embryo mean lower than 50) resulted in a reduction to 582 candidates.

From these the 15 most differentially expressed candidates (indicated by the log2Fold) were analysed in more depth. Eight of these candidates had homologs in at least one other species and an open reading frame covering at least 300 base pairs (Table 9). Among these candidates were predicted homologs of the homeobox protein unc4 from $D$. melanogaster, one odd-skipped (odds) homolog of the human (odds-related 2 OSR2), the Kinesin-like calmodulin-binding protein homolog (KCBP) of the Asian rice plant Oryza sativa subsp. japonica and the MDS1 and EVI1 complex locus protein, Evi1 from the mouse. The other four candidates only had homologs in the gene set of P. tepidariorum. Except for $c 105236$ and the unc4 homolog these candidates were successfully cloned (Table 9 columns filled with green) and subsequently analysed for their expression patterns during embryonic development of $P$. phalangioides (see below).

Table 9: Final candidates and their annotation

\begin{tabular}{|l|r|r|l|l|l|l|l|}
\hline Cluster & baseMean & $\begin{array}{l}\text { log2Fold } \\
\text { Change }\end{array}$ & padj & transcript & length & aug_pred. & sprot_Top_BLASTX \\
\hline $\begin{array}{l}\text { Cluster- } \\
82327.0\end{array}$ & 17607,64 & $-5,75$ & $3,13 \mathrm{E}-04$ & c95092_g2_11 & 2143 & aug3.g14955.t1 &. \\
\hline $\begin{array}{l}\text { Cluster- } \\
60373.0\end{array}$ & 2035,79 & $-4,72$ & $3,47 \mathrm{E}-04$ & c109662_g1_i1 & 4554 & aug3.g26302.t1 &. \\
\hline $\begin{array}{l}\text { Cluster- } \\
88654.1\end{array}$ & 23875,43 & $-4,72$ & $8,71 \mathrm{E}-03$ & c109329_g1_i2 & 2467 & aug3.g27504.t1 &. \\
\hline $\begin{array}{l}\text { Cluster- } \\
91098.5\end{array}$ & 85,93 & $-4,69$ & $1,73 \mathrm{E}-04$ & c102331_g1_i4 & 2211 & aug3.g27262.t1 & OSR2_HUMAN \\
\hline $\begin{array}{l}\text { Cluster- } \\
84832.0\end{array}$ & 273,76 & $-4,6$ & $6,76 \mathrm{E}-04$ & c110656_g1_i4 & 3115 & aug3.g2943.t2 & UNC4_DROME \\
\hline $\begin{array}{l}\text { Cluster- } \\
73819.0\end{array}$ & 545,56 & $-4,31$ & $2,93 \mathrm{E}-22$ & c105514_g1_i1 & 3137 & aug3.g4311.t1 & KCBP_ORYSJ \\
\hline $\begin{array}{l}\text { Cluster- } \\
67258.0\end{array}$ & 915,66 & -4 & $3,68 \mathrm{E}-03$ & c105236_g9_i1 & 1256 & aug3.g22859.t1 & . \\
\hline $\begin{array}{l}\text { Cluster- } \\
72087.1\end{array}$ & 736,78 & $-3,91$ & $2,55 \mathrm{E}-03$ & c109624_g3_i2 & 5505 & aug3.g11431.t2 & EVI1_MOUSE \\
\hline
\end{tabular}




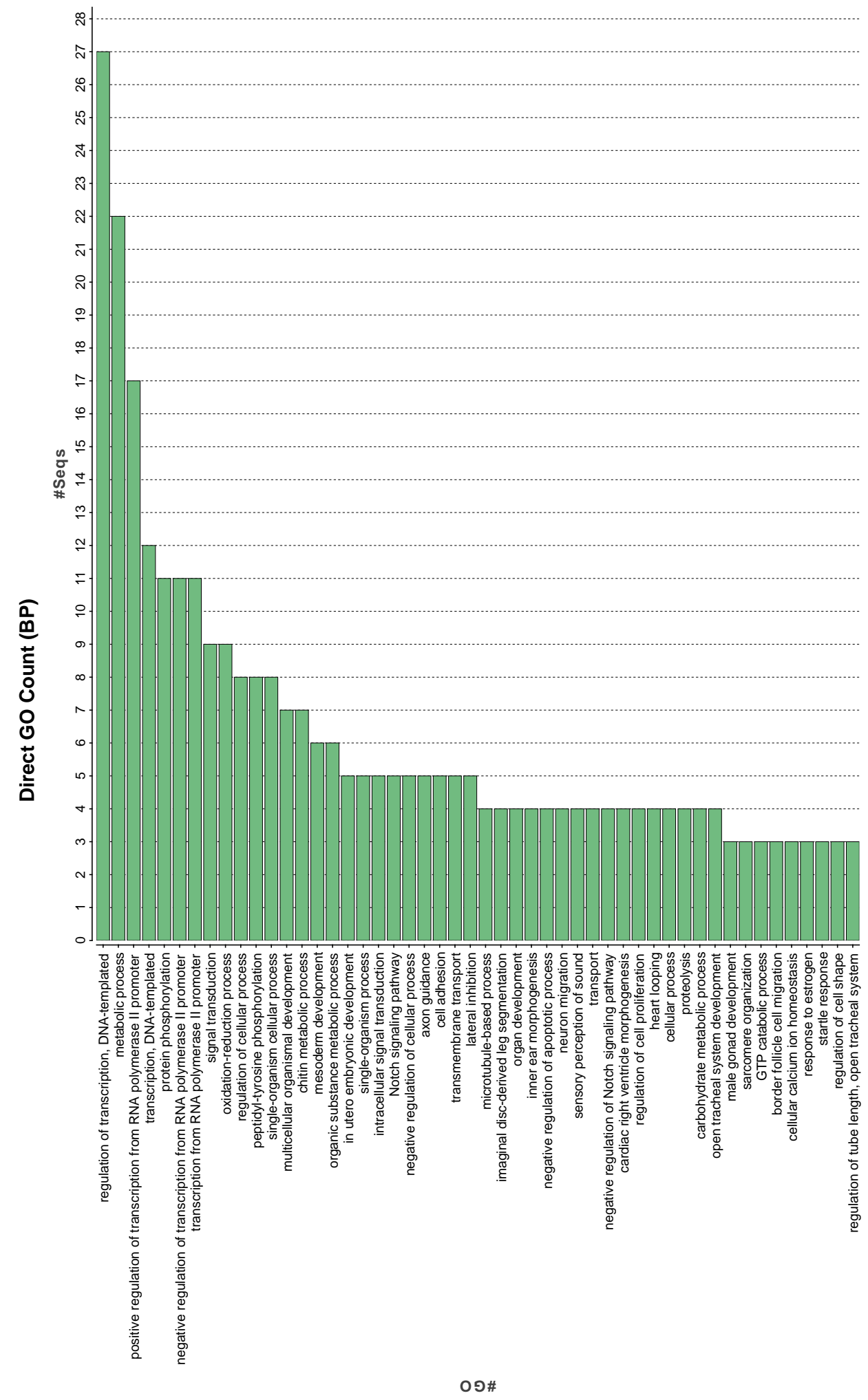

Figure 16: G0-term results for biological processes of the genes up-regulated in the legs Overview of most prominent biological porecesses found in the $\mathbf{1 . 4 9 0}$ up-regulated leg genes after differential gene expression analysis. On the $x$-axis the number of sequences found in the set of up-regulated genes with the certain GO term is depicted. In the $y$-axis the most prominent biological processes are listed. 


\subsubsection{Expression of $c 102331$ in P. phalangioides}

Expression of the odd-skipped ortholog with the transcript ID c102331 starts as a distal ring in the pedipalps and the first walking leg pair with beginning of prosomal limb bud elongation (Figure 17, A arrowhead). During inversion stages this expression also starts in the remaining walking legs (Figure 17,B arrowheads) and broadens during dorsal closure stages (Figure 17, C arrow). This expansion of expression continues during ventral closure stages covering the area proximal to the distal thickening (Figure 17, D arrow), but is not present in the pedipalps, where only the ring of expression remains (arrowhead).
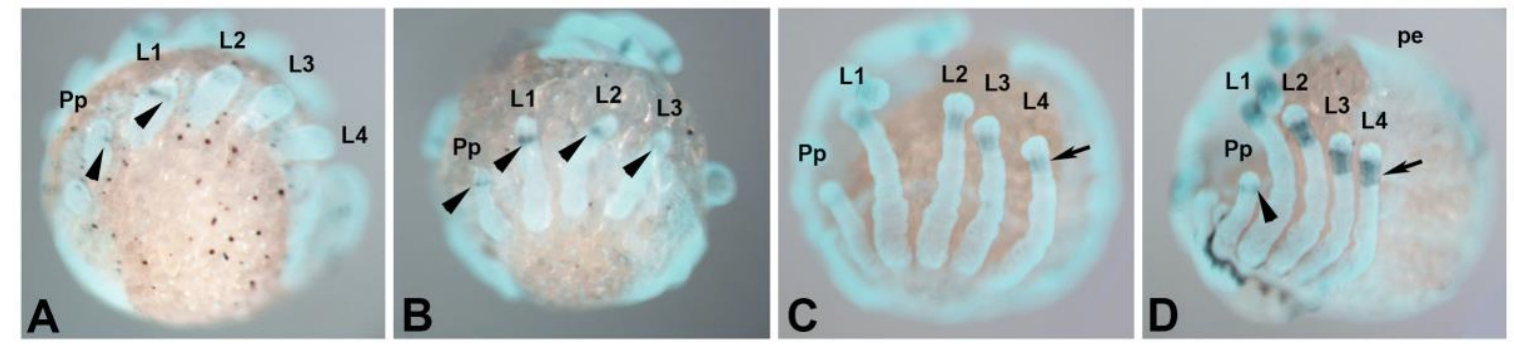

Figure 17: Expression of $c 102331$ Pp-odd skipped in P.phalangioides A: Expression of $c 102331$ in the pedipalps and L1 (arrowhead) of an embryo during prosomal limb bud elongation, lateral view. B: Ventro-lateral view of an embryo during inversion. Some of the walking legs were lost during the washing steps of the in-situ hybridization. C: Ventro-lateral view of an embryo expressing c102331 at dorsal closure. D: Embryo during ventral closure, ventrolateral view. For detailed explanation of expression see text. Embryos are oriented with the anterior side to the left. Pp: Pedipalps, L1-L4: Walking legs pairs 1-4, pe: posterior end.

\subsubsection{Expression of $c 109624$ in P. phalangioides}

The expression of the P. phalangioides Evi homolog c109624 starts in the ventral neuroectoderm during limb bud elongation stages (Figure 18, A arrow). This expression in the ventral neuroectoderm remains throughout the entire embryonic development, but is always stronger in the head and prosomal part (Figure 18, B and C arrowhead) than in the opisthosoma (Figure 18, G and H arrowhead). During dorsal closure the gene is expressed in a single spot in the distal part of the prosomal appendages and the opisthosomal limb buds on 02,04 and 05 starts (Figure 18, C and $\mathrm{H}$ arrow). In the chelicera c109624 expression resolves into a complex pattern during ventral closure (Figure 18, D arrowhead), but expression in the pedipalps remains as a single spot. In the walking legs however, additional spots of expression appear in the distal thickening (Figure 18, D arrow). 

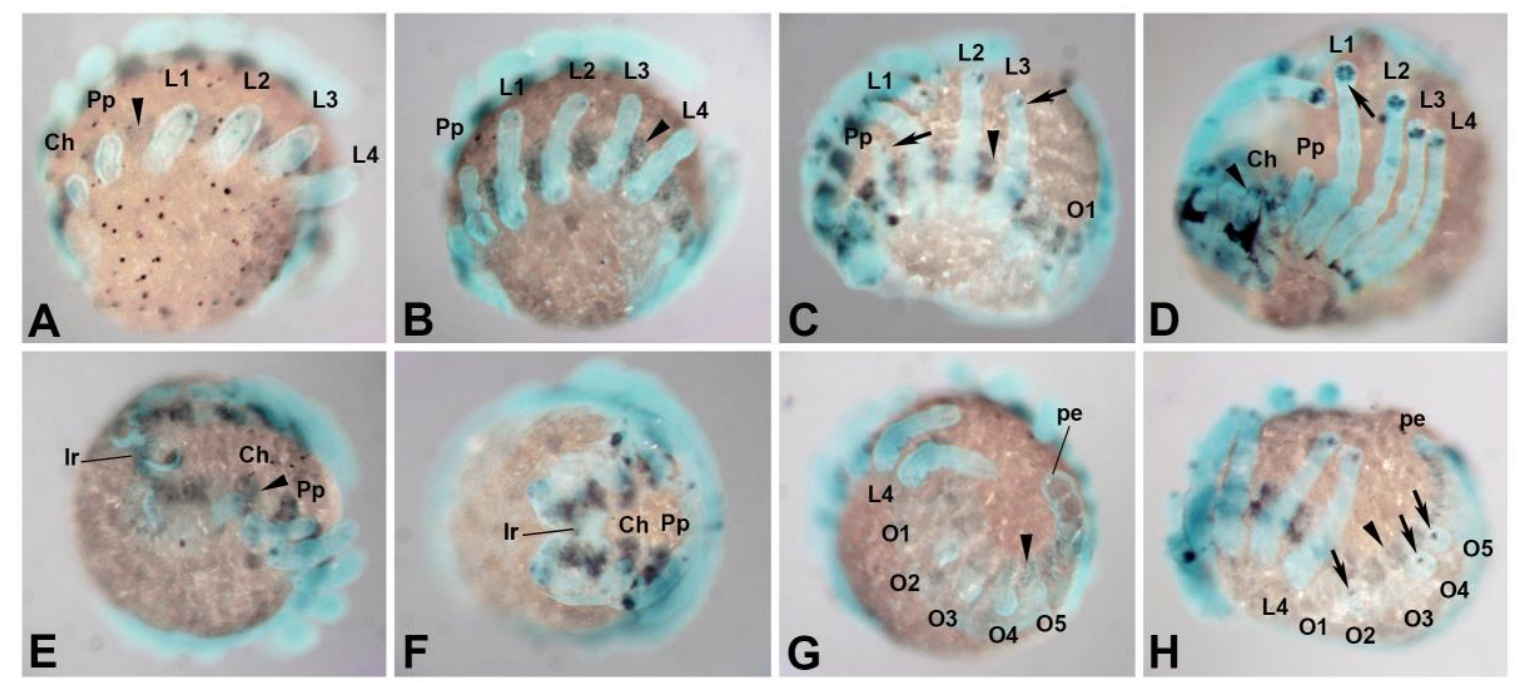

Figure 18: Expression of c109624 in P. phalangioides

A: Expression of $c 109624$ in the ventral neuroectoderm (arrowhead) of an embryo during prosomal limb bud elongation, lateral view. B: Ventro-lateral view of an embryo during inversion. C: Lateral view of an embryo at dorsal closure expressing c109624 in the ventral neuroectoderm (arrowhead) and in a spot in pedipalps and walking legs (arrow). L4 was lost during the staining of the embryo D: Embryo during ventral closure, anterior-lateral view. For detailed explanation of expression in the prosomal appendages see paragraph text. E: Expression of c109624 in the neurogenic ectoderm of the head at inversion of the embryo, frontal-lateral view. F: Neurogenic expression of c109624 in the head of an embryo during dorsal closure, frontal view. G: Posterior-lateral view of the opisthosoma during inversion. Weak c109624 expression in the opisthosomal-ventral neuroectoderm (arrowhead). H: Lateral view of $c 109624$ expression in the ventral neuroectoderm (arrowhead) and limb buds (arrow) of the opisthosoma. A-D and G -H: Embryos are oriented with the anterior side to the left. $\mathrm{E}$ and $\mathrm{F}$ : Embryo is shown in frontal aspect, walking legs pointing right. Ir: Labrum, Ch: Chelicera, Pp: Pedipalps, L1-L4: Walking legs pairs 1-4, 01-05: Opisthosomal segments 1-5, pe: posterior end.

\subsubsection{Expression of c109329, c105514 and c95092 in P. phalangioides}

The genes c109329, c95092 and the $n c d$ homolog c105514 are not expressed in the walking legs of P. phalangioides during embryonic development. For Pp-c105514 no embryonic expression was observed at all. c95092 and c109329 are expressed in the chelicera in late embryonic stages. c95092 is expressed in the distal part of the chelicera when dorsal closure begins (Figure 19, A and B arrow), and later during ventral closure this expression restricts to a small spot (Figure 19, C arrow). c109329 is expressed as a spot in the distal part of the chelicera and starts during ventral closure (Figure 20, C arrow). 

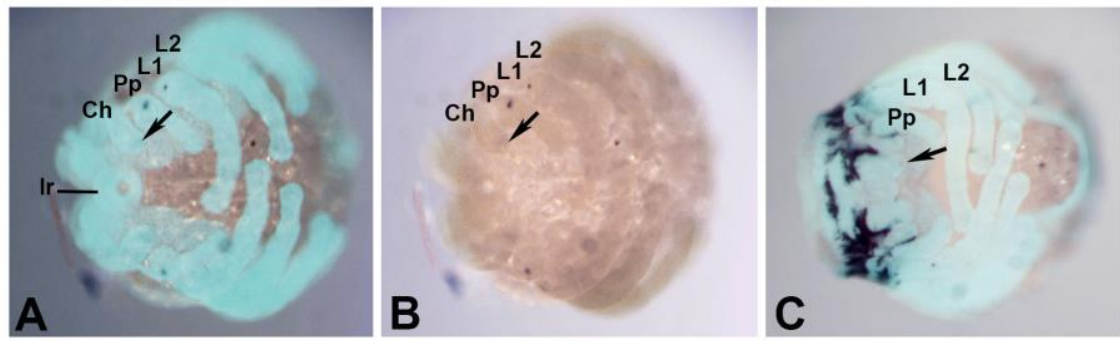

Figure 19: Expression of $c 95092$ in P. phalangioides

A: Dorsal closure of an embryo expressing $c 95092$ in the chelicera and nuclear staining with Sytox Green, frontal view. B: The same embryo without nuclear staining. C: Frontal view of an embryo during ventral closure. Dark areas are unspecific staining of the forming cuticle in the head and lateral at the base of the walking legs. All embryos are oriented with the anterior side to the left. Ir: Labrum, Ch: Chelicera, Pp: Pedipalps, L1-L4: Walking legs pairs 1-4.
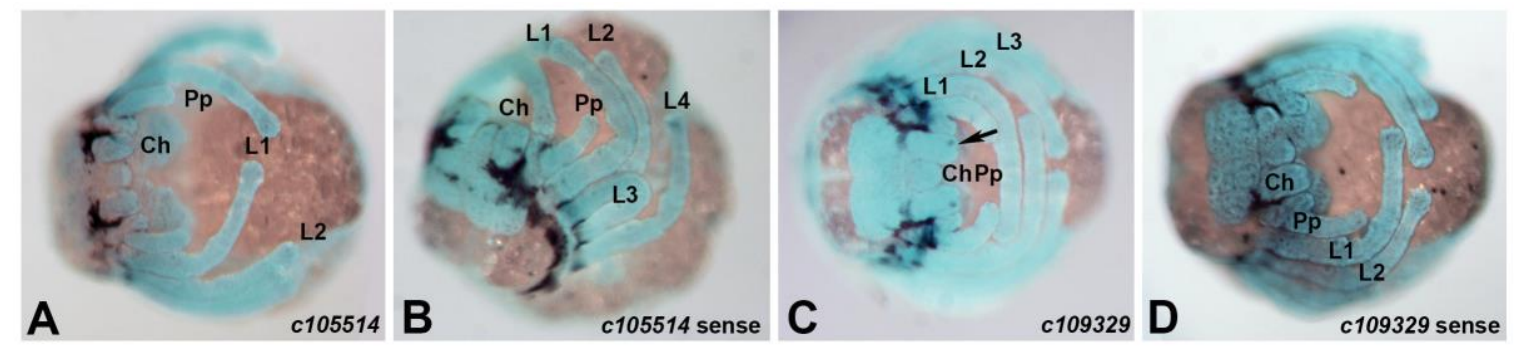

Figure 20: Expression of c105514 and c109329 in P. phalangioides

A-D: Embryos undergoing ventral closure shown in frontal view. A: Embryo after in-situ hybridization with an antisense probe of Pp-c105514 and nuclear staining with Sytox-Green. B: Embryo after in-situ hybridization with a sense probe of Pp-c105514 and nuclear staining with Sytox-Green. C: Embryo with expression in the chelicera after in-situ hybridization with an antisense probe of Pp-c109329 and nuclear staining with Sytox-Green. D: Embryo after in-situ hybridization with a sense probe of $P p$-c109329 and nuclear staining with Sytox-Green. Dark areas in the head and lateral at the base of the walking legs depict unspecific staining of the forming cuticle also present after negative control in-situ hybridizations with sense probes. All embryos are oriented with the anterior side to the left. Ch: Chelicera, Pp: Pedipalps, L1-L4: Walking legs pairs 14.

\subsubsection{Expression of $c 109662$ in P. phalangioides}

The expression of $c 109662$ starts during dorsal closure very strong covering the labrum and the anterior part of the head lobes. In addition a weak ring of expression in the medial part of the walking legs starts (Figure 21, A arrow). During ventral closure the strong labral expression remains unchanged and additional expression domains appear in the gnathendite and two rings in the pedipalps itself. With proceeding ventral closure more rings at the joints of the developing walking legs appear. 


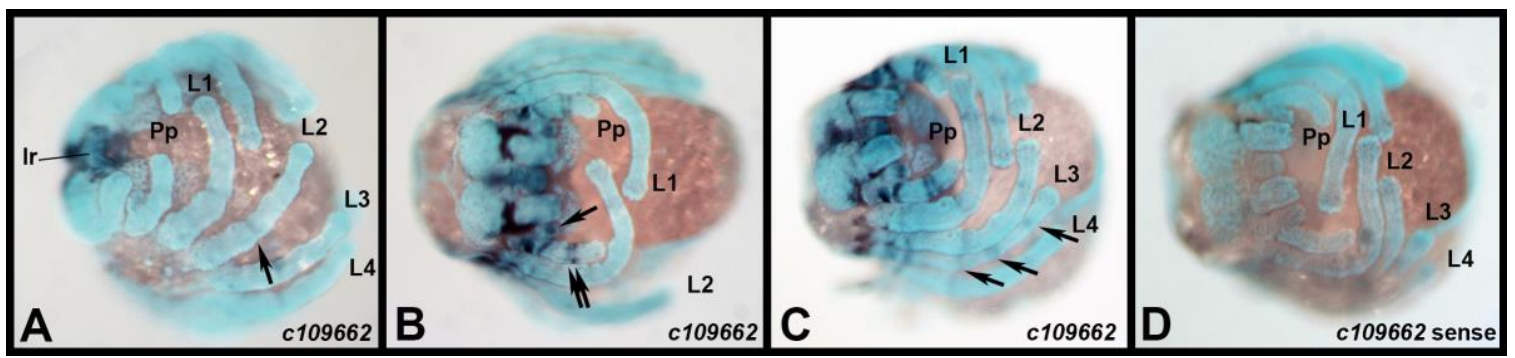

Figure 21: Expression of 109662 in P.phalangioides

Shown are embryos during dorsal (A) or ventral closure (B-D). A: Frontal-lateral view of an embryo expressing $c 109662$ in the walking legs (arrow). B: Frontal view of an embryo with Pp_c109662 expression in the pedipalps and gnathendite (arrow). C: Additional expression of Pp-c109662 starts in the walking legs during ventral closure, frontal-lateral view. D: No expression can be detected after in-situ hybridization with a Pp-c109662 sense probe. All embryos are oriented with the anterior side to the left. Ch: Chelicera, Pp: Pedipalps, L1-L4: Walking legs pairs 1-4, 01-05: Opisthosomal segments 1-5, pe: posterior end. 


\subsection{Discussion and future work}

\subsubsection{Two early waves of cell dispersal}

The formation of a primary thickening and the migration of the cumulus are features common to all spiders and have previously been described for P. phalangioides as well (Claparède 1862; Emerton 1872). However, to our knowledge we provide here the first evidence that this process involves two independent waves of cell aggregation and dispersal in P. phalangioides. These waves are difficult to be appreciated from still images, because they directly follow each other and thus the transition between them is easily missed. Our time-lapse recording of this process shows that the first wave leads to the formation of the primary thickening and its dissolution by cell dispersal. The second wave includes some former primary thickening cells, that reverse their movement, come together a second time at the center of the germ disc (secondary aggregation), but then disperse into the posterior growth zone.

Although this second wave of accumulation and segregation of former primary thickening cells has not been described previously in entelegyne spiders, we think that mechanistically there may be no huge difference between germ band formation in haplogyne and entelegyne spiders. This is because the result of this process is virtually identical in haplogyne and entelegyne spiders: (1) In both spider groups, the majority of primary thickening cells, by dispersion, end up as a "second layer" below the germ disc epithelium (summarized in Edgar et al. 2015). (2) In both groups, the posterior growth zone is formed by a small portion of former primary thickening cells at the center of the germ disc, either because these cells do not disperse like the other primary thickening cells (Akiyama-Oda and Oda 2003), or because already dispersed cells return to the disc center and then disperse again into the larger structure of the posterior growth zone (this study). (3) In both groups, the migrating cumulus ends up at a position in the extra embryonic area of the egg and then disappears (overview in Andersson 1973). Note, however, that Edgar et al. (2015) have recently devised a new model of spider development that proposes that the cells of the migrating cumulus also end up in the posterior growth zone. This model is based on studies of germ band formation in the entelegyne species Latrodectus mactans, Latrodectus geometricus, and Cheiracanthium 
mildei, but is not compatible with the results from Parasteatoda tepidariorum (AkiyamaOda and Oda 2003) and P. phalangioides (this study).

\subsection{2 "Preabdomen-postabdomen" subdivision of the opisthosoma}

Most studies of spider germ band development have focused on entelegyne spiders and this also includes all recent models for developmental genetic studies (e.g. Abzhanov and Kaufman 2000, Akiyama-Oda and Oda 2003, McGregor et al. 2008). In these spiders the opisthosoma develops from a growth zone that remains attached to the yolk surface throughout development. Therefore all opisthosomal segments that are formed by the growth zone lie on the yolk surface as well, and the opisthosoma gradually grows around the yolk as segmentation proceeds. By contrast, in P. phalangioides the formation of the opisthosoma is a two-phase process: the first three segments are formed like in entelegyne spiders, but then the growth zone bulges away from the body and therefore produces a "segmented tail". Intriguingly, this developmental mode has not only been reported from P. phalangioides (Claparède 1862; Emerton 1872), but also from several other haplogyne spiders (Holm 1940), Mygalomorphae (bird spiders) (Yoshikura 1958; Crome 1963; Crome 1964) and even from the Mesothelae (segmented spiders), the most primitive spider group (Yoshikura 1954). Thus, although this mode appears to be more complicated and is unusual when compared to other chelicerates and other arthropods, it appears to be the primitive mode of posterior segment addition in spiders.

Holm (1940) has coined the terms "preabdomen" and "postabdomen" for those segments on the yolk and those segments in the tail, respectively. He suggested that these two parts of the opisthosoma in non-entelegyne spiders are a remnant of a phylogenetically older subdivision of the arachnid opisthosoma into two subtagmata, and he compares the spider "pre-" and "postabdomen" to the mesosoma and metasoma of scorpions. Our data do not support this notion, because the initial border between "preabdomen" and "postabdomen" is between the third and the fourth opisthosomal segment, and this border does not correspond to the subdivision of the scorpion opisthosoma. In addition, the border between "preabdomen" and "postabdomen" shifts during development (see Figure 10) thus making a clear distinction of the two parts 
anyway difficult. We note, however, that an opisthosomal border between the third and fourth opisthosomal segment is indeed present in Ricinulei (hooded tickspiders). This border could thus be homologous to the initial border between "pre-" and "postabdomen" in the non-entelegyne spiders, but to our knowledge no embryological data is so far available for Ricinulei.

\subsubsection{Extreme leg length in pholcid spiders}

The length of spider legs in relation to body length varies significantly between different species. Pholcid spiders are remarkable for their extremely elongate walking legs. Interestingly, the allometric growth that leads to the extremely long legs sets in relatively late in embryogenesis. Before dorsal closure no remarkable difference in leg growth to species with shorter legs is detected, e.g. Parasteatoda tepidariorum and Cupiennius salei. A dramatic increase in leg growth then, however, occurs after dorsal closure and the formation of the prosomal shield. Therefore, future studies of the genetic control of leg length in P. phalangioides will have to focus on these stages.

\subsubsection{Conserved developmental steps for comparative expressional studies}

The detailed study of $P$. phalangioides embryogenesis revealed several differences between the embryonic development of entelegyne spiders, like $P$. tepidariorum and $C$. salei and haplogyne spiders (Mittmann and Wolff, 2012; Wolff and Hilbrant, 2011). These differences include the early organization of the germ disc, differently shaped germ band stages, as the result of the ancestral mode of opisthosoma development and $P$. phalangioides specific extreme leg growth staring during ventral stages. Also the overall developmental time is different for the different spider species, thus hours after egg deposition is not suited to compare temporal expression dynamics of genes. It is,

however, important to identify general processes, which are similar during embryogenesis to perform spatial and temporal comparison of candidate genes involved in appendage patterning processes in the two spiders. In both P. tepidariorum and $P$. phalangioides prosomal limb bud development starts after germ band formation, which 
is followed by limb bud elongation (Mittmann and Wolff, 2012). During inversion and dorsal closure of the embryo the prosomal appendage are further elongated and start differentiating in both species. This differentiation is continued during ventral closure and ends with growth of the appendages, especially the walking legs, which is more drastically observed in $P$. phalangioides compared to the entelegyne spider species observed so far.

In summary this embryonic development study shows, that P. phalangioides, C. salei and P. tepidariorum, share enough main developmental tissue rearrangements, which are sufficient to compare the onset and dynamic of expression pattern between species, especially at early leg development.

\subsubsection{General transcriptome optimization}

The genome of the fruit fly D. melanogaster consists of about 13,000 genes (Adams et al., 2000). Previous findings in the group of chelicerates indicate that their genomes contain a large number of duplicated genes (Di et al., 2015; Nossa et al., 2014; Schwager et al., 2007). A total number of about 30.000 genes were predicted for the genomes of the velvet spider Stegodyphus mimosarum (Sanggaard et al., 2014) and the scorpion Mesobuthus martensii (Di et al., 2015) and in the current transcriptome of the entelegyne spider $P$. tepidariorum roughly 40.000 genes were predicted (Posnien et al., 2014). The

preliminary transcriptome of P. phalangioides (Janssen et. al, 2015) contained 444.103 transcripts and 320.428 predicted genes. This transcriptome was sufficient to search for gene homologs, thus a majority of the genes presented in my thesis are based on this preliminary version of the transcriptome. The number of predicted genes is too high to represent the actual gene set of this species and would hamper the identification of differentially expressed genes. The number of predicted genes was reduced to 233.353 in the second assembly, but still higher than observed for the other spider species. Busco analysis of the improved P. phalangioides transcriptome revealed that $33 \%$ of the genes were duplicated. A similar percentage of duplicated genes was found in the currently annotated genome of the main spider model organism $P$. tepidariorum (personal communication with Dr. Nico Posnien and unpublished data, courtesy of Alistair 
McGregor, co-ordinator of the $P$. tepidariorum sequencing consortium). Thus the increased number of genes in the P. phalangioides transcriptome compared to the other spiders is unlikely caused by these duplicated genes. Two possible scenarios could have led to this large number of predicted gene: (1) wrongly or incomplete assembled transcripts. (2) Isoforms of one gene were assigned as different genes. To improve the number of genes and transcripts lowly supported transcripts were removed and transcripts probably representing isoforms were grouped into possible gene models based on the raw reads mapped to the assembly. This resulted in the reduction of the overall transcript number by especially losing weakly supported short transcripts, and in the reduction of predicted genes to 100.000. This is still higher than observed in the other chelicerate species and is probably caused by a larger amount of input sequences and the use of different sequencing methods.

\subsubsection{Up-regulated genes in the walking legs of $P$. phalangioides}

Transcriptomic resources can, in contrast to genomes, also be used to reveal the actually transcribed set of genes in different stages and tissues. To use the transcriptome of $P$. phalangioides not only to facilitate gene cloning, but also for differential gene expression studies, libraries of different nymphal tissues, including walking legs, the prosoma and the opisthosoma were generated and sequenced in addition to the mixture of embryonic stages. The differential expression analysis, comparing all these nymphal tissues to the embryonic data set, showed the largest number of differentially expressed genes, indicating the drastic expressional shift from embryonic to post-embryonic development. Thus this dataset provides a useful resource to study changes in gene regulation during moulting (for example cuticle formation) and has the potential to be used as a platform to identify genes involved in the transition from embryonic to postembryonic development. Comparisons of different the nymphal tissues identified a large number of differentially expressed genes for the prosoma and the opisthosoma. This can be used for further studies focusing on special characters of these particular tissues, like the pedipalps on the prosoma, or the spinnerets and breathing organs on the 
opisthosoma. The most restricted sample tissue were the walking legs of the nymphs. Accordingly, the number of up and down regulated genes is lowest for this tissue. Since my work has focused on the analysis of the genetic cues involved in the development of the special leg characteristics found in P. phalangioides, the 1.490 upregulated genes were studied in more depth. Previous studies from D. melanogaster and C. salei showed that the Notch pathway and its downstream factors are required for joint formation and leg growth (Celis et al., 1998; Prpic and Damen, 2009). This agrees perfectly with the fact that differential transcriptome analysis of the leg tissue in $P$. phalangioides also identified members of the Notch signalling pathway.

However, not all of these genes could be studied in greater detail and the number of candidate genes was filtered using the above-mentioned cut-offs and restrictions. It could be discussed if the filter criteria were appropriate to find possible gene targets, or if other criteria would have revealed better candidates. Alternative filtering with less restricted cutoffs (e.g. embryo mean $=1.000$ ) largely resulted in candidate genes already known for general leg development function during embryonic development including Dll, Notch and dpp (data not shown) (Celis et al., 1998; Goto and Hayashi, 1997; Wu and Cohen, 1999). This again reveals the huge potential of this transcriptomic resource to identify genes involved in leg development. The decision to discard all candidates lacking any annotation in any other organisms was based on two facts: First of all, many of the presumptive $P$. phalangioides specific genes did not show a proper ORF and thus might represent wrongly assembled transcripts; secondly, functional analysis in $P$. phalangioides is challenging and comparison to the expression and function in $P$. tepidariorum would be beneficial.

Four of the identified candidates genes did not show any similar sequences in any other species except for the spider P. tepidariorum genome. Also manual blast search did not lead to the identification of homologs in other species. Thus these candidates might represent newly evolved spider specific genes. Three of these candidates were successfully cloned. The expression pattern of the spider specific genes c95092 and c109239 did not show any expression in the walking legs, but had a similar expression in the cheliceres. The cutoff for the mean embryo counts was set to a relative low value (50). It is possible that the up regulation of these genes in the walking legs starts at 
nymphal stages, which would explain the lack of walking leg expression during embryonic stages. Comparisons of reverse transcriptase PCR for late embryonic stages and legs of early nymphal stages could support this hypothesis. However, it would be interesting to study a possible expression of these genes in walking legs of nymphs. This demands the establishment of an in situ hybridization protocol for these tissues, which is not available yet. The spider specific $c 109662$ is expressed in a ring like manner in the pedipalps and walking legs. Expression in a ring like manner suggests a role in leg patterning or segmentation (Prpic and Damen, 2009). A detailed phylogenetic study including more chelicerate and arthropod species as well as comparison to the expression in P. tepidariorum, and functional studies would thus be interesting to clarify the function and origin of this possibly newly evolved leg developmental gene.

The remaining four final candidates were predicted to be homologs of unc-4, odd-skipped (odds), a Kinesin-like calmodulin-binding protein homolog (KCBP) and the MDS1 and EVI1 complex locus protein, Evi1. The homeodomain protein unc-4 is not intensively studied in D. melanogaster; the only findings are based on embryonic expression data, where it is found in postmitotic neurons (Tabuchi et al., 1998). Consistent with these findings, data from C. elegans shows that unc-4 regulates the synaptic input of motor neurons by regulation of neurotransmitter vesicles (Lickteig et al., 2001; Miller et al., 1992). More recent findings show that the initial unc-4 gene was duplicated in the Drosophila genome and gained a new function in the male testis and is responsible for hybrid male sterility (Ting et al., 2004). Thus a functional comparison to the fly homologs would be interesting. Unfortunately cloning of this gene was not successful.

KBPC probably represents a gene with a conserved role during cell division indicated by microtubule motor activity and supported by findings from the plants Arabidopsis and tobacco as well as the unicellular flagellate green alga Chlamydomonas (Bowser and Reddy, 1997; Dymek et al., 2006). The P. phalangioides homolog c105514 is the only gene with no detectable expression during embryonic development at all. As mentioned above further studies will be performed to analyse a possible late onset of this gene during nymphal stages. In addition a phylogenetic analysis with similar genes from other species would be interesting to identify animal homologs and their function. 
The homolog of Evi1 c109624 was expressed in a spot-like manner in the legs. This kind of expression pattern usually hints to a neuronal function of the gene. Also the expression in the ventral neuroectoderm in the head, prosomal and opisthosoma suggest a role in neurogenesis. Previous findings for the zinc finger transcription factor Evi1 in mouse showed that this gene controls cell proliferation processes (Hirai, 1999) and it thus plays an important role in general embryonic development. Strong expression was for example found in the urinary system, bronchial epithelium, focal areas within the nasal cavities and cardiac tissues, but also in the developing limbs (Perkins et al., 1991). Furthermore it was found to be essential for proliferation/maintenance of hematopoietic stem cells (Goyama et al., 2008; Yuasa et al., 2005) and thus an interesting oncogene to study leukemogenesis (Hirai, 1999; Mucenski et al., 1988). Also in zebrafish it is involved in hematopoietic stem cells regulation (Konantz et al., 2014, 2015) and nephrogenesis together with Notch signalling (Li et al., 2014). Results for the chicken suggests that its function is required for the size of cartilage elements in the limbs (Celá et al., 2013). These functions in various vertebrates hint to a conserved role of this gene in the control of growth via regulation of cell proliferation and thus it is a promising candidate to study the extreme leg growth of $P$. phalangioides. Many studies were performed to understand its oncogene function in vertebrates (Goyama et al., 2008; Hinai and Valk, 2016). Studies in arthropod species could reveal general regulatory functions of this gene with other developmental genes. However, functional studies are required to analyse the exact function of the spot-like expression of $c 109624$ in P. phalangioides.

The other candidate is a homolog of odd-skipped (odds). odds has a conserved function during leg patterning and development (Angelini et al., 2012), especially in the distal part of the fly leg (Hao et al., 2003). The expression pattern in the tarsal region of the walking legs of $P$. phalangioides thus agrees perfectly with previous findings. Because $P$. phalangioides is one of only a few spider species with tarsus segmentation we performed preliminary expressional studies of two odds related genes in P. tepidariorum. Both genes are expressed in the walking legs of P. tepidariorum. The paralogs are expressed in differing number of rings and throughout the entire walking legs (Supplemental Figure 2), but always different to the expression found in P. phalangioides. This expressional difference of the genes in P. phalangioides and P. tepidariorum suggests that they could 
indeed drive tarsus developmental differences. However, three odds related homologs are found for the other entelegyne spider species C. salei (Damen et al., 2005). Thus it cannot be excluded that there is a possible third P. tepidariorum odds, which is more similar to the one studied in P. phalangioides. Unfortunately expressional data for the odds homologs the walking legs of $C$. salei are lacking. However, a preliminary phylogenetic analysis (Supplemental Figure 1) grouped the odds homolog Locus_17047 of P. tepidariorum and the P. phalangioides odds homolog studied here into one monophyletic group. Thus functional studies of the odds homologs in both spider species would be interesting to study a potential function involved in the different tarsus morphologies found for the two spiders.

In summary the expression pattern analysis showed that three of the six studied candidates showed expression in the legs. More generally the outcome of the GO enrichment and the identification of gene candidates involved in cell division, cell proliferation and limb development and the suggested unique odds expression in the P. $p$ tarsus indicate that the unbiased transcriptome approach and the methodical pipeline led to previously neglected interesting candidates. 


\section{Leg gap genes in appendage development of spiders}

Previous research showed that the formation of the proximo-distal axis in walking legs of arthropods is conserved and requires the leg gap gene function of the genes, homothorax (hth) and extradenticle (exd), dachshund (dac) and Distal-less (DIl) (e.g. Janssen et al., 2010). Previous findings from the spiders Cupiennius salei (C. salei) and Acanthoscurria geniculata (A. geniculata) showed that exd and $h$ th are duplicated in these spiders (Pechmann and Prpic, 2009; Prpic et al., 2003). In the following I present the phylogenetic relationships, expression patterns and gene function analysis of $h t h$, exd and dac in two additional spider species Parasteatoda tepidariorum (P. tepidariorum) and Pholcus phalangioides (P. phalangioides). Dll is in contrast to exd and hth not duplicated and was already studied in P. tepidariorum and P. phalangioides (Pechmann et al., 2011.)

\section{1 homothorax and extradenticle}

The gene hth in Drosophila melanogaster (D. melanogaster) encodes a protein that contains two highly conserved protein domains. The MEIS domain is a protein-protein binding domain that mediates the binding between $H$ th and its co-factor Exd (Pai et al., 1998; Rieckhof et al., 1997). In addition to the MEIS domain the Hth protein also contains a modified homeodomain (TALE-HD) that facilitates the binding of Hth to DNA (e.g. Jacobs et al., 1999; Mann and Affolter, 1998). In many cellular contexts Hth first binds to Exd in the cytoplasm, and the pair is then translocated to the nucleus where they bind DNA together with several Hox proteins. The different Hox proteins recognize very similar DNA sites and thus must rely on protein partners like Hth and Exd to obtain additional binding specificity. Hth and Exd cooperate with most Hox genes and are also known to team up with other DNA binding proteins as well. Therefore, Hth and Exd are involved in the formation and function of a large number of organs and tissues, for example leg and antenna specification, eye development, renal tubule growth, muscle fiber identity, and regulation of neuron differentiation (Bryantsev et al., 2012; Casares 
and Mann, 2001; Dong et al., 2002; Hasegawa et al., 2011, 2011; Pai et al., 1998; Wang et al., 2004).

Hth and Exd are widely conserved in the Metazoa and homologs have been described from all major arthropod clades, onychophorans, and from vertebrates, where $h$ th is called Meis and exd is called PBX (e.g. Janssen et al., 2010). Interestingly, hth and exd are duplicated in two spider species $C$. salei and A. geniculata and the duplicates are expressed in a pattern differing from the first paralog in the walking legs, which is most drastic for hth2 (summarized in the Introduction and Pechmann et al., 2010). The novel expression pattern of the exd2 duplicate is conserved in these two spider species, but the duplicated $h$ th gene is expressed in contrast to its paralog in numerous segmental rings of differing number in the walking legs of $C$. salei and A. geniculata. This expression pattern suggests that one hth copy has retained the original functions of this highly pleiotropic gene, whereas the second hth copy has undergone diversification after neofunctionalization. To study the grade of diversification and to confirm the conservation of the exd after duplication we studied these genes in two additional spider species P. tepidariorum and P. phalangioides. In the next section first the phylogenetic analysis and expression of $h$ th will be described and followed by the study of exd.

\subsubsection{Duplicated $h t h$ and genes in $P$. tepidariorum and $P$. phalangioides}

Previous studies in the spiders Cupiennius salei (C. salei) and Acanthoscurria geniculata (A. geniculata) have shown that $h$ th is duplicated in these spider species (Pechmann and Prpic, 2009; Prpic et al., 2003). In order to investigate whether a set of two hth genes is a general character of spiders and probably of arachnids as a whole, we searched for $h$ th sequences in the transcriptomes of two additional spiders and scorpions, the cellar spider $P$. phalangioides (Janssen et. al 2015) and the common house spider $P$. tepidariorum (Posnien et al. 2014). And in the genomes of the Chinese scorpion Mesobuthus martensii (M. martensii) (Di et al., 2015) and the Arizona bark scorpion Centruroides sculpturatus (C. sculturatus) (I5K Project, Bark Scorpion Genome Project). These searches and the subsequent large phylogenetic analysis to identify only true chelicerate $h$ th orthologs were performed as explained in the methods section. As 
expected from previous studies in spiders, we found two hth homologs in the transcriptomes of $P$. phalangioides and $P$. tepidariorum (group of hth homologs is depicted in red, Supplemental Figure 5 and Supplemental Figure 6). In the large phylogenetic analysis we also included previously cloned sequence fragments of $P t-h t h 1$ (Khadjeh et al., 2012), Pt-hth2 (cloned by Sara Khadjeh with degenerated primers described in Prpic et al., 2003) and Pp-hth1 (also cloned from degenerated primers and prolonged with RACE PCR). The phylogenetic analysis shows that they are largely identical with the sequences found in the transcriptomes of $P$. tepidariorum and $P$. phalangioides. In the genome of $C$. sculpturatus we were able to identify three sequences with similarity to hth, from one of which, CSCU004313, is largely comparable to the previously described C. sculpturatus hth homolog (Sharma et al., 2015). In the genome of the scorpion M. martensii only two sequences were identified with similarity to $h$ th. An additional sequence was found for M. martensii when searched with the $h$ th paralogs of P. phalangioides MMa00258, which is only short and thus might be an incompletely assembled fragment.

In the previous studies of $h$ th genes in C. salei and A. geniculata (Pechmann and Prpic, 2009; Prpic et al., 2003), the hth genes were assigned to two groups, named hth1 and $h$ th2, on the basis phylogenetic analyses and similarities of the expression patterns. The duplicated hth genes from P. phalangioides and P. tepidariorum can also be placed in these two groups (see next chapters). Interestingly, however, the $h$ th1 genes from all four spider species are much more similar amongst themselves than the $h$ th2 genes: pairwise alignments of the full-length spider $h t h$ protein sequences show that the $h t h 1$ homologs have a sequence similarity ranging from $84.1 \%$ to $95.5 \%$, whereas the hth 2 homologs range between 72.5 to 90.3\% (Supplemental Table 8 and Supplemental Table 9). The same trend is true for pairwise comparisons with hth of Drosophila that shows less sequence similarity to the hth2 paralog protein sequences than to hth1 (Supplemental Table 6 and Supplemental Table 7). In summary, the $h$ th1 genes form a more homogeneous group than the $h$ th2 group, which is more diverse, and the genes in the $h$ th1 group are also more similar to the single Drosophila ortholog than the genes in the $h$ th2 group. 


\subsubsection{Phylogenetic analysis reveals duplication of $h t h$ in spiders}

In order to investigate the relationship of the $h t h$ sequences from $P$. phalangioides and $P$. tepidariorum with the previously known arachnid sequences and the $h$ th-related genes from other metazoans, we performed a more detailed phylogenetic analysis with the $h t h$ homologs found in the transcriptomes of P. phalangioides, P. tepidariorum (Supplemental Figure 6) and hth protein sequences from other chelicerates (M. martensii, Limulus polyphemus, Phalangium opilio, Centruroides sculpturatus, Archegozetes longisetosus), mandibulates, onychophorans and vertebrates (Figure 22). The vertebrate Meis sequences form a monophyletic group in the tree and are clearly separated from the $h t h$ sequences from arthropods and the onychophoran. The insect and crustacean sequences also form a monophyletic group that is well separated from the group that contains the chelicerate sequences. Interestingly, the chelicerate sequences are divided into two separate monophyletic groups. The first group contains all the sequences from the horseshoe crab, scorpions, harvestman, and mite, plus the previously described hth1 copy from C. salei and A. geniculata, and one hth copy each from P. tepidariorum and $P$. phalangioides, that we therefore also denote as $h$ th1 (blue shading, Figure 22).

The second group (green shading, Figure 22) exclusively contains the hth2 copy from $C$. salei and A. geniculata, and the second copy from P. tepidariorum and P. phalangioides (therefore denoted as hth2). These data indicate that, although other existing hth duplicates are found in other chelicerates (e.g. in scorpions), the hth2 gene is an hth duplication specific to spiders.

\subsubsection{Expression of $h$ th1 and hth2 during $P$. tepidariorum and $P$. phalangioides embryogenesis}

Previous studies in C. salei and A. geniculata have already suggested that the two hth duplicates differ significantly in their expression patterns (Pechmann and Prpic, 2009; Prpic et al., 2003). We therefore investigated whether this distinction can be confirmed by the gene expression patterns of hth 1 and $h$ th 2 in P. tepidariorum and P. phalangioides. In the developing germ band of P. tepidariorum and P. phalangioides hth1 is strongly 
expressed throughout the ventral neuroectoderm, in the anterior part of the head and in the proximal part of the prosomal limb buds.

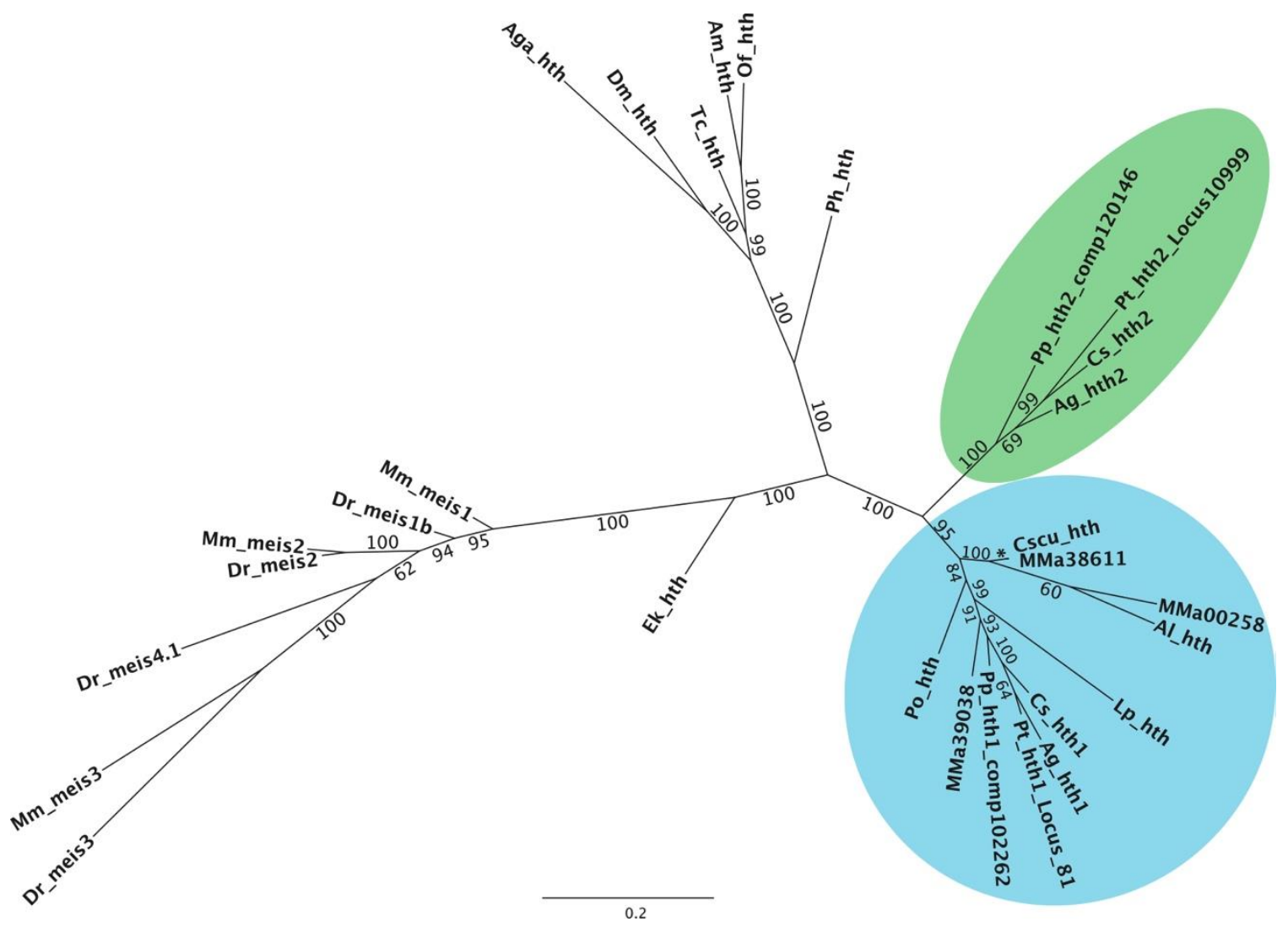

Figure 22: Unrooted 50\% phylogenetic majority rule consensus tree of hth orthologs

The phylogenetic analysis resulted in three major groups of $h t h$. The vertebrate group is shown on the left, the insect $h$ th group is at the top and the chelicerate homologs of $h$ th are forming two branches in the right. The two paralogs of $h$ th after duplication in spiders are present in two distinct phylogenetic groups. The conserved hth1 forms a monophyletic group with all other chelicerate hth homologs (blue circle). The spider hth2 paralogs cluster together forming a spider specific $h$ th2 group (green cycle) separate from the remaining chelicerate hth1 paralogs. For $C$. sculpturatus only the previously described hth homolog (Sharma et. al. 2015) was used due to missing knowledge about the quality of the assembled $C$. sculpturatus transcriptome. Branch lengths of the tree represent the substitutions per site. Numbers at the edges of the phylogram give the probability of each clade indicated by clade credibility values. The Jones amino acid substitution model was chosen. The average standard deviation of split frequencies dropped below 0.01 after reaching 179.000 generations. A total number of 718 trees were written in two files (each file contained 359 trees of which 270 were sampled). Abbreviations are given in Supplemental Table 5 
During brain differentiation the expression in the head becomes restricted to the nonneurogenic ectoderm, the labrum, the stomodeum and two stripes next to the stomodeum (Figure $23 \mathrm{D}$ and Figure $24 \mathrm{D}$ ). When the prosomal limb buds start developing, $h$ th1 is expressed in the entire prosomal appendages excluding the most distal part (Figure $23 \mathrm{~A}$ and Figure $24 \mathrm{~A}$ ). This strong expression in the prosomal appendages remains until late dorsal closure stages, but then decreases slightly, especially in the distal portion, mainly in the metatarsus (Figure $25 \mathrm{~F}$ and Figure $26 \mathrm{~F}$ ). The expression of $h t h 1$ in the head and prosoma is thus virtually identical in both species throughout embryonic development. However, we found differences in the opisthosomal expression patterns at the beginning of inversion. Pt-hth1 is expressed in the entire opisthosoma at same strength except for the posterior end and one spot next to the limb bud of the second opisthosomal segment (O2 Figure 23 E). In P. phalangioides the expression in the opisthosoma is more restricted to presumptive ventral tissue and no expression can be detected in the developing heart tissue (Figure $24 \mathrm{E}$ ).
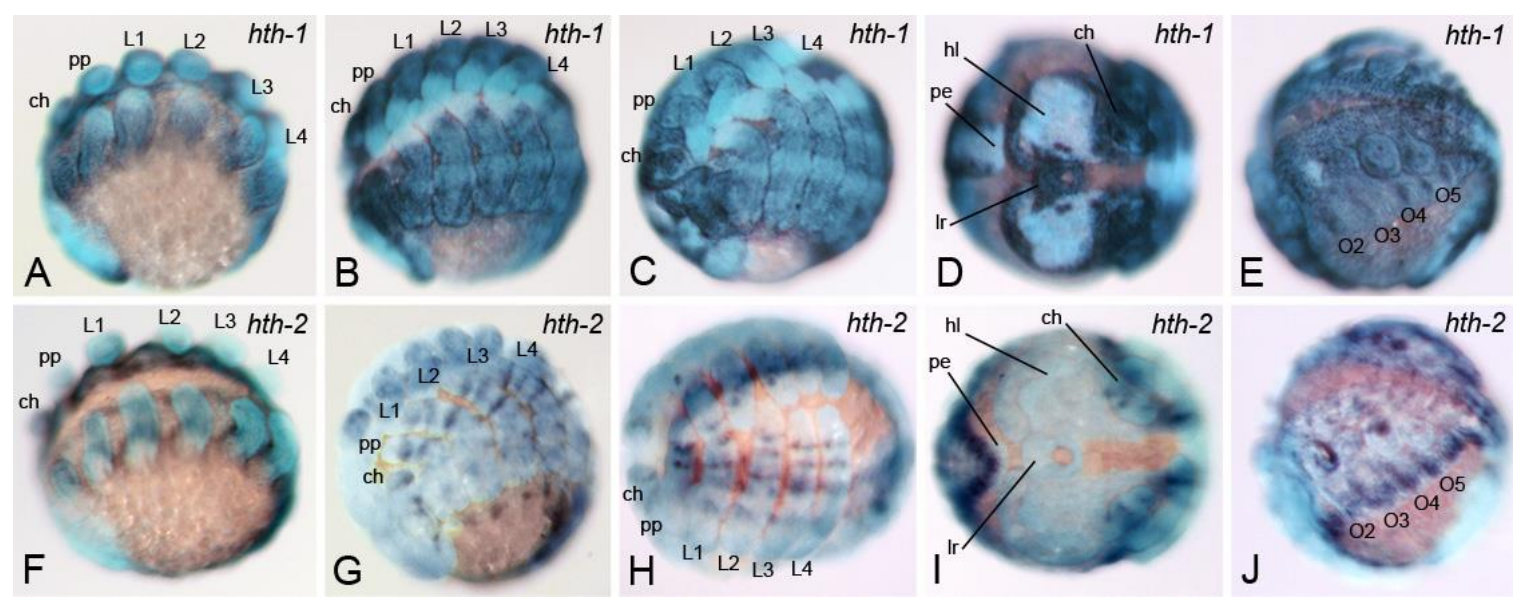

Figure 23: Expression of $h$ th paralogs in $P$. tepidariorum

Expression of Pt-hth1 in the prosomal appendages of embryos during limb bud elongation (A), inversion (B), and dorsal closure (C), all in lateral view. D: Frontal view of Pt-hth1 expression in the head of an embryo during inversion. E: Posterior view of opisthosomal expression of $h t h 1$ in $P$. tepidariorum during inversion. F: Lateral view of Pt-hth2 expression in the ventral neuroectoderm during limb bud elongation. G: Rings of $h t h 2$ are expressed in the prosomal appendages during inversion, lateral view. H: Ventral view of $h t h 2$ ring-like expression in the pedipalps and walking legs during dorsal closure. I: No expression of Pt-hth2 in the head during inversion, frontal view. J: Opisthosomal expression of $h t h 2$ in $P$. tepidariorum during inversion, posterior view. Embryos shown in A-D and F-I are oriented with the anterior side to the left. hl: head lobes, lr: labrum, ch: Chelicera, pp: Pedipalps, L1-L4: Walking legs pairs 1-4, 01-05: Opisthosomal segments 1-5, pe: posterior end. 
Expression of $h t h 2$ differs from $h t h 1$ in terms of timing and pattern. At germ band stages in P. tepidariorum hth2 is expressed more ventrally and weaker as Pt-hth1 in the neuroectoderm and an expression domain in the head tissue or the developing appendages is absent (Figure 23 F). First expression of Pt-hth2 in the prosomal appendages is present at early inversion stages, which then differentiates until ventral closure stages (see next chapter), but is always different from hth1 expression (Figure 23 G). In the opisthosoma Pt-hth2 has a more diffuse expression pattern and can be found adjacent to the limb buds, with strongest expression near the second and fourth opisthosomal limb bud (02 and 04) and dorsally in the tissue of the developing heart (Figure $23 \mathrm{~J}$ ). In P. phalangioides hth2 expression starts in the ventral neuroectoderm at germ band stages comparable to Pt-hth2 (Figure $24 \mathrm{~F}$ ). Also the onset of expression in the prosomal appendages of $P p-h t h 2$ is comparable to Pt-hth2, starting during the inversion stage, whereas the pattern of expression is different (Figure $24 \mathrm{G}$, see also next chapter).
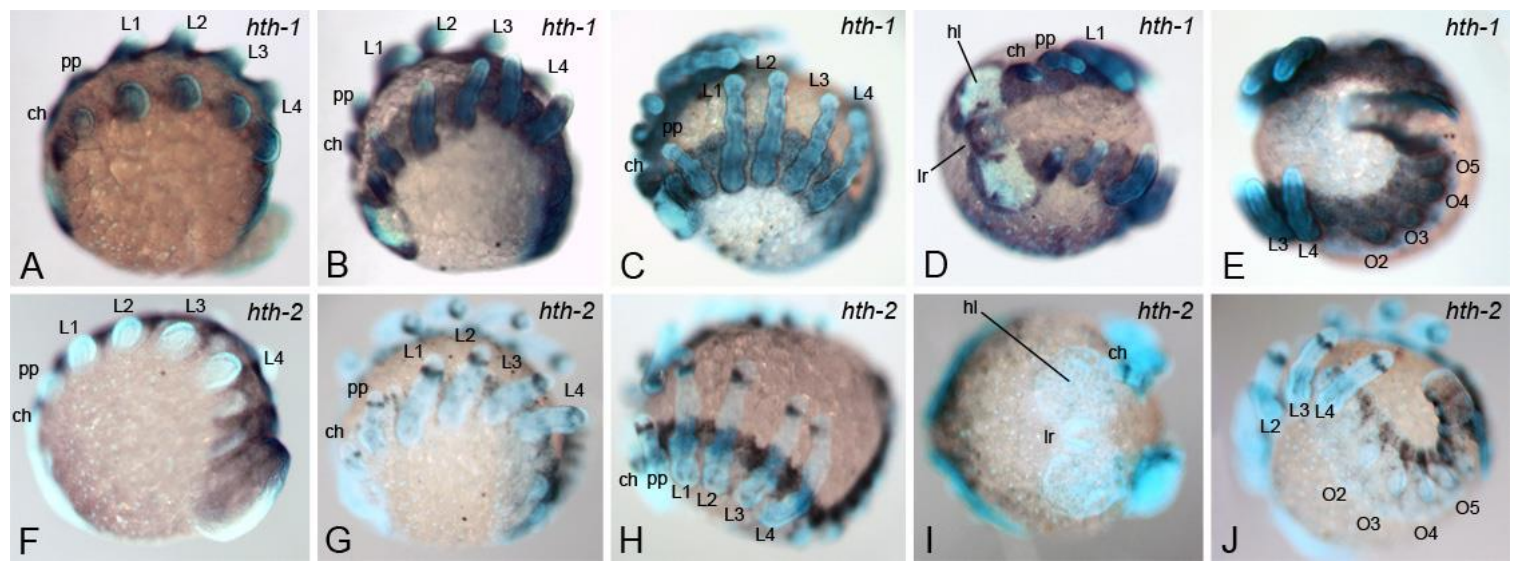

Figure 24: Expression of $h$ th paralogs in $P$. phallangioides

Embryonic expression of Pp-hth1 in the prosomal appendages during germ band stages (A), inversion (B), and dorsal closure (C), all in lateral view. D: Frontal view of $P p$-hth1 expression in the head of an embryo during inversion. E: Posterior view of opisthosomal expression of hth1 in $P$. phalangioides during inversion. F: Lateral view of $P$ - $h$ th 2 expression in the ventral neuroectoderm during limb bud elongation. G: One distal ring of $h$ th2 is expressed in the prosomal appendages during inversion, lateral view. H: Ventral view of the distal Pp-hth2 ring in the pedipalps and walking legs during dorsal closure. I: No expression of $P p$-hth2 in the head during inversion, frontal view. J: Opisthosomal expression of hth2 in P. phalangioides during inversion, posterior view. All embryos (except panel E) are oriented with the anterior side to the left. hl: head lobes, lr: labrum, ch: Chelicera, pp: Pedipalps, L1-L4: Walking legs pairs 1-4, 01-05: Opisthsomal segments 1-5. 
The expression of $h t h 2$ in the opisthosoma differs significantly between P. phalangioides and P. tepidariorum. In P. phalangioides hth2 is not expressed in heart tissue on the presumptive dorsal side, but expression is restricted to the ventral side of the neuroectoderm and a spot ventrally to each opisthosomal limb bud (Figure $24 \mathrm{~J}$ ).

Because $h t h$ expression not necessarily also reflects the region of the functional protein and no antibodies are available for $h t h$ homologs of spiders, we tried to examine the gene function using gene knockdown via parental RNAi. Although two different dsRNA injection procedures were tried no obvious phenotype was observed so far.

\subsection{4 hth expression in the prosomal appendages of spiders}

The largest expression differences of the $h$ th paralogs in spiders were found in the prosomal appendages (chelicerae, pedipalps and walking legs). To analyse these differences in more detail we studied dissected prosomal appendages of $P$. tepidariorum and $P$. phalangioides to compare them with the known expression profiles in C. salei and A. geniculata (Pechmann and Prpic, 2009; Prpic et al., 2003). Expression of hth1 is very similar in P. tepidariorum and P. phalangioides. Until early dorsal closure stages hth1 is strongly expressed in the proximal and medial domain of the pedipalps and the walking legs, but is excluded from the tip of both appendage types. This expression profile is retained throughout most of development; only in embryos undergoing dorsal closure the medial expression becomes slightly weaker (Figure $25 \mathrm{D}$ and F and Figure $26 \mathrm{D}$ and F). Strong expression of $h t h 1$ can also be observed within almost the entire chelicera throughout embryonic development (Figure 25 A-B and Figure 26 A-B).

Expression of $h$ th2 in the chelicera of $P$. tepidariorum starts as a relatively diffuse domain (Figure $25 \mathrm{H}$ ), which later becomes more restricted forming two stripes (Figure $25 \mathrm{I}$ ) and finally forms an irregular ventral domain of expression at late ventral closure stages (Figure $25 \mathrm{~J}$ ). In the pedipalps of $P$. tepidariorum hth2 expression starts as a weak ring at the distal end of the medial portion of the pedipalp (asterisk, Figure $25 \mathrm{~L}$ ), that increases in expression levels during further development. In addition, a second ring appears (arrow, Figure $25 \mathrm{M}$ ), and also becomes stronger during development (Figure $25 \mathrm{~N}$ ). In 
contrast to the expression in the pedipalps, Pt-hth2 in the legs is activated as two weakly expressed rings (asterisks, Figure $25 \mathrm{P}$ ). The remaining dynamics of the expression pattern, however, are similar in pedipalps and legs: the initial rings become stronger during further development and an additional ring appears proximal to the initial two rings.

The expression pattern of hth2 in P. phalangioides also comprises ring domains, but differs in its dynamics from hth2 in P. tepidariorum. In the chelicera Pp-hth2 is first expressed as a ring in the medial part, which broadens and partially dissolves during development (Figure $26 \mathrm{G}$ and $\mathrm{H}$ ). In the pedipalp Pp-hth2 expression starts as one ring in the distal part, which then dissolves into a diffuse patchwork of distal expression, and in addition a number of weak segmental rings appear more proximally (Figure 26 I and $J)$. In the walking legs $P p$ - $h$ th2 expression also starts as a ring in the distal part and is in addition expressed in a spotted manner along the ventral side (Figure $26 \mathrm{~K}$ ). The strongly expressed distal $h$ th2 ring in the walking legs broadens during further development, and additional very weak rings appear near the future leg joints (Figure 26 L).

We have also restudied here the expression profile of hth2 in C. salei, because in the original publication (Prpic et al., 2003), only the expression pattern in the fully formed embryonic leg was shown, but the expression profile of this gene is actually more complex. Cs-hth2 has a diffuse expression in the chelicera with a faint medial ring (Figure $27 \mathrm{~A}$ ). In early pedipalps it is weakly expressed in two rings in the medial pedipalp part (arrowhead and arrow, Figure $27 \mathrm{~B}$ ). The proximal one of these two rings broadens (arrow and bar, Figure 27 D) during further development and the more distal ring divides giving rise to two rings (arrow and bar, Figure 27 C-D). Additionally one distal expression domain appears de novo (asterisks, Figure $27 \mathrm{C}$ ). In later stages these four rings of expression in the pedipalp become stronger and better distinguishable (Figure 27 D). Cs-hth2 in early walking legs is expressed in a proximal, medial and distal ring (arrowhead, arrow and asterisks, respectively, Figure 27 E). All three rings broaden during development (indicated by bars in Figure $27 \mathrm{~F}$ and G), and then split into two rings (Figure $27 \mathrm{H}$ ). This results in the presence of one ring near each of the developing leg joints in late stages of embryonic development 


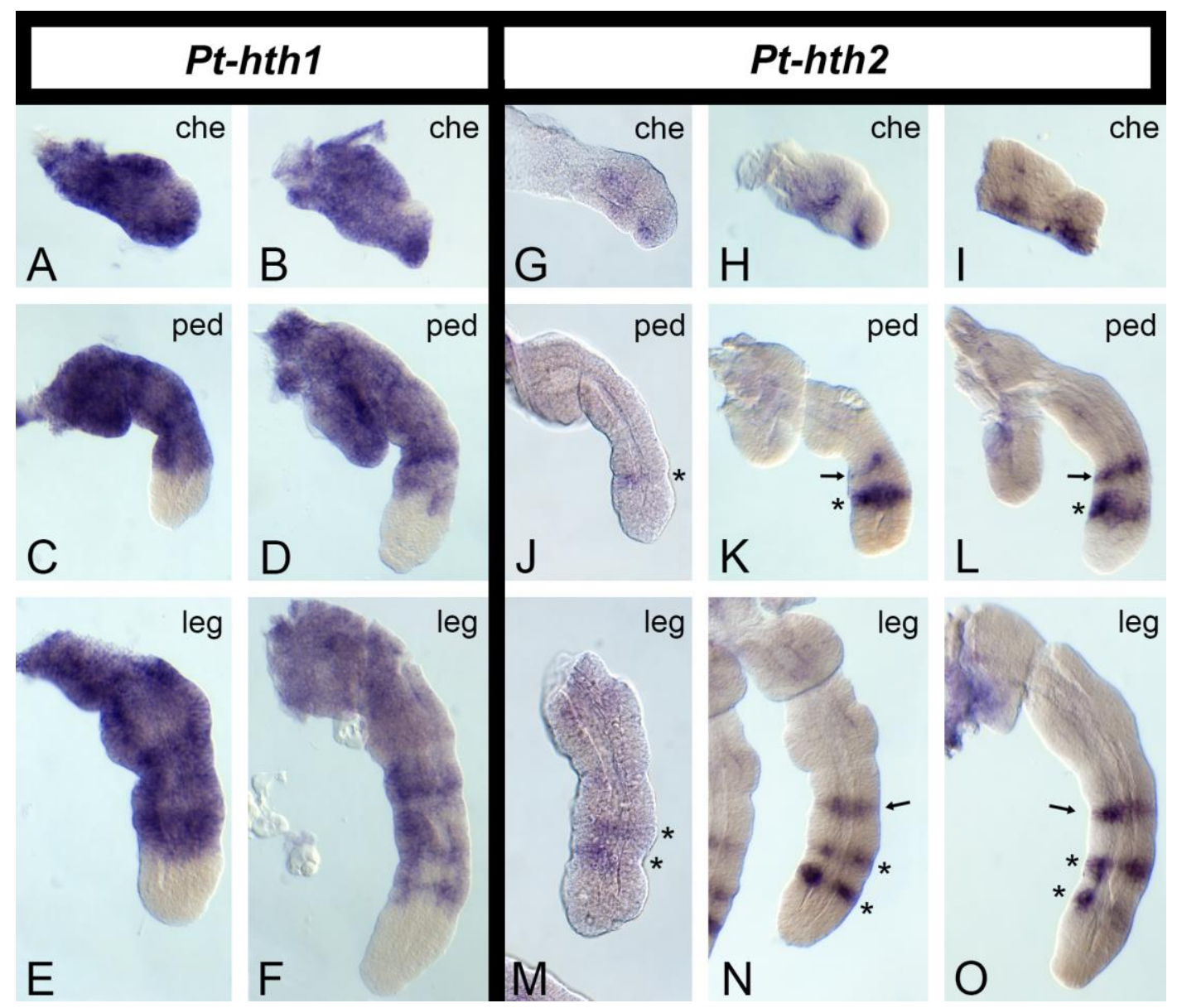

Figure 25: Expression of $h$ th paralogs in prosomal appendages of $P$. tepidariorum

A-F: Expression of hth1 in prosomal appendages of $P$. tepidariorum. A and B: Preparations of chelicera during inversion (A) and ventral closure (B). C and D: Expression of $h$ th1 in the pedipalps during inversion (C) and ventral closure (D). E and F: Walking legs during inversion (E) and ventral closure (F). G-O: Expression of $h$ th2 in prosomal appendages of $P$. tepidariorum. G-I: Preparations of chelicera during early inversion (G), late inversion (H) and ventral closure (I) J-L: Preparations of pedipalps during early inversion $(\mathrm{J})$, late inversion $(\mathrm{K})$ and ventral closure (L). The asterisk indicates the $h t h 2$ ring in the distal part of the pedipalp starting during early inversion. The arrow points to the second $h$ th2 ring appearing during late inversion. M-0: Preparations of walking legs during early inversion $(\mathrm{M})$, late inversion $(\mathrm{N})$ and ventral closure $(0)$. The asterisk marks the start of hth2 walking leg expression starting directly with two rings during early inversion. The arrow marks the start of expression in a third $h t h 2$ in the walking legs proximal to the two initial rings. che: chelicera. ped: pedipalp. leg: walking leg. 


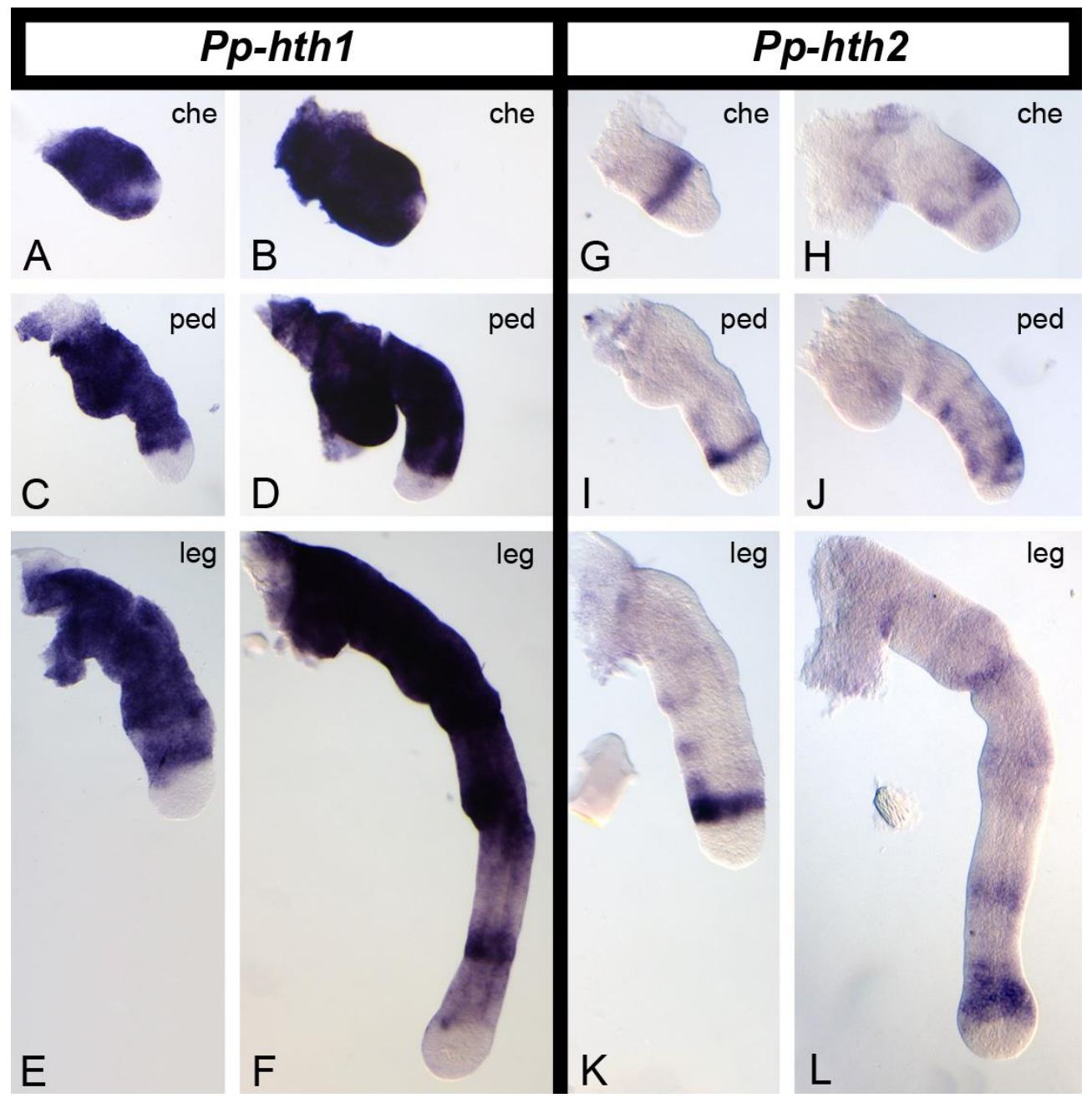

Figure 26: Expression of $h$ th paralogs in prosomal appendages of $P$. phalangioides

A-F: Expression of hth1 in prosomal appendages of $P$. phalangioides. A and B: Preparations of chelicera during limb bud elongation (A) and ventral closure (B). C and D: Expression of hth1 in the pedipalps during limb bud elongation (C) and ventral closure (D). E and F: Walking legs during limb bud elongation (E) and ventral closure (F). G-L: Expression of $h t h 2$ in prosomal appendages of $P$. phalangioides. G and H: Preparations of chelicera during inversion (G) and ventral closure (H). J and I: Expression of exd1 in the pedipalps during inversion (C) and ventral closure (D). $K$ and $L$ : Walking legs during inversion (E) and ventral closure (F). For detailed description of the expression pattern please see text. che: chelicera. ped: pedipalp. leg: walking leg. 


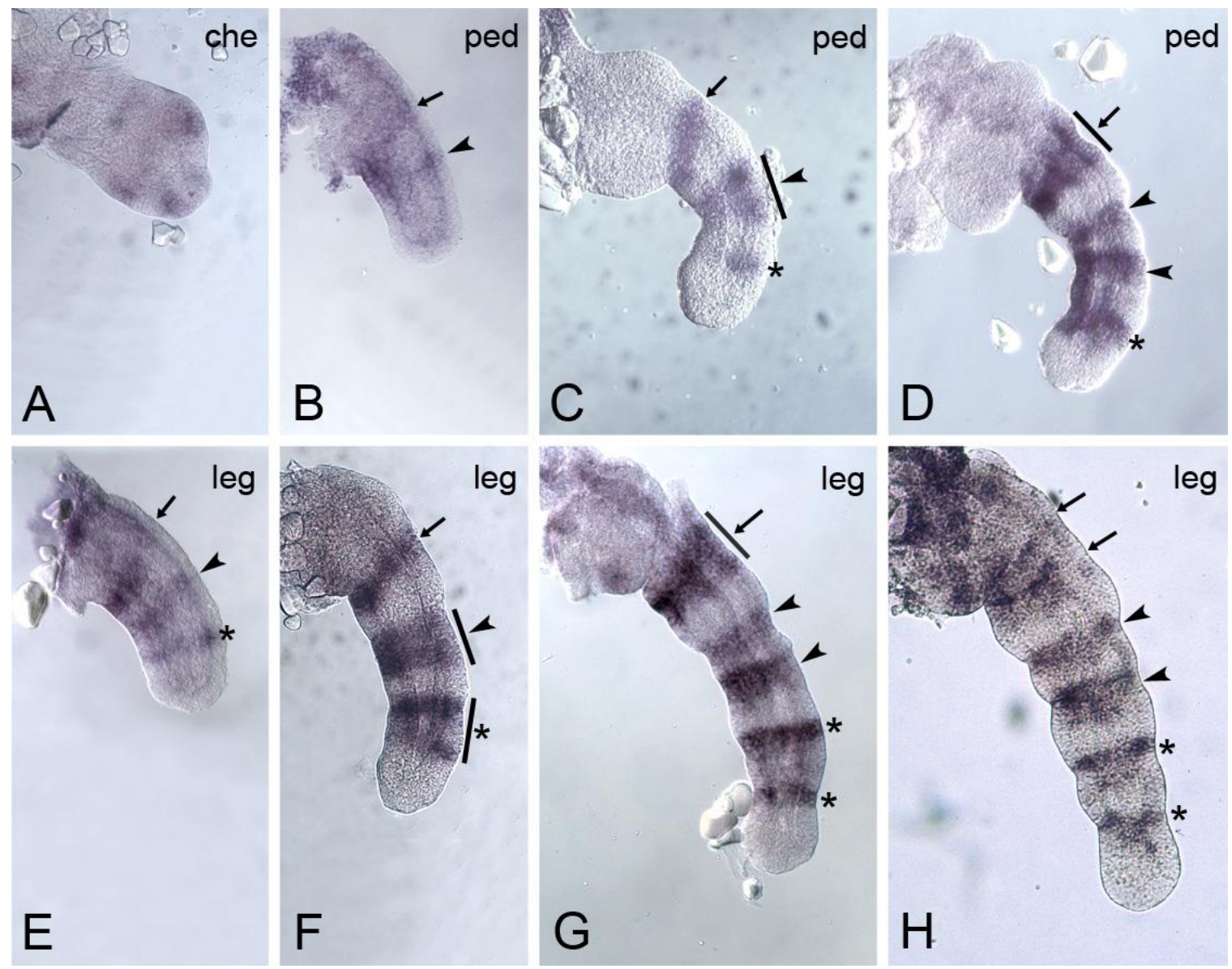

Figure 27: Expression of $h$ th 2 in prosomal appendages of $C$. salei

A: Weak expression of $h t h 2$ in the medial and distal domain of the chelicera during late inversion. B-D: Expression of $h$ th2 in the pedipalps of $C$. salei during limb bud elongation (B), during germ band elongation (C) and during mid inversion (D). The arrow points to the proximal hth2 ring of the pedipalp, which broadens prior to presumptive division during mid-inversion (indicated by the bar). The arrowhead points to the medial $h t h 2$ ring, which broadens shortly before inversion (bar in C) and is then present as two rings during mid-inversion (two arrowheads in D). The asterisk marks the distal hth2 ring staring to be expressed shortly before inversion. E-F: Walking legs expressing hth2 during germ band elongation (E), shortly before inversion (F), during midinversion (G), and during dorsal closure (H). The arrow marks the proximal hth2 ring and its descendent. The arrowhead marks the medial $h$ th2 ring and the asterisk marks the distal $h$ th2 ring and its descendants after divisions. Bars always indicate broadening of the ring expression. A division of the initial ring follows this broadening and results into two independent $h$ th 2 rings. Staining and preparation of the appendages was performed by Dr. Nikola-Michael Prpic-Schäper. che: chelicera. ped: pedipalp. leg: walking leg. 


\subsubsection{Duplication of exd genes in P. tepidariorum and P. phalangioides}

Previous studies of the spiders A. geniculata and C. salei also found a duplication of exd (Pechmann and Prpic, 2009; Prpic et al., 2003). Cloning and expressional analysis of the exd homologs in P. tepidariorum and $P$. phalangioides was already started before transcriptomic resources were available. With the use of degenerated primers (as described in Prpic et al., 2003) short fragments of two exd homologs were obtained in each spider used here. For $P$. phalangioides it was also possible to prolong these sequences using RACE PCR. To identify longer fragments of these exd homologs, they were searched in the transcriptomes of $P$. phalangioides (Janssen et. al 2015) and $P$. tepidariorum (Posnien et al. 2014), after they became available. With the standard search procedure merely two possible candidates were found in both spider transcriptomes. Thus the e-value threshold was lowered to $\mathrm{e}^{10}$. To reveal the time-point of the exd duplication in the lineage of the chelicerates we also searched for exd homologs in the genomes of two scorpions M. martensii (Di et al. 2014) and C. sculpturatus (I5K Project, Bark Scorpion Genome Project). The large phylogenetic tree to identify true homologs of hth (indicated in red) and exd (indicated in black) clearly shows that the cloned exd paralogs are largely identical with the sequences found in the transcriptomes of $P$. tepidariorum and P. phalangioides and that there are the only two exd homologs present in these spiders (Supplemental Figure 6). In the genomes of the scorpions C. sculpturatus and M. martensii I in contrast identified five sequences with similarity to exd. Whether this is the result of additional scorpion specific duplication events or caused by incompletely assembled transcripts or if these are simply isoforms of one gene was not further investigated here. The paralogs found for P. tepidariorum and P. phalangioides were named according to sequence identity (Supplemental Table 2) to the sequences previously described for $C$. salei, which is also reflected by similar expression patterns described in the following parts. Thus comp_102492 and Locus_1_Transcript_5866/166847 and the according clones are referred to as exd1 and comp122137 as well as Locus_1_Transcript_58675/166847 as exd2. Similar as found for the conserved hth1 homologs we see large sequence similarities when comparing sequence identities after multiple exd alignment. But in case of exd this is true for both 
paralogs. Pairwise alignments of the spider exd protein sequences show that the exd1 homologs have a sequence similarity ranging from $87.33 \%$ to $96.09 \%$ (Supplemental Table 3), and also the exd2 homologs range within 87.56 to $90.39 \%$ (Supplemental Table 4). Even in the multiple alignments of both spider exd paralogs the similarities are at least $83.40 \%$ (Supplemental Table 2).

\subsubsection{Phylogenetic analysis of exd homologs in spiders and chelicerates}

This high sequence similarity of and also between the exd paralogs is also reflected in the large phylogenetic tree that was performed to identify exd and hth orthologs in the spiders P. tepidariorum and P. phalangioides. Although 9002 trees were sampled after 3.000.000 generations the phylogenetic relationships are not properly resolved for the different chelicerate sequences. All chelicerate exd (indicated in black, Supplemental Figure 5 and Supplemental Figure 6) are resulting in a polytomy without forming proper paralog clusters indicated by low support values of the posterior probabilities. This is an indication that the paralogs among themselves but also across species have not diversified as rapidly as $h$ th 2 and are more similar instead. To clarify the phylogenetic relationships in more detail we performed a second tree from a subset of exd protein sequences (Supplemental Figure 3 and Supplemental Figure 4). This tree is slightly better resolved and three major groups are formed: the vertebrate group containing all PBX paralogs and one outlier from the horseshoe crab L. polyphemus; the pancrustacean group including the insect and Parhyale hawaiensis exd homologs; and one group including all chelicerate sequences. However, all branches are only supported with low posterior probabilities. Within the chelicerate group, the exd1 paralogs cluster together, but the support value for this clade is with 50 also very low. In summary also this phylogenetic tree shows that exd is in general very conserved on the protein level, as already indicated by similarities after pairwise comparisons, which is true for both paralogs. Thus no prediction of the timing of the duplication event in the lineage leading to the spiders can be clearly stated. 


\subsubsection{Expression of exd during embryonic development of $P$. tepidariorum and $P$. phalangioides}

We observed the expression of the two exd paralogs, exd1 and exd2 in P. tepidariorum and P. phalangioides. Especially the expression in the prosomal appendages differs between the two paralogs, but this is conserved for both spider species. Expression of exd1 starts with early limb bud development in the ventral neuroectoderm, the entire chelicera and pedipalps as well as the proximal base of the walking legs (detailed description below) in both spider species (Figure 28, A and arrow, Figure 29, A). In contrast to similar expression in the prosomal appendages, the expression in the head and opisthosoma shows differences. exd1 is strongly expressed in the labrum of $P$. tepidariorum (Figure 28, E and F), but only very weak in P. phalangioides (Figure 29, E). In the opisthosomal neuroectoderm exd1 is expressed in a more defined way for $P$. tepidariorum compared to P. phalangioides. Pt-exd1 is expressed in a ventral $(\mathrm{H}$, arrow) and a dorsal ( $\mathrm{H}$, arrowhead) stripe as well as in a specific pattern for each opisthosomal limb buds, Pp-exd1, however, is expressed in a broad dorsal domain. The expression of the second exd paralog, exd2, differs compared to its paralog, exd1, but is very much conserved between the two observed spider species. The expression of exd2 starts at early limb bud development in P. phalangioides, covering the proximal part of the chelicera and as a medial stripe in the other prosomal appendages (arrow, Figure 31, A). In P. tepidariorum expression starts slightly later during limb bud elongation, but in a visual identical pattern (detailed prosomal appendage expression description see below). Exd2 expression in the head of both spiders starts with early inversion in the labrum and as a rim surrounding the head lobes (arrow, Figure $30 \mathrm{~F}$ and arrowhead, Figure $31 \mathrm{~F}$ ). The rim remains in this early anterior head tissue during rearrangements of the head tissue throughout embryonic development (arrow, Figure $30 \mathrm{D}$ and arrowhead, Figure $31 \mathrm{E}$ ). The opisthosomal expression of exd2 in P. tepidariorum is first covering the entire dorsal neuroectoderm (arrowhead, Figure $30 \mathrm{G}$ ) and gets restricted to a medial stripe in the neuroectoderm covering the opisthosomal limb buds during inversion (arrowhead, Figure 30, $\mathrm{H}$ and asterisk, Figure 31, H). In the opisthosoma of $P$. phalangioides exd2 is expressed similarly to Pt-exd2 prior to inversion (Figure $31 \mathrm{G}$ ), but 
differentiates differently during dorsal closure. Pp-exd2 is then expressed in the opisthosomal neurogenic tissue dorsally to the limb buds (asterisk, Figure $31 \mathrm{H}$ ) and ventrally on the limb bud on the fourth opisthosomal segment (arrowhead, Figure $31 \mathrm{H}$ ). In summary the expression of each of the exd paralogs is conserved in most tissues between the two spider species, but differs when the two paralogs are compared to each other.

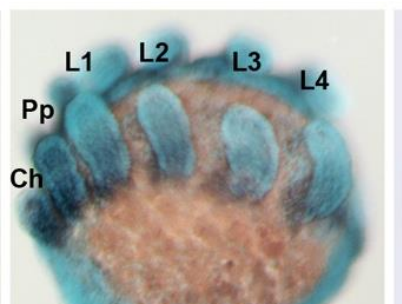

A

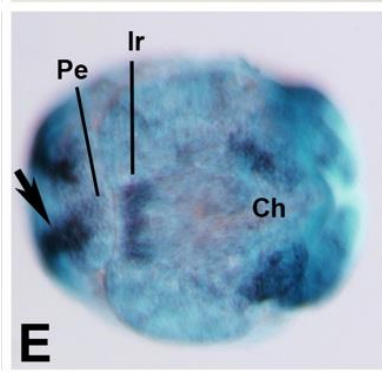

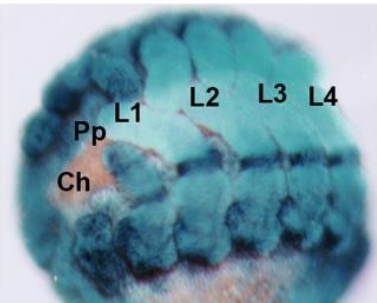

B

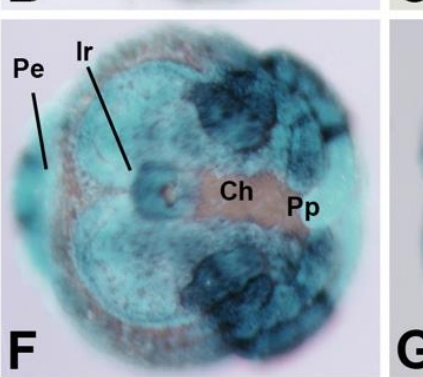

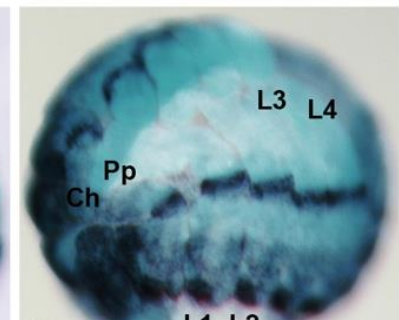

C

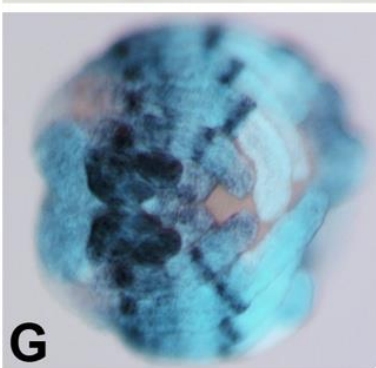

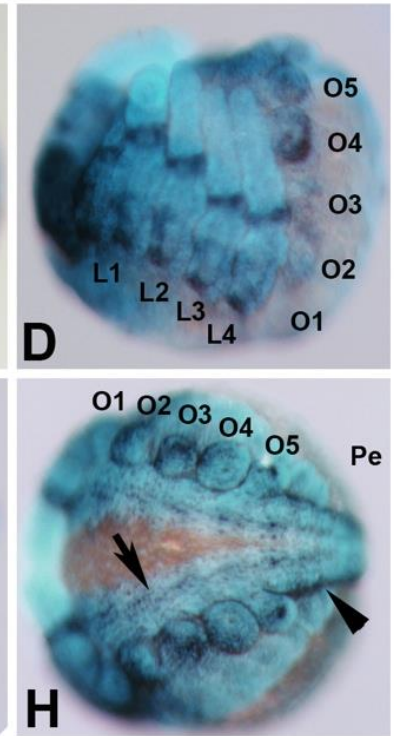

Figure 28: Expression of exd1 in P. tepidariorum

A: Lateral view of a P. tepidariorum embryo during early limb bud development expressing exd1 in the ventral neuroectoderm and the prosomal appendages. Ring-like Pt-exd1 expression in the medial part of the pedipalps and walking legs during inversion (B) and dorsal closure (C), lateral view. D: Lateral view of Pt-exd1 expression in the prosomal appendages and the opisthosoma during ventral closure. E: Frontal view of Pt-exd1 expression in the labrum during early limb bud development. F: This Pt-exd1 expression in the labrum stays but becomes weaker during inversion, frontal view. G: Strong exd1 expression in the chelicera and labrum after head rearrangements in $P$. tepidariorum during ventral closure, frontal view. H: Posterior view of opisthosomal Pt-exd1 expression during inversion. The arrow points to expression in the ventral neurogenic ectoderm, the arrowhead to the expression in the dorsal neurogenic ectoderm. Expression is present in all four opisthosomal limb buds, but in a distinct pattern. All embryos are oriented with the anterior side to the left, except panel H. Ir: labrum, Ch: Chelicera, Pp: Pedipalps, L1-L4: Walking legs pairs 14, 01-05: Opisthosomal segments 1-5, pe: posterior end. 

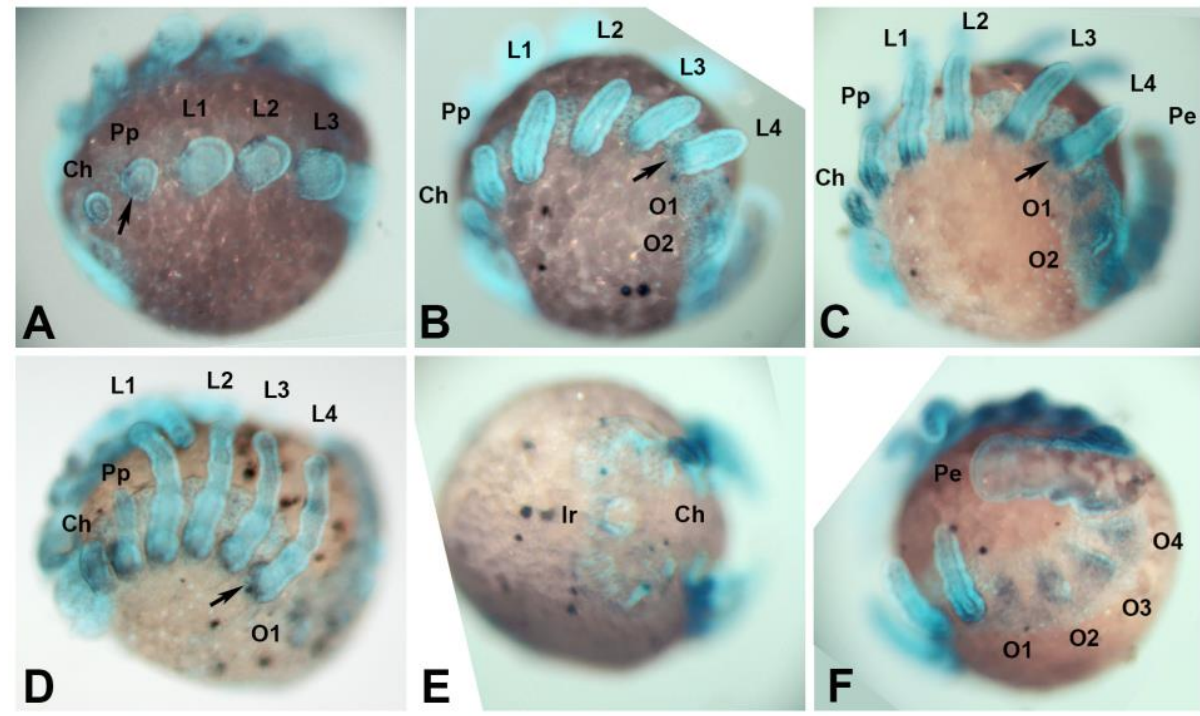

Figure 29: Expression exd1 in P. phalangioides

A-D: Lateral view of embryos expressing Pp-exd1 on the proximal base of the prosomal appendages (arrow) during early limb bud development (A), limb bud elongation (B), inversion (C) and dorsal closure (D). The embryos in A-D are oriented with the anterior side to the left. E: Frontal view of a P. phalangioides embryo during inversion with weak exd1 expression in the labrum. F: Posterior view of opisthosomal Pt-exd1 expression in the dorsal part. Ir: Labrum Ch: Chelicera, Pp: Pedipalps, L1-L4: Walking legs pairs 1-4, 01-05: Opisthosomal segments 1-5, Pe: posterior end.
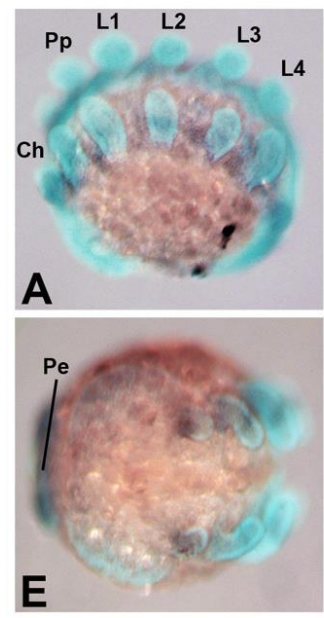
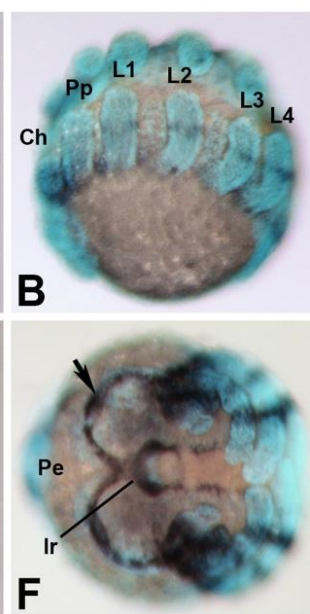
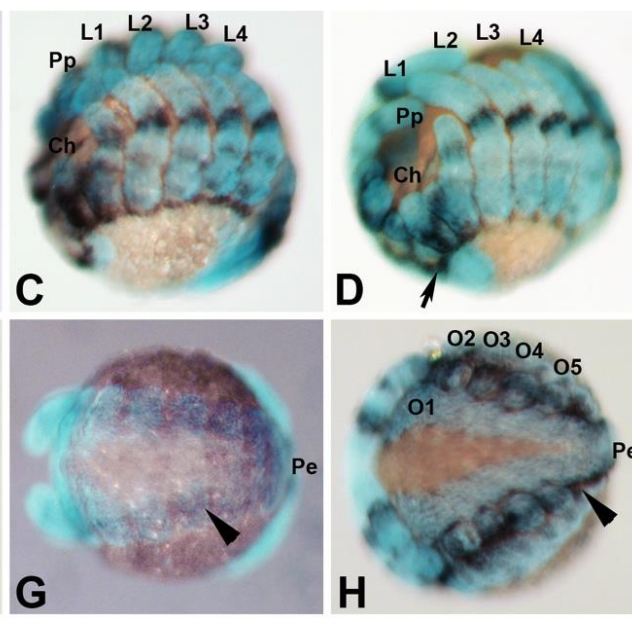

Figure 30: Expression of exd2 in P. tepidariorum

A: Lateral view of a P. tepidariorum embryo during early limb bud development expressing exd2 in the ventral neuroectoderm. Ring-like Pt-exd2 expression in the medial part of the pedipalps and walking legs during limb bud elongation (B), inversion (C) and dorsal closure (D), lateral view. The arrow in D points to Pt-exd2 expression on the former anterior rim of the non-neurogenic ectoderm overgrowing the neurogenic ectoderm. E: Frontal view of the head during early limb bud development, no visible Pt-exd2 expression. F: Onset of Pt-exd2 expression during inversion surrounding the head lobes anteriorly (arrow) as well as in the labrum, frontal view. G: Posterior view of broad opisthosomal Pt-exd2 expression (arrowhead) during early limb bud development. H: Posterior view of medial Pt-exd2 stripe (arrowhead) in the opisthosoma during inversion. All embryos are oriented with the anterior side to the left, except panels $G$ and $H$. Abbreviations same as Figure 28. 


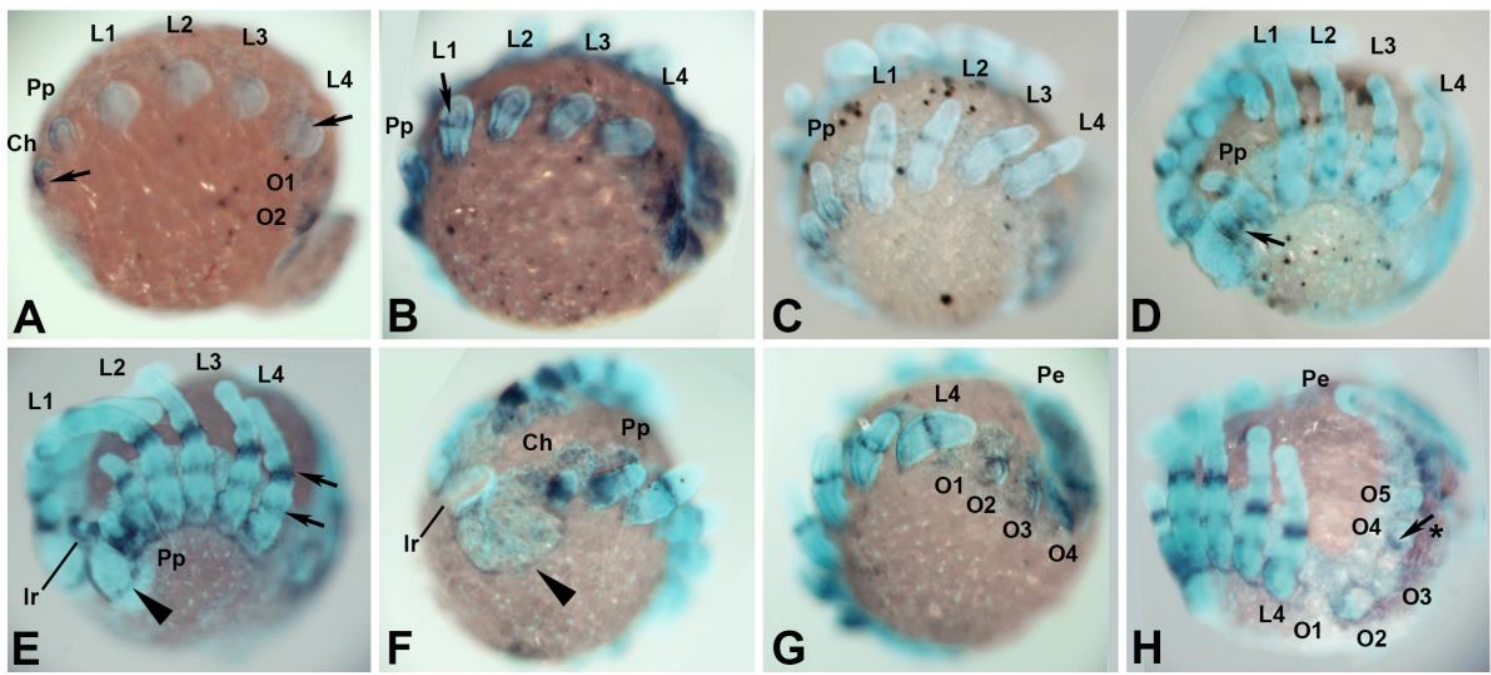

Figure 31: Expression exd2 in P. phalangioides

Lateral view of $P$. phalangioides embryos with ring-like exd2 expression in the medial part of the prosomal appendages (arrow) during early limb bud development (A), limb bud elongation (B), early inversion (C) and early and late dorsal closure (D and E), lateral view. The arrowhead in $E$ points to Pp-exd2 expression on the former anterior rim of the non-neurogenic ectoderm overgrowing the neurogenic ectoderm. F: Frontal view of the Pp-exd2 head expression during inversion surrounding the head lobes anteriorly (arrow) as well as in the labrum. G: Lateral posterior view of broad opisthosomal Pp-exd2 expression during limb bud elongation. H: Posterior view opisthosomal Pp-exd2 expression in the dorsal part (arrowhead) and ventrally on the limb bud on 04 during inversion. All embryos are oriented with the anterior side to the left, except panels $G$ and $H$. Abbreviations same as Figure 28.

\subsubsection{Expression of exd in prosomal appendages}

In the walking legs of $P$. tepidariorum and $P$. phalangioides exd1 shows the conserved expression exclusively covering the proximal leg part with beginning of early limb bud development (bar, Figure 33, G and arrow, Figure 29, A). This proximal expression in the walking legs remains present throughout embryonic development (arrowhead, Figure 32, E and F and bar, Figure 33, $\mathrm{H}$ and I). With the beginning of inversion and prosomal limb bud differentiation a strong ring of exd1 expression appears in the former patella region of the walking legs and pedipalps (arrow, Figure 32, D, E and F and arrow, Figure 33, E, F, H and I). The pedipalps of $P$. phalangioides strongly express exd1 also in the gnathendite (arrowhead, Figure 32, C and D). This is also true for Pt-exd1, which is in addition moderately expressed in the distal and medial part of the pedipalps (asterisk, Figure 33, D, E, and F). From beginning of prosomal limb bud development exd1 is 
strongly expressed within the entire chelicera and only slightly vanishes during ventral closure (arrow, Figure 32, A and B, Figure 33, A, B and C). The expression of exd2 differs from its paralog exd1 for all three prosomal appendage types. In the pedipalps and walking legs, exd2 expression directly starts as a ring in the former patella region at early limb bud developmental stages, but no proximal expression domain is visible (arrow, Figure 31, A and arrow, Figure 33, P). During late inversion a Pt-exd2 and Pp-exd2 expression domain appears in the coxa and as a ring in the developing femur in the walking legs and the pedipalps, as well as in the gnathendite (arrowhead and arrow, Figure 32, I and $\mathrm{K}$ and arrow, Figure 33, Q and N). In P. tepidariorum this expression in the pedipalps and walking legs remains unchanged until ventral closure is completed (Figure 33, R). In P. phalangioides by contrast all three exd2 leg expression domains broaden during embryonic leg growth at ventral closure stages (bar, Figure 33, L). At the same time $P p$-exd2 in the pedipalps vanishes from the coxa and an additional expression appears distal to the patella ring. In the chelicera exd2 is expressed in the proximal half similarly for both spiders until ventral closure. Whereas this expression remains unaltered throughout embryonic development of $P$. phalangioides, a distal expression domain of exd2 appears during ventral closure in P. tepidariorum.

Taken together as already observed in the comparison of the expression in whole mount embryos, there is a general trend that the patterns are highly conserved for the orthologous duplicates between the two spider species studied here, but differs substantially between the paralogs within each species.

As stated above the expression pattern of a gene not necessarily also reflects the region of the functional protein. Also for exd no working antibodies are available for spiders, and thus we directly tried to knock down the gene function using parental RNAi in $P$. tepidariorum. Similar to $h$ th different dsRNA injection procedures were tried each time in three females, but also for the exd paralogs no obvious phenotype was observed, so that the exd RNAi requires improvement as well. 


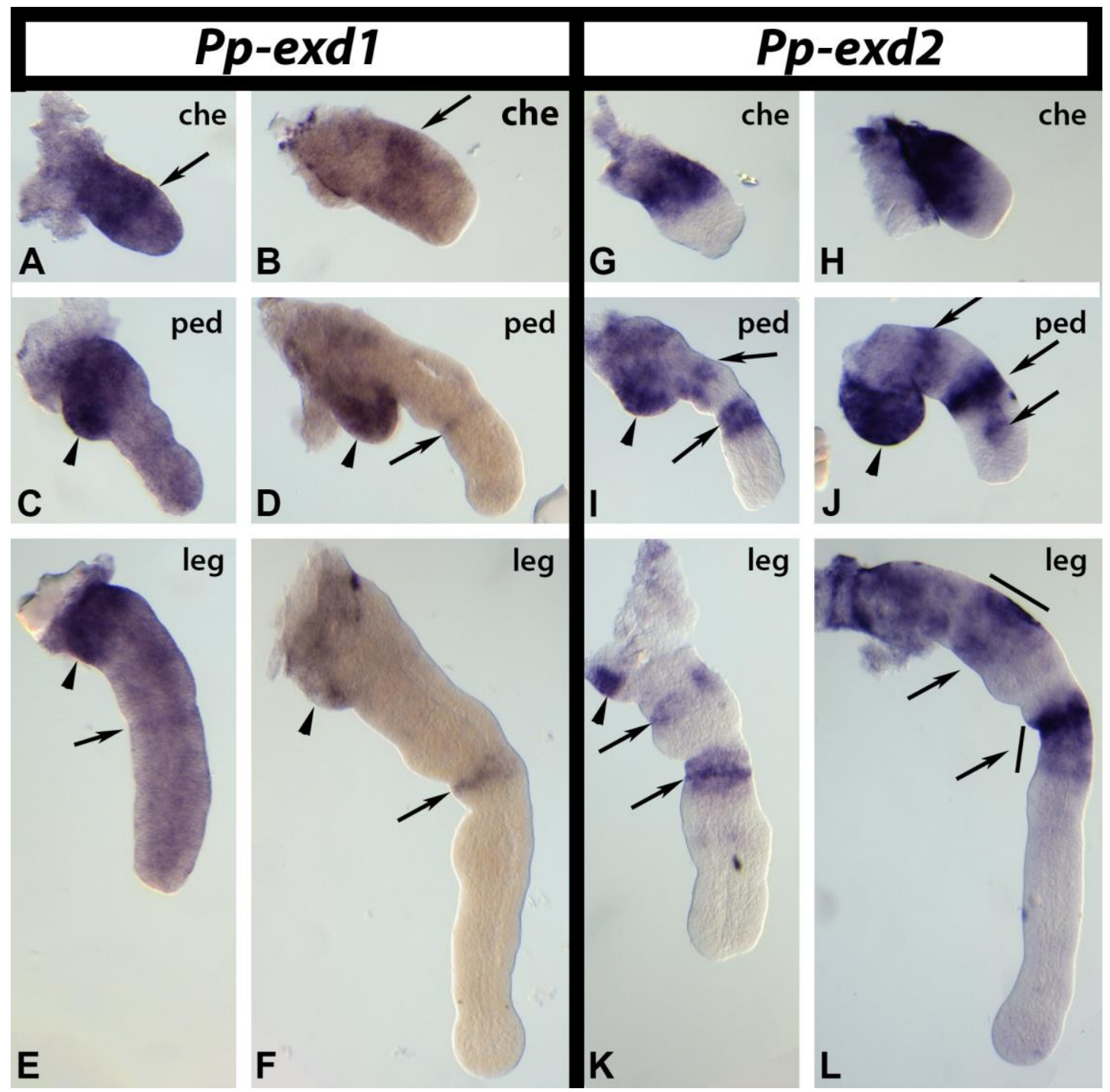

Figure 32: exd expression in prosomal appendages of $P$. phalangioides

A-F: Expression of exd1 in prosomal appendages of P. phalangioides. A and B: Preparations of chelicera during limb bud elongation (A) and ventral closure (B). The arrow points to the proximal exd1 expression, which later is found more medially. C and D: Expression of exd1 in the pedipalps during limb bud elongation (C) and ventral closure (D). The arrowhead points to the expression in the gnathendite. The arrow marks exd1 ring in the patella. E and F: Walking legs during limb bud elongation (E) and ventral closure (F). The arrowhead marks the proximal exd1 domain and the arrow the ring of exd1 in the patella. G-L: Expression of exd2 in prosomal appendages of $P$. phalangioides. $G$ and $H$ : Preparations of chelicera during late inversion $(G)$ and ventral closure $(H)$. I and J: Expression of exd1 in the pedipalps during late inversion (C) and ventral closure (D). The arrowhead points to the expression in the gnathendite. The arrows mark ring-like expression of exd1. $K$ and L: Walking legs during late inversion (E) and ventral closure (F). The arrowhead marks the proximal exd1 spot and the arrow the rings of exd1 in the walking legs, which broaden during ventral closure (bar). che: chelicera. ped: pedipalp. leg: walking leg. 


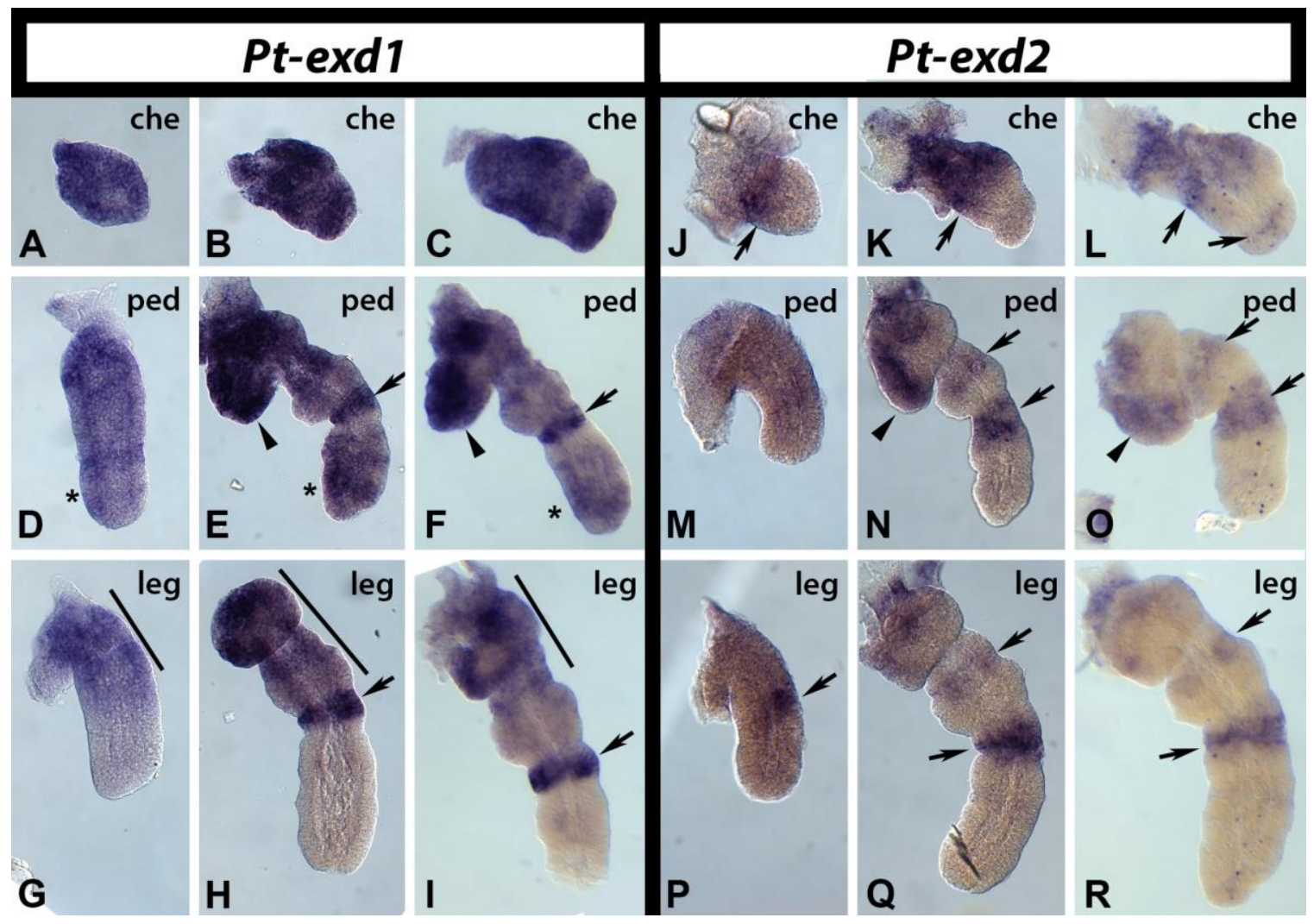

Figure 33: exd expression in prosomal appendages of $P$, tepidariorum

A-I: Expression of exd1 in prosomal appendages of $P$. tepidariorum. J-R: Expression of exd2 in prosomal appendages of $P$. tepidariorum. A-C and J-L: Expression of exd paralogs in the chelicera. The arrows indicate medial and distal expression of exd2. D-F and M-0: Expression of exd paralogs in the pedipalps. The arrowhead points to expression in the gnathendite. The arrow points to rings of expression. The asterisk marks broad distal exd1 expression. G-I and P-R: Expression of exd paralogs in the walking legs. The bar marks the broad, conserved expression of exd1 during limb bud development. The arrows indicate expressed ring of the exd paralogs. A, D, G, J, M and P show prosomal appendages during early limb bud development. B, E, $\mathrm{H}, \mathrm{K}, \mathrm{N}$ and $\mathrm{Q}$ show prosomal appendages during inversion. $C, F, I, L, O$ and $R$ : depict prosomal appendages during ventral closure. che: chelicera. ped: pedipalp. leg: walking leg. 
For the following paragraph and the corresponding discussion section the text, figures and figure legends are taken from (Turetzek et al., 2016). (With kind permission Oxford Journals. Media: Molecular Biology and Evolution, Neofunctionalisation of a duplicate dachshund gene underlies the evolution of a novel leg segment in arachnids (2016), Volume 33, Issue 1. Pages: 109-121, authors N.Turetzek, M. Pechmann, C. Schomburg, J. Schneider and N. M. Prpic, copyright (c) by Oxford Journals and the authors.

\subsection{Neofunctionalisation of a duplicate dachshund gene underlies the evolution of a novel leg segment in arachnids}

\subsubsection{Introduction}

Gene duplication is regarded as the main process how new genes emerge during evolution. At first, the duplicate of the parental gene is superfluous and therefore most duplicates are lost again (Ohno 1970). There are, however, three principal possibilities that may lead to the retention of the duplicated gene (reviewed in Conant and Wolfe 2008; Innan and Kondrashov 2010; Magadum et al. 2013): first, the doubled amount of gene product may have beneficial effects, e.g. because the higher dosage leads to functional stability. In this case, the parental gene and the duplicate retain the same original function. Second, the duplicate may take over a subfunction of the parental gene (subfunctionalisation), thus leading to the evolution of tissue-specific genes and a reduction of pleiotropy. Third, the duplicate may gain a new function (neofunctionalisation) that makes the new gene essential for the organism.

Previous studies of the genetic basis of embryonic development in spiders have shown that many developmental genes are duplicated in spiders, for example the t-box genes optomotor-blind ( $\mathrm{omb}$ ) and H15 (Janssen et al. 2008), or the TALE homeobox genes homothorax (hth) and extradenticle (exd) (Prpic et al. 2003). Indeed, the recent study of the complete developmental transcriptome of the spider Parasteatoda tepidariorum indicates that the genome contains over 40000 genes (Posnien et al. 2014), and the recently published genome sequences of the velvet spider Stegodyphus mimosarum (Sanggaard et al. 2014) and the scorpion Mesobuthus martensii (Cao et al. 2013) contain 
around 30000 predicted genes. Compared to e.g. only 13000 genes in the genome of the fly Drosophila melanogaster (Adams et al. 2000), the high gene numbers in the genomes of arachnids suggest the presence of a large number of duplicated genes.

It has been suggested earlier that the high number of duplicated genes might be correlated with the evolution of many specific features and evolutionary novelties of arachnids (Cao et al. 2013; Sanggaard et al. 2014). Here, we provide evidence that supports this notion using the duplication of the dachshund (dac) gene. The dac gene has been identified first in Drosophila as a factor important for leg development (Mardon et al. 1994). The fly leg is composed of five principal segments, named coxa, trochanter, femur, tibia and tarsus. The $d a c$ gene is expressed in the developing fly leg in a broad domain in the medial portion of the leg comprising the femur and tibia. In dac mutant flies these leg segments are missing, thus resulting in shortened legs (Mardon et al. 1994). This patterning function of dac is widely conserved in the arthropods. A broad medial expression domain in the legs has been confirmed in representatives of all major arthropod groups, e.g. the myriapod Glomeris marginata (Prpic and Tautz 2003), the crustaceans Porcellio scaber (Abzhanov and Kaufman 2000) and Triops longicaudatus (Sewell et al. 2008), diverse insect species (e.g. Angelini and Kaufman 2005), and a number of arachnid species (e.g. Abzhanov and Kaufman 2000; Prpic et al. 2003; Sharma et al. 2013). These data indicate that $d a c$ is an important conserved factor in the evolution and diversification of the medial segment types in the arthropod leg.

We show here, however, that spiders also have a second dac gene, that has originated in the arachnid lineage, and that this duplication is linked to the evolution of a novel medial leg segment in arachnids that is not present in other arthropod groups. In this study, we use the spider species Parasteatoda tepidariorum and Pholcus phalangioides. These two spider species belong to two separate clades in the spider phylogeny: $P$. tepidariorum is a member of the Entelegynae, which comprise most of the extant spider species, whereas P. phalangioides belongs to the Haplogynae, which contain approximately 3500 species worldwide (see simplified cladogram in Figure 3). Thus, conserved aspects in these two species must derive from the spider lineage before the split between entelegyne and haplogyne spiders and therefore are valid for most of the extant species of spiders. We show that in both species the dac gene is present in two paralogous genes that trace from 
a duplication event before the diversification of the extant arachnid groups. The dac1 paralog shows the conserved patterning function that is known from other arthropods as described above. The second dac paralog, dac2, however, has undergone neofunctionalisation and is expressed in the patella, which is a leg segment that is only present in arachnids. We demonstrate that when dac2 function is removed via RNA interference (RNAi), then the patella is joined with the tibia and does not form as a separate leg segment anymore. Thus the loss of dac2 function removes the genetic cues to form a separate patella segment and reverts the spider leg to a state of segment composition before the evolution of the patella.

\subsubsection{Duplicated dachshund genes in spiders}

Previous studies in spiders have isolated the homolog of the Drosophila dachshund gene by using degenerate primers to amplify fragments from cDNA preparations (Abzhanov and Kaufman 2000; Prpic et al. 2003; Pechmann and Prpic 2009). These studies have consistently isolated a single dachshund homolog. However, our recent study of the full developmental transcriptome of $P$. tepidariorum (Posnien et al. 2014) has revealed the presence of a second paralogous dac gene, termed Pt-dac2, that has escaped previous gene cloning attempts. Based on the sequences from P. tepidariorum, we have also isolated two dac orthologs from another spider species, the cellar spider Pholcus phalangioides (termed Pp-dac1 and Pp-dac2). An alignment of the conceptually translated amino acid sequences of the two Dachshund proteins from both species shows that dac1 and dac2 from both spider species encode very similar proteins. All proteins contain the conserved domains that were identified previously (Figure 34). The DD1 and DD2 domains were defined on the basis of sequence similarity between murine and Drosophila Dachshund proteins (Davis et al. 1999) and contain more restrictively defined domains based on similarities between human and murine homologs (DachboxN, Dachbox-C; Hammond et al. 1998), or between Dachshund proteins from diverse metazoan animals and the closely related Ski/Sno proteins (DS domain; Kozmik et al. 1999). Interestingly, the DD1 domain of Dac2 in both spider species contains a novel insertion of about 30 amino acid residues (Figure 34), which is not known from other 
spider Dachshund proteins. In addition, the Dac2 protein of P. phalangioides appears to be truncated at the $\mathrm{C}$-terminus. We cannot exclude the possibility that this truncation is an artifact that occurred during PCR or cloning, but we note that the presence of a standard stop codon at this position supports the notion that this really represents the Cterminus of this protein. The truncation not only interrupts the DD2 domain, but also removes a large portion of the hypothetical alpha-helical coiled-coil domain predicted by Hammond et al. (1998) (Figure 34).

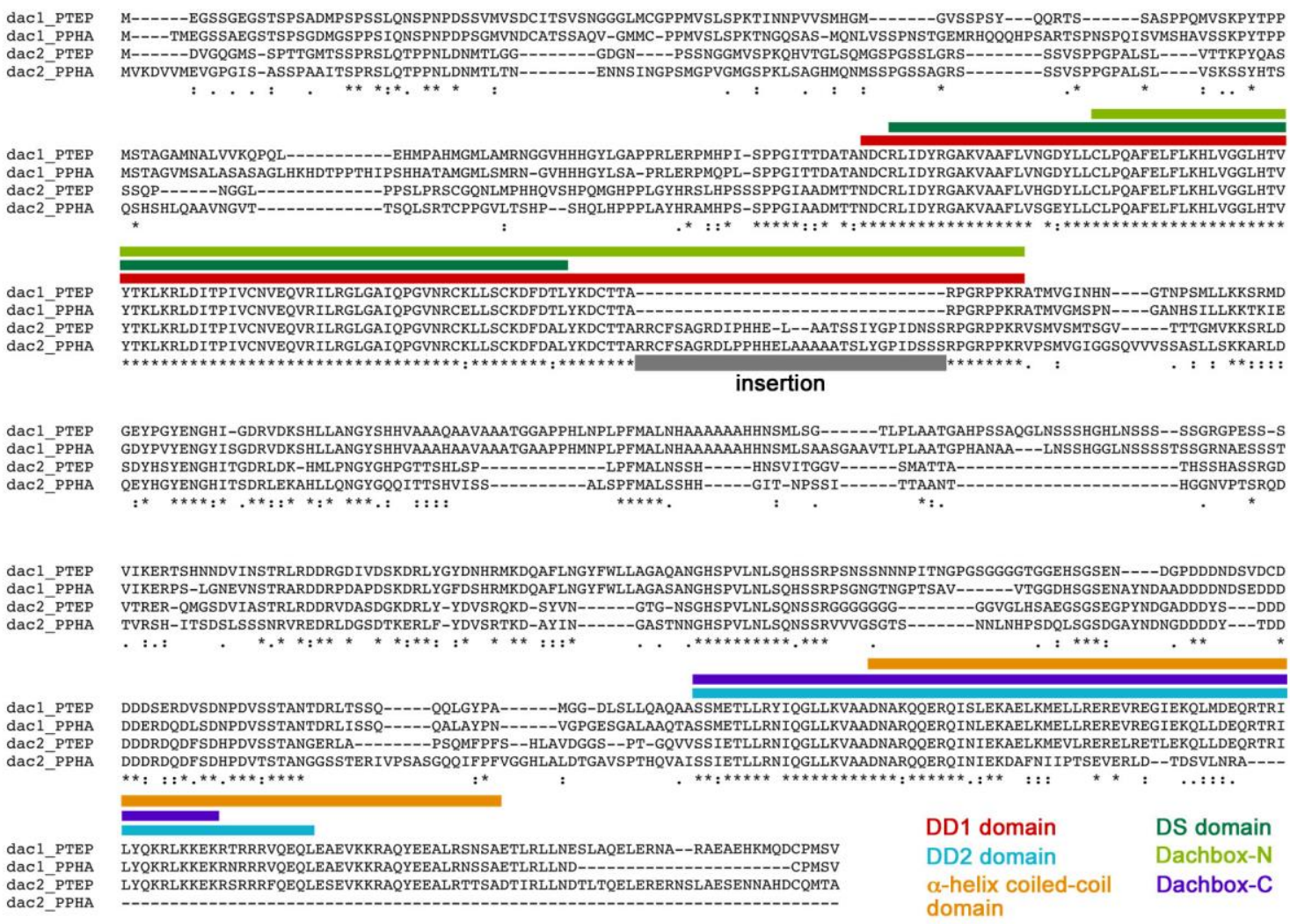

Figure 34: Alignment of the duplicated dac genes from $P$. tepidariorum (abbreviated PTEP in the figure) and $P$, phalangioides (abbreviated PPHA in the figure).

The colored bars indicate conserved regions in the proteins (colors are explained in the figure). The grey bar indicates an insertion in the DD1 domain that is common to dac2 genes, but lacking in dac1 genes. In the annotation directly below the alignment, the asterisks denote identical amino acids, the colons indicate conservative amino acid substitutions, and the dots indicate semiconservative amino acid substitutions. 


\subsubsection{Phylogenetic analysis of dachshund gene evolution and duplication}

All previously isolated dac genes from spiders appear to be orthologs of dac1, whereas dac2 orthologs were missed in gene cloning studies. Duplicated dac genes are also known from vertebrates (named dach1 and dach2; e.g. Davis et al. 2001; Davis et al. 2006; Davis et al. 2008), and the available genome sequence of the scorpion Mesobuthus martensii (Cao et al. 2013) contains three (albeit short and therefore likely incomplete) predicted dac homologs. In order to establish the orthology between the spider dac genes and their relationships with other dac genes, we have performed a phylogenetic analysis of the duplicated $d a c$ genes in spiders, and $d a c$ genes from other protostomes and deuterostomes (Figure 35). The dac1 genes from P. tepidariorum and $P$. phalangioides cluster together with the previously known spider dac genes, demonstrating that these are indeed genuine dac1 genes (dark grey shading in Figure 35), whereas the dac2 genes form a separate clade (light grey shading in Figure 35). The sequences MMa07100 and MMa52425 from the M. martensii genome cluster together with the spider dac1 orthologs, but the MMa07092 sequence is an ortholog of spider dac2. The spider dac genes do not form a cluster with the vertebrate dach genes. Instead, the spider dac genes form a monophyletic group with the dac genes from other arthropods and are the sister group of the clade comprising dac genes from myriapod, crustaceans and insects. By contrast, the vertebrate dac genes form a separate clade in the tree and cluster together with the dac genes from other deuterostomes. This shows that the duplication events that produced the paralogous genes in spiders and vertebrates are entirely unrelated. 


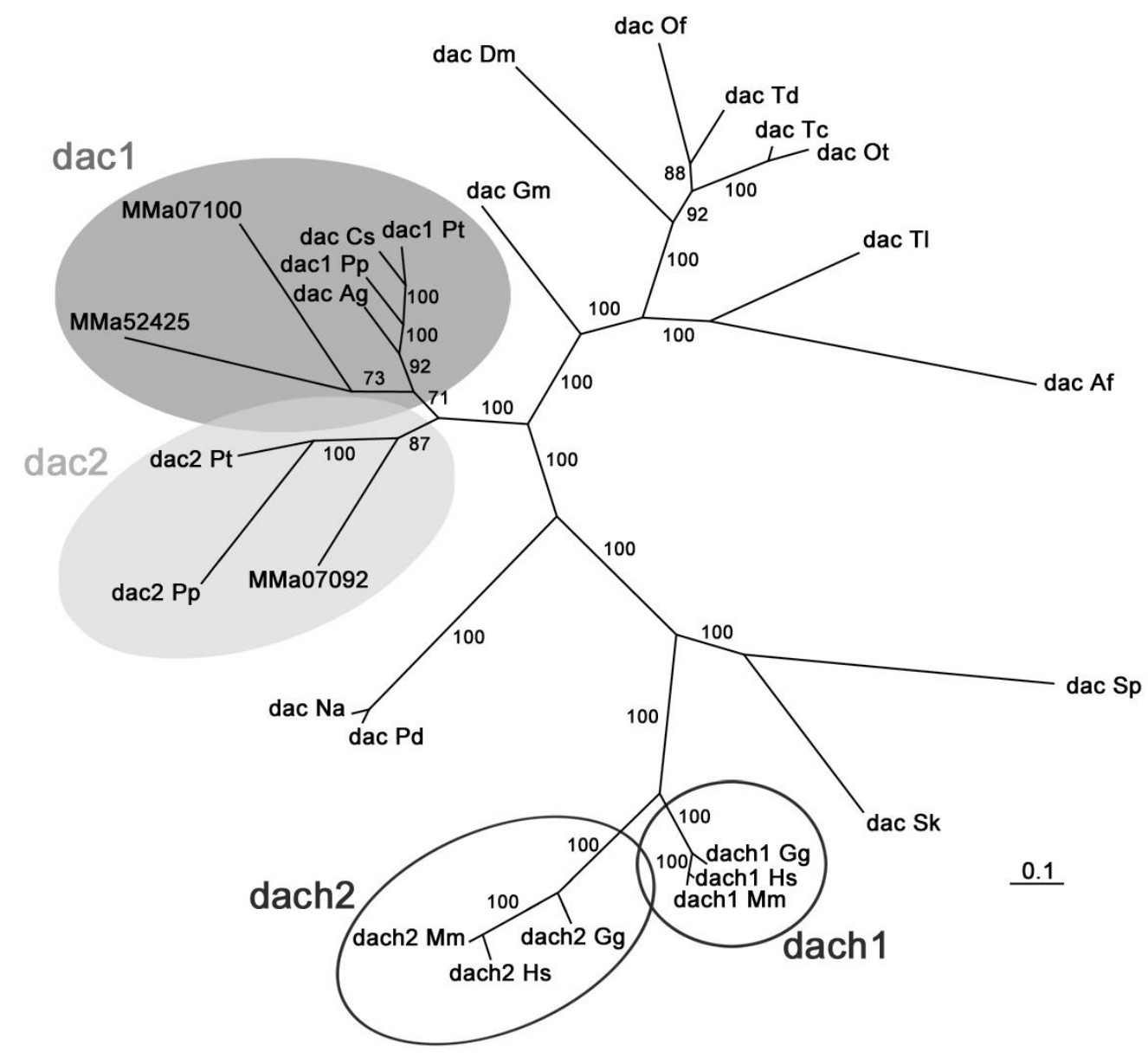

Figure 35: Unrooted 50\% majority rule consensus tree after Bayesian Markov chain Monte Carlo analysis.

Branch lengths in the phylogram give the expected substitutions per site. Numbers at the tree edges are clade credibility values giving the probability of each clade in the tree. The duplicated dac genes in arachnids are indicated in dark grey (dac1) and light grey (dac2). The duplicated dach genes in vertebrates (dach1 and dach2) are denoted by open circles. For species abbreviations and sequence accession numbers please see Supplemental Material 11.4.3 dachshund.

\subsubsection{Expression of dac1 in Parasteatoda tepidariorum and Pholcus phalangioides}

The phylogenetic analysis shows that the dac1 genes of $P$. tepidariorum and $P$. phalangioides are orthologs of the previously isolated dac genes from other spider species that show an evolutionarily conserved expression pattern which is also known from other arthropods (summarized in Angelini and Kaufman 2005). We therefore studied the dac1 expression pattern in P. tepidariorum and P. phalangioides to confirm that they show the evolutionarily conserved expression pattern, too. The most prominent expression domain of dac1 in $P$. tepidariorum is in the medial part of the 
pedipalps and walking legs. This expression is initiated as soon as the limb buds have formed in the prosoma and is retained throughout embryonic development (Figure 36, A-D). At the beginning of germ band extension Pt-dac1 expression is also initiated in the central nervous system (Figure 36, E) (see also Schomburg et al. 2015). The pattern in the head lobes comprises two domains in the center of the brain primordium and a domain at the rim of the head lobe (Figure 36, E). Expression in the ventral neurogenic ectoderm is initially restricted to the pedipalpal segment, the four walking leg segments and the first three opisthosomal segments (Figure 36, E, F). During further development, however, the neurogenic ectoderm of the remaining opisthosomal segments expresses Pt-dac1 as well (Figure 36, G). Additional expression domains of Pt-dac1 appear later in development in dorsal cells that will contribute to the heart, and in cells of the hindgut primordium (Figure 36, H).

The expression of dac1 in $P$. phalangioides is very similar to the dac1 ortholog of $P$. tepidariorum. There is prominent expression in the developing pedipalps, legs and in the central nervous system throughout development (Figure 37, A-G). The expression pattern in the head lobes is virtually identical to the expression in P. tepidariorum, except that the expression domain at the lateral rim of the head lobes is very strong (Figure 37, C). During germ band inversion there is a dorsal expression in the presumptive heart tissue (Figure 37, H), similar to the expression in P. tepidariorum. 


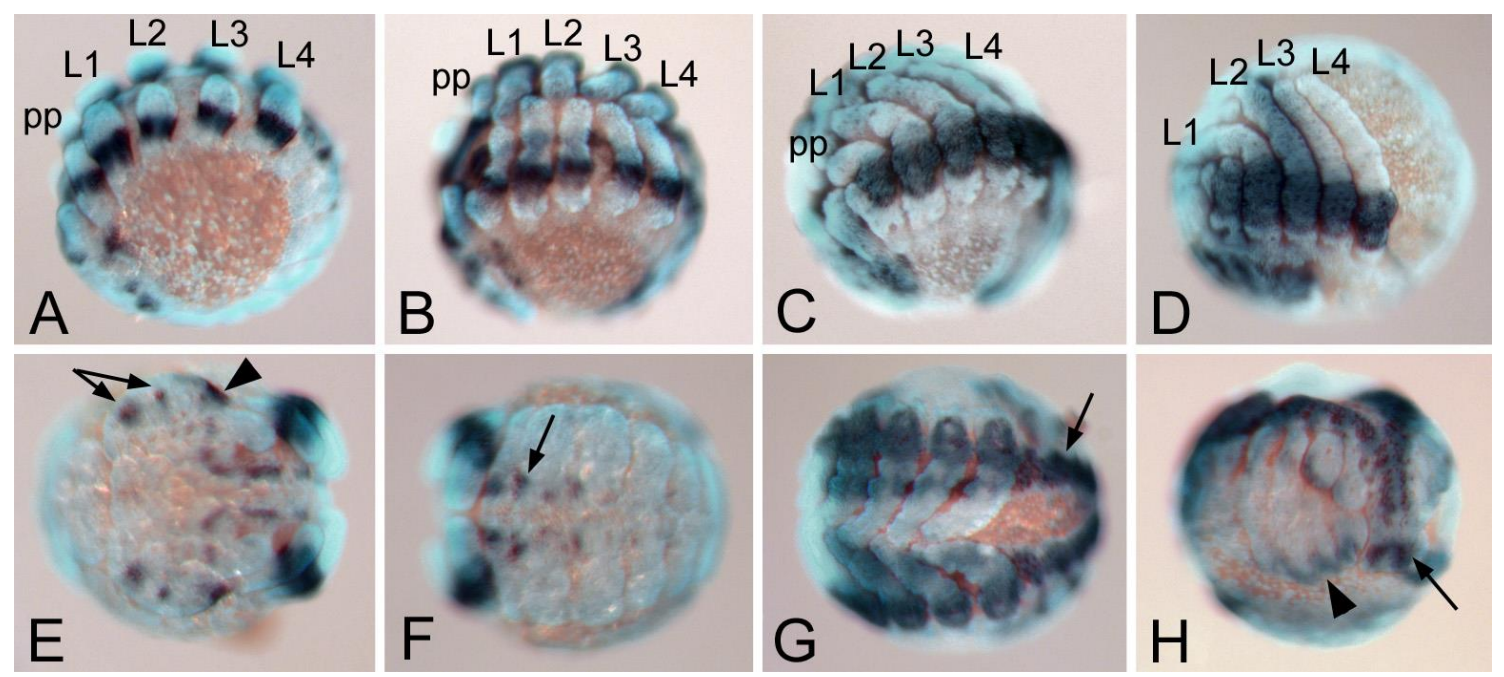

Figure 36: Expression of dac1 in $P$. tepidariorum

(A) Embryo during germ band elongation. Lateral view. (B) Fully elongated embryo. Lateral view. (C) Embryo at the beginning of germ band inversion. Lateral view. (D) Embryo at the end of germ band inversion and after dorsal closure. Lateral view. (E) Embryo during germ band elongation. Frontal view of the head. The arrows point to domains in the center of the brain primordium. The arrowhead denotes expression at the rim of the head lobe. (F) Embryo during germ band elongation. Ventral view of the opisthosoma. The arrow points to expression in the ventral neurogenic ectoderm. (G) Embryo at the beginning of germ band inversion. Ventral view of the prosoma and anterior portion of the opisthosoma. The arrow points to expression in the ventral neurogenic ectoderm. (H) Embryo at mid-germ band inversion, before dorsal closure. Posterior view of the opisthosoma and the hindgut primordium. The arrow points to expression in the hindgut primordium. The arrowhead denotes expression in presumptive dorsal tissue that will contribute to the heart. All embryos are shown with anterior to the left. Abbreviations: pp, pedipalps; L1-L4, walking legs 1 to 4.

\subsubsection{Expression of dac2 in Parasteatoda tepidariorum and Pholcus phalangioides}

In order to establish whether the dac2 paralog in spiders also shows the evolutionarily conserved expression pattern, or has changed its expression during evolution, we have studied the expression of dac2 in P. tepidariorum and P. phalangioides. Pt-dac2 is expressed in the pedipalps and walking legs during germ band extension and during further development (Figure 38, A-D). This appendicular expression pattern, however, differs significantly from the expression of Pt-dac1 (see details below). Expression of Pt$d a c 2$ in the ventral neuroectoderm is entirely restricted to the pedipalpal segment and the anterior half of the first walking leg segment, and there is no early expression in the head lobes (Figure 38, E-F). After dorsal closure, however, expression of Pt-dac2 is activated in the primordia of the lateral eyes (Figure 38, D; see also Schomburg et al. 2015). During early inversion of the embryo there is expression in presumptive dorsal 
ectoderm near the bases of the opisthosomal limb buds (Figure 38, G), and after dorsal closure there is very faint expression in dorsal segmental stripes that possibly correspond to the embryonic septa that divide the yolk mass within the developing opisthosoma (Figure 38, H).
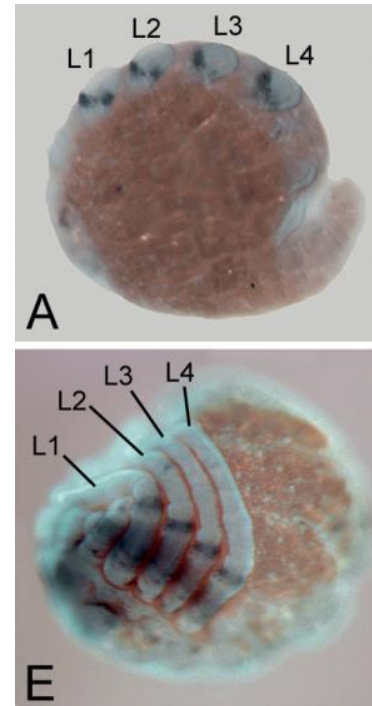
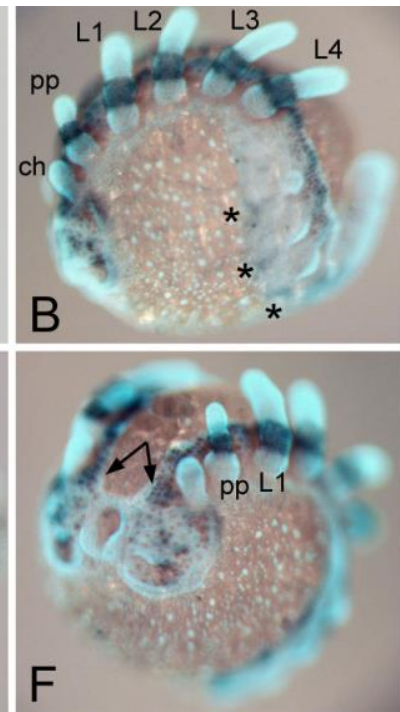
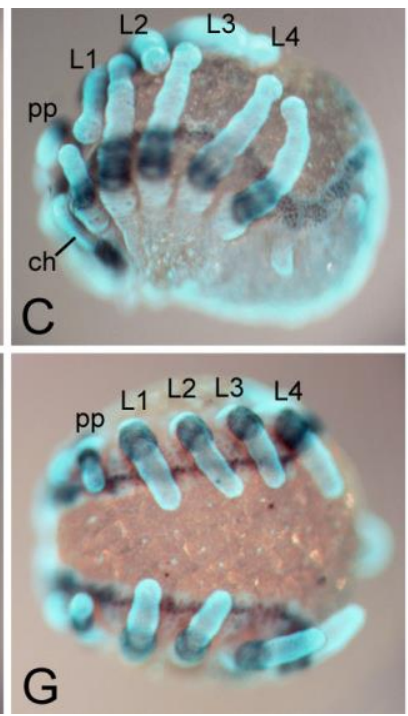

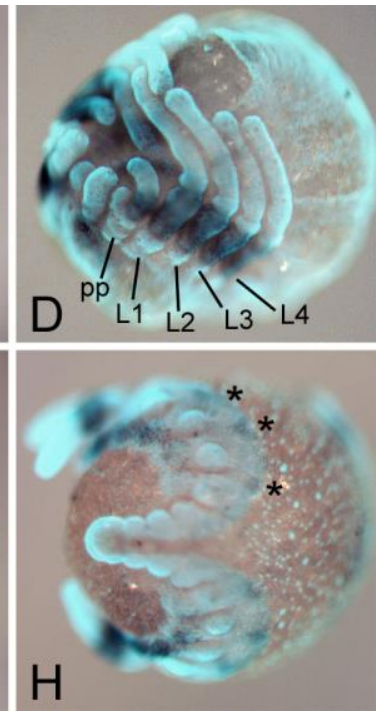

Figure 37: Expression of dac1 in P. phalangioides

(A) Embryo during germ band elongation. Lateral view. (B) Fully elongated embryo at mid-germ band inversion. Lateral view. The asterisks denote expression in presumptive dorsal tissue that will contribute to the heart. (C) Embryo approaching the end of germ band inversion. Lateral view. (D) Embryo at the end of germ band inversion and after dorsal closure. Ventro-lateral view. (E) Embryo after ventral closure. Lateral view. (F) Embryo at mid-germ band inversion. Latero- frontal view. The arrows point to expression in the ventral neuroectoderm. (G) Embryo at mid-germ band inversion. Ventral view of the prosoma. (H) Embryo at mid-germ band inversion, before dorsal closure. Posterior view of the opisthosoma and the hindgut primordium. All embryos are shown with anterior to the left. Abbreviations: ch, chelicera; pp, pedipalp; L1-L4, walking legs 1 to 4.

The expression of Pp-dac2 is very similar to the expression of dac2 in P. tepidariorum (Figure 39, A-E). The expression in the appendages is virtually identical (see details below), and the expression in the ventral neurogenic ectoderm is also restricted to the pedipalpal segment and the anterior part of the first walking leg segment (Figure 39, F, G). At stages after dorsal closure, $P p-d a c 2$ is expressed in the primordia of the lateral eyes (Figure 39, E). Similar to the homologous gene in P. tepidariorum, Pp-dac2 is also expressed in faint stripes in the opisthosoma (Figure 39, H). 


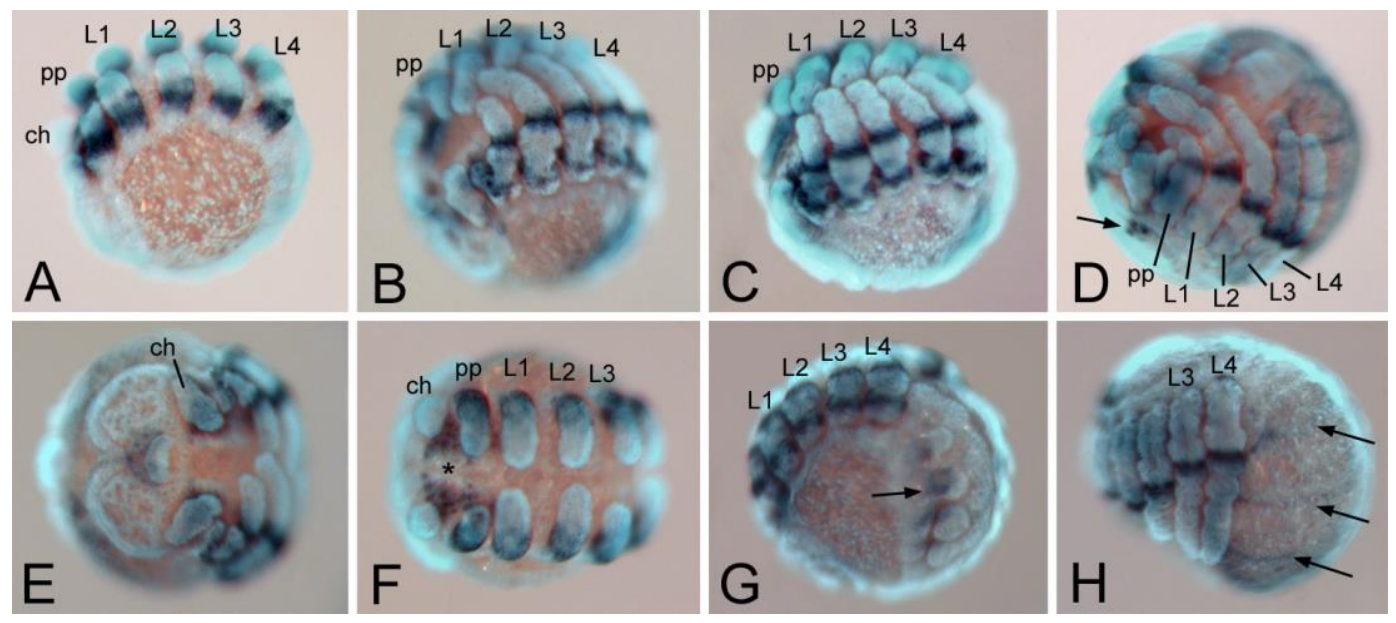

Figure 38: Expression of dac2 in P. tepidariorum

(A) Embryo during germ band elongation. Lateral view. (B) Fully elongated embryo at the beginning of germ band inversion. Lateral view. (C) Embryo at mid-germ band inversion. Lateral view. (D) Embryo after dorsal closure and shortly before ventral closure. Ventro-lateral view. The arrow points to expression in the primordia of the lateral eyes. (E) Fully elongated embryo at the beginning of germ band inversion. Frontal view of the head. (F) Embryo during germ band elongation. Ventral view. The asterisk denotes expression in the ventral neuroectoderm of the pedipalpal segment. (G) Embryo at mid-germ band inversion. Ventro-lateral view of the opisthosoma. The arrow points to expression near the bases of the opisthosomal limb buds. (H) Embryo after dorsal closure and shortly before ventral closure. Lateral view. The arrows point to expression on the dorsal side, presumably in the intralecithal septa. All embryos are shown with anterior to the left. Abbreviations: ch, chelicera; pp, pedipalp; L1-L4, walking legs 1 to 4.

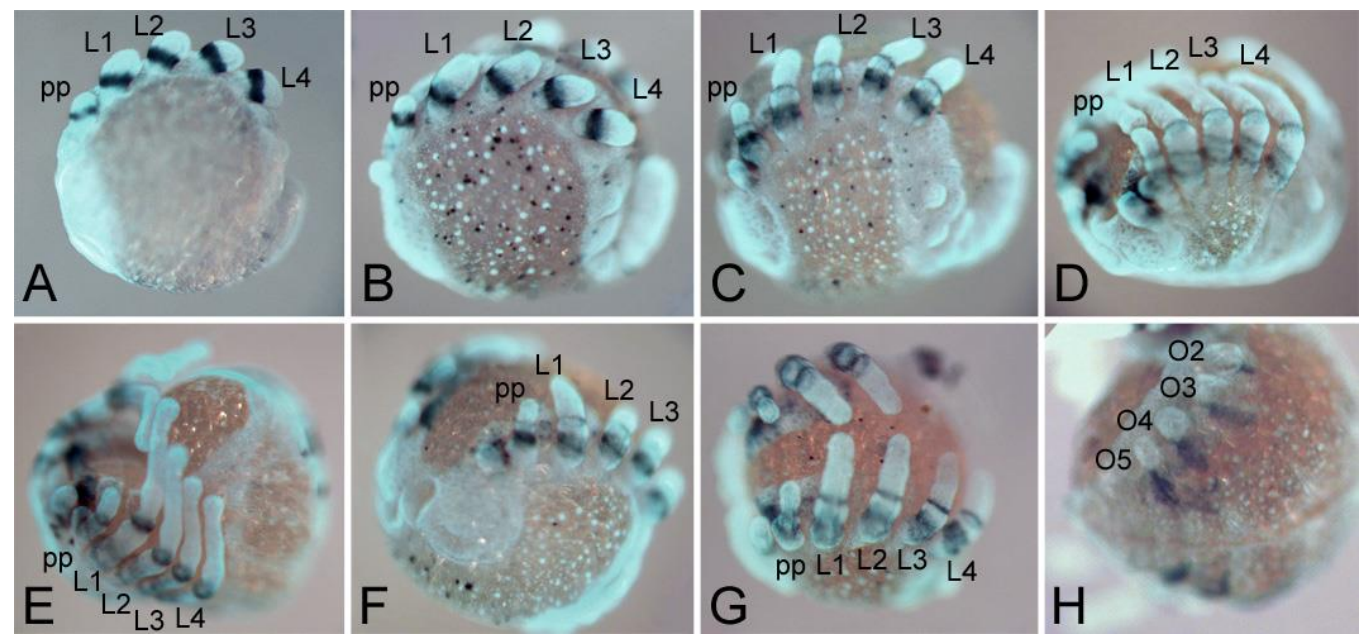

Figure 39: Expression of dac2 in P.phalangioides

(A) Embryo during germ band elongation. Lateral view. (B) Fully elongated embryo. Lateral view. (C) Embryo at mid- germ band inversion. Lateral view. (D) Embryo after dorsal closure. Lateral view. (E) Embryo after dorsal closure and shortly before ventral closure. Ventro-lateral view. (F) Embryo at mid-germ band inversion. Frontal-lateral view. (G) Embryo at mid-germ band inversion. Ventro-lateral view of the opisthosoma. (H) Embryo after dorsal closure. Dorsal view of the opisthosoma. All embryos are shown with anterior to the left. Abbreviations: pp, pedipalp; L1-L4, walking legs 1 to 4 ; 02-05, opisthosomal segments 2 to 5 . 


\subsubsection{Expression profile of dac1 and dac2 in the prosomal appendages}

The most significant differences in the expression profile of the two dac paralogs are found in the developing prosomal appendages and we have studied these in more detail. The expression of the homolog of the dac1 gene in the prosomal appendages has been analysed previously in three other spider species, Acanthoscurria geniculata (Pechmann and Prpic 2009), Cupiennius salei (Prpic et al. 2003), and Steatoda triangulosa (Abzhanov and Kaufman 2000). Our results in P. tepidariorum and P. phalangioides show that the expression of this gene is highly conserved in spiders (Figure 40, A-F and Figure 41, A-F). In the appendages, the dac1 gene of both species is expressed in the pedipalps and all four walking leg pairs in a broad ring-shaped domain that comprises the trochanter and the femur (Figure 40, C-F and Figure 41, A-F). The dac1 gene is not expressed in the gnathal endite of the pedipalps (Figure 40, D and Figure 41, D). In the chelicera, there is only very faint expression within the base of the chelicera in P. tepidariorum (Figure 40, A). A similar expression domain has been reported from C. salei (Prpic and Damen 2004), and is possibly related to the development of the peripheral nervous system within the chelicera. This expression domain disappears during germ band inversion in $P$. tepidariorum (Figure 40, B) and is completely lacking in the chelicera of $P$. phalangioides (Figure 41, A and B). In both species dac2 is expressed in all prosomal appendages, including the chelicera (Figure 40, G-L and Figure 41, G-L). In early stages, there is a single broad basal domain in the pedipalps and the legs comprising the tissue of the distal coxa and the trochanter (Figure 38, A and Figure 39, A and B). A second domain appears in both species in the tissue of the patella in early germ band inversion stages (Figure 40, I and $\mathrm{K}$ and Figure 41, I and K). The patella domain strongly increases its expression intensity during further development, whereas the expression in the distal coxa and trochanter becomes weaker (Figure 40, J and L and Figure 41, J and L). In the pedipalp, the vanishing proximal domain extends into the gnathendite and there retains a higher expression level (Figure 40, J and Figure 41, J). In the chelicera there is a proximal ectodermal domain along the ventral side in both species (Figure 40, G and $\mathrm{H}$ and Figure 41, G and H). This domain is weak in earlier stages (Figure 40, G and Figure 41, G), but becomes stronger in older stages (Figure 40, $\mathrm{H}$ and Figure 41, H). 


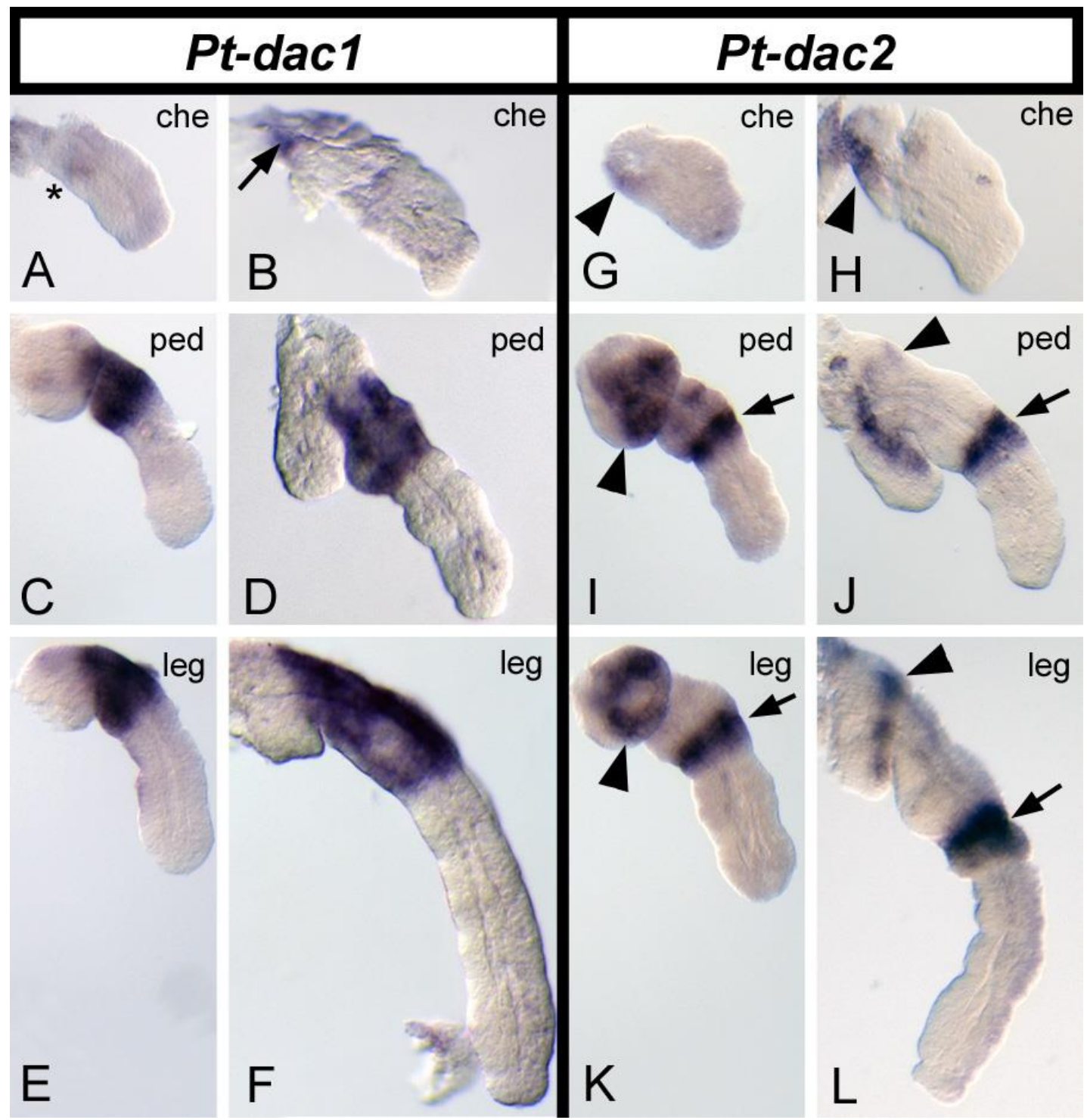

Figure 40: Detection of $\operatorname{dac} 1(\mathrm{~A}-\mathrm{F})$ and $\operatorname{dac} 2(\mathrm{G}-\mathrm{L})$ expression in dissected appendages of $P$. tepidariorum.

(A, B) Chelicera of an embryo during germ band elongation (A) and after dorsal closure (B). The asterisk in $A$ denotes expression of dac1 in tissue at the base of the chelicera. The arrow in $B$ points to expression in brain tissue that has not been completely removed during dissection. (C, D) Pedipalp of an embryo during germ band elongation (C) and after dorsal closure (D). (E, F) Leg of an embryo during germ band elongation (E) and after dorsal closure (F). (G, H) Chelicera of an embryo during germ band elongation (G) and after dorsal closure (H). The arrowheads denote expression of $d a c 2$ in ectodermal tissue at the base of the chelicera. (I, J) Pedipalp of an embryo during germ band elongation (I) and after dorsal closure (J). The arrowheads denote a proximal domain in the coxa. The arrows point to a distal domain in the patella. $(K, L) L e g$ of an embryo during germ band elongation $(\mathrm{K})$ and after dorsal closure (L). The arrowheads denote a proximal domain in the coxa. The arrows point to a distal domain in the patella. 


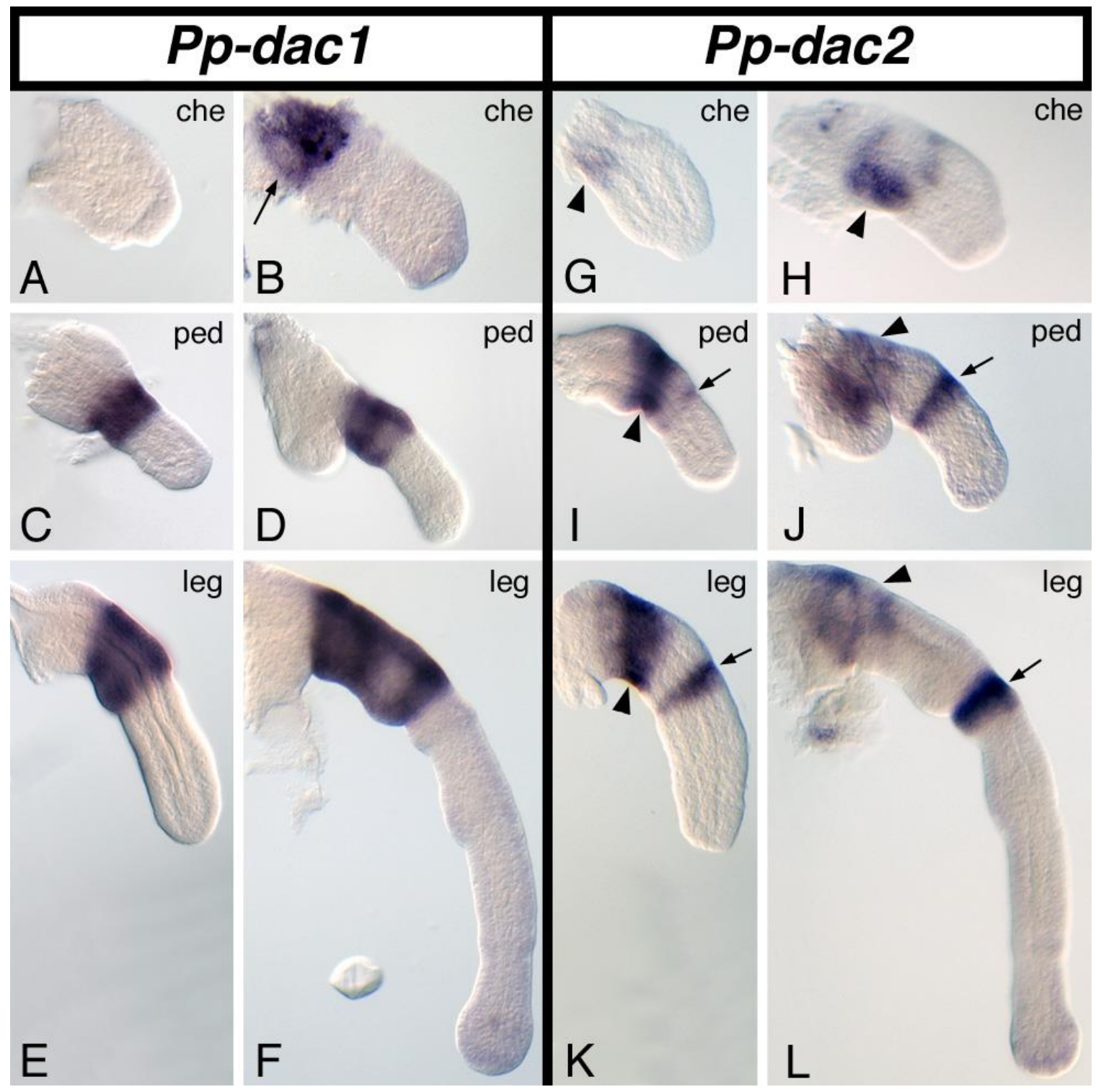

Figure 41: Detection of $\operatorname{dac} 1(\mathrm{~A}-\mathrm{F})$ and $\operatorname{dac} 2(\mathrm{G}-\mathrm{L})$ expression in dissected appendages of $P$. phalangioides.

(A, B) Chelicera of a fully elongated embryo (A) and after dorsal closure (B). The arrow in B points to expression in brain tissue that has not been completely removed during dissection. (C, D) Pedipalp of a fully elongated embryo (C) and after dorsal closure (D). (E, F) Leg of a fully elongated embryo (E) and after dorsal closure (F). (G, H) Chelicera of fully elongated embryo (G) and after dorsal closure (H). The arrowheads denote expression of $\mathbf{d a c} 2$ in ectodermal tissue at the base of the chelicera. (I, J) Pedipalp of a fully elongated embryo (I) and after dorsal closure (J). The arrowheads denote a proximal domain in the coxa. The arrows point to a distal domain in the patella. (K, L) Leg of a fully elongated embryo (K) and after dorsal closure (L). The arrowheads denote a proximal domain in the coxa. The arrows point to a distal domain in the patella. 


\subsection{7 dac2 is required for morphogenesis of the patella leg segment in P. tepidariorum}

The strong and persistent expression of $d a c 2$ in the patella of both spider species studied here is unique to this dac paralog and suggests that dac2 might have a role in the development of this leg segment. To test this idea, we have used parental RNA interference (pRNAi) that is available in P. tepidariorum to downregulate dac2 during embryonic development, and have studied the effects of dac2 loss for the morphology of the legs in the first nymphal stage. At this stage, the cuticle is fully differentiated and the joints between the leg segments are complete and functional. In the wild type nymphs the patella is a short leg segment between the long leg segments femur and tibia (Figure 42, A-D). The patella has a characteristic shape that is reminiscent of a bell, with the narrow end towards the femur and the wide end towards the tibia (Figure 42, B-D). After dac2 parental RNAi 20\% of the nymphs show a strong malformation of the patella leg segment (Figure 42, I). In the dac2 RNAi animals, the shape of the patella is no longer bell-shaped (Figure 42, E-H). In most cases, the proximal end of the patella is almost as wide as the distal end, much like e.g. in the tibia (Figure 42, G and H). In addition to the changes in shape, the patella in dac2 RNAi animals is also shortened and the joint between patella and tibia is fused. At the fused junction between patella and tibia most legs show one or two short bulges (Figure 42, F-H), pointing to an excess of cells at the fused joint. 


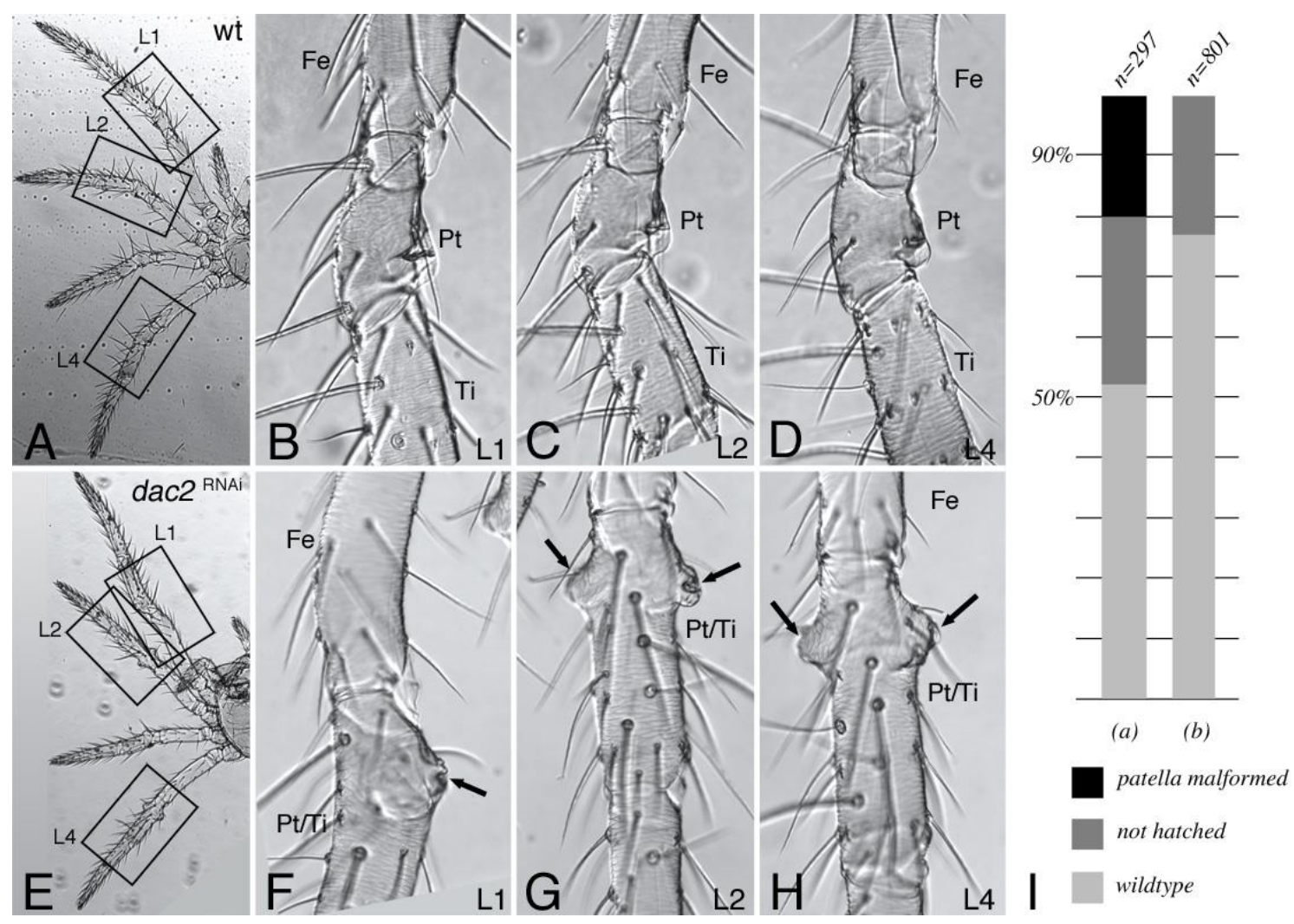

Figure 42: Malformation of the patella after dac2 RNAi in P. tepidariorum. (A-D) Wild type nymph.

(A) Overview of the right half in ventral view. Boxed are those leg regions that are magnified in B-D. (B-D) Magnifications of the area between femur, patella, and tibia of the L1 leg (B), L2 leg (C) and L4 leg (D). (E-H) Nymph with malformations of the patella after dac2 RNAi. (E) Overview of the right half in ventral view. Boxed are those leg regions that are magnified in F-H. (F-H) Magnifications of the area between femur, and the fused patella/tibia of the L1 leg (F), L2 leg (G) and L4 leg (H). (I) Summary of the dac 2 RNAi experiment. The diagram in (a) shows the phenotype distribution after dac2 dsRNA injection. The diagram in (b) shows the phenotype distribution after GFP dsRNA injection as a control. For both analyses the offspring that developed in the second cocoon of the injected females were pooled and scored for phenotypes. Please see the Materials and Methods section for details. 


\subsection{Discussion and future work}

\subsubsection{Gene duplication of spider dachshund genes}

Our search for genes with sequence similarity to dac in the spiders $P$. tepidariorum and $P$. phalangioides revealed the presence of two paralogs in both species. The phylogenetic analysis clearly shows that the duplicated dach genes in vertebrates and the duplicated dac genes in spiders did not originate in the same gene duplication event (see Figure 35). The duplicated $d a c$ genes are present in spiders from two main spider lineages, the Entelegynae and the Haplogynae (see Figure 3 for a simplified overview of spider phylogeny), and this indicates that the duplication event is older than the split between haplogyne and entelegyne spiders. Indeed, the presence of three dac-related fragments in the genome sequence of a basal arachnid, the scorpion $M$. martensii, which are distributed in the phylogenetic analysis to the two dac clades, strongly suggests that the duplication of the parental dac gene into dac1 and dac2 occurred before the radiation of the arachnid orders: the original $d a c$ gene has been duplicated in the arachnid lineage even before the split between scorpions and spiders giving rise to dac1 and dac2, and dac1 has been duplicated again more recently in the scorpion lineage.

\subsubsection{Neofunctionalisation of dac2 in the arachnids}

The $d a c 1$ and $d a c 2$ genes are thus the product of a phylogenetically old duplication event and are common to all extant arachnid orders. The expression of dac1 in spiders is similar to dac genes in other arthropods, for example in the myriapod Glomeris marginata (Prpic and Tautz 2003) and the insects Tribolium castaneum (Prpic et al. 2001) and Oncopeltus fasciatus (Angelini and Kaufman 2004). Thus, dac1 preserves the original patterning function of the parental dac gene. By contrast, dac2 expression differs from the conserved expression pattern, and especially in the appendages shows novel expression domains that are not present in the dac1 pattern. This strongly suggests that dac2 has acquired a novel function and therefore escaped gene loss by neofunctionalisation. The novel expression domains indicate that neofunctionalisation involved evolutionary changes in the regulatory region of dac2. However, the insertion 
within the DD1 domain of the dac2 protein that is conserved in both species (see Figure 34) suggests that the properties of this functional domain are altered in dac2, and thus this indicates that evolutionary changes in the coding region of dac2 have contributed to neofunctionalisation as well.

\subsubsection{Evolution of the arachnid patella: neofunctionalisation of dac2 underlies the evolution of a morphological novelty}

The appendages of the arthropods are divided into segments that are separated by flexible joints. Usually, the appendages are composed of a combination of long and short segments to improve the mechanical properties of the appendages. Especially the short segments determine the degree of appendage flexibility and maneuverability, by providing pivotal points for the movement of the longer segments. In addition, short segments increase the degrees of freedom within a short portion of the appendage. All arthropod groups have a proximal short segment, the coxa, and in most groups this segment is followed by a second short segment, the trochanter (Figure 43). Arachnids, however, have a third short segment, the patella, that is intercalated between two long segments, the femur and the tibia (indicated in black in Figure 43). The patella is therefore a morphological novelty that has evolved in the arachnid lineage. Our results show that dac2 is expressed in the developing patella of two spider species and is required for the proper formation of the patella. Moreover, if dac2 function is removed via RNAi, then the patella is malformed and is no longer morphologically separated from the following segment, the tibia. Thus, removing dac2 function experimentally restores the segment composition of the arthropod leg before the origin of dac2 in the arachnid lineage. In this way, we are able to provide a link between the origin and neofunctionalisation of $d a c 2$ and the evolution of a morphological novelty in the arachnid appendage. The alternative interpretation that the duplication of dac2 already occurred in the ancestral arthropod, is less parsimonious than the interpretation that dac2 has evolved in the arachnid lineage, because it requires two evolutionary events: the origin of $d a c 2$ at the base of the arthropods, and the subsequent loss of dac2 in the Mandibulata lineage. In addition, the early origin of $d a c 2$ and thus the emergence of the 
patella at the base of the arthropods is not supported by the leg morphology of the known Palaeozoic arthropods (overview in Ortega-Hernandez et al. 2013): neither marellomorphs (Whittington 1971; Garcia-Bellido and Collins 2006) or trilobites (Whittington 1975; Ramsköld and Edgecombe 1996), nor other basal arthropods like Sidneyia inexpectans (Stein 2013) or Shankouia zhenghei (Waloszek et al. 2005) show evidence for a patella segment in their legs.

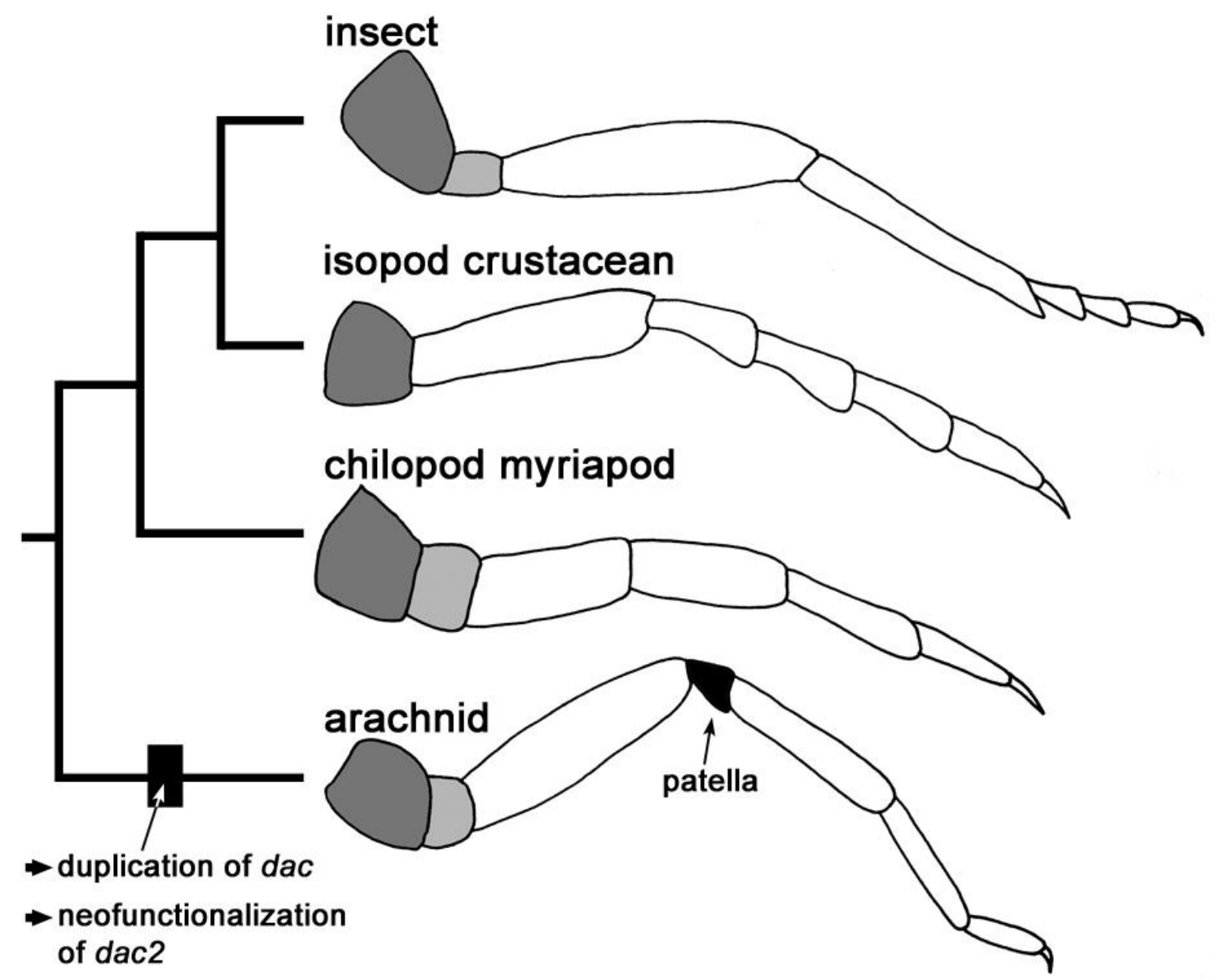

Figure 43: Evolution of a novel leg segment in arachnids linked to dac duplication and neofunctionalisation.

The drawings on the right show the leg segment composition in insects, isopod crustaceans, chilopod myriapods and arachnids (including spiders). The tree on the left depicts a simplified phylogenetic tree showing the interrelationships of these arthropod taxa (after Rota-Stabelli et al. 2011). All taxa possess a proximal short segment (coxa; shown in dark grey), and most taxa also have an additional short segment (trochanter; shown in light grey). But only arachnids have a third short leg segment (patella; shown in black) that is intercalated between two longer leg segments. Our data show that the proper formation of this unique leg segment requires the function of dac2 and thus link the origin of the patella to the duplication and neofunctionalisation of the dachshund gene in arachnids (black rectangle in the tree; autapomorphy of the arachnid clade). 


\subsubsection{Conservation of exd after duplication}

As already found for the bird spider A. geniculata and the entelegyne spider C. salei, and for the previously described leg gap gene dac, the study of exd in the two additional spider species here also revealed the duplication of this leg gap gene. For the two scorpions included in the phylogenetic study even five exd were found. In contrast to the duplication event of $d a c$, the phylogenetic relationship of these duplicated exd paralogs resulted in a polytomy and thus the origin of the gene duplication events could not be properly resolved. Alignments of the protein sequences of the spider exd homologs show a high similarity of the protein also indicating a strong sequence conservation, and this not only true for the first paralog, but also for exd2. The expression in the walking legs was similar to previous findings in spiders (Pechmann and Prpic, 2009; Prpic et al., 2003). Expression of exd1 is restricted to the proximal part during early limb bud developmental stages, reflecting its conserved role in proximal patterning function. The later expression as a ring in the patella, which was found in chelicerate species including C. salei, A. geniculata and the harvest man Phalangium opilio but not in the mite Archegozetes longisetosus, was also present in both spiders studied here (Barnett and Thomas, 2013; Sharma et al., 2012). Thus this ring, which was found to be regulated by the Notch pathway, is a conserved trait of chelicerates (Prpic and Damen, 2009), lost or not detected in the mite. The more detailed expressional analysis of exd1 in the pedipalps of P. tepidariorum showed an additional domain of expression in the distal pedipalp. Such domains were also found in the previously studied spiders, especially in C. salei (personal communication Dr. Prpic-Schäper) but were thought to be the cause of background staining. The presence of this overall pedipalpal staining in an additional spider hints to a differing role of exd1 in the pedipalps and legs.

The second paralog of exd, exd2, differs in expression pattern and dynamics in the walking legs compared to its paralog. The expression starts as a ring in the patella and only later two additional rings arise more proximally. These differences in expression pattern and especially timing suggest a derived function of the duplicate different from the conserved leg gap gene function. To understand if it has also undergone neofunctionalisation similar to e.g. dac, functional studies are required. Although 
different RNAi procedures were tried no visible knockdown phenotypes for exd2 were observed so far, thus future experiments will include the optimization of exd2 RNAi in $P$. tepidariorum. Similar to dac the diverged expression of exd2 after the duplication event is conserved in all four spider species studied so far. This also hints to the evolution of a new function, important for walking leg development in chelicerate species, which was therefore conserved as usual after neofunctionalisation.

\subsubsection{Spider specific hth duplication}

$h$ th is well studied in many arthropod species and is involved in several developmental processes e.g. in eye and limb development (e.g. (Pai et al., 1998; Prpic et al., 2003; Wu and Cohen, 2000)). Consisting with previous studies in the bird spider A. geniculata and C. salei, a member of the entelegyne group from the higher spiders (Pechmann and Prpic, 2009; Prpic et al., 2003), we also found two hth homologs in P. tepidariorum and in P. phalangioides. Although gene duplications in the chelicerates are also found in more basal groups like scorpions and horseshoe crabs (Di et al., 2015; Nossa et al., 2014) it is still not certain how they are related to the duplication found in spiders. Findings for dac paralogs revealed that this duplication is ancestral within the arachnids and that the new function has given rise to an innovative morphologic trait in these species. The study on the phylogeny of the hth duplication in contrast showed that although a duplication event also occurred in scorpions it occurred independent from hth duplication in spiders and therefore hth duplication is not ancestral to the arachnids. The phylogenetic tree shows that the spider $h t h 2$ form a well supported separate clade, not connected with all other chelicerate $h$ ths. All $h$ th1 orthologs show the extremely conserved expression in the proximal and medial part of prosomal appendages excluding the chelicera. This shows that the original gene kept its function after gene duplication. Already in the protein sequence level the spider specific hth2 paralog in contrast shows less conservation than its paralog hth1. Fittingly, like observed for exd and dac, also hth2 is differentially expressed in the walking legs when compared to the conserved expression of $h$ th1. This again indicates a possible neofunctionalisation of this leg gap gene after duplication. 


\subsubsection{Rapid diversification of hth2 after neofunctionalization}

In accordance with diversification at the protein sequence level after the young duplication event in the spider lineage, we found that also the expression pattern of $h$ th2 differs for the three spider species used in this study and the previously published data for the bird spider A. geniculata (Pechmann and Prpic, 2009; Prpic et al., 2003). The four spider species represent different groups with different levels of phylogenetic relatedness (see Figure 3). The largest expression differences for $h$ th2 are found in the prosomal appendages. Our detailed analysis shows that although $P$. tepidariorum and $C$. salei belong to the same group, they do not show a more similar expression pattern compared to the bird spider and the haplogyne spider. This indicates that the possible neofunctionalization and following rapid diversification could have led to the development of diversified morphologies of spider legs. All four spider species have hth2 expressed in a different final number of rings in the fully differentiated walking legs comparing embryos during ventral closure. $C$. salei has six rings in every leg joint, $P$. tepidariorum in contrast has only the three distal rings. In P. phalangioides we find strong hth2 expression only in the most distal ring, whereas in the bird spider we can find ring in every joint, except for the most distal leg joint (Figure 44). Analysis of the expression dynamics further shows that not only the final embryonic walking leg expression is different for the $h t h 2$ homologs in the different species, but that also the development of the rings differs. Usually genes conserve their new function after neofunctionalization and thus do not show diversification after neofunctionalisation (Ohno, 1970). The functional and expressional data for the duplication of exd and dac nicely fit into this picture. Findings for $h t h$ in contrast show that genes can rapidly diversify after neofunctionalization and somehow escape the pressure of conservation, which is even more surprising taking into account that the duplication event of $h$ th is spider specific and thus very young in terms of evolutionary age. Interestingly a recent study in the mouse and the bat Miniopterus schreibersii also showed a diversified function after duplication of the hth vertebrate homologs Meis in the development of the limbs in mammals (Dai et al., 2014). However, functional studies of the $h$ th2 homologs are needed to clearly state that these genes actually have undergone neofunctionalisation. Another 
possible scenario causing the differing expression patterns of hth 2 is the loss of gene function after duplication. A gene without any function could actually show an extreme fast change during evolution because no selective pressure lasts on it. Thus the young $h t h$ duplication might serve as playground for evolution, and being on its way of being lost or gaining a novel function. Genes without function are though to be rapidly lost during evolution, which usually begins with the loss of the open reading frame due to accumulations of mutations caused by lacking selective pressure. The presence of proper open reading frames for all the spider $h$ th 2 homologs contradicts this scenario. However, functional studies are required to reveal the different $h t h 2$ functions in spider. Different RNAi experimental settings were tried, but did not lead to any result yet. A possibility to improve the RNAi for $h t h$ might be an even earlier mating prior to the injection and the injection into more females.

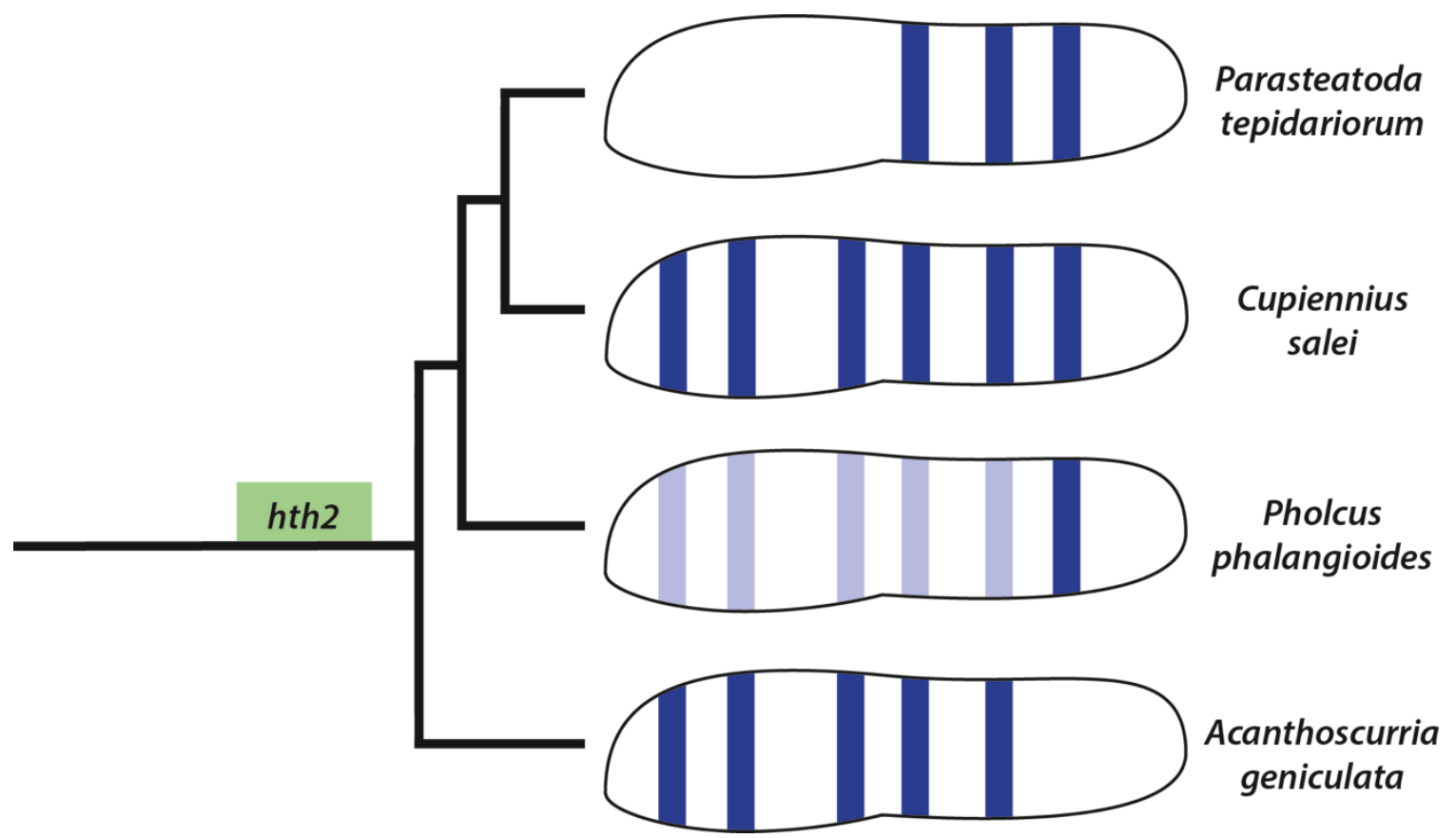

Figure 44: Overview spider $h$ th 2 walking leg expression

The drawings represent a schematic overview of hth2 expression in the walking legs during late embryogenesis in four different spider species. Blue bars indicate strong expression in the waling legs, light blue bars represent weaker expression doamins. Indicated by the green box is the spider specific duplication event of $h t h$ as plesiomorphic trait of all spiders. 


\subsubsection{Leg gap gene conservation and duplication}

Findings in several arthropod species already revealed a strict mode of leg gap gene conservation in patterning the main proximo-distal axis in walking legs (Angelini and Kaufman, 2005; Janssen et al., 2010; Pechmann and Prpic, 2009; Prpic and Tautz, 2003; Prpic and Telford, 2008; Prpic et al., 2003; Sharma et al., 2012; Wu and Cohen, 1999). I studied the medial and proximal leg gap genes $h t h$ exd and dac in the appendages of two additional spider species $P$. tepidariorum and P. phalangioides. I found that all three original leg gap genes observed here were duplicated. The findings also indicate that after the duplication the more conserved first duplicate always kept the conserved leg gene function required for proper PD axis development, but that the other paralog probably had the ability to evolve new functions undergoing neofunctionalisation after the duplication event. This again shows the importance of gene duplications for development novel or alteration of conserved morphological traits. The time point of the duplication event in the chelicerate lineage as well as the conservation after the possible neofunctionalisation, however, was found to be different in all three cases. 


\section{Tarsus segmentation candidate genes in spiders}

In the first part of the following chapter I will present the phylogenetic and expressional analysis in the two spider models $P$. tepidariorum and P. phalangioides of the gene candidates apterous, Lim1, spineless, trachealess and aristaless that are known to be involved in tarsus segmentation of D. melanogaster (Kojima, 2004; Tajiri et al., 2007). The genes rotund, bric-a-brac, chip and tango are known to be involved in the distal patterning of the fly leg as well, but did not show any staining during embryonic development of the two spiders and therefore were excluded from the thesis. The second part of this chapter is concerned with the phylogenetic and detailed expressional analysis of the spider homolog of the D. melanogaster pretarsus gene clawless, also known as C15. The function in the legs of the developing nymphs of one P. tepidariorum cll paralog was studied after gene knockdown via RNAi and is included in that second part as well. The third part describes the phylogenetic relationships of the spider paralogs of another distal segmentation gene candidate, BarH and their expression as well as its function in P. tepidariorum. Finally all findings will be discussed in a separate part at the end of this chapter.

\section{1 apterous in P. tepidariorum and P. phalangioides}

apterous ( $a p$ ) is a homeodomain transcription factor from the Lim-type family (Cohen et al., 1992). A search for homologs in the transcriptomes of $P$. tepidariorum and $P$. phalangioides and subsequent phylogenetic analysis (Supplemental Figure 11 and Supplemental Figure 12) identified three homologs for each spider, which were named according to their phylogenetic relationship with the ap homologs from C. salei (Damen et al., 2002). The phylogenetic analysis showed that the ap duplicate $a p 2$ from $C$. salei, is present in two copies for both P. tepidariorum and P. phalangioides. Only ap1 of $P$. tepidariorum and P. phalangioides, as well as $P p$-ap2.2 could be successfully cloned and used for whole mount in situ hybridization. 
Expressional analysis of the cloned paralogs was performed to investigate their expression in the prosomal appendages and especially in the tarsus during embryonic development. Interestingly contrary to the phylogenetic analysis the expression pattern of Pt-ap1 is more similar to Pp-ap2.2, than to Pp-ap1. Pp-ap2.2 and Pt-ap1 expressions both start with the development of the prosomal limb buds and stay at the base of these appendages during entire limb bud development (arrow, Figure $45 \mathrm{~A}-\mathrm{C}$ and Figure $47 \mathrm{~A}$ C).

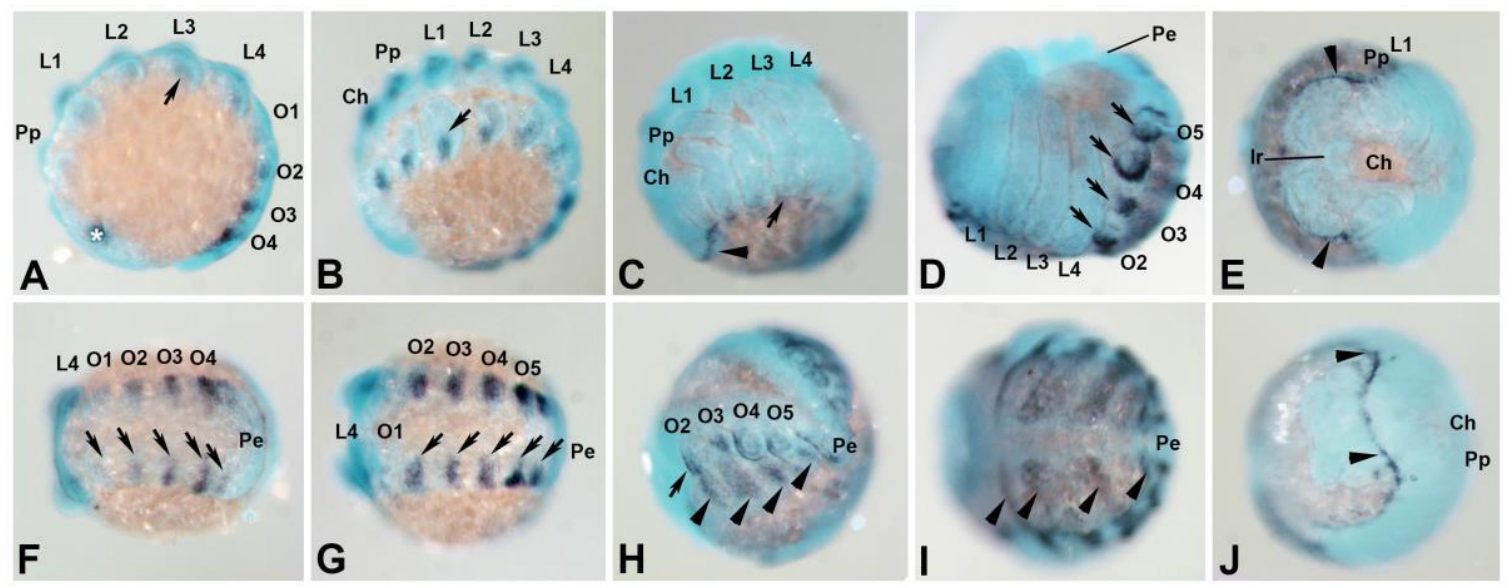

Figure 45: Expression of ap1 in P. tepidariorum

A: Embryo at full germ band elongation, lateral view. The arrow indicates beginning ap1 expression in the prosomal appendages. B: Lateral view of an embryo during limb bud elongation with expression at the base of the appendages (arrow). C: Embryo during inversion expressing ap1 laterally on the head (arrowhead) and proximally in the prosomal appendages (arrow), lateral view. D: Embryo during ventral closure, lateral view. The arrows point to ap1 expression in the opisthosomal limb buds. E: Embryo during inversion, shown in frontal aspect with the walking pointing to the right. Arrowheads indicate ap1 expression laterally surrounding the head lobes. F: Posterior view of an embryo with fully elongated germ band. G: Posterior view of an embryo during limb bud elongation. The arrows mark segmental expression of ap1 in the opisthosoma. H: Embryo during inversion, posterior view. The arrow points to ap1 expression in the opisthosomal limb buds, the arrowhead marks stripes of ap1 expression in the dorsal part of the opisthosoma. I: Dorsal view of an embryo after dorsal closure with stripes of ap1 expression in the dorsal part excluding the heart tissue. J: Frontal view of an embryo during ventral closure. The arrowheads point to the expression of ap1 on the edge of the non-neurogenic ectoderm overgrowing the neural head tissue. The embryos in A-E are oriented with anterior on the left. The embryos in F-I are oriented with posterior to the right. Ir: Labrum, Ch: Chelicera, Pp: Pedipalps, L1-L4: Walking legs pairs 1-4, 01-05: Opisthosomal segments 1-5, pe: posterior end.

With beginning of ventral closure the P. phalangioides ortholog, ap2.2 is additionally expressed as two spots in the patella segment and one spot near the joint of the metatarsus and tarsus (arrow, Figure $47 \mathrm{D}$ and E). Pp-ap1 is in contrast not expressed in any of the prosomal appendages at all. Like expression in the prosomal appendages also 
the opisthosomal expression is more similar between Pt-ap1 and Pp-ap2.2 and strongly resembles that of the ap1 ortholog studied in C. salei. Opisthosomal expression of Pt-ap1 starts at late germ-band stages as stripes in every opisthosomal segment (arrow, Figure 45 F). During limb bud elongation expression in the first opisthosomal segment vanishes and additional expression in 06 appears (arrow, Figure 45 G). During dorsal closure the expression pattern becomes more differentiated and is now present in all opisthosomal limb buds and as stripes in the dorsal areas of the opisthosomal segments. This expression of Pt-ap1 stays during ventral closure of the embryo, but is absent from the most dorsal opisthosomal tissue, the developing heart. In P. phalangioides strong ap2.2 expression in the opisthosomal limb buds begins during limb bud elongation and is similar to Pt-ap.1 (arrowhead, Figure $47 \mathrm{G}$ ). The expression in the dorsal opisthosomal tissue is weaker and only visible during dorsal closure stages (arrowhead, Figure $47 \mathrm{H}$ ). In addition we observe a specific opisthosomal expression for Pp-ap2.2, which was not previously reported for C. salei or observed for the P. tepidariorum paralog (Damen et al., 2002).
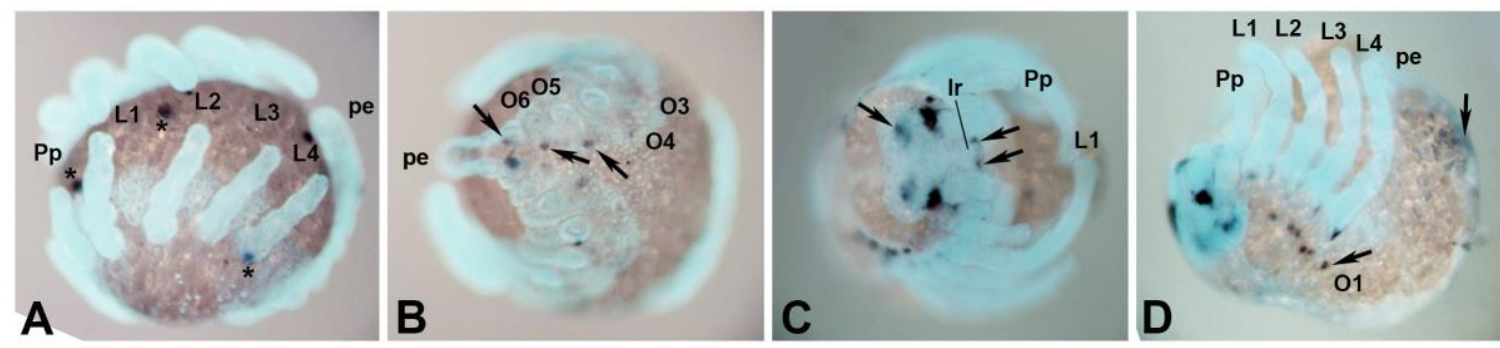

Figure 46: Expression of ap1 in P. phalangioides

A: Lateral view of an embryo during inversion without ap1 expression. The asterisk unspecific staining caused by precipitation. B: Posterior view of an embryo during inversion. The arrows indicate spots of ap1 expression. C: Embryo during ventral closure shown in frontal aspect. The arrow points to expression in the neurogenic ectoderm of the head and two ap1 spots in the labrum. D: Embryo during ventral dorsal closure, lateral view. The arrows mark ap1 spots laterally to the walking legs and a diffuse domain of ap1 expression in the posterior part of the opisthosoma. Ir: Labrum, Ch: Chelicera, Pp: Pedipalps, L1-L4: Walking legs pairs 1-4, 01-05: Opisthosomal segments 1-5, pe: posterior end.

Early during formation and prolongation of the opisthosomal tail, Pt-ap2.2 is expressed as a semicircle near the posterior end (arrow, Figure 47, F and G). During inversion when the opisthosomal tail begins to be folded down on the yolk this semicircle disappears and dorsal spots of expression are found in the opisthosomal segments posterior to 05 
(arrow, Figure $47 \mathrm{H}$ ). A similar expression pattern is observed in the opisthosomal segments 05-07 for the Pp-ap1 paralog (arrow, Figure 46, B). During ventral closure, however, distinct Pp-ap1 expression is present as spots on the lateral side of the prosoma, dorsal to the last three walking legs and in a broad domain in the posterior opisthosoma (arrow, Figure 46, D). The absence of this specific opisthosomal expression pattern in both entelegyne spiders might be correlated with the haplogyne specific opisthosomal organization, but this awaits functional tests in P. phalangioides. The only expression, which is more similar for the two P. phalangioides paralogs compared to Ptap1 is found in the head. Pt-ap1 is expressed as an outer rim surrounding the head region during inversion (arrowhead, Figure 45, E). This Pt-ap1 expression stays on the rim of the non-neurogenic ectoderm when this overgrows the neurogenic ectoderm during head tissue rearrangement at ventral closure (arrowhead, Figure 45, J). In contrast $P p$-ap1 expression in the head starts not prior to ventral closure and is present in two defined areas in the neurogenic ectoderm of the head and in the labrum. The expression of Pp-ap.2.2 in the head starts earliest. First expression is found in a medial head region during limb bud elongation stages (arrowhead, Figure 47, I). During dorsal closure this expression is found near the anterior furrow (arrowhead, Figure 47, J) and later during ventral closure Pp-ap2.2 is expressed in a broad domain in the neurogenic ectoderm and strongly in two defined lateral head regions anterior to the chelicera (arrowhead, Figure 47, E).

In summary expression in the walking legs is only found for Pt-ap1 and Pp-ap2.2 and is very similar for the two genes. Expression in the head is different for all three genes studied here, but more similar for the two paralogs of $P$. phalangioides. The opisthosomal expression of $P p-a p 2.2$ is the most complex and shares pattern elements with both other genes observed here. 


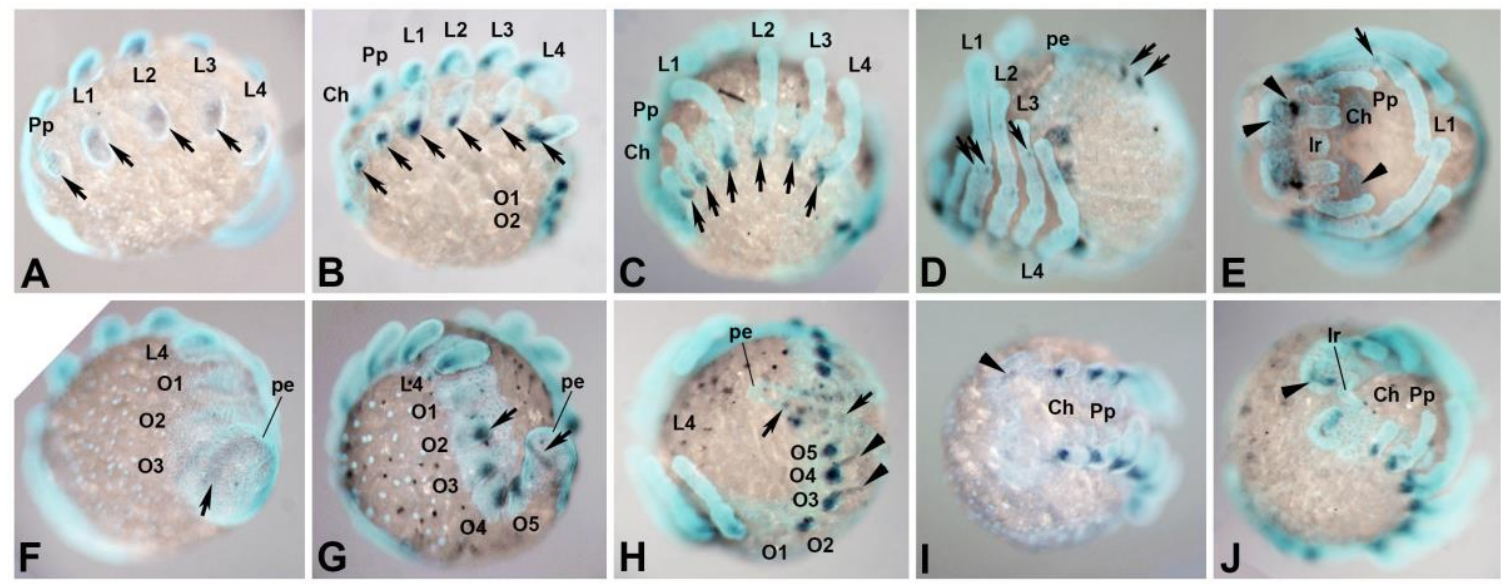

Figure 47: Expression of ap2.2 in P. phallangioides

A: Embryo at beginning of prosomal limb bud development, lateral view. The arrow marks starting expression of ap2.2. B: Lateral view of an embryo during limb bud elongation. C: Embryo during late inversion, lateral view. The arrows in B and C indicate ap2.2 at the base of the prosomal appendages. D: Embryo during ventral closure, lateral view. The arrows indicate spotted ap2.2 expression in the legs and two ap2.2 stripes in the posterior opisthosoma. E: Frontal view of an embryo during ventral closure. The arrow points to the spotted ap2.2 in L1, the arrowheads point on the expression of ap2.2 in the head and the ventral neurogenic tissue. F: Posterior view of opisthosomal expression of ap2.2 in the posterior end (arrow) during beginning of prosomal limb bud development. G: Posterior view of an embryo during limb bud elongation, walking legs pointing to the top. The arrows mark ap2.2 expression in the opisthosomal limb buds and as a semicircle on the posterior end. $\mathrm{H}$ : Posterior view of an embryo during late inversion, walking legs pointing to the left. The arrows point to the spotted ap2.2 expression in the dorsal part of the opisthosomal segments. The arrowheads mark the opisthosomal ap2.2 stripes expanding dorsally from the limb buds. I: Embryo during limb bud elongation, shown in frontal aspect. J: Frontal view of an embryo during late inversion. The arrowhead in I and $J$ points to the ap2.2 expression near the anterior furrow. Embryos are oriented with anterior to the left, exceptions are H-J. lr: Labrum, Ch: Chelicera, Pp: Pedipalps, L1-L4: Walking legs pairs 1-4, 01-05: Opisthosomal segments 1-5, pe: posterior end.

\subsection{Lim1 in P. tepidariorum and P. phalangioides}

Lim1 from D. melanogaster is the ortholog of Lhx1/Lhx5 from vertebrates and like ap another member from the Lim-type gene family (Lilly et al., 1999).

In the transcriptomes of $P$. tepidariorum and $P$. phalangioides two paralogs were identified. The phylogenetic tree (Supplemental Figure 9 and Supplemental Figure 10) shows that this duplication is probably common for spiders, because also two predicted paralogs were found for another spider S. mimosarum. However, to answer the question if the duplication is ancestral in arachnids or chelicerates a more detailed phylogenetic analysis is needed. Nevertheless the phylogenetic tree performed reveals that the 
duplication events in spiders and vertebrates are unrelated, because vertebrate Lhx1 and Lhx5 form a separate monophyletic group.

Both paralogs for P. tepidariorum and one for P. phalangioides (Pp-Lim1.2) were successfully cloned and their expression was studied. The two paralogs of Lim1 found in P. tepidariorum are similarly expressed during early embryonic development. With beginning of limb bud development expression starts at the proximal base of the legs and the pedipalps (early limb bud stages not shown) (asterisk, Figure 48, A and F) including the gnathendite (arrowhead, Figure 48, E, I and J). This expression at the proximal base remains unchanged until the end of embryonic development for both paralogs.

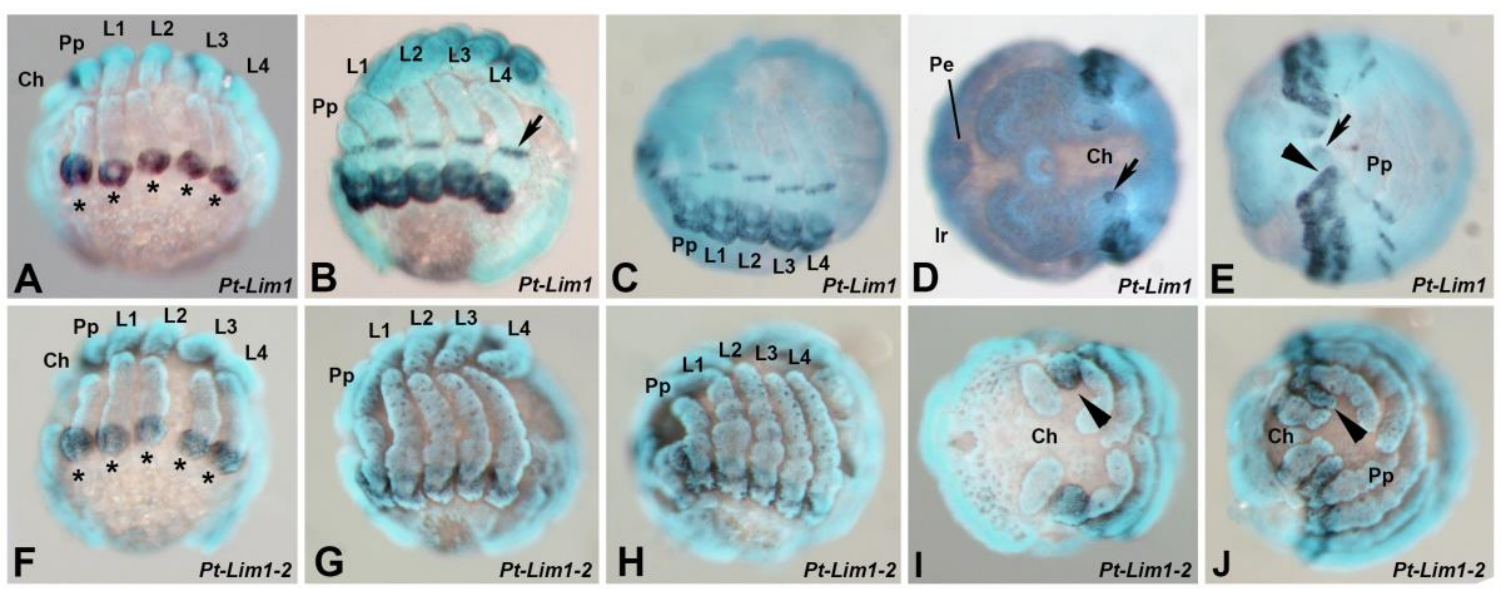

Figure 48: Expression of Lim1 paralogs during embryonic development of $P$. tepidariorum Expression of Lim1 in the prosomal appendages of $P$. tepidariorum embryo during limb bud elongation (A), inversion (B) and ventral closure (C), all in lateral view. The arrow points to the Lim1 ring in the pedipalps and walking legs. D: Frontal view of the head during inversion. The arrow points to Lim1 expression in the ventral part of the chelicera. E: Embryo during ventral closure, frontal view. The arrow points to Lim1 expression in the ventral part of the chelicera. The arrowhead marks the expression of Lim1 in the gnathendite. Expression of Lim1.2 in the prosomal appendages of $P$. tepidariorum embryo during limb bud elongation (F), inversion (G) and ventral closure $(\mathrm{H})$, all in lateral view. I: Frontal view of the head during inversion. J: Embryo during ventral closure, frontal view. The arrowhead in I and J mark the expression of Lim1 in the gnathendite. The asterisk in $A$ and $F$ indicate the start of expression at the base of the pedipalps and walking legs. All embryos are oriented with anterior to the left. Ch: Chelicera, Pp: Pedipalps, L1-L4: Walking legs pairs 1-4, pe: posterior end.

However, during inversion stages an additional ring of Pt-Lim1 expression begins in the medial part of the legs and the pedipalps (arrow, Figure 48, B). At the same time Pt-Lim1 expression starts on the ventral part of the chelicera (arrow, Figure 48, D and E). The Lim1 paralog of $P$. phalangioides, Pp-Lim1.2, is expressed largely similar to its $P$. 
tepidariorum paralog Pt-Lim1.2 in the pedipalps and walking legs. The only expressional difference in these appendages is a ventral stripe of expression (red arrow, Figure 49 B, $\mathrm{C}$ and $\mathrm{H}$ ). The expression of Pp-Lim1.2 in the other embryonic tissues is more distinct between the two spider species. Before strong expression starts at the proximal base of the pedipalps and walking legs it is already expressed in the ventral neurogenic ectoderm anterior to the chelicera (arrowhead, Figure 49, A). This neurogenic expression of Pp-Lim1.2 stays and gets further defined during head differentiation (arrowhead, Figure 49, E and F). Another expression uniquely found for the $P$. phalangioides gene starts just before inversion in the proximal base of the chelicera. Also in the developing limb buds of the opisthosoma Pt-Lim1.2 is expressed throughout development. RNAi of Pt-Lim1 did not lead to any obvious phenotype in the freshly hatched nymphs. A repetition of this RNAi experiment would be required to clarify the gene function.
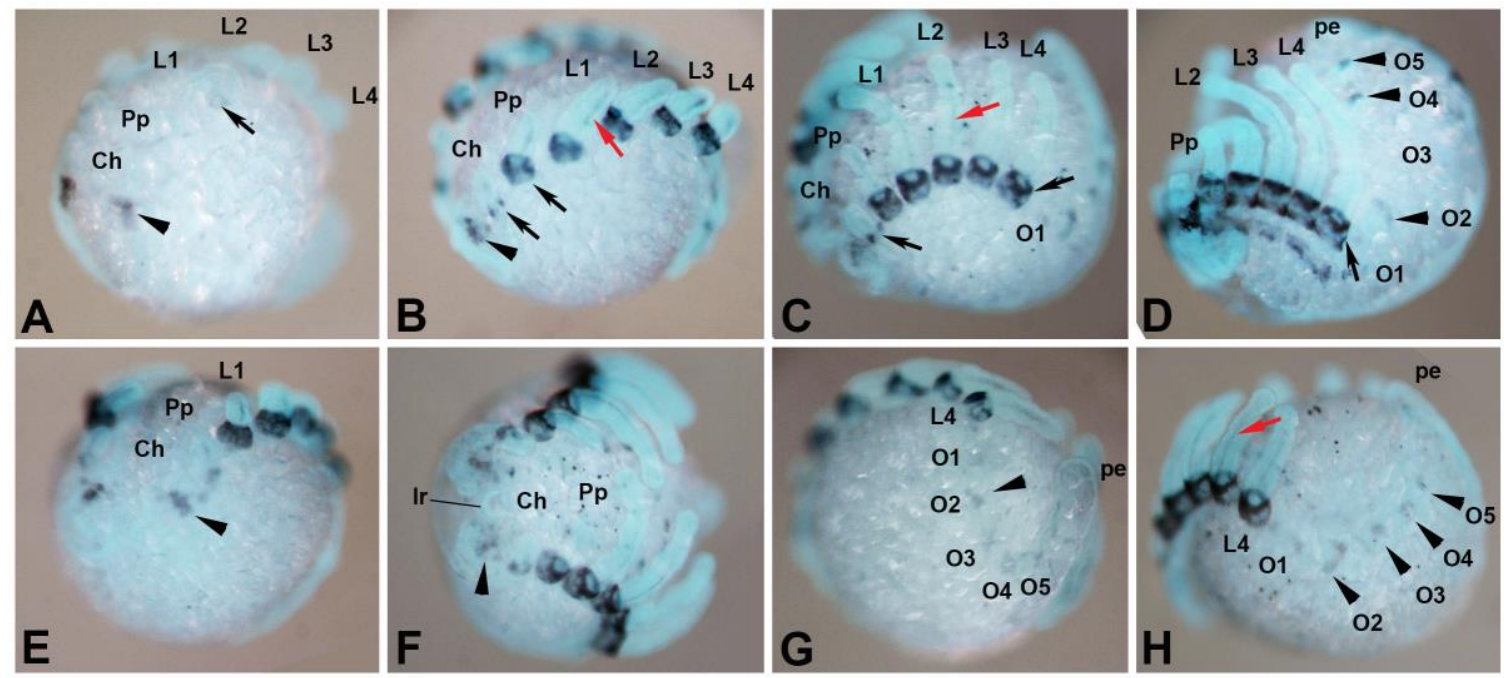

Figure 49: Expression of Lim1.2 in P. phalangioides

Expression of Lim1.2 in during embryonic development of $P$. phalangioides at early limb bud development (A), inversion (B), dorsal (C) and ventral closure (D), all in lateral view. E: Embryo during inversion shown in frontal aspect. F: Embryo during dorsal closure, frontal view. G: Posterior view of an embryo during inversion. $\mathrm{H}$ : Embryo during dorsal closure in posterior view. The arrowhead in A, B, E and F points to a domain of Lim1.2 expression anterior to the chelicera, later giving rise to part of the neurogenic ectoderm. The black arrows in A, B, C and D point to the proximal expression domain of Lim1.2 in the prosomal appendages. The arrowheads in $D$, $G$ and $H$ mark Lim1.2 expression in the opisthosomal limb buds. All embryos are oriented with anterior to the left. The staining was performed by Jana Holtzheimer. Ir: Labrum Ch: Chelicera, Pp: Pedipalps, L1-L4: Walking legs pairs 1-4, 01-05: Opisthosomal segments 1-5, pe: posterior end. 


\section{3 spineless in P. tepidariorum and P. phalangioides}

spineless (ss) is a transcription factor and belongs to the family of basic-helix- loophelix-PAS (bHLH-PAS) domain proteins. The name PAS derives from the founding members of this family, namely: Period, Aryl hydrocarbon receptor (Ahr) and singleminded (for review see (Crews, 1998; Crews and Fan, 1999)). Early studies in $D$. melanogaster found that it is, as it name says, involved in correct bristle formation and also development of distal antenna and leg regions (Emmons et al., 1999; Struhl, 1981). Due to high sequence similarity caused by the PAS domain the phylogenetic analysis for ss was performed in combination with another bHLH-PAS gene, trachealess that is also known to be involved in tarsus segmentation of D. melanogaster (Tajiri et al., 2007). The phylogenetic analysis (Supplemental Figure 13 and Supplemental Figure 14) indicates that two spineless paralogs are present in the spider species $P$. tepidariorum and $P$. phalangioides (depicted in red). This duplication is probably ancient for spiders because two paralogs are also present in the spider S. mimosarum. Consistent with their gene family relationships the spider genes form a monophyletic group with $s s$ homologs from onychophorans and insects, as well as with their vertebrate homologs, the Ahr proteins. The other candidates found in the transcriptomes of the two spiders studied here cluster together with other bHLH-PAS domain proteins like clock (depicted in violet), similar (depicted in green), trachealess and its vertebrate homolog neuronal-PAS-containing protein (dark red) and single minded (depicted in blue).

I cloned and performed in situ hybridization for all four ss paralogs identified in the phylogenetic tree. In P. tepidariorum expression of $s s 1$ in the prosomal appendages starts during limb bud elongation with one spot at the distal part of the chelicera and two spots in the walking legs (arrow, Figure 50, A). During limb bud differentiation additional expression in the gnathendite and corresponding tissue in the ventral neuroectoderm under the legs starts and medial spots of expression arise in the walking legs (arrowhead, Figure 50, B). The expression in the distal part becomes stronger and more spots of expression arise, covering the distal part of the walking legs and pedipalps in a ring like manner (arrowhead, Figure 50, B). During inversion and dorsal closure stages a broader expression domain arises underneath the spotted expression in the distal part of 
the walking legs and pedipalps (red bar, Figure 50, C and D). In addition more spots of expression arise in the medial part of the pedipalps, the walking legs and the chelicera (Figure 50, D). Expression of ss1 in the prosomal appendages during early embryonic development of $P$. phalangioides is comparable but not identical to P. tepidariorum. Just after beginning of inversion Pp-ss1 is expressed in the ventral neuroectoderm at the basis of the walking legs (arrowhead, Figure 51, B) and in a spotted manner in the distal part of the walking legs and pedipalps (arrow, Figure 51, B). During dorsal closure additional medial spots arise in the walking legs, whereas the distal expression does not broaden as in P. tepidariorum (arrow, Figure 51, C). In addition only one single spot of $P p$-ss1 is expressed in the chelicera during dorsal closure (arrow, Figure 51, G). However, during ventral closure the expression in the chelicera is virtually identical to $P$. tepidariorum showing a complex spotted expression pattern in the chelicera (Figure 51, H).

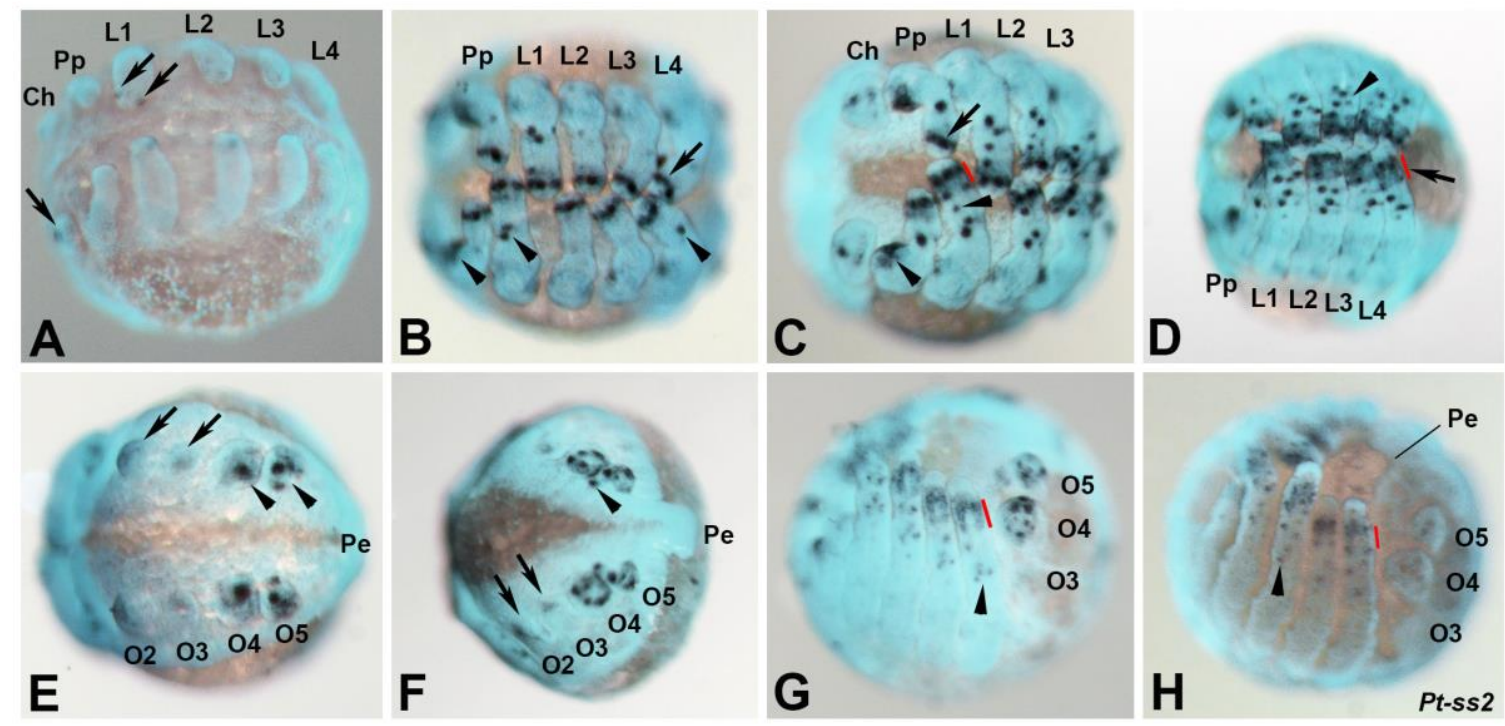

Figure 50: Expression of spineless paralogs in P. tepidariorum

A-G: Expression of $s s 1$ in P. tepidariorum. A: Embryo during limb bud elongation, lateral view. Ventral view of the prosomal appendages during limb bud differentiation (B), inversion (C) and dorsal closure (D). E: Embryo during limb bud elongation, ventral view. F: Embryo during dorsal closure, posterior view. G: Lateral view of an embryo during ventral closure. H: Embryo expressing Pt-ss2 during ventral closure, lateral view. Detailed expression of $s s$ paralogs is described in the text. Embryos in A-D and $G$ and $H$ are oriented with anterior to the left, $E$ and $F$ are oriented with posterior to the right. Jana Holtzheimer helped by the stainings of Pt-ss2. Ch: Chelicera, Pp: Pedipalps, L1-L4: Walking legs pairs 1-4, 01-05: Opisthosomal segments 1-5, pe: posterior end. 
In contrast to the similar expression of $s s 1$ in the chelicera of late embryonic stages, the expression in the remaining prosomal appendages differs drastically between the two spiders during ventral closure. Whereas the ss1 expression pattern in P. tepidariorum remains similar to earlier stages, it changes significantly for P. phalangioides. With the beginning of ventral closure Pp-ss1 is expressed in a complex ring like manner in the pedipalps and very strongly in the entire walking legs excluding the areas of joint formation (Figure 51, $\mathrm{H}$ and D). In the opisthosoma the expression pattern of ss1 is more comparable for the two spider species, and differences are only observed concerning the temporal expression profile. In P. phalangioides opisthosomal ss1 expression already starts after early prosomal limb bud formation and covers the dorsal part of the developing opisthosomal tail bud (arrow, Figure 51, A). During inversion this opisthosomal expression is restricted to the four opisthosomal limb buds (arrows, Figure 51, E) in P. phalangioides. Later expression in the second and third opisthosomal limb bud, however, first fades (dorsal closure, Figure $51 \mathrm{~F}$, arrow) and finally completely disappears for the limb bud on 03 (ventral closure, Figure 51, D). The expression in the limb buds on 04 and 05 in contrast differentiates into a spotted expression pattern (arrowhead, Figure 51, D). This differentiation (arrowhead) and fading (arrows) can also be observed in $P$. tepidariorum but starts already during limb bud elongation and continues during dorsal closure (Figure 50, E and F).

The expression of the second paralog found in both spider species starts remarkably later and weaker. Pt-ss2 starts during ventral closure stages and is virtually identical to its paralog $P t$-ss1. $P p$-ss2 expression already starts during dorsal closure similar to Pp-ss1 in the distal part of the walking legs (arrow, Figure 51, I) and in the limb buds on 04 and 05 (arrow, Figure 51, J). During ventral closure the distal expression in the walking legs becomes stronger and additional spots in the medial part arise (arrow, Figure 51, K). Expression also arises in the chelicera (arrow) and the gnathendite of the pedipalps (arrowhead, Figure 51, L). Hence, $P p$-ss2 does not change so dramatically as its paralog $P p$-ss 1 and is thus more comparable to the expression found for the two P. tepidariorum paralogs. RNAi of Pt-ss1 did not lead to any obvious phenotype in the freshly hatched nymphs. A repetition of this RNAi experiment including analysis of older nymphal stages is currently in progress. 

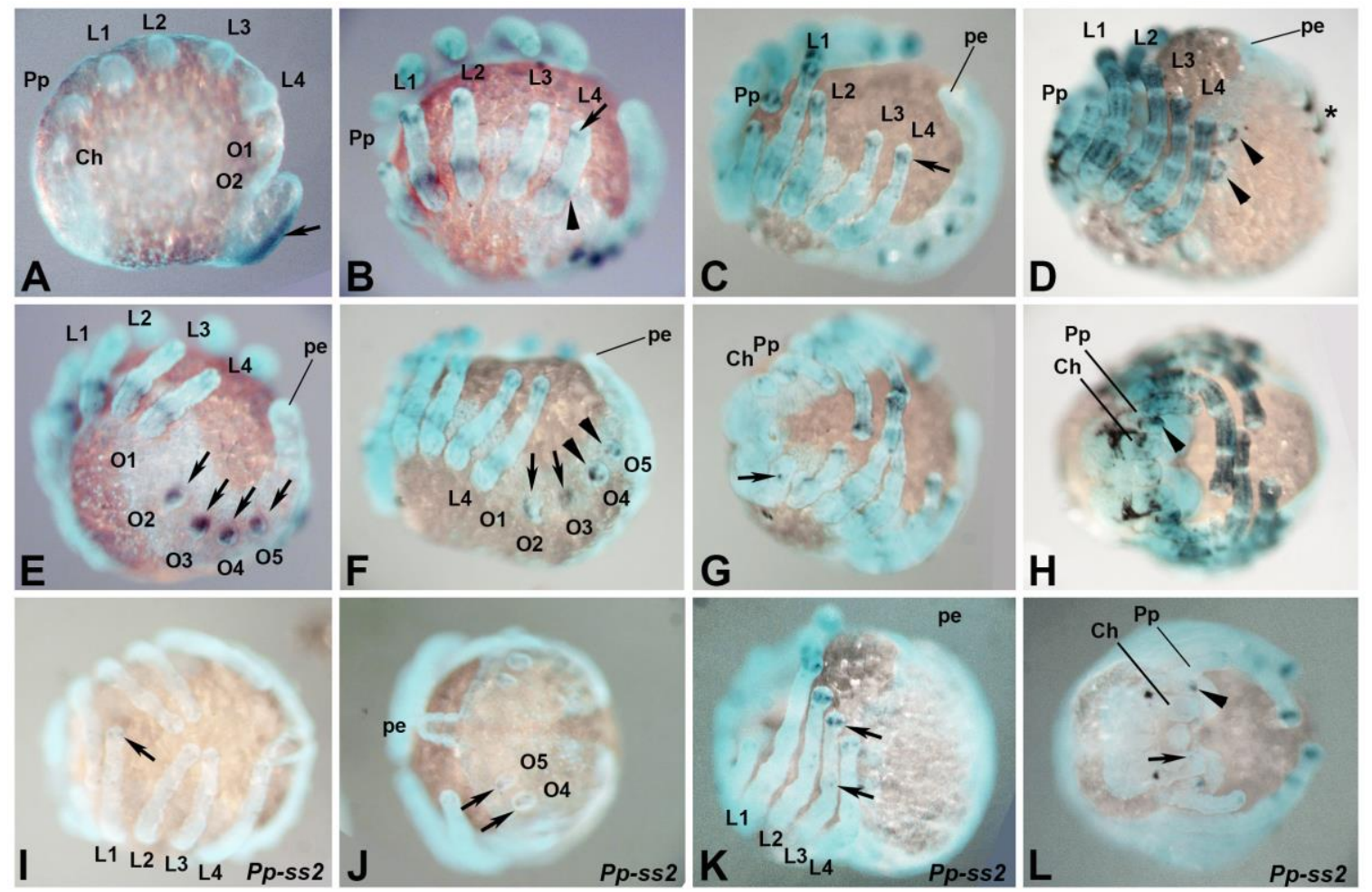

Figure 51: Expression of spineless paralogs in P. phalangioides

A-H: Expression of $s s 1$ during embryonic development of $P$. phalangioides. I-L: Expression of $s s 2$ in embryos of $P$. phalangioides. A: Embryo during early limb bud formation. B: Embryo at inversion. C: Embryo during dorsal closure. D: Embryo during ventral closure. The asterisk marks unspecific staining in the forming cuticle tissue. E: Opisthosoma of an embryo during inversion. F: Opisthosoma of an embryo during dorsal closure. The embryos in A-F are all shown in the lateral view. G: Head of an embryo in frontal view during dorsal closure. H: Frontal view of an embryo during ventral closure. I: Ventral view of an embryo during dorsal closure. J: Posterior view of an embryo during dorsal closure. K: Embryo during dorsal closure in lateral view. L: Embryo during ventral closure shown in the frontal aspect. Detailed expression of $s s$ paralogs is described in the text. All embryos are shown with anterior to the left, except for panel J. Ch: Chelicera, Pp: Pedipalps, L1-L4: Walking legs pairs 1-4, 01-05: Opisthosomal segments 1-5, pe: posterior end. 


\section{4 trachealess in P. tepidariorum and P. phalangioides}

trachealess (trh), encodes like ss, a PAS domain protein and is thus a member of the bHLH-PAS family (Córdoba and Estella, 2014).

Two paralogs of trh were identified after detailed phylogenetic analysis in the transcriptomes of P. tepidariorum and P. phalangioides (Supplemental Figure 13 and Supplemental Figure 14). Although not many representatives of other chelicerate species were found in the blast search the phylogeny nevertheless supposes that the paralogs of $P$. tepidariorum and P. phalangioides cluster together into two distinct chelicerate trh groups. The time point of a possible trh duplication in the lineage leading to the spiders needs to be further studied including more representatives from the chelicerate group. The phylogenetic tree suggests that the duplication of trh in chelicerates and the duplication of the vertebrate homologs of the neuronal PAS proteins were two independent events.

According to the clustering found for the spider trh homologs, the expression is more similar for orthologs of the different species, than for paralogs of one spider species. trh1 is expressed in a less complex pattern than its paralog and shows a very comparable pattern in the walking legs of both spider species. In P. tepidariorum expression starts in two medial rings in the walking legs during early inversion (arrow, Figure 52, A). Later during inversion one ring of Pt-trh1 expression also starts in the pedipalps (arrowhead) and the distal ring in the walking legs broadens and starts to split (bar, Figure 52, B). With the onset of dorsal closure and during ventral closure, thus three rings of Pt-trh1 are present in the medial part of the legs (arrows, Figure $52 \mathrm{C}$ and D). The proximal ring is expressed in the joint between the leg segments femur and patella, whereas the two distal rings are both expressed distally in the tibia. For P. phalangioides this late expression pattern of trh1 in the walking legs is similar, but shows differences in terms of timing in earlier stages as well as in the other embryonic tissues. Pp-trh1 already starts prior to inversion as one medial ring in the first walking leg pair (arrow, Figure 53, A), which is later during inversion visible in the other legs pairs, but never in the pedipalps (arrow, Figure 53, B). Also splitting of the Pp-trh1 distal ring occurs slightly later, during ventral closure. However, the three rings of Pp-trh1 are expressed in 
comparable leg segments as observed for P. tepidariorum (arrows, Figure 52 and 53, C). In addition to these comparable trh1 expression patterns, Pp-trh1 has also unique expression different to Pt-trh1. During ventral closure Pp-trh1 is also expressed at the proximal part of the pedipalps and walking legs as well as in the limb bud on the third opisthosomal segment during inversion (arrow, Figure 53, D). In summary trh1 is expressed in a very conserved pattern in the walking legs of these two spiders and only the exact timing and expression in the opisthosoma is different.

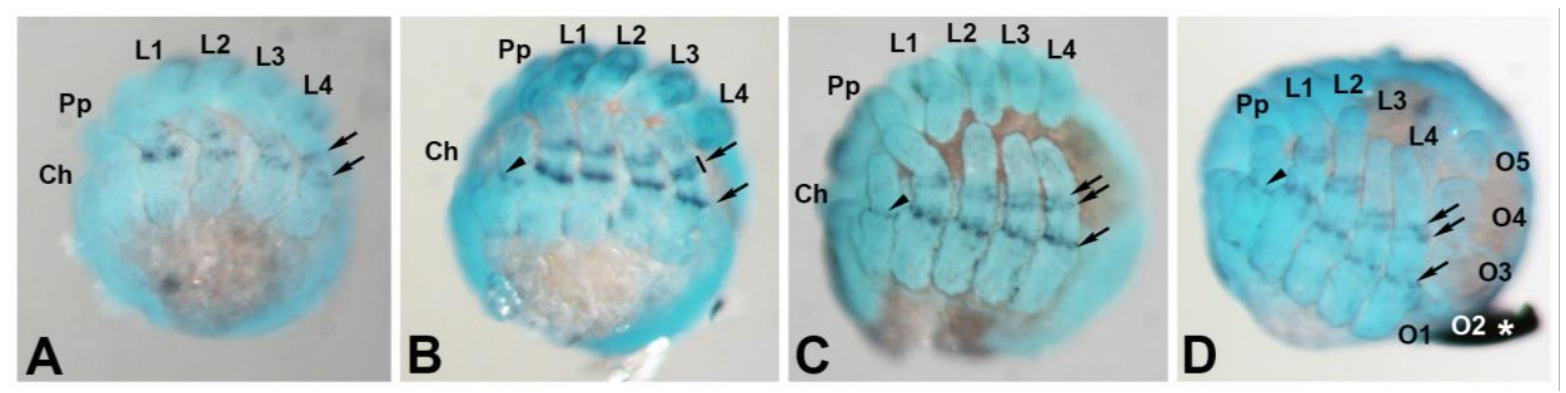

Figure 52: Expression of trh1 in P. tepidariorum

Expression of $t r h 1$ in prosomal appendages during embryonic development of $P$. tepidariorum at limb bud elongation (A), inversion (B), dorsal closure (C) and ventral closure (D). D: The asterisk marks remaining vitelline membrane which was accidentally not removed before the in-situ hybridization and shows unspecific staining. Detailed explanation of the expression pattern is found in the text. All embryos are shown in lateral view and oriented with anterior to the left. Ch: Chelicera, Pp: Pedipalps, L1-L4: Walking legs pairs 1-4, 01-05: Opisthosomal segments 1-5.
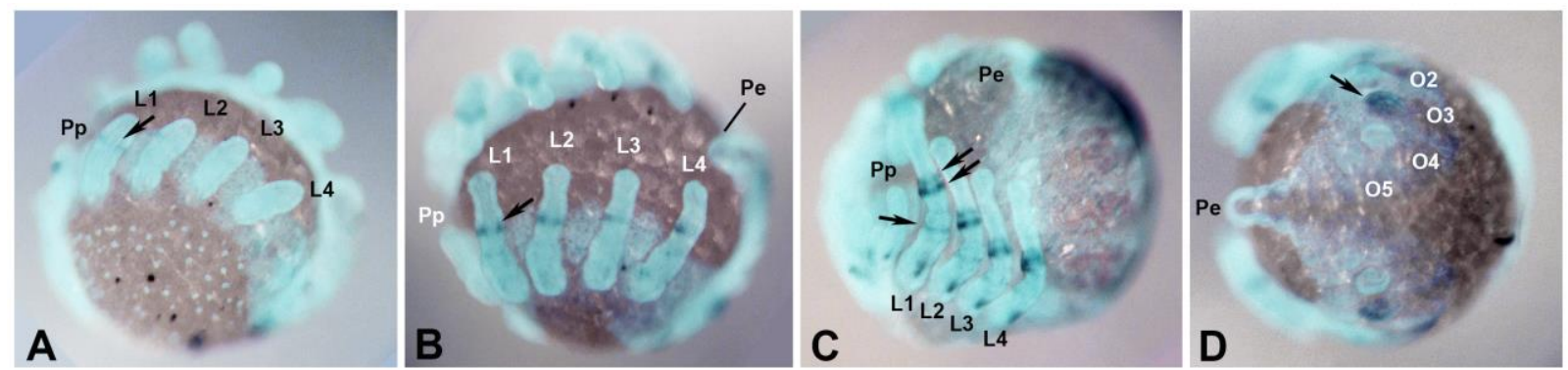

Figure 53: Expression of trh1 in P.phalangioides

Expression of trh1 in prosomal appendages of $P$. phalangioides during limb bud elongation (A), inversion (B) and ventral closure (C). A-C: All embryos are shown in lateral view and oriented with anterior to the left. D: Dorsal view of the opisthosoma during inversion. Detailed explanation of the expression pattern is found in the text. Pp: Pedipalps, L1-L4: Walking legs pairs 1-4, 01-05: Opisthosomal segments 1-5, pe: posterior end. 
The expression pattern of trh2 is in general more complex in the prosomal appendages and the opisthosoma when compared to its paralog trh1. For trh2 ring-like expression in the prosomal appendages starts only very weak prior to dorsal closure (arrow, Figure 54, B and Figure 55, B). At later stages an extensive expression throughout the entire pedipalps and walking legs starts for both spider species. Strong Pt-trh2 expression starts during dorsal closure stages with a complex pattern in the entire walking legs, including a ring-like pattern in the medial part (arrows, Figure 54, C). During ventral closure this complex Pt-trh2 expression in the walking legs remains ring-like but is now strongest in the distal part (arrow, Figure 54, D). In P. phalangioides a medial ring of expression becomes slightly stronger during dorsal closure (arrow, Figure 55, C). During ventral closure a sudden increase of expression starts and is then even stronger and more defined than for P. tepidariorum. Pp-trh2 during these late stages is expressed in a ring-like manner in the pedipalps and in the entire walking legs, excluding the areas of joint formation (arrow, Figure 55, D). Not only in the walking legs but also in the chelicera trh2 is differently expressed in the two spiders. Whereas Pp-trh2 starts very late in the medial part of the chelicera during ventral closure (arrow, Figure 55, D), Pptrh2 expression already begins with a spot proximal on the ventral side during inversion (arrow, Figure 54, H). Afterwards a second spot of Pt-trh2 appears more medially (arrows, Figure 54, I and J). In contrast to the prosomal appendages the expression pattern of trh2 in the opisthosoma is more comparable in P. tepidariorum and $P$. phalangioides and differs only in timing and strength. First trh2 expression starts at the ventral rim of the opisthosoma during limb bud elongation stages in P. tepidariorum (arrowhead, Figure 54, F). In P. phalangioides trh2 expression starts slightly later, during inversion, also at the ventral part of the opisthosoma (asterisk and arrows, Figure 55, B and E), but is already additionally present in the third opisthosomal segment (arrowhead, Figure 55, E). During dorsal closure the ventral expression in $P$. phalangioides vanishes in the more anterior stages, but remains in the more posterior ones and the limb bud on 03 (arrowhead and arrows, Figure 55, F). During ventral closure the expression in opisthosomal limb bud is covered by the legs and cannot be observed. The expression of Pt-trh2 is stronger compared to Pp-trh2, but also found in the third opisthosomal limb bud during later stages (arrow on 03, Figure 54, G). In 
contrast to Pp-trh2 it stays also in the ventral part of the opisthosoma, as well as in the posterior end and is additionally expressed in the more posterior opisthosomal limb buds after inversion. During dorsal closure Pt-trh2 is expressed on the ventral rim of the limb bud on 04 and remains there during ventral closure (arrow on 04, Figure 54, G and E). At this stage two additional spots of Pt-trh2 are present in the limb bud on 04 and 05 (arrows on 05, Figure 54, E). Unique is the expression of Pt-trh2 at the anterior rim of the head during these late embryonic stages (arrowhead, Figure 54, I and J).

In summary trh2 in both spiders is expressed stronger in the prosomal appendages and the opisthosoma compared to its paralog, especially during late stages. The pattern in these tissues is similar but not entirely identical and differs in timing.

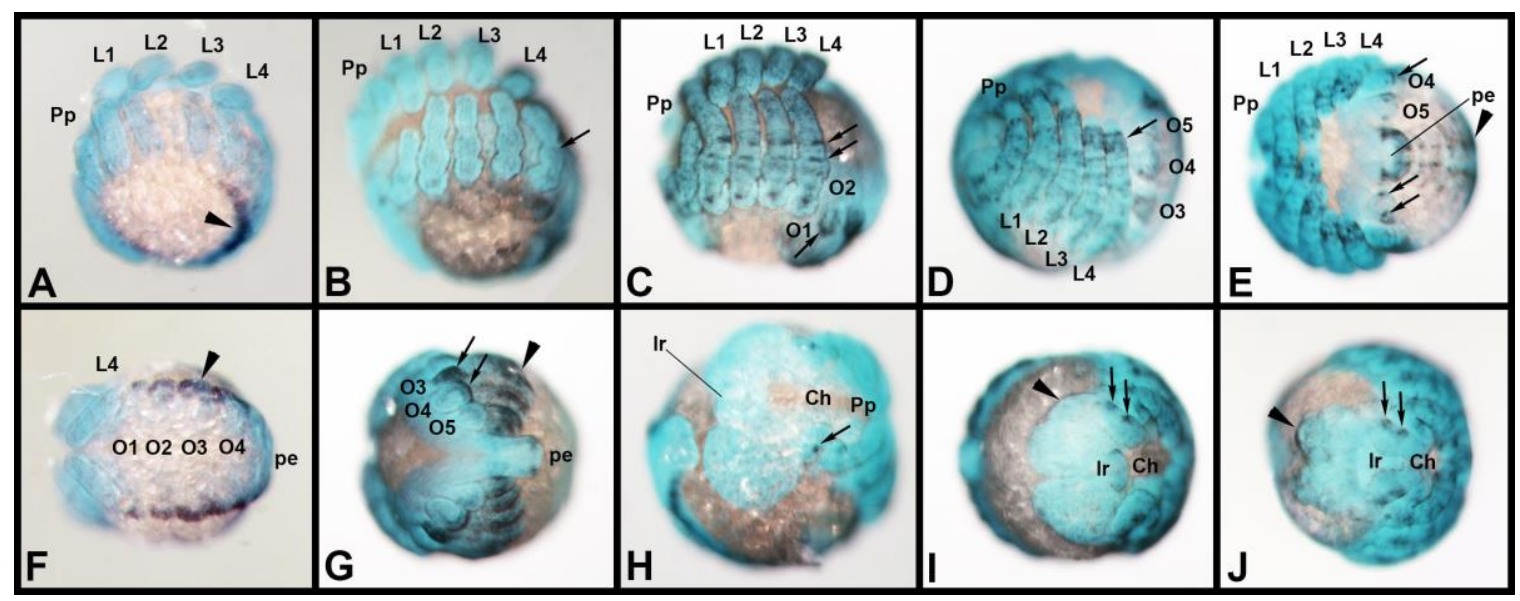

Figure 54: Expression of trh2 in P. tepidariorum

Expression of trh2 in prosomal appendages during embryonic development of $P$. tepidariorum at limb bud elongation (A), inversion (B), dorsal closure (C) and ventral closure (D). The embryos are shown in lateral view. E: Ventral view of an embryo during ventral closure. F: Ventral view of the opisthosoma during limb bud elongation. G: Opisthosoma of an embryo during dorsal closure in posterior view. H: Embryo during inversion shown in frontal aspect with posterior in the left. I: Frontal view with legs pointing to the right of an embryo during dorsal closure. J: Embryo during ventral closure, frontal view walking legs pointing to the right. Detailed explanation of the expression pattern is found in the text. The embryos in A-E are oriented with anterior to the left, in $F$ and $G$ the posterior end is pointing to the right. Ch: Chelicera, Pp: Pedipalps, L1-L4: Walking legs pairs 1-4, 01-05: Opisthosomal segments 1-5, pe: posterior end. 


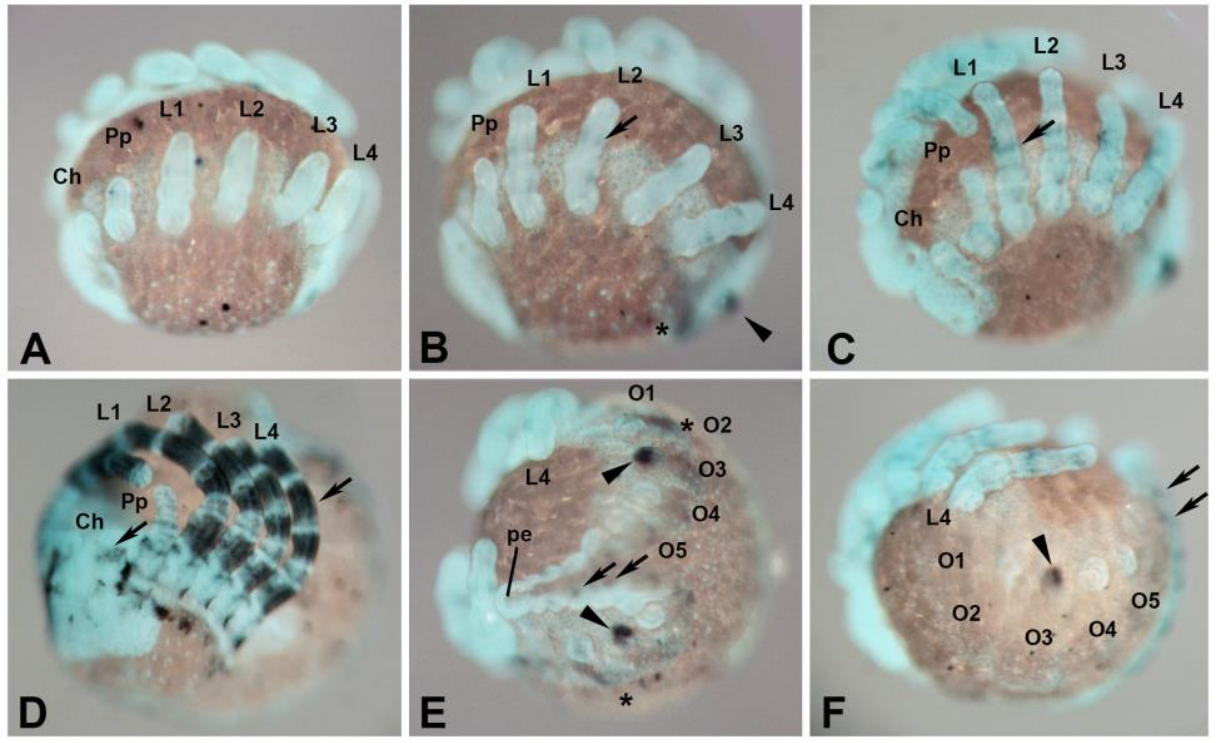

Figure 55: Expression of trh2 in P. phalangioides

Expression of trh2 in prosomal appendages of $P$. phalangioides during limb bud elongation (A), inversion (B) and beginning dorsal closure (C) and ventral closure (D). The embryos are shown in lateral view. F: Posterior view of an embryo during inversion, the walking legs pointing to the left. F: Dorso-lateral view of the opisthosoma during dorsal closure. Detailed description of expression in the text. All embryos are oriented with anterior to the left, except for panel E. Ch: Chelicera, Pp: Pedipalps, L1-L4: Walking legs pairs 1-4, 01-05: Opisthosomal segments 1-5.

\section{5 aristaless in P. tepidariorum and P. phalangioides}

The paired-type homeobox gene aristaless (al) is required for the development of the distal elements of the antenna and walking legs (Campbell and Tomlinson, 1998).

The phylogenetic analysis of al homolog candidates found in the transcriptomes $P$. phalangioides (Janssen et. al., 2015) and P. tepidariorum (Posnien et al., 2014) showed that both spiders have only one genuine al homolog (depicted in red, Supplemental Figure 7 and Supplemental Figure 8). Only one homolog was found for another spider $S$. mimosarum. A number of additional genes that are similar to aristaless were found in the transcriptome sequences, but have been excluded from further analysis, because their relationship to aristaless is not clear from the phylogenetic analysis.

The al homologs were cloned for P. tepidariorum and P. phalangioides. Expression of Ptal starts as spots in the distal part of the first, third and fourth walking legs at beginning of limb bud development (arrowhead, Figure 56, A). During limb bud elongation stages the spot-like expression of $P t-a l$ is visible in the distal part of all legs and the pedipalps 
(arrowhead, Figure 56, B) and a broader distal expression domain in the chelicera. Additional expression starts as a ring in the medial part of the walking legs (arrows, Figure 56, B) and pedipalps and a spot on the proximal base of these appendages (asterisk, Figure 56, B). During early inversion stages a second ring of expression appears proximal to the earlier ring (arrow, Figure 56, C). Beginning in late inversion stages until ventral closure these two medial rings and the proximal spots diffuse and give rise to a very complex expression pattern covering the proximal and medial part of the pedipalps and legs (Figure 56, D-F). The spot-like Pt-al expression in the distal part of the appendages is then also present as three spots at the very tip of the legs and pedipalps. By contrast, Pt-al expression in the chelicera and the opisthosomal limb buds is more uniform during embryonic development (see below). Early expression of $a l$ in $P$. phalangioides is visually identical with $P$. tepidariorum. With the start of limb formation on the germ band $P p-a l$ is expressed as spots at the distal tip of pedipalps and legs (arrowheads, Figure 57, A). As in P. tepidariorum the medial ring and proximal spot of Pp-al expression (arrows, Figure 57, B) starts during limb bud elongation. With the beginning of inversion this medial ring broadens and proximal to it a domain of $P p-a l$ starts, but not so clearly ring shaped as in P. tepidariorum. Starting with dorsal closure until ventral closure the expression of $P p$ - $a l$ becomes more diffuse covering the proximal and medial region of pedipalps and legs, similar to P. tepidariorum. In contrast to the similar expression of $a l$ in the pedipalps and the walking legs, $P p-a l$ never is expressed in a broad distal domain in the chelicera but as a spot which later divides identical to the remaining prosomal appendages (arrowheads, Figure 57, E and F). The expression in the head and opisthosoma, however, is mainly similar for the two observed spider species. 


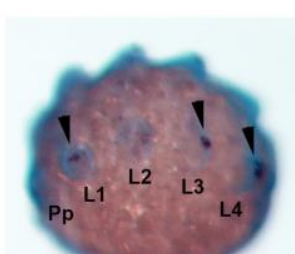

A

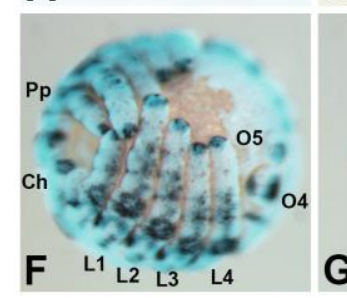

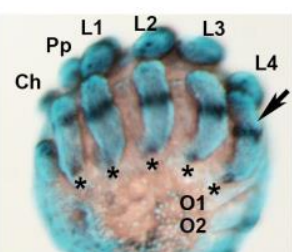

B

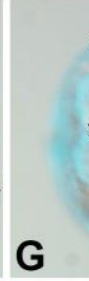

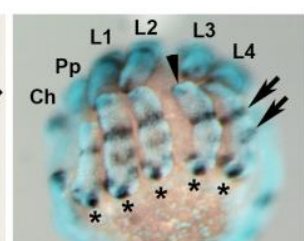

C

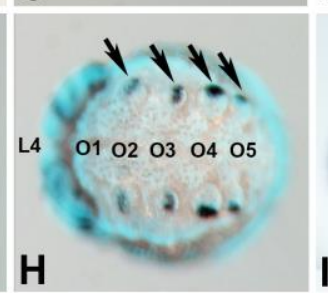

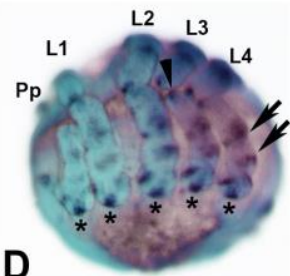

D

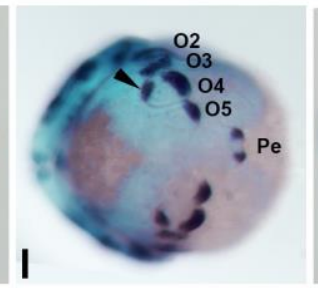

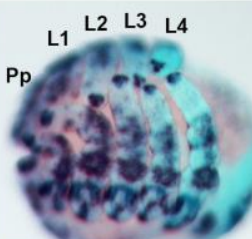

E

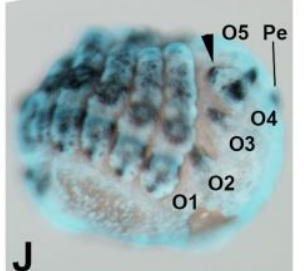

Figure 56: Expression of aristaless in P. tepidariorum

A: Fully elongated germ band embryo, lateral view. Arrowheads point starting al expression on the forming limb buds. Expression of al during limb bud elongation (B), early inversion (C), late inversion (D) and dorsal closure (E), all embryos in lateral view. Asterisk mark al expression at the proximal base of the pedipalps and walking legs. The arrows point to ring-like al expression in these appendages. The arrowhead marks the distal $a l$ spot in the walking legs and pedipalps. F: Embryo during ventral closure expressing three distal al spots in the walking legs, ventral view. G: Frontal view of embryo during ventral closure with al expression around the eye field. H: Ventral view of the opisthosoma, posterior to the right. The arrows point to al expression in the opisthosomal limb buds. I: Posterior view of an embryo during dorsal closure. J: Embryo during ventral closure, lateral view. The arrowhead in I and $J$ points to the additional ventral expression domain on the limb bud on 04 . All embryos are oriented with anterior to the left, except for the embryos shown in $\mathrm{H}$ an $\mathrm{I}$. Ch: Chelicera, Pp: Pedipalps, L1-L4: Walking legs pairs 1-4, 01-05: Opisthosomal segments 1-5, pe: posterior end.

Until ventral closure $a l$ is not expressed in the head, but then starts surrounding the fields of lateral eye development in the head lobes (Figure 56, G and Figure $57 \mathrm{~F}$, arrowheads). With the beginning of opisthosomal limb bud development al is expressed as a stripe in the opisthosomal limb buds on the second and third opisthosomal segment in both spiders (the precursors of the book lung and tubular trachea) (arrow, Figure 56, $\mathrm{H})$. In the opisthosomal limb bud of the fourth and fifth opisthosomal segment, which will give rise to the spinnerets, al is expressed exclusively on the dorsal part. With beginning of dorsal closure in P. tepidariorum an additional ventral expression of al is visible at the limb bud of the fourth opisthosomal segment (arrowhead, Figure 56, I and J). During the same stage expression of al starts as two spots at the posterior end of the $P$. tepidariorum embryo. In P. phalangioides expression of al on the limb bud on 03 disappears with invagination of the tubular trachea (asterisks, Figure 57, H). 

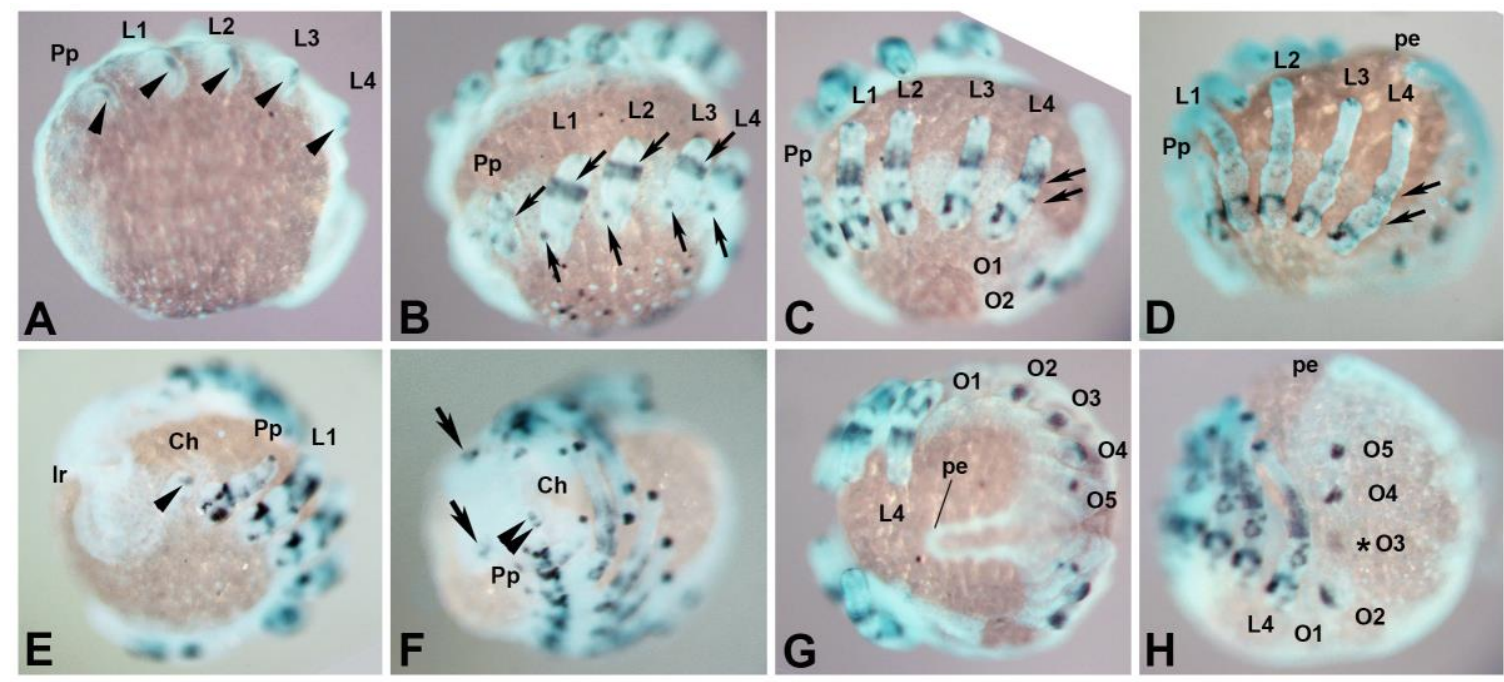

Figure 57: Expression of aristaless in P. phallangioides

A: Embryo during early limb bud development, lateral view. The arrowheads point to distal al expression in the pedipalps and walking legs. B: Embryo of $P$. phalangioides during limb bud elongation, lateral view. The arrows mark proximal $a l$ spot in the walking legs and appearing $a l$ ring in the pedipalps and walking legs. $C$ and $D$ : Lateral view of embryos during inversion and dorsal closure. The arrows point to two al ring-like expressions. E: Embryo during inversion shown in frontal aspect. The arrowhead points to distal al expression in the chelicera. F: Frontal view of an embryo during ventral closure. The arrows point to al expression around the developing eyes and the arrowheads indicate the division of the distal al spot. G: Posterior view of the embryo during inversion. $\mathrm{H}$ : Embryo during ventral closure in lateral view. The asterisk marks the disappearing al expression during invagination of the opisthosomal limb bud on 03. All embryos are oriented with anterior to the left, except for the embryo shown in $\mathrm{G}$ : here ventral is located left and dorsal right. Ir: Labrum, Ch: Chelicera, Pp: Pedipalps, L1-L4: Walking legs pairs 1-4, 01-05: Opisthosomal segments 1-5, pe: posterior end. 


\subsection{Clawless}

clawless (cll) encodes for a homeodomain protein, and was first identified as a member of the 93DE homeobox cluster located on the third chromosome of D. melanogaster and initially named C15 (Dear and Rabbitts, 1994; Dear et al., 1993). The name cll was given in accordance to its D. melanogaster mutant phenotype lacking the tarsal claws of the walking legs (Campbell, 2005; Kojima et al., 2005).

\subsubsection{Phylogenetic analysis of clawless duplicates in spider}

I searched for cll sequences in the transcriptomes of $P$. phalangioides (Janssen et. al 2015) and P. tepidariorum (Posnien et al. 2014) and performed a large phylogenetic analysis for all candidates with an e-value below $\mathrm{e}^{-15}$. This phylogenetic tree is shown in Supplemental Figure 15 and Supplemental Figure 16 and revealed a duplication event of cll in the two spiders used in this study (depicted in red). In this large phylogenetic analysis a monophyletic group with the highest support value is formed for the cll homologs, which are further subdivided into two distinct groups: cll homolog sequences from insects and vertebrates (for vertebrates three homologs are present and called Tcell-leukaemia homeobox (TLX) protein) form one group which is further subdivided giving rise to two branches within this group; the second group includes the cll homolog of an onychophoran branching of from another subgroup including all chelicerate sequences. The paralogs found for three spiders (P. tepidariorum, P. phalangioides as well as $S$. mimosarum) form two separate clusters. The paralogs in the distinct clusters were named according to sequence similarity to the D. melanogaster homolog. Thus comp116449 and Locus_15589 are referred to as cll1 and comp 121099 and Locus_21582 as cll2. For the other spider S. mimosarum a third $c l l$ is found which clusters together with the other spider cll2 sequences, but was not further studied. To analyse the origin of the cll duplication event in chelicerates in more detail cll homologs were additionally searched in the genome of the scorpion M. martensii (Di et al., 2014). Then a less inclusive Bayesian phylogenetic tree was computed with a subset of the cll homologs identified in the large phylogenetic tree, including also sequences from one more basal bilaterian, the purple sea urchin Strongylocentrotus purpuratus. 


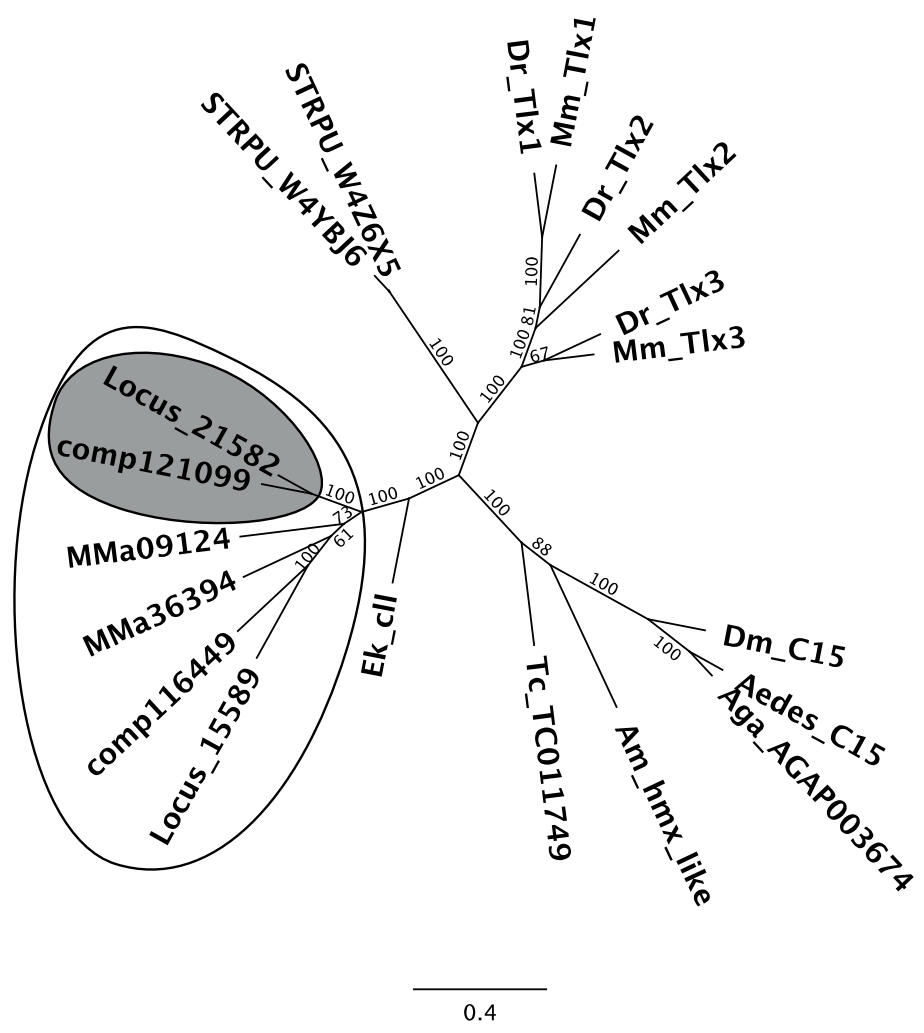

Figure 58: Unrooted 50\% phylogenetic majority rule consensus tree of cll orthologs

The phylogenetic analysis resulted in three major groups of $c l l$ homologs. One branch includes the sea urchin homologs branching of from the TLX homologs of vertebrates, shown on the top. The insect $c l l / C 15$ group is located at the right. The third branch includes the cll homolog of the onychophoran Euperipatoides kanangrensis forming a sister branch to all chelicerate cll (marked by black circle) located in the left. The two cll paralogs from spiders are present in two distinct phylogenetic groups after duplication. cll1 forms a monophyletic group with the scorpion homologs, whereas cll2 of $P$. tepidariorum and $P$. phalangioides cluster together more basally branching in the chelicerate group (Grey circle). Branch lengths of the tree represent the substitutions per site. Numbers at the edges of the phylogram give the probability of each clade indicated by clade credibility values. Abbreviations and accession numbers are given in Supplemental Table 10.

In the unrooted tree (Figure 58) the cll sequences form three major clades supported with the highest posterior probability value: the two cll homologs of the sea urchin cluster together and form one clade with the vertebrates TLX paralogs, the other clade includes all insects $c l l$ sequences and the onychophoran $c l l$ homolog is predicted as the sister group of the chelicerates forming the third group. This phylogenetic analysis clearly shows that the duplication events of the vertebrate TLX and spider cll paralogs are entirely unrelated. As already observed in the large phylogenetic analysis, the spider paralogs cluster together in two separate groups. The two scorpion cll homologs, 
MMa09124 and MMa36394, cluster together with the spider cll1 sequences, but do not form a separate group, which leads to the assumption that the duplication occurred twice within the lineages leading to scorpion and spider and is not ancestral for arachnids. To clearly state whether cll2 has evolved in a spider specific duplication event additional chelicerate sequences are required.

\subsubsection{Expression of clawless}

The expressional analysis of the two cll paralogs revealed that both paralogs are strongly expressed in the prosomal appendages with beginning of early limb bud development until late ventral closure stages in both spiders. Their specific pattern, however, differs between the two paralogs. The first paralog, cll1, is only similarly expressed in the prosomal appendages until ventral closure. The second paralog, cll2, however, is similarly expressed throughout entire walking leg development, but not in the pedipalps and chelicera (detailed description of $c l l$ expression in the prosomal appendages in the next section). Pt-cll1 and Pp-cll1 expression starts in the distal tip of all prosomal appendages at very early limb bud development (arrowhead, Figure 59, A and Figure 60, A). This distal expression stays during limb bud elongation (arrowhead, Figure 59, B and Figure 60, B), but then divides and gives rise to two rings of distal expression in the walking legs during inversion stages in both species (arrows, Figure 59, C and Figure 60, C). Whereas this expression of cll1 remains unchanged in the walking legs of $P$. tepidariorum until the end of embryonic development (arrows, Figure $59 \mathrm{D}$ and E), $P p$ cll1 expression changes to a single distal spot during ventral closure (arrow, Figure 60, D). The only expression of cll1 found in the head of both spider species is present in the developing labrum with beginning of inversion until ventral closure (Figure 59, F arrow, $\mathrm{G}$ arrowhead and $\mathrm{H}$, and Figure 60, E and D). In the opisthosoma cll1 is also similarly expressed in both spiders. Opisthosomal expression starts during inversion stages covering the most dorsal opisthosomal tissue in the presumptive area of the heart (asterisk, Figure 59, I and arrowhead, Figure 60, F). In P. phalangioides the dorsal cll1 expression is strongest in the first opisthosomal segment already at these stages. Slightly later with beginning of dorsal closure opisthosomal cll1 expression is restricted to this 
segment in both spiders (asterisk, Figure 59, J and arrowhead, Figure 60, G). After rearrangement of the embryonic tissue during dorsal and ventral closure, cll1 expression covers the former first opisthosomal tissue in the field of the developing heart (asterisk, Figure 59, E and arrowhead, Figure 60, H). In summary cll1 is similarly expressed in the head, opisthosoma and early walking legs in both spider species studied here, but differentiates during late walking leg development.

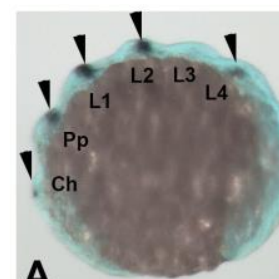

A

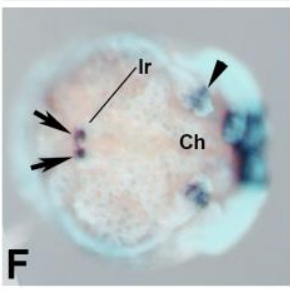

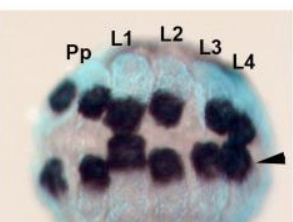

B

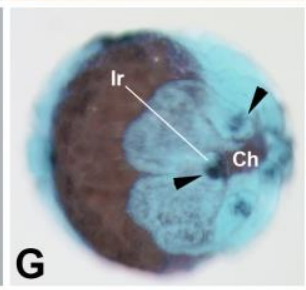

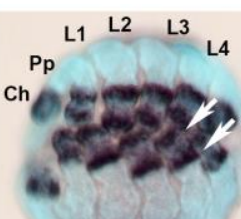

C

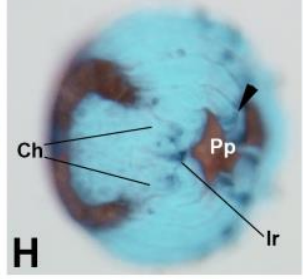

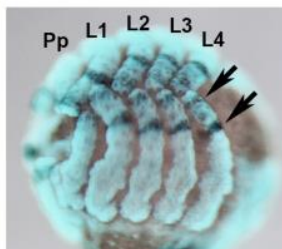

D

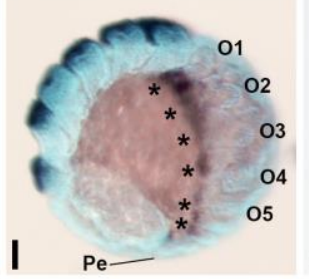

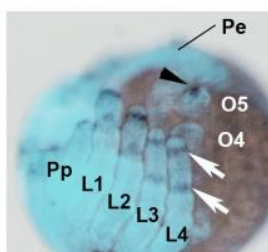

E

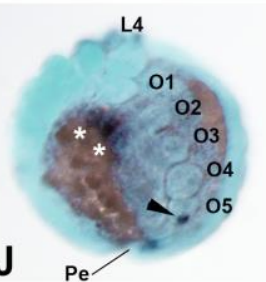

Figure 59: Expression of cll1 in P. tepidariorum

A: Lateral view of a germ band stage embryo with Pt-cll1 expression in all developing prosomal appendages excluding L3 (arrowheads). B: Ventral view of an embryo during limb bud elongation with strong distal cll1 expression in all prosomal appendages. C: Ventral view during of an embryo inversion. D: Ventral view of an embryo during dorsal closure. E: Lateral view of an embryo during ventral closure. F: Anterior view of the head during inversion. G: Anterior view of the head during beginning of dorsal closure of the embryo. H: Anterior view of the head of an embryo undergoing ventral closure. I: Lateral view of the embryo depicted in B. J: Lateral view of an embryo during dorsal closure. More detailed description of expression in the text. Anterior is always to the left. Jana Holtzheimer was involved in the staining of the embryos. Ch: Chelicera, Pp: Pedipalps, L1-L4: Walking legs pairs 1-4, 01-05: Opisthosomal segments 1-5, pe: posterior end. 


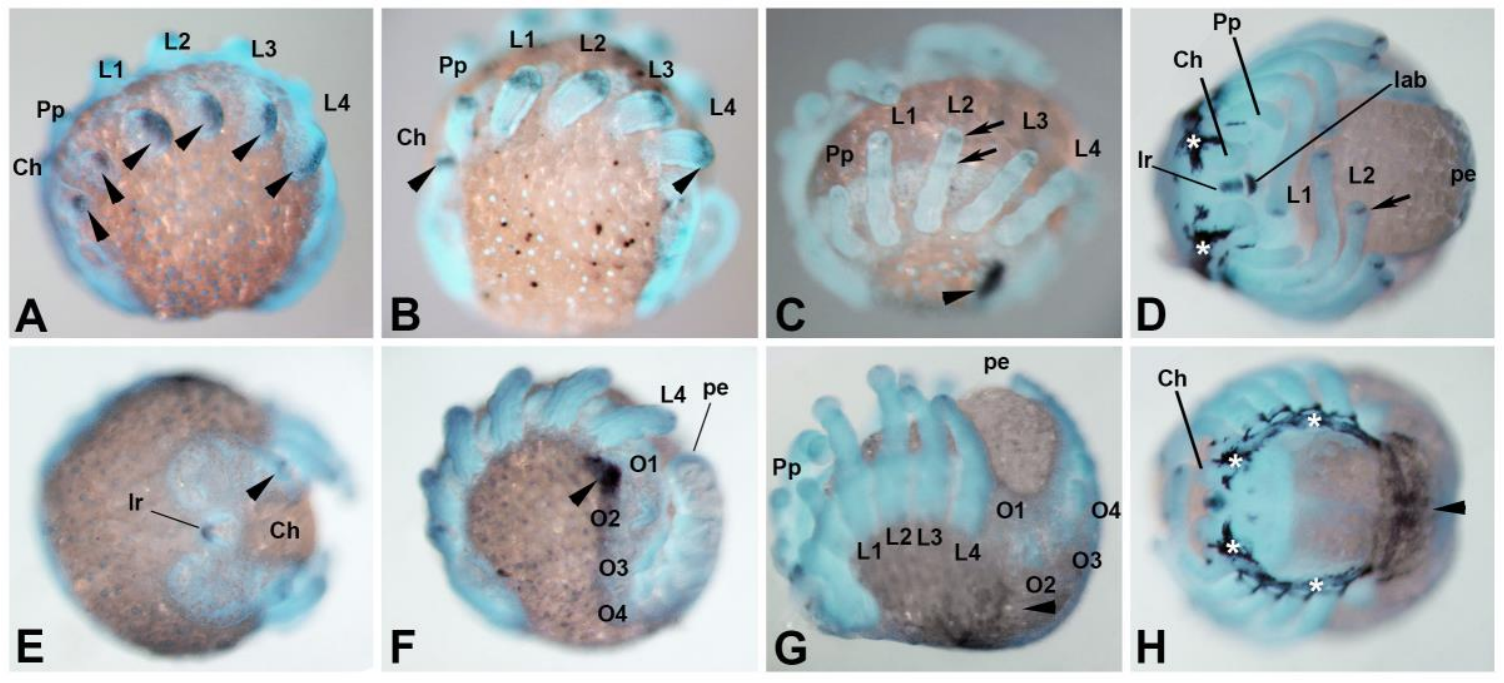

Figure 60: Expression of cll1 in P. phalangioides

Expression of cll1 in prosomal appendages of $P$. phalangioides during limb bud development (A) and limb bud elongation (B), lateral view. C: Ventro-lateral view of an embryo during inversion D: Ventral view of an embryo during ventral closure. E: Embryo during inversion shown in frontal aspect. F: Lateral view of an embryo during inversion. G: Lateral view of an embryo during dorsal closure. Dorsal view of an embryo during ventral closure. The asterisk in $D$ and $H$ marks unspecific staining of the probe in the forming cuticle. The other marks are described in the according text section. Anterior is always to the left, except for the embryo shown in E, this embryo is oriented with the walking legs to the right. Ir: Labrum, Ch: Chelicera, Pp: Pedipalps, L1-L4: Walking legs pairs 1-4, 01-04: Opisthosomal segments 1-4, pe: posterior end.

The next part will focus on the comparison of the expression patterns between the paralogs of both spiders (Cloning, probe synthesis and whole mount in situ hybridization of Pp-cll2 and Pt-cll2 was performed with the help of Jana Holtzheimer). In contrast to cll1, cll2 is not expressed in the opisthosoma of P. phalangioides. In P. tepidariorum cll2 is expressed in the opisthosoma, but different to its paralog cll1. Pt-cll2 is expressed at the posterior end of the opisthosoma starting at limb bud elongation until the end of ventral closure (Figure 61, F, G and E). In the head cll2 is also differently expressed compared to cll1, but similar for both spiders. With beginning of limb bud elongation cll2 is expressed at the anterior rim of the head (arrowhead, Figure 61, $\mathrm{H}$ and Figure 62, F). This expression remains unchanged during early dorsal closure stages (arrowhead, Figure 61, I and Figure 62, G). cll2 expression in the prosomal appendages, especially the walking legs, is like the expression in the head on the one hand different for the two paralogs but on the other hand largely comparable for the two spider species. Although the start of cll2 expression at the distal tip of the walking legs in P. phalangioides (arrowhead, Figure 
62, A) still somehow resembles that of cll1, it is quickly modified and becomes different during limb bud elongation. Directly at this stage not the complete distal tip is expressing cll2, as observed for cll1, but in contrast one distal ring of expression is present in the pedipalps and walking legs of both spiders (expression in the chelicera will be explained in the following section) (arrows and arrowhead in Figure 61, A and Figure 62, B). This distal ring of expression stays throughout the rest of embryonic development (arrows, Figure 61, B-E and Figure 62, B-D). Taken together cll2 is expressed in a comparable pattern in the head and the walking legs for both spiders, but not in the opisthosoma and the other prosomal appendages and is not comparable to its paralog cll1.
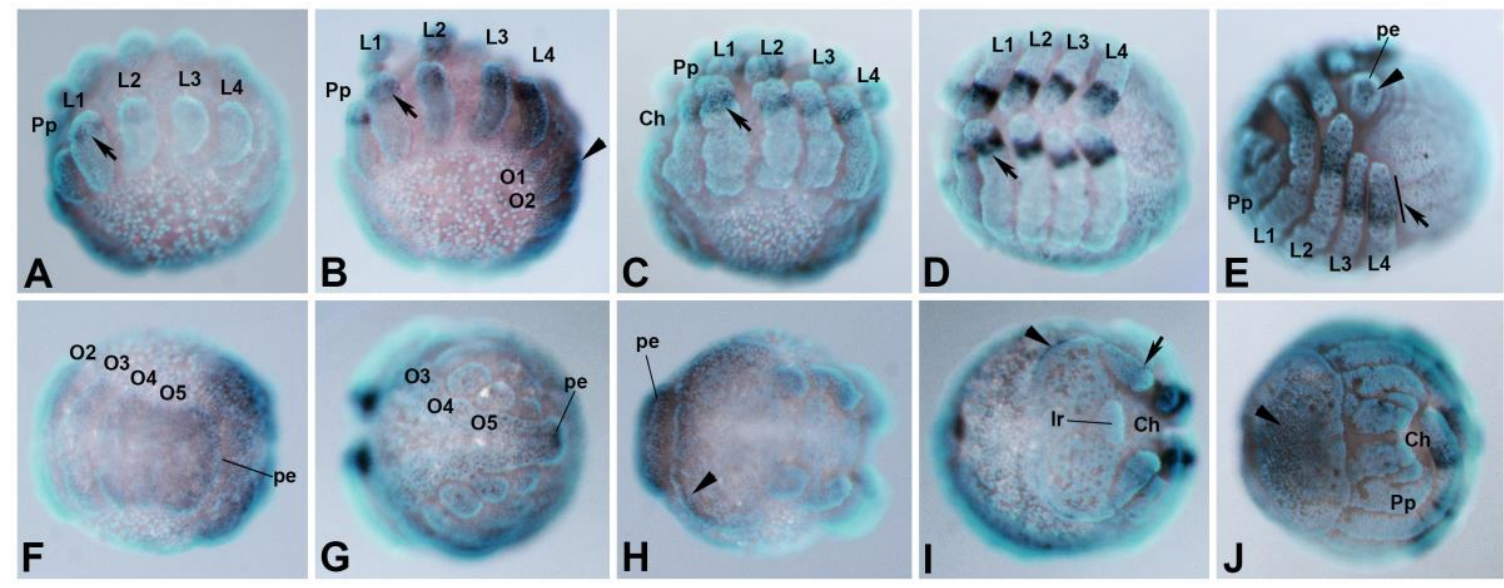

Figure 61: Expression of cll2 in P. tepidariorum

A, F and $H$ show the same embryo during early limb bud elongation in lateral view, ventral view of the opisthosoma and ventral view of the head. B: Embryo during late limb bud elongation, lateral view. C: embryo during inversion, lateral view. D, G and I show the same embryo during early dorsal closure in ventral view of the walking legs, opisthosoma and head. E and J: Embryo during ventral closure in Ventro-lateral and frontal view. Detailed explanation of the expression pattern is found in the text. Anterior is pointing to the left for A-E, $\mathrm{H}$ and $\mathrm{J}$. F and G posterior is on the right and in I posterior points to the left. Ir: Labrum, Ch: Chelicera, Pp: Pedipalps, L1-L4: Walking legs pairs 1-4, 01-05: Opisthosomal segments 1-5, pe: posterior end. 

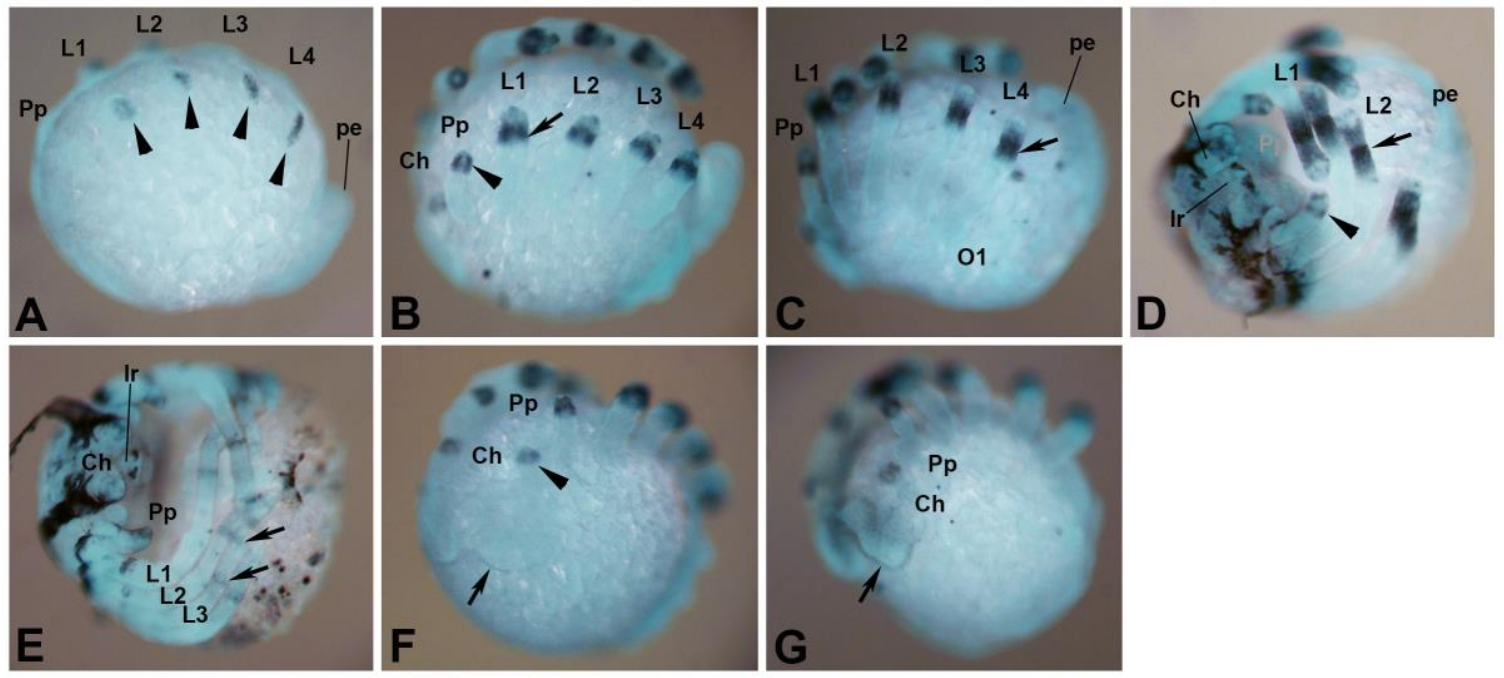

Figure 62: Expression of cll2 in P. phallangioides

Expression of cll2 in prosomal appendages of $P$. phalangioides during limb bud development (A), limb bud elongation (B) and dorsal closure (C), all in lateral view. D: Embryo during ventral closure shown in anterior-ventral view. E: Embryo after ventral closure also in ventro-frontal view. F: Embryo during limb bud elongation shown in frontal aspect. G: Embryo during dorsal closure shown in dorsal-anterior view. Detailed description of cll2 expression in the text. All embryos are oriented with anterior to the left. Ir: Labrum, Ch: Chelicera, Pp: Pedipalps, L1-L4: Walking legs pairs 1-4, 01: first opisthosomal segment, pe: posterior end.

\subsubsection{Expression of clawless in prosomal appendages}

In the previous section it was already stated that the expression in the early walking legs is different for the two intraspecific paralogs but similar for interspecific orthologs. In the following the expression in the prosomal appendages will be described and compared in greater detail. As observed for the walking legs the expression of Pt-cll1 is present also as a strong distal domain in the more anterior prosomal appendages during limb bud elongation (bars and arrow, Figure 63, A, E and I). This domain divides during early inversion in the walking legs and pedipalps (bar and arrow, Figure 63, F and J), but not in the chelicera, where it only moves more proximal (bar, Figure 63, B). In the pedipalps the more proximal portion of the dividing domain quickly vanishes during inversion and only one distal Pt-cll1 ring is present in this appendage during dorsal and ventral closure (arrow, Figure 63, G and $\mathrm{H}$ ). In the legs, however, the division of the distal domain gives rise to two distal rings in the area of the joints forming between tibia and metatarsus and metatarsus and tarsus (arrows, Figure 63, K and L). In the chelicera Ptcll1 is expressed in a medial ring-like domain (arrowhead, Figure 63, C). In $P$. 
phalangioides the chelicera specific expression in the medial part starts already during limb bud elongation, when Pp-cll1 expression is still in the distal domain of the pedipalps and walking legs. Later during ventral closure $P p$-cll1 expression is different for all three prosomal appendage types compared to Pt-cll1. In the pedipalps it remains absent and in the chelicera and walking legs the expression is restricted to a small spot ventrally on the distal tip of these appendages.

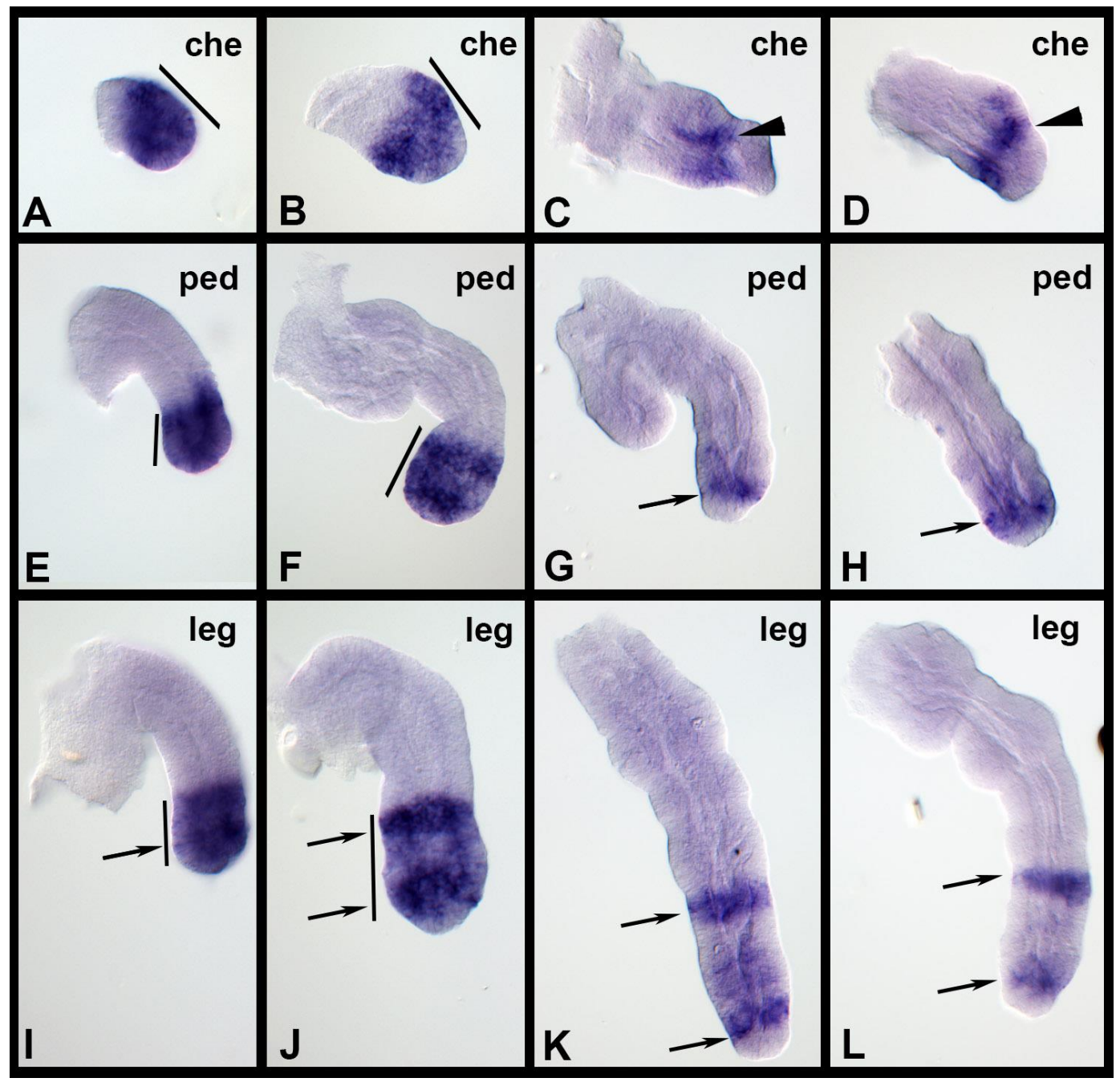

Figure 63: Expression of cll1 in prosomal appendages of $P$. tepidariorum

A-D: Expression cll1 in the chelicera of $P$. tepidariorum. E-H: Pt-cll1 expression pattern in the pedipalps. I-J: Expression of $\mathrm{Pt}$-cll1 in the walking legs. A, E and I shows prosomal appendages during limb bud elongation. $B, F$ and $J$ show prosomal appendages during early inversion of the embryo. C, G and $\mathrm{K}$ depict expression in prosomal appendages during dorsal closure. D, H and $\mathrm{L}$ show prosomal appendages during ventral closure. For an explanation of the bars and arrows, please see text. che: chelicera. ped: pedipalp. leg: walking leg. 


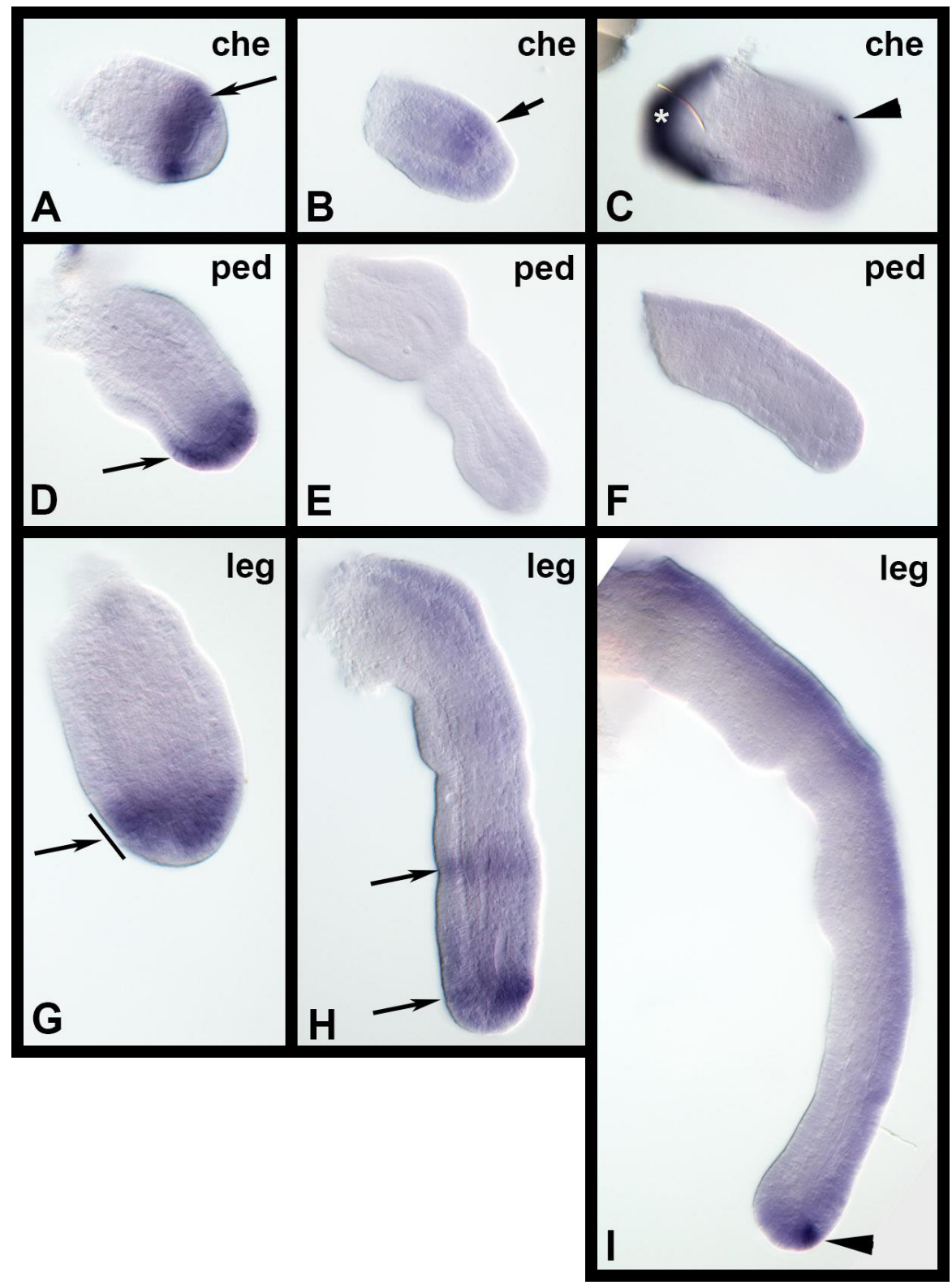

Figure 64: Expression of cll1 in prosomal appendages of $P$. phallangioides

A-C: Expression cll1 in the chelicera of $P$. phalangioides after in situ hybridization. D-F: Preparations of pedipalps after in situ hybridization with Pp-cll1. G-I: Pp-cll1 expression in the walking legs. A, D and $G$ show prosomal appendages during limb bud elongation. $B, E$ and $H$ depict these appendages during inversion of the embryo. C, F and I present the stained prosomal appendages during ventral closure stages. Asterisk in picture $C$ marks unspecific staining of the forming cuticle at the chelicera. Detailed explanation of the expression pattern is found in the text. che: chelicera. ped: pedipalp. leg: walking leg.

As stated before the expression of cll2 is more similar also in late stages of the developing walking legs in the two spiders, which it not true for the more anterior appendages. Whereas it is expressed in the entire chelicera of P. tepidariorum during 
limb bud elongation (Figure 65, A), it is at comparable stages expressed in the distal part of this appendage in P. phalangioides (arrow, Figure 66, A) and thus more similar to Ptcll1. During dorsal closure cll2 is expressed in a medial domain similarly to its paralog cll1 in P. tepidariorum and P. phalangioides (arrow, Figure 65 and Figure 66, B). Later this expression broadens in the chelicera of $P$. phalangioides embryos during ventral closure (arrow, Figure 66, C), but shows a ubiquitous expression in the chelicera of $P$. tepidariorum embryos of comparable stages (Figure 65, C).

cll2 is expressed in the distal domain of the pedipalps of $P$. phalangioides during limb bud elongation (bar, Figure $66 \mathrm{D}$ ) and stays there until dorsal and ventral closure of the embryo (bar, Figure 66, E and F). During these stages the distal domain of Pp-cll2 in the pedipalps differentiates slightly (arrow). The expression of $c l l 2$ in the pedipalps of $P$. tepidariorum is a bit more differentiated already during limb bud elongation stages but similar beginning with inversion (arrow, Figure 65, D and E). During ventral closure the same ubiquitous expression as in the chelicera is present. In the walking of both spiders cll2 is expressed in a distal ring throughout entire prosomal limb bud development, which broadens during later stages (arrow, Figure 65 and Figure 66, G-I). This ring is present in the joint between the metatarsus and tarsus segment. Only subtle differences are present for the walking leg expression of cll2: in P. tepidariorum weak ubiquitous expression in the entire leg is present during prosomal limb bud elongation and ventral closure stages; in P. phalangioides strongest expression is as stated found in the most distal joint but also in neighbouring tissues diffusing proximally and distally from this joint (bar, Figure 66, G-I). 


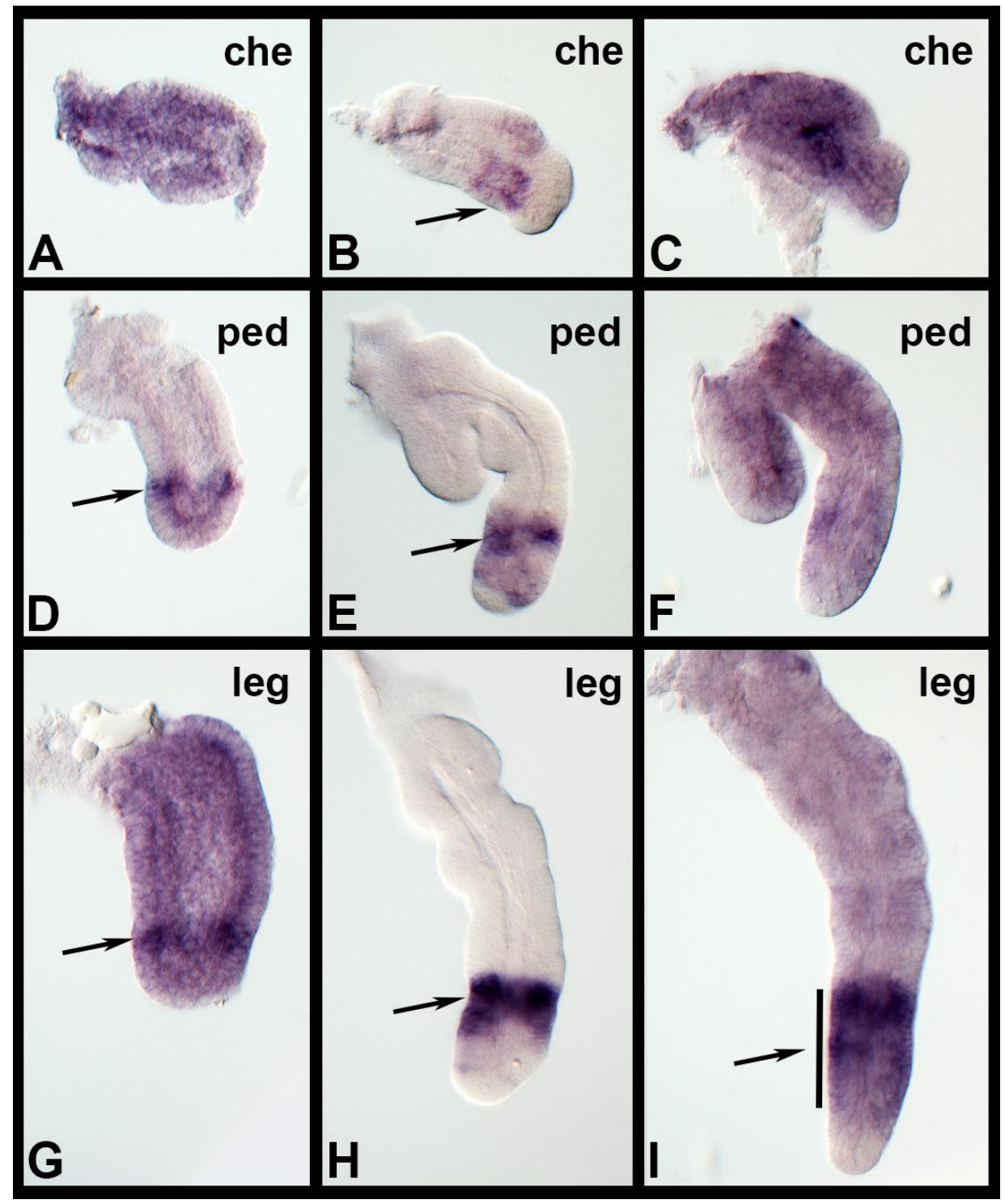

Figure 65: Expression of cll2 in prosomal appendages of $P$. tepidariorum

A-C: Expression cll2 in the chelicera of $P$. tepidariorum. D-F: Preparations of pedipalps after in situ hybridization with Pt-cll2. G-I: Pt-cll2 expression in the walking legs. A, D and G show prosomal appendages during limb bud elongation. $B, E$ and $H$ depict these appendages during inversion of the embryo. C, F and I present the stained prosomal appendages during ventral closure stages. Detailed explanation of the expression pattern is found in the text. che: chelicera. ped: pedipalp. leg: walking leg. 


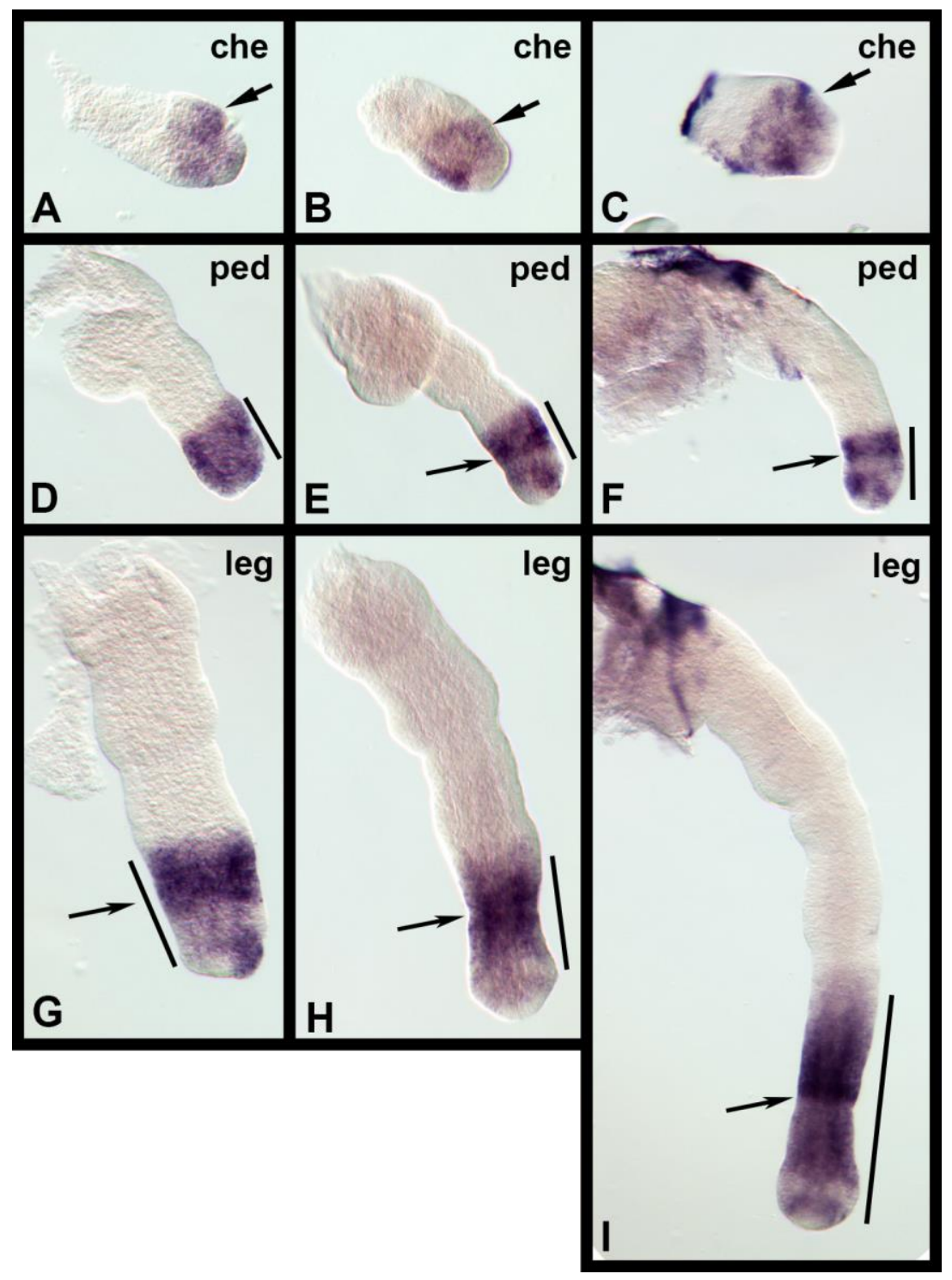

Figure 66: Expression of cll2 in prosomal appendages of P. phalangioides

A-C: Expression cll2 in the chelicera of $P$. phalangioides. D-F: Preparations of pedipalps after in situ hybridization with Pp-cll2. G-I: Pp-cll2 expression in the walking legs. A, D and G show prosomal appendages during limb bud elongation. $B, E$ and $H$ depict these appendages during inversion of the embryo. C, F and I present the stained prosomal appendages during ventral closure stages. Detailed explanation of the expression pattern is found in the text. che: chelicera. ped: pedipalp. leg: walking leg.

\subsubsection{Function of cll1 in P. tepidariorum}

To study the function of cll1 in the walking leg of $P$. tepidariorum parental RNAi was performed using the cloned gene fragment (1161 Bp). The exact procedure of injection is described in the chapter Materials and Methods. Observation of freshly hatched nymphs 
from the cocoons produced by injected females revealed two different phenotypes after Pt-cll1 knockdown (Overview in comparison to control, Figure 68).

In weak phenotypes (Figure 68, C and D) the joint between the metatarsus and tarsus segment is missing, which leads to a fusion of these distal leg segments. For this weak phenotype two different variations were found: (1) lateral outgrows on the position of the presumptive joint area (arrow, Figure 68, C and red arrow, Figure 69, B and E), comparable to the phenotypes after Pt-dac2 knockdown, which are missing the joint separating the patella from the tibia. These outgrows are probably caused by missing cues for the dividing cells in this area. (2) bending of reminiscent tarsal tissue resulting in a foot-like shaped distal leg part (arrowhead, Figure 68, C and D and red arrow, Figure $69, \mathrm{C}$ and F), probably caused by uncontrolled growth of cells on one side of the leg usually forming the joint. In both variations a disturbed bristle pattern in the area where normally the joint should be was observed. This suggests that the joints are needed to guide normal development of the tissue in the segments and missing the joint removes these cues.

In strong phenotypes (Figure 68, E and F and Figure 70, B and D) the complete tarsus segment and its neighbouring joints are entirely lost. In more severe cases of this strong phenotype the tissue distal to the metatarsus including all bristles, sensory organs and the claw, was absent completely (arrowhead, Figure 70, E). In all other phenotypic variations the claw located on the most distal tip of the walking legs was unaltered (indicated by $\mathrm{Cl}$ plus arrow, Figure $69 \mathrm{E}$ and $\mathrm{F}$ and Figure $70 \mathrm{D}$ ) and develops the typical hook and claw shape needed for holding on the silk thread (Foelix, 1996). In accordance to $c l 11$ expression in the pedipalps, defects in the distal segments of this appendage were observed as well. Resembling the weak leg phenotypes, affected pedipalps show fusion of the tibia and tarsus segment and disrupted joint formation causing lateral outgrows (black arrow Figure 71, C and D). Like in the legs the trident shaped claw of the pedipalpus is not altered in these phenotypes (red arrow Figure 71, D). Penetrance of the Pt-cll1 knockdown was analysed for the different cocoons produced by females after dsRNA injections (left panel, Figure 67) and compared to control females, which were equally treated with injection buffer (right panel, Figure 67). The amount and onset of weak and strong phenotypes was very diverse for the different females (ranging from 
only one affected cocoon to strong effect in all of the observed cocoons), but did not vary in accordance to the different injection numbers and mating time points (data not shown). The characteristics of the observed phenotypes were, however, comparable. Thus the results of all females were pooled and included in the statistic. This combination shows that weak (green) and strong (light blue) phenotypes are present in all first five cocoons produced by females treated with Pt-cll1 dsRNA. The amount of these phenotypes is lowest in the first cocoon (around 20\% Figure 67, C1) and highest in the third cocoon (more than 40\%, Figure 67, C3). The number of normally developed nymphs (Wt, light violet, Figure 67) is lower compared to the control in each of the observed cocoons. In contrast the amount of embryos which were not able to hatch from the embryonic egg-shell (not hatched, dark violet, Figure 67) lies within the usual range of $20-30 \%$ and is consistent with previous findings for both knockdown and control offspring.

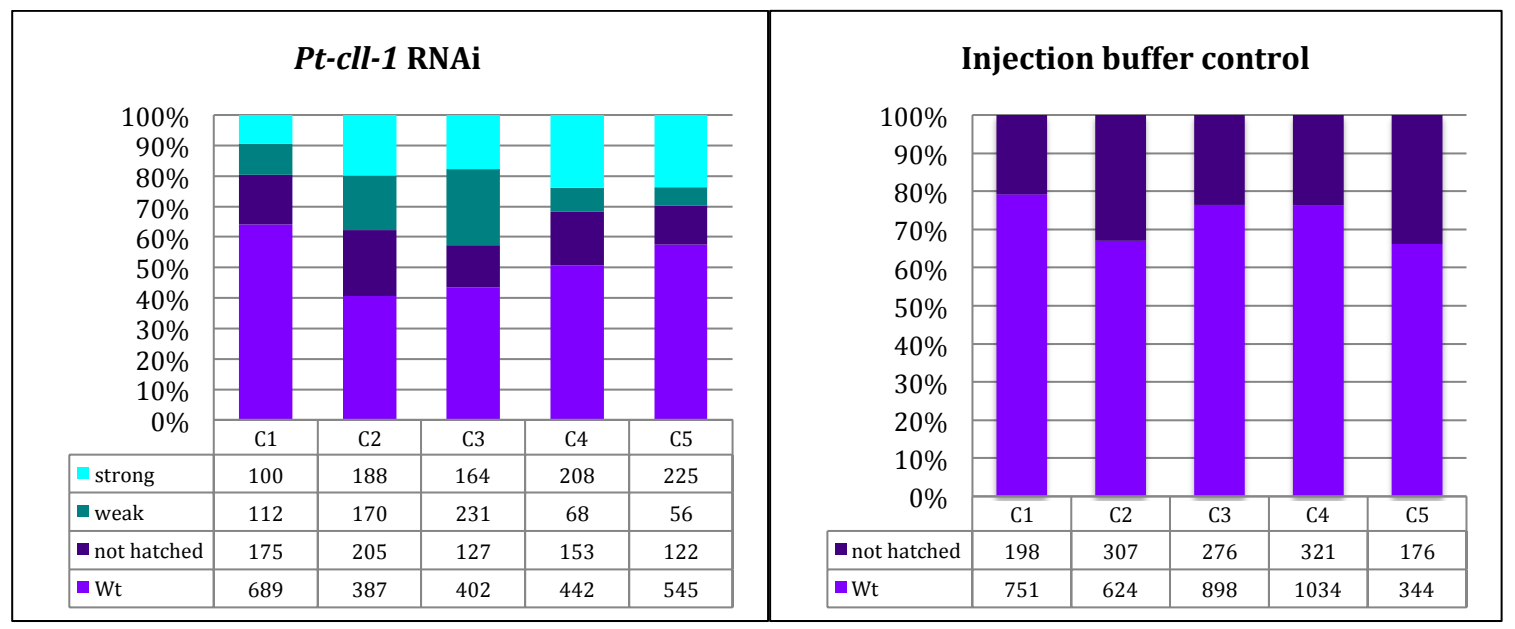

Figure 67: Overview of phenotypes in the different cocoon after cll1 RNAi and control injection The graph on the left gives an overview of the phenotypes in freshly hatched and third instar nymphs after knockdown of Pt-cll1 in the first five cocoons produced by females after injection. The graph on the left shows the first five cocoons produced by females after control injections with injection buffer. In the control the amount of unhatched phenotypes ranges between $20-30 \%$, thus usually at least $70 \%$ of the offspring in each cocoon developed without any visible abnormalities (indicated by $\mathrm{Wt}$ : wild-type appearance marked in light violet). After cll1 the highest amount of normally developed nymphs is found in the first cocoon with approximately $60 \%$. The other cocoons, especially the second and third cocoon, are more severely affected by the RNAi. 


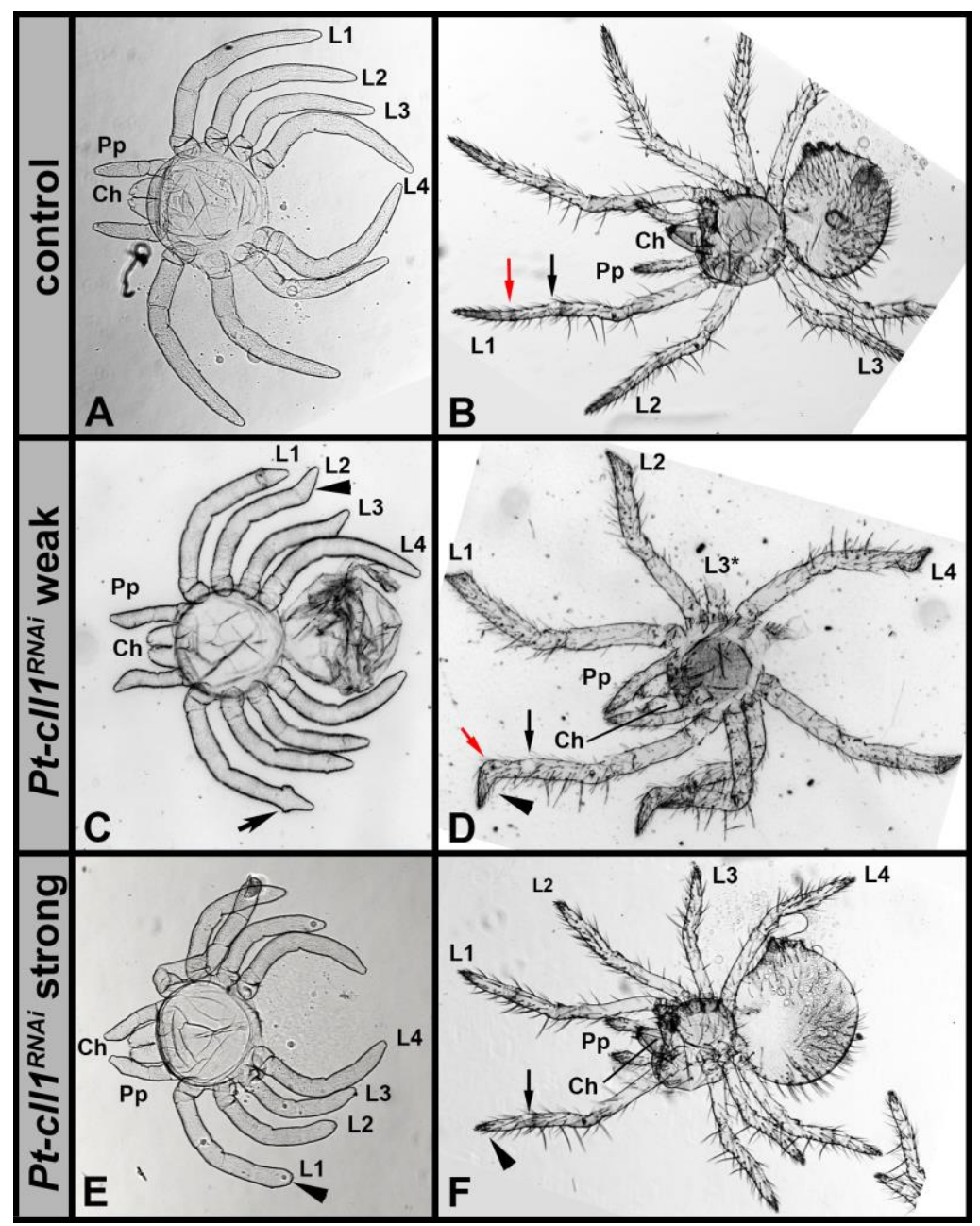

Figure 68: Overview of phenotype in nymphs of $P$. tepidariorum after cll1 RNAi

Depicted are cuticle preparations with Hoyer's medium of different nymphal stages of $P$. tepidariorum. A: Prosoma of a freshly hatched nymph from a control female treated with injection buffer according to the parental RNAi procedure. B: Third instar nymph from a female injected in the same way as in the cll1 RNAi with injection buffer. The black arrow points to the fully developed joint between the tibia and metatarsus. The red arrow marks the most distal joint between the metatarsus and tarsus segment. C: Prosoma of a freshly hatched nymph after parental with Pt-cll1, showing the weak cll1 phenotype in the pedipalps and walking legs. The arrow indicates the malformed joint between the metatarsus and tarsus leading to lateral outgrow at the presumptive side of the joint. The arrowhead points to another variation of a weak phenotype where the disrupted distal joint causes bending of the reminiscent tarsus tissue. D: Cuticle preparation of a third instar nymph after cll1 RNAi with weak phenotype in all walking legs. The red arrow indicates the disrupted joint, which is not properly formed between the metarsus and tarsus. The black arrow point to the intact joint of the tibia and metatarsus. The arrowhead points to the bending of presumptive tarsal tissue after fusion. E: Prosoma of a freshly hatched nymph after parental with Pt-cll1, showing the strong cll1 phenotype in the walking legs. The arrowhead marks the tip of the leg missing the tarsal segment. F: Cuticle preparation of a third instar nymph after cll1 RNAi with strong phenotype in all walking legs. The black arrow point to the intact joint of the tibia and metatarsus. The arrowhead point to the intact claw although missing of the tarsus segment. Freshly hatched nymphs are shown in ventral view and third instar nymphs in ventro-lateral view, but all nymphs are oriented with anterior to the left. Ch: Chelicera, Pp: Pedipalps, L1-L4: Walking legs pairs 1-4. 


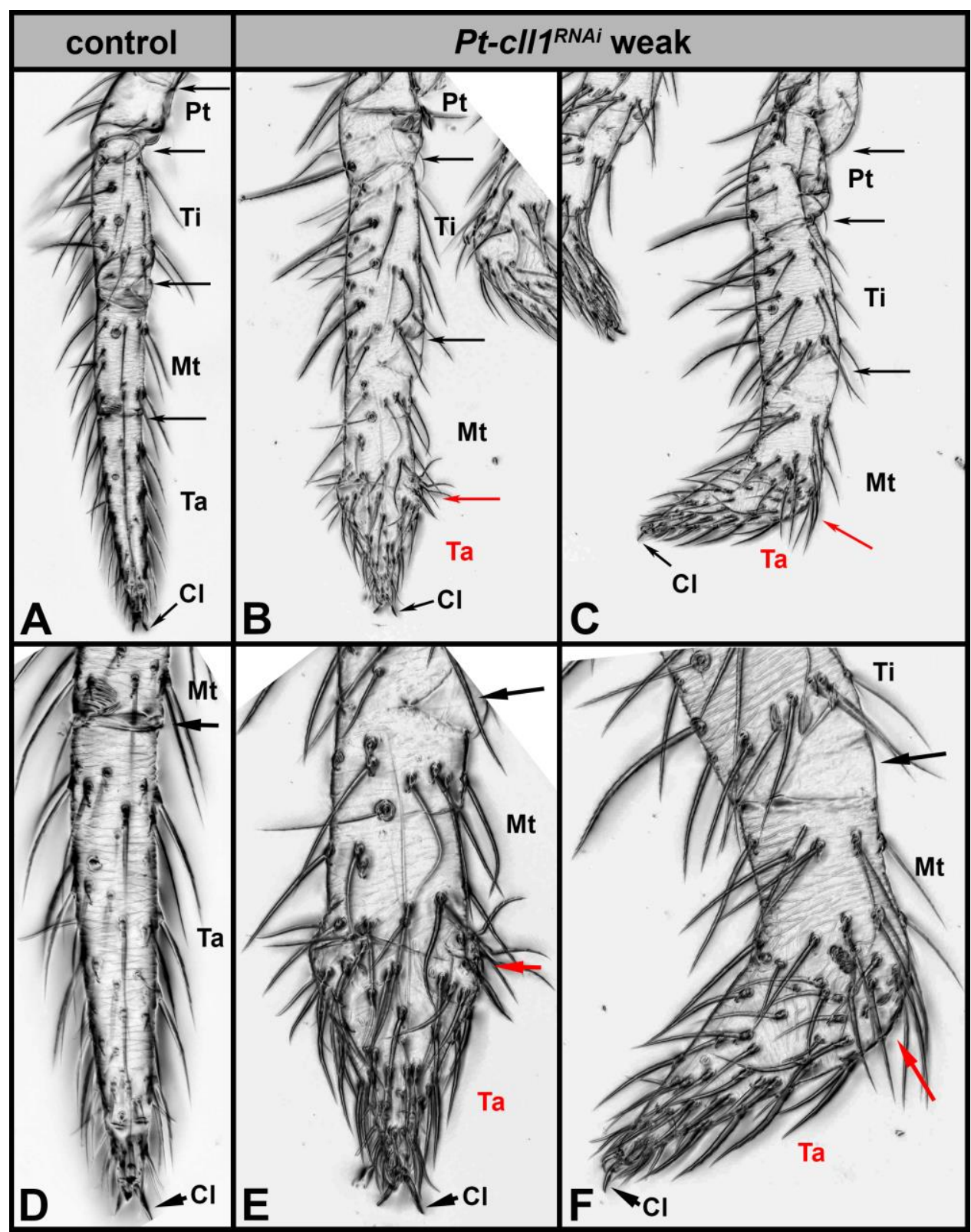

Figure 69: Weak Pt-cll1 RNAi phenotype in the walking legs

Depicted are close ups from cuticle preparation of walking legs from the third nymphal stage of $P$. tepidariorum. A: Walking leg of third instar nymph offspring from control females injected with injection buffer instead of dsRNA. B and C: Walking legs of third instar nymph offspring after cll1 RNAi showing two different forms of weak phenotypes, indicated by fusion of the metatarsus and tarsus segment. B: This legs shows lateral outgrowth in the presumptive area of the missing joint. C: Bending of reminiscent tarsal tissue after distal segment fusion. D: Close up of the leg shown in A. E: Close up of the leg shown in B. F: Close up of the leg shown in C. The red arrow indicates the missing joint between the metatarsus and tarsus segment and malformed tissue causing outgrows in this area. This causes fusion of the tarsus and metatarus segment, the remaining tissue, which would usually belong to the tarsus, is indicated by Ta written in red. The black arrows in all pictures point to properly shaped and formed joints between the femur and patella, patella and tibia, tibia and metatarsus and metatarsus and tarsus. All leg preparations are oriented with proximal at the top and distal to the bottom. Cl: Claw, Pt: patella, Ti: tibia, Mt: metatarsus, Ta: tarsus. 


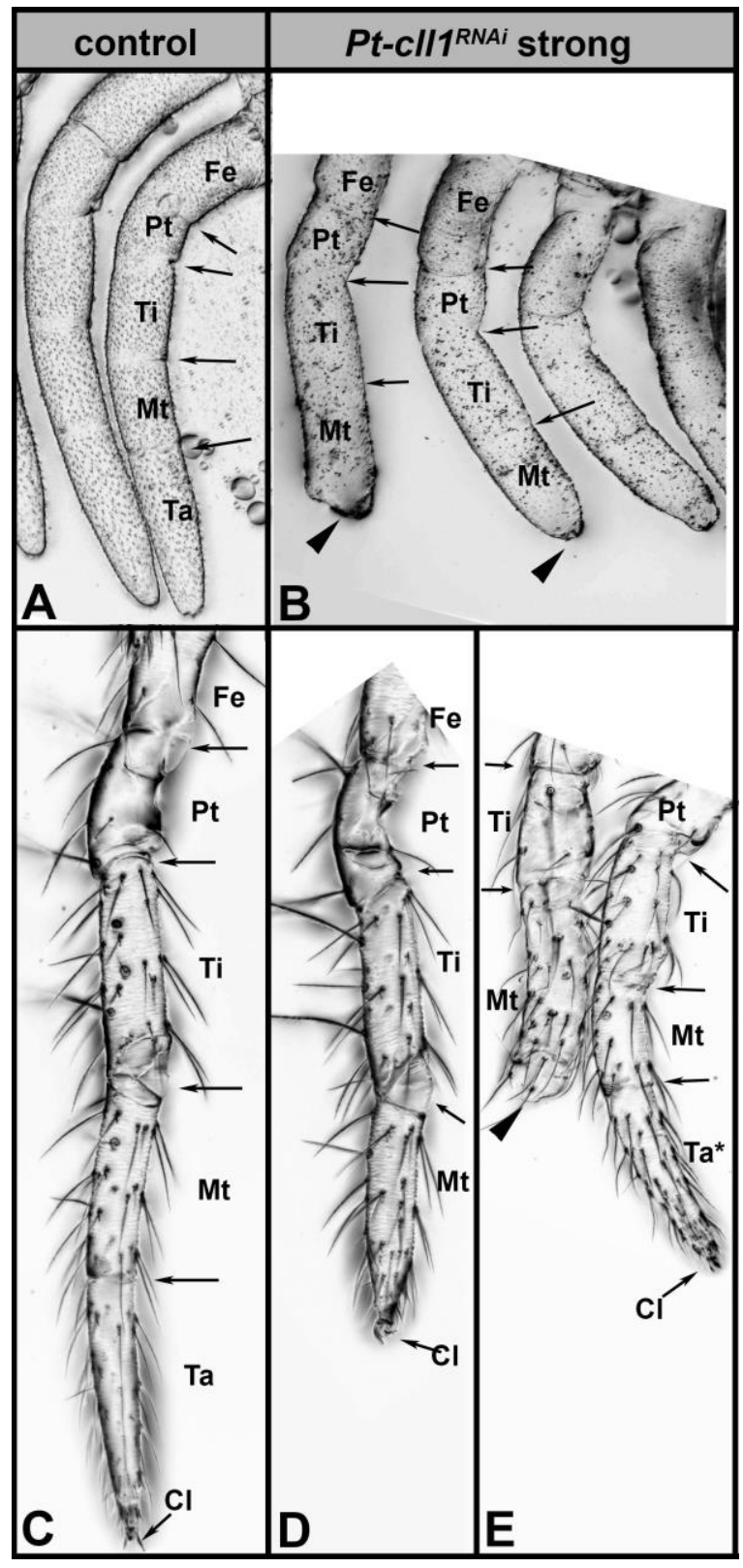

Figure 70: Strong Pt-cll1 RNAi phenotype in the walking legs

Depicted are close ups from cuticle preparations of walking legs of different nymphal stages of $P$. tepidariorum. A: Walking leg of second instar nymph offspring from control females injected with injection buffer instead of dsRNA. B: Walking legs of second instar nymph offspring after cll1 RNAi with strong phenotypes, indicated by absence of the tarsus and disrupted distal tip. C: Walking leg of third instar nymph offspring from a control female. D and E: Walking legs of third instar nymph offspring after cll1 RNAi with different variations of the strong phenotype. D: The walking leg is missing the entire tarsal segment, whereas the claw is still present and properly shaped. E: Depicts two variants of walking legs observed in a specimen with strong cll1 knockdown phenotype; on the right normally developed leg with all segments; on the left affected leg missing the entire distal tissue distally of the metatarsus. The black arrows in all pictures point to properly shaped and formed joints between the different leg segments. The arrowhead marks disrupted distal-most leg structures in most severe phenotypes. All leg preparations are oriented with proximal at the top and distal to the bottom. Cl: Claw, Pt: patella, Ti: tibia, Mt: metatarsus, Ta: tarsus. 


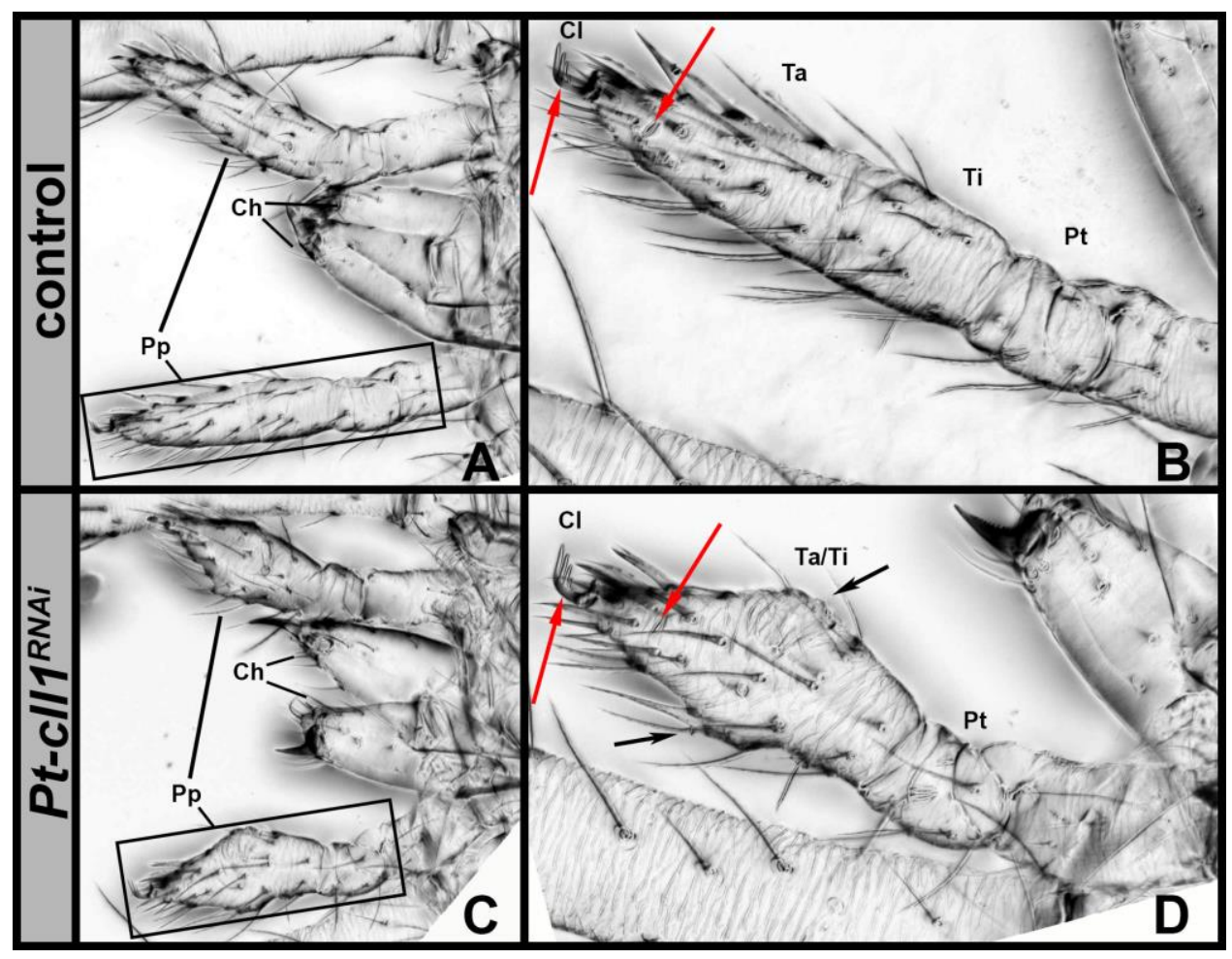

Figure 71: Pt-cll1 RNAi effect on nymphal pedipalps

Depicted are close ups from cuticle preparation of pedipalps from the third nymphal stage of $P$. tepidariorum. A: Chelicera and pedipalps of third instar nymph offspring from control females injected with injection buffer instead of dsRNA. The rectangle marks the area including one pedipalp magnified in B. B: Magnification of the pedipalp shown in A. The segments are marked by the according abbreviations. The red arrows point to the lyriform organ and the claw on the distal tip of the pedipalps. C: Chelicera and pedipalps of third instar nymph offspring from females injected cll1 dsRNA. The rectangle marks the area including one pedipalp magnified in D. D: Magnification of the pedipalp shown in C. The segments are marked by the according abbreviations. The black arrow points to the malformed joint between the tibia and tarsus segment. The red arrows point to the unaltered lyriform organ and the properly shaped claw with tree hooks on the distal tip of the pedipalps. Ch: chelicera, Pp: pedipalps, Cl: Claw, Pt: patella, Ti: tibia, Ta: tarsus, Ta/Ti: fusion of the leg segments tibia and tarsus. 


\subsection{BarH in P. tepidariorum and P. phalangioides}

BarH transcription factors are members of the Bar class of homeobox genes and part of the highly conserved group of Q50-homeoproteins . Homologs of the BarH genes were first identified in D. melanogaster (Kojima et al., 1991) and also found in a number of vertebrates (reviewed in (Reig et al., 2007)). Homeodomain transcription factors can bind to a variety of downstream targets, whereas specificity is mediated by posttranslational modifications and co-factor interactions depending on the cellular environment. This fine-tuning of transcription factor specificity serves as a versatile toolkit for functional evolution. Accordingly, aside from their conserved function in cell fate determination during retinal neurogenesis in D. melanogaster and vertebrates (Kojima et al., 1991, reviewed in Reig et al., 2007), BarH genes show various unique functions in distinct species. These include tarsus segmentation in distal leg of $D$. melanogaster (Kojima et al., 2000; Kozu et al., 2006), cell survival in either ear or mesoderm development in the mouse or Xenopus laevis respectively, as well as cell migration in the mouse cerebellum.

\subsubsection{Phylogenetic analysis of Bar in P. tepidariorum and P. phalangioides}

Genuine BarH homologs were searched in the transcriptomes available for the two spider species observed in this study and, in contrast to the other genes used in this study, three transcripts with high similarity to D. melanogaster BarH were found. The large phylogenetic study (Supplemental Figure 17 and Supplemental Figure 18) includes all candidates found in the spider transcriptomes as well as their blast hits from the nonredundant protein sequence database of chosen taxa (for details see methods section). It shows, that one of the P. phalangioides candidates rather clusters into the sister group of BarH-like proteins branching off the true Bar clade more basally (depicted in dark red, see Supplemental Figure 17). The true Bar group (depicted in red) includes all sequences from vertebrates, insects and chelicerates. These homologs are further subdivided forming a monophyletic group of the vertebrate paralogs, which cluster together forming two distinct vertebrate Bar groups (BarH-like-1 and BarH-like-2) and a polytomy for the other proteins. In this polytomy the insect homologs form a cluster, although missing the 
T. $c$ homolog. In the insect cluster the Dm-Bar paralogs are closely related. Next to the insect cluster two distinct clusters for the spider Bar homologs were found, including $P$. tepidariorum, P. phalangioides and S. mimosarum. The grouping of the D. melanogaster, vertebrate and spider paralogs into taxa specific groups indicates that a Bar gene duplication evolved several times independently, but before the split of the Entelegynae and Haplogynae spider groups.

In addition many predicted sequences were found e.g. from the horseshoe crab $L$. polyphemus, the scorpion M. occidentalis, and a sequence from T. castaneum that all proved to be difficult to place in the phylogenetic tree and usually formed separate but weakly supported clades. Together with the insect polytomy and the difficulties to properly resolve the insect homologs this strongly suggests that a more specific phylogenetic analysis of the BarH homologs with additional arthropod species is necessary to clarify their phylogenetic relationships on a greater level.

However, Pp-comp120407 clusters together with the transcript Locus_1_Transcript_157093/166847 of $P$. tepidariorum (in the following referred to as BarH2) and Pp-comp116578 with Locus_3013, Locus_3015 and Locus_3016 (referred to as BarH1), which were named according to sequence similarity to D. melanogaster paralogs. The existence of three BarH1 transcripts in P. tepidariorum and thus a possible species-specific duplication was examined in more detail. RACE PCR was performed to rule out that these transcripts might represent two isoforms of one BarH1 paralog, wrongly identified as three separate genes during de novo assembly of the $P$. tepidariorum transcriptome (data not shown). Unfortunately this did not help in understanding the origins of these sequences. As mentioned in the Introduction current work includes genome assembly of $P$. tepidariorum (unpublished data, courtesy of Alistair McGregor, co-ordinator of the P. tepidariorum sequencing consortium), thus the corresponding gene was searched in a preliminary version of this genome. The fulllength protein found in the genome revealed that Locus_3013, Locus_3015 and Locus_3016 represent parts of two isoforms of one BarH homolog (Augustus prediction Pt-aug3.g8250.t1 and Pt-aug3.g8250.t2). One of these augustus predictions was included into the large phylogenetic analysis subsequently. There it resolves with the three previously found transcripts, validating the assumption that only one Pt-BarH1 paralog 
is present in P. tepidariorum. The gene fragment cloned from the transcriptome sequence Locus_3013 was used for the expressional and functional analysis.

\subsubsection{Expression of BarH homologs in P. tepidariorum}

Expressional analysis of the two BarH paralogs in P. tepidariorum showed that they are similarly expressed in the prosomal appendages and parts of the head, but that BarH1 has in addition unique expression details during early embryonic development and in parts of the head and opisthosoma. The expression in the prosomal appendages starts with their formation on the germ band (Figure 72, E and F, data not shown for Pt-BarH2). During limb bud elongation both paralogs are expressed in a medial and distal domain in the chelicera and remains unchanged throughout appendage development (arrowhead, Figure 74, D-F and Figure 73, A-C). During limb bud elongation in the walking legs and pedipalps both paralogs are expressed as a distal spot (arrow, Figure $72 \mathrm{G}$ and Figure 74, A). This expression in the distal part of the legs further changes during development. At the beginning of inversion an additional spot of expression appears (arrow, Figure 72, $\mathrm{H}$ and Figure 74, B). Later during dorsal closure the expression pattern in the distal part changes to an intensive expression domain in the most distal part (arrow, Figure 72, I and Figure 74, C) and a diffuse spot-like pattern proximal to this distal domain. Also similar for both Bar homologs is the strong expression in the gnathendites of the pedipalps and the corresponding endite region at the base of the walking legs (arrowhead, Figure 72, G-J and Figure 74, A-C). Although the expression pattern for both $P$. tepidariorum paralogs is strongly conserved in the prosomal appendages, the expression in the head shows some differences between Pt-BarH1 and Pt-BarH2. Both paralogs are expressed in the neurogenic ectoderm posterior to the anterior furrow in the head and later during inversion also in the labrum (Figure 73 and Figure 74, arrow E and F). After the non-neurogenic ectoderm has overgrown the neurogenic ectoderm the most anterior domain in the neurogenic ectoderm is hardly visible for Pt-BarH1 (Figure 73, anterior arrow C) and has fully disappeared for Pt-BarH2. Pt-BarH1 is expressed adjacent to the lateral furrow and as a stripe in the neurogenic ectoderm anterior to the cheliceral segment at prosomal limb bud developmental stages. These expression details 
are not present in the Pt-BarH2 pattern and start already during early germ band elongation stages (arrowhead, Figure 72, F). In addition, Pt-BarH1 shows several other unique expression details that differentiate it from the expression pattern of Pt-BarH2. Starting with opisthosomal limb bud development it is strongly expressed in the limb bud developing on the second opisthosomal segment (red arrow, Figure 72, G and I), which will give rise to the book lung after invagination during further development. Pt-BarH1 is also expressed very early as a ring surrounding the outer rim of the germ disc (Figure 72, A). This expression remains in this tissue and then opens on one side (asterisk, Figure 72 B) during the break of radial symmetry. During early germ band stages the ring of expression is therefore present as a stripe (arrow, Figure 72, C), which is moving posteriorly (arrow, Figure 72, D). However, no segmental stripe of Pt-BarH1 expression can be detected after germ band elongation. The two paralogs found in the transcriptomes of $P$. phalangioides were cloned (Jerome Janssen) but in situ hybridization of whole mount embryos resulted in staining of low quality, which is probably attributable to problems during probes synthesis and thus needs to be repeated.

\subsubsection{Function of BarH1 in P. tepidariorum}

Parental RNAi was performed with the cloned BarH paralog fragments of Pt-BarH1 (813 Bp) and Pt-BarH2 (726 Bp). $4 \mu \mathrm{g}$ ds RNA was injected five times in adult females spiders, which were mated after the third injection. The knockdown of Pt-BarH2 did not lead to any obvious phenotype (data not shown). Knockdown of Pt-BarH1 resulted in a large number of embryos, which were unable to hatch from the embryonic envelope (depicted in green, Figure 75) or died during embryogenesis (depicted in dark violet, Figure 75). This was observed already in the first cocoon laid by RNAi females after injection with dsRNA and did not change for at least seven cocoons. The most prominent phenotype was fully developed embryos that were unable to hatch, especially in cocoon 4 (over 90 \%), cocoon 5 (over 70\%) and cocoon 6 (more than 80\% in Figure 75). 

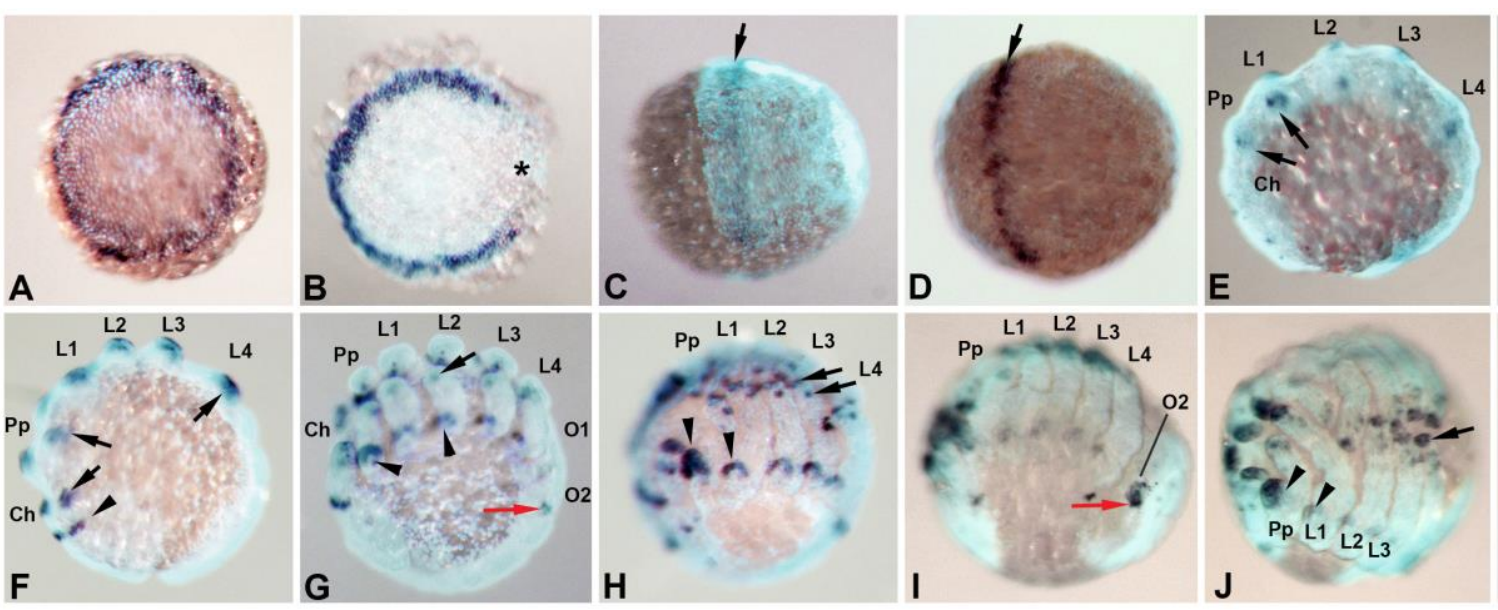

Figure 72: Expression of BarH1 throughout embryonic development of P. tepidariorum

A: Germ disc surrounded with expression of BarH1. B: Opening of germ disc. The asterisk marks the area of opening. C: Embryo during early germ band formation. D: Embryo during late germ band formation. E: Fully elongated germ band embryo, lateral view. The arrows point to distal BarH1 expression in the areas where prosomal limb buds will form. F: Embryo during inset of limb bud development, lateral view. The arrows point to BarH1 expression distally in the limb buds. The arrowhead indicates BarH1 bilateral stripe anterior to the chelicera. G-I: Lateral view of embryos during limb bud elongation (G), inversion (H) and dorsal closure (I). J: Ventral view of the shown in I. The arrowheads in G-J point to BarH1 expression in the gnathendite and the corresponding tissue at the base of the legs. The back arrows point to distal BarH1 spots in the prosomal appendages. The red arrow marks BarH1 expression in in the second opisthosomal limb bud. All embryos are oriented with anterior to the left, except for the germ disc stages which are oriented in top view of the germ disc. Ch: Chelicera, Pp: Pedipalps, L1-L4: Walking legs pairs 1-4, 01-02: Opisthosomal segments 1-2.
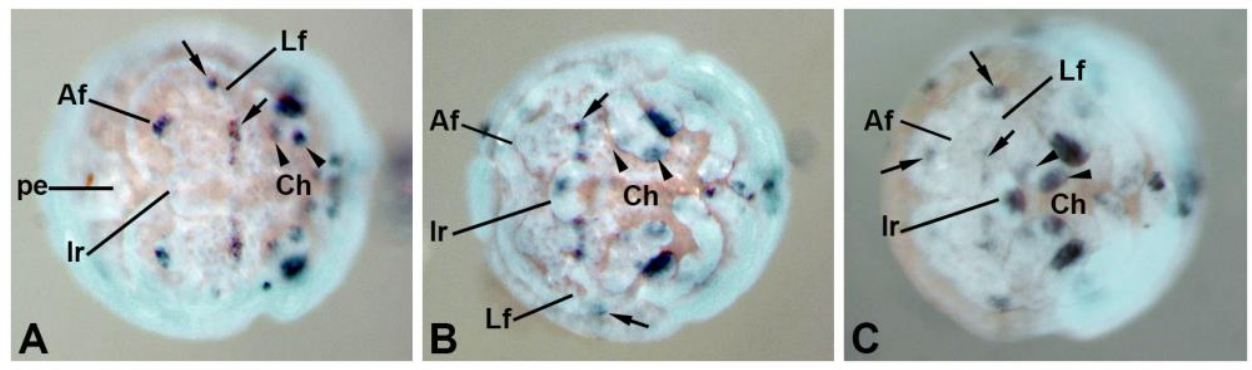

Figure 73: Expression of BarH1 in the head of P. tepidariorum

A: Frontal view of the head during limb bud elongation with Pt-BarH1 expression in the chelicera (arrowheads), the lateral furrow and two stripes anterior of the chelicera segment (arrow) as well as in the anterior furrow. B: Head of the embryo during inversion of $P$. tepidariorum, shown in frontal aspect. The arrowheads point to expression in the chelicera, the arrowheads indicate segmental BarH1 stripes anterior to the chelicera and a domain lateral to the lateral furrow. C: Frontal view of an embryo during dorsal closure. Arrowheads as described before, arrows indicate same expression as in B and additional expression anterior to the anterior furrow. All embryos are oriented with anterior to the left. lr: Labrum, Lf: Lateral furrow, Af: Anterior furrow, Ch: Chelicera, Pp: Pedipalps, L1-L4: Walking legs pairs 1-4, 01-05: Opisthosomal segments 1-5, pe: posterior end. 


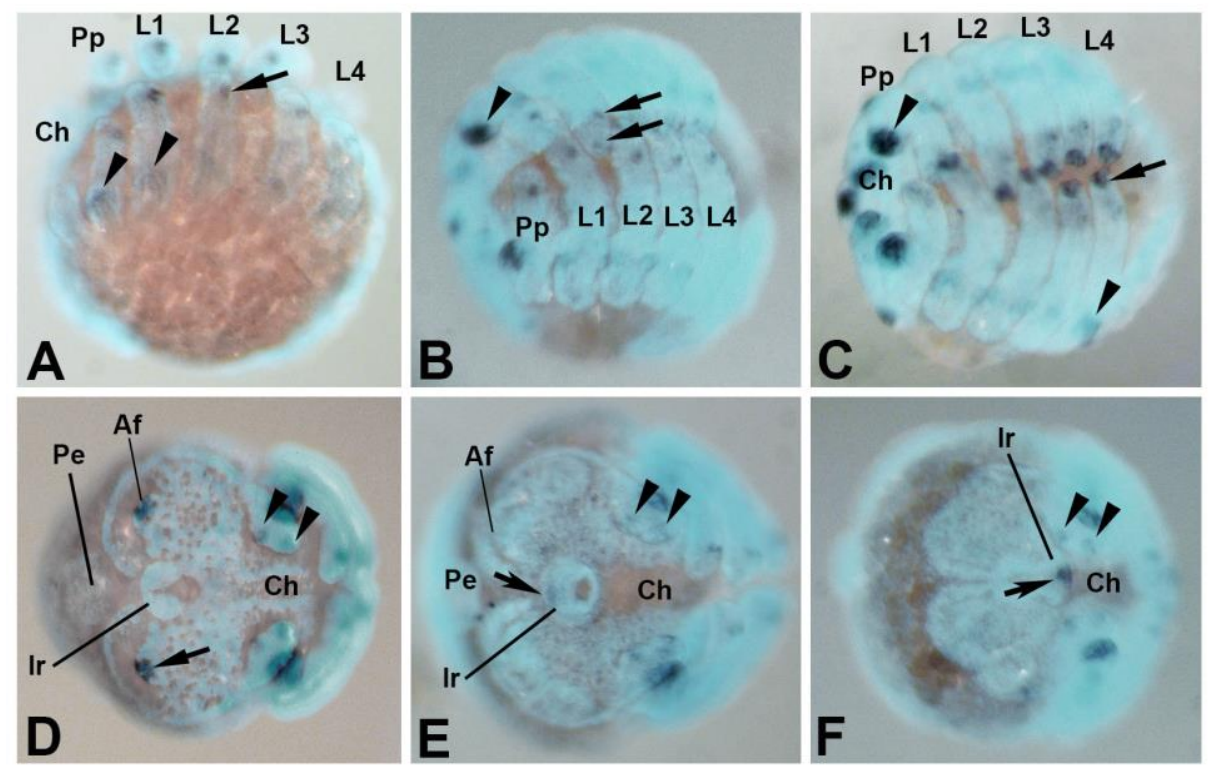

Figure 74: Expression of BarH2 in P. tepidariorum

A: Lateral view of an embryo during limb bud elongation. B: Embryo during inversion shown in ventro-lateral view. C: Embryo during dorsal closure also in Ventro-lateral view. A-C: The arrowheads point to expression of $\mathrm{Pt}$-BarH2 in the gnathendite of the pedipalps and corresponding tissue at the base of the legs. The Arrows point to distal spotted Pt-BarH2 expression in the pedipalps and walking legs. D: Embryo during limb bud elongation with Pt-BarH2 expression in the anterior furrow (arrow), shown in frontal aspect. E and F: Embryo during inversion and dorsal closure shown in frontal aspect. The arrowheads point to two spots of $\mathrm{Pt}$-BarH2 expression in the chelicera. The Arrow indicates Pt-BarH2 expression in the labrum. All embryos are oriented with anterior to the left. Af: Anterior furrow, Ir: Labrum, Ch: Chelicera, Pp: Pedipalps, L1-L4: Walking legs pairs 1-4, 01-05: Opisthosomal segments 1-5, pe: posterior end.

The weak phenotype found in freshly hatched nymphs showed the absence of of the frist walking leg pair or the entire the first walking leg segment (depicted in Figure 76, C and D, strongest in cocoon six, but less than $10 \%$ ).

In general the RNAi of Pt-BarH1 showed a high penetrance with over $60 \%$ phenotypes in the cocoons 2-7 (Figure 75). More detailed analysis of the unhatched embryos from the affected cocoons showed that these embryos had several defects concerning anterior segmentation or showed completely disrupted embryonic development (other defects indicated in black, Figure 77). Strongest phenotypes were missing the head segments, including the cheliceral, pedipalpal and at least first leg segment (Figure 76, E and F). More common (approximately $20-40 \%$ in Cocoon 2-4, Figure 77) were phenotypes with ventral fusion or absence of at least one or different combinations of several prosomal appendages (usually chelicera, pedipalps or the first walking leg, example in Figure 76, 
D), which resembles the weak phenotype observed in the hatched nymphs. The reason why animals missing the first walking leg were able to hatch, but animals lacking the pedipalps were not able to hatch is the absence/presence of the egg tooth. This structure is located on the pedipalps and is required for the initial bursting of the embryonic envelope (Mittmann and Wolff, 2012).

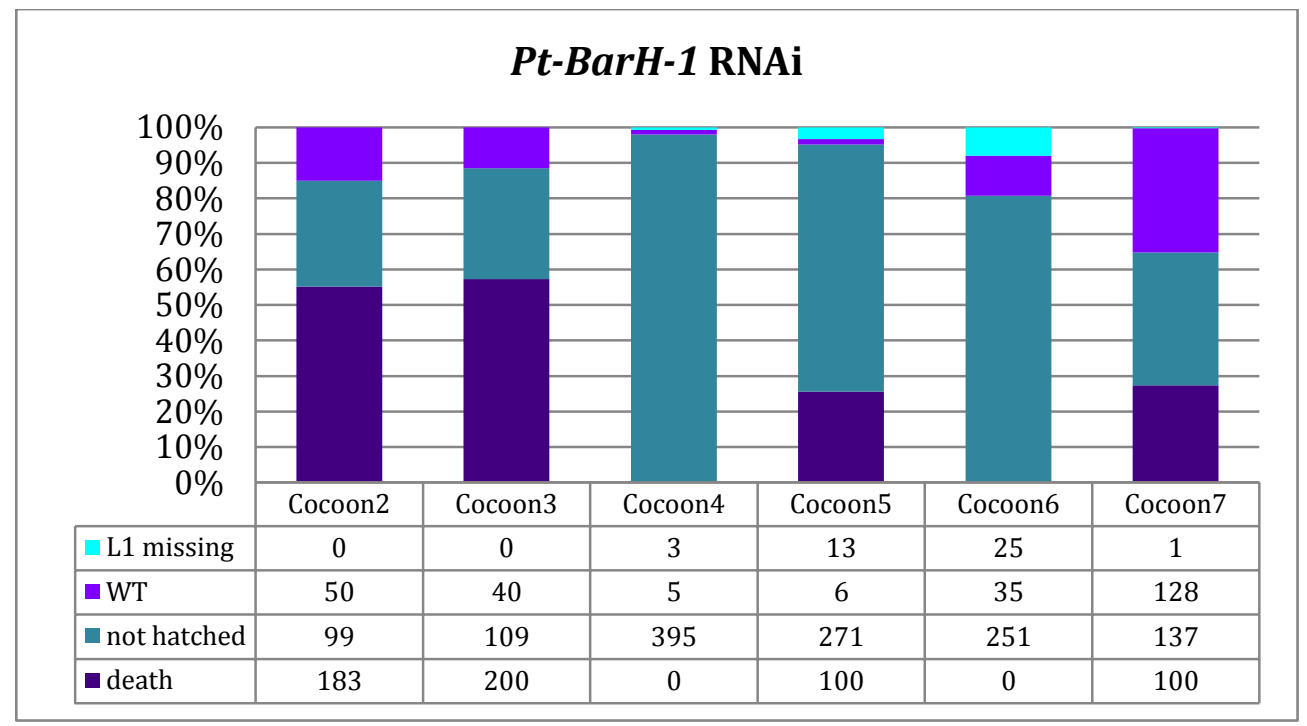

Figure 75: Phenotype of Pt-BarH1 knockdown in the different cocoons

The graph gives an overview of the phenotypes in freshly hatched nymphs after knockdown of PtBarH1 in the cocoons 2-7 produced by females after injection. Up to the sixth cocoon a majority of offspring are affected (over $\mathbf{8 0 \%}$ ) and were not able to develop normally (Wt: indicated in light violet). The offspring of cocoon 4 were nearly completely unable to hatch from the embryonic envelope. But also the cocoons 5 and 6 are severely affected with total number of unhatched embryos ranging between $60-80 \%$. The only phenotype which was observed after moulting into first instar nymphs shows absence the first walking legs segment as depicted in Figure $76 \mathrm{C}$. This phenotype is found in offspring of the cocoons 4 to 7 , but in a relatively low amount (highest in cocoon 6 with less than $10 \%$ ). 

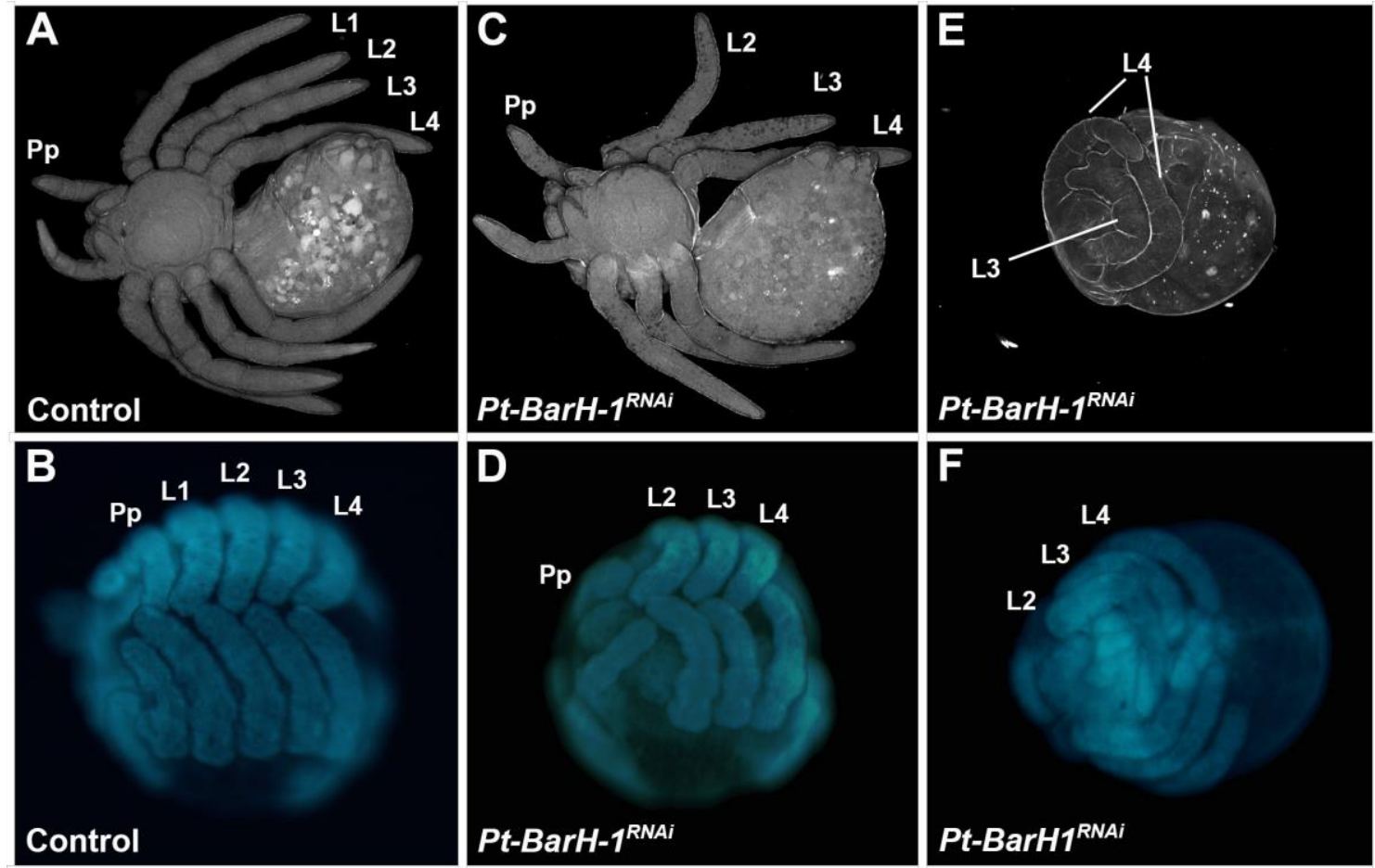

Figure 76: Embryonic and nymphal phenotypes from Pt-BarH1 knockdown

A: Freshly hatched first instar nymph from a cocoon produced by an untreated female, mounted in Voltalef oil. B: Embryo during inversion stained with Sytox-Green from a female without any treatment. C: Freshly hatched first instar nymph (mounted in Voltalef oil) showing the weak PtBarH1 phenotype indicated by absence of the first walking leg segment. D: Embryo after knockdown of Pt-BarH1 with weak phenotype missing the first walking leg pair on the corresponding segment stained with Sytox-Green. E: Embryo after completion of embryonic development unable to hatch from the embryonic envelop due to missing egg tooth usually located on the pedipalps. The individual was mounted in Voltalef oil after the embryonic eggshell was removed by hand. It represents a very strong phenotype missing all segments anterior to the third walking leg segment. The third walking leg segment carries only one leg, which is transformed into a trident at the distal portion. F: Embryo probably during or shortly after ventral closure after knockdown of Pt-BarH1. This embryo represents a weaker variation of the strong phenotype, missing all segments anterior to the second walking leg segment, but with the three posterior walking leg pairs fully developed. All specimens are oriented with anterior to the left and in ventrolateral view. Ch: Chelicera, Pp: Pedipalps, L1-L4: Walking legs pairs 1-4. 


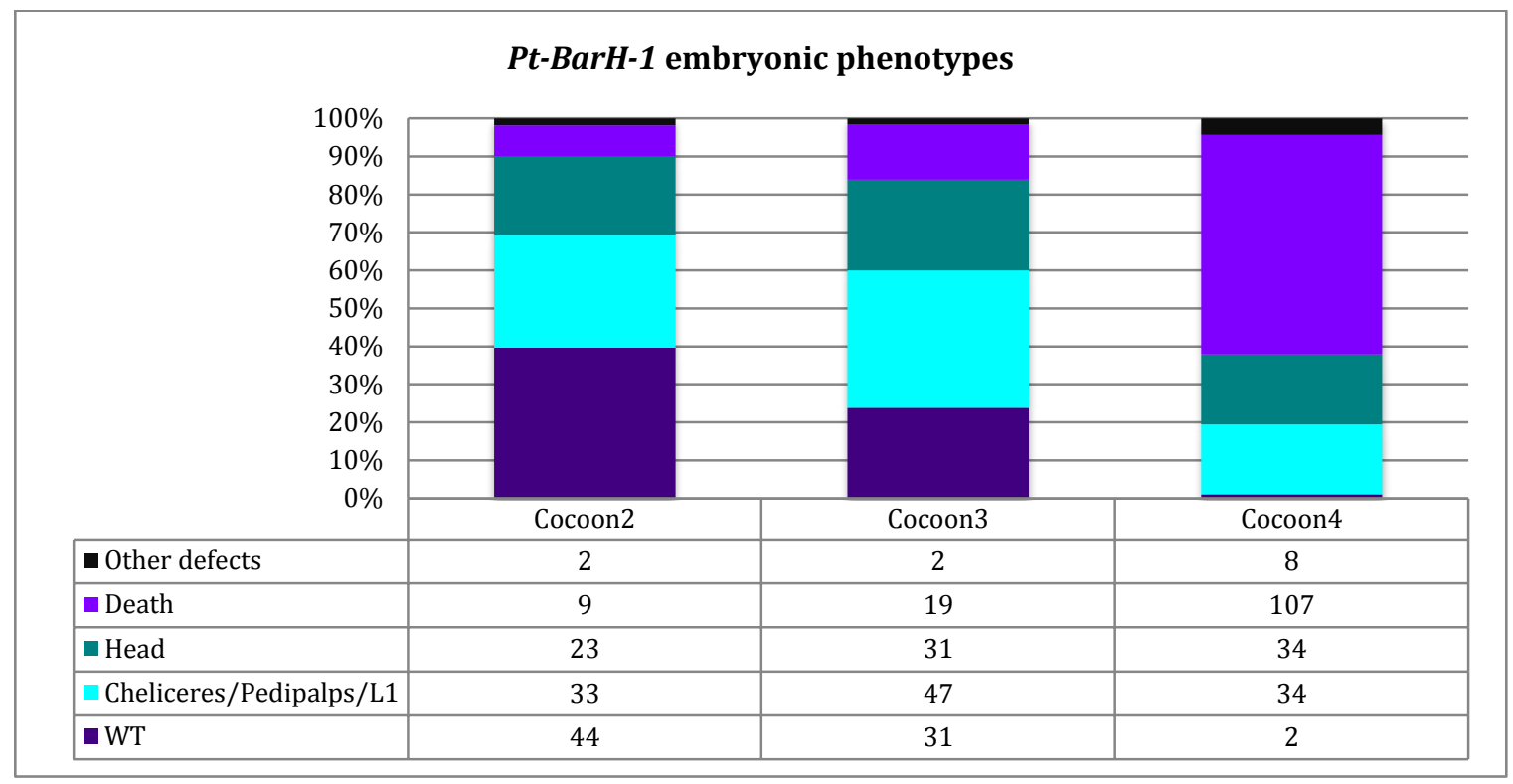

Figure 77: Overview of embryonic phenotypes in cocoon 2-4 after Pt-BarH1 knockdown The graph shows the analysis of embryos from the cocoons 2-4 to reveal the reason of the hatching problem observed in a majority of individuals after knockdown via parental RNAi of Pt-BarH1. The number of properly developed embryos is indicated by Wt and marked in dark violet. It decreases from nearly $\mathbf{4 0 \%}$ in the second cocoon to nearly no individual in the fourth cocoon. In the fourth cocoon the majority of embryos died during the course of development. In the two previous cocoons the majority of individuals show either strong or weak defects, including missing the complete head or deletion or fusions of the anterior prosomal appendage segments. Other defects, like axis duplication and multiple opisthosoma represent the minority of phenotypes in these cocoons with less than $10 \%$ in each.

\subsubsection{Expression of head and leg developmental genes in Pt-BarH1 RNAi embryos}

To verify which walking legs were missing in the different weak phenotypes we examined the expression of the Hox gene Sex-combs-reduced in embryos after Pt-BarH1 knockdown. Expression of this gene is absent in the first walking legs pair, and has a legspecific pattern for each of the walking leg pairs 1-3 of spiders (shown in Figure 78, D and in previous findings (Khadjeh et al., 2012; Schwager et al., 2007)). The expression of Pt-Scr supports the conclusion that the first walking leg is missing, whereas the other three walking legs are able to develop normally in weakly affected Pt-BarH1 knockdown phenotypes (Figure 78, H). Furthermore the expression in these more posterior walking legs shows that they can develop normally although the complete head including the first walking leg segment is missing (Figure 78, L). The expression of Pt-Dll in very weak phenotypes (Figure $78 \mathrm{G}$ ) shows that in some cases the segment of the first walking leg is 
still intact and only the pair of legs is reduced. In contrast Pt-Dll expression in strong PtBarH1 knockdown phenotypes indicates that no appendage primordia are present after depletion of the head tissue.

orthodenticle (Pechmann et al., 2009) and the early function of Pt-Dll (Pechmann et al., 2011), were previously found to be involved in the head development of P. tepidariorum, their interaction and the genetic mechanism, however, is not known. To ascertain the position of Pt-BarH1 in the head patterning network of $P$. tepidariorum the expression of these genes was observed in early embryos after Pt-BarH1 knockdown. Due to lacking anterior tissue in the Pt-BarH1 phenotypes it was challenging to predict in which stage of development the observed embryo were fixed. The embryos (Figure 78 (E-F and I-J), could either represent late stage six or late stage seven embryos, which is hard to elaborate due to missing anterior tissue. However, in general the staining of Pt-otd in these phenotypes shows a disrupted and patchy otd expression after knockdown of $P t$ BarH1 (Figure 78, E and F). The expression of Pt-Dll in Pt-BarH1 knockdown embryos seems at first glance less affected compared to expression of Pt-otd, indicated by a strong anterior stripe of expression. Comparison to early stage seven control embryos, however, indicates the complete absence of the head tissue anterior to the Pt-Dll stripe in the knockdown phenotypes. Another possible alteration of Pt-Dll caused by Pt-BarH1 is the suppression of the splitting of the Dll stripe (not shown but previously described in (Pechmann et al., 2011)) later during stage seven. 


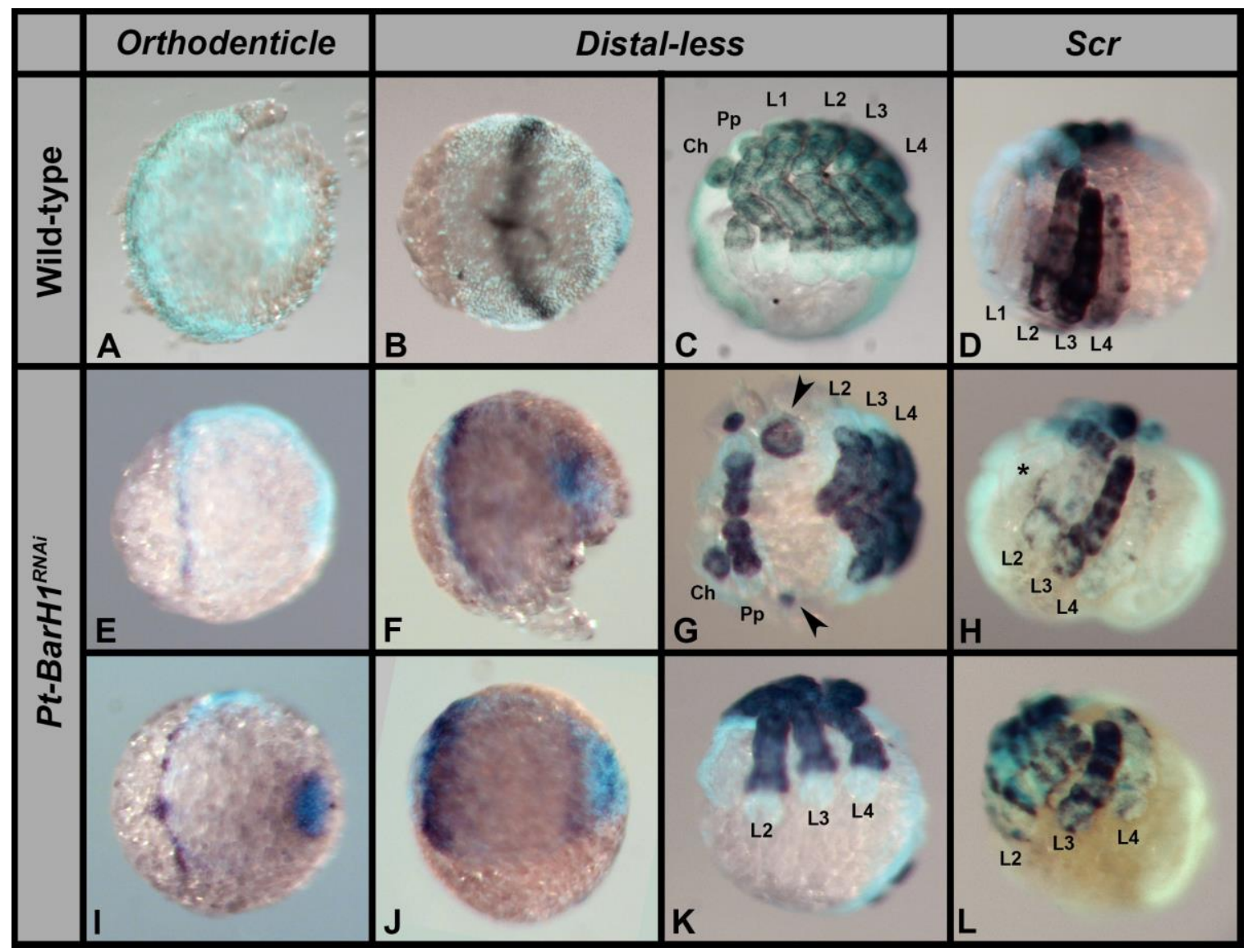

Figure 78: Expression of otd, Dll and Scr in embryos after Pt-BarH1 RNAi

A-D: Expression of otd (A), DII (B and C) and Scr (D) in untreated P. tepidariorum embryos at different stages. E-L: Expression of otd (E and I), DII (F-G and J-K) and Scr (H and L) after knockdown of BarH1 at different stages of embryonic development in P. tepidariorum. The embryos shown in A and $B, E$ and $F$ as well as $I$ and $J$ represent embryos after break of radial symmetry on the way to germ band stages. C,G and K: Expression of Pt-DIl in prosomal appendages during early inversion. The arrowheads point to remaining Pt-Dll expression in reminiscent leg tissue on an intact first walking leg pair segment. The embryos are shown in lateral ( $C$ and $K$ ) and ventral view (G) D, $H$ and L: lateral view of embryo with expression of Pt-Scr during dorsal closure. The asterisk indicates the fusion of the first walking leg pair into a single appendage. All embryos are shown with anterior to the left. Ch: chelicera, Pp: pedipalps, L1-L4: walking leg 1-4. 


\subsection{Discussion and future work}

\subsubsection{Distal limb development is not conserved for insects and spiders}

Findings from the insects D. melanogaster and T. castaneum and the onychophoran $E$. kanangrensis (Angelini et al., 2012; Estella et al., 2012; Kojima, 2004; Oliveira et al., 2014), the sister group of arthropods suggested that patterning of the distal limb development is conserved in panarthropods and thus similar genes could be used for tarsus segmentation in P. phalangioides. However, the lack of any tarsus segmentation in most arthropods and especially in chelicerates instead suggests that an unsegmented tarsus is the ancestral state in arthropods. Therefore I studied several tarsus segmentation candidate genes in this spider and compared their expression to $P$. tepidariorum, a spider without segmentation of the tarsus. The first evidence that the genetic cascade involved in distal limb patterning in insects was not already present in the last common ancestor of the panarthropods (including onychophorans and arthropods) is provided by the lack of expression of the genes rotund, bric-a-brac, chip and tango in P. tepidariorum.

This is further supported by the absence of distal expression of all ap homologs, the Lim1 homologs and the trh1 orthologs in the walking legs of both spiders. Only very few of the candidate genes revealed a possible function in the distal part of the walking leg. The homologs of al, both paralogs of $s s$ in P. tepidariorum and P. phalangioides, as well as both BarH homologs in P. tepidariorum are expressed in the distal tips of the walking legs. To define sharp borders between the segments, genes involved in leg or tarsus segmentation should be expressed in broad domains in the segment of their function or in rings at the joints (Angelini and Kaufman, 2005; Prpic and Damen, 2009). All these genes, however, show a spotted expression in the distal leg, that suggests some function in this portion of the legs, but does not immediately suggests a role in tarsus segmentation. Spot-like expression usually is found for genes involved in neurogenesis, defining neural precursors (Doeffinger et al., 2010), which could develop into sensory organs, like bristles or lyriform organs. Thus also these genes are probably not conserved for distal leg development. Only in two cases a ring-like, or broad domain of 
expression was observed. Expression of trh2 in P. tepidariorum is present in a ring-like manner. However, this expression was also observed in more proximal part of the developing walking legs. Thus trh homologs might have a general role in leg development but not exclusively in the distal segment. The other case are the two cll paralogs in both spiders. These were expressed exclusively in the distal part of the developing prosomal appendages. In addition, knockdown of cll1 in P. tepidariorum revealed that it is required for proper tarsus formation. In summary my data suggest that the similarities between insects and onychophorans are rather the results of a parallel co-option of a plesiomorphic gene regulatory network or caused by misinterpreted leg expression in the onychophoran. The expression patterns of the tarsus segmentation candidates in the distal part of the spider walking legs, except for cll, are clearly different from D. melanogaster and do not suggest any role in distal segmentation. Thus neither the distal patterning functions known from insects nor tarsus segmentation are phylotypic traits of arthropods or panarthropods.

\subsubsection{Cross-regulation of al, cll and Lim1 is not conserved in arthropods}

Only the gene al was not duplicated in the spiders studied in this thesis and in the predicted genome sequences of the spider S. mimosarium. Although many al related genes were found these were not grouped into the clade containing genuine $a l$ homologs from other model organisms. The high support value of the monophyletic al group including homologs from insects, chelicerates and vertebrates rather suggests that no duplication event occurred for this gene in the lineage leading to spiders or that a possible duplicate was secondarily lost. $a$ l has a complex expression pattern in the entire walking legs during embryonic development of $P$. tepidariorum and P. phalangioides and might therefore be used for different purposes. The early ring-like expression hints to a possible role in segmentation, because rings of expression are often found for genes involved in patterning and segmentation processes (Prpic and Damen, 2009). The spotlike $a l$ expression at the base and in the tip of the walking legs probably define neural precursor cells. Although the expression pattern of al drastically changes during embryogenesis and shows a very complex pattern in the late walking legs, the pattern 
and temporal profile of the expression is virtually identical in both spider species. Comparisons to al expression in Gryllus bimaculatus, D. melanogaster, Manduca sexta and T. castaneum show two general patterns found within insects (Beermann and Schröder, 2004; Campbell and Tomlinson, 1998; Miyawaki et al., 2002; Tanaka and Truman, 2007). Whereas the expression in G. bimaculatus and T. castaneum also shows a complex pattern in the developing legs, al expression in M. sexta and D. melanogaster is restricted to the coxa and the most distal part of the leg. Because G. bimaculatus and T. castaneum are less derived insect species it is most probably that the loss of medial al leg expression was lost in the lineage leading to D. melanogaster and M. sexta. Furthermore $G$. bimaculatus is the only representative from the hemimetabolous group that does not involve the derived pupal stage, and thus its general mode of development might be more similar to spiders than to holometabolous insects. Despite the divergent expression patterns, however, functional studies in T. castaneum showed that knockdown of al results in the absence of the pretarsus segment carrying the claw as it was also shown for D. melanogaster (Angelini et al., 2012; Beermann and Schröder, 2004). If knockdown of the distal expression in spider legs would lead to similar phenotypes, one could suggest that the spot-like expression is required for claw formation and that this function is conserved in all arthropods. Otherwise the function in claw development has evolved after the split of spiders and insects. The expression in the distal tip of the onychophoran hints to a phylogenetically old conservation of this gene in the leg (Oliveira et al., 2014). I note, however, that the expression was only observed in very young embryos and is extremely weak. It might therefore be premature to draw any conclusions about gene function in onychophorans. Rather, functional studies of al in spiders and onychophorans are required to understand the role of $a l$ in distal leg development. It is also interesting that activation of Lim1 by al is dependent on protein complex formation with cll demonstrated in D. melanogaster (Campbell, 2005; Miyazono et al., 2010). In P. tepidariorum and P. phalangioides al and cll are never co-expressed and it is not likely that transcription factors diffuse over larger distances in cellularised environments. This could be explained in two ways: (1) If the formation of the $\mathrm{Al}$ and Cll protein complex is ancestral and required for correct binding of specific DNA targets neither al nor cll would be functional in the leg of spiders. (2) In spiders $\mathrm{Al}$ and $\mathrm{Cll}$ have other co-factors, which 
mediate binding to DNA targets. The second scenario is more likely, because genes without function would not show such a conserved expression pattern like al and cll. In addition, the tarsal phenotypes obtained after knockdown of $c l l$ demonstrate that at least cll is not an unfunctional gene in the tarsal segment.

Lim1 was not expressed distally in the walking legs, in contrast to E. kanangrensis and D. melanogaster (Kojima, 2004; Oliveira et al., 2014; Pueyo, 2004). As stated above data from the onychophoran are based on expression patterns only and are in addition not observed for the entire walking leg development as performed in this thesis. Knockdown of Lim1 in T. castaneum resulted in fusion of femur and tibia but no defects in the distal part of the leg (Angelini et al., 2012). Even double or triple knockdown together with al and cll only showed additive defects, but no role in distal patterning (Angelini et al., 2009). Unfortunately expressional data for Lim1 during embryonic development of $T$. castaneum is lacking. In summary this suggests that Lim1 in spiders and T. castaneum likely represent the ancestral function, which is independent from interaction with al and cll.

\subsubsection{Conservation of distal function of clawless}

Both cll paralogs in P. tepidariorum and P. phalangioides show expression in the tarsus segment. However, the exact pattern differs in late embryonic stages for both paralogs. Tarsus segmentation presumably starts at late embryonic stages. Thus differentiation of the paralogs in the two spiders might reflect differing roles in tarsus segmentation of $P$. tepidariorum and P. phalangioides. The distal cll1 expression in the walking leg of both spiders divides into two rings during inversion, but not in the pedipalps, which is consistent with the fact that the pedipalps lack the metatarsal segment. Interestingly, the further pedipalpal and walking leg expression profiles of cll1 differ between $P$. tepidariorum and P. phalangioides indicating that cll1 might be involved in the formation of species specific differences in pedipalp and leg morphology between the two species. cll2 is more similarly expressed in the pedipalps and walking legs and thus is unlikely to be involved in the development of morphological differences between these two appendage types. Unfortunately, RNAi is not yet available in P. phalangioides and 
therefore a possible role of either cll gene of this species in the special tarsus segmentation uniquely found in P. phalangioides is unclear at the moment and awaits future gene function studies.

Knockdown of cll1 in P. tepidariorum demonstrate that it is necessary for proper development of the tarsal tissue. Depending on the timing of RNAi effect, we see either a disruption of the formation of the joint or the ablation of the entire tarsal segment. This shows that cll is conserved in defining most distal tissues in insects and at least one spider (Campbell, 2005; Cande et al., 2009; Miyazono et al., 2010). Together with findings from the onychophoran (Oliveira et al., 2014), where cll expression in the distal tip is more defined and stronger than the other distal genes observed in the same study, this hypothesis is supported. Lack of distal phenotypes after RNAi in T. castaneum might be caused by problems of the RNAi procedure itself. This is additionally supported by strong expression found in the distal tip of developing embryos of this species (Cande et al., 2009; Grossmann and Prpic, 2012).

\subsubsection{Other conserved functions of tarsus segmentation genes}

In contrast to different or absent expression of tarsus candidate genes in the tarsus of $P$. phalangioides and $P$. tepidariorum, conservation of these genes involved in other processes was observed. Both ss paralogs in both spiders for example showed spot-like expression in all prosomal appendages as well as in the limb buds on the opisthosoma. As previously mentioned spot-like expression hints to a possible role for defining neural precursors, including sensory organs and bristles. Apart from its function in distal leg patterning of D. melanogaster, ss is required for the development sex combs on the first tarsal segment (Kuzin et al., 2014), correct bristle formation in the antenna and legs (Emmons et al., 1999) and for dendrite diversification in dendritic arborization of sensory neurons (Kim et al., 2006). Thus the role of ss in distal leg development apparently represents the derived mode of this gene, whereas its role in defining neurogenic tissues or its descendants is probably conserved. This is supported by findings in C. elegans where the ss homolog, named aryl hydrocarbon receptor 1, mediates 
the differentiation of neuronal fates (Huang et al., 2004). Functional studies to clarify the exact role of $s s$ in spiders are in progress, but did not show any results yet.

Also the expression of the trh homologs in the spiders showed interesting parallels to previously described functions. Both $P$. phalangioides paralogs and trh2 from $P$. tepidariorum are expressed in the opisthosomal limb bud on the third opisthosomal segment. This limb buds invaginate during embryonic development and then gives rise to the tubular trachea of the spider. Interestingly, trh in D. melanogaster is required for tubulogensis and was found to act up-stream regulating most genes involved in trachea development (Chung et al., 2011; Isaac and Andrew, 1996). Although functional data in the spiders are lacking, the expression in the precursor of the spider trachea suggests, that this gene has a conserved function in trachea development. This is even more surprising because morphological studies so far suggested that the tracheae of spiders and flies are analogous structures. But already comparative studies of the respiratory organs of D. melanogaster and crustacean gills showed that trh is expressed similar in both tissues and thus might originate from the same precursor, although they are morphologically diverged (Franch-Marro et al., 2006; Sánchez-Higueras et al., 2014) .

For $a p$ we did not find tarsal expression in either spider species. The restriction of the expression ventrally to the most proximal part suggests that $a p$ in spiders does not have a significant role in walking leg patterning. However, the expression in the opisthosoma and the opisthosomal limb buds is similar to the previously studied ap homologs of $C$. salei (Damen et al., 2002). That study concluded that the opisthosomal appendages of spider and the wings of insects might be homologous appendages evolved from ancestral gills, but without more detailed and especially functional analysis of this gene this hypothesis remains open for debate. The co-expression $a p$ and the other Lim-type family gene Lim1 in the legs or in the opisthosomal limb buds are more compatible with a role in neural differentiation or muscle precursor diversification as already known from the annelid Neanthes arenaceodentata (Winchell and Jacobs, 2013) and also from D. melanogaster (Bourgouin et al., 1992).

Surprisingly, the expression of Pp-ap2.2 is more similar to ap.1 of P. tepidariorum and $C$. salei, whereas $P p$-ap1 shows a different expression pattern in the opisthosoma of $P$. phalangioides. This calls into question my classification of the orthology of these 
duplicates in the spiders, and suggests that the phylogenetic analysis was not able to properly resolve the phylogenetic relationships of the spider ap homologs. The phylogenetic tree also suggests that ap2 was duplicated once more in the $P$. phalangioides and P. tepidariorum but not in C. salei. Whether this duplication of ap2 did not take place in C. salei or just escaped previous gene cloning attempts or is also the result of a problematic phylogenetic analysis is currently unclear. The second scenario is supported because also not all cloning attempts for ap2 lead to successful cloning in $P$. tepidariorum and P. phalangioides. Findings based on the expressional data of the different P. phalangioides tissues during the transcriptomic approach further support this hypothesis: no read counts were found for Pp-ap2.1 in the embryonic tissue, but only in the opisthosoma of the nymphs. Thus it is probably missing in the cDNA libraries synthesized from embryonic stages and thus escaped cloning attempts. In addition, a second copy of ap2 is present in an additional spider, Stegodyphus mimosarum ( $S$. mimosarum), as revealed during the phylogenetic analysis. S. mimosarum, $P$. tepidariorum and C. salei all belong to the entelegyne spiders. P. phalangioides is placed into the group of haplogyne spiders. The presence of two copies of ap2 in members of both entelegyne and haplogyne spider, but not in C. salei thus can only be explained (if escaping cloning attempts could be ruled out as most likely cause) by loss of this gene in C. salei. More expressional and also functional analysis would be helpful, but also the study of the transcriptome of $C$. salei and additional chelicerate species would help to have more profound understanding of the phylogenetic relationships of the ap paralogs within this lineage.

\subsubsection{Possible role for $s s$ and $t r h$ in growth regulation during late embryogenesis}

As previously mentioned unbiased approaches using transcriptomics representing a valuable opportunity to identify novel genes involved in morphological diversification. This was supported by various findings in different species (Armisén et al., 2015; Dong et al., 2015; Gilad et al., 2009; Pantalacci and Sémon, 2014; Tom et al., 2014; Yu et al., 2014) and in this work. However, the analysis of the tarsus candidate genes $s s$ and trh revealed an extremely interesting expression during late leg development of $P$. phalangioides. The 
analysis of the embryonic development of $P$. phalangioides showed that the extreme growth of the walking legs starts and continues during ventral closure stages. ss 1 and trh2 show an extreme increase in expression at exactly that developmental step. The genes are very strongly expressed in the entire walking leg excluding the short segments and the areas of the joints, thus areas where growth should be enhanced or repressed, respectively. This expression pattern was only found in P. phalangioides, thus the spider species with long legged morphology and not in P. tepidariorum, which develops shorter leg compared to the overall body size. At least Pt-trh2 also shows an increase of expression during late embryonic stages, however, the pattern is ring-like and not covering the entire segment. A possible role of $s s$ and trh paralogs of $P$. phalangioides during leg growth are further supported by comparison to their expression in the other prosomal appendages. The pedipalps and chelicera of $P$. phalangioides are not prolonged like the walking legs. Also the expression of $s s$ and trh in these appendages shows a ringlike pattern similar as observed for Pt-trh2 or D. melanogaster. Thus the candidate approach might have revealed two genes, which could have a role in the extreme leg growth of the long-legged spider species, although this was not the purpose of the study. The influence of these genes in growth control, however, must now be verified by functional studies.

\subsubsection{Novel role for BarH1 in head patterning of $P$. tepidariorum}

For the tarsus candidate gene BarH1 I also found an unexpected expression apart from appendage patterning. BarH1, but not BarH2, is expressed in the periphery of the developing germ disc during early embryonic development of P. tepidariorum. For this gene it was even possible to verify the function caused by this expression. Knockdown of the gene resulted in embryos lacking at least the first walking leg segment or even the complete head. A similar role has been previously observed for another gene of $P$. tepidariorum usually involved in leg patterning, Dll (Pechmann et al., 2011). Dll knockdown embryos lack the first and sometimes also the second walking leg segment. The phenotype after BarH1 RNAi thus affects also more anterior segments as Dll. In addition to Dll, orthodenticle (otd) is known to be involved in head development of $P$. 
tepidariorum (Pechmann et al., 2009). Knockdown of otd resulted in the loss of all head segments anterior to the first leg segment. It was shown that the formation of a proper head requires a dynamic fine tuned expression of the anterior genes (Pechmann et al., 2009). Because the BarH1 RNAi phenotype represents a combination of both otd and Dll phenotypes it might be involved in orchestrating these early dynamics required for proper head formation. Preliminary data of otd and Dll expression in early BarH1 knockdown embryos indeed showed partial loss of the strong anterior otd expression and a possible alteration of Dll expression dynamics. Whether BarH1 acts upstream of otd and Dll or if these alterations are an artefact caused by missing the anterior tissue remains to be tested in further experiments. Testing this would require a detailed examination of the early expression dynamics of these genes in the anterior part embryo of $P$. tepidariorum, as well as combinations of loss and gain of function studies. 


\section{General Discussion}

\subsection{Hourglass model of walking leg development}

The aim of my work was to study which genetic mechanisms are important to drive the immense arthropod appendage diversity. Despite this extreme diversity the different appendages all need to undergo some crucial developmental steps, like specification of primordia, development of the proximo-distal axis, further patterning and segmentation as well as growth (Angelini and Kaufman, 2005). The walking leg development of $D$. melanogaster is studied in great detail. It was shown that the legs are developed following a cascade of gene networks required for these different steps (Detailed description in the Introduction) (reviewed in Estella et al., 2012; Kojima, 2004). Thus the question is which genetic modules of this cascade are free for alteration and which are crucial and need to be conserved to ensure proper walking leg development in general. To answer this question my work and previous studies in other representatives of the arthropods studied the gene networks of this cascade and compared the level of conservation.

A summary of the previous findings by Prpic and Damen 2008 supposed, that an hourglass could represent the genetic cascade involved in walking leg development. The early steps like appendage primordia specification and initialization of the proximodistal axis at the beginning of the cascade require some similar genes, but the exact function of these genes is different between species. These different modes of early appendage development are reflected by differences of the gene regulatory networks involved in appendage primordia specification (e.g. Khadjeh et al., 2012; Pechmann et al., 2011, Angelini and Kaufman, 2005; Hughes and Kaufman, 2002b). In contrast to the early steps of the walking leg development cascade, the following step, patterning the proximo-distal axis and development of the joints are extremely conserved within different species. During this step the leg gap genes organize the regionalization of the developing limbs into proximal, medial and distal parts (Angelini and Kaufman, 2005, 
Janssen et al., 2010). Previous studies showed that the leg gap gene function is conserved in all arthropods and also found to be required during vertebrate limb development (Janssen et al., 2010; Prpic and Damen, 2004; Prpic and Telford, 2008; Prpic et al., 2001, 2003; Sharma et al., 2012). The study of these genes in $P$. tepidariorum and $P$. phalangioides further supports the constrained role of these genes for the development of walking legs. It was suggested by Prpic and Damen, that this developmental stage might represent the constraint podotypic stage of locomotory legs. The correct regionalization thus represents a crucial step during walking leg development, which should not be altered to diversify appendage morphologies. The data on the leg gap gene exd1, however, showed that this only concerns early functions of these genes, whereas later function, like the patella ring observed for this gene are again suited to be altered (Prpic and Damen, 2009).

Now the question arises where changes could occur in genetic cascade to drive the evolution of novel morphologies. The next step in the cascade of walking leg development is the development of joints between the leg segments. The segmented leg is a common trait of all arthropods and is most likely one of the keys to their evolutionary success. Because of this important role of leg segmentation, the genetic mechanism behind it should be conserved as well and is probably not the basis for diversification. Expressional and functional data for the Notch signalling pathway largely reflect this genetic conservation and thus indicates that also this module of the cascade belongs to the podotypic leg state of arthropods (Bishop et al., 1999; Celis et al., 1998; Rauskolb and Irvine, 1999; Angelini et al., 2012; Mito et al., 2011; Prpic and Damen, 2009). Thus the morphological diversification must occur after these crucial developmental steps. The next process in the hierarchy involves additional patterning processes refining the leg gap gene borders during leg elongation, especially in the distal leg part. The distal-most segment, the tarsus, is further subdivided into numerous distal segments in many insects and the haplogyne spider model studied here. The secondary PD axis patterning gene battery is initiated by $w g$ and $d p p$ interactions and Dll and involves EGFR signalling and its downstream targets (summarized in Estella et al., 2012). In contrast to leg gap genes and Notch mediated joint formation secondary proximodistal patterning is only intensively studied in D. melanogaster and T. castaneum 
(Angelini et al., 2012; Beermann and Schröder, 2004; Grossmann and Prpic, 2012; Kojima, 2004). Data for representatives of the other three arthropod clades a scarce and data from an onychophoran species are only based on expressional data of not fully elongated legs (Hao et al., 2003), Comparison of tarsus segmentation between $T$. castaneum and D. melanogaster showed only some functional differences of this patterning process. The adult leg morphologies of these two species are, however, also relatively similar. Thus studies in species with morphologically more different walking legs are required to verify the hypothesis that the distal segmentation module is less constrained and therefore drives morphological diversification. Furthermore the findings of $T$. castaneum were only based on RNAi studies, mostly lacked whole mount embryonic expressional observations and did not include the complete set of tarsus segmentation genes identified in D. melanogaster. In my work I studied the gene battery involved in tarsus segmentation in two spider species. One, like D. melanogaster, with tarsus segmentation, and the other one, without any subdivision of the tarsus. When the further subdivision of the most distal part of the walking legs would be ancestral for all arthropods these genes should be conserved at least between $P$ phalangioides and $D$. melanogaster and only different in P. tepidariorum.

My data show that this hypothesis can clearly be rejected. Some genes were not expressed during embryonic development at all and thus are probably not conserved in distal patterning of arthropods. However, other genes were expressed in the prosomal appendages, but not in the distal part. Even if expression in the distal part was present, the pattern of expression suggested a different function than found for D. melanogaster. Furthermore these genes were similarly expressed in the walking legs during embryonic development of the two spiders. Thus the late steps of the genetic cascade of walking leg development is in contrast to the podotypic stage not strictly functionally conserved and represent the "edge of the hourglass", and are therefore good candidates driving the evolution of walking leg diversification. However, functional studies and more species are required to study the exact diversification of these genes and other possible factors involved in the development of novel traits. Such novel factors might be missed by candidate gene based studies. The use of unbiased transcriptomic approaches is suited to reveal genes causing developmental differences. Indeed, my study of differentially 
expressed genes in the walking legs of $P$. phalangioides identified candidates with conserved roles in cell proliferation. Control of allometric growth driven by cell proliferation is one of the last steps after patterning of the walking legs. Another interesting candidate from the transcriptomic approach is the gene odd skipped. It is known to be involved in tarsal development of the fruit fly and shows distal expression in the walking legs of the two spider species studied here. Thus it might represent a conserved gene required for distal leg patterning, in contrast to the mostly insect specific tarsus segmentation genes. Interestingly the distal odd-skipped expression is different in P. phalangioides and P. tepidariorum and thus might be involved in the special tarsus segmentation of the haplogyne spider. All these hypotheses are based on expressional data and of course await future functional studies.

The gene $c l l$ is the only tarsus segmentation gene candidate, which is expressed in the distal part of both spider species. For this gene it was possible to perform knockdown experiments, which showed that it is required for the formation of the tarsus in $P$. tepidariorum. This indicates a conserved role for cll in distal patterning. The exact function of this gene must, however, be different to the one known in D. melanogaster, because the co-factors and downstream targets are not present in the spider legs.

During the study of the duplicates of the leg gap gene I found that the dachshund duplicate is necessary for proper formation of the arachnid specific leg segment the patella. Thus this duplication led to the development of a novel morphological trait. The $h$ th duplicate is differentially expressed in the walking legs of four different spider. Intriguingly, gene duplications represent a further possibility how novel traits might evolve on the genetic level.

All these data indicate that the diversification of walking leg morphologies probably happens after the establishment of the crucial podotypic state that includes the highly conserved gene patterning networks and is driven by a combination of different genetic causes, namely (1) altered roles of conserved gene (cll and odds), (2) evolution of novel gene function of existing genes (most tarsus segmentation gene of D. melanogaster), (3) differences in cell proliferation and (4) new genes (duplications) (summarized in Figure 79). In summary my study supported the suggested hourglass model and provided hints of the previously unknown genetic causes acting at the edge of the hourglass to drive 
morphological differences and showed that these are probably positioned in the most downstream area of the walking legs development cascade.

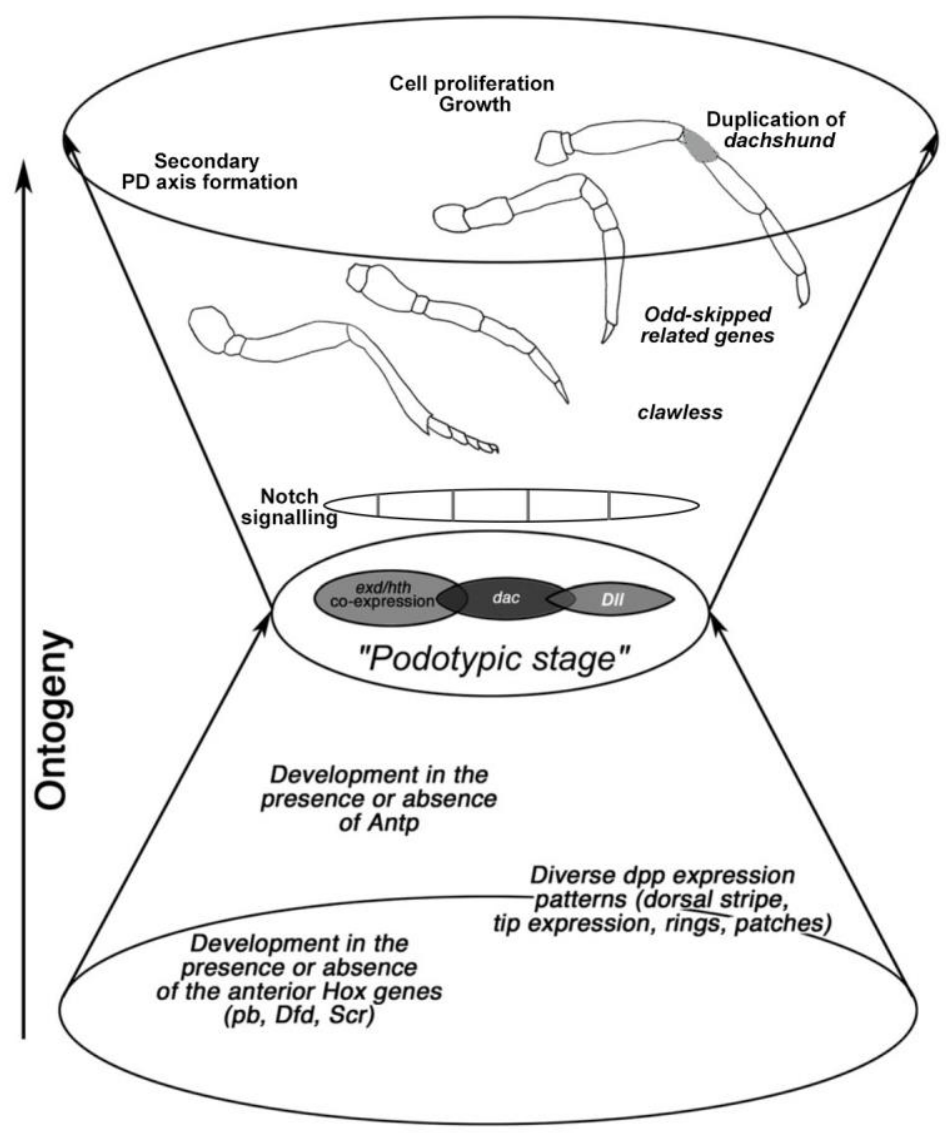

Figure 79: Hourglass model of walking leg diversification development

Illustration of the walking leg development cascade. Early steps include specification of walking leg primordia on the anterior-posterior axis, controlled by $d p p$ and the Hox genes. These early steps show a certain level of genetic diversification. The patterning of the proximo-distal axis and the development of the joints requires the leg gap genes and Notch signaling. This is the most conserved step in the walking leg development cascade of arthropods and might represent a podotypic stage. During my work I was able to show that the genetic mechanisms more downstream in the walking leg development cascade involved in secondary PD axis formation and tarsus patterning are not conserved. This can explain the development of diversified walking legs despite the constrained genetic network within the podotypic stage. Modified from Prpic and Damen, 2008.

\subsection{Duplication of genes facilitates diversification}

One other main finding of my work was that nearly all of the studied candidates genes were duplicated in the two spider species. Diversification during evolution requires diversification also at the molecular level. To diversify gene functions, genes can be 
altered on the regulatory level or in their coding region. Because most genes have several functions (so-called pleiotropic genes), changes in their sequence or in their regulation usually cause alterations in different organs or tissues (Rose, 1982). This bears the risk of disturbing finely tuned gene regulatory networks crucial for proper development of the individual. Providing new genetic material by gene duplications can facilitate evolution of novel traits, because the gene duplicates are initially redundant and therefore are free to evolve new functions (Innan and Kondrashov, 2010; Kondrashov et al., 2002; Ohno, 1970). In vertebrates extensive gene and genome duplication are in the focus of research for many years (Baskaran et al., 2015; Holland, 2013; Holland et al., 1994; Kasahara, 2013; Marlétaz et al., 2015). Although arthropods are the phylum with the largest species number displaying an enormous morphological diversity, only few examples of gene duplications are known from this phylum. Gene duplications similar in magnitude to those observed in vertebrates are so far only known in the clade of the chelicerates (Di et al., 2015; Nossa et al., 2014; Sharma et al., 2014). These gene duplications have been linked to the evolution of the repertoire of silk and venom genes in spiders (Haney et al., 2016), and to specific sensory organs in scorpions.

A general course after duplication of genes is loss, sub- or neofunctionalisation (Conant and Wolfe, 2008). The study of gene paralogs during my study showed that paralogs within one species were usually less similar than the orthologs between different spiders. Most intensively studied were the duplications of the leg gap genes and their expression and function in the walking legs. Also this study clearly shows that one gene homolog kept the original gene function. The paralog, however, was free to differentiate in expression and function after the duplication event. Most of the studied genes were again conserved after neo- or subfunctionalizaton due to the importance of their new function. hth2 in contrast showed differing expression patterns in the walking legs of all four spider studied so far. Thus also differences in the conservation of duplicated genes might represent a playground for the evolution to develop novel traits. For all duplicated genes of the two spiders we found that the duplication event evolved independently from the duplication events in the vertebrates. In addition, the phylogenetic analyses revealed that the timing of gene duplications in the spiders differs between the studied genes, indicating that they were duplicated in several waves, instead of a single genome 
duplication. Due to the technical advances in sequencing technology, the number of available transcriptomes and genomes is increasing steadily and provides the basis for more detailed studies of duplication events in the group of chelicerates. However, gene predictions by automatic annotation based on blast searches often lead to wrongly predicted genes and detailed phylogenetic analysis are needed to clearly identify genuine homologs. The duplication of the dachshund gene was found to be older than the split between scorpions and spiders and is linked with the evolution of a novel leg segment, the patella. The duplication of $h$ th in contrast was found to be spider specific, which is astonishing because of the diversified expression of $h t h 2$. The protein sequences after duplication of exd, however, were shown to be so similar that they caused a polytomy even after intensive phylogenetic studies. These findings show that also the time-point of the duplication might be as diverse as the conservation and the use of the duplicates.

In summary our data show how important gene duplications are in terms of evolving diversity of morphologic traits and that it is of significant importance to study more duplication events in more depth to gain insight into how they might have been used in the evolution of diversification. In addition, this shows that the astonishing diversity in the animal kingdom presumably is caused by a similar diverse use of molecular diversity rather than by simple changes in the protein coding regions or on the regulatory level. 


\section{References}

Abu-Shaar, M., and Mann, R.S. (1998). Generation of multiple antagonistic domains along the proximodistal axis during Drosophila leg development. Development 125, 38213830 .

Adams, M.D., Celniker, S.E., Holt, R.A., Evans, C.A., Gocayne, J.D., Amanatides, P.G., Scherer, S.E., Li, P.W., Hoskins, R.A., Galle, R.F., et al. (2000). The Genome Sequence of Drosophila melanogaster. Science 287, 2185-2195.

Akiyama-Oda, Y. (2003). Early patterning of the spider embryo: a cluster of mesenchymal cells at the cumulus produces Dpp signals received by germ disc epithelial cells. Development 130, 1735-1747.

Anders, S., and Huber, W. (2010). Differential expression analysis for sequence count data. Genome Biol. 11, R106.

Angelini, D.R., and Kaufman, T.C. (2005). Insect appendages and comparative ontogenetics. Dev. Biol. 286, 57-77.

Angelini, D.R., Liu, P.Z., Hughes, C.L., and Kaufman, T.C. (2005). Hox gene function and interaction in the milkweed bug Oncopeltus fasciatus (Hemiptera). Dev. Biol. 287, 440455.

Angelini, D.R., Kikuchi, M., and Jockusch, E.L. (2009). Genetic patterning in the adult capitate antenna of the beetle Tribolium castaneum. Dev. Biol. 327, 240-251.

Angelini, D.R., Smith, F.W., and Jockusch, E.L. (2012). Extent With Modification: Leg Patterning in the Beetle Tribolium castaneum and the Evolution of Serial Homologs. Genes|Genomes|Genetics 2, 235-248.

Armisén, D., Nagui Refki, P., Crumière, A.J.J., Viala, S., Toubiana, W., and Khila, A. (2015). Predator strike shapes antipredator phenotype through new genetic interactions in water striders. Nat. Commun. 6, 8153.

Baanannou, A., Mojica-Vazquez, L.H., Darras, G., Couderc, J.-L., Cribbs, D.L., Boube, M., and Bourbon, H.-M. (2013). Drosophila Distal-less and Rotund Bind a Single Enhancer Ensuring Reliable and Robust bric-a-brac2 Expression in Distinct Limb Morphogenetic Fields. PLoS Genet. 9, e1003581.

Bairoch, A., and Apweiler, R. (2000). The SWISS-PROT protein sequence database and its supplement TrEMBL in 2000. Nucleic Acids Res. 28, 45-48.

Barnett, A.A., and Thomas, R.H. (2013). The expression of limb gap genes in the mite Archegozetes longisetosus reveals differential patterning mechanisms in chelicerates: 
Archegozetes longisetosus reveals differential patterning mechanisms in chelicerates. Evol. Dev. 15, 280-292.

Baskaran, P., Rödelsperger, C., Prabh, N., Serobyan, V., Markov, G.V., Hirsekorn, A., and Dieterich, C. (2015). Ancient gene duplications have shaped developmental stage-specific expression in Pristionchus pacificus. BMC Evol. Biol. 15, 185.

Beermann, A., and Schröder, R. (2004). Functional stability of the aristaless gene in appendage tip formation during evolution. Dev. Genes Evol. 214.

Bell, S.M., Schreiner, C.M., Waclaw, R.R., Campbell, K., Potter, S.S., and Scott, W.J. (2003). Sp8 is crucial for limb outgrowth and neuropore closure. Proc. Natl. Acad. Sci. 100, 12195-12200.

Bennett, R.L., Brown, S.J., and Denell, R.E. (1999). Molecular and genetic analysis of the Tribolium Ultrabithorax ortholog, Ultrathorax. Dev. Genes Evol. 209, 608-619.

Berthelsen, J., Kilstrup-Nielsen, C., Blasi, F., Mavilio, F., and Zappavigna, V. (1999). The subcellular localization of PBX1 and EXD proteins depends on nuclear import and export signals and is modulated by association with PREP1 and HTH. Genes Dev. 13, 946-953.

Bishop, S.A., Klein, T., Arias, A.M., and Couso, J.P. (1999). Composite signalling from Serrate and Delta establishes leg segments in Drosophila through Notch. Development 126, 2993-3003.

Bitsch, J. (2001). The hexapod appendage : Basic structure, development and origin. In Annales de La Société Entomologique de France, (Société entomologique de France), 37, 175-193.

Bolger, A.M., Lohse, M., and Usadel, B. (2014). Trimmomatic: a flexible trimmer for Illumina sequence data. Bioinforma. Oxf. Engl. 30, 2114-2120.

Bolker, J.A. (1995). Model systems in developmental biology. BioEssays 17, 451-455.

Borner, J., Rehm, P., Schill, R.0., Ebersberger, I., and Burmester, T. (2014). A transcriptome approach to ecdysozoan phylogeny. Mol. Phylogenet. Evol. 80, 79-87.

Bourgouin, C., Lundgren, S.E., and Thomas, J.B. (1992). Apterous is a Drosophila LIM domain gene required for the development of a subset of embryonic muscles. Neuron 9 , 549-561.

Bowser, J., and Reddy, A. s. n. (1997). Localization of a kinesin-like calmodulin-binding protein in dividing cells of Arabidopsis and tobacco. Plant J. 12, 1429-1437.

Bryantsev, A.L., Duong, S., Brunetti, T.M., Chechenova, M.B., Lovato, T.L., Nelson, C., Shaw, E., Uhl, J.D., Gebelein, B., and Cripps, R.M. (2012). Extradenticle and Homothorax Control Adult Muscle Fiber Identity in Drosophila. Dev. Cell 23, 664-673. 
Campbell, G. (2005). Regulation of gene expression in the distal region of the Drosophila leg by the Hox11 homolog, C15. Dev. Biol. 278, 607-618.

Campbell, G., and Tomlinson, A. (1998). The roles of the homeobox genes aristaless and Distal-less in patterning the legs and wings of Drosophila. Development 125, 4483-4493.

Cande, J.D., Chopra, V.S., and Levine, M. (2009). Evolving enhancer-promoter interactions within the tinman complex of the flour beetle, Tribolium castaneum. Development 136, 3153-3160.

Caravas, J., and Friedrich, M. (2010). Of mites and millipedes: Recent progress in resolving the base of the arthropod tree. BioEssays 32, 488-495.

Casares, F., and Mann, R.S. (2001). The Ground State of the Ventral Appendage in Drosophila. Science 293, 1477-1480.

Celá, P., Balková, S.M., Bryjová, A., Horáková, D., Míšek, I., Richman, J.M., and Buchtová, M. (2013). Expression, function and regulation of Evi-1 during embryonic avian development. Gene Expr. Patterns 13, 343-353.

Celis, J.F. de, Tyler, D.M., Celis, J. de, and Bray, S.J. (1998). Notch signalling mediates segmentation of the Drosophila leg. Development 125, 4617-4626.

Chung, S., Chavez, C., and Andrew, D.J. (2011). Trachealess (Trh) regulates all tracheal genes during Drosophila embryogenesis. Dev. Biol. 360, 160-172.

Claparède, R. (1862). Recherches sur l'évolution des araignées. (Natuurkundige Verhandelingen uuitgegeven door het Provinciaal Utrechtsch Genootschap von Kunsten en Wetenschappen. Deel I. Stuk 1. C. van der Post Jr., Utrecht.).

Cohen, S.M. (1993). Imaginal disc development. In The Development of Drosophila Melanogaster 2, pp. 747-841.

Cohen, S.M., and Jürgens, G. (1989). Proximal-distal pattern formation in Drosophila: cell autonomous requirement for Distal-less gene activity in limb development. EMBO J. 8, 2045-2055.

Cohen, B., McGuffin, M.E., Pfeifle, C., Segal, D., and Cohen, S.M. (1992). apterous, a gene required for imaginal disc development in Drosophila encodes a member of the LIM family of developmental regulatory proteins. Genes Dev. 6, 715-729.

Cohen, B., Simcox, A.A., and Cohen, S.M. (1993). Allocation of the thoracic imaginal primordia in the Drosophila embryo. Development 117, 597-608.

Cohen, S.M., Brönner, G., Küttner, F., Jürgens, G., and Jäckle, H. (1989). Distal-less encodes a homoeodomain protein required for limb development in Drosophila. Nature 338, 432434. 
Conant, G.C., and Wolfe, K.H. (2008). Turning a hobby into a job: how duplicated genes find new functions. Nat. Rev. Genet. 9, 938-950.

Conesa, A., Götz, S., García-Gómez, J.M., Terol, J., Talón, M., and Robles, M. (2005). Blast2GO: a universal tool for annotation, visualization and analysis in functional genomics research. Bioinformatics 21, 3674-3676.

Consortium, T.U. (2015). UniProt: a hub for protein information. Nucleic Acids Res. 43, D204-D212.

Córdoba, S., and Estella, C. (2014). The bHLH-PAS Transcription Factor Dysfusion Regulates Tarsal Joint Formation in Response to Notch Activity during Drosophila Leg Development. PLoS Genet. 10, e1004621.

Crews, S.T. (1998). Control of cell lineage-specific development and transcription by bHLH-PAS proteins. Genes Dev. 12, 607-620.

Crews, S.T., and Fan, C.-M. (1999). Remembrance of things PAS: regulation of development by bHLH-PAS proteins. Curr. Opin. Genet. Dev. 9, 580-587.

Dai, M., Wang, Y., Fang, L., Irwin, D.M., Zhu, T., Zhang, J., Zhang, S., and Wang, Z. (2014). Differential Expression of Meis2, Mab21l2 and Tbx3 during Limb Development Associated with Diversification of Limb Morphology in Mammals. PLoS ONE 9, e106100.

Damen, W.G.M. (2007). Evolutionary conservation and divergence of the segmentation process in arthropods. Dev. Dyn. 236, 1379-1391.

Damen, W.G., Saridaki, T., and Averof, M. (2002). Diverse adaptations of an ancestral gill: a common evolutionary origin for wings, breathing organs, and spinnerets. Curr. Biol. 12, 1711-1716.

Damen, W.G., Janssen, R., and Prpic, N.-M. (2005). Pair rule gene orthologs in spider segmentation. Evol. Dev. 7, 618-628.

Davidson, N.M., and Oshlack, A. (2014). Corset: enabling differential gene expression analysis for de novo assembled transcriptomes. Genome Biol. 15, 410.

Dear, T.N., and Rabbitts, T.H. (1994). A Drosophila melanogaster homologue of the T-cell oncogene HOX11 localises to a cluster of homeobox genes. Gene 141, 225-229.

Dear, T.N., Sanchez-Garcia, I., and Rabbitts, T.H. (1993). The HOX11 gene encodes a DNAbinding nuclear transcription factor belonging to a distinct family of homeobox genes. Proc. Natl. Acad. Sci. U. S. A. 90, 4431-4435.

Di, Z., Yu, Y., Wu, Y., Hao, P., He, Y., Zhao, H., Li, Y., Zhao, G., Li, X., Li, W., et al. (2015). Genome-wide analysis of homeobox genes from Mesobuthus martensii reveals Hox gene duplication in scorpions. Insect Biochem. Mol. Biol. 61, 25-33. 
Diogo, R. (2015). Where is the evo in Evo-Devo (evolutionary developmental biology)? J. Exp. Zoolog. B Mol. Dev. Evol. n/a - n/a.

Doeffinger, C., Hartenstein, V., and Stollewerk, A. (2010). Compartmentalisation of the precheliceral neuroectoderm in the spider Cupiennius salei : Development of the arcuate body, the optic ganglia and the mushroom body. J. Comp. Neurol. 518, 2612-2632.

Dong, P.D.S., Dicks, J.S., and Panganiban, G. (2002). Distal-less and homothorax regulate multiple targets to pattern the Drosophila antenna. Development 129, 1967-1974.

Dong, Y., Dai, F., Ren, Y., Liu, H., Chen, L., Yang, P., Liu, Y., Li, X., Wang, W., and Xiang, H. (2015). Comparative transcriptome analyses on silk glands of six silkmoths imply the genetic basis of silk structure and coloration. BMC Genomics 16.

Dymek, E.E., Goduti, D., Kramer, T., and Smith, E.F. (2006). A kinesin-like calmodulinbinding protein in Chlamydomonas: evidence for a role in cell division and flagellar functions. J. Cell Sci. 119, 3107-3116.

Edgecombe, G.D. (2010). Arthropod phylogeny: An overview from the perspectives of morphology, molecular data and the fossil record. Arthropod Struct. Dev. 39, 74-87.

Emmons, R.B., Duncan, D., Estes, P.A., Kiefel, P., Mosher, J.T., Sonnenfeld, M., Ward, M.P., Duncan, I., and Crews, S.T. (1999). The spineless-aristapedia and tango bHLH-PAS proteins interact to control antennal and tarsal development in Drosophila. Dev. Camb. Engl. 126, 3937-3945.

Estella, C., and Mann, R.S. (2008). Logic of Wg and Dpp induction of distal and medial fates in the Drosophila leg. Development 135, 627-636.

Estella, C., and Mann, R.S. (2010). Non-Redundant Selector and Growth-Promoting Functions of Two Sister Genes, buttonhead and Sp1, in Drosophila Leg Development. PLoS Genet. 6, e1001001.

Estella, C., Rieckhof, G., Calleja, M., and Morata, G. (2003). The role of buttonhead and Sp1 in the development of the ventral imaginal discs of Drosophila. Development 130, 59295941.

Estella, C., Voutev, R., and Mann, R.S. (2012). A Dynamic Network of Morphogens and Transcription Factors Patterns the Fly Leg. Curr. Top. Dev. Biol. 98, 173-198.

Foelix, R.F. (1996). Biology of spiders (Oxford: Oxford University Press.).

Franch-Marro, X., Martín, N., Averof, M., and Casanova, J. (2006). Association of tracheal placodes with leg primordia in Drosophila and implications for the origin of insect tracheal systems. Dev. Camb. Engl. 133, 785-790. 
Friedrich, M., and Tautz, D. (1995). Ribosomal DNA phylogeny of the major extant arthropod classes and the evolution of myriapods. Nature 376, 165-167.

Fristrom, D.F.A.J.W., and Fristrom, J.W. (1993). The metamorphic development of the adult epidermis. In The Development of Drosophila Melanogaster 2, pp. 843-897.

Galindo, M.I. (2002). Leg Patterning Driven by Proximal-Distal Interactions and EGFR Signaling. Science 297, 256-259.

Galindo, M. i., Bishop, S. a., and Couso, J. p. (2005). Dynamic EGFR-Ras signalling in Drosophila leg development. Dev. Dyn. 233, 1496-1508.

Gentleman, R.C., Carey, V.J., Bates, D.M., Bolstad, B., Dettling, M., Dudoit, S., Ellis, B., Gautier, L., Ge, Y., Gentry, J., et al. (2004). Bioconductor: open software development for computational biology and bioinformatics. Genome Biol. 5, R80.

Gilad, Y., Pritchard, J.K., and Thornton, K. (2009). Characterizing natural variation using next-generation sequencing technologies. Trends Genet. 25, 463-471.

Giorgianni, M.W., and Mann, R.S. (2011). Establishment of Medial Fates along the Proximodistal Axis of the Drosophila Leg through Direct Activation of dachshund by Distalless. Dev. Cell 20, 455-468.

Giribet, G., Edgecombe, G.D., and Wheeler, W.C. (2001). Arthropod phylogeny based on eight molecular loci and morphology. Nature 413, 157-161.

Godt, D., Couderc, J.L., Cramton, S.E., and Laski, F.A. (1993). Pattern formation in the limbs of Drosophila: bric a brac is expressed in both a gradient and a wave-like pattern and is required for specification and proper segmentation of the tarsus. Development $119,799-812$.

Gonzalez-Crespo, S., and Morata, G. (1995). Control of Drosophila adult pattern by extradenticle. Development 121, 2117-2125.

Goto, S., and Hayashi, S. (1997). Specification of the embryonic limb primordium by graded activity of Decapentaplegic. Development 124, 125-132.

Goyama, S., Yamamoto, G., Shimabe, M., Sato, T., Ichikawa, M., Ogawa, S., Chiba, S., and Kurokawa, M. (2008). Evi-1 Is a Critical Regulator for Hematopoietic Stem Cells and Transformed Leukemic Cells. Cell Stem Cell 3, 207-220.

Grossmann, D., and Prpic, N.-M. (2012). Egfr signaling regulates distal as well as medial fate in the embryonic leg of Tribolium castaneum. Dev. Biol. 370, 264-272.

Haas, B.J., Papanicolaou, A., Yassour, M., Grabherr, M., Blood, P.D., Bowden, J., Couger, M.B., Eccles, D., Li, B., Lieber, M., et al. (2013). De novo transcript sequence 
reconstruction from RNA-seq using the Trinity platform for reference generation and analysis. Nat. Protoc. 8, 1494-1512.

Haney, R.A., Clarke, T.H., Gadgil, R., Fitzpatrick, R., Hayashi, C.Y., Ayoub, N.A., and Garb, J.E. (2016). Effects of gene duplication, positive selection and shifts in gene expression on the evolution of the venom gland transcriptome in widow spiders. Genome Biol. Evol. evv253.

Hao, I., Green, R.B., Dunaevsky, O., Lengyel, J.A., and Rauskolb, C. (2003). The odd-skipped family of zinc finger genes promotes Drosophila leg segmentation. Dev. Biol. 263, 282295.

Harding, K., Rushlow, C., Doyle, H.J., Hoey, T., and Levine, M. (1986). Cross-regulatory interactions among pair-rule genes in Drosophila. Science 233, 953-959.

Hasegawa, E., Kitada, Y., Kaido, M., Takayama, R., Awasaki, T., Tabata, T., and Sato, M. (2011). Concentric zones, cell migration and neuronal circuits in the Drosophila visual center. Development 138, 983-993.

Hilbrant, M., Damen, W.G.M., and McGregor, A.P. (2012). Evolutionary crossroads in developmental biology: the spider Parasteatoda tepidariorum. Development 139, 26552662.

Hinai, A.A., and Valk, P.J.M. (2016). Review: Aberrant EVI1 expression in acute myeloid leukaemia. Br. J. Haematol. n/a - n/a.

Hirai, H. (1999). The transcription factor Evi-1. Int. J. Biochem. Cell Biol. 31, 1367-1371.

Holland, L.Z. (2013). Evolution of new characters after whole genome duplications: insights from amphioxus. Semin. Cell Dev. Biol. 24, 101-109.

Holland, P.W., Garcia-Fernàndez, J., Williams, N.A., and Sidow, A. (1994). Gene duplications and the origins of vertebrate development. Development 1994, 125-133.

Holm, Å. (1940). Studien über die Entwicklung und Entwicklungsbiologie der Spinnen. Zoologiska Bidrag från Uppsala 19, 1-214. Plates 1-11.

Huang, X., Powell-Coffman, J.A., and Jin, Y. (2004). The AHR-1 aryl hydrocarbon receptor and its co-factor the AHA-1 aryl hydrocarbon receptor nuclear translocator specify GABAergic neuron cell fate in C. elegans. Development 131, 819-828.

Hughes, C.L., and Kaufman, T.C. (2002a). Exploring the myriapod body plan: expression patterns of the ten Hox genes in a centipede. Development 129, 1225-1238.

Hughes, C.L., and Kaufman, T.C. (2002b). Hox genes and the evolution of the arthropod body plan1. Evol. Dev. 4, 459-499. 
Innan, H., and Kondrashov, F. (2010). The evolution of gene duplications: classifying and distinguishing between models. Nat. Rev. Genet. 11, 4.

Isaac, D.D., and Andrew, D.J. (1996). Tubulogenesis in Drosophila: a requirement for the trachealess gene product. Genes Dev. 10, 103-117.

Jacobs, Y., Schnabel, C.A., and Cleary, M.L. (1999). Trimeric Association of Hox and TALE Homeodomain Proteins Mediates Hoxb2 Hindbrain Enhancer Activity. Mol. Cell. Biol. 19, 5134-5142.

Janssen, R., Eriksson, B.J., Budd, G.E., Akam, M., and Prpic, N.-M. (2010). Gene expression patterns in an onychophoran reveal that regionalization predates limb segmentation in pan-arthropods: Appendage patterning in onychophorans. Evol. Dev. 12, 363-372.

Janssen, R., Schönauer, A., Weber, M., Turetzek, N., Hogvall, M., Goss, G.E., Patel, N.H., McGregor, A.P., and Hilbrant, M. (2015). The evolution and expression of panarthropod frizzled genes. Evol. Dev. Biol. 3, 96.

Jaw, T.J., You, L.-R., Knoepfler, P.S., Yao, L.-C., Pai, C.-Y., Tang, C.-Y., Chang, L.-P., Berthelsen, J., Blasi, F., Kamps, M.P., et al. (2000). Direct interaction of two homeoproteins, Homothorax and Extradenticle, is essential for EXD nuclear localization and function. Mech. Dev. 91, 279-291.

Johnston, D.S., and Nüsslein-Volhard, C. (1992). The origin of pattern and polarity in the Drosophila embryo. Cell 68, 201-219.

Kasahara, M. (2013). Impact of whole-genome duplication on vertebrate development and evolution. Semin. Cell Dev. Biol. 24, 81-82.

Kawakami, Y., Esteban, C.R., Matsui, T., Rodríguez-León, J., Kato, S., and Belmonte, J.C.I. (2004). Sp8 and Sp9, two closely related buttonhead-like transcription factors, regulate Fgf8 expression and limb outgrowth in vertebrate embryos. Development 131, 47634774 .

Kensley, and Schotte (1989). GT MARINE ISOPOD CRUSTACEA (Smithsonian Inst Press).

Khadjeh, S., Turetzek, N., Pechmann, M., Schwager, E.E., Wimmer, E.A., Damen, W.G., and Prpic, N.-M. (2012). Divergent role of the Hox gene Antennapedia in spiders is responsible for the convergent evolution of abdominal limb repression. Proc. Natl. Acad. Sci. 109, 4921-4926.

Kim, M.D., Jan, L.Y., and Jan, Y.N. (2006). The bHLH-PAS protein Spineless is necessary for the diversification of dendrite morphology of Drosophila dendritic arborization neurons. Genes Dev. 20, 2806-2819. 
Kim, S.-N., Jung, K.I., Chung, H.-M., Kim, S.H., and Jeon, S.-H. (2008). The pleiohomeotic gene is required for maintaining expression of genes functioning in ventral appendage formation in Drosophila melanogaster. Dev. Biol. 319, 121-129.

Kojima, T. (2004). The mechanism of Drosophila leg development along the proximodistal axis. Dev. Growth Differ. 46, 115-129.

Kojima, T., Ishimaru, S., Higashijima, S., Takayama, E., Akimaru, H., Sone, M., Emori, Y., and Saigo, K. (1991). Identification of a different-type homeobox gene, BarH1, possibly causing Bar (B) and Om(1D) mutations in Drosophila. Proc. Natl. Acad. Sci. U. S. A. 88, 4343-4347.

Kojima, T., Sato, M., and Saigo, K. (2000). Formation and specification of distal leg segments in Drosophila by dual Bar homeobox genes, BarH1 and BarH2. Development 127, 769-778.

Kojima, T., Tsuji, T., and Saigo, K. (2005). A concerted action of a paired-type homeobox gene, aristaless, and a homolog of Hox11/tlx homeobox gene, clawless, is essential for the distal tip development of the Drosophila leg. Dev. Biol. 279, 434-445.

Konantz, M., Grzywna, S., Pereboom, T.C., Carroll, K.J., Esain, V., Kanz, L., North, T.E., and Lengerke, C. (2014). Multiple Roles for the Zebrafish Homologue of the Murine Evi1 Gene during Primitive Myelopoiesis and HSC Development. Blood 124, 2901-2901.

Konantz, M., Müller, J., Lenard, A., Esain, V., North, T.E., and Lengerke, C. (2015). The zebrafish homologue of the murine EVI1 gene critically regulates hsc development. Exp. Hematol. 43, S74.

Kondrashov, F.A., Rogozin, I.B., Wolf, Y.I., and Koonin, E.V. (2002). Selection in the evolution of gene duplications. Genome Biol 3, 8-1.

Kozu, S., Tajiri, R., Tsuji, T., Michiue, T., Saigo, K., and Kojima, T. (2006). Temporal regulation of late expression of Bar homeobox genes during Drosophila leg development by Spineless, a homolog of the mammalian dioxin receptor. Dev. Biol. 294, 497-508.

Kubota, K., Goto, S., Eto, K., and Hayashi, S. (2000). EGF receptor attenuates Dpp signaling and helps to distinguish the wing and leg cell fates in Drosophila. Development 127, 3769-3776.

Kuzin, B.A., Nikitina, E.A., Cherezov, R.O., Vorontsova, J.E., Slezinger, M.S., Zatsepina, O.G., Simonova, O.B., Enikolopov, G.N., and Savvateeva-Popova, E.V. (2014). Combination of Hypomorphic Mutations of the Drosophila Homologues of Aryl Hydrocarbon Receptor and Nucleosome Assembly Protein Family Genes Disrupts Morphogenesis, Memory and Detoxification. PLoS ONE 9, e94975.

Langmead, B., and Salzberg, S.L. (2012). Fast gapped-read alignment with Bowtie 2. Nat. Methods 9, 357-359. 
Lecuit, T., and Cohen, S.M. (1997). Proximal-distal axis formation in the Drosophila leg. Nature 388, 139-145.

Li, H., Handsaker, B., Wysoker, A., Fennell, T., Ruan, J., Homer, N., Marth, G., Abecasis, G., Durbin, R., and Subgroup, 1000 Genome Project Data Processing (2009). The Sequence Alignment/Map format and SAMtools. Bioinformatics 25, 2078-2079.

Li, Y., Cheng, C.N., Verdun, V.A., and Wingert, R.A. (2014). Zebrafish nephrogenesis is regulated by interactions between retinoic acid, mecom, and Notch signaling. Dev. Biol. $386,111-122$.

Lickteig, K.M., Duerr, J.S., Frisby, D.L., Hall, D.H., Rand, J.B., and Miller, D.M. (2001). Regulation of Neurotransmitter Vesicles by the Homeodomain Protein UNC-4 and Its Transcriptional Corepressor UNC-37/Groucho inCaenorhabditis elegans Cholinergic Motor Neurons. J. Neurosci. 21, 2001-2014.

Lilly, B., O'Keefe, D.D., Thomas, J.B., and Botas, J. (1999). The LIM homeodomain protein dLim1 defines a subclass of neurons within the embryonic ventral nerve cord of Drosophila. Mech. Dev. 88, 195-205.

Liu, T., Yu, L., Liu, L., Li, H., Li, Y., Liu, T., Yu, L., Liu, L., Li, H., and Li, Y. (2015). Comparative Transcriptomes and EVO-DEVO Studies Depending on Next Generation Sequencing, Comparative Transcriptomes and EVO-DEVO Studies Depending on Next Generation Sequencing. Comput. Math. Methods Med. Comput. Math. Methods Med. 2015, 2015, e896176.

Mann, R.S., and Affolter, M. (1998). Hox proteins meet more partners. Curr. Opin. Genet. Dev. 8, 423-429.

Mardon, G., Solomon, N.M., and Rubin, G.M. (1994). dachshund encodes a nuclear protein required for normal eye and leg development in Drosophila. Development 120, 34733486.

Marlétaz, F., Maeso, I., Faas, L., Isaacs, H.V., and Holland, P.W. (2015). Cdx ParaHox genes acquired distinct developmental roles after gene duplication in vertebrate evolution. BMC Biol. 13, 56.

McGinnis, W., and Krumlauf, R. (1992). Homeobox genes and axial patterning. Cell 68, 283-302.

McGregor, A.P., Hilbrant, M., Pechmann, M., Schwager, E.E., Prpic, N.-M., and Damen, W.G.M. (2008). Cupiennius salei andAchaearanea tepidariorum: Spider models for investigating evolution and development. BioEssays 30, 487-498.

McKay, D.J., Estella, C., and Mann, R.S. (2009). The origins of the Drosophila leg revealed by the cis-regulatory architecture of the Distalless gene. Development 136, 61-71. 
Miller, D.M., Shen, M.M., Shamu, C.E., Bürglin, T.R., Ruvkun, G., Dubois, M.L., Ghee, M., and Wilson, L. (1992). C. elegans unc-4 gene encodes a homeodomain protein that determines the pattern of synaptic input to specific motor neurons. Nature 355, 841845.

Mitchell, B., and Crews, S.T. (2002). Expression of the Artemia trachealess gene in the salt gland and epipod. Evol. Dev. 4, 344-353.

Mito, T., Shinmyo, Y., Kurita, K., Nakamura, T., Ohuchi, H., and Noji, S. (2011). Ancestral functions of Delta/Notch signaling in the formation of body and leg segments in the cricket Gryllus bimaculatus. Development 138, 3823-3833.

Mittmann, B., and Wolff, C. (2012). Embryonic development and staging of the cobweb spider Parasteatoda tepidariorum C. L. Koch, 1841 (syn.: Achaearanea tepidariorum; Araneomorphae; Theridiidae). Dev. Genes Evol. 222, 189-216.

Miyawaki, K., Inoue, Y., Mito, T., Fujimoto, T., Matsushima, K., Shinmyo, Y., Ohuchi, H., and Noji, S. (2002). Expression patterns of aristaless in developing appendages of Gryllus bimaculatus (cricket). Mech. Dev. 113, 181-184.

Miyazono, K., Zhi, Y., Takamura, Y., Nagata, K., Saigo, K., Kojima, T., and Tanokura, M. (2010). Cooperative DNA-binding and sequence-recognition mechanism of aristaless and clawless. EMBO J. 29, 1613-1623.

Mucenski, M.L., Taylor, B.A., Ihle, J.N., Hartley, J.W., Morse, H.C., Jenkins, N.A., and Copeland, N.G. (1988). Identification of a common ecotropic viral integration site, Evi-1, in the DNA of AKXD murine myeloid tumors. Mol. Cell. Biol. 8, 301-308.

Nakamura, T., Mito, T., Miyawaki, K., Ohuchi, H., and Noji, S. (2008). EGFR signaling is required for re-establishing the proximodistal axis during distal leg regeneration in the cricket Gryllus bimaculatus nymph. Dev. Biol. 319, 46-55.

Neuwirth, E. (2011). RColorBrewer: ColorBrewer palettes. R package version 1.5.

Nossa, C.W., Havlak, P., Yue, J.-X., Lv, J., Vincent, K.Y., Brockmann, H.J., and Putnam, N.H. (2014). Joint assembly and genetic mapping of the Atlantic horseshoe crab genome reveals ancient whole genome duplication. GigaScience 3, 9.

Ohno, S. (1970). Evolution by Gene Duplication. (New York, Heidelberg, Berlin: SpringerVerlag.).

Oliveira, M.B., Liedholm, S.E., Lopez, J.E., Lochte, A.A., Pazio, M., Martin, J.P., Mörch, P.R., Salakka, S., York, J., Yoshimoto, A., et al. (2014). Expression of arthropod distal limbpatterning genes in the onychophoran Euperipatoides kanangrensis. Dev. Genes Evol. 224, 87-96. 
Oppenheim, S.J., Baker, R.H., Simon, S., and DeSalle, R. (2015). We can't all be supermodels: the value of comparative transcriptomics to the study of non-model insects. Insect Mol. Biol. 24, 139-154.

Pai, C.-Y., Kuo, T.-S., Jaw, T.J., Kurant, E., Chen, C.-T., Bessarab, D.A., Salzberg, A., and Sun, Y.H. (1998). The Homothorax homeoprotein activates the nuclear localization of another homeoprotein, Extradenticle, and suppresses eye development in Drosophila. Genes Dev. $12,435-446$.

Panganiban, G., Irvine, S.M., Lowe, C., Roehl, H., Corley, L.S., Sherbon, B., Grenier, J.K., Fallon, J.F., Kimble, J., Walker, M., et al. (1997). The origin and evolution of animal appendages. Proc. Natl. Acad. Sci. 94, 5162-5166.

Pantalacci, S., and Sémon, M. (2014). Transcriptomics of developing embryos and organs: A raising tool for evo-devo. J. Exp. Zoolog. B Mol. Dev. Evol. 00B, 1-9.

Pechmann, M. (2011). Comparative studies of appendage and segment formation in arthropods. PhD Thesis. Georg-August-University Göttingen.

Pechmann, M., and Prpic, N.-M. (2009). Appendage patterning in the South American bird spider Acanthoscurria geniculata (Araneae: Mygalomorphae). Dev. Genes Evol. 219, 189198.

Pechmann, M., McGregor, A.P., Schwager, E.E., Feitosa, N.M., and Damen, W.G.M. (2009). Dynamic gene expression is required for anterior regionalization in a spider. Proc. Natl. Acad. Sci. U. S. A. 106, 1468-1472.

Pechmann, M., Khadjeh, S., Sprenger, F., and Prpic, N.-M. (2010). Patterning mechanisms and morphological diversity of spider appendages and their importance for spider evolution. Arthropod Struct. Dev. 39, 453-467.

Pechmann, M., Khadjeh, S., Turetzek, N., McGregor, A.P., Damen, W.G.M., and Prpic, N.-M. (2011). Novel Function of Distal-less as a Gap Gene during Spider Segmentation. PLoS Genet. 7, e1002342.

Peel, A.D., Chipman, A.D., and Akam, M. (2005). Arthropod Segmentation: beyond the Drosophila paradigm. Nat. Rev. Genet. 6, 905-916.

Perkins, A.S., Mercer, J.A., Jenkins, N.A., and Copeland, N.G. (1991). Patterns of Evi-1 expression in embryonic and adult tissues suggest that Evi-1 plays an important regulatory role in mouse development. Development 111, 479-487.

Posnien, N., Zeng, V., Schwager, E.E., Pechmann, M., Hilbrant, M., Keefe, J.D., Damen, W.G.M., Prpic, N.-M., McGregor, A.P., and Extavour, C.G. (2014). A Comprehensive Reference Transcriptome Resource for the Common House Spider Parasteatoda tepidariorum. PLoS ONE 9, e104885. 
Prpic, N.-M., and Damen, W.G.M. (2009). Notch-mediated segmentation of the appendages is a molecular phylotypic trait of the arthropods. Dev. Biol. 326, 262-271.

Prpic, N.-M., and Damen, W.M. (2004). Expression patterns of leg genes in the mouthparts of the spider Cupiennius salei (Chelicerata: Arachnida). Dev. Genes Evol. 214, 296-302.

Prpic, N.M.P., and Damen, W.G.M. (2008). Arthropod appendages: a prime example for the evolution of morphological diversity and innovation. In Evolving Pathways: Key Themes in Evolutionary Developmental Biology., (Cambridge University Press, Cambridge), pp. 381-398.

Prpic, N.-M., and Tautz, D. (2003). The expression of the proximodistal axis patterning genes Distal-less and dachshund in the appendages of Glomeris marginata (Myriapoda: Diplopoda) suggests a special role of these genes in patterning the head appendages. Dev. Biol. 260, 97-112.

Prpic, N.-M., and Telford, M.J. (2008). Expression of homothorax and extradenticle mRNA in the legs of the crustacean Parhyale hawaiensis: evidence for a reversal of gene expression regulation in the pancrustacean lineage. Dev. Genes Evol. 218, 333-339.

Prpic, N.-M., Wigand, B., Damen, W., and Klingler, M. (2001). Expression of dachshund in wild-type and Distal-less mutant Tribolium corroborates serial homologies in insect appendages. Dev. Genes Evol. 211, 467-477.

Prpic, N.-M., Janssen, R., Wigand, B., Klingler, M., and Damen, W.G.. (2003). Gene expression in spider appendages reveals reversal of exd/hth spatial specificity, altered leg gap gene dynamics, and suggests divergent distal morphogen signaling. Dev. Biol. $264,119-140$.

Prpic, N.-M., Schoppmeier, M., and Damen, W.G.M. (2008a). Whole-mount in situ hybridization of spider embryos. CSH Protoc. 2008, pdb.prot5068.

Prpic, N.-M., Schoppmeier, M., and Damen, W.G.M. (2008b). Collection and Fixation of Spider Embryos. Cold Spring Harb. Protoc. 2008, pdb.prot5067 - pdb.prot5067.

Pueyo, J.I. (2004). Chip-mediated partnerships of the homeodomain proteins Bar and Aristaless with the LIM-HOM proteins Apterous and Lim1 regulate distal leg development. Development 131, 3107-3120.

Pueyo, J.I., and Couso, J.P. (2008). The 11-aminoacid long Tarsal-less peptides trigger a cell signal in Drosophila leg development. Dev. Biol. 324, 192-201.

Pueyo, J.I., Galindo, M.I., Bishop, S.A., and Couso, J.P. (2000). Proximal-distal leg development in Drosophila requires the apterous gene and the Lim1 homologue dlim1. Development 127, 5391-5402. 
Rauskolb, C., and Irvine, K.D. (1999). Notch-Mediated Segmentation and Growth Control of the Drosophila Leg. Dev. Biol. 210, 339-350.

Rauskolb, C., Smith, K.M., Peifer, M., and Wieschaus, E. (1995). extradenticle determines segmental identities throughout Drosophila development. Development 121, 3663-3673.

Regier, J.C., Shultz, J.W., Zwick, A., Hussey, A., Ball, B., Wetzer, R., Martin, J.W., and Cunningham, C.W. (2010). Arthropod relationships revealed by phylogenomic analysis of nuclear protein-coding sequences. Nature 463, 1079-1083.

Reig, G., Cabrejos, M.E., and Concha, M.L. (2007). Functions of BarH transcription factors during embryonic development. Dev. Biol. 302, 367-375.

Rieckhof, G.E., Casares, F., Ryoo, H.D., Abu-Shaar, M., and Mann, R.S. (1997). Nuclear Translocation of Extradenticle Requires homothorax, which Encodes an ExtradenticleRelated Homeodomain Protein. Cell 91, 171-183.

Roberts, M.J. (1995). Collins Field guide SPIDERS of britian and Nothern europe (London: Harper Collins Publishers).

Ronquist, F., and Huelsenbeck, J.P. (2003). MrBayes 3: Bayesian phylogenetic inference under mixed models. Bioinformatics 19, 1572-1574.

Rose, M.R. (1982). Antagonistic pleiotropy, dominance, and genetic variation. Heredity $48,63-78$.

Rota-Stabelli, O., Campbell, L., Brinkmann, H., Edgecombe, G.D., Longhorn, S.J., Peterson, K.J., Pisani, D., Philippe, H., and Telford, M.J. (2011). A congruent solution to arthropod phylogeny: phylogenomics, microRNAs and morphology support monophyletic Mandibulata. Proc. R. Soc. Lond. B Biol. Sci. 278, 298-306.

Sánchez-Higueras, C., Sotillos, S., and Castelli-Gair Hombría, J. (2014). Common Origin of Insect Trachea and Endocrine Organs from a Segmentally Repeated Precursor. Curr. Biol. $24,76-81$.

Sanggaard, K.W., Bechsgaard, J.S., Fang, X., Duan, J., Dyrlund, T.F., Gupta, V., Jiang, X., Cheng, L., Fan, D., Feng, Y., et al. (2014). Spider genomes provide insight into composition and evolution of venom and silk. Nat. Commun. 5, 3765.

Schneuwly, S., Kuroiwa, A., and Gehring, W.J. (1987). Molecular analysis of the dominant homeotic Antennapedia phenotype. EMBO J. 6, 201-206.

Schomburg, C., Turetzek, N., Schacht, M.I., Schneider, J., Kirfel, P., Prpic, N.-M., and Posnien, N. (2015). Molecular characterization and embryonic origin of the eyes in the common house spider Parasteatoda tepidariorum. EvoDevo 6, 15. 
Schwager, E.E., Schoppmeier, M., Pechmann, M., and Damen, W.G. (2007). Duplicated Hox genes in the spider Cupiennius salei. Front. Zool. 4, 10.

Sharma, P.P., Schwager, E.E., Extavour, C.G., and Giribet, G. (2012). Evolution of the chelicera: a dachshund domain is retained in the deutocerebral appendage of Opiliones (Arthropoda, Chelicerata): Harvestman leg gap genes. Evol. Dev. 14, 522-533.

Sharma, P.P., Schwager, E.E., Extavour, C.G., and Wheeler, W.C. (2014). Hox gene duplications correlate with posterior heteronomy in scorpions. Proc. R. Soc. B Biol. Sci. 281, 20140661-20140661.

Sharma, P.P., Tarazona, O.A., Lopez, D.H., Schwager, E.E., Cohn, M.J., Wheeler, W.C., and Extavour, C.G. (2015). A conserved genetic mechanism specifies deutocerebral appendage identity in insects and arachnids. Proc. R. Soc. Lond. B Biol. Sci. 282, 20150698.

Sievers, F., Wilm, A., Dineen, D., Gibson, T.J., Karplus, K., Li, W., Lopez, R., McWilliam, H., Remmert, M., Söding, J., et al. (2011). Fast, scalable generation of high-quality protein multiple sequence alignments using Clustal Omega. Mol. Syst. Biol. 7, 539.

Simão, F.A., Waterhouse, R.M., Ioannidis, P., Kriventseva, E.V., and Zdobnov, E.M. (2015). BUSCO: assessing genome assembly and annotation completeness with single-copy orthologs. Bioinformatics 31, 3210-3212.

Smith, F.W., Angelini, D.R., Gaudio, M.S., and Jockusch, E.L. (2014). Metamorphic labral axis patterning in the beetle Tribolium castaneum requires multiple upstream, but few downstream, genes in the appendage patterning network: Labral metamorphosis in $T$. castaneum. Evol. Dev. 16, 78-91.

Sonnenfeld, M., Ward, M., Nystrom, G., Mosher, J., Stahl, S., and Crews, S. (1997). The Drosophila tango gene encodes a bHLH-PAS protein that is orthologous to mammalian Arnt and controls CNS midline and tracheal development. Dev. Camb. Engl. 124, 45714582 .

Stapley, J., Reger, J., Feulner, P.G.D., Smadja, C., Galindo, J., Ekblom, R., Bennison, C., Ball, A.D., Beckerman, A.P., and Slate, J. (2010). Adaptation genomics: the next generation. Trends Ecol. Evol. 25, 705-712.

Struhl, G. (1981). A homoeotic mutation transforming leg to antenna in Drosophila. Nature 292, 635-638.

Suzek, B.E., Huang, H., McGarvey, P., Mazumder, R., and Wu, C.H. (2007). UniRef: comprehensive and non-redundant UniProt reference clusters. Bioinforma. Oxf. Engl. 23, 1282-1288.

Tabuchi, K., Yoshikawa, S., Yuasa, Y., Sawamoto, K., and Okano, H. (1998). A novel Drosophila paired-like homeobox gene related to Caenorhabditis elegans unc-4 is 
expressed in subsets of postmitotic neurons and epidermal cells. Neurosci. Lett. 257, 4952.

Tajiri, R., Tsuji, T., Ueda, R., Saigo, K., and Kojima, T. (2007). Fate determination of Drosophila leg distal regions by trachealess and tango through repression and stimulation, respectively, of Bar homeobox gene expression in the future pretarsus and tarsus. Dev. Biol. 303, 461-473.

Tanaka, K., and Truman, J.W. (2007). Molecular patterning mechanism underlying metamorphosis of the thoracic leg in Manduca sexta. Dev. Biol. 305, 539-550.

Ting, C.-T., Tsaur, S.-C., Sun, S., Browne, W.E., Chen, Y.-C., Patel, N.H., and Wu, C.-I. (2004). Gene duplication and speciation in Drosophila: evidence from the Odysseus locus. Proc. Natl. Acad. Sci. U. S. A. 101, 12232-12235.

Tom, M., Manfrin, C., Chung, S.J., Sagi, A., Gerdol, M., Moro, G.D., Pallavicini, A., and Giulianini, P.G. (2014). Expression of cytoskeletal and molt-related genes is temporally scheduled in the hypodermis of the crayfish Procambarus clarkii during premolt. J. Exp. Biol. 217, 4193-4202.

Treichel, D., Schöck, F., Jäckle, H., Gruss, P., and Mansouri, A. (2003). mBtd is required to maintain signaling during murine limb development. Genes Dev. 17, 2630-2635.

Tsuji, T., Sato, A., Hiratani, I., Taira, M., Saigo, K., and Kojima, T. (2000). Requirements of Lim1, a Drosophila LIM-homeobox gene, for normal leg and antennal development. Development 127, 4315-4323.

Turetzek, N. (2011). On the role of hox genes and co-factors in body plan specification in spiders. Msc Thesis. Georg-August-University Göttingen.

Turetzek, N., Pechmann, M., Schomburg, C., Schneider, J., and Prpic, N.-M. (2016). Neofunctionalization of a Duplicate dachshund Gene Underlies the Evolution of a Novel Leg Segment in Arachnids. Mol. Biol. Evol. 33, 109-121.

Untergasser, A., Cutcutache, I., Koressaar, T., Ye, J., Faircloth, B.C., Remm, M., and Rozen, S.G. (2012). Primer3-new capabilities and interfaces. Nucleic Acids Res. 40, e115.

Wang, J., Kean, L., Yang, J., Allan, A.K., Davies, S.A., Herzyk, P., and Dow, J.A. (2004). Function-informed transcriptome analysis of Drosophila renal tubule. Genome Biol. 5, R69.

Whittington, H.B., and Almond, J.E. (1987). Appendages and Habits of the Upper Ordovician Trilobite Triarthrus eatoni. Philos. Trans. R. Soc. Lond. B Biol. Sci. 317, 1-46.

Wilkins, A. S. (2002). The evolution of developmental pathways. (Sunderland, Massachusetts, USA: Sinauer Associates Inc.). 
Winchell, C.J., and Jacobs, D.K. (2013). Expression of the Lhx genes apterous and lim 1 in an errant polychaete: implications for bilaterian appendage evolution, neural development, and muscle diversification. EvoDevo 4, 4.

Wittkopp, P.J., Haerum, B.K., and Clark, A.G. (2004). Evolutionary changes in cis and trans gene regulation. Nature 430, 85-88.

Wolff, C., and Hilbrant, M. (2011). The embryonic development of the central American wandering spider Cupiennius salei. Front. Zool. 8, 15.

Wu, J., and Cohen, S.M. (1999). Proximodistal axis formation in the Drosophila leg: subdivision into proximal and distal domains by Homothorax and Distal-less. Development 126, 109-117.

$\mathrm{Wu}$, J., and Cohen, S.M. (2000). Proximal distal axis formation in the Drosophila leg: distinct functions of teashirt and homothorax in the proximal leg. Mech. Dev. 94, 47-56.

Yu, Y., Fuscoe, J.C., Zhao, C., Guo, C., Jia, M., Qing, T., Bannon, D.I., Lancashire, L., Bao, W., $\mathrm{Du}, \mathrm{T}$., et al. (2014). A rat RNA-Seq transcriptomic BodyMap across 11 organs and 4 developmental stages. Nat. Commun. 5, 3230.

Yuasa, H., Oike, Y., Iwama, A., Nishikata, I., Sugiyama, D., Perkins, A., Mucenski, M.L., Suda, T., and Morishita, K. (2005). Oncogenic transcription factor Evi1 regulates hematopoietic stem cell proliferation through GATA-2 expression. EMBO J. 24, 1976-1987. 


\section{References of Publications and Manuscripts}

\subsection{Turetzek et al. (2016). Neofunctionalization of a Duplicate dachshund Gene Underlies the Evolution of a Novel Leg Segment in Arachnids.}

Abzhanov A, Kaufman TC. 2000. Homologs of Drosophila appendage genes in the patterning of arthropod limbs. Dev Biol. 227:673-689.

Adams MD, Celniker SE, Holt RA, Evans CA, Gocayne JD, Amanatides PG, Scherer SE, Li PW, Hoskins RA, Galle RF, et al. 2000. The genome sequence of Drosophila melanogaster. Science 287:2185-2195.

Angelini DR, Kaufman TC. 2004. Functional analyses in the hemipteran Oncopeltus fasciatus reveal conserved and derived aspects of appendage patterning in insects. Dev Biol. 271:306- 321. Angelini DR, Kaufman TC. 2005. Insect appendages and comparative ontogenetics. Dev Biol. 286:57-77.

Cao Z, Yu Y, Wu Y, Hao P, Di Z, He Y, Chen Z, Yang W, Shen Z, He X, et al. 2013. The genome of Mesobuthus martensii reveals a unique adaptation model of arthropods. Nat Commun. 4:2602. doi: $10.1038 /$ ncomms3602.

Conant GC, Wolfe KH. 2008. Turning a hobby into a job: How duplicated genes find new functions. Nature Rev Genet. 9:938-950.

Davis RJ, Shen W, Heanue TA, Mardon G. 1999. Mouse Dach, a homologue of Drosophila dachshund, is expressed in the developing retina, brain and limbs. Dev Genes Evol. 209:526- 536. Davis RJ, Shen W, Sandler YI, Amoui M, Purcell P, Maas R, Ou CN, Vogel H, Beaudet AL, Mardon G. 2001. Dach1 mutant mice bear no gross abnormalities in eye, limb, and brain development and exhibit postnatal lethality. Mol Cell Biol. 21:1484-1490.

Davis RJ, Pesah YI, Harding M, Paylor R, Mardon G. 2006. Mouse Dach2 mutants do not exhibit gross defects in eye development or brain function. Genesis 44:84-92.

Davis RJ, Harding M, Moayedi Y, Mardon G. 2008. Mouse Dach1 and Dach2 are redundantly required for Müllerian duct development. Genesis 46:205-213.

Garcia-Bellido DC, Collins DH. 2006. A new study of Marrella splendens (Arthropoda, Marrellomorpha) from the Middle Cambrian Burgess Shale, British Columbia, Canada. Canadian J Earth Sci. 43:721-742.

Hammond KL, Hanson IM, Brown AG, Lettice LA, Hill RE. 1998. Mammalian and Drosophila dachshund genes are related to the Ski proto-oncogene and are expressed in eye and limb. Mech Dev. 74:121-131. 
Innan H, Kondrashov F. 2010. The evolution of gene duplications: classifying and distinguishing between models. Nature Rev Genet. 11:97-108.

Janssen R, Feitosa NM, Damen WGM, Prpic NM. 2008. The T-box genes H15 and optomotor-blind in the spiders Cupiennius salei, Tegenaria atrica and Achaearanea tepidariorum and the dorsoventral axis of arthropod appendages. Evol Dev. 10:143-154.

Kozmik Z, Pfeffer P, Kralova J, Paces J, Paces V, Kalousova A, Cvekl A. 1999. Molecular cloning and expression of the human and mouse homologues of the Drosophila dachshund gene. Dev Genes Evol. 209:537-545.

Magadum S, Banerjee U, Murugan P, Gangapur D, Ravikesavan R. 2013. Gene duplication as a major force in evolution. J Genet. 92:155-161.

Mardon G, Solomon NM, Rubin GM. 1994. dachshund encodes a nuclear protein required for normal eye and leg development in Drosophila. Development 120:3473-3486.

Ohno S. 1970. Evolution by Gene Duplication. New York: Springer-Verlag.

Ortega-Hernandez J, Legg DA, Braddy SJ. 2013. The phylogeny of aglaspidid arthropods and the internal relationships within Artiopoda. Cladistics 29:15-45.

Page RD. 2002. Visualizing phylogenetic trees using TreeView. Curr Protoc Bioinformatics Chapter 6:Unit 6.2. doi: 10.1002/0471250953.bi0602s01.

Pechmann M, Prpic NM. 2009. Appendage patterning in the South American bird spider Acanthoscurria geniculata (Araneae: Mygalomorphae). Dev Genes Evol. 219:189-198.

Pechmann M, Khadjeh S, Turetzek N, McGregor AP, Damen WGM, Prpic NM. 2011. Novel function of Distal-less as a gap gene during spider segmentation. PLoS Genet. 7:e1002342. doi: 10.1371/journal.pgen.1002342.

Posnien N, Zeng V, Schwager EE, Pechmann M, Hilbrant M, Keefe JD, Damen WGM, Prpic NM, McGregor A, Extavour CG. 2014. A comprehensive reference transcriptome resource for the common house spider Parasteatoda tepidariorum. PLoS One 9:e104885. doi: 10.1371/journal.pone.0104885.

Prpic NM, Wigand B, Damen WGM, Klingler M. 2001. Expression of dachshund in wild- type and mutant Distal-less Tribolium corroborates serial homologies in insect appendages. Dev Genes Evol. 211:467-477.

Prpic NM, Janssen R, Wigand B, Klingler M, Damen WGM. 2003. Gene expression in spider appendages reveals reversal of exd/hth spatial specificity, altered leg gap gene dynamics, and suggests divergent distal morphogen signaling. Dev Biol. 264:119-140.

Prpic NM, Tautz D. 2003. The expression of the proximodistal axis patterning genes Distal- less and dachshund in the appendages of Glomeris marginata (Myriapoda: Diplopoda) suggests a special role of these genes in patterning the head appendages. Dev. Biol. 260:97-112. 
Prpic NM, Damen WGM. 2004. Expression patterns of leg genes in the mouthparts of the spider Cupiennius salei (Chelicerata: Arachnida). Dev Genes Evol 214:296-302.

Prpic NM, Schoppmeier M, Damen WGM. 2008a. Dissecting spider embryos for light microscopy. CSH Protocols 3, 950-954. doi: 10.1101/pdb.prot5072

Prpic NM, Schoppmeier M, Damen WGM. 2008b. Whole-mount in situ hybridization of spider embryos. CSH Protocols 3:933-936 (2008b). doi: 10.1101/pdb.prot5068

Ramsköld L, Edgecombe GD. 1996. Trilobite appendage structure - Eoredlichia reconsidered. Alcheringia 20:269-176.

Ronquist F, Huelsenbeck JP. 2003. MrBayes 3: Bayesian phylogenetic inference under mixed models. Bioinformatics 19:1572-1574.

Rota-Stabelli O, Campbell L, Brinkmann H, Edgecombe GD, Longhorn SJ, Peterson KJ, Pisani D, Philippe H, Telford MJ. 2011. A congruent solution to arthropod phylogeny: phylogenomics, microRNAs and morphology support monophyletic Mandibulata. Proc R Soc B. 2011 278:298306. doi: 10.1098/rspb.2010.0590.

Sanggaard KW, Bechsgaard JS, Fang X, Duan J, Dyrlund TF, Gupta V, Jiang X, Cheng L, Fan D, Feng Y, et al. 2014. Spider genomes provide insight into composition and evolution of venom and silk. Nat Commun. 5:3765. doi: 10.1038/ncomms4765.

Schomburg C, Turetzek N, Schacht MI, Schneider J, Kirfel P, Prpic NM, Posnien N. 2015. Molecular characterization and embryonic origin of the eyes in the common house spider Parasteatoda tepidariorum. EvoDevo 6:15. doi: 10.1186/s13227-015-0011-9.

Sewell W, Williams T, Cooley J, Terry M, Ho R, Nagy L. 2008. Evidence for a novel role for dachshund in patterning the proximal arthropod leg. Dev Genes Evol. 218:293-305. doi: 10.1007/s00427-008-0220-5.

Sharma PP, Schwager EE, Giribet G, Jockusch EL, Extavour CG. 2013. Distal-less and dachshund pattern both plesiomorphic and apomorphic structures in chelicerates: RNA interference in the harvestman Phalangium opilio (Opiliones). Evol Dev. 15:228-242. doi: 10.1111/ede.12029.

Sievers F, Higgins DG. 2014. Clustal Omega, accurate alignment of very large numbers of sequences. Methods Mol Biol. 1079:105-116. doi: 10.1007/978-1-62703-646-7_6.

Stein M. 2013. Cephalic and appendage morphology of the Cambrian arthropod Sidneyia inexpectans Walcott, 1911. Zool Anz. 253:164-178.

Waloszek D, Chen J, Maas A, Wang Y. 2005. Early Cambrian arthropods-new insights into arthropod head and structural evolution. Arthropod Struct Dev. 34:189-205.

Whittington HB. 1971. Redescription of Marrella splendens (Trilobitoidea) from the Burgess Shale, Middle Cambrian, British Columbia. Geol Survey Canada Bull. 209:1-24. 
Whittington HB. 1975. Trilobites with appendages from the Middle Cambrian, Burgess Shale, British Columbia. Fossils Strata. 4:97-136.

\subsection{Turetzek and Prpic in preparation. Observations on germ band development in the cellar spider Pholcus phalangioides}

Abzhanov A, Kaufman TC 2000. Homologs of Drosophila appendage genes in the patterning of arthropod limbs. Dev Biol 227, 673-689.

Akiyama-Oda Y, Oda H 2003. Early patterning of the spider embryo: a cluster of mesenchymal cells at the cumulus produces Dpp signals received by germ disc epithelial cells. Development $130,1735-1747$.

Akiyama-Oda Y, Oda H 2006. Axis specification in the spider embryo: dpp is required for radialto-axial symmetry transformation and sog for ventral patterning. Development 133, 2347-2357.

Akiyama-Oda Y, Oda H 2010. Cell migration that orients the dorsoventral axis is coordinated with anteroposterior patterning mediated by Hedgehog signaling in the early spider embryo. Development 137, 1263-1273.

Andersson DT 1973. Embryology and phylogeny in annelids and arthropods. Pergamon Press, Oxford.

Claparède É 1862. Recherches sur l'évolution des araignées. Natuurkundige Verhandelingen uuitgegeven door het Provinciaal Utrechtsch Genootschap von Kunsten en Wetenschappen. Deel I. Stuk 1. C. van der Post Jr., Utrecht.

Crome W 1963. Embryonalentwicklung ohne "Umrollung" (=Reversion) bei Vogelspinnen (Araneae: Orthognatha). Deutsche Entomologische Zeitschrift Neue Folge 10, 83-95.

Crome W 1964. Eikokon, Embryonalstadien und frühe Jugendformen von Conothele arboricola Pocock (Araneae: Ctenizidae). Zoologische Jahrbücher Abteilung für Systematik, Ökologie und Geographie der Tiere 91, 411-450.

Edgar A, Bates C, Larkin K, Black S 2015. Gastrulation occurs in multiple phases at two distinct sites in Latrodectus and Cheiracanthium spiders. EvoDevo 6:33.

Emerton JH 1872. Observations on the development of Pholcus. Proceedings of the Boston Society of Natural History 14, 393-395. Plate II.

Holm A 1940. Studien über die Entwicklung und Entwicklungsbiologie der Spinnen. Zoologiska Bidrag från Uppsala 19, 1-214. Plates 1-11.

Huber BA 2011. Revision and cladistic analysis of Pholcus and closely related taxa (Araneae, Pholcidae). Bonner Zoologische Monographien 58, 1-509. 
McGregor AP, Hilbrant M, Pechmann M, Schwager EE, Prpic NM, Damen WG 2008. Cupiennius salei and Achaearanea tepidariorum: Spider models for investigating evolution and development. Bioessays 30, 487-498.

Pechmann M, Khadjeh S, Turetzek N, McGregor AP, Damen WG, Prpic NM 2011. Novel function of Distal-less as a gap gene during spider segmentation. PLoS Genet. 7:e1002342.

Turetzek N, Pechmann M, Schomburg C, Schneider J, Prpic NM 2016. Neofunctionalization of a Duplicate dachshund Gene Underlies the Evolution of a Novel Leg Segment in Arachnids. Mol Biol Evol 33, 109-121.

Wallstabe P 1908. Beiträge zur Kenntnis der Entwicklungsgeschichte der Araneinen. Die Entwicklung der äussern Form und Segmentierung. Zoologische Jahrbücher Abteilung für Anatomie und Ontogenie der Tiere 26, 683-712. Plates 38-39.

Yoshikura M 1954. Embryological studies on the liphistiid spider Heptathela kimurai, part I. Kumamoto Journal of Science Series B (Biology and Geology) 3, 41-48. Plate I.

Yoshikura M 1958. On the development of a purse-web spider, Atypus karschi Dönitz. Kumamoto Journal of Science Series B Section 2 Biology 3(2), 73-86. 


\section{Acknowledgments}

This work was supported by the Deutsche Forschungsgemeinschaft (grant numbers PR 1109/4-1, PR 1109/6-1 to N.M.P.). Additional financial backing has been received from the Göttingen Graduate School for Neurosciences, Biophysics and Molecular Biosciences (GGNB), the Göttingen Center for Molecular Biosciences (GZMB), and the University of Göttingen (GAU). Further support was given by Christiane-Nüsslein-Volhard-Foundation fellowship and a "Women in Science" Award by L'Oréal Deutschland and the Deutsche UNESCO-Kommission. I thank Beate Preitz and Felix Quade for help with microscopy. Cloning and in situ hybridization experiments were performed with support of my student assistants, Julia Golldamm and especially Julia Schneider. 


\section{Supplemental Material}

\section{1 engrailed}

Pp-engrailed was cloned using degenerated Primers for the initial PCR, followed by RACE PCR (Turetzek, 2011). The probes were synthesized from the 5'RACE clone with a length of $386 \mathrm{bp}$ (coloured in light blue).

acgcggggagtgactcaagagaaaccacctcccatagaccgctcacttcaattctgatt ttttttgagacaggagtacagcaacattcgacctcaaggcattcattggagaacaaga ctatttcgttttcgtccacgtgctgctttcogcttgctattacttaattagtctgtgat tgtatgtttttttgtttgtttttttcacggtgaattattatggtggaaagaaaaat agcattctcccgtcctctgtgttagaacgttcttcagaatcttatcttcaggcctctttg aattttgaaacgtagatcattttgcaagactttctatcgaatcaacgtaagtttgaaga acttctaggcgtgttagttgatcaacacttatttaatgatcgcttattccacatcagtga tgaaggaatgacgagtgtgcctggagaagacttttcccgaagagtcagcagggcctgatt catattctcactcaagtggcaaaacggaaacattttgtcgccacctgcagcctcgagt tatcagttttggtatcaacgtagcaacctgttgtctaatgcaaagaatccgtttcctttc tgggactttgctacttgctcttcgtccgctggtgagccactgaccgtggactcttctttc cagaggtcgaaattcagcccagcgatgaaactgatctcgataatttccagctaagtt gcttcaatattggactgtcgtctagaataccaggatttaggactatggctctggacatg M A L D M gagcgaccaagctctgtgcacagtccagagcagcattcattatccggcggaagtagcctc $\begin{array}{llllllllllllllllllll}E & R & P & S & S & V & H & S & P & E & Q & H & S & L & S & G & G & S & S & L\end{array}$ ggcggtctaccggccagtcaaatgcacgaacgtcggtcatcggaaggtcgccggtcgacg $\begin{array}{llllllllllllllllllll}G & G & L & P & A & S & Q & M & H & E & R & R & S & S & E & G & R & R & S & T\end{array}$ agtcctgaggatgagcgatcgagcagcgaaggaacagaaccgccgcacagagcacaccac $\begin{array}{lllllllllllllllllllllllll}S & P & E & D & E & R & S & S & S & E & G & T & E & P & P & H & R & A & H & H\end{array}$ ccagcggtccagggacagccgctaccgactactgcgcattcccttaagttttcaatcgaa $\begin{array}{llllllllllllllllllll}P & A & V & Q & G & Q & P & L & P & T & T & A & H & S & L & K & F & S & I & E\end{array}$ aaatactctctccggactttggacgcttggtatccagggacaggagagattcgtttcga $\begin{array}{llllllllllllllllllll}K & I & L & S & P & D & F & G & R & L & V & S & R & D & R & R & D & S & F & R\end{array}$ gaagagacttccgtagcgctcaataataaagttaactccaaagaatctacgaactctagc $\begin{array}{llllllllllllllllllllllllll}E & E & T & S & V & A & L & N & N & K & V & N & S & K & E & S & T & N & S & S\end{array}$ aagcaaagttcggaaaacaaaatacggctcctttgggactcgcagaccctaccgattcc $\begin{array}{llllllllllllllllllll}K & Q & S & S & E & N & K & N & T & A & P & L & G & L & A & D & P & T & D & S\end{array}$ ggaaaaatgtattgtggccagcatggtgctattgtacaaggttcagcgatcgcccctca $\begin{array}{llllllllllllllllllll}G & K & N & V & L & W & P & A & W & C & Y & C & T & R & F & S & D & R & P & S\end{array}$ tcaggtcctcgatcacgacgagtgaaaagaaggacaagaagcccgatgaaaagaggcct $\begin{array}{llllllllllllllllllll}S & G & P & R & S & R & R & V & K & K & K & D & K & K & P & D & E & K & R & P\end{array}$ aggactgctttcacggccgaacaattggctcggttgaaacaagaatttcaagaaatcgg $\begin{array}{llllllllllllllllllll}R & \mathrm{~T} & \mathrm{~A} & \mathrm{~F} & \mathrm{~T} & \mathrm{~A} & \mathrm{E} & \mathrm{Q} & \mathrm{L} & \mathrm{A} & \mathrm{R} & \mathrm{L} & \mathrm{K} & \mathrm{Q} & \mathrm{E} & \mathrm{F} & \mathrm{Q} & \mathrm{E} & \mathrm{N} & \mathrm{R}\end{array}$ tatctgacagagaaacggcgacaggatttggcccgtgacttgaaacttcacgaatctcaa $\begin{array}{llllllllllllllllllll}Y & \mathrm{~L} & \mathrm{~T} & \mathrm{E} & \mathrm{K} & \mathrm{R} & \mathrm{R} & \mathrm{Q} & \mathrm{D} & \mathrm{L} & \mathrm{A} & \mathrm{R} & \mathrm{D} & \mathrm{L} & \mathrm{K} & \mathrm{L} & \mathrm{H} & \mathrm{E} & \mathrm{S} & \mathrm{Q}\end{array}$ attaagatatggtttcagaaccgtcgtgcgaaactgaaaaagctagtggtcaaagaat $\begin{array}{lllllllllllllllllllll}I & K & I & W & F & Q & N & R & R & A & K & L & K & K & A & S & G & Q & R & N\end{array}$ cctctcgctctccaacttatggcccaaggattgtataaccatagcaccattcctttgagg $\begin{array}{llllllllllllllllllll}P & \mathrm{~L} & \mathrm{~A} & \mathrm{~L} & \mathrm{Q} & \mathrm{L} & \mathrm{M} & \mathrm{A} & \mathrm{Q} & \mathrm{G} & \mathrm{L} & \mathrm{Y} & \mathrm{N} & \mathrm{H} & \mathrm{S} & \mathrm{T} & \mathrm{I} & \mathrm{P} & \mathrm{L} & \mathrm{R}\end{array}$ gatgaagacgatgatgagaggccaaatcgtcttcgtcttcttaatagatggaaactttc $\begin{array}{lllllllllllllll}D & E & D & D & D & E & R & P & K & S & S & S & S & S & -\end{array}$

caagtttctaacttgacaatgcttcaccggtggtgtaatactaaggcctagtggtagacc 
agaaatcttggaactccctgtgtaagaactgcacaaccgaattcacaataacatatgact tgatgacgagtagagatgcgagtagaaaaggactagaatgttttgactaaactactcgct aacatcactataatagcagaaacgaaatcatcactcttagatgaacttggattttatc cttgtacaacataatataggaaagagcccagtttaatcattcttacgagcagggacacga tattctggttgatcatgattaatttaagtatgggggaacaatcaaccatctttgtctctt aagcaccatcgtagtgcccagttgagcagcactgttcgattcaccacgtgcagtcagcct acgtactgaaaaaaaatcaaactgtgataatttctgaagaaaactttttcttccttgc tatggtgtgaagtatttatcactgatttccgcagattccattttttttctgagcagcct cttggtttccattgaaaatagcctataccattactttccttactatactcgtaccttca tctaatgntcacatagtgaatatactcaagatcacatgcgtg 


\subsection{Timing of embryonic development of $\boldsymbol{P}$. phalangioides}

Supplemental table 1: Timing of developmental events during germ band formation and differentiation in embryos of $P$, phalangioides at $25^{\circ} \mathrm{C}$

Please note that all time points given in the table are approximate values. Abbreviation: $h$ AED, hours after egg deposition

$48 \mathrm{~h}$ AED

$58 \mathrm{~h}$ AED

$70 \mathrm{~h}$ AED

$72 \mathrm{~h}$ AED

82 h AED

$92 \mathrm{~h}$ AED

96 h AED

106 h AED

119 h AED

$130 \mathrm{~h}$ AED

143 h AED

$154 \mathrm{~h}$ AED

$167 \mathrm{~h}$ AED

$178 \mathrm{~h}$ AED

$191 \mathrm{~h}$ AED

215 h AED

$225 \mathrm{~h} \mathrm{AED}$

239 h AED

$263 \mathrm{~h}$ AED
Primary thickening forms in germ disc center

Cumulus migration starts

Primary thickening cells disperse

Connection between cumulus and remnant of primary thickening ruptures

Remaining primary thickening cells congregate again: formation of secondary agglomeration

Cumulus reaches rim of germ disc

Cells of the secondary agglomeration disperse: formation of the posterior growth zone

Radial symmetry is broken: bilaterally symmetric germ band forms

First segments are visible in the posterior portion

Embryo attains bean-shape

All prosomal segments are morphologically visible

Cumulus slowly disappears

Prosomal segments well delineated

First opisthosomal segment visible

Cumulus not visible anymore

Prosomal limb buds formed

Tail bud forms

Three opisthosomal segment anlagen present

Prosomal limbs elongate

Tail elongates

Three opisthosomal segments well developed and lying on the yolk

Ventral sulcus appears

Buds of book lungs appear

Tail segments clearly delineated

First tail segment (04) opens and folds down on yolk

Brain differentiation begins

Dorsal tissue develops on opisthosoma: start of inversion

Tail adds more segments

Opisthosomal limb buds are well formed

Next tail segment (05) opens and folds down on yolk

Mid inversion: dorsal tissue grows

Tail shortens by folding down more segments to the yolk

Late inversion: dorsal tissue overgrows yolk

Tail shortened; only two segments

Dorsal closure

Tail gone; all segments folded down on yolk

Prosomal shield begins differentiating

Ventral closure begins

Prosomal shield fully formed; pulls legs apart

Ventral prosoma closed

Ventral closure complete

Dramatic leg length growth starts

Legs wrap body

Eyes are pigmented 


\subsection{RNA seq genes}

\subsubsection{Evi1}

$>$ c109624_g3_i2 CAAAAAAGAAACCCCCACAGGTGAGAGAAAAAGAGTAGTAGACCTTGTTTTTCTTTTTTTCAATCAGAGCCTTACCTGTTCCATAGAGTAAAGT AGAGAGAGAGAGAGACGCACTCTTTAACGCGGCTGCTCTCACTTTACTTACTTTACTCTCTCTGGAAATAAGGGAGGAGTCCCCGCAAAGTGGGA CATGAAATGCCCCGATCTGATTCCAACAACCGCGCGCACACACACACAACAAGTAATACTCAACAACGAGACCCAACCAAAGCCGGTGTATAATT GGGCAGACACTGCTGTTTACCTGGTGGTGGTGGTGGCAATAAAAAAAAAAGAAGAACAACAACAAGAAACGAGAAGAGAGAGAGAGAGAGGAG AAGAAAAAGTCCAACTCACTCACTCTCTCTACATTTGTGTGTGTAATCTCTATCTGTGGGAGAGAAAAAAAAAAGGGGCGGTGGCAGCGGCAAG GCATGGAAACTCTGTGAAGCATGGACGTCCAGGTACGGCAGTCCTGTCATGAATCTCGTCCGAGTAACACACAACACCACACACACACACACACA ATTGACGGTTTTCTGAGGAAATTTACTCATGGGTCCGTCCATCTACTAGCTTGACGGGTGTCGTTTGATTTGCCTGCTGATTACTGGTGTGATAT CCGCACCGCGATCCGGAAACAATATTCTGTCGCGTGAGTGTGTTCTCGACACTTGAGACATTGTGTGTTGTCAACTCGGCAAATGTGCACATGAA ATTGATCTCTCATGAAAGTGTGTGTCAAGTGCGCGTTGAGTGTGGAAATGACTCTGATGAAGAAAGGGGGTGCCGATTTCCCACCGAATTGCCAC GTGATAATGCCGAGAGCCTACAGCTACTGACGGTCAGCAACGGATATTTCCGGTGACATTGTCTCGATCTTCGGCATCTTCGCCAGCCAAAGAAT AGTTTTAAGCCACCGTCGATTTCGAAGAAGAAGGATATTTTTCGATAAGAAAAGACAAAACTCCATCTTTTCTAGGAGACTTACCAATCGATGA ATTTTTGTCCAGGACTCGAAACTGATTGAGAGGATTTCGTTTTTGCAAAAGAACCCTATTGTGATTCCTATCGATATCGACCAAATCGAATATTT AAGTGTGACTTTTGTGGATAAGAATCAAAGGGAATTGAATCTACTTAGTTTGGCTCAAGGCTAAACGAGCATATTGGAGCGAATTCCCACCTCT CTGAAGACAGCGGTCAAGGCGAGAGGGAATACAAGTGCCCGCAGTGCCCGAAAGTCTTCAACTGGAAATCAAACCTCATACGTCACCAGGTCGCC CACGACGAGAGCCGAAGGTACACCTGCGAGAACTGCAAGAAAGTCTTTACAGACCCGAGTAACCTACAGCGGCACATCAGGTCCCAGCATATAGG AGCTAGGTCTCACGCCTGTCCCGAATGTGGCAAGACCTTTGCCACTTCCAGCGGACTCAAGCAGCACACCCACATTCACAGCAGCGTAAAGCCGT TTAGGTGCGAGGTATGCTTCAAGGCGTATACGCAGTTCTCGAACTTGTGCCGGCACAAGCGCATGCACGCGACCTGCCGCATGCAGATTAAGTGT CACAAATGCGGTCAAGCGTTCAGTGCCGTTACTTCTCTGTCGAAGCATAAGAGGTTCTGTGAAGGTACTTCAGCGAACAGTAACTCGGCATCGAC GACGTCGACGGGTCAACAAAAGCAGCAACAGTCGGCATCGGATCAAGGCACCGGTTTCGAACCTAGCAAGGCTGCTCCTATGAGCCTAGCAGCAC CGTCTAACCCCCTGCTGTTCTATCCTCGACCTGGTTTCCCGCTCTATCCTCCATCTTTATTCGCTTACCCTTTGTTTCCTGGCCCGGGTTTACCCGC ACCTCCACCATTGATCCCGGACAACAGTTCTGTGGGACAGACGAACGCGAGCAACAGCTACGCTGCGGCGAGCCAAAGTGTGCAAGAATTCCAAA GGCAACAGTTATCTACCAATAGCTCCTCCGATGTCACGCAAAAAGCACAGGACTCTTGTTCTTCGCCAAAGCCATCGGAGAACGTGAATTCGTCG CCAACCAAACACGATAAGAGAAACGACAACAACTCGGAAGGTTCTGAATCTGACGCGTCTGAGGACTTGAGTTCCGAAACGGGTGGTGAAGACA TCAGTTCTATCAGTGACGCTGAGTCCGATCCAGATAATATCAGGTCTCCGAGTCCTACGCCTGTGGCGAAAAGCAGCAGCACCCCGACGAAAAGT GAAATGTCAGCCATATTCCCGAATCACAGGCAGGACTCACCAAAGCAAGGGTCAGTCTGCCCAGACCCATCTTTGATTATGTCACCAATGAACTC GAACGGTCACATCAGATCGATGATGACCAACGGCATGACCAAGTCAGACAACGACATGCCATTCGACTTGTCCCGGAACAGCAAAGGAGGCCGTT CCGAAGAATCGGACACTTATAATTCAAAGTGCCAGGACGACACCAAGTTGGACGACCAGCCACTGGACTTGAGAGTGACCCACAAAAAATCTTTT CAGCCCGTCGAGAAAGAGGATTTGTGCAAGAGCCCAATCAGCACCAAAGACAGCGAACAAACGCGCAACAACGATTTCGCGTGTGCACCGAAAGG CACCAACAGCATACTATCTACGGTACCTCCGCCACTCCCACCGCTGGAACTGCCAAAACCGGACAGTCCTATCCCCCAGTCACCGAGTAAGCTACA GATGGCCTACCCCAGGCCGATACATCCGATGTTCCTCGAGTCCATGTACAGGCTGCAACAAGAGAAACAGGCCTACAACTTGTTCCCGGGCCACG ACCGACTGATGCCGCCTTTCCCGGCACGATATCCGTTTTTGGGTCCACTACTGGGTACGAACCCTTCCTTCGATTTCATGAGGGCCCACATGGAGA AAGTCGGCAAGCCCATGCACGACATGATGTCCCCTCACCTGGGGAAGACGAAAGAACGTTACTCGTGTAAGTTCTGCGGCAAGATTTTCCCGAGG TCCGCAAACCTGACGCGGCACCTGCGGACGCACACCGGCGAGCAGCCCTACAAATGCAAGTACTGCGAGAGATCGTTCAGCATATCATCGAACCT CCAGCGTCACGTCCGCAACATACACAACAAGGAGAAACCTTTCAAATGTCCGCTCTGCGACAGATGCTTCGGCCAGCAGACCAATTTGGACAGGC ACCTCAAGAAGCACGAAGCAGACGGTCCGACCATACTCGACGACTCGCCCAAGACCATAGAAGTCGACGACAAGGACGAGACTTACTTCGACGAG ATCCGGAGCTTCATCGGAAAAGTCACGAACAGCAACCACATGGGAGCAGCGCCGCCGGGTCCGCTGCCGCTGATGGGCGTGGCGATGGACGACAG GAAGCGGGAACGCGACATGATGCTGCTGGCCTCCTTGTCCGCCAGGCACAGTCCCGGGAGCGGACACAGCGACAATCTGGACGAGGAAACCGACG GTATTAGCCCCTCGGACCAGGACGAGGAACCTTCCATCAAAAAGCACCGGTCCTCCGAGCGCCTGTCCTCTCCCAGCGGCGAAAGCAGCAGCAACC GGAGCCCGGACTCGACGAAAGACCCGGAGCACCGAAACGAATCGAGTCCACGCCATCATCACCACCACCATCACAACAGCAACAACCATCACCAT CATCTCAGGAACGGCGATTACAAGTCGGCAAGTCACCAGCAACAGCAGCAGAAGGCCCTCGCGTACGAGGTGATGATGACCCTGAACCATAAGAT CGACAACATCAACGGCATCGACAGGAAACGACGACAGAGCGTGGCCGGTAGCAACGACACGGAATACGGAGAGGAGTCCAGCGACGAGGACGAG ATCGTGGACCCCGTCGACGACGACGACGAGGAAGTCGAGATCGACGTCGACTCAAGGAAAGAGGTAAAAGTCGACTGATGTCATTGTCGCAGTA AATTCGGGTAGTAGAATCAAAAGACACAACCTAAAGAAAAGGCATAGCGGGGGCCCGTTCCCAGGGTCACAACCGCTGCCTCAGCCCAGAACGCG ACGGGACGTTCACTGCAACCGAGAAAACTCGGCGGAAACCGACTCGTGACGGACATTGAGTCCTCATTTCTTGATGACTCATAGGACTCAAAAAT CATCTTCCCTATAACGAACTCACGTGCGTGGAATGAAACTGTATCCGGACTTCGGTTAAGACGAATACGTCAGTCACATTGGGATACGTCACAAT TTTTTTCCCATATCAAAATTTCAAAAAAATGTTGTTGTTTTTTTTAAATAGATGCGCGTGAAAATGCTAGTCACAAAAAAAAAAGTTATATATA TACATAAAATATACATATATAATATCTATATCCCTGCGTTTAGCCGTTGCAACCCCCGTTTTATGTGAAGCCTAATGATGCCCTCGAAGACATTT TGTAGAAACTGTGACGTTTCCCCCCACTCGAAGAAAATCGCGTGATGAAACTGAAATGATGGACGTATTGAGTCAAATCAAAGTGATTGAAATG AACATGATATCGTGACATGCAAGTGACTCTCGTCGTCTCGTCACCGTTTATTTCGTGTTGTTTTTCTCAATCGATTATGTATGGACTGGATCGAT GTCAAAAAAAATTATCCCCGTCTTTTTCTTTCTTTTGTTGATGTGAACATGCGCATTCCTTGATTTTCACTGTAATAACATGATAAGAAATTATT CAATATCCGGTTTTCGGCCACACATGAATTTGTATCGGTGAAATATTATTTGATAATTAATCGAGAGAAAATGTATTCAGATCATGTCCATAGT AGGGAGTATGATTGGCATCCATGAGGGAAAAAGCCATGCTGAATCTTCGGTCGGTTATTTTCTTACCTAGTGATGGGCAAATTACGACCCGCGGG TCAGGTGCGGCCCACCAAGGCTCCCCATCCGGCCCTCCAAAGGTGTTCAGGTAGAAAAGTATCAATGCACATACCACAATAAATACCTAGGCTTA CCATATTTCCTTCATGAAAATACGGGAAATAGACAATCAGGATCCATATGGGATATCACAAATTATCTCCAAAAAAAAAATTGTCAAATGCAAG TTAATTTGATAAATACATCCAAAAAATTTTCAAATGCAAGCAAAGTTGTCAATTATAACCAAAAATTTGTCAAAGACAAGAAAAGTTGCCAACT ACCACTGAAATTGCTGAAAAATCGCCAATAGCGGGAAGTAGGGATCTTCCACAAGACGCGATGTGTAAAAATTTATACTTCACCAAACAAAACG TATGTAGTTGACAAATATGTGAGATGAATGTGGCTAAAATTAATTATGATATAAATTATCTTGTGATAATTATGACTAAGCGTGAAAAAAAAA TCTTAATTATTGATTTCAAAAGTTCAAGTAACGCTCATTGAAAATAAGAGAGAAAACAGGAAATTTTTTAAAAACATTAAAAGTCCAAAATTAC GGAAACAT 


\subsection{2 $\mathrm{c} 105514$}

>c105514_g1_i1 GGAGCGTAAAAGGTTTACGCTCTGAGAAACGCGTGACAGGGGTCCTTAAGTGAGGAAGAGAAACAACATTTTCTCGTATTTGTGAGTGTGTATC AATCATTTTCGTGAAATAGTTTTGGGCTATCGGTCGGAGGAGGATTTCTTGTATTTGATCCTGTTCTCTCCATTCATGATCGTGTAATGGGCAAT GTACGCGAGCATCAGGGATCATCATTCGGCTAATAATCATTTAACAGTCATCGTGTTTTTCTTTAGTGTTTTTTATTCATGTAGTGTGTGTGGAA ATAATACCTTTATAGGAAACGTAAATCTCGTGCACTTTTCATGTGTCTAGATATGGGAAGATGGATTATCAAAAGATGTTTTGGCTCTGGTAGC TAGCACAGAGTTGTTCGTGCACCGTCCGATGTCCTTTCGTGCGAATTTGCCGTCGGAGAAAGGATCGTTTTCGATAGCCAGTCTAGCATCTGCTG TGGGCTGCAAAGTTCTCGAAAAAGTCTTTGTTCCGACAGCCGTCCAAGCTATCTTGAAGCAAGAGCTAATCCAAGCGATGGGTAACGGTAATTCT AAAGCCATAACATCCAGCAAGTTCTTGTCAAATGTCGGCATCAAGAAGCAATCTCCGAGCTACGCCTTGCAGCGCAGGCGCTACGGCCCGAGGGA GGTGCACCGCAACATCTGGCGTTTAGAGCGGAAAACTTTCGGCAAACCTGGCAAAACTGTCAAAGATCCCGAAACGGAGGAGAAAAATGTCAGA CCTGAAGTACCGAAGAGATCGACATATAAGAAACGCAAGGCAGTGTCAAAGGAAAAAGCCATTGAAGTCGTGGTTAAGGAGCTCGAAGTAAAGG TTCCATGTGCAGCGCCAAATCCCGATTCCCTGCCATCGAGATCGGAAGTCCTTCCCCCTGATGGCGATTCATTACGTTCAGATGCCGAAAAGAAG CATCAATCCTCCGAAGGGCACAGCAGATGCCCATCCTGTACTTCTTCGCAACTGAAGCTTTCGAAAGCGTACGCTCAGAAAGGAGCTGTTGCTGT GACCTTGGAGAAGACTCAGAGGGATTTGAATAATGCTCACACGAGGATTAAGGATCTCGAGTTCGAATTGGAAGACTGCAAGAAGTCCGTTGAA GAACTCTCAGCGACCATTAAAGACTTGGAAACAGAGATGTGCCAACTTCAGAAAGACAAAGCTTTGCTCCAGCAGGAATTGATGGTCACTAGGA AACAACTGACGAGGATTCTCGACCAGTCGGATTCTTCCGATTCATCTCATGTGAGCTCGGTGTTTGTTTCCGGGTCTCAGAACCTCACTGACGAC GGACTCGGTGACGAGACGACAATGACCCCTGACGAGGACGGTGACGGACACCCGGAAGATGATGATGACGACGAGGACATCGAAGTGCACTCAC AGCAAGACCGCAAGAATGCCAAGACGAGGTTGGAGCAGAATAATTTCGATATATCGCGGCTAGAAGACTTGCAAATGAAAAATGAAGAGCTCGA ATTGACGATTAGGCATTGCGAGGAACGGATAATCGAACTCGAGGAACTACTTAGACAAGCCACTACCCGTATCGATATGAGAGACGTGACGACC CAAACATTCACGAGAGACGGTACTTTCACGAGACCAGAGAAAACAAAAATTCCTAAAAGGATGCTCGGTCATCACAGCTCGTTACCTGTGATAA GTGACCCGAACAACCTTGGTAGATGCAAAACCCCAACTGGTAGCTTGACGAGATTGGAAAACCTACAGCGAAGCTTACGGGTGAAAGAAAGAGA GATCACAACATTGAAGAAGGTGATAGCAACTATGGAAATGAATGAAGTCGGTAAGGAATTGGAAAAGCACAAAGAAAAAGAGGTGATGATCCA GAATTCGAGGATCGGTGAACTTGCATCTGAGAACCGCAGGCTTTTGAATGAGTTGCAGATCGTCAAGGAGAACTACCAGAGAGAGACGGTATTG AGAAAGAAGTATTTCAACATTGCCGAAGATCTCAAAGGCAAAATTCGAGTTTTTTGCCGGATAAAGCCAATGGCAATGATGCACAGGAATGCAC CTGTCCTTGAAGTACCTGATGAATATACGGTTATGTTACGAACCCACAGAGCACAATACGAATTTACATTTGATGGTGTCTTCGATGGGGATCGA TCTCAAGAGGATGTGTTCAGTGATGTTCAAAACTTGGTGCAGTCTGCAGTAGACGGCTACAATGTTTGTATATTTGCGTACGGCCAAACGGGATC CGGAAAGACCTACACTCTAGTAGGAAATGAGTCCCAACCTGGAATAGGACCGAGAGCTTTCCAGAAGATTTTCGATTTAGTCCGTGAGAACCAA TGTGAACTTGACTTCCGAATAACTGTATCTGTTTATGAACTGTACAACGAACGTCTGATTGATTTATTGAAAGATTCAACTGAAGACAACCTAA AGATAAGGCGAGATAATTATGGTCTAGTGTTTGTTCAAGGTTCAGAATCTAGAGAGGTCAAAAATGAACGGCAACTGTCAGAAATCTTTGAGGA AGCCTGTCGGAATCGTCACGTCGGAGCAACAAGAATGAACACGGAGAGCTCAAGATCTCACCTCGTAATAACTATACGCGTCGAAAGTACAAACA AAATAACTGGAACTGTTTCCCGGGGGAAACTTAGCATAATTGACTTGGCTGGAAGTGAAAGAGTTGGAAAGACAGCGGCTGTAGCACAGCAGTT GAAGGAAGCCAATTCGATCAACAAATCATTATCCGCTCTTGGTGACGTGATAGCCGCCCTGAGCTCGGAGCAACAGCACGTGCCATATAGGAATA ACAAGCTGACACTTCTCATGCAGGATTCTCTCGGAAGCAATGCCAAGACTCTCATGTTTGTCAATATCTCATCATCTGAACAAAACGTCGAAGAA TCACTCAACTCTTTAACGTATGCTTCAAGGGTCAAACAGATCACCAATTCGGCATCACGAATGGCAGAAAGCAAAGAAGTCGCAAGGTTACGATC GATTATAATGAAGCTTTCGAAGGAAAACAAATCGAAGAATTGACGCTTTCATGAAATTATTACTCAGTATTATGCACGTGATCAATCAATTTTG TCTTTTTAATTTCATGGAAATA

\subsection{3 c95092}

$>$ c95092_g2_i1

GCGGGGGGTTTGTATGATCCAGTCTCAGGTAAGTTGTTATAAAGGTCATCCTAACAACTTCCTCACTTCTTCGTGTTCCGATTGGACCACATAAA CGATAATGGAGGAAAAAAAAAAATAAAGAGAAATTTTTTCACATGTAATTTTTTTCGTTTCGAGAGAGTTTTTTCGGTGCGTGTGTATAATAAG GGGTGTGTGTGACGTTTTTACAAGTCAAGGTTGACCAACTTTCTCCCTCAGCTGACGGGTACTAGTACGACCATCACCAGCGACTATTTCGCATT CGGCATCAGACCATCAGCCCGAGAGTTTACAAACACCAGTCGGGTCTAATTTTTTTCTTACTCTTACGACTTTTTCACGATAAAATTCGAACAAA AACAAAAATGACTTTCAGCTTCAAGACCATCGTTTTCATTTCTGGTCTAGTCGCCGCAGTCATGGGCCAGGAGTATAGGTACCAGCAGCAGCAAC AGCGTCAGTTCAGGCAACAACAGCAGCAGAAAGAGAATTGCAACATCGAAGAATGTCTCATGAAGCTCAGGGACCCCGACCCTGTCAAGGAAGG AGGAGACATCAAAATTTTCTGCGCCCGAACTGCTGAAGGATTGGCCTGCTTGGACAGATGCCTGGACTCACCGCTGTACCAGGCCACCAGCCCAA TCGTTATGGGAGGAGTTAAGCAGCTGCTGTCCGAAATATGCAGTCCTGGATCTAGTCTTGGCAAACGTTACATGCAGGAGTCCAAGTGCCTGAAC CACCAGAACACGACAGTCATGGACTGCGCAACCTCCATGATCGAGAAGTATCCAGCTGTTCTCACCCGTCCCGAACCCGAGGCCATCGTCCGAGTC TTCTGTTGTTCAATCGACAGAACCGAAGAATGCATCATCGAACGTGTCCACAAAGACTGCGGACGTGGAGCTGCTAAACTCGTCGACGAAATGAT GTCCAAGGCTTTTTACCCCATCAACCAAGTCATTTGCTATTTCAATGATCCATCGCAGTGTCCTCAATTCTAGAGGAAAAAAAGACTCATTGTTT TTAAAACTCTTCATCATTTTTAAATATGTGTAAATATATTTTTTTAAATAATCACATCATCCCCTCCATCATTTTTCTTCTACATACAAACAAAA TATTCATCTCTGCATGTGTTTTTTTTTTCTTTATGTAAAAAGGAATTTCTCATCGATTATTTTGAAAGTTGAATGTAATATAAATAAGGCCGGG ATTATATATATATACACTTTAAAAGTTTGATCAATGCTCTTTCACAAAACTTCAAAAATGCAACAACAAACAAGCGTATCGGTTACATTTGAGC GAATTTAACCAATAGGCTTATTGGTGTTTTTTAAGTTTTGAAAAAGATCTTTTATCAAACTTTCAGAGTGTAATCTCGGTCTAAGGAAGATATA ATCTTCAGTTTTTAAAATTTCATTAAAGCTAGGATTACGCTATAAAAGTTGTTTTATGAAATTCGAAAAATTCAACCAATAAGCGTTTAATAAA GTTAAATAAAATTATTTAGCAACCAATAAGTTTTCTAGCAAATGGAACAGATAAAATTGTTGGTTGCATTTTGATGTTTAAATTTGTCGAAAAA ATTTTCATAAAACTTCTATAGTGCAATCCCGCCTTAAGAATTTCTTGACATATTTAAGTTCCACCCGAAAAAGAATTGGAGATATACATATATG TGACCCACATAAAATATTTAAATTTAGTATATGTATGACATTGATACAATGACGAGGTCCAATCTTTAGCTGAAAGCTAGACGCGGCGAATACA ATGTCAGAGATTGGAAAACTTGAAGACATCAAGTAGCTTGGTGACATGGCTACAAATACTCCTCGTCAATTCGAATAAAACCAATCCATGATGA AATGTAATTTGCATCACACTCATTTCGCTATACTGATTATCAATTAAAAAATTACTACTGTCACTTTTCAAAGCTCTCTGTGATAAAAATCGTGA AAAACAAGTACAACATAATTATGACATATTAGTGAACTTGTTCGGTTTCCTCCTCACACCAATGCGAAATGTCAATCAATATTTTTTTATGTGCA ATCATTTTGTAAATAGCTGAGATTTGTCAATAAATATTCATCCATTCACCAAAAAAAAAAAA 


\subsection{4 c109329}

$>$ c109329_g1_i2 TTTTTTTACAGAAACAAACTCTTTTTTTTATTATTTTACAAAACATTCTGTAGGAAAATAACTGCGTGCGTACTCGACAACAAAAGAATTTCCA AGAAAGAAAAAAAAATGTACAGCTCATACTGATATTTTTTTTTACATTGACGTTGTACAAAGCAATTCATCTTCTCGACTAAAATATTCAGTCT GATCAACGCATTACAGCAACAAAAAAAACAAGCGTACAGCCATTATTTTGTAATACTGCATATTTTTTGTTCACATATCTTTGTTGGTCCCATTA AGAACATTCATGTATAGATGAAACATCATTGCAGAATTTCAAAGAAAAAAAAGAAAATTTTTTACACATATTTTTCGATGAAATCATTTCAAAT TATCAAAGCGTCTCTTATCCATGCTTTGTATATTTTCCCACATTTTTCTCCTTATTTGACAAGAAAAAATTTGATTGTCCCCAGTCGTAATTCTT CGCGTCTTGCGTCAAATTCGCAAGATGGAAGACAACACTGGGTCTTATGCGTCACCGGTTTTTATTTTTGAATTAACGTTCATTTTGTTTTCCGA ATCGTGGCACCCTTGTAATTATGGAAAAGAAGAAGAAACTATCAATTGTTAGTAAGTAGTTAGTATACCCTAATTTTGGCCCCAATCAATCATT ACTTTGTGGAAGAATTTTCGTTATCATGCAATTGTTTTCAATTATGGCAGAATTCGGAGTGTCGGGTGAATTAGAGGCACCTCAGATGCGATAC CTCCATTTTAGAGCAGAGATGCCATTTCATTTTCAAAGAGTTTATAAAATGTACATAGTACATCGAAATATAGTTAGGACATGGTGATTTTTCT AAATTTACAACTATCCACACCCACAGAAATTTCTTGCGACGATCTGATATCTTTTGAATGGTTTTTCAATAGGCAATTTTTGATACCGATGTTTT TTTCTTTTGTCATGAAACTTATGGCATCCCTGCTGCCACTTATGGATTTTTAGAAGCACAAATTTACAAAAAAAGTGAGATATGTGTGTGTAGA GAGGATAAAGGAATGAGATGCCCACCGTTCTCTTGATACAAATGCCTCTTCGATGTCTGCGGTGGTGTCTTGAGTGACAAAAGGGAGGGACGGG ACGGGATGGGGAGATCTTTTATTTCACATCAAAGCTCGTGAATGTCCTCTCTTCCAAATATTCTACTGAGGTGATGGAAATGGTACGTAGACTGG ATGAAGCTCACTGGCGTCGGCCCTGGACGGATGAGATACTTGTTGGATCATTCTCTGTTGGTGATGGGCCGGGATGATGCGGAGTTGCTGTTGGA CTATTTCAACGATCCTCGGAGATCTCATTTGGGATGGCATCGGTTGCATTTGAGCAACTTGACGGTTGGCCTGTCTTGCCATCAGCAGTTCTCTA AGGAGCCTGATTCGCTGCAGAGGGCTCATCACTGGTGGCCTTACGACTGGTGGGTGCGGCATGAACATAGGTGGCCTCTGCGCTACCTGTTCGAC CTCGTCGGTAGGAACTAGGAGAAGGACTTCTTGATCGTCGTCGTCAGCTTCTTCTTCGGGGTCCTCCCTCACGAAGATGACTCGTTCTTCTTCTT GCGGGACTGGGATCTGAATCATTTGGCGGTGGAACATCGGCATCTGTTGCTGGACGATCGGGAGCTGCTGTGGACGATGGAACACTGGTATTTGG TGTTGCGACTGGTGCATAAGAGGAACGTGGGATGGCATCGGTGACATCATAAGCATGACTGGATGAAGAGGCAACTGGGGAGGCCTGATCATTC TAGGAAGGACACGTGGAGCTGGTTCTGGAACTGGTCTACGCAGCATGGGGCTGATGAACTGGTGTGGCACTTGTTGAATGAAATGGGGTCGTGG CTGAGGCATCGGAACTCGTACCATTGGGTTTGGTACTGGCATGCGTCTGGCCATAGTTGGGTGAGGTTGCTGCACGGGCATCCTCCTTGGCATTG GCCTCATGACTGGGCGAGGAACGTTTGGAAGAGGGATCAGTATGGGGGCGTGCATCGACGGTTGGGGCATAGGCATGATTGGTGACTGTACGGG TACCATGTGGTGCATGTGCATTATCTGCTGTTGTTGCACTGGATACATATTCTGCTGTTGTTGCACTGGATACATATTCTGCTGTTGTTGCACTG GATACATATTCTGCTGTTGTTGCACTGGATACATATTCTGCTGGACTGGCATGATAGGGATGAGGTTTTGCATTGGTGGTTGGATGGGAATCAG ATGTTGCATGGGGGAAAGGGGCAACATTGGTGGTGGTTGAGATCGTGGCTGTGGTCGGCTCATGATGTTGTGCATGATCTGCTGCGCCAAGGGT AGGAATAAAGCTACTGGTGCCAGTGGTGGTACTGGTAGCCTGGGTCGGTTTGCAGCAGGCATTTGTCGTGGGACTGGGAAAGGTAGGGGCAATG GGAATCGTCGG

\subsection{5 $\mathrm{c} 109662$}

$>$ c109662_g1_i1 TTCACACATACCAGTTTTTTCTCTACATATAATGGGATATTGAAATCTTGAATATTTCAAGAATCATTCGGGGGAAAAAAATCATTGTTGAAGG AAAAGAAACTGTTTTTTTTTCACTTGAGAAGAATCCGGTTTTATTGTGAGGAGCGTATGTGTGTGTGGAAAAGAGACACAAAAGGCACCAAACC ACCACCACCACCAACCTAAATTCGTTAATAATAAAAGAAAAGTGAGGTAGAGAAAACAAAATAAAAAACTATATACCAGTGAAGTGAGTGGTAT GTACTACTAGGCTCTTGGGAACGAAACGGCGGTGGCGGCGCGAGGAAGAGAAGCCCGAATAAATATATAATAATAATAACCATAATCGTAAGGA GTTTACCTCTCAGGCGATGAATCTCATGGTAGCGGACGTTATAACGGTATAAGACTTTCCTCGGGGTACATACAGGTTGAGAGAGAAGCTCAAG ACGCGGGAGTAAACAACGTAAGATAGACGAATTGTGCCAAATTTTGAGAGATAAATTTAAAAAAAAAACTATGGATTTTCGACTGCTGGTTCTA GCGGTCGCCGTCTGTTTGCTCGATGTCGGTCTCGCACAAGACAGGACAAGAAGCCGGTACGTACCATCTTCTCCACAAGAGGATGTCGATGCCGC TTACAGCAGGGGTGAGGACAGACTGGTCACTCGTCAACCGGTCGTCAGGCAGCCATCATCGTCATCCCGAAGGAGACAGCCGTCGGTGTCCACTG GTGGGCAACGCCAGCATTCTGCAACGGCCGACGACGATACGGCTGTGGCCCCAAACCCTCGGCTAGAGGATCGTCTACCAGTCGAGCGTGAACTG AAGGACAGCTATGACTTGAGGGGACCTATCGACAACAGGCCGTCATATCACGAATCGGTCGTAGGTGAAGCACCTGGAACAGACGCCCCACCAGC CGACAACAGCCAGAGACGCAGGAAGAGGCCCAAGAAGCGAAGGAGGCCTAAACCCGAATCCAAGCAGCCAGAGCTTTCCGATCCCGAAGAACACG CTGAAGACCGCCTGCCACAAGCCCCGGGCGCATCGATAGAAGATGATTACCATGCGTACAGGAAACCTCAGACACCATCCCCAGTATCTGAGAGC CAATACTCAGCACCGAGAGGGCGCCAACAGCACGAATCAAACTCAGACACACGCGGCCAGAGGCAAGCAACGAGACCAGCGGTATCCTCGAGGGT TCCGGAATCGAGGTCTTCCAGGAGACAGCCTACCGCGCCGGTGAACCGTCTTCCAGTGGAGCCACCTAGTCGTTCCTTGAGGAACAGGCCCTCGG TACCGGAGGCTCCGGCTAGTGTGCACCAACCTTATCAGCCAGACGGCGACGACACGAGCATTTCTGGAGGTTACGAAACGAATTACGCGGTTTCT GACGACTCCGATCCGTCTCGGCTACCAGTAAACGACGAAACTGGTCAGACGAGCTTTCTGAATCATAGGCGGCCGTTACCGGTCCATTCTGACGG AATAGACTCCGCCGAAAAGTCGTCTGGTCAACAGCACGGATCAGAGTTTTTACCTACCCCAAATCGTCTTAACGATGCCTCAGTACCATCATCAC CTTTCATCGATCCTCCACGTCATCTGCCTCCGCCCGTAATCAGAGACATCGACTTCCAAGCTGATGTGGTAACTAGGCAGCCAGAGGGACTGTCCG ACCGCCTTCCTAACGATCAGTACCTGAGGGCCTTGCCGATCAGAGAAGCTCTACCGCACGACAGACTTCCGGAACCCGAAGGTCGAAACCCCGAA CCAGAAAGGATCGCCGCTCGAGTTCCCTTGGAATCGACGAGAGGTGGTTCCCGCTACCAGGCCGCGGAGCAACCCGAACCAGCTCGCATACAGCC TCCGACCGACCAACGTCGTAGGCAGGTGCCCTCGTCGAGATCCAGGTCCGGTATAGAAAATGAAGAAGTCGCCAAGAATACTGTACGGTCTTCCA ACAACGACAGGCCAGTCGGCGCTCGATTGCAGAGGACCGGTTCCCGTTACCAGCCTGAAGATCCCGAAGTCACACGGACCAGTCCCTCCAGGGGA CCCCAAAGAGTTTCTGAACCAGCAGTCAACGAAAGAGCCCCTCAAAGGAACCAGCGCACTCGGTCACGACAAGAAGAGCCGAAGCCCCAACCCAC ACCGACCAGATCCTCGAGGACGAGGTCCAGATACCAACCCGAACCTGCACCCGTAGAAGAGACTTACAGCGCCCCGATAGAAGAGCCGGCTCGAC AGAGGACGGTCCCGAGACCAGACAGGTCCAGGAGTCGGAGCCAGTCGGCTCCTGCTGCCAGGCCGGAGCCAGTCGATGACAGCGAGGAATTCCAT CGCACGGGACCTCAGTATGAATCTGACGTCCGAGGTCCCCAGCCACAACCAGAGGCTTACGTGCCTCAATACCAGAATGTTCCGAGGACAACGCA ACGACGACCGGAACCCGCACAAGTTGGTCATTCGTCTTCGTCACGTGGTGCTGACGCTTCTCCGAGGCAGAGACGTCCATCCTCAAGGCCTCCTCA GGAAGAGCCTCCAAGCAGGTCGACGCCACGACAGAATCAGCAACAACCTCCGCGAAGCAGTAGCAGCGGGTCATCCTTCGAATGCCCGAATCCCT ACGGCTTCTACCCTGATCCAAACCAGTGCGACAAGTACTACGAATGCCGCAACGACACTGCTGAGGAAGGACTTTGTCCGGACGGTCTTGCTTTC AACCCCATCAGCGCCCCGACTTACTTGAGGTGCGATTCCGTCAGGGACATCGACTGCAGTGCCAGGCCCGAATTGCAACCTCCTCAATCGACAAA GAACTGTCCTCGACGATTTGGTCTGTTCCCGGACAAAAAAGAATGCACCAAGTTCTGGCACTGCGTTGACGGCACGTCCACTCAAGTGACCTGTC CTCCAGGACTCGTGTACGACGATTCGAAGGGAACCTGTAACTGGGCAGATCTCGTGAAGGATTCCTGTTCCACTGAAGATCTCCTGGGATTCGCT 
TGCCCCGACGAAATACCAGCCAATATCCAGGAAGGGTTGTACCTTCGCTATCGCCATCCGGACAACTGCCAACTGCACTTCACCTGCATCACGGCC GCTGACGGAACCCGCAAGCCTCGCATGCTGTCCTGCAACGAGGGATTCGTGTTTGATCCCGAAACCAGTCAGTGCACTCGTCCAGAGAACGTACC AGGCTGCGAGGAATTCTACGGACCACCGCCACCACCACGTCGGTCATCGAATACGAGGCAAGAGCAACAGAAACCACCGACCCGTGGCTCGCAAC CACCTCCACCGGCAGAATACGCTGAGGAAGAGGACGAGGAGCAACTGTTGCAGAGGACCCAGAGAAGGAGGAGGCCGAGCAGTACGAGGACGAG ATCATGAACGATGTGAAATGTTAATAAGAAAAGCCGAATAAGCAATTAAACGTTTCAAGACTATCTTAAAGAAGCCACCTGTAATGAAAACAAA AATTGCCTCGTCATCTCTTATATAGAACAGTATTAAAACACATTTTTCTATTTAAAAAGAGGATTTCAATCAGCACATTGATCTCACAAACAGA ACCCTATAGGAACCCTTGAACAAAATCTATGCATCAGAAATCTCACATAAACGTATCGATGAAAATCTTCAATAGTTTGGAGGTCTTTATTTGA CTCTGGACTCTTTGGCTCACCCGTTAAGGTACCCCTCTGCACTACAGCGTGCCTCCTAGGCATTGCTCCCAAATCCGTCACCCGTAGGCT GAAAAT CCCTCCCTGGTTCAGGATGTTTTGTGCACCCTCAGGTCGGAGCTTTTGGTTGGTTCCCTTCTCTGATTACCTAATTGATTTTTCAGCGATTTAAA AATAATAAGATTCCCGGTCTCTTAGGCAACAGCCTTGTAAACCTCGTACATGTTGGGCGTGCAATGCCCGAAATCCCTCTAGGTGTAGATCATAT TAACATTAACCCTTTGACTACGAATTAAAAAAGAAATTACCTCAGCCTTTTCTAATGAACAAAATAGGTCACAAGTAAGGCGAATTTAGCATTT GGAATTTTTTCTTTTTTGTGGGATATATAAGTCCCGTTACTAGTCAAAGGGTTGAAGGAGATATAGAAGCAAGGAGAATGAATTGCTCCTATAT CTCCTCTAACTAACGAGGCGGTGATTTTGTTTTGGTATTAAACAGTGGCACTCAAACTTTGACAGCAGTGACTCCTTTGAGATAGGGTCAAAATA TCGACTGGAAACAAAAAAACTTACTCTGTCGCCGGGGCCAGTAGCATTCTGTCGCCCCACCCTTCACAATTTTGAACAGAAAAAGAGTAAACTCA ACACGCTTCTGTTCCCTAGTTGCGGCCCTGCATAGGATAGCAGCGGCTTGACATAGCGACGAAAAGTGCGCTGTCTTGGACCTCGCTACACAATA TACAGGTGTGTCGACCGCCCAATAATTACACATAGATTCCTTCTGAAAAACTAACGTGCAAGAGTGTTTTCGGTTTTTTTTTTTTGATTTGATCA AT

\subsection{6 oddskipped}

$>$ c102331_g1_i4

AGAGGAACATGGACGAGGTGCATGTGTGTGGTTCAGTAGTAGTAGTTCTTCGAGTCAAATCGGGAGCCCTACGTTATTCGAGAATTTTGTTTGT TCCGAGATCATATAATTTTTCATAAATTATTTTCCCACTGAATATGTATTTTTCATTAGTTCTGAGAAATTATTTTTCATTAAATATGAATTTC ATAAATCTTGATTACTCCCACTAAAATATCAAATTGCCCCTTTCAAAAATCATATTGCCCCTCCCCCCTGTGGGGCGTGGGCCTCACGTTGGGAA ACAGGGCTCTAGAAAATTGATGTGTGAGATCGGCCCGGGTGGGCCCATGTATTAACACGGCAGTGTCTGCATTATAATGTTTCCTCAGTCTCTCA GTTGTTATAAAAATACTTCTATATACTGTTACATTATAATGTAGCGACAGGTTAAGAATTTCAGTTTTAATTGATACTTGAGAAGCGTTGCAAA TTAGACGATCACAAAAATTACATTGGCTCTATTGCACATAATTCTTTGATATAACCTCGTGAGATATACATTCCATTGTGTACGCGTTACATATT TCTTAGTATATTTGTCTCTTTAAAAATGGGTTAAATCAATGAATAAGAATTTTTTTATTTAGTATTTTTTTAAGCGAACTTCTCTTGCCGCGGA GTAAGAAAAGAACAGGCGATGTTTCACGACAGACACTTTCGAAAAAGGACGGACGGCACACGCGGGGGTGGGAAGAGCATAATTTCGATTGTTT CAGTTTTCAACGTCGACGATATCAGAGTCGATGTCGGAGCACGAGAGATCGCTATCGTGATCTTGATCGCCGCTCTCTAATCCAGAGTCTGCGGG AGACGGCGGCGAACATTTTTCAGCGGCGTTATTGACGTCGTGGATGAGTAGATGTCTCCTCAAGTCACAATTGCGTCTGAACTCTTTGCCGCAGG ACGAGCAGTTGTAAGGCTTTATGTCCGTATGCGTGAGTAGATGAGTTTTCAAATTGCTGCGCTGGTTGAAAGAACGTCCGCAGGTAGCGCACTTG TGCGGAGAATCTTCCATGTGAAGAATCCGGTGCACCGCCAACGTTCTCGACTGGCAGAAACCCTTGCCGCATTCGATGCACTTGAAAGGCTTTTC TTTCGAATGAATGTACCTGTGATCTCGAAGATGGTCTTGCCTTCTGAAAGATTTGTGACATATGTCGCAAGTATACGGTCTCTCGTCTGTGTGCG TCCTTTCGTGAATCAGCAAATTGTACGATTTCGTGAACCTTCTTTGGCAGAATTTGCACACGAATTCTTTCCGGGGACGAGAAGTTGCAAAAGGA TTGCCGTGGTGATGCTCATCTCGCCGCTGTTGGATGCAGGATGAAGGCCTCCTTAGTTGAGGTACCGCCGAGTCGGCGGATGCCGTGAACCAAGC GTTAGTTTGGCCAAATGTCTCGAAAAGTTTGTCGCTGGCGATAGATGTCGTACACGGATATTCGCCGTTCATAGACGATGAATCGGCTCGACTGA CGTCAGTCATCGTTGCGGACTCGGCCAATCGGCTGAAATCGAATTTAATGGAACGTCTCATTTCCGGATTAAAAAGAGGCGATACCGCGTGGGGG AAATTTTGATCAACGAGAGGATAACAGTCAACCGCTGGGGGTGCACCGACTTGGCACTCTCCAAGGAAAGACGACTTTTTACGTCCTTCGAGACT AAGAGCACAATGCGATCTCGGAAACTGTCGTTGATCCAGAATCTGAGGCAAACAACGCATCGTAGATTCGCCATCGTCAATGTCCGACCCCAGCG TCCAACAGTGCATGTCACAAACATTCAATACGAACGGATGCTGCCCGTGACGAGCTGCTACTGGCCAGAGAATACGCTGGTTCGAAGAGCCGCTC GAAGGAAGATTGTGTTGTTGATGATACATCATGTGTGACATCAAGTAAATCGACTTCACTTCATATCGTCGTGCTTTTCAGCGTAATCTGATTGC AAAGGAGATAACGGTGACACTCCCGCAGCGAAAATAACAGGAAGCAACTACATTATGGATACCACAGGCAGCTACATATGATATACCATATCCT ACTGCTTAAATTTGCCACAGTTACTGTAAGCACAGCGTCGGTGGAGTAGTATCCAGGGTTTGTATTTAACTCTTGCGTCATTCAACTCAGTCAAA TTCTGACTAGCGGGTGACATCGATTCCGTGGG 


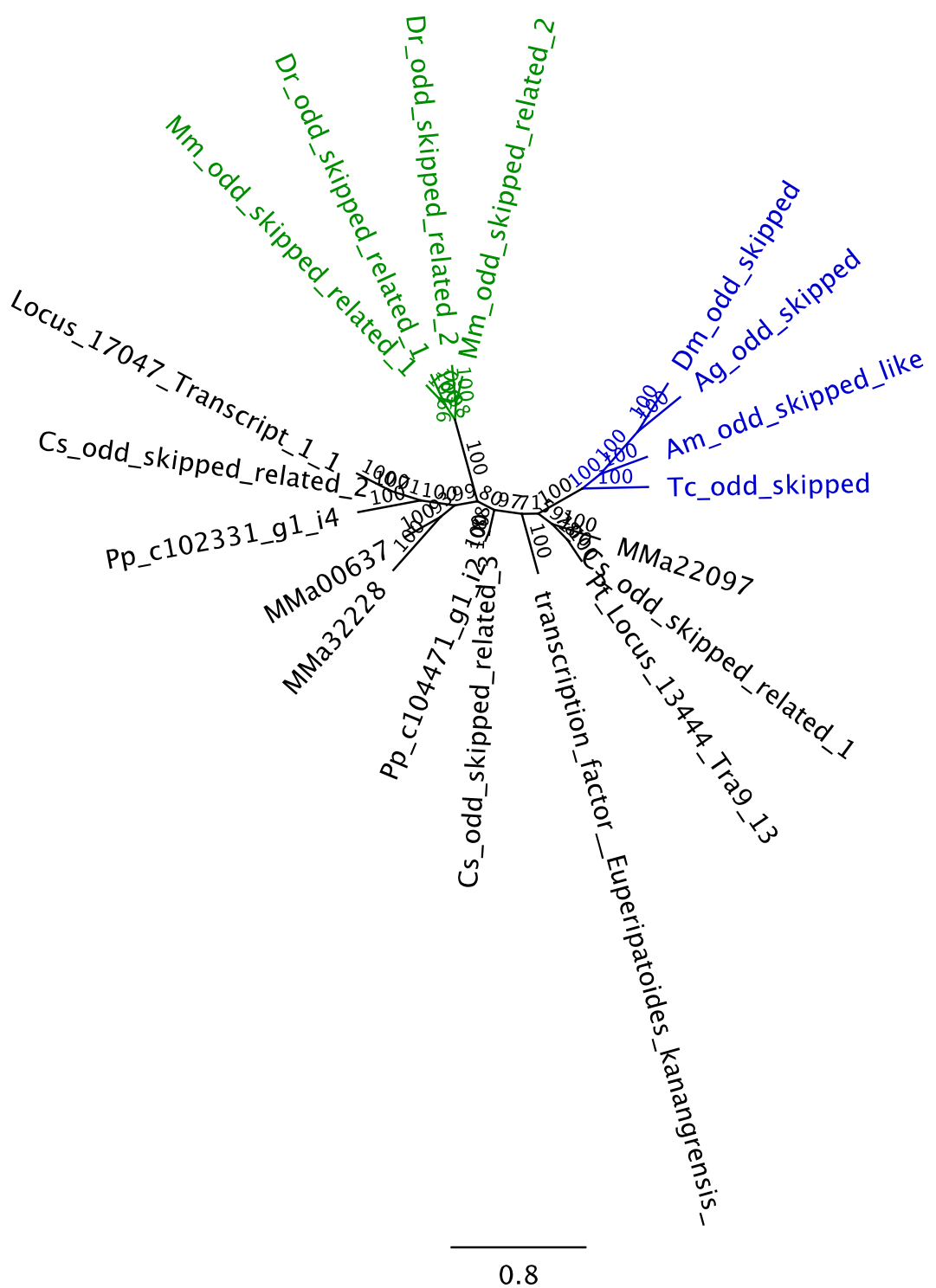

Supplemental Figure 1: Preliminary phylogenetic analysis of odds-related homologs

Rooted $50 \%$ majority rule consensus tree from total number of 670 trees written in two files. Each file contained 335 trees of which 252 were sampled. Mixed model testing indicated that the amino acid substitution model Blosum was the best suited model and the majority consensus tree was generated from 167000 generations after reaching the average standard deviation of split frequencies below 0.01 . Shown in black are all the chelicerate and one onychophoran sequences. In blue all the insect sequences are grouped and in green the odds homologs of vertebrates are depicted. 


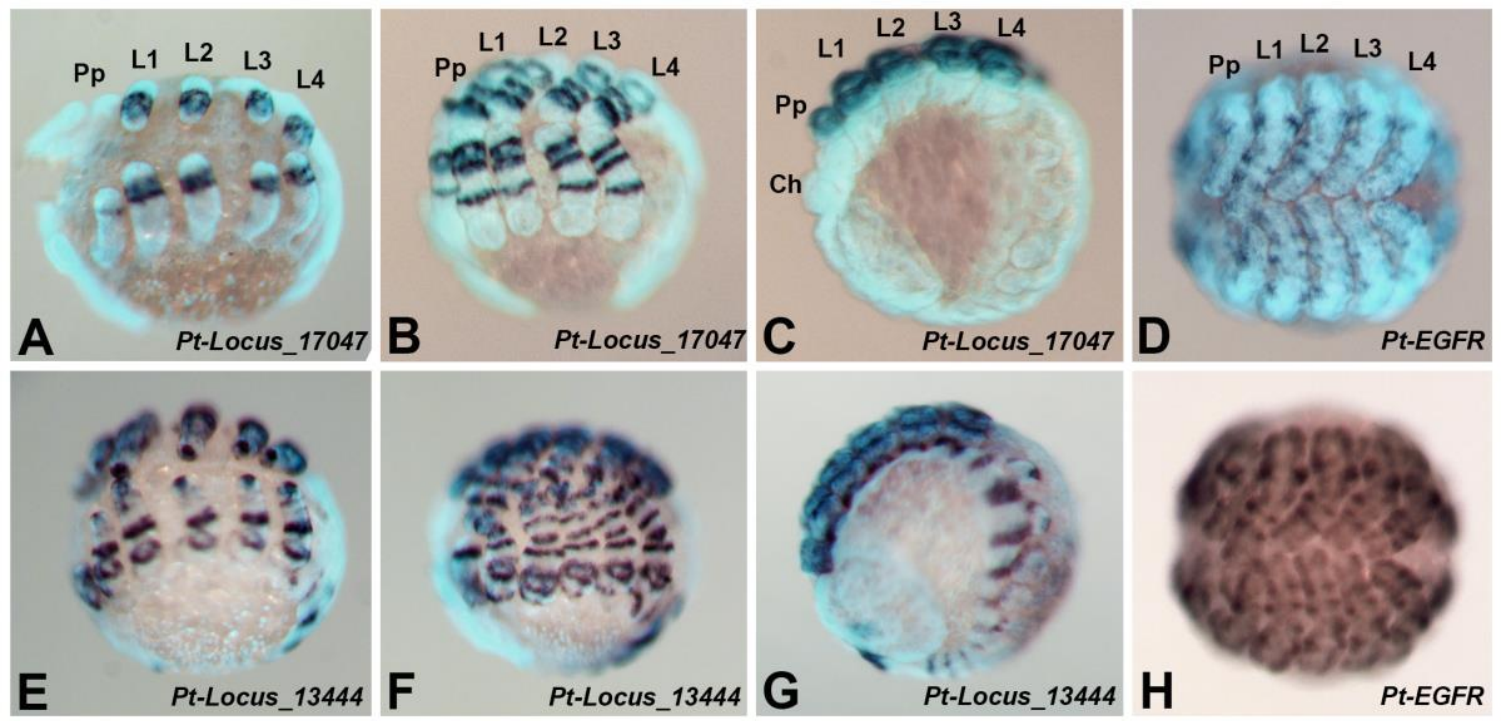

Supplemental Figure 2: Expression of odds and EGFR homologs in P. tepidariorum

\subsection{Leg gap genes}

\subsection{1 extradenticle}

In the following you will find the nucleotide and protein sequences, which were cloned with degenerated primer and RACE PCR of the spider exd homologs observed in this study. To verify that these are the only paralogs of exd and hth in the two spider species observed here, a large phylogenetic analysis was performed, with these sequences and additional data obtained from the now available transcriptomes. In most of the times the sequences obtained from transcriptomic data include more sequence information and result in longer protein sequences. For the detailed phylogenetic trees to clarify phylogenetic relationships of the duplicated paralogs of spiders and other chelicerates usually these longer sequences obtained from the transcriptomic resources were used. The probe for Pp-exd1 was synthesized from the clone Nt_251

\subsubsection{1 exd1 paralog sequences}

>comp102492_c1_seq1

AGCGATTGGACGAGGCTTTTAGTGACGTCAGGAGGTAGCGCCACGGCACCGACGCTCATCTTTTATGGCGAAGTGGCTTGACTGGCATTATCGCT CAAACCCTCCTGCGCTGTCCGTCAAAAACGATTTCCCCCATCAAAACACTCAATTGAGAGCACAAACCAAGCAGTGCTAGAGGCTCGAGCCCCAC GGCGTGTCGCGCGGCCTCGTCTCCACATTTTTCACAATGGATGCTGCTGCTCGACTACGAACGGGCCGCTAAATGAAACATAAACACGCTAATAA AAAACGAACGAAGGCTTTTGTTTCACAACTGTTGACGTCGACGTAGAATGGACGATCAGCAAAGAATGATGCACCCGGTATCGCAGCACCCTTCA TCGGCAGCAGCTAGCATGGGCGGCCACGTCGTGCCCCAGCACGGGTACGGACTACCGGGCCCACACGTTGTTGACCCGGGCTCGCAACAGAGCACT CCAGACCATCAAGATTCTCGAAAACACGACATCTCCGAGATCCTTCAACAAATCATGAACATCACAGATCAAAGCCTGGACGAAGCTCAGGCGAG 
GAAGCACACCCTGAACTGTCATCGCATGAAACCGGCGTTGTTTAACGTCCTCTGTGAAATCAAGGAAAAAACAGTTTTAAGTCTGCGGAATACAC AAGAAGATGAGCCACCCGATCCGCAGCTGATGCGGCTCGACAATATGCTCATAGCTGAAGGTGTCGCGGGTCCCGAGAAAGGGGGAGGCGCCGGT GCCGCGGCCAACGCGACGGCGGCGACAGCGGCCGGTGGCCCCAATCAGCCGGAAAATGCCATTGAACATTCCGACTACCGGGCGAAACTGGCCCA AATCAGACAGATATACCACCAAGAATTAGAGAAATACGAACAGGCGTGCAACGAGTTCACGACGCACGTCATGAACTTACTACGAGAGCAAAGC CGCACTCGCCCTATCACGACGAAAGAGATTGAAAGAATGGTGCAGATCATCCACAAGAAATTCAACTCCATCCAAGTCCAGCTGAAGCAGAGCAC GTGTGAAGCCGTCATGATTCTCAGGTCCAGGTTTCTGGATGCGAGACGGAAGCGAAGGAATTTCAGCAAGCAGGCCACAGAAATACTCAATGAG TATTTCTATTCTCATCTGAGCAATCCCTATCCGAGTGAAGAAGCGAAAGAGGAATTAGCCCGGAAGTGTGGTATAACAGTCTCACAGGTTTCAA ATTGGTTTGGGAACAAGCGTATTCGATATAAAAAAAATATCGGCAAGGCCCAAGAAGAGGCGAACCTGTATGCTGCGAAAAAAGCAGATTACAT CAATGACTGTTATTCCCCACCGTCTGCGGCAGGTTCCTCGCCCTACAGCTTAGGTCCTTCTTCTCAGGGGCAAATGATGAGCCCTCCACCTGGTCC AGCAACGCCGCAAGATTCTATGTATAACATGTCTATGAACGGTAGCGACTCTTACTCCTCTATGGGGGCAAATGTGCAGTCTCAGGCAAACGCAT TGAGGCACGTCATTTCTCAAACTGCTGGTTACGGTGATGGGATGCCGCCGCCATCTTCAGCTTCATCTATATACGACCCCGGCATGCATCAGACT TCCGAACTTCACCCATAGTAAAGCTGAGCACTAGCAGCCAGGCAATCTGTGTACAGAATGGTCTGATTAAGGCCTACCAAAAAATAAAGAAAAA AAAAGATAAATAAAAAAAGAAAAATAAAATGAAACAAGGGCTTCAATCAATATACGTTTTGTTCTCATACCTCAAAAAAAAAAAGATTTACAA AATAAAAACACCATGTATCATTATTAAAAATTGGAAAGAAAGGAAAGAAAAATGATGGAAAAACGGACAATTTTCACGACATTATGGGTATAT ATGTGTATATAAAAACTCTGATGGAACGTTTTACATGATTATATATATAAATAATATACCCGGTCGTTGTACTGTGAAAGAACACAAAAATATC TGAAAAGAAGAAAAAATGGAAAATTTAAAAAAAAAAAACTGGAAAGTGTGTGTGTACAGGAATTCAGCGTTTAAGAAAAAAACATAAAAAAAA TAATATGTAGACAGAATAATTGTGCTTTAAAAAATCCAGATTTCGTGATTTTTGATGAACATCCCGTGGTGTTGAATTTTTTTTTTTTTTTTTT

$\mathrm{T}$

>comp102492_c1_seq1

MDDQQRMMHPVSQHPSSAAASMGGHVVPQHGYGLPGPHVVDPGSQQSTPDHQDSRKHDISEILQQIMNITDQSLDEAQARKHTLNCHRMKPAL FNVLCEIKEKTVLSLRNTQEDEPPDPQLMRLDNMLIAEGVAGPEKGGGAGAAANATAATAAGGPNQPENAIEHSDYRAKLAQIRQIYHQELEKYE QACNEFTTHVMNLLREQSRTRPITTKEIERMVQIIHKKFNSIQVQLKQSTCEAVMILRSRFLDARRKRRNFSKQATEILNEYFYSHLSNPYPSEEAK EELARKCGITVSQVSNWFGNKRIRYKKNIGKAQEEANLYAAKKADYINDCYSPPSAAGSSPYSLGPSSQGQMMSPPPGPATPQDSMYNMSMNGSD SYSSMGANVQSQANALRHVISQTAGYGDGMPPPSSASSIYDPGMHQTSELHP

$>$ Pp_exd1_RACE_Nt249_Nt251

ACATGGGGATCAAAACACTCAATTGAGAGCACAAACCAAGCAGGGCTAGAGGCTCGAGCCCCACGGCGTGTCGCGCGGCCTCGTCTCCACATTTT TCACAATGGATGCTGCTGCTCGACTACGAACGGGCCGCTAAATGAAACATAAACACGCTAATAAAAAACGAACGAAGGCTTTTGTTTCACAACT GTTGACGTCGACGTAGAATGGACGATCAGCAAAGAATGATGCACCCGGTATCGCAGCACCCTTCATCGGCAGCAGCTAGCATGGGCGGCCACGTC GTGCCCCAGCACGGGTACGGACTACCGGGCCCACACGTTGTTGACCCGGGCTCGCAACAGAGCACTCCAGACCATCAAGATTCTCGAAAACACGA CATCTCCGAGATCCTTCAACAAATCATGAACATCACAGATCAAAGCCTGGACGAAGCTCAGGCGAGGAAGCACACCCTGAACTGTCATCGCATGA AACCGGCGTTGTTTAACGTCCTCTGTGAAATCAAGGAAAAAACAGTTTTAAGTCTGCGGAATACACAAGAAGATGAGCCACCCGATCCGCAGCTG ATGCGGCTCGACAATATGCTCATAGCTGAAGGTGTCGCGGGTCCCGAGAAAGGGGGAGGCGCCGGTGCCGCGGCCAACGCGACGGCGGCGCCAGC GGCCGGTGGCCCCAATCAGCCGGAAAATGCCATTGAACATTCCGACTACCGGGCGAAACTGGCCCAAATCAGACAGATATGCCACCAAGAATTAG AGAAATACGAACAGGCGTGCAACGAGTTCACGACGCACGTCATGAACTTACTACGAGAGCAAAGCCGCACTCGCCCTATCACGACGAAAGAGATT GAAAGAATGGTGCAGATCATCCACAAGAAATTCAACTCCATCCAAGTCCAGCTGAAGCAGAGCACGTGTGAAGCCGTCATGATTCTCAGGTCCAG GTTTCTGGATGCGAGACGGAAGCGAAGGAATTTCAGCAAGCAGGCCACAGAAATACTCAATGAGTATTTCTATTCTCATCTGAGCAATCCCTATT CGAGTGAAGAAGCGAAAGAGGAATTAGCCCGGAAGTGTGGTATAACAGTCTCACAGGTTTCAAATTGGTTTGGGAACAAGCGTATTCGATATAA AAAAAATATCGGCAAGGCCCAAGAAGAGGCGAACCTGTATGCTGCGAAAAAAGCAGATTACATCAATGACTGTTATTCCCCACCGTCTGCGGCA GGTTCCTCGCCCTACAGCTTAGGTCCTTCTTCTCAGGGGCAAATGATGAGCCCTCCACCTGGTCCAGCAACGCCGCAAGATTCTATGTATAACAT GTCTATGAACGGTAGCGACTCTTACTCCTCTATGGGGGCAAATGTGCAGTCTCAGGCAAACGCATTGAGGCACGTCATTTCTCAAACTGCTGGTT ACGGTGATGGGATGCCGCCGCCATCTTCAGCTTCATCTATATACGACCCCGGCATGCATCAGACTTCCGAACTTCACCCATAGTAAAGCTGAGCA CTAGCAGCCAGGCAATCTGTGTACAGAATGGTCTGATTAAGGCCTACCGAAAAAAAAAAAAAAAAAAAAAAAGT

$>$ Pp_exd1_RACE_Nt249_Nt251

MDDQQRMMHPVSQHPSSAAASMGGHVVPQHGYGLPGPHVVDPGSQQSTPDHQDSRKHDISEILQQIMNITDQSLDEAQARKHTLNCHRMKPAL FNVLCEIKEKTVLSLRNTQEDEPPDPQLMRLDNMLIAEGVAGPEKGGGAGAAANATAAPAAGGPNQPENAIEHSDYRAKLAQIRQICHQELEKYE QACNEFTTHVMNLLREQSRTRPITTKEIERMVQIIHKKFNSIQVQLKQSTCEAVMILRSRFLDARRKRRNFSKQATEILNEYFYSHLSNPYSSEEAK EELARKCGITVSQVSNWFGNKRIRYKKNIGKAQEEANLYAAKKADYINDCYSPPSAAGSSPYSLGPSSQGQMMSPPPGPATPQDSMYNMSMNGSD SYSSMGANVQSQANALRHVISQTAGYGDGMPPPSSASSIYDPGMHQTSELHP

>Locus_1_Transcript_58666/166847_Confidence_1.000_Length_605

AGAAGTTCAACTCCATCCAAGTCCAGCTCAAACAGAGCACGTGTGAAGCCGTCATGATCCTTAGGTCGAGGTTTCTAGACGCCAGGAGAAAAAGG CGGAACTTCAGCAAGCAGGCGACGGAGATCCTGAATGAGTATTTCTATTCTCATCTGAGCAATCCTTACCCTAGTGAAGAAGCAAAGGAAGAGCT TGCCAGGAAGTGTGGAATCACAGTCTCTCAGATATCAAATTGGTTTGGTAATAAAAGGATACGATATAAAAAGAACATAGGCAAAGCTCAAGAA GAGGCCAACCTTTATGCTGCGAAAAAAGCAGATTACATAAACGACTGCTACTCACCTCCTTCAGCAGCAGGTTCTTCACCTTACAGTCTGGCTCC TTCTTCGCAGGGCCAGATGATCAGTCCACCCCCGGGTTCGGGAACTCCTCAAGATTCCATGTACAATATGAGTATGAATGGTGGTGACTCCTATT CCTCTATGGGAGCCAACGTACAGTCACAGGCGAATGCGTTGAGGCACGTGATCTCTCAAACGGCGGGATATCCTGATGGCCTGCCACCACAATCT TCAGCCTCTATGTACGATCCTGCAGGCATGCATCAG

>Locus_1_Transcript_58666/166847

MILRSRFLDARRKRRNFSKQATEILNEYFYSHLSNPYPSEEAKEELARKCGITVSQISNWFGNKRIRYKKNIGKAQEEANLYAAKKADYINDCYSPP SAAGSSPYSLAPSSQGQMISPPPGSGTPQDSMYNMSMNGGDSYSSMGANVQSQANALRHVISQTAGYPDGLPPQSSASMYDPAGMHQ 


\subsubsection{2 exd2 paralog sequences}

>comp122137_c6_seq1

GTCTCGCATCCAGAAACCTGGACCGAAGGATCATGACGGCTTCGCACGTGCTCTGCTTGAGCTGGACCTGGATGGAGTTGAACTTCTTGTGGATG ATTTGGACCATTCGCTCGATCTCTTTCCCCGTGATGGGCCTCGTGCGGCTTTGCTCCCGTAACAGGTTCATAACGTGCGTCGTGAACTCGTTACAC GCCTGTTCGTATTTTTCTAGTTCCTGATGATAGATTTGGCGGATTTGGGCCAACTTGGCCCGATAGTCGGAGTGTTCGATGGCGTTTTCGGACAT GGGACCACTGGCCGCGGCCGCCGTCGCGTTAGCCGCTGCCGCTGAACCACCTCCTTTCTCGGGTCCTGCAACCCCTTCGGCTATGAGCATGTTGTC TAGCCTCATCAACTGAGGATCTGGTGGCTCTTCTTCTTGTGTGTTCCTCAAGCTCAAAACTGTTTTTTCTTTGATTTCACATAGAACGTTGAATA GGGCTGTTTTCATGCGATGGCAGTTGAGTGTGTGCTTCCGTGCTTGCGCTTCGTCCAAACTTTGGTCGGTGATGTTCATGATTTGTTGGAGGATT TCCGAGATGTCGTGCTTCCTGGGTTCTTGATCGGTCGGCGGACCCTGGTCGACACCGTGAGGTGGTTGCTGAGGCATTCCGTAACCGTGCTGAGG AACGGCCATGCTGACAGAAGGATGCTGCGATACTGGGTGCATCATTGTTTGTTGATCGTCCATGCTACCTTACGCCGAGCAGCCGCCAACAATCG CGAAGCCGCTATCGGCCGCTTCGCTTCTACAAGAGCTTCGGTCCAGCACTGGGCCGGTTATTTTTTTTTTCCTTCTCACTCTCTCTCTCTCGGGGG TCCTTTTCAGCAGCGAGTAAACACCGGAGACACTCTCGATGCACAACCATCCATAACGACCGCTTCGAAACTTTAACGGCGATATCAAGGGTCCC CTTCGACGCGTCTCCTTGTGTACAACACACACTCACACGCCACTGGAAAAAGTAGTAAACGGGGGGCCCCCACGACTGTCTCGTAATTGAGTGTT TTGATTGGGAATAGCGTTTTTTGACGGACAGCGAGCGGGTTTCTAGGGTGGGTTGAACGCTAATGCCAGTCAAGTCACCTCGCCATAAAAGATG AGCGTCTCGAGTGACGTAGCCGTCG

>comp122137_c6_seq1

MDDQQTMMHPVSQHPSVSMAVPQHGYGMPQQPPHGVDQGPPTDQEPRKHDISEILQQIMNITDQSLDEAQARKHTLNCHRMKTALFNVLCEIK EKTVLSLRNTQEEEPPDPQLMRLDNMLIAEGVAGPEKGGGSAAAANATAAAASGPMSENAIEHSDYRAKLAQIRQIYHQELEKYEQACNEFTTHV MNLLREQSRTRPITGKEIERMVQIIHKKFNSIQVQLKQSTCEAVMILRSRFLDAR

$>$ Pp_exd2_Nt310

ACTTTTTTTTTTTTTTTTTTTTTTTTTTTTTGTGTGAGTATGTGGCATGACTTTGACTTCCAGCGGGATACCAAAAACTAAACTCCGGGACCCAA TGGACAGAGACTAGGGATGCAGTTCCGTCGGCTGATGCATGCTGTCATACATTCCACCTGCTCCATCGGCGTAACCCGCCGTTTGGGAGATGACG TGCCTGAGGGCATTAGCCTGAGACTGTACATTGGCCCCCATGGACTGGTAGGAGTCCCCTCCTCCGTTCAAGCTCATGTTGTACATACTGTCCTG GGGTGCACCAGGTGGCGGCGGACTGATCATCTGACCAGGCTGCTGACCTTGGGACGTTGGCACTAGGTTATAAGGGGAAGAGCCCGCTGCTTTTT TGGCAGCGTAAAGGTTTGCTTCTTCTTGAGCTTTACCAATATTTTTCTTATATCTTATCCTCTTGTTACCAAACCAGTTGGAGACCTGAGAGACC GTGATGCCACACTTCCTCGCCAGTTCCTCTTTTGCTTCTTCACTAGGATAAGGGTTACTGAGGTGCGAATAGAAATACTCATTGAGGATTTCTGT CGCCTGTTTGGAAAAATTCCGCCTCTTCCGTCTCGCATCCAGAAACCTGGACCGAAGGATCATGACGGCTTCGCACGTGCTCTGCTTGAGCTGGA CCTGGATGGAGTTGAACTTCTTGTGGATGATTTGGACCATTCGCTCGATCTCTTTCCCCGTGATGGGCCTCGTGCGGCTCTGCTCCCGTAACAGGT TCATAACGTGCGTCGTGAACTCGTTACACGCCTGTTCGTATTTTTCTAGTTCCTGATGATAGATTTGGCGGATTTGGGCCAACTTGGCCCGATAG TCGGAGTGTTCGATGGCGTTTTCGGACATGGGACCACTGGCCGCGGCCGCCGTCCCGTTAACCGCTGCCGCTGAACCACCTCCTTTTTCGGGTCCT GCAACCCCTTCG

$>$ Pp_exd2_Nt310

MSENAIEHSDYRAKLAQIRQIYHQELEKYEQACNEFTTHVMNLLREQSRTRPITGKEIERMVQIIHKKFNSIQVQLKQSTCEAVMILRSRFLDARRK RRNFSKQATEILNEYFYSHLSNPYPSEEAKEELARKCGITVSQVSNWFGNKRIRYKKNIGKAQEEANLYAAKKAAGSSPYNLVPTSQGQQPGQMISP PPPGAPQDSMYNMSLNGGGDSYQSMGANVQSQANALRHVISQTAGYADGAGGMYDSMHQPTELHP

>Locus_1_Transcript_58675/166847_Confidence_1.000_Length_868

TATTCCCAATCAAAACACTCAATTACCTAGAGTCGGGGCGTTTAGTGGTGCTGTGAACGAACCACCCCAACACACAGTGCATAGAGAACTTTTAT GGATGGTTAGTGTGCGTGGGGATTGTTGAGAAGTAAGATGCTCGTCTGACAGTGAAGTCAGCGAGTGATTTTTATTGTGCTGTTGTGTGAGGCA TGGACGATCCACAACAAAGAATGATGCATCCTGTTTCACAGCATACTTCCGTAAGCATGGCCGGTCATGTTGTACCCCAACACGGCTACGGCATG CCCCAGCAACCGCATGGCGTGGATCCTAATAGTCAACCACCCCCACAAGACCAGGACGTTCGTAAACATGACATCTCTGAAATACTTCAACAGAT AATGAATATCACCGACCAGAGTCTTGACGAAGCGCAAGCACGGAAACATACACTCAACTGTCATCGTATGAAGCCAGCCCTATTCGGCGTGCTGT GTGAAATCAAGGAAAAAACAGTTCTAAGTCTACGGAATACACAAGAAGAAGAACCACCTGACCCTCAGCTAATGCGACTGGACAATATGCTTTT AGCTGAGGGTGTTGCTGGTCCAGAAAAAGGCGGTGGAGCTGGAGCTGCCGCTAATGCTTCAGCCGCAGCCTCAAGCGGTCCTCAGTCAGAAAACG CAATAGAGCACTCCGACTACCGCGCCAAATTAGCACAAATTCGCCAAATATATCACCAAGAGCTAGAGAAATATGAACAGGCATGTAACGAGTT CACGACGCACGTAATGAACCTGCTACGAGAACAAAGTCGAACAAGGCCGATAACGCCGAAAGAAATCGAGCGAATGGTTCAAATAATCCACAAG AAGCTCAACTCCATCCA

>Locus_1_Transcript_58675/166847

MDDPQQRMMHPVSQHTSVSMAGHVVPQHGYGMPQQPHGVDPNSQPPPQDQDVRKHDISEILQQIMNITDQSLDEAQARKHTLNCHRMKPALF GVLCEIKEKTVLSLRNTQEEEPPDPQLMRLDNMLLAEGVAGPEKGGGAGAAANASAAASSGPQSENAIEHSDYRAKLAQIRQIYHQELEKYEQACN EFTTHVMNLLREQSRTRPITPKEIERMVQIIHKKLNSI

$>$ pt_exd_2_Sk12

GTGTGAGATTAAGGAGAAGACAGTTCTAGGTCTACGAAATACACAAGAAGAAGAACCACCTGACCCTCAGCTAATGCGACTGGACAATATGCTT TTAGCTGAGGGTGTTGCTGGTCCAGAAAAAGGCGGTGGAGCTGGAGCTGCCGCTAATGCTTCAGCCGCAGCCTCAAGCGGTCCTCAGTCAGAAAA CGCAATAGAGCACTCCGACTACCGCGCCAAATTAGCACAAATTCGCCAAATATATCACCAAGAGCTAGAGAAATATGAACAGGCATGTAACGAG TTCACGACGCACGTAATGAACCTGCTACGAGAACAAAGTCGAACAAGGCCGATAACGCCGAAAGAAATCGAGCGAATGGTTCAAATAATCCACA AGAAGTTCAACTCCATCCAAGTCCAGTTGAAACAGAGTACGTGTGAAGCAGTCATGATCCTCAGGTCTAGGTTTCTCGACACCAGGAGAAAAAG GCGGAATTTTAGTAAACAAGCAACAGAAATCCTCAACGAATACTTTTATTCCCACCTCAGTAATCCCTACCCTAGTGAGGAGGCCAAAGAAGAAC TCGC 
$>$ pt_exd_2_Sk12_-_ORF_1_(frame_2)_translation

MRLDNMLLAEGVAGPEKGGGAGAAANASAAASSGPQSENAIEHSDYRAKLAQIRQIYHQELEKYEQACNEFTTHVMNLLREQSRTRPITPKEIER MVQIIHKKFNSIQVQLKQSTCEAVMILRSRFLDTRRKRRNFSKQATEILNEYFYSHLSNPYPSEEAKEEL 


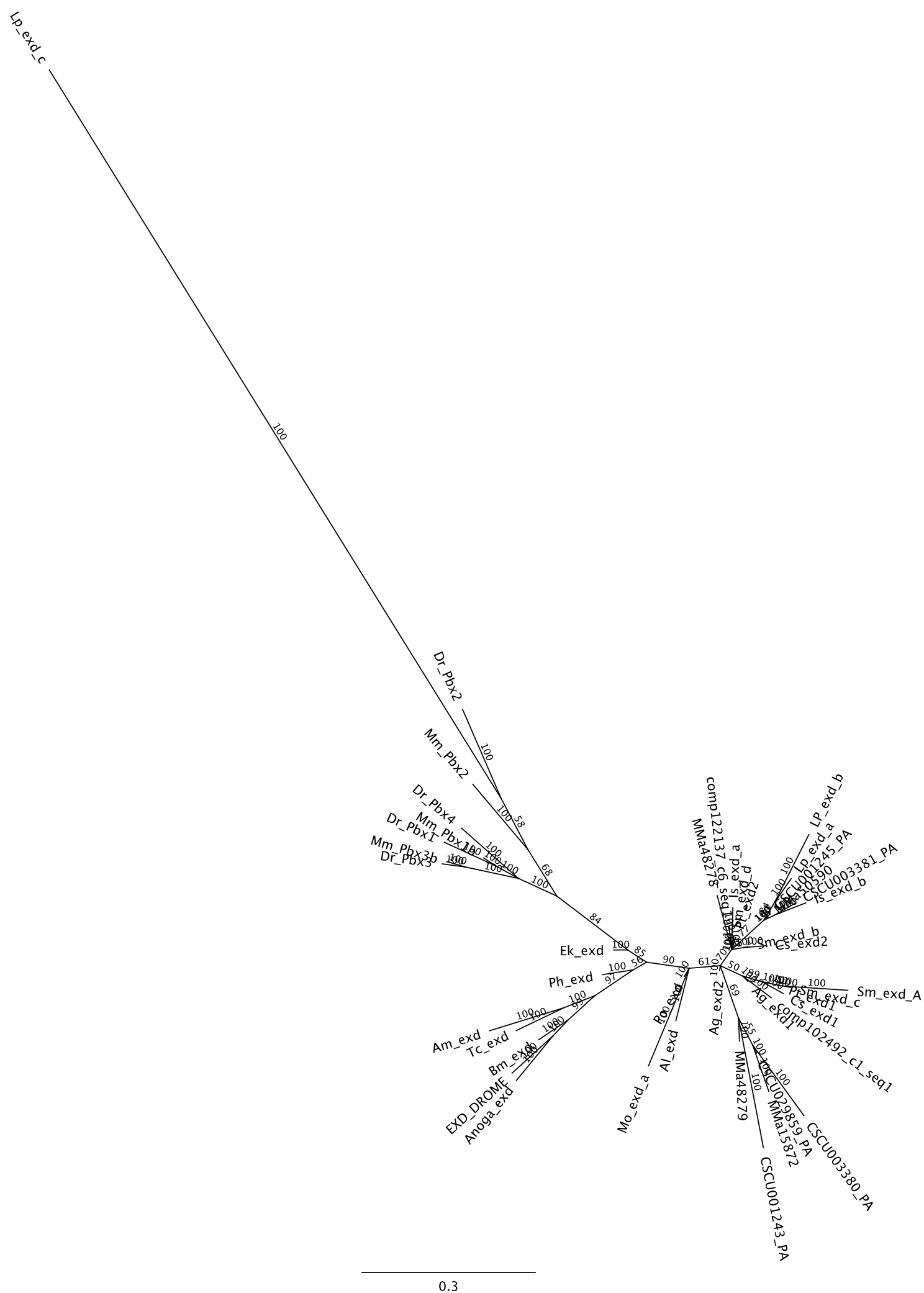

Supplemental Figure 3: Unrooted 50\% majority rule consensus tree to resolve exd relationships of the paralogs

Description according to Supplemental Figure 4. 


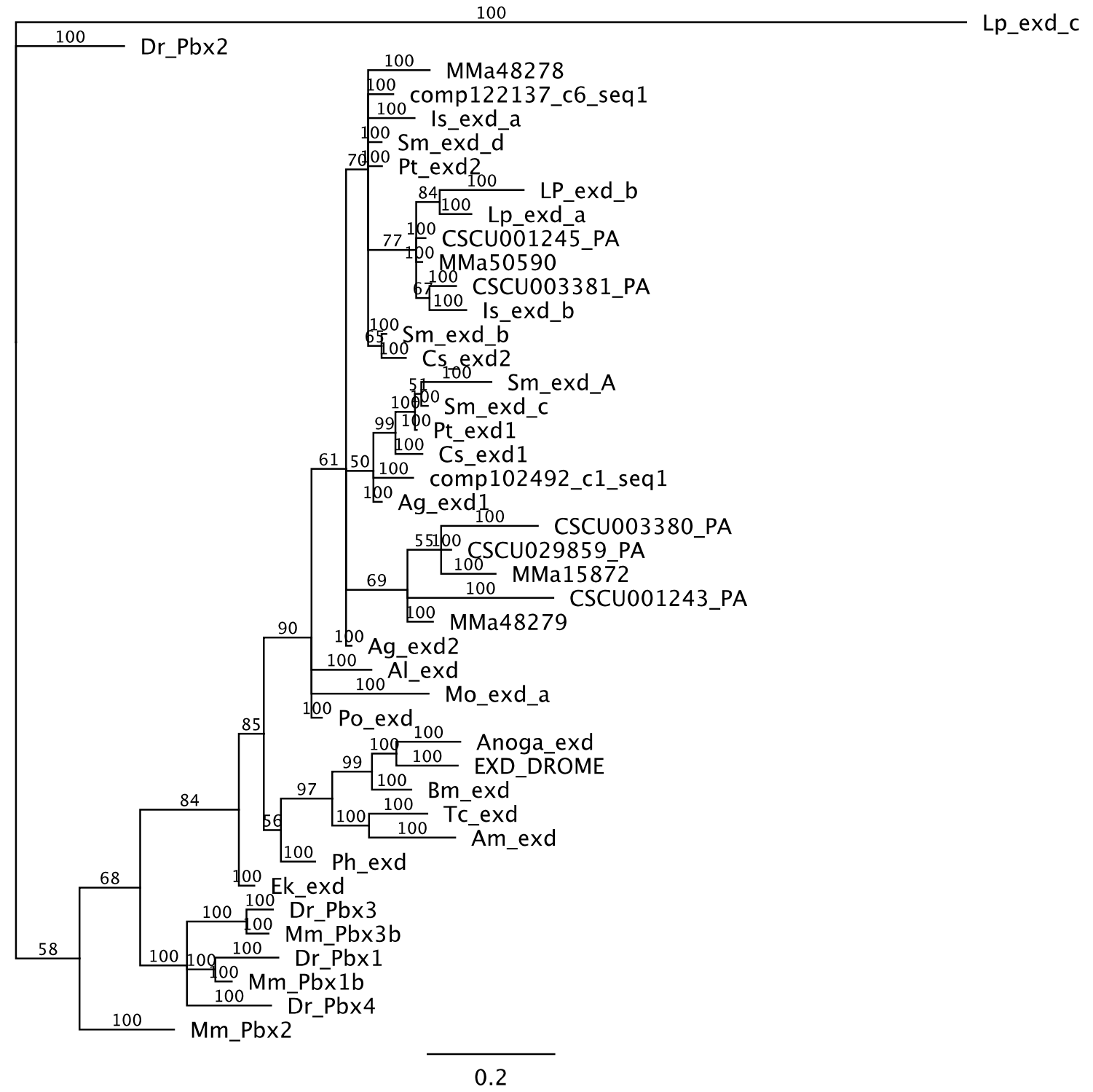

Supplemental Figure 4: Unrooted 50\% majority rule consensus tree to resolve exd relationships of the paralogs

The Jones amino acid substitution model was chosen after mixed model testing. After reaching 3000000 generations a total number of 12002 trees were written in two files (each file contained 6001 trees of which 4501 were sampled). 


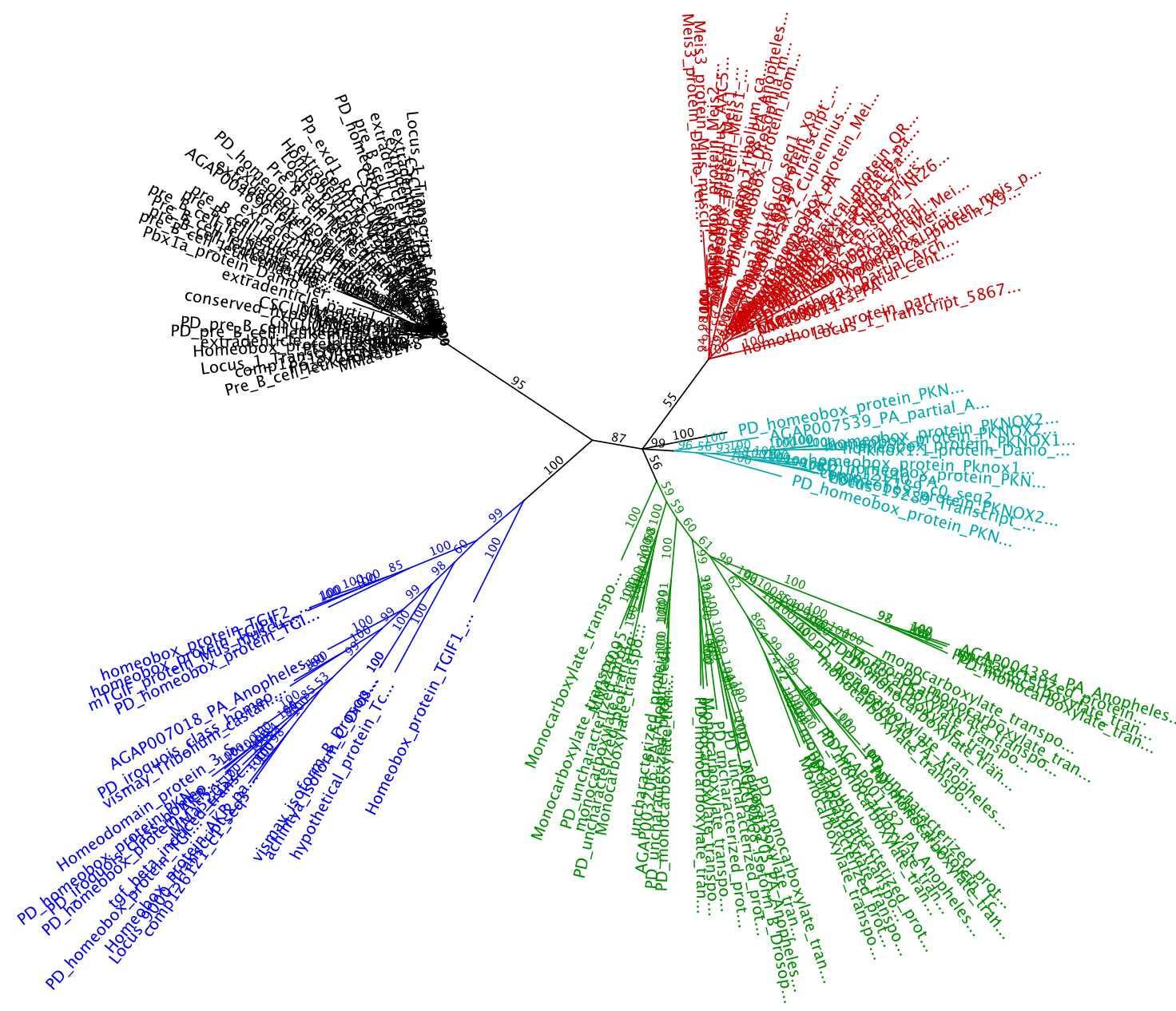

0.7

Supplemental Figure 5: Unrooted 50\% majority rule consensus tree to identify true exd and $h$ th homologs.

The Wag amino acid substitution model was chosen after mixed model testing. After reaching 3000000 generations a total number of 12002 trees were written in two files (each file contained 6001 trees of which 4501 were sampled). Colors indicate monophyletic groups in the tree. 


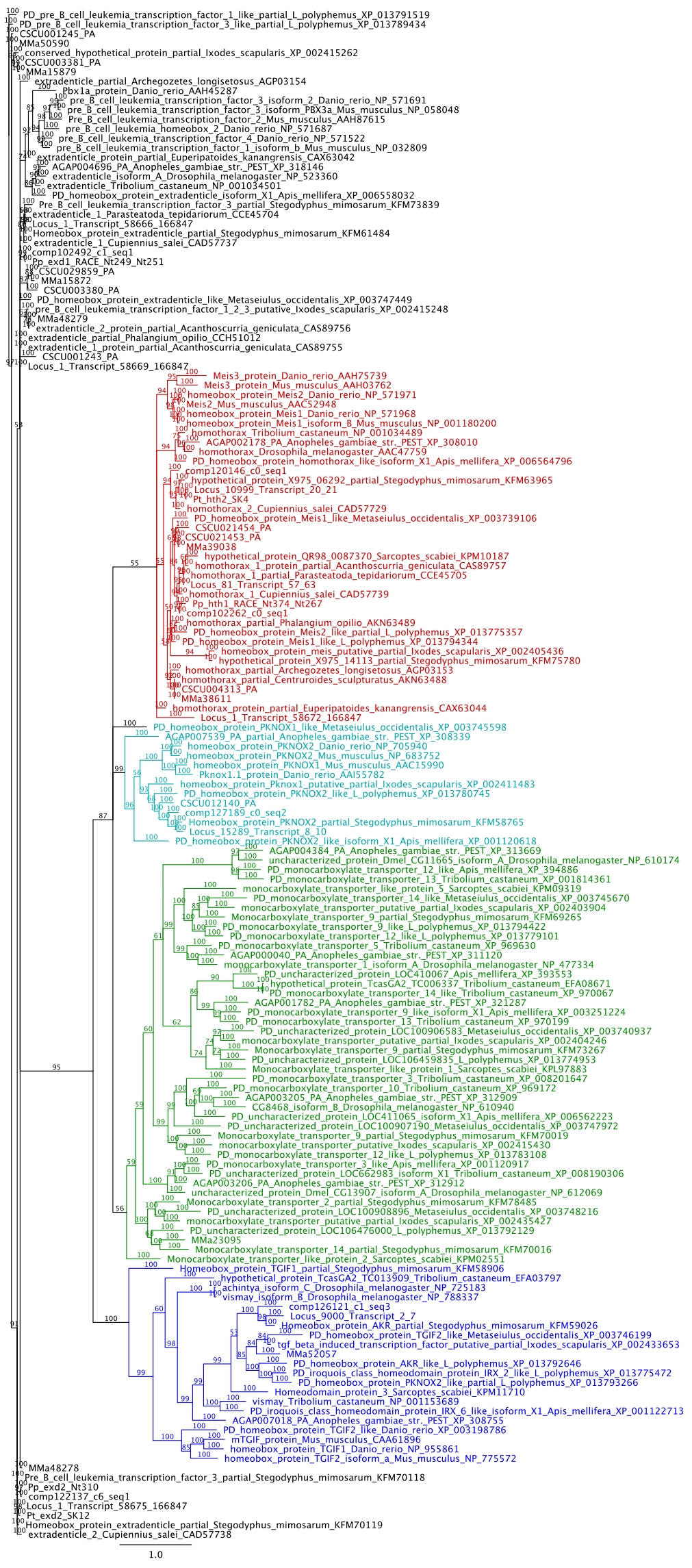


Supplemental Figure 6: Unrooted 50\% majority rule consensus tree to identify true exd and hth homologs.

The same phylogram as in Supplemental Figure 5 in a magnified view to display gene and species names. The third sequence with similarity to hth, Locus_1_transcript_58672 was transcribed into a protein with only 33 amino acids and thus excluded from further studies.

\section{General exd alignment:}

CLUSTAL O(1.2.1) multiple sequence alignment

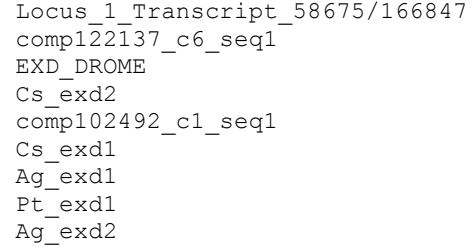

MDDPQQRMMHPVSQHTSV---SMAGHVVPQHGYGMPQQPHGVDPNSQ-PPPQDQDVRKHD -MDDQQTMMHPVSQHPSVS------MAVPQHGYGMPQQPP---HGVDQGPPTDQEPRKHD -MEDPNRML-----------AHTGGMMAPQGYGLSGQDDGQNAGSE-----NEVRKQKD -MDDPQRMMHPVSQHTS---VSMAGHVVPQHGYGMPQQPHDTT---GQAPPHDPDVRKHD -MDDQQRMMHPVSOHPSSAAASMGGHVVPOHGYGLPGP-HVVDPGSQOSTPDHODSRKHD -MNDQQTMMHPVSQMPS---ASMAGHVGAQHGYGLAQTSHV--PGSQGGTPD-QDAPKHD -MNDQQTMMHPVSQLPS---ASMAGHVGAQHGYGLAQPSHVVNQGSQGGTPD-QDAPKHD

ISEILQQIMNITDQSLDEAQARKHTLNCHRMKPALFGVLCE IKEKTVLSLRNTQEEEPPD ISE I LQQ IMNI TDQS LDEAOARKHTLNCHRMKTALFNVLCE I KEKTVLSLRNTQEEEPPD I GE I LQQIMS I SEQS LDEAQARKHTLNCHRMKPALFSVLCE IKEKTVLS IRNTQEEEPPD ISE ILQQIMNITDQS LDEAQARKHTLNCHRMKPALF SVLCE IKEKTVLSLRNTQEEEPPD ISE I LQQ IMNITDQS LDEAQARKHTLNCHRMKPALFNVLCE I KEKTVLSLRNTQEDEPPD ISEI LQQIMNITDQSLDEAQARKHTLNCHRMKPALFSVLCEIKEKTVLSLRNTQEDEPPD ----------------------------------VLSLRNTQEDEPPD ISEILQQIMNITDQSLDEAQARKHTLNCHRMKPALFSVLCEIKEKTVLSLRNTQEDEPPD -VLSLRNTQEEEPPD

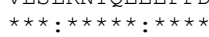

PQLMRLDNMLLAEGVAGPEKGGGAGAAANASAAASS----GPQSENAIEHSDYRAKLAQI PQLMRLDNMLIAEGVAGPEKGGGSAAAANATAAAAS----GPMSENAIEHSDYRAKLAQI PQLMRLDNMLIAEGVAGPEKGGGGAAAASAAAASQGGSLS IDGADNAIEHSDYRAKLAQI PQLMRLDNMLIAEGVAGPEKGAGSAANSAAA-------AGSPQSENAIEHSDYRAKLAQI PQLMRLDNMLIAEGVAGPEKGGGAGAAANATAATAAGG--PNQPENAIEHSDYRAKLAQI PQLMRLDNMP IAEGVAGPEKGGGASAAANASAAAAS---QGSQCENAIEHSDYRAKLAQI PQLMRLDNMLIAEGVAGPEKGGGAGAAANASAAA---SSGPGQAENAIEHSDYGAKLAQI PQLMRLDNMLIAEGVAGPEKGGGAGAAANASAAAASQSPGGPQAENAIEHSDYRAKLAQI PQLMRLDNMLIAEGVAGPEKGGGAGAAANASAAA----SSGPQSENAIEHSDYRAKLAQI

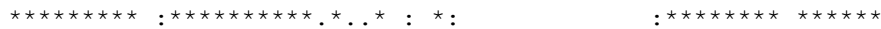

RQIYHQELEKYEQACNEFTTHVMNLLREQSRTRPITPKEIERMVQI I HKKLNSI----- ROIYHOELEKYEOACNEFTTHVMNLLREOSRTRPITGKE IERMVQI I HKKFNS I QVQLKO RQIYHQELEKYEQACNE FTTHVMNLLREQSRTRP I TPKE IERMVQ I I HKKF SS IQMQLKQ RQI YHQELEKYEQACNEFTTHVMNLLREQSRTRPITPKE IERMVQ I I HKKFNS IQVQLKQ RQIYHQELEKYEOACNEFTTHVMNLLREOSRTRPITTKE IERMVO I I HKKFNS I OVQLKO RQIYHQELEKYEQACNE FTTHVMNLLREQSRTRP I TPKE I ERMVQ I I HKKFNS IQVQLKQ RQIYHQELEKYEQACNEFTTHVMNLLREQSRTRPITPKE IERMVQI I HKKFNS IQVQLKQ RQIYHQELEKYEQACNEFTTHVMNLLREQSRTRP ITPKE IERMVQ I I HKKFNS IQVQLKQ RQ I YHQELEKYEQACNE FTTHVMNLLREQSRTRP I T PKE IERMVQ I I HKKFNS I RVQLKQ

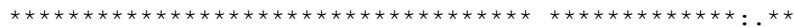
STCEAVMILRSRFLDARRKRRNFSKQASEI LNEYFYSHLSNPYPSEEAKEELARKCGITV STCEAVMILRSRF LDARRKRRNFSKQATE I LNEYFYSHLSNPYPSEEAKEELARKCGITV STCEAVMILRSRFLDARRKRRNFSKQATEI LNEYFYSHLSNPYPSEEAKEELARKCGITV STCEAVMILRSRFLDARRKRRNFSKQATEILNEYFYSHLSNPYPSEEAKEELARKCGITV STCEAVMILRSRFLDARRKRRNFSKQATEILNEYFYSHLSNPYPSSTCEAVMILRSRFLDARRKRRNFSKQATEILNEYFYSHLSNPYPSEEAKEELARKCGITV STCEAVMI LRSRFLDARRKRRNFSKQATEI LNEYFYSHLSNPYPS-------------SQVSNWFGNKRIRYKKNIGKAQEEANLYAAKKAA------------GASPYSMAGPPSGT SQVSNWFGNKRIRYKKNIGKAQEEANLYAAKKAGKPDLCDDFF---------------SQVSNWFGNKRIRYKKNIGKAQEEANLYAAKKADY INDCYSPPSAAGSSPYSLGPSSQGQ SQVSNWFGNKRIRYKKNIGKAQEEANLYAAKKADY INDCYSPPSAAGSSPYSLALSSQGQ SQISNWFGNKRIRYKKNIGKAQEEANLYAAKKADYINDCYSPPSAAGSSPYSLAPSSQGQ 
Locus 1 Transcript 58675/166847 comp $1 \overline{2} 2 \overline{1} 37$ c6_seq1

EXD_DROME

Cs è $\mathrm{exd} 2$

comp102492 c1 seq1

Cs exd1

Ag_exd1

Pt exd1

Ag_exd2

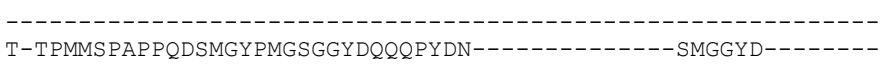

T-TPMMSPAP PQDSMGYPMGSGGY DQQQPYDN-----------SMGGYD------

MMSPPPGPATPQDSM-YNMSMNGSDSYSSMGANVQSQANALRHVISQTAGYGDGMPPPSS MISPPPGAGTPQESM-YNMSMNGGDSYSSMGANVQSQANALRHVISQTAGYADGLPPQST

MISPPPGSGTPQDSM-YNMSMNGGDSYSSMGANVQSOANALRHVISQTAGYPDGLPPQSS

Locus 1 Transcript 58675/166847

comp $1 \overline{2} 2 \overline{1} 37$ c 6 seq1

EXD DROME

Cs exd2

comp102492_c1 seq1

Cs_exd1

Ag_exd1

Pt exd1

Ag_exd2

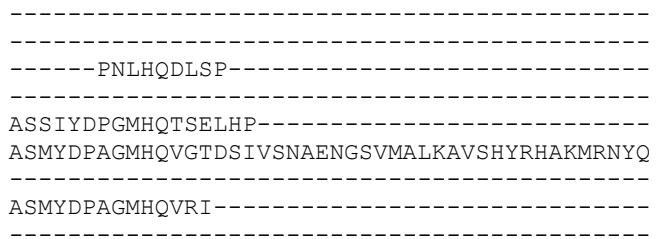

Supplemental Table 2: Identities of exd spider paralogs compared to $D$. melanogaster

\begin{tabular}{|c|c|c|c|c|c|c|c|c|c|}
\hline \multirow{3}{*}{$\begin{array}{l}\text { Locus_1_Transcript_5... } \\
\text { comp122137_c6_seq1 }\end{array}$} & Locus_1_T. & omp 122. & EXD_DROME & Cs_exd2 & comp 102. & Cs_exd1 & Ag_exd1 & Pt_exd1 & Ag_exd2 \\
\hline & & $85.02 \%$ & $69.43 \%$ & $85.15 \%$ & $85.28 \%$ & $84.65 \%$ & $90.55 \%$ & $84.85 \%$ & $95.24 \%$ \\
\hline & $85.02 \%$ & & $71.26 \%$ & $83.40 \%$ & $83.86 \%$ & $83.60 \%$ & $90.00 \%$ & $82.75 \%$ & $93.29 \%$ \\
\hline EXD_DROME & $69.43 \%$ & $71.26 \%$ & & $75.81 \%$ & $65.21 \%$ & $64.73 \%$ & $88.83 \%$ & $65.81 \%$ & $88.83 \%$ \\
\hline Cs_exd2 & $85.15 \%$ & $83.40 \%$ & $75.81 \%$ & & $83.92 \%$ & $83.68 \%$ & $90.34 \%$ & $83.78 \%$ & $92.00 \%$ \\
\hline comp102492_c1_seq1 & $85.28 \%$ & $83.86 \%$ & $65.21 \%$ & $83.92 \%$ & & $87.33 \%$ & $93.30 \%$ & $88.89 \%$ & $92.18 \%$ \\
\hline Cs_exd1 & $84.65 \%$ & $83.60 \%$ & $64.73 \%$ & $83.68 \%$ & $87.33 \%$ & & $93.82 \%$ & $95.09 \%$ & $94.35 \%$ \\
\hline Ag_exd1 & $90.55 \%$ & $90.00 \%$ & $88.83 \%$ & $90.34 \%$ & $93.30 \%$ & $93.82 \%$ & & $96.09 \%$ & $95.45 \%$ \\
\hline Pt_exd1 & $84.85 \%$ & $82.75 \%$ & $65.81 \%$ & $83.78 \%$ & $88.89 \%$ & $95.09 \%$ & $96.09 \%$ & & $94.97 \%$ \\
\hline Ag_exd2 & $95.24 \%$ & $93.29 \%$ & $88.83 \%$ & $92.00 \%$ & $92.18 \%$ & $94.35 \%$ & $95.45 \%$ & $94.97 \%$ & \\
\hline
\end{tabular}

\section{Alignment of exd1 in spider}

CLUSTAL O(1.2.1) multiple sequence alignment

comp102492_c1_seq1

Cs exd1

Ag_exd1

Pt_exd1

comp102492_c1_seq1

Cs_exd1

Ag exd1

Pt_exd1

comp102492_c1_seq1

Cs_exd1

Ag exd1

Pt_exd1

comp102492_c1_seq1

Cs exd1

Ag_exd1

Pt_exd1

comp102492_c1_seq1

Cs exd1

Ag_exd1

Pt_exd1
MDDQQRMMHPVSQHPSSAAASMGGHVVPQHGYGLPGP-HVVDPGSQQSTPDHQDSRKHDI MNDQQTMMHPVSQMPS---ASMAGHVGAQHGYGLAQTSHV--PGSQGGTPD-QDAPKHDI MNDQQTMMHPVSQLPS---ASMAGHVGAQHGYGLAQPSHVVNQGSQGGTPD-QDAPKHDI

SEILQQIMNITDQSLDEAQARKHTLNCHRMKPALFNVLCEIKEKTVLSLRNTQEDEPPDP SEILQQIMN ITDQSLDEAQARKHTLNCHRMKPALFSVLCE IKEKTVLSLRNTQEDEPPDP --------------------------------------------VLSLRNTQEDEPPDP SEILQQIMNITDQSLDEAQARKHTLNCHRMKPALFSVLCEIKEKTVLSLRNTQEDEPPDP

QLMRLDNMLIAEGVAGPEKGGGAGAAANATAATAAGG--PNQPENAIEHSDYRAKLAQIR QLMRLDNMPIAEGVAGPEKGGGASAAANASAAAAS---QGSQCENAIEHSDYRAKLAQIR QLMRLDNMLIAEGVAGPEKGGGAGAAANASAAA---SSGPGQAENAIEHSDYGAKLAQIR QLMRLDNMLIAEGVAGPEKGGGAGAAANASAAAASQSPGGPQAENAIEHSDYRAKLAQIR

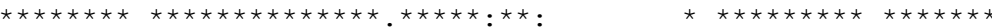

QIYHQELEKYEQACNEFTTHVMNLLREQSRTRP ITTKE IERMVQI I HKKFNS IQVQLKQS QIYHQELEKYEQACNEFTTHVMNLLREQSRTRP ITPKEIERMVQI I HKKFNS IQVQLKQS QIYHQELEKYEQACNEFTTHVMNLLREQSRTRP ITPKEIERMVQI I HKKFNS IQVQLKQS QIYHQELEKYEQACNEFTTHVMNLLREQSRTRP ITPKEIERMVQI I HKKFNS IQVQLKQS

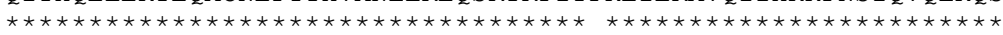

TCEAVMILRSRFLDARRKRRNFSKQATEILNEYFYSHLSNPYPSEEAKEELARKCGITVS TCEAVMILRSRFLDARRKRRNFSKQATEILNEYFYSHLSNPYPSEEAKEELARKCGITVS TCEAVMILRSRFLDARRKRRNFSKQATEILNEYFYSHLSNPYPS---------------TCEAVMILRSRFLDARRKRRNFSKQATE ILNEYFYSHLSNPYPSEEAKEELARKCGITVS

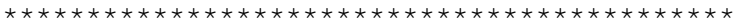




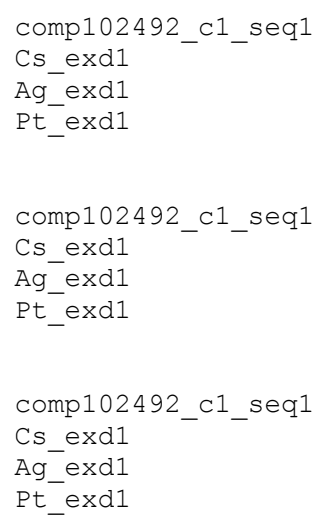

QVSNWFGNKRIRYKKNIGKAQEEANLYAAKKADYINDCYSPPSAAGSSPYSLGPSSQGQM QVSNWFGNKRIRYKKNIGKAQEEANLYAAKKADY INDCYSPP SAAGSSPYSLALSSQGQM

QISNWFGNKRIRYKKNIGKAQEEANLYAAKKADYINDCYSPPSAAGSSPYSLAPSSQGQM

MSPPPGPATPQDSMYNMSMNGSDSYSSMGANVQSQANALRHVISQTAGYGDGMPPPSSAS ISPPPGAGTPQESMYNMSMNGGDSYSSMGANVQSQANALRHVISQTAGYADGLPPQSTAS

ISPPPGSGTPQDSMYNMSMNGGDSYSSMGANVQSQANALRHVISQTAGYPDGLPPQSSAS

SIYDPGMHQTSELHP---------------------------

MYDPAGMHQVGTDSIVSNAENGSVMALKAVSHYRHAKMRNYQ

MYDPAGMHQVRI---

Supplemental Table 3: Identities of spider exd1

\begin{tabular}{|c|c|c|c|c|}
\hline \multirow[b]{2}{*}{ comp102492_c1_seq1 } & comp $102 \ldots$ & Cs_exd1 & Ag_exd 1 & Pt_exd1 \\
\hline & & $87.33 \%$ & $93.30 \%$ & $88.89 \%$ \\
\hline Cs_exd1 & $87.33 \%$ & & $93.82 \%$ & $95.09 \%$ \\
\hline g_exd1 & $93.30 \%$ & $93.82 \%$ & & $96.09 \%$ \\
\hline _exd1 & $88.89 \%$ & $95.09 \%$ & $96.09 \%$ & \\
\hline
\end{tabular}

\section{Alignment of exd2 in spiders}

CLUSTAL O(1.2.1) multiple sequence alignment

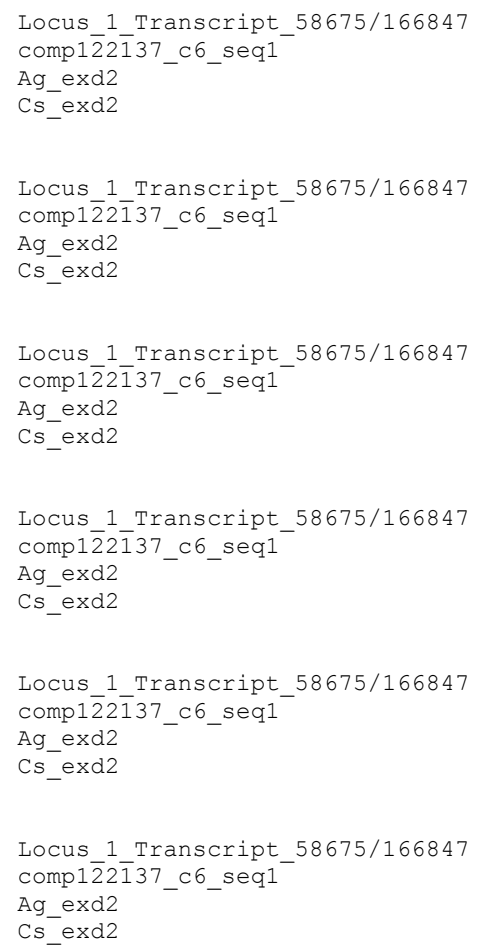

MDDPQQRMMHPVSQHTSVSMAGHVVPQHGYGMPQQPHGVDPNSQPPPQDQDVRKHDISEI -MDDQQTMMHPVSQHPSVSMA---VPQHGYGMPQQPPHG--VDQGPPTDQEPRKHDISE I -MDDPQRMMHPVSQHTSVSMAGHVVPQHGYGMPQQPHDT--TGQAPPHDPDVRKHDISEI

LQQIMNITDQSLDEAQARKHTLNCHRMKPALFGVLCEIKEKTVLSLRNTQEEEPPDPQLM LQQ IMN ITDQSLDEAQARKHTLNCHRMKTALFNVLCEIKEKTVLSLRNTQEEEPPDPQLM

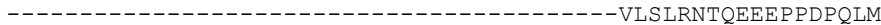
LQQIMNITDQSLDEAQARKHTLNCHRMKPALFSVLCEIKEKTVLSLRNTQEEEPPDPQLM

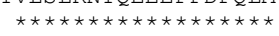

RLDNMLLAEGVAGPEKGGGAGAAANASAAASSGPQSENAIEHSDYRAKLAQIRQIYHQEL RLDNMLIAEGVAGPEKGGGSAAAANATAAAASGPMSENAIEHSDYRAKLAQIRQIYHQEL RLDNMLIAEGVAGPEKGGGAGAAANASAAAS SGPQSENAIEHSDYRAKLAQIRQIYHQEL RLDNMLIAEGVAGPEKGAGSAAN---SAAAAGSPQSENA IEHSDYRAKLAQIRQI YHQEI

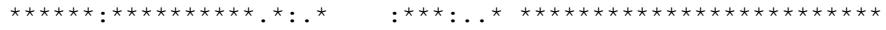

EKYEQACNEFTTHVMNLLREQSRTRPITPKEIERMVQI I HKKLNS I-------------EKYEQACNEFTTHVMNLLREQSRTRP ITGKE IERMVQ I I HKKFNS IQVQLKQSTCEAVMI EKYEQACNEFTTHVMNLLREQSRTRP ITPKE IERMVQ I I HKKFNS IRVQLKQSTCEAVMI EKYEQACNEFTTHVMNLLREQSRTRP ITPKE IERMVQI I HKKFNS IQVQLKQSTCEAVMI

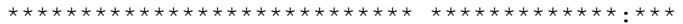
LRSRFLDARRKRRNFSKOATEI LNEYFYSHLSNPYPS - LRSRFLDARRKRRNFSKQATEI LNEYFYSHLSNPYPSEEAKEELARKCGITVSQVSNWFG

NKRIRYKKNIGKAOEEANLYAAKKAGKPDLCDDFF 
Supplemental Table 4: Identities of spider exd2

\begin{tabular}{|c|c|c|c|c|}
\hline \multirow{3}{*}{$\begin{array}{l}\text { Locus_1_Transcript_5... } \\
\text { comp122137_c6_seq1 }\end{array}$} & Locus_1_T... & comp $122 \ldots$ & Ag_exd2 & Cs_exd2 \\
\hline & & $87.56 \%$ & $98.39 \%$ & $88.89 \%$ \\
\hline & $87.56 \%$ & & $95.24 \%$ & $88.62 \%$ \\
\hline Ag_exd2 & $98.39 \%$ & $95.24 \%$ & & $93.71 \%$ \\
\hline Cs_exd2 & $88.89 \%$ & $88.62 \%$ & $93.71 \%$ & \\
\hline
\end{tabular}

\subsection{2 homothorax}

\subsubsection{1 hth1 paralog sequences}

>comp102262_c0_seq1 TTTTTTTTTAACATTTCACTTTTATTTTTGAATATTTTTTTTTAATTTAACAGGTTACAACAATACAATCATAAGCACGTCACATACAAATGCAG AGTCACCTATATGAGAAAAGTTCTATACAGATTACCACCGTTATATATATAATCAAACGGACAGAAGAAATAATAATAATAATTTCCAATTCAA ATATTTCTAATTATTCCCATCAAAACTGAAAAAAAAATATACTGTATAACATTAATATAATAATTAATAAGTGCCATGATTAAAACAATAACGG GGGTAAAAAAAAAAATCATTATATCCATCGCTAATAATGTCTCCATAAGAAATAAACGGAATCAATCACAGCACCATCATAGATAAAATAATAA TGAGCATTATTTTAATTAAAATCATTAATTATTTAAAATTATATTGCAGCAGCAGTAAGTGGCAATAATCATCAATATCTAATCACTATTGTAA AAATCGAGAAAAAATGTAAAAACGACCGTTTTTTTTTTTTTGTTCCTTGGTAAATTTGCAAAAAACCAATAAAAAATAACACGCCGTAACACAT GATTATTTTTATAAATTATTACAACAACATTGAAAAAATGTGCAGCTCCAAAATAAGAACAGAAAAAGCGAGCGAGAAGATACAGTATTGTATA TTATATATGCAGTATTGTCTATTCTTTTTAAATTTCAACATTATTTCAAATAATGTCCATACCGACATGACCACAATGAATTCTCGGGGTAAAGG GGAAGTAAAATTACACCGAATGAAATATAATGTGTATTATATTACATATAAAGAACTGGCCAACAGTCTCAATTCTCAAAACTCAACAGCAAAC AATTGTCATCTATAATAATAATTAATAATAATAATTAACGGAGCATCATCATCATTCAAGAGTTCGTTTTTCATCTGATTGGACTGGACAATCA ATTATTTTTATCATGATCAAATAGAGATAAGAGATAAAATACATTATCCATTATACACACACACGTGGCATCGACATGTAGAGTAGAGATAATA ATTATCATTACACATTATTTATCATTATTATTACAACGGCACATCTATAAAGAGAAGGGTTATATATAATTATGGCCATTTAGAAACACAAAAT AATAATTCGAATGTTTCAATCAAGCACAAAAAAATTTTTTTTGGGAGATTCATGTTTAATTTTATAGTTATAATAATTATTTTCGGCAACATGT AAGTATTTTTTTGTTCTATAGTTTTGCATTTCCCATGAAATTCCAGAGACATAATTTTTATGGGAATTTTTTGGGAAGAGGGTTAAAAATTTTT GCGGTAGATGAGGTGGGAAAAATCTTAAAAATTATAGTTTAATTATTTTTTTTTCCTGTTGGAAACAATGAATTTTTTTCCTCTAAAAAATTTT TTTTCCCTAAATTTTTGTATTTTTCATTTTTTAAAAAGGGACTTGTTTGTGTGATATAAGCTCCGAGGAAAAAAATGGCTACTTGCCGATTTAT TTTCATTTTTTTTTTTTTAGCAATAGCCCCATTTTTGAGGAGAAAAGGGTTTTTTGTTGTTATTGTCGTATAATTGTTTGTTTCTTGTAAGTTGA AATTGTTTAACTAGCGTGGATATCCATGATGTGCCCACCGGAACCGTCGTACGGAGATCCTTGCGGTGGCAATCCGGGATGTCCCATGGGGCCGT GCGCCATCATCATAGCGTGGGGATGTCCCGGAAGAAGCATTGCTTGCGAGTGAACGGGTGAACGCAGCTGAGACATCTGAGAATATCCGCCCATT CCTCCCATGTGGCCCATGCCCATGGCTCCGTCACTGCATGAAAGATTCTGCATACCTGGTGGGCGGATGTGCATCTGCTGGCTTCCATCCATCATG TATCCCATACCTGCGCCATCGGGTCCGTACGCAGCTGTAGCTCCACCAGCTCTGTTGGATTGGTCAATCATAGGCTGAACTATTCGCCGTCTTGCA TTGATAAACCAATTATTCACTTGCAGAATCGTCAGACCAGTATCTTGAGCAAGTTGCTTCTTCTGATCTTCAGAAGGGTATGGATGCGTAAGATG CTGGAAGAGCCAGGCTCTCATGATATTCGTGGCGACCTTCGGGAAGATTCCTCTCTTCTTCTGGCGTTTCTTACTTCTGTCGTCGTCGTCGTCTTC GCCGGTTCCATCTCCGGATCCAATACTAGCATCGCCGTAAGTCGAGACATCGGCTTCACTGTTGTTGTCGGTGCTCATCTGCGAACTCGGTTGTT GGGATATAGGTCCCGGTGTCCCTGTCGAATCAGCGGGCGACCTTGCATCCTCTCCTCCTGTGCTGTACGAATTCAGGGACTGCGACGGGGGCCTCT GGTCAGGCGTCGACGAATTGTCCGAACTGTGACCAGTCGTATCGGGATTGCCTCTGCCACCATTGCCACCAGCACCACCGCTTCCACCACCGGCGC CACCACCATTGCCACTGCCGCCATTGCTGCTGTTGTTGTTGTCACCGAGATCACCCGGTTTGGAATCTCTTTCGTCGATGACCAAATCGATGGGCA TCTTACCTTTCAGGCAACTGATATATCGTTGACAGAAGTTGTCGCATAACTCGTGCACCTTTTCCAGTTCCAACAGATGGAATCTCAAGACCTGA ATAGCTTGTACCATAAGGCTGTCTAATTCGGGATTTGGGGCATAATATGGTTTTTCTTGCCTTATCTGTTTGGCAAATACTGTAATGTCTTCATT GAAAGATTCCGAAGAGCAAACATCTCCACCAGCAATTCCAGGCTCTCGTGGAGTACACGTAGCTAATTCGCATTTCTCAAAAATTAGAGCCAACA ATGGAAACAATGGATGTCCGTATATAGCGTCTTTGTCGCGCTTGTTGTGAACATCGGGTACGGAACCCATAATATGATTGGACATGACACCGGAC ACATGATTACCGTGGTATTGATGCATAGATGGTGTGTGATTCATATGTGGAGCGTGTCCCAGCGATTGCATGGCCCTACTGTGGCCATGCGGATC GTAGAGCGACCCAGGTCCGTCCATGGACCCGTAATGCGACATACCTTCGTCGTACTGCATGGTGGCGAACGGGTGTTCCACAGGGTAAGGCTCAC TCTTCATAAAGAAGCTGCACACATTCACTACTTGAAAACAACTCTATAATTTAAATATTATCCTATTATTATTTTTTTAGAATATTTCTCAAAA AATTGATTCTTTTCCCGTGAATAAATACGAAAAATCTTCTGGAATCACTTTTTTTTAGATATATATATATCGATCGGAAGAGGTTAATGCCTGC AATATTAAAATCTTTTATTTTATTATTATCTTTTTTTTCACAAGAAACCAATATTAGAGAAGTTGTCACGCAATTTATAGATTCAATGGCTTCC TTTCACTTTTCGCAGCAAATTCTTATCTAGTTCTTCAATCGAAGTTATTACTTCAACCGTAACAACAACACCAACTGTTGTACAATAAATATTCT GTCGAAGAAAAAATCCTTCAAGATGGTAGTAGTAGCCACTTATGTGTATATAATATAATACACAGAAACACACACAAACACACTCACACACAAG AAACACCACTAACCGGTCTTCAAACTGGTTATCACACTTTTTCTCTCTCTTCTTTGAAGAAAAAAAAATTATATATAATAATAATAACAATTTTT TTCAGCAGCAACACCACAGGTCAGTCAGTAAATCGATGATGATGATGATGATAATAATAATGATAACAATAATATCATATATGTCCAGTCCGAT ATAAAACACAGGAAATTTCCAATAAATTTTTCTTACCGATCATAATTTTTTTTTTTTCCTCTTGGAGGGAAAAGGGGATGAAATAATTTTTTTT TTTAAATAATAAAATACGTCGATCGATTAAATTAATTAATTTTCAAATGCGAGGAATTATTTAATTAATTAATTTATTTTTTTTTGCAAGATCT AAACGCGCGCGAAGTTAAGAAGGAGCCGAGATGGAGAAGAAGTGCAAAGTGCGAATTGAATGGGAAATGCGCTTTTTGCGCTCTTTTTCCCCTC CCCGGTGGGTGGTTTTACCGCCAGTTGGCGACGGCAGCGCCTCCCCGTGGCGACGAAACTCAACTTGGAGATTATCTTCTATCTCTTTCCCCACTT TCTCCTTTCGCCAAGGGTAGGAAATAATGTGGAAAAAGAAATACAATTCATTAACTTAAATTTTTTTTTTTTACTAATTTATTTAAAATTTATC ATCCGTCTTAAAAATTATTCATTAGATGATTATTCTCTTAAAATTCTTTCGTTAGCGCTTTTTCTTCTCCCCGTGCTGAAAATTTTCCGATATTT TTAGGTAAAAAGAAGGGAAAAGTTTGGAAGAGCTGATTGGTTAG 
>comp102262_c0_seq1_-_ORF_16_(frame_2)_translation

MKSEPYPVEHPFATMQYDEGMSHYGSMDGPGSLYDPHGHSRAMQSLGHAPHMNHTPSMHQYHGNHVSGVMSNHIMGSVPDVHNKRDKDAIY GHPLFPLLALIFEKCELATCTPREPGIAGGDVCSSESFNEDITVFAKQIRQEKPYYAPNPELDSLMVQAIQVLRFHLLELEKVHELCDNFCQRYISCLK GKMPIDLVIDERDSKPGDLGDNNNSSNGGSGNGGGAGGGSGGAGGNGGRGNPDTTGHSSDNSSTPDQRPPSQSLNSYSTGGEDARSPADSTGTPG PISQQPSSQMSTDNNSEADVSTYGDASIGSGDGTGEDDDDDRSKKRQKKRGIFPKVATNIMRAWLFQHLTHPYPSEDQKKQLAQDTGLTILQVNN WFINARRRIVQPMIDQSNRAGGATAAYGPDGAGMGYMMDGSQQMHIRPPGMQNLSCSDGAMGMGHMGGMGGYSQMSQLRSPVHSQAMLLPG HPHAMMMAHGPMGHPGLPPQGSPYDGSGGHIMDIHAS

>Pp_hth1_RACE_Nt374_Nt267

TGTTTTCAAGTAGTGAATGTGTGCAGCTTCTTTATGAAGAGTGAGCCTTACCCTGTGGAACACCCGTTCGCCACCATGCAGTACGACGAAGGTAT GTCGCATTACGGGTCCATGGACGGACCTGGGTCGCTCTACGATCCGCATGGCCACAGTAGGGCCATGCAATCGCTGGGACACGCTCCACATATGA ATCACACACCATCTATGCATCAATACCACGGTAATCATGTGTCCGGTATCATGTCCAATCATATTATGGGTTCCGTACCCGATGTTCACAACAAG CGCGACAAAGACGCTATATACGGACACCCATTGTTTCCATTGTTGGCTCTAATTTTTGAGAAATGCGAATTAGCTACGTGTACTCCACGAGAGCC TGGAATTGCTGGTGGAGATGTTTGCTCTTCGGAATCTTTCAATGAAGACATTACAGTATTTGCCAAACAGATAAGGCAAGAAAAACCATATTAT GCCCCAAATCCCGAATTAGACAGCCTTATGGTACAAGCTATTCAGGTCTTGAGATTCCATCTGTTGGAACTGGAAAAGGTGCACGAGTTATGCGA CAACTTCTGTCAACGATATATCAGTTGCCTGAAAGGTAAGATGCCCATCGATTTGGTCATCGACGAAAGAGATTCCAAACCGGGTGATCTCGGTG ACAACAACAACAGCAGCAATGGCGGCAGTGGCAATGGTGGTGGCGCCGGTGGTGGAAGCGGTGGTGCTGGTGGCAATGGTGGCAGAGGCAATCC CGATACGACTGGTCACAGTTCGGACAATTCGTCGACGCCTGACCAGTCTTTCGTTCCTTACCAGAGGCCCCCGTCGCAGTCCCTGAATTCGTACAG CACAGGAGGAGAGGATGCAAGGTCGCCCGCTGATTCGACAGGGACACCGGGACCTATATCCCAACAACCGAGTTCGCAGATGAGCACCGACAACA ACAGTGAAGCCGATGTCTCGACTTACGGTAAGAATATATTTCAATTTATTCATTTATTTAAAAACCAATATGTTTCTTGTATTGTTGCTCAGTCT TTCATGGTTTGATGGTGAAATACAGTCAAACATACACACACACACACTCACTCACACTCTGTTATAATGACATTGCAGATTCCACGAAATTTGGT CGTTATAACCGAGGCTGACATTTTCAAGACCTTTTGACGAAAAATGTAATCAGGCGAAATGAAAAATATATACTGAGCATGAAGTGTTATTTCA TTTCATCAAATTATGTTGACCATATTTGATCGAGAAAAATGCGAGAAATGCGTGTGTGGAGATAATTCATACGCGACATACTTGATTCTCACCA AACATGTGGCCAATAGTCGGTGAAATCGCCAAATATTGCTTATGAGTCACCCATGGCGAGAAATACATTGCTACAGCAAGTCTGCTAAAATGCA TTTTACGGTGGAAAATTGAACATGATCCAATATGTGAGATGAAAATTTATAATACACGTGCTCAACCCCATGCACCACGATTTCAAACTTAATT TTGTAAAAAAATGGACTAAATAGCACAGTCCCAGATCACTTGAGTGTCCTGCGGCACAGTTCCAAATCCCTCAAGCGGCAAACGACATATTTTCA GAACACTTGAGGGACCCTTGCAGTTCCACATCACTGAAGCGGAGCACAACACGTTTTCAGATAACTTTAGTATCTCACGGCACATTTACAGATCT CAAGTAACTTGTGGTGCTGTTCCAGATCACTTAGTGGCCCGCGACACGTTTTCAAATCAATGAAATGGCCCGCGTACTCTGCGTTGATACCACTG CTTTCC

$>$ Pp_hth1_RACE_Nt374_Nt267

MKSEPYPVEHPFATMQYDEGMSHYGSMDGPGSLYDPHGHSRAMQSLGHAPHMNHTPSMHQYHGNHVSGIMSNHIMGSVPDVHNKRDKDAIYG HPLFPLLALIFEKCELATCTPREPGIAGGDVCSSESFNEDITVFAKQIRQEKPYYAPNPELDSLMVQAIQVLRFHLLELEKVHELCDNFCQRYISCLKG KMPIDLVIDERDSKPGDLGDNNNSSNGGSGNGGGAGGGSGGAGGNGGRGNPDTTGHSSDNSSTPDQSFVPYQRPPSQSLNSYSTGGEDARSPADST GTPGPISQQPSSQMSTDNNSEADVSTYGKNIFQFIHLFKNQYVSCIVAQSFMV

>Locus_81_Transcript_57/63_Confidence_0.052_Length_1558

TCAGAAATCTTGGAAGGATTGCGCCGGACAATAAGGGACGGTGCGCGGAATTTTTTCTTTAGCTTGTAGCCGGTGTGTGTTTGTGTATGTGTTG TCATTTGCAAGTGATTTAAGAGGTCAAAGTATACTTATAAAAATAATATAAAAAAGCACCGATTTGATACCGTTCTTAAAGGAAAACTGGATTT TTCGTAAGGAAGTTAATAAACAAAACTTTCCGGAAAGAAAAAAAAAAGTTTTGCAAAGTTTACTTGTTCACCTGTTTCGGCGCAATCCTTCCAA GATTTCTGATTTGTATATTATTTATTAAATGCTGTGATCGATTTATTTTGTGATCCTTGAAGGATAAAAATCGTCTGCCAAAAGGATATAATCG TTTGAAATATTAATTTAAATTATCTTTTGAGTGGTACGTTTGGTAAGTTAATCCTTTCGTGCGACAGCTATCTTTGACAGATTCTTGTAATTTG AATGGCCATAACAGACCGTTGCTTATGAAGAGTGAACCATACCCAATTGACCATTCCCTTGCTATGCAATACGAAGATGGTATGCCGCACTACGG CGGGATGGACGGTCCAACGTCCCTATACGACCCGCACGGGCATCGGGCAATGCAACCCCTATCTCATGCCCCACATATGAATCACACACCCTCAAT GCACCAGTATCACAGCAATCATGTATCCATGTCTAACCATATTATGGGAACTGTACCAGATGTTCATAAGAGAGATAAGGATGCCATATATGGG CATCCTCTGTTCCCTCTGCTAGCACTGATATTTGAAAAGTGTGAACTGGCCACGTGTACCCCCAGGGAGCCCGGTATAGCTGGCGGAGATGTCTG CTCGTCTGAATCTTTCAATGAAGACATAGCTGTCTTCGCCAAACAGATTAGACAAGAAAAACCATATTATTCTCCCAATCCCGAGTTAGACAGTC TTATGGTTCAAGCGATTCAAGTATTAAGGTTCCATTTATTAGAGTTAGAAAAGGTACACGAATTGTGCGATAACTTCTGCCAGCGTTACATTAG CTGTTTAAAAGGCAAAATGCCAATTGATTTAGTCATCGATGAAAGGGACAGCAAACCTGGTGACTTGGGAGACAATAATAATAACAGCAGTAAC GGTGGTGGTGGTGCTGGTGGTGGTAACAGTGGGGCAGGAGGTGGAAGGGGTAACCCTGACACCACTGGACACAGCTCAGACAACTCATCCACTCC AGACCAATCTTTTATACCCTACCAGAGACCACCTTCACAATCTCTAAACTCCTATAGTACCGGACCTGATGATGCCAGATCACCCGCCGGATCTAC AGGAACTCCAGGCCCTATTTCACAACAACCTAGTTCACAACTTAGTACTGACAACAACAGTGAAGCAGGTGATGCGAGTATCGGGTCTGGTGATG GAACGGGTGAGGATGATGATGATGATAGGAGTAAAAAACGTCAAAAGAAAAGAGGCATATTTCCAAAAGTAGCCACAAACATAATGAGGGCAT GGCTTTTTCAACATCTAACGCATCCCTATCCATCCGAAGATCAAAA

>Locus_81_Transcript_57/63_Confidence_0.052_Length_1558_-_ORF_1_(frame_3)_translation MTDSCNLNGHNRPLLMKSEPYPIDHSLAMQYEDGMPHYGGMDGPTSLYDPHGHRAMQPLSHAPHMNHTPSMHQYHSNHVSMSNHIMGTVPD VHKRDKDAIYGHPLFPLLALIFEKCELATCTPREPGIAGGDVCSSESFNEDIAVFAKQIRQEKPYYSPNPELDSLMVQAIQVLRFHLLELEKVHELCD NFCQRYISCLKGKMPIDLVIDERDSKPGDLGDNNNNSSNGGGGAGGGNSGAGGGRGNPDTTGHSSDNSSTPDQSFIPYQRPPSQSLNSYSTGPDDA RSPAGSTGTPGPISQQPSSQLSTDNNSEAGDASIGSGDGTGEDDDDDRSKKRQKKRGIFPKVATNIMRAWLFQHLTHPYPSEDQ

\subsubsection{2 hth2 paralogs sequences}

$>$ comp120146_c0_seq1

TAAAAAGTTAACAGAAACCAAACTCCTGTTATTACACGCACTACACGGAAAAACTTGGTCGGGTGAAGTCGCTTTCACGCGCGAGCGCCATCTGT GAGAATTCCGCGGCGGCACCTTCCACAAGTTTATCCGCCGGTTATCGGGTGGACTTCGGTGGTGACCATTTCTCTCGTGTTTATTTGTGTCTTTT TTTTCAATCGTTGAATAAATTGGTTTCGGGCTTTCCTTTGGAATAATAAAATAACGACGTACGATAATGCAGTATAACGAAGACGGCATCCCTC 
ACCCTTACGGAGTGGACGGTGGCGGGCCGCCGTCCCTTTACGACCCGCACCGGCCGATGCCGAACCTGTCCCATCACATGAACCACGGACCGTCCA ATAATCTCCATCAGTATGGCAACAGTCATGTCAACATCGCCAACCACGTCATGGGGTCCATGCCGGACGTTCACAAACGGGACAAAGACGCTATA TACGGGCATCCATTGTTTCCATTGTTGGCGCTAATCTTCGAAAAATGTGAACTGGCCACTTGTACGCCGAGGGAACCAGGCATACCCGGCAACGA TGTCTGTTCATCGGAGTCATTCAACGAAGACATCGCAGTTTTT GCCAAACAGATTAGGCAGGAGAGACCTTACTACAGCCCCGACGAGGAACTCG ACAGCATCATGGTACAAGCAATCCAAGTCTTGAGATTCCATCTATTGGAATTGGAGAAAGTCCACGAGCTGTGCGACAATTTCTGCCAGAGATAC ATCAGCTGCCTGAAGGGCAAGATGCCCATAGATTTGGTCATCGAGGAGAGGGACACCAAACCCGAACTCGGCGACACCAACAACAACAGCAACGG CAGCAGCTACTGCGGGGGCCCGCCGTGTGTCCCCAGGGGTATGATGGACACTTCGGGACACAGCACGGACAGCGCGTCCACTCCTGATCAGAGAC CACCATCGCAGAGTTTGAATTACGGTCCGGTCAGCGATGACGTCCGGTCGCCGGCGTCCGCGGGCACACCCTGCCCATTGTCCCAACAGCCCGCGT CCCAGCAGAGCACGGACAACAACAGCGAAGTCGGTGAGTGGGACGCCAGCATCGGATCTGGAGAAGGGACCGGCGACGAAGACGACGACGACAG AGCCAAGAAGAACCAGAAGAAACGTGGGATATTCCCCAAGGTCGCTACTAACATCATGAGGGCTTGGCTCTTCCAGCATCTCACGCATCCATATC CATCCGAGGACCAGAAGAAGCAGCTCGCTCAGGATACGGGTCTCACGATCCTTCAAGTCAACAATTGGTTCATCAATGCCAGGCGTAGAATTGTA CAGCCGATGATTGATCAGTCGAACAGAGCAGGTGGCAGTATCGGTCCACCAGGAGCGTCTTACAGCCCGGAGTCTTCTATGGGATACCTGATGGA TGGTGTGCCGCAGATGCATATACGACCAGGTCTCCAGGGATTGCCGGATTCGTCGATGGGTCACATGGGCTACTCCCAGCTCCGTTCTCCTGTCCA TTCCCAGGCCATGCTGATCCCGGGACACCACGCCATGATGATGTCTCACCCTGGGTTGCCGCCTCCACCTCCTCACGGATCGCCTTATGACAGTTC GCCTCCGAACATCATGGACCTTCACTCGAGCTGAGACTGTTCACTTCAAAAATATTATAAATTCACAATATATATATAATATATGTATCTCGATG TGTGAAAGAGATCGTTATGCGTGACTTAATATAACATAGATTTGTGGCGCCATCTATATATATGCTACCAGTTTTTTTTTTGTCTTGCTTTCTTT CCCATTGCACTCAACTCACTCATTCGTATGATGATAATATACGTATTTGTATGTACATAAACGAGAATTTATCGGTCGGTAATGTGTAAATAAG TAATTAGTGGCGTCTTGACGGGGCCCCGGGCCCCCGTAAACAGTCGAAAGTTCTTAATTTCTTCAATATCTCATTTCGTTTTGTTATTGCAATTT GATTCTTTGTCTCTCATTGCGATTAGATATCCTTTATAAATGTTGAAAAAATGCGGGCCACAAAGTTTTTGGACCCATGTACATGTTGTGTTCAA AAGTCCTCGCCTATAATGTGGGATTGATTTCCGATTTCTCCCATGTTGTCCCAGCATGTGGCAATATTTGGCACATTCCAGATGTAATACTAGAT GCATTCCTAAATGCAAGGCCGTTTTAAACACATGGACTCACTGGGGGAAGGGGTGCACAAAGGGCCGGCTCACTTGGCATCATTGTGGCCGGCTA GTAAGGCAAAATTTGTCGGCCATATGCGTGGTCAGAATTGTATGCGTCACTGTCGCACACATTGGAATTGTGATAGGATTGTACTCACCGCTGTG TTTGTGTATATTAGGAACTGCAGCAGGCTAGTAAGGGCCACAGTTCCACATGAATCGGCATCAGTTGCAGCAGTCTATAAGGGCCACAGTTCTCT GCAGATTGGCATTAATAGCAGTAGGGTTTTCGGTGCAATTGAAATTGCTTGTGGTAGGTTTGAAACAGTCCTATCTTATACGCATTTGCATTAA TCGTGACAGGATTGTAAAGACATGCCATTGTGTGCCATTGTGCCGTCACATTAGCACCAGTTATGACTGAATGTCATGCGTCACTGTTACATACA CGTTAGCACCAATAGTCGCCGGCTGGAAAGAGGCCACATATCCACATGAGTCGTCATCAGTTGTAGCATGGTGTAAGAGCCACAGTTTTCTGCAT ATTGTCATTAATATCGGCAGGATTACAGGCGCATTGCATTGAGATCAATTGCTGCAACAGTCCTATCCTATGAATTAATTCTAGCAGGTTTGTAA GGGCCACTGCATTATACATAGTGGCACCAACTCTGGGAGTATTTTATGCACCACTGTGTTACACACACACACACACACATAAGAAGCAATTACGG CCACAATCCTCCACACATCGGCATCGCTTTTGACAGGTTGCAAGAAACATAGTTCTCTACAAATTAATATTAATTTCAGCAAGACTTGCAACAGC CCTATCTTTTGCGCATTAGCATTTGTGGCAGAATTTCACCCACCGCTGTCCATCAATTATGACAAGCTAGTAATTGCCACAGTTCTCTACACATT GGTTGCGGTAGGGTTGTGGGCATTATATGATATTGACATCAGTTTTAGATTGTTTGTAACCTATCCTATGCAAATGTGAATTAATTCTAGCAGG TTTGTCGGCATGCTATGCACATTCGCATCAAATGATGCGGGAAAAATTGTATTCGATGACATGCACATATTCATAGGCATCAATTGTGGTAAAC CAGTTAGGGTCAAAGTTCTCTACATAATTAGAATCGATTGTGGAAGGGTTGCACTCGTCACACAAATCGACTCCAGTTGTCACATGCTTGTAAGA GCGAGCTTAAGTGGTACAACATAAATGGCTGTGCCACATCAGCCCAAAACCCCCGGAATGCCAGGAAGAGAGCCCGTAGTCCCCCAGAATTTCTG CGATCAATTTCCCAGCTGAAACACGTACTGTGTGAAGCCACATTCCTTGTCACATTCCCGGAAATAGCTTTGAATTACTTAATTTTTAAACTTTT GGAGTATAAAAATGAGTATAATTGTAATATTTCGCTTTTTAAATGATTTTATATAGTTAAGGACTATAATTATCTTTCATGTGTATTGAATTCG GAGGCACGAACGAAAAGGAATAATTTCTCAGAAATGTAACTTGAAATAATGTTGGAAATACATCGAGGGTATTCTTATTCCTATACATTTTTTG ATAAATATTCAAAGTAGAAATTTATGTATAGCTCCAATATTTGATGTAATAAAATCTTTCTCTCTCTCTCTTCAAAAAAAA

>comp120146_c0_seq1

MQYNEDGIPHPYGVDGGGPPSLYDPHRPMPNLSHHMNHGPSNNLHQYGNSHVNIANHVMGSMPDVHKRDKDAIYGHPLFPLLALIFEKCELATC TPREPGIPGNDVCSSESFNEDIAVFAKQIRQERPYYSPDEELDSIMVQAIQVLRFHLLELEKVHELCDNFCQRYISCLKGKMPIDLVIEERDTKPELGD TNNNSNGSSYCGGPPCVPRGMMDTSGHSTDSASTPDQRPPSQSLNYGPVSDDVRSPASAGTPCPLSQQPASQQSTDNNSEVGEWDASIGSGEGTG DEDDDDRAKKNQKKRGIFPKVATNIMRAWLFQHLTHPYPSEDQKKQLAQDTGLTILQVNNWFINARRRIVQPMIDQSNRAGGSIGPPGASYSPES SMGYLMDGVPQMHIRPGLQGLPDSSMGHMGYSQLRSPVHSQAMLIPGHHAMMMSHPGLPPPPPHGSPYDSSPPNIMDLHSS

>Locus_10999_Transcript_20/21_Confidence_0.221_Length_2489

CTCCCAACTTCCCAAAAGCGAACAAATTATGCAGGAAAGAAAGTTTGTGATTTTCGCCAACCCTCGCCAAGCATTCCACGATTATTTATCATTCC GTTCAAGTTGACGGTGGGTGCAAAGAAAGGGGTGGGTATAAAAGCGCCCCCTACAGGAAACCGGCTGAGCGAGGGGCGCGAAATTTTCTCGTGT TGTTTTGATCGTCAGATCCAACACGTGGTTGGAAGAGTAACGTTGTCGACATGCAATATCCTGAAGATGGGATGCCTCATTACGGCCATGGGGAT GGATCGGCCGGCGGTCTATATGACCCCCATAGGCAAAATCTTATGAACCACCATGGTGTTTATCATGCCAATCATGTGTCTATTGCTAATCATGT CATGGGATCTACACCTGATGTCGGAAAAAGGGATAAAGATGCCATTTTTGGACACCCGCTTTTTCCTCTGTTAGCCTTGATCTTTGAAAAATGTG AGCTGGCCACCTGCACCCCTAGGGAACCAGGCATCGCTGGGGGAGACGTCTGTTCCTCAGAGTCTTTCAATGAGGATATCGCCTGCTTTGCAAAA CAGATTAAGGAAGAAAGACCATTGTATGATGCCAATCCTGAACTAGATAGCCTTATGGTGCAAGCAATCCAAGTGCTTAGATTCCATTTGTTAG AATTGGAAAAAGTTCATGAGCTCTGTGATAACTTTTGTCAGCGTTACATCAGCTGCCTAAAGGGTAAGATGCCTATTGATTTGGTTATCGAAGA GAGAGATACTAAACCTGAGTTAGGGGACACCAATAACAACAGCAACGGTAGCAGTTTCTGTGGTGGTCCTCCCTGTGTCTCCAGGGGTATGCTGG ATACTTCCGGCGGACATAGTACTGATAGTGGCTCTACACCAGATCAGGGCCACTATGAAGACATGTCTGTGGGAAATATGGAAAGGCTAGGGAG ACCACCTTCACAATCCCTGAATTATGGTTCCGTAGGCGATGATGTTCGGTCCCCCACGGGTTCAACTGGCACTCCATGTCCTCTGTCACAACAGCC TTCTTCCCAACAAAGTACAGACAATAACAGTGAAGCTGGTGATGCGAGTATTTGTTCAGGAGAAGGCTCAGGTGATGAGGATGATGATGAAAGG GGTGGTAAGAAAAGGCAGAGGAAGAGGGGTATTTTTCCTAAAGTAGCGACAAACATACTGAGGGCATGGCTATTTCAGCATCTTACGCACCCGT ATCCATCTGAAGATCAAAAAAAGCAGCTTGCTCAAGACACAGGTCTTACGATTCTACAGGTAAACAATTGGTTTATCAATGCCCGTAGAAGAAT AGAGTCTTTAAATTCTGGCTCCAATTGTACTCATATTTTGAGGATTAAAATTTTTAATCCGGCAGGGAAAGCATAACCATGTTTTTGAGAGGTA ATTGATCTCTAACACCAAGTAAAATGCTTAAAATTTACTCTTCAAACCATAGTTTAATAAGTGAAAATTGTGTCATTACAGGTTTATCAATGCC CGTAGAAGAATAGTTCAACCTATGATTGATCAGTCAAACAGAGCTGGAGGGACTATGGGAACACCAGGACCGACATACAGCCCTGAATCCTCTG GTATGGGCTATATGATGGATGGAGTGTCGCCCATGCATATCAGATCATCCAGCCTGCAGAATTTGCCTTGCCCAGAGAACATGACCATTGGCCAT ATGGCAGGATATTCTCAGCTCAGGTCACCGGTCCACTCACAAGGAATGCTTCTTTCTGGACACCCTATGATGATGTCACATGGTGCGCTACCCCC ACCTCCGCATGGATCGCCTTATGATAGTTCTCCCCCCAGTATTATGGACTTGCACAATAGTTAGTTTATAAACCAATATTCTTGATATGGATAAT TTTGTGTTGTGTAGAAATTTGTCCATTTTGTATACCTCCCCCAAAAAAACCTTAATCATGTTCATTTTTGTAGTGCAATAATAATTTTATTTCAA GTGTCATAAATTTATTGTTACTGTGGCTCTTCCACTGCAATAATGCTCGTCAATTCTACAGGTTTTAAAATTATTAAGTTAAAGAAAGAAT TTT 
TTTAGCATAACTTTAGTTCTTATCCTTTATTGTTAATAACTTCGTCTGAAATTAAACATATACGTATTAACAATATAACTTAAAAAACAATATT TTAAAATGCTACAAATTTTAACCACTTACTTAAAAGAAATCAATTATTTAAATACATTTTAGCTGATATATGTTGTTAATTTTAATATAGTTTT TATCATTATAAATTGTGTATTTTATGTTTTTAAAAAAAATAGTTTGTGTTCAAATAATTGTCGGAATGTTTAGTGATTTTTGTACAAATGAATG GTCATGTTAAGTTAAAGTGTGTATCTTCTTAGAAAAATGTTTTAAAATGTGATGATGATTGCTGTAAAACGTCTTTGTATATAAATATAACACT GAATAAATTAATCAGTATTAAATTAAAAAAAAA

>Locus_10999_Transcript_20/21_Confidence_0.221_Length_2489_-_ORF_2_(frame_3)_translation MQYPEDGMPHYGHGDGSAGGLYDPHRQNLMNHHGVYHANHVSIANHVMGSTPDVGKRDKDAIFGHPLFPLLALIFEKCELATCTPREPGIAGGD VCSSESFNEDIACFAKQIKEERPLYDANPELDSLMVQAIQVLRFHLLELEKVHELCDNFCQRYISCLKGKMPIDLVIEERDTKPELGDTNNNSNGSSF CGGPPCVSRGMLDTSGGHSTDSGSTPDQGHYEDMSVGNMERLGRPPSQSLNYGSVGDDVRSPTGSTGTPCPLSQQPSSQQSTDNNSEAGDASICSG EGSGDEDDDERGGKKRQRKRGIFPKVATNILRAWLFQHLTHPYPSEDQKKQLAQDTGLTILQVNNWFINARRRIESLNSGSNCTHILRIKIFNPAG KA

>Pt_hth2_sk4_complete_extraction

GACAAGGATGCAATATACGGTCACCCGCTTTTTCCTCTGTTAGCCTTGATCTTTGAAAAATGTGAGCTGGCCACCTGCACCCCTAGGGAACCAGG CATCGCTGGGGGAGACGCCTGTTCCTCAGAGTCTTTCAATGAGGATATCGCCTGCTTTGCAAAACAGATTAAGGAAGAAAGACCATTGTATGAT GCCAATCCTGAACTAGATAGCCTTATGGTGCAAGCAATCCAAGTGCTTAGATTCCATTTGTTAGAATTGGAAAAAGTTCATGAGCTCTGTGATA ACTTTTGTCAACGTTACATCAGCTGCCTAAAGGGTAAGATGCCCATTGATTTGGTTATCGAAGAGAGAGATACTAAACCTGAGTTAGGGGACAC AAATAACAACAGCAACGGTAGCAGTTTCTGTGGTGGTCCTCCCTGTGTCTCCAGGGGTATGCTGGATACTTCCGGCGGACATAGTACTGATAGTG GCTCTACACCAGATCAGGGCCACTATGAAGACATGTCTGTGGGAAATATGGAAAGGCTAGGGAGACCACCTTCACAATCCCTGAATTATGGTTCC GTAGGCGATGATGTTCGGTCCCCCACGGGTTCAACTGGCACTCCATGTCCTCTGTCACAACAGCCTTCTTCCCAACAAAGTACAGACAATAACAG TGAAGCTGGTGATGCGAGTATTTGTTCAGGAGAAGGCTCAGGTGATGAGGATGATGATGAAAGGGGTGGTAAGAAAAGGCAGAAGAAGAGGGG TATTTTTCCTAAAGTAGCAACAAACATAATGAGGGCATGGCTATTTCAGCATCTTACGCACCCGTATCCATCTGAAGATCAAAAAAAGCAGCTTG CTCAAGACACAGGTCTTACGATTCTACAGGTCAACAACTGGTTCATCAATGC

$>$ Pt_hth2_sk4_complete_extraction_translation DKDAIYGHPLFPLLALIFEKCELATCTPREPGIAGGDACSSESFNEDIACFAKQIKEERPLYDANPELDSLMVQAIQVLRFHLLELEKVHELCDNFCQ RYISCLKGKMPIDLVIEERDTKPELGDTNNNSNGSSFCGGPPCVSRGMLDTSGGHSTDSGSTPDQGHYEDMSVGNMERLGRPPSQSLNYGSVGDDV RSPTGSTGTPCPLSQQPSSQQSTDNNSEAGDASICSGEGSGDEDDDERGGKKRQKKRGIFPKVATNIMRAWLFQHLTHPYPSEDQKKQLAQDTGL TILQVNNWFIN

Supplemental Table 5: Accession number, gene names and organism used for the phylogenetic tree of $h$ th

\begin{tabular}{|l|l|l|}
\hline Name & Accession & Description \\
\hline Ag_hth1 & CAS89757 & Acanthoscurria geniculata hth1 partial \\
\hline Ag_hth2 & CAS89758 & Acanthoscurria geniculata hth2 partial \\
\hline Aga_hth & XM_308010 & Anopheles gambiae str. PEST AGAP002178-PA \\
\hline Al_hth & AGP03153 & homothorax, partial [Archegozetes longisetosus] \\
\hline Am_hth & XM_006564734 & $\begin{array}{l}\text { PREDICTED: Apis mellifera homeobox protein } \\
\text { homothorax-like }\end{array}$ \\
\hline Cs_hth1 & CAD57739 & Cupiennius salei-hth1 \\
\hline Cs_hth2 & CAD57729 & Cupiennius salei-hth2 \\
\hline Cscu_hth & AKN63488 & homothorax, partial [Centruroides sculpturatus] \\
\hline Dm_hth & AAN13474 & Drosophila melanogaster hth isoform c \\
\hline Dr_meis1b & NP_571968 & Danio rerio-meis1b \\
\hline Dr_meis2 & NP_571971 & Danio rerio-meis2 \\
\hline Dr_meis3 & NP_571853 & Danio rerio-meis3 \\
\hline Dr-meis4.1 & AF376049_1 & Danio rerio-meis4.1 \\
\hline Ek_hth & CAX63044 & $\begin{array}{l}\text { Euperipatoides kanangrensis partial mRNA for homothorax } \\
\text { protein (hth gene), isoform A }\end{array}$ \\
\hline
\end{tabular}




\begin{tabular}{|l|l|l|}
\hline Lp_hth & AKN63487 & homothorax, partial [Limulus polyphemus] \\
\hline Mm_meis1 & NP_034919 & Mus musculus-meis1 \\
\hline Mm_meis2 & NP_001153040 & Mus musculus-meis2 \\
\hline Mm_meis3 & AAI17533 & Mus musculus-meis3 \\
\hline Of_hth & AAS93633 & Oncopeltus faciatus hth \\
\hline Ph_hth & CA098908 & Parhyale hawaiensis hth \\
\hline Po_hth & AKN63489 & homothorax, partial [Phalangium opilio] \\
\hline Tc_hth & NP_001034489 & Tribolium castaneum hth \\
\hline
\end{tabular}

Supplemental Table 6: Identity of $h$ th 1 paralogs to $D$. melanogaster $h t h$

\begin{tabular}{l|l|}
\cline { 2 - 2 } & \multicolumn{1}{c}{ Dm_hth } \\
\cline { 2 - 2 } Dm_hth & \\
\cline { 2 - 2 } Pp_hth1_comp102262 & $50.9 \%$ \\
\cline { 2 - 2 } Cs_hth1 & $51.7 \%$ \\
\cline { 2 - 2 } Ag_hth1 & $53.0 \%$ \\
\hline Pt_hth1_Locus_81 & $51.2 \%$ \\
\hline
\end{tabular}

Supplemental Table 7: Identity of $h$ th 2 paralogs to D. melanogaster hth

\begin{tabular}{l|l|}
\cline { 2 - 2 } & \multicolumn{1}{c}{ Dm_hth } \\
\cline { 2 - 2 } Dm_hth & \\
\cline { 2 - 2 } Pp_hth2_comp120146 & $49.9 \%$ \\
\cline { 2 - 2 } Pt_hth2_Locus10999 & $47.3 \%$ \\
\hline Ag_hth2 & $41.1 \%$ \\
\hline Cs_hth2 & $45.9 \%$ \\
\hline
\end{tabular}

\section{Spider hth1 alignment:}

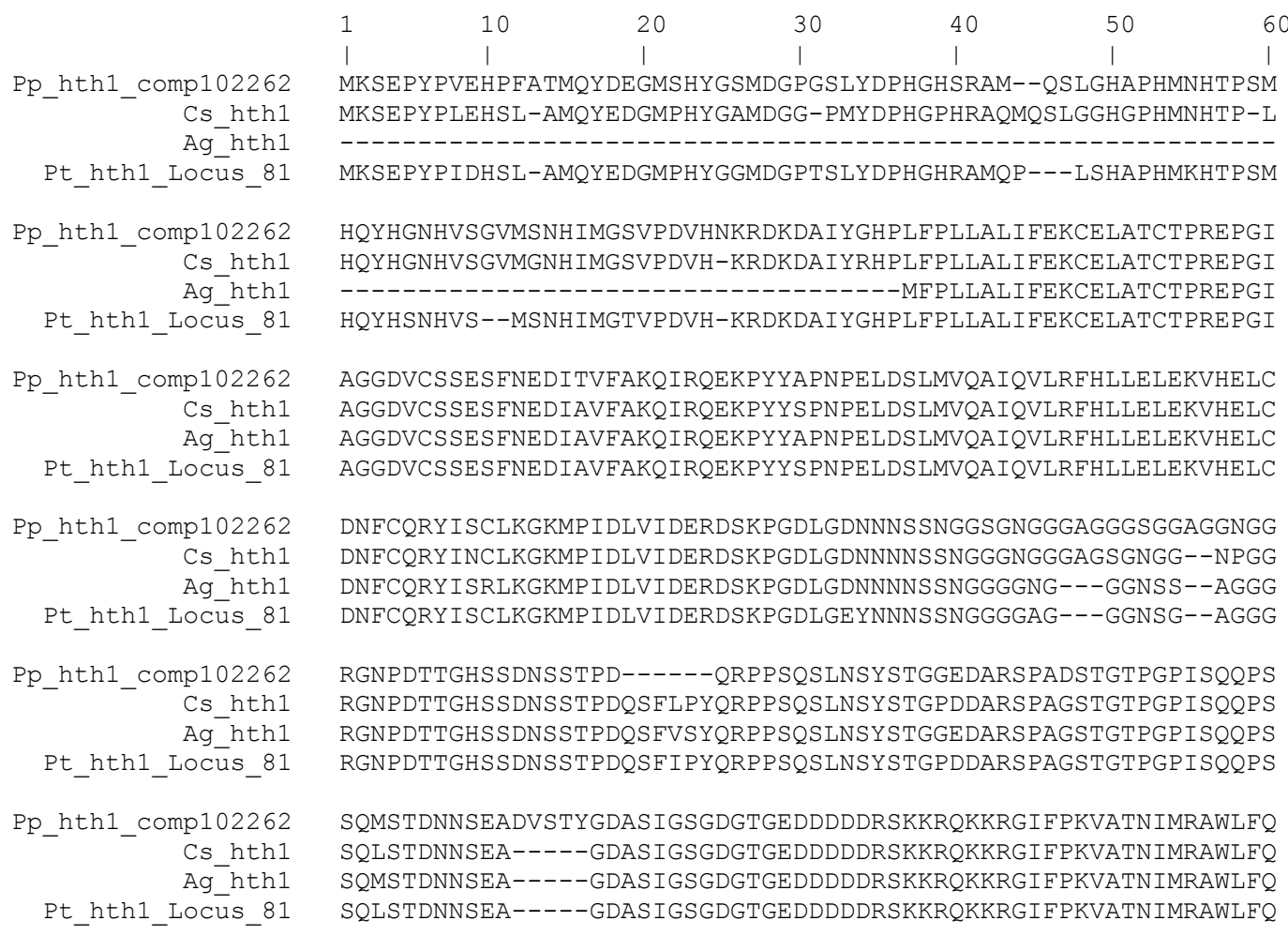




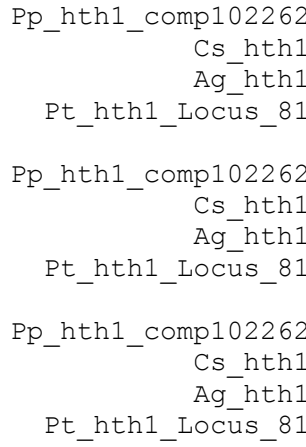

HLTHPYPSEDQKKQLAQDTGLTILQVNNWF INARRRIVQPMIDQSNRAGGATAAYGPDGA HLTHPY PSEDQKKQLAQDTGLT ILQVNNWF INARRR IVQPMI DQSNRAGGASAAYGPEGA

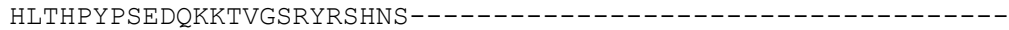

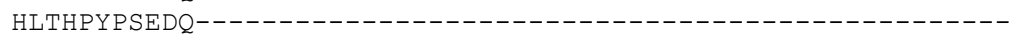
GMGYMMDGSQQMHIRPPGMQNLSCSDGAMGMGHMGGMGGYSQMSQLRSPVHSQAMLLPGH GMGYMMDGAQQMH IRPPGMQNLSCSEGAMGMGHMGGMGGYSQMSQLRSPVHSQAMLLPGH -

PHAMMMAHGPMGHPGLPPQGSPYDGSGGHIMDIHAS PHAMMMAHGPMGHPGLPPQGSPYDASGGHIMDIHAS -

Supplemental Table 8: Identity of $h$ th 1 spider paralogs

\begin{tabular}{|c|c|c|c|c|}
\hline & Pp_hth1_c... & Cs_hth1 & Ag_hth1 & Pt_hth1_Lo... \\
\hline Pp_hth1_comp 102262 & & $88.372 \%$ & $85.417 \%$ & $84.054 \%$ \\
\hline Cs_hth1 & $88.372 \%$ & & $89.324 \%$ & $88.950 \%$ \\
\hline Ag_hth1 & $85.417 \%$ & $89.324 \%$ & & $95.472 \%$ \\
\hline Pt_hth1_Locus_81 & $84.054 \%$ & $88.950 \%$ & $95.472 \%$ & \\
\hline
\end{tabular}

\section{Spider hth2 alignment:}

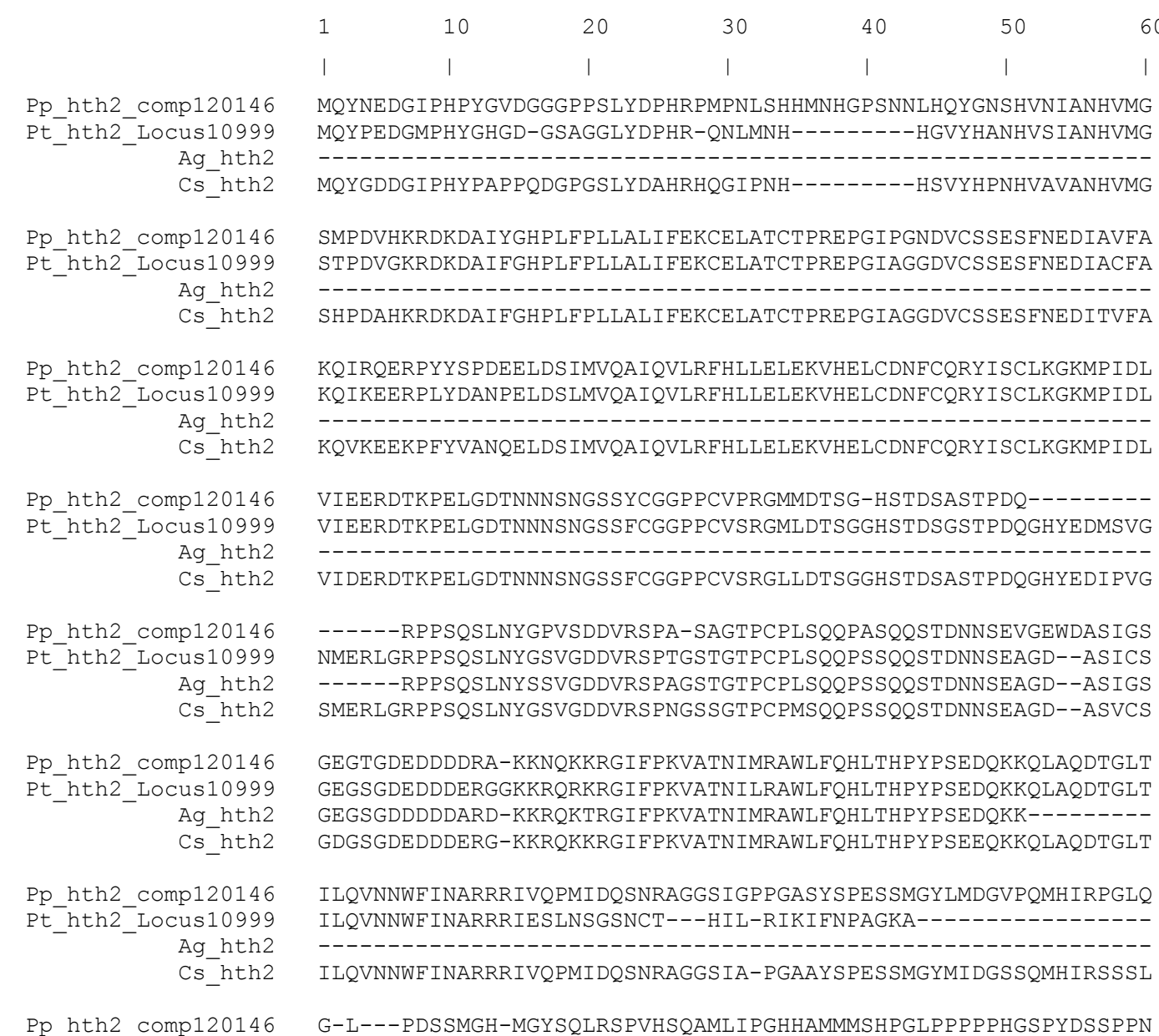




\begin{tabular}{|c|c|}
\hline $\begin{array}{r}\text { Pt_hth2_Locus10999 } \\
\text { Ag_hth2 }\end{array}$ & 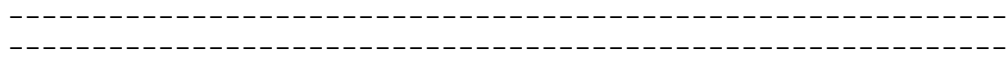 \\
\hline Cs_hth2 & QNLSCPENMAIGHMAGYSQLRSPVHSQAMLLPGHP-MMMSHPSLP--PPHSSPYDSSPPS \\
\hline Pp hth2 comp120146 & IMDLHSS \\
\hline Pt hth2 Locus10999 & ------- \\
\hline Ag hth2 & ------ \\
\hline Cs_hth2 & IMDLHSS \\
\hline
\end{tabular}

Supplemental Table 9: Identity of $h$ th2 spider paralogs

\begin{tabular}{l|c|c|c|c|}
\multicolumn{1}{c}{ Pp_hth2_c... Pt_hth2_Lo... } & Ag_hth2 & \multicolumn{1}{c}{ Cs_hth2 } \\
\cline { 2 - 5 } Pp_hth2_comp120146 & & $72.457 \%$ & $84.615 \%$ & $75.309 \%$ \\
\cline { 2 - 5 } Pt_hth2_Locus10999 & $72.457 \%$ & & $90.291 \%$ & $81.586 \%$ \\
\cline { 2 - 5 } Ag_hth2 & $84.615 \%$ & $90.291 \%$ & & $88.235 \%$ \\
\hline Cs_hth2 & $75.309 \%$ & $81.586 \%$ & $88.235 \%$ & \\
& &
\end{tabular}

\section{General hth alignment:}

CLUSTAL O(1.2.1) multiple sequence alignment

\begin{tabular}{|c|c|}
\hline Dm_hth & 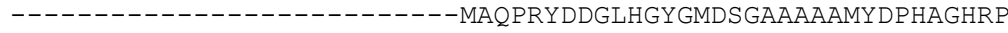 \\
\hline Pt-hth1 & MTDSCNLNGHNRPLLMKSEPYPIDHSL-AMQY-EDGMPHYGGM--DGPTSLYDPHGHRAM \\
\hline Cs_hth1 & $---------------M K S E P Y P L E H S L-A M Q Y-E D G M P H Y G A M--D G-G P M Y D P H G P H R A$ \\
\hline Pp_hth1 & $--------------M K S E P Y P V E H P F A T M Q Y-D E G M S H Y G S M--D G P G S L Y D P H G H S R A$ \\
\hline Pp-hth2 & -----------------------------MQYNEDGI PHPYGVDGGGPPSLYDPHRPMPN \\
\hline Cs_hth2 & --------------------------- MQYGDDGI PHYPAP PQDGPGSLYDAHRHQGI \\
\hline Pt-hth2 & 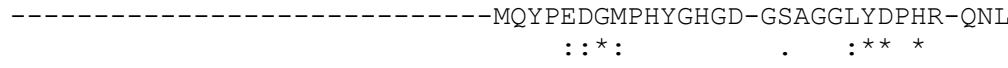 \\
\hline
\end{tabular}

Dm_hth

Pt-hth1

PGLQGLPSHHSPHMTHAAAAAATVGMHGYHSGAGGHGTPSHVSPVGNHLMGAI PEVH-KR $-\mathrm{QP}---\mathrm{L}-\mathrm{SHAPHMNHTP}------$ SMHQYHSN-------HVS--MSNH IMGTVPDVH-KR -QMQSLG-GHGPHMNHTP-------LHQYHGN-------HVSGVMGNHIMGSVPDVH-KR --MQSL--GHAPHMNHTP------SMHQYHGN-------HVSGVMSNHIMGSVPDVHNKR $------\mathrm{L}-\mathrm{SH}--\mathrm{HMNHGPSN}----\mathrm{NLHQYGNS}-------\mathrm{HVN}--$ IANHVMGSMPDVH-KR $------\mathrm{P}-\mathrm{NH}---------------\mathrm{H}$ SVYHPN-------HVA--VANHVMGSHPDAH-KR $------\mathrm{M}-\mathrm{NH}---------------$ HGVYHAN-------HVS--IANHVMGSTPDVG-KR

Pp hth 1

$\mathrm{Pp}-\mathrm{hth} 2$

Cs hth2

Pt-hth2

$$
:{ }^{\star *}:{ }^{*}:{ }^{*}: \text {. }
$$

Dm hth

Pt-hth1

Cs hth1

Pp_hth1

Pp-hth2

Cs hth2

Pt-hth2

DKDAIYEHPLFPLLALIFEKCELATCTPREPGVQGGDVCSSESFNEDIAMFSKQIRSQKP DKDAIYGHPLFPLLALIFEKCELATCTPREPGIAGGDVCSSES FNEDIAVFAKQIRQEKP DKDAIYRHPLFPLLALIFEKCELATCTPREPGIAGGDVCSSES FNEDIAVFAKQIRQEKP DKDA IYGHPLFPLLALI FEKCELATCTPREPGIAGGDVCSSESFNEDITVFAKQIRQEKP DKDA IYGHPLFPLLALI FEKCELATCTPREPG I PGNDVCSSES FNEDIAVFAKQIRQERP DKDAIFGHPLFPLLALI FEKCELATCTPREPGIAGGDVCSSESFNEDITVFAKQVKEEKP DKDAIFGHPLFPLLALI FEKCELATCTPREPGIAGGDVCSSESFNEDIACFAKQIKEERP

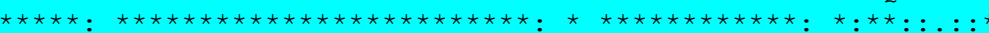

Dm hth

Pt-hth1

YYTADPEVDSLMVQAIQVLRFHLLELEKVHELCDNFCHRY I SCLKGKMPIDLVIDERDTT YYSPNPELDSLMVQA IQVLRFHLLELEKVHELCDNFCQRY I SCLKGKMP I DLVIDERDSK YYSPNPELDSLMVQAIQVLRFHLLELEKVHELCDNFCQRY INCLKGKMP I DLVIDERDSK YYAPNPELDSLMVQA IQVLRFHLLELEKVHELCDNFCQRY I SCLKGKMP IDLVIDERDSK YYSPDEELDS IMVQAIQVLRFHLLELEKVHELCDNFCQRY ISCLKGKMPIDLVIEERDTK FYVANQELDS IMVQA IQVLRFHLLELEKVHELCDNFCQRY ISCLKGKMP I DLVIDERDTK LYDANPELDSLMVQA IQVLRFHLLELEKVHELCDNFCQRY I SCLKGKMP I DLVIEERDTK

$\mathrm{Pp}$ hthl

Pp-hth2

Cs hth2

Pt-hth2

Dm hth

Pt-hth1

KPPELGSANGE-

GRSNAD--STSHTDGASTPD-------PGDLGDNNNNSSNGGGGAGG-----GNSGAGGGRGNPDT-TGHSSDNSSTPDQS----Cs hth1 Pp hth1 $\mathrm{Pp}-\mathrm{hth} 2$ Cs hth2 -PGDLGDNNNNSSNGGGNGGGAGSGNG--GNPGGRGNPDT-TGHSSDNSSTPDQS-----PGDLGDNNNSSNGGSGNGGGAGGGSGGAGGNGGRGNPDT-TGHSSDNSSTPD-------

-PE-LGDTNNNSNGSSYCGGPPCV---------PRGMMDTSG-HSTDSASTPDQ------PE-LGDTNNNSNGSSFCGGPPCV--------SRGLLDTSGGHSTDSASTPDQGHYEDI -PE-LGDTNNNSNGSSFCGGPPCV---------SRGMLDTSGGHSTDSGSTPDQGHYEDM Pt-hth2 
Dm_hth Pt-hth1 Cs hth1 Pp hth1 Pp-hth2 Cs hth2 Pt-hth2

Dm_hth Pt-hth1 Cs_hth1 Pp_hth1 Pp-hth2 Cs_hth2 Pt-hth2

Dm_hth Pt-hth1 Cs_hth1 Pp_hth1 Pp-hth2 Cs hth2 Pt-hth2

Dm_hth Pt-hth1 Cs_hth1 Pp_hth1 Pp-hth2 Cs hth2 Pt-hth2

Dm_hth Pt-hth1 Cs hth 1 Pp hth1 Pp-hth2 Cs_hth2 Pt-hth2

Dm_hth Pt-hth1 Cs_hth1 Pp hth1 Pp-hth2 Cs hth2 Pt-hth2
-------VRPPSSSLSYGGAMNDDARSP-GAGSTPGPLSQQPPALDTSDPDGKFLSSLN ----FI PYQRPP SQS LNSYSTGPDDARS PAGSTGTPGPISQQPS SQLSTDNN-----------FLPYQRPPSQSLNSYSTGPDDARSPAGSTGTPGPISQQPSSQLSTDNN--------------QRPPSQSLNSYSTGGEDARSPADSTGTPGPISQQPSSQMSTDNN---------------RPPSQSLN-YGPVSDDVRSPA-SAGTPCPLSQQPASQQSTDNN-------PVGSMERLGRPPSQSLN-YGSVGDDVRS PNGSSGTPCPMSQQPSSQQSTDNN-------SVGNMERLGRPPSQSLN-YGSVGDDVRSPTGSTGTPCPLSQQPSSQQSTDNN--------

$$
\star \star \star \star * \star . \quad{ }^{\star} \cdot{ }^{\star \star \star}:{ }^{\star \star \star}{ }^{\star \star \star \star \star}:::^{\star}:
$$

PSELTYDGRWCRREWSSPADARNADASRRLYSSVFLGSPDNFGTSASGDASNASIGSGEG ------------------------------------ SEA-----GDAS IGS GDG -- - - - - - -

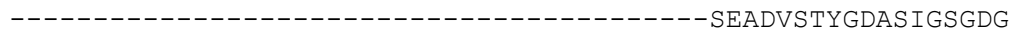
-----------------------------------SEAGD-----ASVCSGDG . $\quad * \star: * \star: *$

TGEEDDDA-SGKKNQKKRGIFPKVATNILRAWLFQHLTHPYPSEDQKKQLAQDTGLTILQ TGEDDDDDRS-KKRQKKRGI FPKVATNIMRAWLFQHLTHPYPSEDQ-----------TGEDDDDDRS-KKRQKKRGIFPKVATNIMRAWLFQHLTHPYPSEDQKKQLAQDTGLTILQ TGEDDDDDRS -KKRQKKRGI F PKVATNIMRAWLFQHLTHPYPSEDQKKQLAQDTGLTILQ TGDEDDDDRA-KKNQKKRGIF PKVATN IMRAWLFQHLTHPY PSEDQKKQLAQDTGLTILQ SGDEDDDERG-KKRQKKRGIFPKVATNIMRAWLFQHLTHPYPSEEQKKQLAQDTGLTILQ SGDEDDDERGGKKRQRKRGIFPKVATN I LRAWLFQHLTHPYPSEDQKKQLAQDTGLTILQ

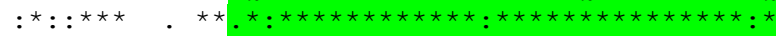

VNNWFINARRRIVQPMIDQSNRAVYTPHPGPSGYG--HDAMGYMMDSQAHMMHRPPGDPG VNNWFINARRRIVQPMIDQSNRAGGASA----AYGPEGAGMGYMMDGAQQMHIRPPGMQN VNNWF INARRRIVQPMIDQSNRAGGS I GP PGASYSP-ESSMGYLMDGVPQMH IRPGLQGVNNWF INARRRIVQPMIDQSNRA GGS IA-PGAAYSP-ESSMGYMIDGSSQMHIRSS SLQN VNNWFINARRRIESLNSGSNCT---HIL-RIKIFNP-AGKA------------------

FH--------------QGYPHYPPAEYY--GQHL-------------------------LSCSEGAMGMGHMGGMGGYSQMSQLRSPVHSQAMLLPGHPHAMMMAHGPMGHPGLPPQGS LSCSDGAMGMGHMGGMGGYSQMSQLRSPVHSQAMLLPGHPHAMMMAHGPMGHPGLPPQGS L---PDS-SMGH----MG---YSQLRS PVHSQAMLI PGHHAMMMSH---PGLPPPPPHGS LSCPENM-AIGHM---AG---YSQLRS PVHSQAMLLPGHP-MMMSH---PSLP--PPHSS 


\subsection{3 dachshund}

\section{Species abbreviations}

Af $=$ Artemia fransciscana (brine shrimp)

$\mathrm{Ag}=$ Acanthoscurria geniculata (bird spider)

$\mathrm{Cs}=$ Cupiennius salei (American wandering spider)

$\mathrm{Dm}=$ Drosophila melanogaster (fruit fly)

$\mathrm{Gg}=$ Gallus gallus (chicken)

$\mathrm{Gm}=$ Glomeris marginata (pill millipede)

Hs = Homo sapiens (human)

$\mathrm{Mm}=$ Mus musculus (house mouse)

$\mathrm{MMa}=$ Mesobuthus martensii (bark scorpion)

$\mathrm{Na}=$ Neanthes arenaceodentata (polchaete worm)

Of $=$ Oncopeltus fasciatus (milkweed bug)

$\mathrm{Ot}=$ Onthophagus taurus (horned beetle)

$\mathrm{Pd}=$ Platynereis dumerilii (polychaete worm)

$\mathrm{Pp}=$ Pholcus phalangioides (cellar spider)

$\mathrm{Pt}=$ Parasteatoda tepidariorum (common house spider)

$\mathrm{Sk}=$ Saccoglossus kowalevskii (acorn worm)

$\mathrm{Sp}=$ Strongylocentrotus purpuratus (sea urchin)

$\mathrm{Tc}=$ Tribolium castaneum $($ red flour beetle $) \mathrm{Td}=$ Thermobia domestica $($ firebrat $)$

$\mathrm{Tl}=$ Triops longicaudatus (fairy shrimp)

\section{Primer sequences}

Initial amplification of $d a c 2$ fragments from Pholcus phalangioides have been performed with these degenerate primers:

dacPCR1-Fw: 5'-TGY YTN CCN CAR GCN TTY GAN YTN TT-3'

dacPCR1-Rev: 5'-ARN AMR TCC ATY TTN ARY TCN GYY TTY TC-3'

An aliquot of this PCR has been used in a second round of PCR with the following nested primers:

dacNest-Fw: 5'- CCN GTN GTN TGY AAY GTN GAR CAR GT-3'

dacNest-Rev: 5'-CKN GCR TTR TCN GCN GCN ACY TT-3'

Additional sequence information was acquired via RACE PCR using the following primers: Pp-dac1-3'RACE-1: 5'-GGT GCT GCC GTG ACC TTG CCC TTG-3'

Pp-dac1-5'RACE-1: 5'-CCG GGC TGT GTC CAT TAG CAG AAG C-3' Pp-dac2-3'RACE-1: 5'-GCC CAT CTT CTC CAA AAC GGT TAC GGT C-3' Pp-dac2-5'RACE-1: 5'-CAT TAT AAG CAC CGT CAC TGC CGC TCA G-3' 


\section{Sequences and sequence accession numbers}

>dac1 Parasteatoda tepidariorum| Accession number LN877883

MEGSS GEGSTSPSADMPS P SS SLQNS PNPDSSVMVSDC ITSVSNGGGLMCGP PMVS LS PKT INNPVVSMHGMGVSS PSYQQRTSSASP PQMVSKPYTPPMSTAGAMNALVVKQPQLEHMPAHMGMLAMRNGGVHHHGYLGAPPRLERPMHP IS P PGITTDATANDCRL I DYRGAKVAAFLVNGDYLLCLPQAFELFLKHLVGGLHTVYTKLKRLDITP IVCNVEQV RILRGLGAIQPGVNRCKLLSCKDFDTLYKDCTTARPGRP PKRATMVGINHNGTNPSMLLKKSRMDGEYPGYENGH IGDRVDKSHLLANGYSHHVAAAQAAVAAATGGAPPHLNPLPFMALNHAAAAAAHHNSMLSGTLPLAATGAHPSSA QGLNSSSHGHLNSSSSS GRGPESSSVIKERTS HNNDVINSTRLRDDRGDIVDSKDRLYGYDNHRMKDQAFLNGYF WLLAGAQANGHS PVLNLSQHSSRPSNS SNNNP ITNGPGSGGGGTGGEHSGSENDGPDDDNDSVDCDDDDSERDVS DNPDVSSTANTDRLTSSQQQLGYPAMGGDLSLLQAQAASSMETLLRYIQGLLKVAADNAKQQERQISLEKAELKM ELLREREVREGIEKQLMDEQRTRI LYQKRLKKEKRTRRRVQEQLEAEVKKRAQYEEALRSNSAETLRLLNESLAQ ELERNARAEAEHKMQDCPMSV

>dac2_Parasteatoda tepidariorum| Accession number KP725072 MDVGQGMSSPTTGMTSSPRSLQTP PNLDNMTLGGGDGNPSSNGGMVS PKQHVTGLSQMGSPGSSLGRSSSVSPPG PALSLVTTKPYQASSSQPNGGLPPSLPRSCGQNLMPHHQVSHPQMGHP PLGYHRS LHPSSS P PGIAADMTTNDCR LIDYRGAKVAAFLVHGDYLLCLPQAFELFLKHLVGGLHTVYTKLKRLDI TP IVCNVEQVRI LRGLGA IQPGVNRC KLLSCKDFDALYKDCTTARRCFSAGRDIPHHELAATSS I YGP I DNSSRPGRPPKRVSMVSMT SGVTTTGMVKKSR LDSDYHSYENGH ITGDRLDKHMLPNGYGH PGTTSHLS PLPFMALNSS HHNSVITGGVSMATTATHSSHASSRGDV TRERQMGSDVIASTRLRDDRVDAS DGKDRLYYDVSRQKDSYVNGTGNSGHS PVLNLSQNSSRGGGGGGGGGVGLH SAEGSGSEGPYNDGADDDYSDDDDDDRDQDFS DHPDVSSTANGERLAPSQMFPFSHLAVDGGSPTGQVVSS IETL LRNIQGLLKVAADNARQQERQINIEKAELKMEVLRERELRETLEKQLLDEQRTRI LYQKRLKKEKRSRRRFQEQL ESEVKKRAQYEEALRTTSADTIRLLNDTLTQELERERNSLAESENNAHDCQMTA

>dac1_Pholcus_phalangioides| Accession number LN877884 MEGSSĀEGSTSPS STMGSPPS IQNS PNPDP S GMVNDCATSSAQVGMMCP PMVSLS PKTNGQSASMQNLVSSPNST GEMRHQQQHP SART S PNS PQISVMSHAVS SKPYTP PMSTAGVMSALASASAGLHKHDTPPTHI PSHHATAMGMLS MRNGVHHHGYLSAPRLERPMQPLSP PGITTDATANDCRLIDYRGAKVAAFLVNGDYLLCLPQAFELFLKHLVGGL HTVYTKLKRLDITP IVCNVEQVRI LRGLGAIQPGVNRCELLSCKDFDTLYKDCTTARPGRPPKRATMVGMSPNGA NHS I LLKKTKIEGDYPVYENGYISGDRVDKSHLLANGYSHHVAAAHAAVAAATGAAP PHMNPL PFMALNHAAAAA AHHNSMLSAASGAAVTLPLAATGPHANAALNSS HGGLNSSSSTS S GRNAESSSTVIKERPS LGNEVNSTRARDDR PDAPDSKDRLYGFDSHRMKDQAFLNGYFWLLAGASANGHSPVLNLSQHSSRPSGNGTNGPTSAVVTGGDHSGSEN AYNDAADDDDNDSEDDDDDERDQDLSDNPDVSSTANTDRLISSQQALAY PNVGPGESGALAAQTASSMETLLRNI QGLLKVAADNARQQERQ INLEKAELKMELLREREVREG IEKQLLDEQRTRI LYQKRLKKEKRNRRRVQEQLEAEV KKRAQYEEALRNSSAETLRLLNDCPMSV

>dac2_Pholcus_phalangioides| Accession number LN877885

MEVGPG ISASSPAĀITS PRSLQTP PNLDNMTLTNENNS INGP SMGPVGMGS PKLSAGHMQNMS SPGS SAGRS SSV SPPGPALSLVSKSSYHTSQSHSHLQAAVNGVTTSQLSRTCPPGVLTSHPSHQLHP P PLAYHRAMHPS SPPGIAAD MTTNDCRLIDYRGAKVAAFLVSGEYLLCLPQAFELFLKHLVGGLHTVYTKLKRLDITPIVCNVEQVRILRGLGAI QPGVNRCKLLSCKDFDALYKDCTTARRCF SAGRDLPPHHELAAAAATS LYGPIDSSSRPGRPPKRVPSMVGIGGS QVVVSSASLLSKKARLDQEYHGYENGH ITS DRLEKAHLLQNGYGQQITTSHVISSALSPFMALSSHHGITNPSS I TTAANTHGGNVPTSRQDTVRSHITSDSLSS SNRVREDRLDGS DTKERLFYDVSRTKDAY INGASTNNGHSPVLNL SQNSSRVVVGSGTSNNLNHPSDQLSGSDGAYNDNGDDDDYTDDDDDRDQDFSDHPDVTSTANGGSSTERIVPSAS GQQIFPFVGGHLALDTGAVSPTHQVAISS IETLLRNIQGLLKVAADNARQQERQINIEKDAFNI I PTSEVERLDT DSVLNRA

>gi|313661346|ref|NP_001186375.1| dachshund homolog 1 [Gallus gallus] MAVPAALIPPAPLVPPQPPVSTSAACTTTTTTSSAAATSSPAPAIAPPPPASGTNLFRPEPIAAPAAAAA TVTSTATTTTTGGSGGGGGSGGGGGSPSLGSGTGGGTSGTSTPNAGAAGSL L PKPVYSTPS PVENTPQNN ECKMVDLRGAKVASFTVEGCELICLPQAFDLFLKHLVGGLHTVYTKLKRLEITPVVCNVEQVRILRGLGA IQPGVNRCKL ISRKDFETLYNDCTNASSRPGRP PKRTQSVTS PENSH IMPHSVPGLMS PGI I P PTGLTAA AAAAAAATNAA IAEAMKVKK I KLEAMSNYHANNNQHGADSENGDLNS SVGLELPFMMMPHPLI PVSLPPA SVTMAMSQMNHLSTIANMAAAAQVQSP PSRVETSVIKERVPDSPS PAPSLEEGRRPGSHPSSHRSSSVSS 
SPARTESSSDRI PVHQNGLSMNQMLMGLSPNVLPGPKEGDLAGHDMGHESKRIHIEKDETPLSTPTARDS LDKLSLTGHGQPLP PGFPS PFLFP DGLSS IETLLTNIQGLLKVAIDNARAQEKQVQLEKTELKMELFRER ELRETLEKQLAVEQKNRA I IQKRLKKEKKAKRKLQEALEFETKRREQAEQTLKQAASTDSLRVLNDSLTP EIEADRSGGRTDAERTIQDGRLYLKTTVMY

>gi|45382177|ref|NP_990126.1| dachshund homolog 2 [Gallus gallus] MAVSAPPVIAATSSGGGAGĞGAGLFRADPLYSSPAESPRLTNSLVNSFLSAGGGGTGAGGGGGGGNECKM VDLHGVKVASFLVEGQELICLPQVFDLFLKHLVGGLHTVYTKLKRLDI S PVVCTVEQVRILRGLGA IQPG VNRCKLITRKDFETLYNDCTNASSRPGRPPKRSLGVAIQENARLLPHGVPGLLSPGLISPTGLTAAAMAE AMKLQKMKLMAMNSLHGSGSQNGTESENEELNSNAGGSESSWDKDKLQSPITTGSQHS I GHPTLSGQSSL GSAHPLSPLQQNHLLTNR I DLP FMMMPHPLLPVSLP PASVAMAMNQMNHLNT IANMAAAAQMHSPLSRAG ASVIKERIQDSPSPAPSLEDSQRPGSHASSHQSSSVSSSP SQLDNTPDRIAMLTNSREGELIDQETGTSL KKMQKEKEEVQIAIPIMKPTLDKVQLAGQALP PGFPAPFLFADGLSSVETLLTNIQGLLKVAVDNARVQE KQIQQEKKELKMELCRERELRESLERQLTAELQSRATIQKRLKKEKKAKRKLQEALEFESKRREQVEQAL KQATSGDGLRMLNDAGI PDMEIEHNGTQHDSAAMQENRTYIKPTIMY

>gi|629266050|ref|NP_542937.3| dachshund homolog 1 isoform a [Homo sapiens] MAVPAALIPPTQLVPPQPPI $\bar{S} T S A S S S G T T T S T S S A T S S P A P S I G P P A S S G P T L F R P E P I A S A A A A A A T V$ TSTGGGGGGGGSGGGGGSSGNGGGGGGGGGGSNCNPNLAAASNGSGGGGGGISAGGGVASSTPINASTGS SSSSSSSSSSSSSSSSSSSSSSSCGPLPGKPVYSTPSPVENTPQNNECKMVDLRGAKVASFTVEGCELIC LPQAFDLFLKHLVGGLHTVYTKLKRLEITPVVCNVEQVRILRGLGAIQPGVNRCKLISRKDFETLYNDCT NASSRPGRPPKRTQSVTS PENS H IMPHSVPGLMS P G I PPTGLTAAAAAAAAATNAAIAEAMKVKK IKLE AMSNYHASNNQHGADSENGDMNSSVGLELP FMMMP H PLIPVSLP PASVTMAMSQMNHLST IANMAAAAQV QSPPSRVETSVIKERVPDSPSPAPSLEEGRRPGSHPSSHRSSSVSSSPARTESSSDRI PVHQNGLSMNQM LMGLSPNVLPGPKEGDLAGHDMGHESKRMH IEKDETPLSTPTARDSLDKLSLTGHGQPLPPGFPSPFLFP DGLSS IETLLTNIQGLLKVAIDNARAQEKQVQLEKTELKMDFLRERELRETLEKQLAMEQKNRAIVQKRL KKEKKAKRKLQEALEFETKRREQAEQTLKQAASTDSLRVLNDSLTPE IEADRSGGRTDAERTIQDGRLYL KTTVMY

>gi|16876441|ref|NP_444511.1| dachshund homolog 2 isoform a [Homo sapiens] MAVSASPVISATSSGAGVPGGGLRAEPLYSTPREP PRLTPNMINSFVVNNHSNSAGGGGRGNTNTNECRM VDMHGMKVASFLMDGQELICLPQVFDLFLKHLVGGLHTVYTKLKRLDIS PVVCTVEQVRILRGLGAIQPG VNRCKLITRKDFETLFTDCTNARRKRQMTRKQAVNSSRPGRP PKRSLGVLQENARLLTHAVPGLLSPGLI TPTGITAAAMAEAMKLQKMKLMAMNTLQGNGSQNGTESEPDDLNSNTGGSESSWDKDKMQSPFAAPGPQH GIAHAALAGQPG I GGAPTLNPLQQNHLLTNRLDL P FMMMP H P L L PVS L P PASVAMAMNQMNHLNT IANMA AAAQI HS PLSRAGTSVIKERIPESPSPAPS LEENHRPGSQTS SHTS S SVS S S P SQMDHHLERMEEVPVQI PIMKSPLDKIQLTPGQALPAGFPGPFIFADSLSSVETLLTNIQGLLKVALDNARIQEKQIQQEKKELRLE LYREREIRENLERQLAVELQSRTTMQKRLKKEKKTKRKLQEALEFESKRREQVEQALKQATTSDSGLRML KDTGIPDIEIENNGTPHDSAAMQGGNYYCLEMAQQLYSA

>gi|6681129|ref|NP_031852.1| dachshund homolog 1 isoform 1 [Mus musculus] MAVPAALIPPTQLVPPQP P ISTSASSSGTTTSTSSATSSPAPS IGPPASSGPTLFRPEPIASSASSSAAA TVTSPGGGGGGSGGGGGSGGNGGGGGSNCNPSLAAGSSGGGVSAGGGGASSTPITASTGSSSSSSSSSSS SSSSSSSSSSSSSSSCGPLPGKPVYSTPSPVENTPQNNECKMVDLRGAKVASFTVEGCELICLPQAFDL FLKHLVGGLHTVYTKLKRLEITPVVCNVEQVRILRGLGAIQPGVNRCKLISRKDFETLYNDCTNASSRPG RPPKRTQSVTS PENSH IMPHSVPGLMSPG I I PTGLTAAAAAAAAATNAA IAEAMKVKKIKLEAMSNYHA SNNQHGADSENGDMNSSVGSSGGSWDKETLHSPPSQGSQAPVAHARMPAAFS LPVSHPLNHLQHSHLP PN GLELPFMMMP HPL I PVSLPPASVTMAMSQMNHLSTIANMAAAAQVQSPPSRVETSVIKERVPDSPSPAPS LEEGRRPGSHPSSHRSSSVSSSPARTESSSDRIPVHQNGLSMNQMLMGLS PNVLPGPKEGDLAGHDMGHE SKRIHIEKDETPLSTPTARDS I DKLSLTGHGQPLP PGFPS PFLFPDGLSS IETLLTNIQGLLKVAIDNAR AQEKQVQLEKTELKMDFLRERELRETLEKQLAMEQKNRAIVQKRLKKEKKAKRKLQEALEFETKRREQAE QTLKQAASADSLRVLNDSLTPEIEADRSGGRADAERTIQDGRLYLKTTVMY

>gi|217035136|ref|NP_001136042.1| dachshund homolog 2 isoform 1 [Mus musculus ]

MAVSAPPVISATSSSAGVPGGLFRAEPLYSSPGEPPRLTPNMINSFMANNHNGSVLGGGIGGGSGGSSNT

\section{S30}


NTNECRMVDMHGVKVASFLMDGQELICLPQVFDLFLKHLVGGLHTVYTKLKRLDISPVVCTVEQVRILRG LGAIQPGVNRCKLITRKDFETLFTDCTNASSRPGRP PKRSLGVLQDNARLLPHAVPGLLSPGLITPTGIT AAAMAEAMKLQKMKLMAMNTLQGNGSQNGTESEPDDLNSTTGGSESSWDKDKIQSPLAASGPQHGIAHAA LAGQPGLGGAPTLNPLQQNHLLSNRLDLPFMMMPHPLLPVSLP PASVAMAMNQMNHLNT IANMAAAAQ I H SPLSRAGASVIKERIPESPSPAPSLEESHRPGSQTSSHPSSSVSSSP SQMDHHSERMVMMPNNREELIVD QDNGQS IKKFQRDNKEEVPAQ I PVMKSPLDKIQLAPGQALHPGFPGPFIFADSLSSVETLLTNIQGLLKV ALDNARIQEKQIQQEKKELRIELFREREIRENLERQLAVELQSRSTMQKRLKKEKKAKRKLQEALEFESK RREQVEQALKQATSGDSGLRMLKDSGIPDIEIENSGTPHDSAAMQA

>gi|642919228|ref|XP_008191786.1| PREDICTED: dachshund homolog 1 [Tribolium castaneum]

MDNSGMDSASEHSSHASSGSPAVAVKPSPSP PAANSNTHQVHSPRQASPLGPPMSMAHPLSP P PVSSASL ARSMALGHLPP PGLGLLNSLGVLHSPLDLMAHHAP PRSYNSP P P ISTSDPTANECKLVDYRGQKVAAFI I AGDTMLCLPQAFELFLKHLVGGLHTVYTKLKRLDIVPLVCNVEQVRI LRGLGAIQPGVNRCKLLSCKDFD TLYRDCTTAS SRPGRPPKRAPVGLSLAAS HLQQQQLKKQRLDNGDYPYENGHMGAEF PLDIMMRRDMSRL EKS PLLANGYNHP PHLS HMQFMQLPHPAAAHSALLS PAMPHNLTRHDGSVIKNQGMPTMEAIARSGIWEN CRAAYEDIVKHLERLREERCDPDRPLPLDQKARDLSPRNGSPTDHSPVLNLSKSGGGSAGSLGDGDHSGS EADGPDVPPSPRS PRSAAEDEDDDNISDPEDDDDKDQDMDEGDLPLPAAPGGDSQQAALNYSTLASAVAS ANGPSQDPSISSTETLLRNIQGLLKVAADNARQQERQ INYEKAELKMDVLREREVKDSLERQLVDEQKMR ALVQKRLRRERRARRRLQDQLEAEVKRRTLMEEALKAAGAAEQLRI INEKVAPSEQKLSNPKVTTSSTPN QS ISSERERVSERVERMERERVES P P PAYGQQTVREP P P P PENKPWGYSGIDLMNTGAAFWQNYSESLA QELELERKSRQQQVERDVKSPLQDRSGYYKNSMLFTSTAT

>gi|624296|gb|AAC46506.1| Dachshund [Drosophila melanogaster] MDSVTSEQSENNNNKAGA INNNINSGIERAAGNMNTTAMLMHHQQQQQAAAAAAAAAAQQQQQQHNGRDG SNSSRGANEDRPNGRSSHTGRGSSCSPAS SP SRHPSAVS PVS S LNHSMMQQMQQQQQQQQQQQQQQQHHQ LSP PPHGMPS GNGLPTGLP PRMP HGLPPHSLGLLNSLQMMHHAS P LELMAAAHHHVPPRSYNSPPPISTS DPSANECKLVEYRGQKVAAF I ISNETMLCLPQAFELFLKHLVGGLHTVYTKLKRLDIVPLVCNVEQVRIL RGLGAIQPGVNRCKLLCCKDFDILYRDCTTARCLS IKPPESNSLQFRSSRPGRPPKRGPVGLSLPPTHLS QHPQLKKHRLDNGDYAYENGH IS DMKS PLLANGYNP P P INHMAFMQMNAHH PGAAALMS PGMP PHGLHAR PESQMLKAAAQNAGMSAANMDALARSGIWENCRAAYEDIVKHLERLREERTDERQQAMGVGGAVVDRVGN VMVAEHKPRDLSSRNCSPTRQSPVLNLSKSGGNTDHGGSNCDAGSERSDCHSVAGSPARGGSRSLDEGSR SGVGGGVASHVGGGI IGVEDDEEEEENLSDENQSEVDERCLAKDDEDLSDTERDNLSTGSAAAAAAAAAA AAHQLHQHAAS HHHQTVGLS HLGVVP PHQRGS PSSAEAAAAALQHQRALNYSQLAAAAAVANGAAVGGGA VANGPTGGGGALTPNEALLAANDAAALAGGLALGPLGIDAHAAVPASSTETLLRNIQSLLKVAADNARQQ ERQISYEKAELKMDVLREREVKDSLERQLVDERKLRVLYQKRFRRERKIRIRYQQQLGGGSGAVKGS PNA NGSGGGGSGSACSESEACGGGGGDAKCNNNNNNNNNNNNSNSSSHGGDVETMKENDAGSEKSDKSLASNS SCDVTAGHSAGSVSGSISAGLNCPDSPTHFKREPSSDHEGSVERQPRSSAASLAAHDEDNIKRCGSRDRD ERPPSGSAASVAAEGSLAAAAAAAAAAAAAAASNGKGPWSYPGI DLMATGAFWQNYSES LAQELEMERKS RAANAERDVKSPLSERPTAYYKNSVLFGSAN

>gi|38490436|emb|CAD57736.1| dachshund, partial [Cupiennius salei]

RLERPMLPVSPPGLTTDATANDCRLIDYRGAKVAAFLVNGDYLLCLPQAFELFLKHLVGGLHTVYTKLKR LDITPIVCNVEQVRILRGLGA IQPGVNRCKLLSCKDFDTLYKDCTTASGCCRPGRPPKRATMVGINHNGA TGHGMLLKKPRMDGEYPGYENGH IAGDRVDKSHLLANGYSHHVAAAQAAVATHLNPLPFMALNHAAAAAA AHHNSMLTGALPLAATGAHTPGGALNTSTSSTHLTSASTPARGPESSSVIKERTSHGNDVINSTRLRDER VDLVDSKERLYGFDSHRMKDQAFLNGYFWLLAGGSANGHSPVLNLSQHSSRPSNSSGSNNNLNNNPTTNG PGGPNSGEHSGSENAYNVEGEDDVNDSEDDDDDDREEDLSDNPDVSSTANTDRLTSSQQQLAYPAMMGDM GPLPGQTASS IETLLRNIQGLLKVAADNARHQERQ I SLEKAELKMELLREREVREGIEKQLLDEQRTRIL YQKRLKKEKRSRRRVQEQLEAEVKKRAQYEEALRSNSQETLRLLNESLAQELERERNARAEAEHKMQDCP MSV

>gi|229614606|emb|CAS89751.1| dachshund protein, partial [Acanthoscurria geniculata] RILRGLGAIQPGVNRCKLLSCKDFDTLYKDCTTARPGRP PKRATMVGMS PNGPTPAMLLKKSRLDGEYPA YENGH I GS DRVDKS HMLANGYSHHVAAAQAAVAAATGAP PH INP L PFMALNHAAAAAAHHNSMLSSGVSL PLAATAHGHGLSTSRSDTSVIKERQSHGSDVINSSRLRDERGEPAESKDRLYGFDSHRMKDQAFLNGYFW 
LLAGASAANGHSPVLNLSQHSSRPNATCNGTATGDQSGSETAYNEGPDDDDSEDDEDDDREQDLSDNPDV SSTANTDRLTSTQQALTYPNVSGDANALAAQTASSMETLLRNIQGLL

>gi|91982995|gb|ABE68635.1| dachshund, partial [Triops longicaudatus] RILRGLGAIQPGVNRCKLLSCKDFDILFKDCTTARPGRP PKRS PLMGMTSPHCDALMKMKRPRLDNGDYM DYGNGPVNDRIEKS PLLANGYNP P PTLNPMQFMALNQAAAAAAAHSALLGS P PGMPGSMPGSGLALGPA ARESLSGNTGGNHSSAAGLPGANGSNPSGPNGPVLSFPGP I PGSGGVPRPDGSNVAKAQQP PLALEHMTA ARTGFWENCRAAYEDVVKHLERLKDERSEMERNVESRGRDFTTHNGSGTSPI LNLSKTPSAGGLNGTEEG DRIERGPRDTSADSVSEGARTPHRDDDEEMDRGSDIEEDDKNDMSDAIERGSAHHSDKSVVSNPAGNNHA AAAAAAAAAFNYSAALLGATVTDPTAMLTSTESLLRNIQSLLKVAADNARQQDRQANYEKAELKME ILRE RELRES IEKQVAEEQRIRLLYQKRLKKERRARRKLLEQMDPDGKRRAQLEEVVKNNASSGEALKLISGER DSHCLKEI

>gi|91982993|gb|ABE68634.1| dachshund, partial [Artemia franciscana] RILRGLGAIQPGVNRCKLLSCKDFDALYRDCTTASSRPGRPPKRSPLLGNSPSSSQTEGFKKPRIESDYS SAFQNGRTNEGRYDKALLGTPPSHLHPMQLMAFNAAAAQQAFLGNPGSLHHPGLVGMMHQQPQITQTSLP AHLESQRREQS PNE PNARTGFWENCRAAYEDAVKHFRLKEEKI DCSAPQQATSHSSKGERNAGEASSREN SPALNLSKTCEESS IGGEDSNS IEERSPVHENEESETSDQEGDHDTKTVDAVAQDHVLTPVSEKSHVGVA GNSNPQQAMAAAAAYNYSTLLSAAAAADPSLVLASTETLLCNIQGLL

>gi|33352130|emb|CAD82906.1| dachshund protein, partial [Glomeris marginata]

RILRGLGAIQPGVNRCKLLCCKDFDVLYKDCTTASSRPGRPPKRASLLGMSPGHQETLLKLKKSRLENGD YTSYENGH IAAHLLKGLHRA IDPMMLEPLLTGETRMEKSPLLANGYNHPPTH INPLPEMALNHHGHSATA I LNPATGVPISSSHSMSSRSDGS I IKERAHVADVLAARLKEEKNDCDRS IMALDHRVRKLNQLNGSSNGH SPVLNLSQKSTCAVAGDQSGSDAALNDPCDDDDDNISDVDDDDEKDQDLSDGPDVSCGANGDRSAANATA AAAAAAAYSTLLGETSGGISS IETLLRNIQGLL

>gi|530776472| emb|CDG15335.1| Dachshund, partial [Thermobia domestica] PVVCNVEQVRILRGLGAIQPGVNRCKLLSCKDFDVLYRDCTTASSRPGRPPKRATVGLS IAASQMNNHHL KRHRLENGEYPGFENGHLGDPPRMEKSPLLANGYNHPPTHLNHMQFMQLNHPAAAHTA ILSPAGIPLSSS HGLARADGSI IKNQGI PGI DAIARSGIWENCRAAYEDIVKHLERLREERGEAERALALDQKARDLS S HNG SSNGHSPVLNL

>gi|46405859|gb|AAS93632.1| dachshund, partial [Oncopeltus fasciatus] VRILRGLGAIQPGVNRCKLLSCKDFDILYRDCTTARCLMTNKSTERPPKRAPVGLSLAASHLSQQIKKHR MDNGDYPSSGYENGHISDTPRMEKSPLLANGYNHP PTHLNHMQFMQLNHPGPGHTAILNPQLQHHLIKPP PPMDALSRSGIWENCRAAYEDIVKHLERLREERGETDRALALDQKPRDLSSHNGSSTGQSPVLNLSKSNC EASGSETGGTGPEDEDEEDEGPESDQEEASDKEQELSDREEGGGSPGTVSPHALNYSALGAAGPNSAPTS DPTISSTETLLRNIQGLLK

>gi|91718848|gb|ABE57142.1| dachshund, partial [Onthophagus taurus] RILRGLSAIQPGVNRCKLLSCKDFDTLHKDCTTASSRPGRP PKRAPVGLSLAASHLQHQQLKKQRMDNGD YPYENGHMGD I SRMEKS P LPANGHNHPPHLGHMQ FMQLPHPAAAAHSALLSPAMP HNLGRPDGSVIKNQG MPSMEA IARSGIWENCRAAYEDIVKHLERLREERGDP DRP LPLDQKVRDFSPRNGS PTEDHSPVLNLSKS GGGSAGSMGDADHSGSGADGPDAPPTPRSPRSAADDDEDDNISEPEDDDEKDQDMDEGDLPLPVVPGNDS QQAA INYSTLASAVASANGQNHHQDPSISSTETLLRNIQGLL

>gi|196123803|gb|ACG70190.1| dachshund protein [Saccoglossus kowalevskii] MSLAGIATSLSPPTQVLFKLDKPAYSTPPPVSNDPANNECKMVEYRGAKVASFS IDAREMICLPQAFDLF LKHLVGGLHTVYTKLKRLDITPVVCNVEQVRILRGLGAIQPGVNRCKLVTRDEFDILYNDCTTASSRPGR PPKRTLPP PGLSPSGHFLLKKPKIENGCLTNGIDFSGFSPLGLRDGKTVGIPNGYLSPYLLTTHPSMMPT SLAMVTSHSSMHAS HRHDEP ISLSVNTTAKSDGTSAKS DKS P S S SDLSSPRSGI DRPKEQQCSPPRKEAE KEHERHTDVKS HKDSDNKRNSTDQRDHHHHYHQHSTESEKISS I GNGLNYNQTAAALYNIPENTSS IETL LTNIQGLLKVAADNARQQEKQINLEKAELKMELLREKELREALEKQLVAEQKTRAI IQKRLKKEKKAKRK

\section{S32}


LQEQLEVEQKTRSQTDSSVIKPNTVEALRVINDAKIASKYSYPSTEALSQELKLERNARTDAERKLLDIR GSLQSFTENFLAKNGHMDYSQYRMECKDPRNISGDEIDDDVIDNDIEDNDDDMADIEDED

>gi|390362165|ref|XP_791836.3| PREDICTED: dachshund homolog 1-like [Strongylocentrotus purpuratus]

MKMGSPPPTQVPLSSVLYKLDRVAYSTPP PVSNDPANNECKLIEYRGAKIASFNINGDCMICLPQAFELF LKHLVGGLHTVYTKLKRLS ITPVVCNVEQVRILRGLGA IQPGVNRCKLLTRKQFDILYEDCTTASRPGRP PKRNIPLSLTPTIHGLSRMGPTLDLLKRPKLENGHHRLPFNGYEHLAAAS PYLLATHPAFLPSSLSMPTN HLTASPAKAAEMHHLHHADLGAAHLHNLHKLSVGIPRSSSQPSSHSHASVDIDDHEEDLENGDMSPRSSD GPSPRMGGSQGGLSPKSDSS SAMHEDEDEKESDRI HQEAMAAMEHSLANAREENKP SGMNGALSEGAHSR GEEYEQLHGNSTSQSGMPLPGMTATDILSHQVGPSSMETLLTNIQGLLKVASDNARQRDKQINLEKAELK LELLRERELRESMEKQLVAEQRRFA I LVRRLKREKRSRRRLQEQAESTTQQQQQQREREREQQKMKGSLS DQEPSRSPPIHTIQPIQAVAQES IRALNGGDRRPSCPDVYEKGEWLRGSSLVVS

>gi|295656530|gb|ADG26728.1| dachshund protein, partial [Platynereis dumerilii] MMMEAVPPRPTSLPS PSRTPVLSPQPP PAPMHQGP L LP PPSHS IVSMSS ISTTTSS ILPLKMEKPIYSS PPPAASNPENNTCKMIDYRGAKVAAFKVDGRELICLPQAFELFLKHLVGGLHTVYTKLKRLDITPIVCNV EQVRILRGLGA IQPGVNRCKLISPGEFDVLYDDCTNSSARPGRPPKRSP IAAHPETIEKLKKQRMEGEYP YS PNRLLGQFMFS DPKKSPLFGNGYHYPPHLASMNVP FMPLGH PMMTMAMANHMGMRPDGS I IRERQAPE DLASPRPKDDGRYDENNMEHNNNKPLDKPLNLQMDRPRERRPSLTDKDSGMSTPSDVMTNGQLDLSMKHS APS INEDLDDDSEDDKEDDDDMDDS DAP P SMANS DAADKMAASS LPYQASQMLNTETTGISSVETLLMNI QGLLKVASENARHRERQMNYEKAELKMELMRERELRESLEKQMGDEQRTKVNLQRRLKKKK

>gi|224830721|gb|ACN66455.1| dachshund, partial [Neanthes arenaceodentata] LKRLDITPIVCNVEQVRILRGLGA IQPGVNRCKLISPGEFDVLYDDCTNSSARPGRPPKRSPITANPETI EKLKKQRMEGEYPYS PNRLLGQFMFSDPKKSPLFGNGYHYPPH IASMNVP FMPLGH PMMTMAMANHMSMR PDGSI IRERQAPEDLAS PRPKDDGRYDENSMENNNNKPLDKPLNLQMERTRERRPSLTDKDSGMSTPSDV MTNGQLDLSMKHTAPS INEDLDDDSEDDKEDDDDMDDSDAP P SMANS DQAEKMPQS SLQYQASQMLNNEA TAISSVETLLMNIQGLLKVASENARHRERQMNYEKAELKMELMRERELRESLEKQMGEEQRTKINLQRRL KKEKKARRKLQETLEQMGAKTHPMEDLPSPKPLSTESHRSLNDGMSSQDSERDRASCHS SPDRRLPGSAS LYTYDAHSGLQNLMETFLGPQKSCNGSTNGYLHPPVIKATESV

$>$ MMa07092। Cao et al. 2013

MSAFYFVSRPGRPPKRASMIGMHVSTSGHTTLLKKTRLESEYSAYENGHITGERIDKSHSLLANGYSHHSSTTAG APPHISPI PFMALNHPSSHPNSMVPVSLPLSATSHSHSHQLTTSRTDASLNKDRSSHDVIVSSRVRDDRVDNLDA KERLYNLECHRVKDQYMNGTSVNCHSPVLNLSQNSSRRGGGEHSGSETAYNDCQEDDYNDSEDDEEDDREQDFSD NPDVTSTGNSERLTNQALFY PHVQVDGMTATGQVVSS IETLLRNIQGLLKVAADNARQQDRQISVEKAELKMEVM RERELRENLEKQLLEEQRIRILYQKRLKKEKKSRRRIQEQLESEMKKRVQYEEALRNSSAETIRLLNDSLAQELE RAERNVRNDTEHKVQGTVFKS

$>$ MMa07100| Cao et al. 2013

MDDSRGHCTPSPTSGLASPHSLQTP PNLEPGVIVTESETLSPKHGQQTSTMLAS PCSTLETPPRRSPVSPAGHGN VLPQGSQHKPYPLGMAGMNGGIPKHCLDAYHPQTTVSLAARTPVSAPAGLAYSNRPMHPASPPGVPADISVNECR LIDYRGAKVAAFLVNTEYLLCLPQAFELFLKHLVGGLHTVYTKLKRLDITP IVCNVEQVRILRGLGA IQPGVNRC KLLSCKDFDTLYKDCTTARSTH

$>$ MMa52425 | Cao et al. 2013

MDGSSGEGTISPSGDLSSPQSLQTSPNPDSSGLPGDSGQILAGGSPKPVTSMAGLPGTGSQVIGGNRSSPVGSPG SAMPPVSVAKSYAAS IVSAGALSGCGPKHPLEPLPLGLTAPRPGAPTQVNSYGLHSGRIHTASPPGLTTDATAND CRLIDYRGAKVAAFLVNGEYLLCLPQAFELFLKHLVGGLHTVYTKLKRLDITP IVCNVEQVRILRGLGAIQPGVN RCKLLSCKDFDSLYKDCTTARHLWPGRYKSNSYWKEQRPGGLPGVASVESWPGLIFFELGLHTHTSRSEVTWERE KRDRYPITTKLYELE 


\section{5 $\underline{\text { Tarsus segmentation genes }}$}

\subsection{1 aristaless}

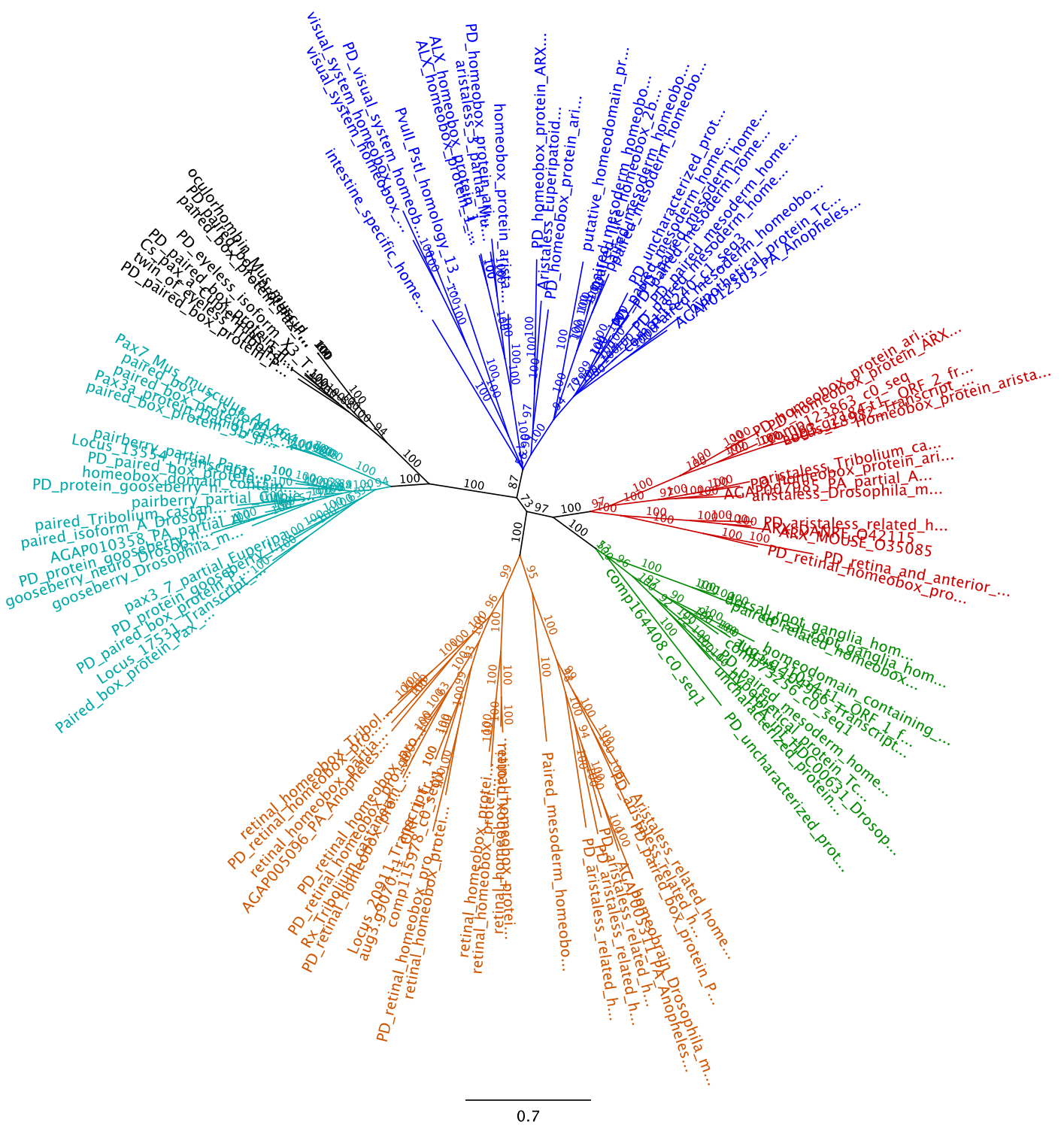

Supplemental Figure 7: Unrooted 50\% majority rule consensus tree to identify true al homologs.

The Jones amino acid substitution model was chosen after mixed model testing. After reaching 3000000 generations a total number of 12002 trees were written in two files (each file contained 6001 trees of which 4501 were sampled). Colors indicate monophyletic groups in the tree. 


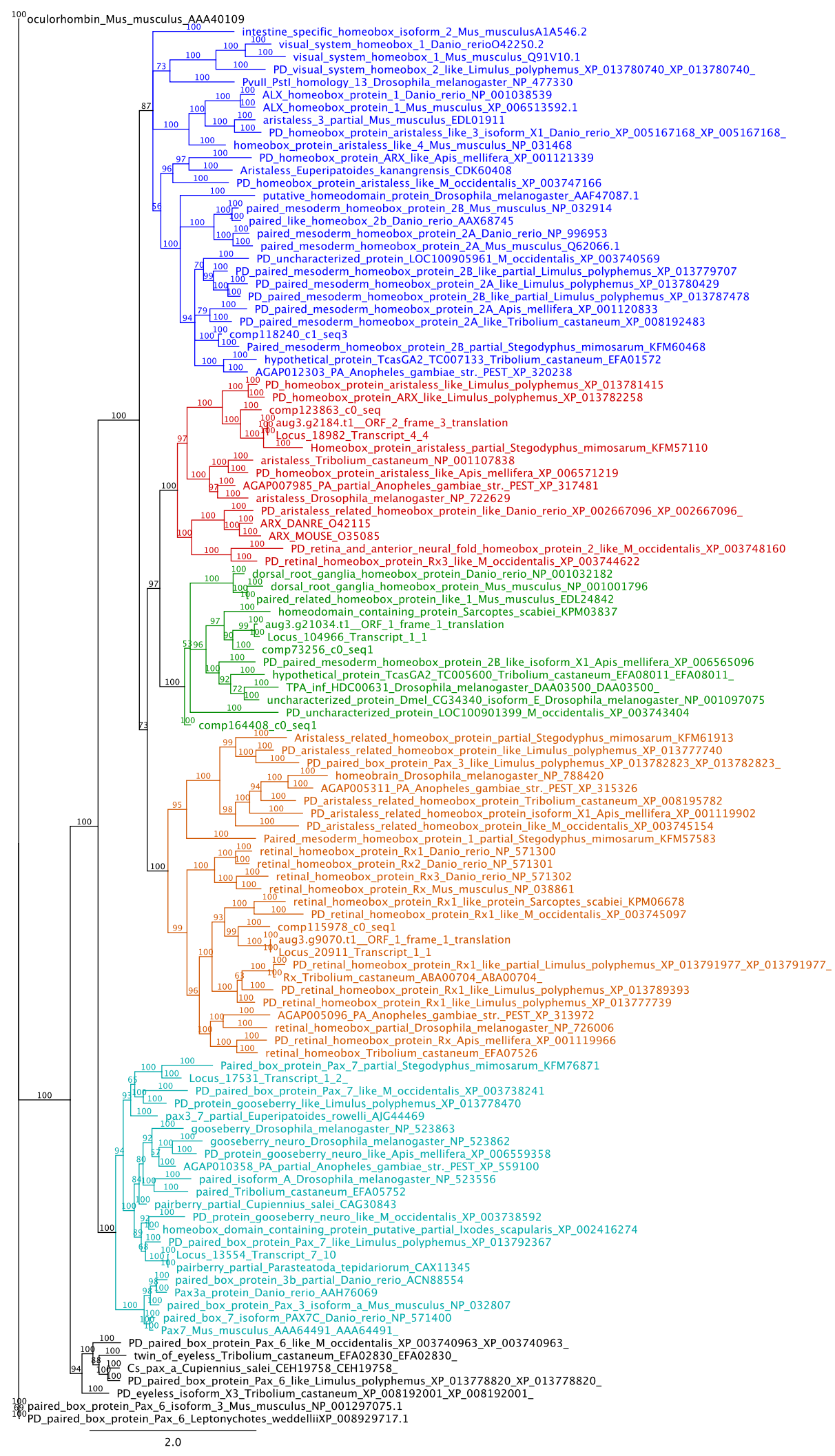

Supplemental Figure 8: Unrooted 50\% majority rule consensus tree to identify true al homologs. The same phylogram as in Supplemental Figure 7 in a magnified view to display gene and species names. 
>comp123863_c0_seq1

GTCCCGCCCAGCACTCAGTAGTGTGTTGGTTTGTGTACCTCGAAGCATCACCGGTTCTCTCCTTCGATATTGTTGTCTCTTTCAACAACTGAAGG GGAAAAGAGAGAGAAACTGAATCATTTGATGTGTGTGTGCGTTCGTGCATGTTGTGATCATAACAGACAAGTGATCGTCTTCGGTTTCCCAGTC AGTGATCCGGGGACTTCCATCTGAAAATCTCTTTCTTTTTTTTTGCCGCGGACATTCATGTTGTTTTCAAGCTGTGGCCGGTCGAATCAAGTAAA GTTCCAAGACCAGCTCATCTTTCGAGTGCCTTGTCGTTCAACAACGTCCATCTGCTGCCACCAATTCGAGGAGGACAGGACAAAGGAAGAGAAGG AGAAGCAGAGTCCTCACCGTCGCCCCCACCATCATCTGCCGGACTGAACGCCGTAAACTTCTCCTTGCTTCCTCCACAATATCACGGTTACCACCA GTTCCATCATTACTTCAACAGCTGTAATATGGGAATATTGGACAAAGTCGAGACCCACCACTGCTTAAGCGAATCCGCCCGAACACTGAACATGA CCACCAAACACGAATCAGTCAATCACCAAAACAAGGGACAGCTCACGGTTATGCGACTGATCGGTGACAAATCTCCGCCGTTGGCCAACACCCCG AGTCCAAAGTCCATATCGCAGGAAGTGAAGGGCAATCCGTCATCGGCATACGGAACGAAGGATAATCCTTCGTCACCTCCCGAGGACTTGACCAT GACTTCTGTGGTGGACGTCGTCGCGGACAGTGACCACGCGGTGACGGAAGACGAGGACGAAACCGACGGTAAAATTCCTGGAAACGGCAACGAA GTTCCGTCCAACGACAACTTACTTGAAGGTGAGGATTTCCCGAAGAGGAAGCAGAGACGTTACAGAACGACTTTCACGAGCTTCCAGCTGGAGGA ACTCGAGAAAGCCTTTGCGAGAACCCATTATCCTGACGTGTTCACTCGGGAAGAACTAGCCATGAGAGTTGACCTCACAGAAGCTAGAGTACAGG TTTGGTTCCAGAACCGCCGAGCAAAATGGCGCAAACAAGAGAAAGCGGCCGGTGGACAGTCTCAAAACCAGGGATACAATCCTTATTCGTCATCA ACAACTCTGCCGTCCATACCAGCGACCAGTCCAGTATCGTCAAAGTCCTTCAACCCTCTAGGATACCCTAGAACGTACGACCTGAGTCTTTTCAAC ACAGCTCATCACCATCATCCTCAGTTTCCTCCGCCTTACCTGCCACCACCCGGGCTATCGCTGTTCCGCCCGGCTCCGAACTTCCTCGCACCTGCTG GTTACAGCCTCAGGGACCTACCATACCCAGCTGGACTGTTCTCGGCAGCTGCAGCGGCCACTCTATCCTCACCATACAGTGCGTTCCCTCCGACAT CGTTCCAAAGTCTCTTGGTTAACCTATCGAGTCAGAACCGACCTAAACTCCCGGTGCCTACTGACCTTACCACCGGGGAAGCTTACACCGGTTTGC TGACGGCTTCCTCCGTGACAACACCCACCGCTGCTTCGGCTACGTCTACGACGTCCCAACCTTCACCGACTGGAACTGTATCACCAGTGGGATCTT TACCAGGATCTGGTTCACCAAATCCACCACCGTTGCCACCAACAACAACGTCTGCAACAGCAGCAGCGTCGTCATCAGCAGCACCTCAGACAAGC GTCGAGCTGGACAGACGAAGTTCCAGCATCGCTGCTCTACGCCTCAAAGCGAGGGAACACGAGGTCAGGATGGAAATGCTGAGGAAGTCCAACGG CGAAGTCAGTTGAAAGATTATATGTGGGGTCGTTACCAGACAGCTGGGAAGGACCTGCTTAATTGAAATTTGTGAGATTCTCCAGTGAAAAAAA ACCGTCCAAAGAACGAGTCAAGAACAGGAAAAATATAAGACAAACTTCCTCCTATTTATTTTTGCTTGAAAAACTTTTCATACACACTATCCGTG AAGATGCCTTACATGAGCATTGAAAAACGGTCATTTTCATCATGTAGTGTCTGACTGGTCTATTTTCCGTCCTAAATAACGTCCTATTTACAGCT ATTCTTGATGTTCAACGTGAACATGAGACACTGAAGCGCTGAATCAGTGCTATTTTTTTCCTCACTAAAAACGCACATTCATTTAAGACTGGTTT AGAAACAGTGAACTATCCTATCATATGGGACATCGATTTCCGTTTTGCACTTTGAATGATCATTTTACCAGGCGAGGATCCAGGGTTATGTGTCG GGGGAGGGGGTCACTCATGTGCATTAAAGGTCAATAGTGTCCCGTATTTCACCAGACAGGCCCATCTCCCCATATGATCGGGTTCTTGAAGAAGA GCATCGCCGACTCTGGATCAGCGCCTGTATCTTGATATAATAATACCATGAACTTTCTCATATCAATAACTGTTATGTATTGTGGTGTGAATATA CCCATGGATAAGGCGCCTAGAGAGGCAGGAGTCATCCTCTATCATACTCTATGTTAGAGCGACTAAACTAGGGCTCCTTAAAACCTCAGGTTAGC TTATCCGCCATCTTCGAGCGAAGTAGGGGACGTCAGTATTATCACTATAGCCCAGAATTTCCCAAAGTGAATAAAATCAATCCCTTGGGGTTGAT GAAAAGGACCATGTGGACGATAGAATCGATGGGTTTAGTATAGGATCCTATACGTTAGCTTTTGAGCTGGATGAGACGATGAAGAAAAAAAATT GAAGAAGTGGGGAGATGATGAAGCAGAAAATTTGGGAAACTATGCTATAGCCTAGGAATGCATAAACTTTTCAGTCCGCGGGCCAAATATGTGG TGTTTCTGCCTCATTTTTTGTTTCTTTAAAAATGCGCGTTTTCTTATTTACAATGAGGTTTTACTTTAATTTTCCAAATAAAAAATAATGATTAT TTTCATGACATAAATTA

>Locus_18982_Transcript_4/4_Confidence_0.600_Length_6071

CGTTAAGCCTCCAATCCCTACACGTAACAGGGGTTTCGTTCTGATAACGGTAAAGTAATGCGGCGTTGTATTATCTATTTGTGCTTTTATATAAT AAAATGATTATCTTACCGTGATACAAATGATAAAATTGATTGAAAGTAGCTTCCTCGGTCTTGGTAATTAACTACCATATTTTAATGTGCCTTT CTCCAAGAAAAAATACTATTTGCCTTATTTGTACCCCGTCCAGCTCATTTGCGTTCCACGTTGTCCGTTCTCGGAGGTCCAATCCAGACCACGGA AGGTGGCATCGTGCCGGATTTGACCGACCACCACTCCAACACTTTTAAAAATACTATGGGCATATCAGATAAGATCGAACGTCAGGAGTGCATTA GCGAAGCCGCCCGCACCCTTAACCTCATGGAAGCCCACCAAAAGGATCAACAGCCTCATCATCGAAAACCAATTCATCATCATCACAAACTATCT GTTGTGCACCTTGTAGGGGATAAATCCCCTAGACCAGCTTCAAGTCCAGAACAAATGAAACCATGCCAACAAGATGCTGTGGCAGAG GACTTGTC AGTGACCGGTGGTAATGATGAGGTGGACAGTGGTGATGAAAATATCAGTGTGGCTGATGATGATGGTGAGAACAACAAAGGATCAGCTTCTGCA GAATCTTTGATGGAAGGAGATGATTTTCCAAAGAGGAAGATACGTAGATACAGGACAACTTTTACCAGTAATCAACTTGATGAATTGGAAAGAG CTTTTGCACGGACTCATTATCCTGATGTTTTTACAAGAGAAGAACTTGCTATGAGGGTTGACTTGACTGAAGCCAGAGTTCAAGTATGGTTTCA AAATCGCCGAGCAAAATGGCGGAAGCAAGAAAAAGCAGCTGGTGGACAATCACAAAGTCAAGGCTACAATCCCTATTCAGCATCTACAGCGCTA CCGTCCTTACCAGCATCTGCACCAGGATCTGGAAAACCATTCAGTGCTCTGAGCTATCCTAGATCGTATGACCTTGGTCTTTTGAACGCAGCAGC AGTAGCTCACCATCACCATCATCCACAATTTCCTCCACCTTATTTACCACCGCCTGGCTTATCAGGACTGTTTCGCCCAGCTCCTAGTTTCTTGAC TCAAGCTGGCTATTCCCTTCGAGATTTGCCTTACACTAGCTTATTTCCAGCAGCATTATCTTCACCTTATTCCGTAACTGGTTCATTTCCTCCAAC ATCATTTCAAACATTACTCGCTAACTTATCAGCACAGAACAGGCCAAAGCTTACAACTTCTCCTGAACTAAGTCCTGTTGCCACGGAACCATATC CTAGCCTACTTGCGACGAGCACTGCAAGTGCTAATTCTGCTCCTGCAACTGTTTCACAACCATCCCCTACTGTAACTCCACCCGCAGCATTGCCAC GTTCATCAACTGGTTCTCCCGTATCACCACCAGCTACAAGTCCTGTGGATTTTGATCGAAGAAGTTCGAGCATAGCTGCTCTAAGACTTAAAGCT CGAGAACATGAAGTGCGTATGGAGTTGTTGAGAAAAGCGAATGGGGAAGTGAGTTGAGTTAAATGAGCGACACAAAAACTTATAGAATATTTA TAATACTGTGGCTGAGAGAAAATGCAGCAGTGGATTTTCTAACATGGCAACGTCCAAAGAATTTATCATGAAACTTTAAAACTCGTTTCGTGAA ATTACATTGAACGGACATTCTATTGATTTCATTTGAATAACTGCAGATTGTTTTCATGACTTAAAACTTTTCCAATTTCTTGTCTTTCATCATTT AGTCAAGTTGACAGTAACAGTTCGGGTGGTGGGCAAATGCCATCTTTGACAACAAAATATTTTTGAACTATATGAACAGTTTTGCTTTGTTTGT GCCTCAAACTTTTATAAAACCAAAGGTTGTGTAAATTTGTGTTTATTGTATCATTTATCTGATGACGCTCATTGTGAGTTAGCTCAATAGAGTG GAAAGATCCGACTTTACAAAATACCCTTAAAAAGTCTTTCAATAAAGAAACTACATCAAATGTCACTTATTATATCAAGTGCGTGGTGTTTTGG GAAGCTAATACTAAAAGTCGAGTTTCGAGGAAATAATGCTCTCTTACTTAACAACTTACAGAAAATTCACGACTACGGTTGAGGCGATTGGTTT AAATGCCTACTTTATGTCTTTACAGAGTTTGCATTTGAGTCAATATTTATGATGCTTATTAGAAAGTAATGAGATTGAATAACGCGTAAAGTTA TGTTTTCCTCATCAAAACATTCTAATATTTATGTTAACAGAACTATTTTAAATACGAGCTGAGATGATTTTTGCTAATTTTGTAACAGAAAACT AATGATATGTTTTGTATTGTGGTCGCTGTTTTTATTCAAGAAGCTAAAAAATAAGAAAATAGTTTTTATAATAATATTTTTAAAAATCTAAAGA AAGCTAGTTTAAAAAAATACTTTGGATTATCTATAATAATCTAAGCTGGTTTTAAAACTAAAGATCTCTATTTATCAAAAAAACAGTGATTTTC TTTAAATCTTCCAGTGTGCATTAGTCATTCAAATCTTAAATTCTCCTACTAAGAAAATTAATGAATTTATTTTATTGATTCAAATCTAAAAATA AATTCACTTAAATATTTAATTGCTTTCTTTCAGCAGTCTATAAAAGAAAGTACTCGTGCATTTTCTTCTTCCCTTCAATTAAAATATTTATCAAT CTTTTGAAAAACGGTTTATGCAAATTTAGTTTTAGAGAATTTAGGTTTCAACTTTCTGAAAATGGGAAAAACTTTAATGTAATTGCAAACTTAA GTTTTTGACTTGAATAATAAGCGAGCAATTATATCAGCTCTCTGAAATTTCAGCCAGGTTTTATTTTACTTATCGATTAATGCGAAAAAAAATA ATTAAATATAAATCTACGCGAAATAAATTTGCGAAAATTGTTTCGATGATGTATAATATATTAAATATCAAGGAAAATTTCAGTACCGAACATA GATTTAAAAATTCTGGGAAATTTTTTTTTAGGGTGACTTAGTCTCACTACGTGGTTGGATGAAGGTTAATCAGTTTAAAAAATTGCTGGAATTA TAGACAATTAAAAATCTTAATAGAAATAAATAACATGTGACAATCTGAAAAAGTAAGATATTTGGATTAAAAAACATATTATGGTCTGTTGTA 
TGAATATAACTTTTTTTCTGCCAGGTTAATAATCAAGACGGTGCTGATAACGAATATGTGCTTTGTAAACAATTTCCTGCTGTTGCCAGACTTTT ATTTTTACGCGTGTTTATAAATTCACTAAATTATCACAGCAAACCTTCTTCCCTTAAGTTTTTCAATTGCACCAGTTAAAAAAACAGCTCCGGGG GGGAGGGGGCGAGTGCTCATTGAGTCACAAACAATGTATATCTAATTTTCTCTAAAATTATTTTCTACAAGTCAGACTTTACAGTGAGAGGGAA TAATTTTCGGAGTATAAAAATAAAGTATTCTCTTTAAAATCGTTAAAATATAACACGCCTCTCACAAGCAACTGCAACGCGTCTATCCGGACTGT CCTAAAATCGGACCTGACTGTTCTAAAAACGCGTCACAAAATCACAAAAATAATTCTCTTGTCATAAATTTGACGTAATTAATTTTTAATTACCT TTATTCAAATTTTACCAAGAAAAAAAACACGTAAGTCTTTGGTTGTTAATGAAGTAATTCTTTGATTTTTAAGTTCAACTTAAAAGCAACTTTT CACAATTCCCATAAAAAATTTTAAACTTTTTTACTAGTTTGCATTTTGTAAGTATTTTAGTTAGTATAATAATTCAACGACTTTTTTTAGAATG TTATATCACCTTGATTTTTTTTTTAATATTTTGCGTTACGTCCCAAAATTTAATTTGCTTCAAACAAAAGTTTTGTTATTGAGATTTTAGCACTT TTATTGAAGTTTTCGCAATATTTAAATTCATACCTAAAATATTTTTAAAAATTTGGACTTCAGCCTTACTGATTTTATAAGGGAAGATTCCAAT AAGTTCCCAACTGAGTAAATTGATTATTTAAAATAAACTTTTGGCATTGAAGAAAACAAAGTATCAATCCTGATTCTAATAAACTTTGAATTAT TTTCATTTTTTAGTAAAATCATTGAATAATCTTTGTATCTAAAGACTAAAGGTATCACGTATAATTAATATAGAATTTAGAAAGTAAAAATGAG ACAAAAATCTCGAATTGTAGCCATTACAATAAATTATTGCTATAAACAAAGTACTCATTGATGTATAGATGTAAATGTTGTTACTATTTTACCA AAGTTGAATTTATTTATATGATCATGATCTTGCACTTTCCTGTATTTAATAATAATAAATGTATACCTTTTCATCTTTTAACTTATTACATTACT TTTTAAACATAAATGCAAAATGTAGTAATTTTCAGCAATATTTGTAATATATTATGAAATGATTCCCTTTCGAGACTATTTAACATTTTTTACG GTATAATCTGGAAAAAATATAAGTCAAATAAAAAAAATTGATTTCTGCTTAATAAATTAATTTTAAATTGCACTCGAATCACATCTAACATCTA TCAATAACTTTAATTATTTTTAACAAACGTATTTTAACGCAAAATAATTATACAAGTCGGAAATATAAATTACATTTGATGTCTCCTCGTAAAT ACATCCTGTTTTTAGAATTATTCAACGAATTTATTTTTCAATTGAGAAATGGTTTTTACTTTAAATTAAAGTTATTATAACCCCTTGAGAAGTT TGAAGTTCAAATCTTTGAGACATACAGGGTGATTCATAATGATCACCAATAAAATATATTTAAAAAAAATTTGGCGAAGATAAAGTAAAAAAA TGATACATATTTGGAACGCATAAATTATATTTAAGCGAGGAAAAAGTAAAACGCTGTAGAAATCGGTTGAATCACTACTGAGAAAGATGGAATA TTAAAAAAATGAAGTTTGCATTTATTAAAACATAAAAACATATTGAATTAAACAGTTTCAAATGCAATGTGATGTACAAATATTGTTGCAAATT GATGTATGGTATTAAAAAATACTATGGTATACACATATAATTTATATTTTTTGAATTATGTAACATGCATATAAATGTTCTGTTTTCAGAAACT ATGCAGTGATACTTCATAGTTATATTTTTGTATCAAAGTGTATTGAATGAAACCGTCCAAACAGTACGTTAAATTCCTTAACTAATCCGTCCTGC TAAGCTTTAAAGTAAAGAAAAGTGAAATTTTAAAGAATATGTATTCCTTAGATCAGATATATTTCTTATTTATTTATAAGTGCTCAAAGTTTAT TAAAATCCATAAATTGCTGTATTTTAAAATTTAGTGTAGGAAAATTTTTGTTTTCTTTAAATATCCTTATGTGCTCTGTAATCAATGCAAATAA TATAAATGTTTATGATCCAATAAAAATTGAAACATCCCAATAATATCAAAAATTAAAGCCACTTTCGCACTTGCAGTACTATTTTCCATCTATCG AATGGCTTATTTGTCATGCGCGTTGTGGATGGATCAAAGGATTCTAGATTCATGTGGCTCTACGCTAAAATAATTTATATTCCTTTCTCAAAAAC AAAACAAAAAATCAAAAGAAATGGAAAACAATTCCTAGATATTGTGGAAATTTTCATTTGAATATGCTCTGTCCCACTTTATCAACATTTAATT TTTTTCTTTAATTTTTTTACAAAATTTGTACAGCTTGTTTGAGCATTTGAGTTTAAAGAATGGTCAACAACTCTTAAACTATAAAAATATTTCA CTTTGTAAATATAAAATGTATTTCCGGTGCATAAGTTTCTCTGTTACTTTGAAGGGAAGTTTACCGTCCATTTAATCATTTATTGTTGGAAATG TGATTTGCATATTTAATGGCATATTTAACTTTTACGTTTGCACTCATTCAAATAAAGTGAGTGATTGTCAGTTCTTATGTATTGTAATTTAGGT GACTTTCTGTTAATAAAAATGGGAAAATAAAAA

>aug3.g2184.t1

ATACAAATGATAAAATTGATTGAAAGTAGCTTCCTCGGTCTTGGTAATTAACTGCCATATTTTAATGTGCCTTTCTCCAAGAAAAAATACTATT TGCCTTATTTGTACCCCGTCCAGCTCATTTGCGTTCCACGTTGTCCGTTCTCGGAGGTCCAATCCAGACCACGGAAGGTGGCATCGTGCCGGATTT GACCGACCACCACTCCAACACTTTTAAAAATACTATGGGCATATCAGATAAGATCGAACGTCAGGAGTGCATTAGCGAAGCCGCCCGCACCCTTA ACCTCATGGAAGCCCACCAAAAGGATCAACAGCCTCATCATCGAAAACCAATTCATCATCATCACAAACTATCTGTTGTGCACCTTGTAGGGGAT AAATCCCCTAGACCAGCTTCAAGTCCAGAACAAATGAAACCATGCCAACAAGATGCTGTGGCAGAGGACTTGTCAGTGACCGGTGGTAATGATG AGGTGGACAGTGGTGATGAAAATATCAGTGTGGCTGATGATGATGGTGAGAACAACAAAGGATCAGCTTCTGCAGAATCTTTGATGGAAGGAGA TGATTTTCCAAAGAGGAAGATACGTAGATACAGGACAACTTTTACCAGTAATCAACTTGATGAATTGGAAAGAGCTTTTGCACGGACTCATTAT CCTGATGTTTTTACAAGAGAAGAACTTGCTATGAGGGTTGACTTGACTGAAGCCAGAGTTCAAGTATGGTTTCAAAATCGCCGAGCAAAATGGC GGAAGCAAGAAAAAGCAGCTGGTGGACAATCACAAAGTCAAGGCTACAATCCCTATTCAGCATCTACAGCGCTACCGTCCTTACCAGCATCTGCA CCAGGATCTGGAAAACCATTCAGTGCTCTGAGCTATCCTAGATCGTATGACCTTGGTCTTTTGAACGCAGCAGCAGTAGCTCACCATCACCATCA TCCACAATTTCCTCCACCTTATTTACCACCACCTGGCTTATCAGGACTGTTTCGCCCAGCTCCTAGTTTCTTGACTCAAGCTGGCTATTCCCTTCG AGATTTGCCTTACACTAGCTTATTTCCAGCAGCATTATCTTCACCTTATTCCGTAACTGGTTCATTTCCTCCAACATCATTTCAAACATTACTCGC TAACTTATCAGCACAGAACAGGCCAAAGCTTACAACTTCTCCTGAACTAAGTCCTGTTGCCACGGAACCATATCCTAGCCTACTTGCGACGAGCA CTGCAAGTGCTAATTCTGCTCCTGCAACTGTTTCACAACCATCCCCTACTGTAACTCCACCCGCAGCATTGCCACGTTCATCAACTGGTTCTCCCG TATCACCACCAGCTACAAGTCCTGTGGATTTTGATCGAAGAAGTTCGAGCATAGCTGCTCTAAGACTTAAAGCTCGAGAACATGAAGTGCGTATG GAGTTGTTGAGAAAAGCGAATGGGGAAGTGGCCAAGTGGTTAGCGCGCCTGACTGCGAAGCCAATGGCTGCGGGTTCGAATCCCGCTCTGGATA TGGATGTTTCTCTCTCTCTGTGCTGTATTCTGACGTGTGAATGTATGAATGTGGCCCACCCTATGAACGGGTATTTGTGGCAGTGGGGAGTGGGT AATGTTGCTCGCCTCCGTGACATTGGATCACAGGTGCCCACTGGGTAACGATAAATGAGCAACACTTCCGGCATCTTCTAAGGCGAAGAACTAAA GTTCAGTTCAGTGCCAGCCGTTATTAAAAAAAAAAAAAGAAATACTCAAATTTTAGATTAGTTCACTATAAAAGCTGTGATGTAAT

>aug3.g2184.t1_-_ORF_2_(frame_3)_translation MRSTLSVLGGPIQTTEGGIVPDLTDHHSNTFKNTMGISDKIERQECISEAARTLNLMEAHQKDQQPHHRKPIHHHHKLSVVHLVGDKSPRPASSPE QMKPCQQDAVAEDLSVTGGNDEVDSGDENISVADDDGENNKGSASAESLMEGDDFPKRKIRRYRTTFTSNQLDELERAFARTHYPDVFTREELA MRVDLTEARVQVWFQNRRAKWRKQEKAAGGQSQSQGYNPYSASTALPSLPASAPGSGKPFSALSYPRSYDLGLLNAAAVAHHHHHPQFPPPYLP PPGLSGLFRPAPSFLTQAGYSLRDLPYTSLFPAALSSPYSVTGSFPPTSFQTLLANLSAQNRPKLTTSPELSPVATEPYPSLLATSTASANSAPATVSQ PSPTVTPPAALPRSSTGSPVSPPATSPVDFDRRSSSIAALRLKAREHEVRMELLRKANGEVAKWLARLTAKPMAAGSNPALDMDVSLSLCCILTCE CMNVAHPMNGYLWQWGVGNVARLRDIGSQVPTG

$>$ comp123863_c0_seq1_-_ORF_1_(frame_3)_translation MKISFFFFAADIHVVFKLWPVESSKVPRPAHLSSALSFNNVHLLPPIRGGQDKGREGEAESSPSPPPSSAGLNAVNFSLLPPQYHGYHQFHHYFNSC NMGILDKVETHHCLSESARTLNMTTKHESVNHQNKGQLTVMRLIGDKSPPLANTPSPKSISQEVKGNPSSAYGTKDNPSSPPEDLTMTSVVDVVA DSDHAVTEDEDETDGKIPGNGNEVPSNDNLLEGEDFPKRKQRRYRTTFTSFQLEELEKAFARTHYPDVFTREELAMRVDLTEARVQVWFQNRRA KWRKQEKAAGGQSQNQGYNPYSSSTTLPSIPATSPVSSKSFNPLGYPRTYDLSLFNTAHHHHPQFPPPYLPPPGLSLFRPAPNFLAPAGYSLRDLPY PAGLFSAAAAATLSSPYSAFPPTSFQSLLVNLSSQNRPKLPVPTDLTTGEAYTGLLTASSVTTPTAASATSTTSQPSPTGTVSPVGSLPGSGSPNPPPL PPTTTSATAAASSSAAPQTSVELDRRSSSIAALRLKAREHEVRMEMLRKSNGEVS* 
>Locus_18982_Transcript_4/4_Confidence_0.600_Length_6071_-_ORF_1_(frame_1)_translation MRSTLSVLGGPIQTTEGGIVPDLTDHHSNTFKNTMGISDKIERQECISEAARTLNLMEAHQKDQQPHHRKPIHHHHKLSVVHLVGDKSPRPASSPE QMKPCQQDAVAEDLSVTGGNDEVDSGDENISVADDDGENNKGSASAESLMEGDDFPKRKIRRYRTTFTSNQLDELERAFARTHYPDVFTREELA MRVDLTEARVQVWFQNRRAKWRKQEKAAGGQSQSQGYNPYSASTALPSLPASAPGSGKPFSALSYPRSYDLGLLNAAAVAHHHHHPQFPPPYLP PPGLSGLFRPAPSFLTQAGYSLRDLPYTSLFPAALSSPYSVTGSFPPTSFQTLLANLSAQNRPKLTTSPELSPVATEPYPSLLATSTASANSAPATVSQ PSPTVTPPAALPRSSTGSPVSPPATSPVDFDRRSSSIAALRLKAREHEVRMELLRKANGEVS*

\subsubsection{Lim}

Lim1.2Locus_2322_Transcript_1/1 help by dawid Salamanca

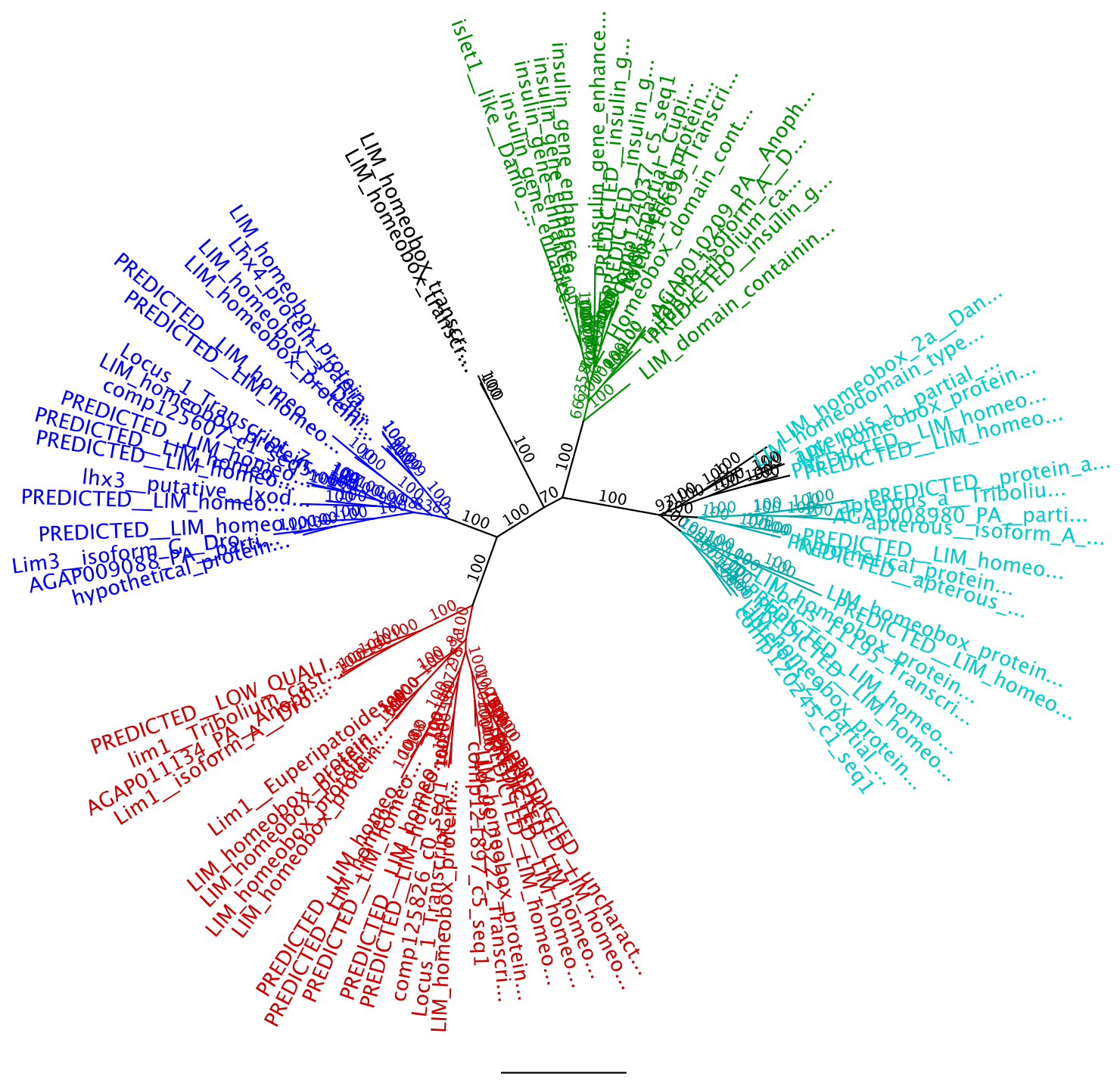

0.7

Supplemental Figure 9: Unrooted 50\% majority rule consensus tree to identify true Lim homologs. The Jones amino acid substitution model was chosen after mixed model testing. After reaching 1078000 generations and an average standard deviation of split frequencies below 0.01 a total number of 4314 trees were written in two files (each file contained 2157 trees of which 1618 were sampled). 


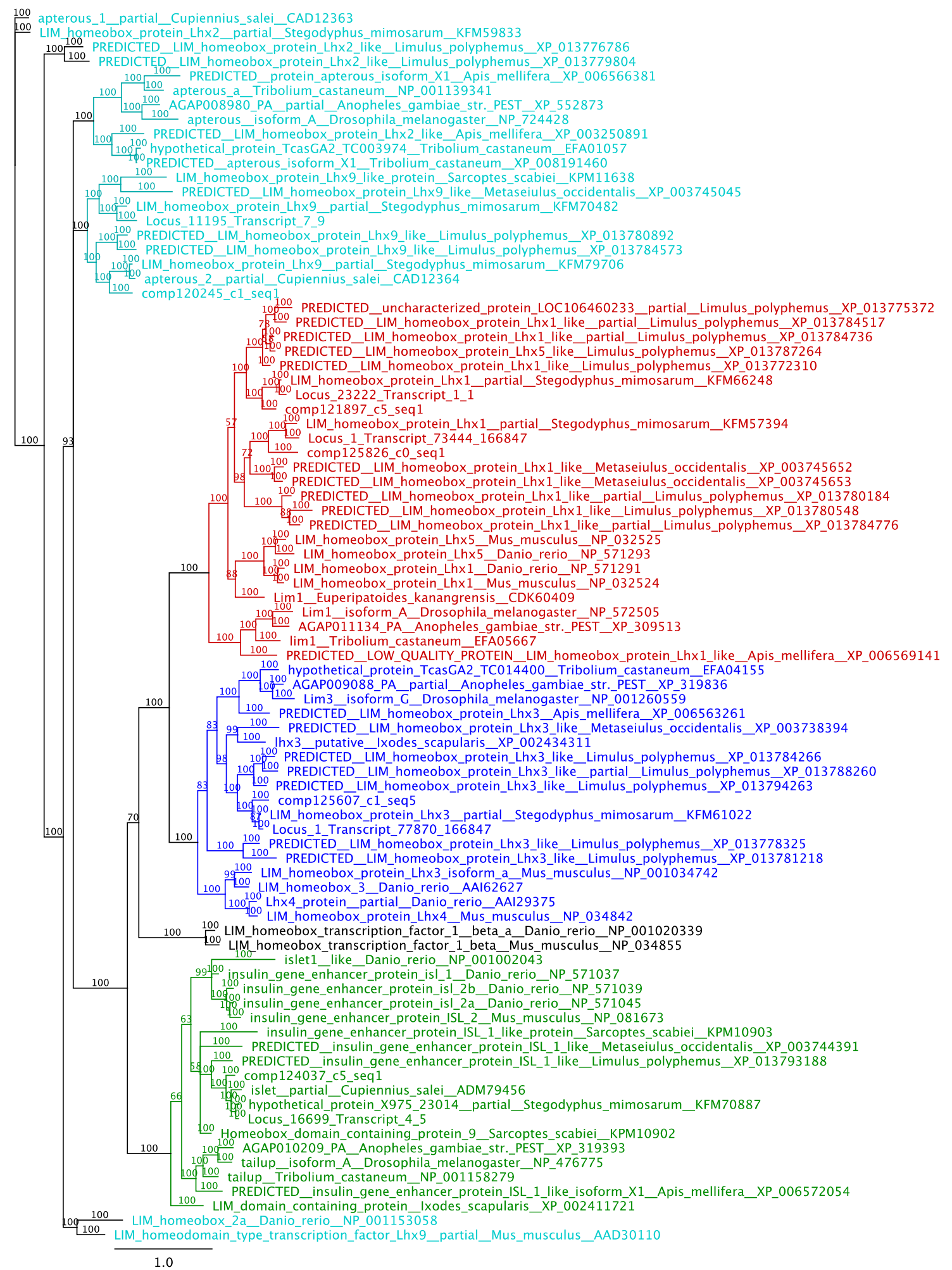

Supplemental Figure 10: Unrooted 50\% majority rule consensus tree to identify true Lim homologs. The same phylogram as in Supplemental Figure 9 in a magnified view to display gene and species names. 
>comp121897_c5_seq1_len=2584

GTATATAATCACCTTGTTTTTTTTTGTTGTTTTTTTTTTTGTTTACCTGTGAGTTTTGGTGTTCTGTGATGGTGGACATCAGTTGACAGTTTGAC GTGTCACTTCCCCCCATGACGTGTCTGTTTACTATCCGACGAGACGAACTGTCCATACCGTGCCGTTGAATCCGAAGATAATTAGCCTGAATTTC AAGAAGGACAGGAGGACGAGGAAGAAGAGAGGACGAGGAGGCGTAAGAGGAAGCGGAGGAGGTCCAGCCGTGCTGTTCCCAACTGTGCCGTACG GGGACGGCCGGTGTCCGGCCGTCGATACCCGCGCCGCGGTGCCGCTGAGGAGTTCGCCCATCAAGGTGGAAGCGTTCGGGGTAAGAACTCGGGTA CCCGGGGGCACTTACGGTGGCCTCTATCCCGCACTCCACCTGCCACAGCTTCGCTTCGCGACGGTGCCGGAACGGGAGTCGAACCCGGAGCCGGAC GCGGCTGCCGTCGACATGAAGATGGTGCAGTGCGCCGGCTGCGAACGGCCGATACTGGACCGTTTCCTGCTGAACGTGCTGGACAGGTCGTGGCA CGCGCGTTGCGTCCAGTGCTGCGAGTGCAAGTGCAATCTCACGGAGAAGTGCTTCACGCGGGAGGGCAAGCTCTACTGCCGGACGGACTTCTTCA AGCGTTACGGCACGAAGTGCTCGGGGTGCTCGCAAGGGATATCTCCGACAGACCTCGTGAGGAGAGCCCGAAGCAAGGTGTTCCACCTGAAGTGC TTTACATGCCTGGTATGTCGAAAGCAGCTGTCCACGGGCGAGGAACTCTACGTCCTGGACGAGAACCGATTCGTCTGTAAAGAGGATTACCTCAA CAGCCGGCATAGCCAAGGTGTACTAGTAGCAAACTCGCCTTCGGACACGAACGATCTGGACGACCTCGGCGACCCGTTAGGCGGCGACCCCGACA GCGGCCACATGAGCGAGAGAGACTCTAGTGTGGAACGCGAAGGCACCGGAATGAACGGGGGCCCGCCGGTCGGTAGCCCGTCGGGGCCCCCACCG GGAGCTCACACGGTACCGCACGGTGCCAACACCACGACCGGACCGGGCAACGGTGGCAATAACAGTGGGACCGAAAACAGCGGGCCCGGGACAAC CGGTGGCCCCGGCGGTGGACCTCCGAACGACGAGAACACGCCCGGGACGAAGAGGAGGGGTCCCCGAACCACCATCAAAGCCAAACAGCTGGAAA CCCTCAAGGCAGCCTTCGCTGCGACACCTAAACCGACGAGGCACATTCGCGAACAGCTTGCGCAGGAGACTGGACTCAACATGCGTGTTATACAG GTATGGTTTCAGAACCGGCGCTCTAAAGAAAGGCGAATGAAACAGCTGAGTACGTTAGGAGCCAGGAGGCATTTCTTCAGGAGTCCACGAAGAG CCATGAGGCCGCTGAGGCCCGGAATGTCTCCGGATGGTTTAGACGACAGCCCAGACATGGTAGCCGGGCCAAATTCTGGATACGCGTATTTCTCG GATTCTGGCAGCCCCGGTGATTTTGGTTACGGTGGTCAGCCTGGTTTTTACGACTTTTTCCCGGGACAACCTCCACCGGATGGTATGGGTTACCC CGGGATCCTCGGCCCTGGACAGAATGTTCCTCCGCCACCGACTAGCATGGAGCAACCTCTACCTTCGGCCATGGGACCTGGTCCGGTGAACCTTGG AGCGGACGCGCCGTACCTGACGCCTCACGGTGACCTCCTGTCGCAGAGATCGAGTCCCGAGAGCGTCCTCAGCCTGCCGTCCGTCAACGACGCGTA CGGCCCGCAGCGGTCGGGGGGTCCCGACGGGTTCCCCGGTGCCCTCGGTGGGCATCCTGCCATAACCGAGACTCCAGTCTGGTGACAACACGCGTC CTCGGTGACTCCCGGGGCGGCAATTAACTTCCTGAAGCGCAACATTCCTCGGCTTGACGCTAATGTCCTCAAAGAGAAGGACAACCTGGACGACA ACTGAAAACAAGAAGAAGAAAAAAAACAAACCACGACGACATGAATCATCATCGTCATCATCATCAAACATCATCATGAACATTCATGTGACGA ACGAAATGTAATTCCGAAAGGAGCGGGAAAAATCGTGCATTGTGTACAGTACAGGGACGAATTGCATCGAGCATTAATCGTGCCATTTGAAAAC AAAAAATTATGTATGTCTCGGTAAATGTGATGAGAATCCGGTTATTATGGATAATCACCGACCATTTTAACAAGTTTGATAAATTTCCCTGAAT TTTTATGACTTTTACCGGTAATTATGCATGATATCTAAGCGTCGTTGGTGAACATGGATAAGTCTTACGAAATTCGTCACGTTTGTCGGTTATTA CGCATAATCACCATTTCGTATTGGTACAAATGGTAAATCGGTTCCGTTGCCATGATATTATTTTTTATTGAGTCGATACCGGTTATAATGAACGC TGATTTAACGAATTTTTCACACACATCCCTGAACTAACTCCTATTTACGAATTCTGAAATCGGACCTCGTTATAAAGAAATCATATTGAACAAAT TTTACGATTGCACCCCGATA

>comp125826_c0_seq1_len=2345

CCGCTGCTGAACGGAGCGGAGCTGAAAGGCTCCGTCGCCGAGTTCTCTCTTTCCCCCCTCCCGTTTTGCGAGATATATTTCCAGTTTTTGTTCCGT TTTTTTTTTTAAATTTTCCGAAAACAGAAATTTCTCAAACGCGTGTTATTTTTTGAAGAAAAAATAATAATTGTGTTTTTTGTACATAATATAT ATATATTGTTTGGCGTTTGTGATGAAGACCCTGGTGCAATGCGCCGGGTGCGAACGGCCTATACTGGATCGCTTCTTGCTGAGCGTCGTCGACAG AGCGTGGCACGTGAAATGCGTCAAATGTTGGGATTGTAAAAGCACTTTGGCTGACAAGTGTTTCTCTAGGGACGGTAAACTCTTTTGCAGGAAA GACTTCTTCAGGCGTTTCGGAACGAAATGCGGTGGCTGTTCCGAAGGGATATCACCGACGGACCTCGTTCGAAGAGCCCGAGGTCAAGTGTTTCA CTTGGCTTGCTTCACTTGCCTGCTGTGCCGCAAGCAGTTGTCCACGGGCGAAGAGCTTTATGTGTTAGACGAGAACCGTTTCATTTGCAAGGAGG ACTACATCACCAGCACCAAGTTCCAGCATCACCACCATCATCATCATCACCAAGCCTGTTCTTCGAGTTCGCCTGTGGATTCGCCGGAGTTCGATG AATCACTCGAAATGCCAGGTACTGACCCCGGTTCTCCCGCAGAGCGGGATAACGAGAGGGATTCCAGTATCGAACGCGATCGGGATCTACACAAC GATCCCGTTTCGGGTGGTTCGGCAGTGAACAGTCCTACTGGGAACGGCAATGGTCAACAGTCCAACATAGTGGACGGCGTGCACTCGTCGGCCAC GGATTCGTGTCTGCAGGGCTCATCTGGACAAGGCGCCAGTGACGACGGCGGTCCCGGCGGTACGAAACGCAGGGGGCCGCGGACAACCATCAAGG CCAAGCAACTAGAGACCCTGAAAGCAGCCTTCGCCGCAACGCCTAAGCCTACGAGGCATATCAGAGAACAACTCGCTCAGGAAACTGGACTCAAC ATGCGAGTTATACAGGTTTGGTTCCAGAATAGAAGATCTAAAGAGAGGCGGATGAAACAGTTGAGTACTCTGGGTTCGAGGCGGCATTTCTTCA GGAATCCCCGGAGAGCTATGAGGCCTCTACGAGCGGGTCTCGGAGCCGATGGTCTCATGGAAGATGTACAGGGACATCATAACGGGCCGTATCCA TATTTTTCAGATTCCAGCAGCCCTAATGATTTCGGATACGGGCCACATCCAGGTTTTTATGAGTACTTCGCTAATCATTCTCAAGAGATGACCGT GTTTCCGGGTACCATGCCAATCGGACCAGCTAGCGTCGACGGACATCCGCTGGATTCACAAAACCCACCGACCGTCGCTGGCATGCCATCCATGGC 
GAATGGAGGCGTCAACCTGGCTTCAGACGCACCATACATGGCACCGAACGGAGATTACGTCCGTCACCACCAATCCAGCCCGAAGAATGGTATGG CCATCCAAATCCAGGCGTCCATGGACGGGTACGCTTCCAGGAGCGCTCAAGAAGCTTATCCGAACATGCCTCAGCAGCACCAAACGCCACAGCTC AGCTCCGACACTCCCGTGTGGTGACCAGAAACACTGCTGGTGTTTTAATGGAAGTGGTGTGAACTAATAGTGAAGTGTTCTCCGTGCCTCAAGAG TTATGTACAGTAATAACACTCGCATAATAATGCTGACACTTTTTCCTTTTTCCTCTTGGTTTTCTTCCACCTCATTCCGCAGACCGATTCGTGCTG CGGCGTTCACGGAAACTACAATAATTTGCATAATTTGACGACAGGAAAACGGGTCTGAGACGCCCCCTGATGGATTTTTTTTCTCAAGTCAATAG ATATTGAAACTGTGATTCATTAATAGAAAAATTTATGTGTGAAATATAAAAAAAATGGTCTACACTTTGCGTGGCTTCCACGAAGAGGATGCTG ACAGGATCCAGGATCCTGACAGGCCAGGAGCCCGGCAGCCACATTTTTTTAGGTCATAAATTCACAAGGTCTCATAGAAGTCCTTGCCAAAAAGC CCACCGAAAATTCCGGCTTAGGAGGTTCGATGGAGGGATTTGGGAATCCTTGATACTCTATAGGAAAGTGGTTCCCAAAGTGTAGGTCGAGTCA ATGAACTTTGGGGGGGGGGAAAGATTCTCCAAAATTAAAACGTCCAAATAAGAAATTCATTTCTACTTTAAAAACGTATCAAAACTAAAAAATG CCTTTACTCAGCTGAGCTAATTACAATGTATCATACAATGTAATTTATCAAATTCTACTTGTACAAT

>Locus_1_Transcript_73444/166847_Confidence_1.000_Length_855

AGGAGGTTTGGTACAAAATGTGGTGGCTGTTCCCAAGGCATATGTCCAACAGATCTGGTACGGAGAGCGAGGGGTCGGGTGTACCATGTGGCCT GTTTCACGTGCCTCCTTTGCCGGAAGCAGCCCTCTACCGGAGAAGAACTTTATGTACTTGACGAAACACGTTTCGTGTGCAAAGATGACTTCAAT AGGCAACAGCAACATGTGTCAAGTTCACCGCTCGATTCACCAGAAGAGTCCCTAGGTAGTCCTCAGGGTGATTCGAGCATCGTTGTCAGCGGTGG CTCAGGAGCTAATAGTCCTCACAACAACACTCCCAACAGTGAAGAACCCGTCGATTGTTTGGTTGCTGGACCTTCTTCGAATGACGAAGCAGGAG GAGGGACTAAAAGAAGAGGTCCTCGTACCACCATCAAGGCTAAACAGCTGGAGACATTGAAGGCTGCTTTCGCCGCAACGCCCAAACCGACAAGG CACATAAGAGAACAATTAGCACAGGAAACGGGACTTAATATGCGTGTCATACAGGTGTGGTTTCAAAACAGGCGTTCGAAAGAGCGAAGAATGA AGCAACTGAGCAACTTGGGAGCAAGAAGACATTTTTTTAGGAATCCAAGGCGAGCGATGCGTCCTCTTAGAGCCGGTCTCATGGACGAAGCTGTC CCACACAACGCCTTTGCCTATTTCTCAGATTCGAACAGTCCAAATGACTTCAACTATGGACCCCATCCAGCTTTCTACGACTATTTTCCCAGTTCT CATCCTCAAGGTGGTATAACAGATTTTCCGGGAACTGTTCCCATAACTGCAGGAGGAAATAATACTCTCGAACATTCGAACACAATCAATAATGG $\mathrm{T}$

>Locus_23222_Transcript_1/1_Confidence_1.000_Length_2928

CATCGACTTTCTTAACAAATTTGTGAGTCAACGTTATTTTATATTAAATTATTTTATAATATATATTAATAAATGATTTGTTAGCAACAAAATA CTAATTATGAAACACTAAAAAAAATTCAATATTTTTTATATTATTACTTAAAATGGTGAAATTATTTATTATACAAAATAAAGAAGTTTTTCCT TTGCTTATACAAAAAAAATTCTTTCTAGTAATTTCTAGAAATTTATTTCAATTTCCCATGCAAGAGAGCAAACATATACTTACATTTAAATTTT TTACCAACATTAATAATTTTTCTTTTTTAAATTAAACTTGTTTAAAAAAATAAATAAATTAAATAAAGTGAATATAACTTCCCCTTTCTTAATG TACTCTCTGTCTTTTTACATTTTTACATTTTTGTTTCCTAATGATAGCAACATAAACAATAAAAGAATCCCTTTTCTATATTTACAAT AAATGAT TTCCATTTCGTATTTAGAAATTTGGCATCCATGTTATTTCACTTCAGTTCTTTTCCTCTCTTCTATGCCGACCACAAGAAAGCTTAGGTGCAGAC GATAAGTTCCTCTGCAAATATGAAATTTTTTCTGTACAGTTTCAGAAATGAAATACGAAGAATTCAGTTCAACAAATCATCAGACGATTCACAG TTTCAATAGTCCAAATTTTATTCGGTGGATACTGACACTGGTGGTGCCAAAATGGCGTGACGTCACCAAACTGGAGTCTCTGACATAGTTGGATG ACCGGATAAGTTAGCCGGAAATCCGGCAGATGATGATCGTGGTGGTCCGTAAGCGTCTGATGCTAGGCTGAGGACACTGTCCGGGCTAGATCGCT GTGACATGAGATCACCATGCGTCGCCAGATATGGTGCATCTGCCCCTAAGTTGACAGGTCCTGGGCCAACAACGGCGGAGAGAGGTTGCTCCATA CTAGTTGGAGGAGGAGGAACATTCTGACCGGGTCCTAGTACACCGTGGTATGCCATGCCATCTGGTGGTGGTTGTCCAGGAAAGAAGTCGTAGA AGCCTGGCTGCCCACCATAGCCAAAGTCTCCCGGACTCCCAGAATCTGAGAAGTAAGAATAGCCAGAATTAGGTCCGGACACCATGTCCGGACTG TCATCAAGGCCATCAGGAGACATTCCCGGCCTAAGTGGTCGCATCGCTCTTCTGGGGCTGCGAAAGAAATGCCTTCTTGCACCCATAGAACTTAG CTGTTTCATTCGCCGCTCTTTCGATCTTCTATTCTGAAACCACACCTGTATAACCCTCATGTTGAGGCCCGTTTCTTGCGCCAACTGTTCTCGTAT ATGTCTCGTTGGCTTAGGCGTTGCAGCGAATGCTGCCTTTAGGGTTTCCAGTTGTTTGGCTTTGATAGTGGTGCGAGGTCCCCTTCTCTTCTGAC CTGGCGTATTTTCGTCATTCTGTGCTGCAGAGTTGGACGAATTGGTGGTTTCACTGCTGCTGTTGTTACCGTTAGGCCCACAGGAAGCAGGATTA GGCCCACTGGCTGGGCCACCAGCACTACCCGGCGTCGAATGCAGATGCATTTGAGGCCCTGTAGGACTGCTAACAGTAGAGGTTCCACCATTAAG GCCATTATGACCTCCGTCTCTCTCAACGCTGGAGTCTCTCTCACTTAAATGTCCATCGTGGTCAGGACCCAAACCGTCTCCAAGATCATCGAGGTC GTTACCGTCTGAAGGGGAACTTGCAGCAAGGACACCTTGACTATTCCTGCTGCTTAGGTAGTCATCTGTAAAGATGAACCTATTTTCGTCCAGTA CATAAAGTTCTTCTCCAGTTGATAACTGCTTTCTACAGACGAGGCATGTAAAGCATTTCAGGTGAAATACTTTATTCCTCGCTCTTCTAACTAGA TCTGTTGGAGATATGCCCTGCGCACACCCCGAACATTTTGTGCCGAAACGCTTGAAAAAATCATTCCGGCAGTAGAGTTTACCTTCCCTGGAATA ACACTTTTCCTGGAGTGTACACTTACATTCGCAGCATACAACGCACTTTGCATGCCATGATCTGTCTAGTACACTGCAAATGTATCTGTCCTTGA TAGGTCTTTCGCAACCGGCACATTGTACCATTTTCATGTCGTCTGTCCGAATTAAGGGCAGGGTCCGGCCAGCCCCGCGCCCACGCCAGGTCCCAA 
GTGTGTCCACCTTGGACGCCCTCAGTCCCGTTGGCTCATAGGACAGACCCAGGGCTGATAGCTCGCTGACCAACGAGGCTTTTGAATCGACCTCCT CCTGGTTCCTCAAGAGGCAAGAGGACTGGGTCGTCAGATAAGTCGAGCAGGTCGCCGTTTTGCTTGGTCAATAACTTATCAGTACCTATATAGAA TTCTCGAACCAAAACTGACGGGAAAAAAATTAGAATCCAAAACAGCTCAAATGAACTACTCTGGTTTATGTTGCTTTATGAAACAGAAATTTTG ATCAGTCATTCATTACAAACTTCTGTCTTGAACAGTTCTTCACCATTAAACTGCGATTATTTTAATTAGCTGATTTACTGCAAAACTTCGAAAGC CGAATAACATTGTATGCTTCTCAAATACCGGTTTGGTAATATTATTTGATGAGTATCATTCATTCTTATAAAAACTATAATAAAAAATTCATAA AAAAAAGCGAACTAATCTGTTAACCAGAAGAGCGGCTGAATCGAAAATGAAGAAAATAGACGATCTGAATCAAGAAGGTAAACAACAGTTTCTC GAACCGTCACCTGACCAGTTTCACGGCATGAAATGGGAACGCTGGGCACTATGCGTGCGTCCGGTATAACAGTTTGCCCCAGACTAGCTTTTTCT ACTGCGACTGCAGCTTGTTTGCAGGAGCAGCGTGTTTTACCGCCCGTTCCCCTTACCACACGCTTCTTTGCTTATCTCTGGCT

>comp121897_c5_seq1_len=2584_0RF_1_(frame_2)_translation MNPKIISLNFKKDRRTRKKRGRGGVRGSGGGPAVLFPTVPYGDGRCPAVDTRAAVPLRSSPIKVEAFGVRTRVPGGTYGGLYPALHLPQLRFATVP ERESNPEPDAAAVDMKMVQCAGCERPILDRFLLNVLDRSWHARCVQCCECKCNLTEKCFTREGKLYCRTDFFKRYGTKCSGCSQGISPTDLVRRA RSKVFHLKCFTCLVCRKQLSTGEELYVLDENRFVCKEDYLNSRHSQGVLVANSPSDTNDLDDLGDPLGGDPDSGHMSERDSSVEREGTGMNGGPP VGSPSGPPPGAHTVPHGANTTTGPGNGGNNSGTENSGPGTTGGPGGGPPNDENTPGTKRRGPRTTIKAKQLETLKAAFAATPKPTRHIREQLAQE TGLNMRVIQVWFQNRRSKERRMKQLSTLGARRHFFRSPRRAMRPLRPGMSPDGLDDSPDMVAGPNSGYAYFSDSGSPGDFGYGGQPGFYDFFPG QPPPDGMGYPGILGPGQNVPPPPTSMEQPLPSAMGPGPVNLGADAPYLTPHGDLLSQRSSPESVLSLPSVNDAYGPQRSGGPDGFPGALGGHPAIT ETPVW

>comp125826_c0_seq1_len=2345_ORF_1_(frame_2)_translation

MKTLVQCAGCERPILDRFLLSVVDRAWHVKCVKCWDCKSTLADKCFSRDGKLFCRKDFFRRFGTKCGGCSEGISPTDLVRRARGQVFHLACFTCL LCRKQLSTGEELYVLDENRFICKEDYITSTKFQHHHHHHHHQACSSSSPVDSPEFDESLEMPGTDPGSPAERDNERDSSIERDRDLHNDPVSGGSA VNSPTGNGNGQQSNIVDGVHSSATDSCLQGSSGQGASDDGGPGGTKRRGPRTTIKAKQLETLKAAFAATPKPTRHIREQLAQETGLNMRVIQVWF QNRRSKERRMKQLSTLGSRRHFFRNPRRAMRPLRAGLGADGLMEDVQGHHNGPYPYFSDSSSPNDFGYGPHPGFYEYFANHSQEMTVFPGTMPI GPASVDGHPLDSQNPPTVAGMPSMANGGVNLASDAPYMAPNGDYVRHHQSSPKNGMAIQIQASMDGYASRSAQEAYPNMPQQHQTPQLSSDTP VW

>Locus_1_Transcript_73444/166847_Confidence_1.000_Length_855_-_ORF_1_(frame_1)_translation RRFGTKCGGCSQGICPTDLVRRARGRVYHVACFTCLLCRKQPSTGEELYVLDETRFVCKDDFNRQQQHVSSSPLDSPEESLGSPQGDSSIVVSGGSG ANSPHNNTPNSEEPVDCLVAGPSSNDEAGGGTKRRGPRTTIKAKQLETLKAAFAATPKPTRHIREQLAQETGLNMRVIQVWFQNRRSKERRMKQ LSNLGARRHFFRNPRRAMRPLRAGLMDEAVPHNAFAYFSDSNSPNDFNYGPHPAFYDYFPSSHPQGGITDFPGTVPITAGGNNTLEHSNTINNG

>Locus_23222_Transcript_1/1_Confidence_1.000_Length_2928_-_ORF_1_(frame_2)_translation

MTTQSSCLLRNQEEVDSKASLVSELSALGLSYEPTGLRASKVDTLGTWRGRGAGRTLPLIRTDDMKMVQCAGCERPIKDRYICSVLDRSWHAKCV VCCECKCTLQEKCYSREGKLYCRNDFFKRFGTKCSGCAQGISPTDLVRRARNKVFHLKCFTCLVCRKQLSTGEELYVLDENRFIFTDDYLSSRNSQG VLAASSPSDGNDLDDLGDGLGPDHDGHLSERDSSVERDGGHNGLNGGTSTVSSPTGPQMHLHSTPGSAGGPASGPNPASCGPNGNNSSSETTNSS NSAAQNDENTPGQKRRGPRTTIKAKQLETLKAAFAATPKPTRHIREQLAQETGLNMRVIQVWFQNRRSKERRMKQLSSMGARRHFFRSPRRAM RPLRPGMSPDGLDDSPDMVSGPNSGYSYFSDSGSPGDFGYGGQPGFYDFFPGQPPPDGMAYHGVLGPGQNVPPPPTSMEQPLSAVVGPGPVNLGA DAPYLATHGDLMSQRSSPDSVLSLASDAYGPPRSSSAGFPANLSGHPTMSETPVW 


\subsection{3 apterous}

apterous2 comp118341 help by Julia Schneider

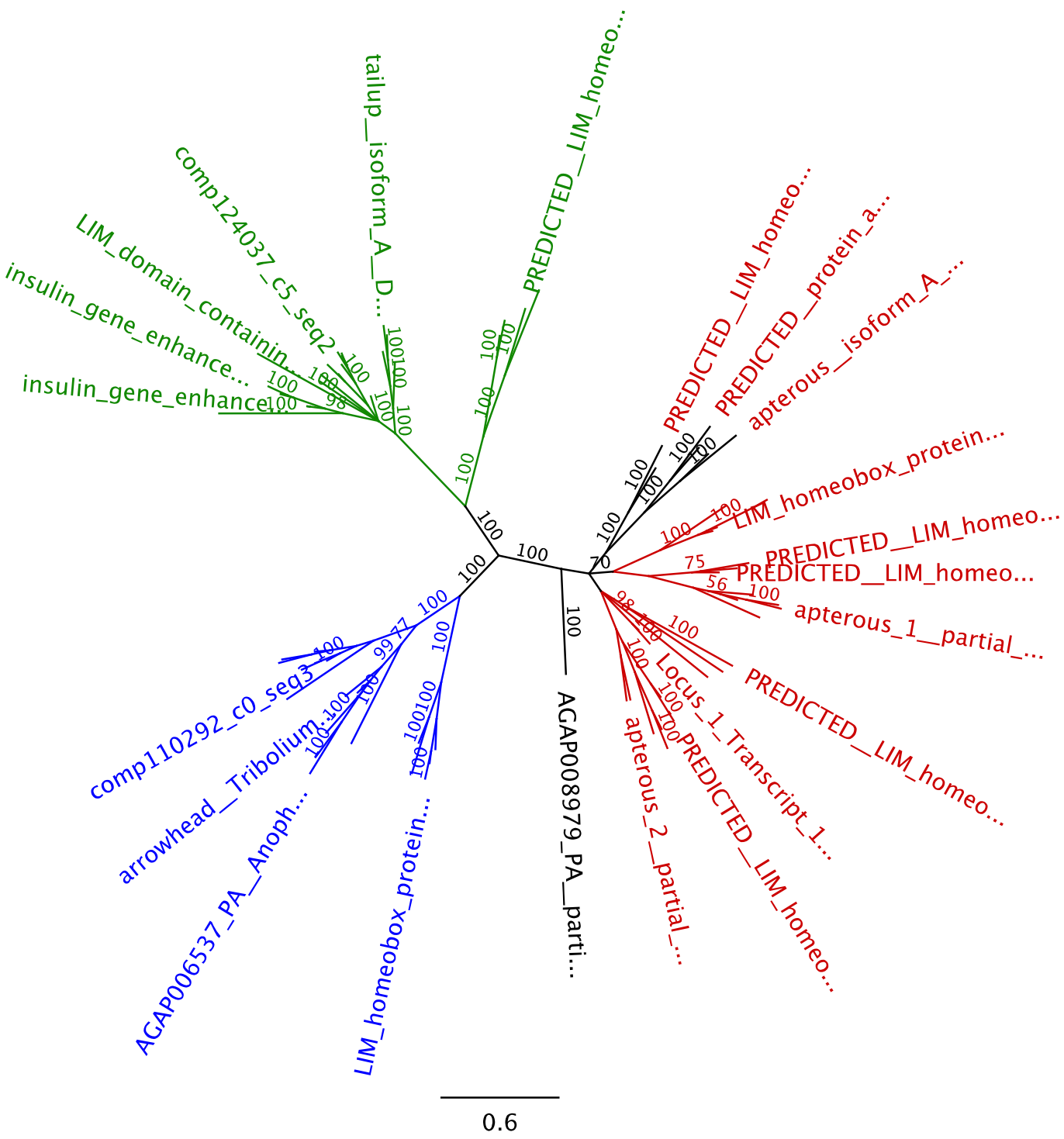

Supplemental Figure 11: Unrooted 50\% majority rule consensus tree to identify true ap homologs. The Jones amino acid substitution model was chosen after mixed model testing. After reaching 3000000 generations a total number of 12002 trees were written in two files (each file contained 6001 trees of which 4501 were sampled). Colors indicate monophyletic groups in the tree. 


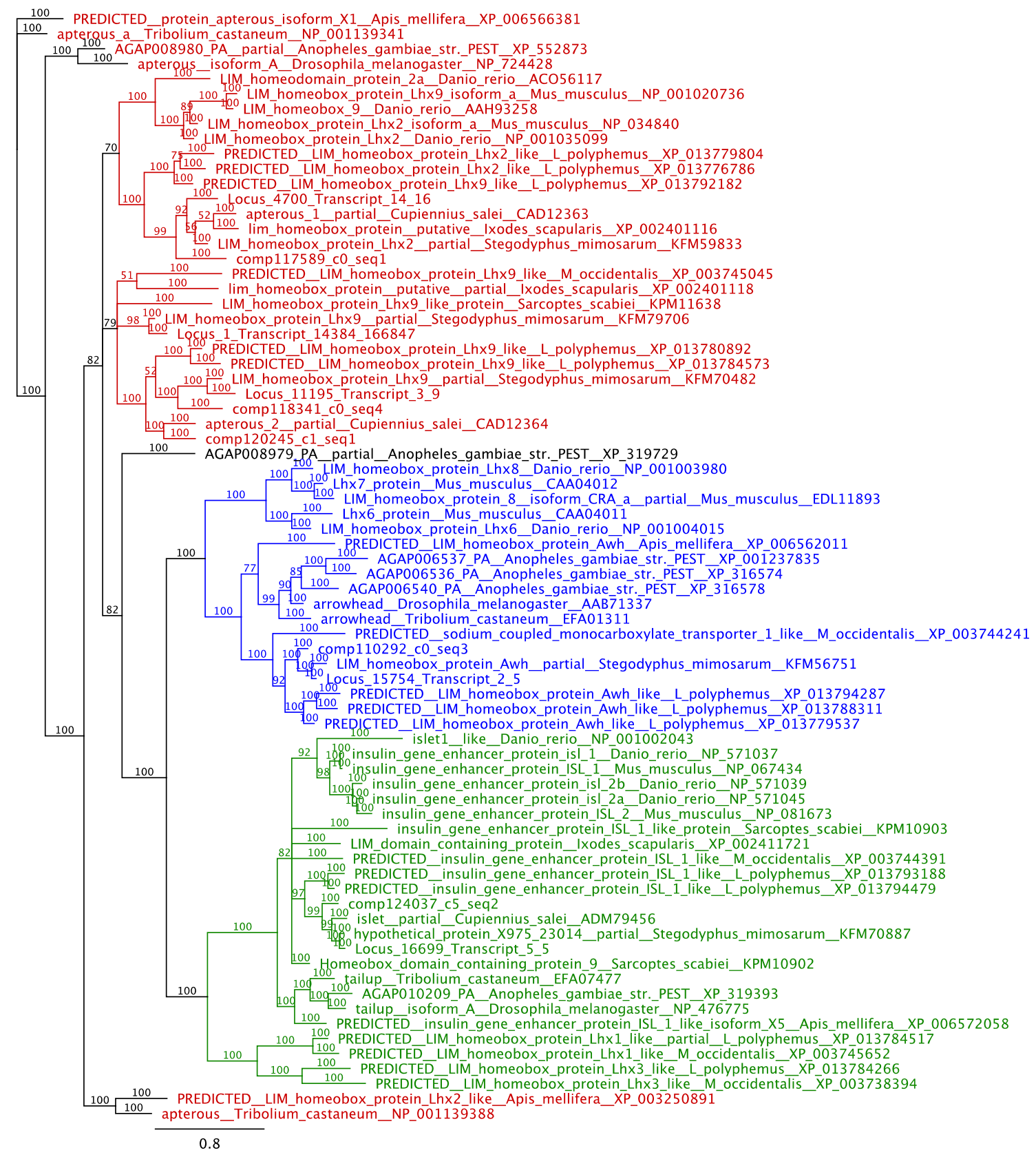

Supplemental Figure 12: Unrooted 50\% majority rule consensus tree to identify true ap homologs. The same phylogram as in Supplemental Figure 11 in a magnified view to display gene and species names.

>comp117589_c0_seq1

CCCCAATAAAAAATTTAATTTAGGAAAAAAAAACAGTAAAGCGCCATCTAGTGTCCCTCATTCTCTTATCGCGGGGCAGCAGCCTCCCTTATCGC GTGGCAGCAAAGGGCAGCAGGTGGGCAGCAAAGGGCAGCACCCATTGTCCCCGAACCGCTAATTTCCCCCTACTCATATGCTCTCATATAACTTG GAAAAAAAGACCACCAAAACTTACAAGAATGTTTGAGAAATGCGGGGGAGGAAAAAAAGGAACATTTATTGGACTCACAAATGAATATCGGTC AAATAAGGTTCGTGAATGTTTGTATGTTTTGTTCTTCCTGCGTGATCTGAGTCGGCTGAACGTCCACCAGAGCCGAAGCGAGTCGTAGAGAGTCG CAGAAGTGAGCCTGCGACGTCAGTTCCGACAGAGGCTTCGAGTCTTGCACTATGTCCGGGCCGTGTATACGCTGTCGTAAGATATTCCGCCTCCA TTTCGCTCTGGCGTTTTGGAACCATACCTGTAGCACTCTCTTCGAAAGCCCAGTCTTGAGGGACAGCTGCTTCAAGTCTTTGGCGTCTGGGTTAT GGTTGATGGCGAAATACGACTTCATGGCCCTCAGCTGGTGGTGTTTGAAAGAAGTCCTCATCCTTTTAGGTCTCATGACTGCCGCGTGTTGTTCG CTGGACGTGCTGCTGTCGCTACCCGTAGCCGTGACGCTACTGCTGCAGTTGGATTGGTGATGGTGGTGTTGTCCCAACGGAGAGAAGTTTGTGCG AGGCAAGGAACAAACACCGTCGGCACAGTTCAGCAAAGACCCTCGAAGGGGAGTGAAAAGGGAAGACGTGAAAAACGATTTGTGCTTCTTGGGA CGACCTTTGAGTGCGTTTTTCTGCTGGACGTTTGCCTTTCCTTTGCCACCACCGCCGCCACCCTGTTGCAGCTGAGCGGCCGGACTGCACGGCCGC 
AACGGATTTGCCGAGCCGGACGCATAGACACGAGACGTAGGAGGAATGGCCTTCTCGTCCGCGAGGCAAGACGAATCCTCGATTTTCAAGTCCTT CATCAGTGATTTCGGACAAATATTTGCGTTTGATCCTTCGTATTGTACGATTATCTCGTAGTGCAACCTGCAGTAAATGACGTCTTCTCTCAGTC CGAAATATTCTCCTTTGGTCAATGGCTTGTTGCAAGTCGCACAGGTGAAGCAATGGAGATGGTACACGTGGTCTCTGGCCCTCATCACCAGTTCC GTCCTGGATATTGACAAGTGACATCGAGTGCAGCATTTCGCCGAGAATAATCTGTGGTAGTCTTTCCTGCAATAAATGTGTCCCTCTCTGGCGAA ACAGCTGATCTCGGTGTCCAGGTGCTCGTTGCACTGGCAGCAGGTGAGGCACTCCAAGTGCCACTGGCGTTCCACCGCGAACAGGTAATAGCGGT CGTATATGTCCCTTCCGCAACCCGCGCACCTGACGCTGCTTCTCTGCACGCCACTGTCATTATTCGACGAGGAAGTGATGTTCTTCTCGCAAGTGC CACCGTTCTCTACTTCCGCTTCCTCATCCCGATCTTCTTCTTCTTCTTCCTCTTCTCGTCTCTGGCTCGATCCCAGAGAATCGTGTCCCCTCCGACC GTATACAACCAACATCAAGAGGACGATGACGATGACGCCGTTTTTCCAAGCTCGATATCTGCTACCTTGGTGGAGGACCTCCAGGTTGGGGAAGA CAATGCCTGGTGTTTGGTCATTCCTTATCCATTTTTTCTTCCCTTATTAGGGAAATTCAAGAATTGCTGCAAGATGTTGATTATTTCCGCCTCAC TGTTGTCACGTAACGGTGAAAAATAAATGAATGAAATAGATAAGGGAAGATGCTCAAGAGGTGGGGGAAAAATGAGAGGCGCAGTAGCGTTGA GAATCCTTTACTATGAATTTTTCCGATTCACTTGCCGTCCAACAACGTGTCTTGCTGTTTCGTGCTCGGGGAATACGATGATCATTAACCGGTGC ATTTTTTGACCTGTTCAAGGGGAAAGTAAAATAGGGGGGCAAAAAAAAGATTATATATAGAAAAATAAAGGGTTCTCGCGCTATAGCGAGGTTC GTAGGGGAAAGGTGGGGAGAGCGTAAAATCGCAAAAGTGGTGTCAGATAGCGTGAGAGGATTTATATTCGAGAAGCCCGGGGTTCTCCGAAGGG GCTTTTATCTTTTGCACAGTGGCCGCCGAATTGAATAGCGTAGTGGAATAATATCTATTGCATATGTCTTTGACGCTGTGTGTGTGTGTGGGTGG TGTGTCCTTCTCTTG

>comp117589_c0_seq1_-_ORF_5_(frame_2)_translation

MEVLHQGSRYRAWKNGVIVIVLLMLVVYGRRGHDSLGSSQRREEEEEEEDRDEEAEVENGGTCEKNITSSSNNDSGVQRSSVRCAGCGRDIYDRYY LFAVERQWHLECLTCCQCNEHLDTEISCFAREGHIYCRKDYHRLFSAKCCTRCHLSISRTELVMRARDHVYHLHCFTCATCNKPLTKGEYFGLRED VIYCRLHYEIIVQYEGSNANICPKSLMKDLKIEDSSCLADEKAIPPTSRVYASGSANPLRPCSPAAQLQQGGGGGGKGKANVQQKNALKGRPKKHKS FFTSSLFTPLRGSLLNCADGVCSLPRTNFSPLGQHHHHQSNCSSSVTATGSDSSTSSEQHAAVMRPKRMRTSFKHHQLRAMKSYFAINHNPDAKD LKQLSLKTGLSKRVLQVWFQNARAKWRRNILRQRIHGPDIVQDSKPLSELTSQAHFCDSLRLASALVDVQPTQITQEEQNIQTFTNLI

$>$ comp118341_c0_seq4

GATACGCCAGCTGGCGGGGGGCGTGGCGTTTTAACCCTGAGTGCCTCGGACGTTTTTCGAGGCGAAGCCCTTTTTACACACGCGCATTACGCAGA CCTCACGAGTCCGAAACACAGACCGCAAAAACACGCGATTCGTCCGACGTCCTTCCCCCCCTTGCTTCTTAACGCTTTATATTTATATATCTACCC TTTTTTTTTCTACTTTTTAAAGAATTTCTACCTTTTTTTTTTGTTGTGTTTTGTTTCTACCTTTGCATAACCGAAATCTTGAAACACACGAAACG AGGAATTCATCGTCGGTGATATTTTTCCGAAGACACCCGCAAAGTGGACATTTTTTTACTGGTTGTGGAGGAAACAGCTTCCGATCGGTGAGAA TAATTATGATTGCGATCGCCGATCAATGACGGAGAAGGATTGATCGACTGATTGATCTCTCTAATTTGCCAGTTCATCGACGTAAAAGATCGATC GCTCGCATCACGCTGGTGCCATACAAGCGCCCTTCCTCATCAGAGCTGTGGTCAGGTTCTGATGAATTTTCGGATGACCAGTGCCAAGACCTGCT GCTGACCACAGAACAACAACACCAATTCATCGTGAGTATCAGCCGGGGTCAGTCTTCGTCCGATATGCCGGTCATATCGCCCGGTGATTACGAAC CGTCTCCGGTGCCCAATTCGTGCGCGGGTTGCGGCACCAGAATAAGTGACAGGTACTACCTGCAGGCCGTGGACCGACTGT GGCACGTCTCGTGC TTGACGTGCTGCCAATGCAAAGTACAGCTGGACTCCGAGAAGACGTGCTTCGCCAAAGACGGTCAGATCTACTGCAGGGAGGATTACTACAGGAT GTTTGCCGTGAAAAGATGTGCGAGGTGTCAGAAAGGTATATTTTCCAATGAACTGGTGATGCGAGTCCGAGGCCTGGTCTTCCATCTGCATTGCT TCACGTGCGCGTGGTGCAATTCGGCGTTACCTCAGGGTGATTACTTCGGACTGAGGGACGACCTCGTCTATTGCAGAACTCACTACGAACTCATA GTGCGCGGCGAGAACGTTTACGCGCCCACGACCGGGGACGATTCCCTGGGATATCCGCCGACGCTCATCCCGCATCTGTCACCGATGATCCCGAGC GAGACGGTCGAACAGCCGACGAGCATCGAGCCACTCACCGACCGGCCGCCGCTCGTCGACGGCAACAGCTTCTCGACGTTTTCGTCCGTCGTGTCC GGTCGCAAAGGGCGACCCAGGAAGCGGAAAAACGTCGAGTTGAGGGTCGAGGGGCTCTCTCCCACGTCTTTAGCTGGTATATCGCCGATGGACGC AGCAGCCTGTAATCTTCATCTCTCACAACTGGACACCAGTTCCGCGGTGTCGCAACATCATCACCACCAGCAACAGGTGTCCCCACCGATAAGCGG AAACAACGGACAGGGCGGACAGCGGACGAAACGGATGAGGACGTCTTTCAAACATCACCAGCTGAGGACTATGAAGTCTTACTTTGCCATCAACC AGAACCCCGATGCCAAGGACCTGAAACAGTTGGCGCAGAAAACTGGACTTTCAAAGAGGGTTCTCCAGGTGTGGTTCCAGAACGCGAGAGCGAA GTGGAGGCGAAACAATCTAAAGCAGCAAGATTCGCACCTCATAAGCAGTGAACAAGGCTCTGGCGGTCCTCACATGCACGGAGGGCCGCACGGGC CACTTTCTGGAATGAACAGCCCCGGAGCCACGAGCTCTTTTTCCGAGTCGAGTCCCGTGCCATCCTGCGGTGGGATACCATCCGACGGCCCGTTGT CGTCTTCACAGACTTCTGGCAATCCTTCGTTGGACTTTGAAGGTAGCAATCCCATGAACCCGATGATGACGACGACGACGCAATCCCACCACGCC ATGGGCGCAGTCCACAACGGCGACGCGACGCCAATTCACATGACTTCCTTCCACGAGATGTTCTGACAAAGAGCGAAGCAAAGAGCCGAACATAT CAAGAAATTGTTCGTAGAGAAATTTCGGTGTTGAAGAAAACATTGTAATAATTTAATTATTCGTGTTTGAAAACCACGAAAACCGATCGAATTC ATTCGAAGAAATGTGGAGCCATTTTTATCGATCGAGTCATCCATTTCGTTCTTCGCGTATGATCAGGGGGAACCACGGCAGACACCATCTATAGC AGAAGGCCTCTGTACAAAGCTCGTGACGTCAATGAAAAAGTTATATCACGTATAGTCGTTGGATATACTGCACAGAGGTGCTATATCCAACGGT TACAGGCGAAATAACTTCCTCAATGACGTCACGAGCTTCGCTG

>comp118341_c0_seq4_-_ORF_2_(frame_2)_translation

MIDLSNLPVHRRKRSIARITLVPYKRPSSSELWSGSDEFSDDQCQDLLLTTEQQHQFIVSISRGQSSSDMPVISPGDYEPSPVPNSCAGCGTRISDRYY LQAVDRLWHVSCLTCCQCKVQLDSEKTCFAKDGQIYCREDYYRMFAVKRCARCQKGIFSNELVMRVRGLVFHLHCFTCAWCNSALPQGDYFGLR DDLVYCRTHYELIVRGENVYAPTTGDDSLGYPPTLIPHLSPMIPSETVEQPTSIEPLTDRPPLVDGNSFSTFSSVVSGRKGRPRKRKNVELRVEGLSP TSLAGISPMDAAACNLHLSQLDTSSAVSQHHHHQQQVSPPISGNNGQGGQRTKRMRTSFKHHQLRTMKSYFAINQNPDAKDLKQLAQKTGLSKR VLQVWFQNARAKWRRNNLKQQDSHLISSEQGSGGPHMHGGPHGPLSGMNSPGATSSFSESSPVPSCGGIPSDGPLSSSQTSGNPSLDFEGSNPMN PMMTTTTQSHHAMGAVHNGDATPIHMTSFHEMF

>comp120245_c1_seq1

CTCTCATCTGGAAGTTTTTTTTTTTTCGCTCCAGAAAGAAGATGAGAGAGAAGTGAAACGCATATACACGGCGTATGGGACGTTATCGGTGGTT TTGTTTTGTTGATAATTATTAGTTATTATTTTTTCAATCATTGTAGTGATCAGTGAGAATTATATGGCAGTGATGATAGAGAGACTTTCCGTGT GAATTGTGCGTTTGATAATTTTTTTTTTTGTCCGCTGAAGGATGCCTTTTATGTGTAATTTTGTGATTGACGCCACTCATGGTCGCATGAAATTT ACGTGTGAATATTTTAATAGTGACTCGTGTTCGTGTGTTGAAAAGCGATGAAAGACCTAAGATGCAAGTCCGCACCGAAAACATCATCGAGAAG TTGACGTTTTCGTGACTTACGAGACGTGGATTATTTCCGTGAAGGGATGATTCGTCCCTGATGTGCTGGTGAATTATCATTATTGTTCTTCTAGT GTGTTAGGTGTGTACAAAGAGAGAGAGAGAGAGAGGGGAAAGAACTTTTTCAAACATAGGAGCTAGACCAGGTGTGCTCAAGAACATAGTCTCC CTAACGGGAAGAAGTGGTGGTGGCGGCAGCGGCGGGACCCTCGAGGGGGACGACCCGACAGGGACCCGGAGTGACGACTCGCCCCCGAAGCGCGG CCCCGTCATGCCAGTCATCAGTATAGGGGATTACGAGCCCCCGAAACCGCACCTGTGCGCCGGGTGCGGGCTGAAGATATCGGATCGCTACTACC TGCTCGCCGTCGACAGGCAATGGCACGCGCGGTGCCTCACATGCAAGGAGTGCCACGTGCCCCTCGACTCCGAACTCACGTGCTTCGCACGCGACG GAAATATTTACTGCAAGGAGGACTATTACAGGTTGTTTGCCGTCAAGAGATGCGCTCGGTGCCAGAGAGGCATCTTCGCCAACGAGATGGTCATG 
AGGGCCAGGGACCTCGTCTACCACCTCCACTGCTTCACGTGCGCCTGGTGCAACACGGCCCTGACTCAGGGCGATTACTTCGGTCTGAGGGACAAC CTGGTCTATTGTAGGGCACATTACGAACTCATGGTGCACGGTGATAATTGCATGGCCTCCGCTGCCGATAGTCCAGAGGGTGCACTCGGACCCCT CGACCACCAGAAACCATTCCCCGGAGTCGCCCTGATGCAATCGAACCACGAGATCCGTAGCCCAGTTGCTGCTACCTCTGCCTACTCGCCTCTACC GGGTGCTCCCACTGGATCATCTGGTGTAAGGAAAGGAAGGCCGAGAAAAAGAAAAAGTTCCGAAGCTGGCGGAGTGATACATCCGCATCCACCC GGTCTGACAATGATGGACGCGGAAGGTTGTGCCCTTCACCTGTCGGTTATGGACCAGAACGCGTCTGGTCTGACAACGGGATCGGCACCTCCTCC GCCGCCTCAAAGAACCAAGAGGATGAGGACGTCGTTCAAGCACCATCAGTTAAGAACCATGAAGTCCTATTTCTCGATCAACCAGAACCCTGACG CGAAGGACCTGAAGCAGCTGGCTCAAAAAACAGGGTTGTCGAAGCGGGTGCTGCAGGTGTGGTTTCAGAATGCTCGGGCAAAATGGCGTAGAAA CAATATGAAGCAAGGCACTGACCCTTCGTCGAACGGTGGTGGTCAGCCGAACCAGACGCAGCAGCAACCGTCTCAGCCACCGCATGTGAGCAGTC CAGGAGCTACCAGCTCTTTCTCGGAGTCGAGCCCCGTCGCTACCTGCGGTGGCGCTTCCACCACCGGCAACAACAACAACAACCCCGATCATCACC CCCAGTCGATAGTGGCCGTCACGCCCACGCAACATCATCATCATCATCACCAACAGACCCAGCACGTACTTCCACCGATCGACTACGAGCCAGCCG GATCGAGCCTGACGCCCCTGCCACCTCAAGCCCGACACATCGCGTCGCACGGCAGCGAGAACTTGCCGTTGACATCTTTCCAGGAACTTTTCTAAA TGATGAAATTCGAATCAGTGGGGGAAGGGGGGCCCCCGGTCAGGCAATGTCAGTCACTTCAGTGTACAAAAAAAGAAGAAGAAAAAGAATCCTA ATGTTTCCCGAATGGTTGTCCACGGCATTTCGGAATCACCTCCAGTATAGACATGGACACACGCCTGGAAAAAAAAAAAAACTTTTGAAACATCC TCTCAAATTTGATCACGTCATTTCTTCTCTATCATGTTGACCAGGGGGTGCCCAACCTCCAGCCCGCGGATCAAATGCAGCCCGCCAGGAAGTTA TAACTGGCCCGCGAAATGTGTTCTGGTAGAAAAGTACAATAATGTAGTATTAGTATAAAAAGTCAATCGCACACAACACGATAAATGCCTATGC TGTAAGCGTGGATCCAGGGTAATGTATCCGGAAGGGTAGTTTATTGTGTAGGCT

>comp120245_c1_seq1_-_ORF_1_(frame_3)_translation MPVISIGDYEPPKPHLCAGCGLKISDRYYLLAVDRQWHARCLTCKECHVPLDSELTCFARDGNIYCKEDYYRLFAVKRCARCQRGIFANEMVMRAR DLVYHLHCFTCAWCNTALTQGDYFGLRDNLVYCRAHYELMVHGDNCMASAADSPEGALGPLDHQKPFPGVALMQSNHEIRSPVAATSAYSPLPG APTGSSGVRKGRPRKRKSSEAGGVIHPHPPGLTMMDAEGCALHLSVMDQNASGLTTGSAPPPPPQRTKRMRTSFKHHQLRTMKSYFSINQNPDA KDLKQLAQKTGLSKRVLQVWFQNARAKWRRNNMKQGTDPSSNGGGQPNQTQQQPSQPPHVSSPGATSSFSESSPVATCGGASTTGNNNNNPDH HPQSIVAVTPTQHHHHHHQQTQHVLPPIDYEPAGSSLTPLPPQARHIASHGSENLPLTSFQELF

>Locus_1_Transcript_14384/166847

AATTGGAGTCCAGTGAGGACAAGTGGGGATGGCCTCCTCTCACAAACTCTCATGATGGAGAGTGGTGAGGGAGGTTGCAATCTCCACTTGTCCTC ACTGGACTCCAATTCGGCGACGTCCACTGGCCTGAACCCATCCAGTGCTTCTCAGCGGACGAAGCGGATGCGGACATCCTTCAAGCATCATCAGT TGCGGACGATGAAATCCTACTTTGCTATCAATCAGAATCCCGATGCCAAAGACCTGGAGCAACTTGCCCAGAAGACTGGGCTTTCCAAAAGGGTG CTCCAGGTGTGGTTTCAGAACGCCCGTGCCAAATGGCGCCGAAATAATCTGAGGCAGGTGGACCTCCAGCAAGGCGGCCAGAACTCTCAAAATCC TCAGCCCTCCTCGCAATCTACGCATCCGAACAGCCCCGGCGCCACAAGTTCCTTCTCAGAGCCCAGCCCGTGCGGGGGCGGATCGGGCATGGGCGC AGAACTCTCTCACGCCTCTTCACTTCCGCCCCCTGCCGCCCAGCCGGGTGTTATGGAATACGAGGGCAACTCCGGTCTGACGTCACTAGTCCCAAG ACACGTCTCCCCCAGTGGTGAACCTTTGCCTTTGACATCATTCCAGGAATTGTTCTAACTGTGAATTTTGTTTTTAACTTTACCCGTAACTTGGA GGAAGAAGGGAAAAAAAAACTATTATCGAACGTCAAGAATTAATATTCATTTTCAGAGTAAATTTTTGACTTGAACTGATTGTGGAGTCAAAAA GGATTGAAATATTAGAGCACTACTATGACTTTCAATTTTTGTTTTTTGTTTTGAACTTCAGAAATCGAAGTTAAAGTTATTTTTTATGCACTCT GTTGGAGTTGGCAATCTACGGCTAACGAGCCGAGTCATGTTCGTGGAATAAATTTGAGGAGTTTGTGAAAAATAGCTTATTGCAATATTTCAAA ATTTGTTTCTAAACTTATTTTTTTATTACTCGTAACGACACCGAATAATGTATATTTTTAGAATCCTCATCAAAAAGAAAGTCAAAAATAGTAC ACACATTAGTGGTGGCAAAGTTTTGAGTGATGAAAAAATAAAACCGCTTTTGAAATTTTAACAAATGCTTTCTGAGAAAAGCGGAGATGAAAGT ATCAAAACCTGTTTGATTGCCAGATA

>Locus_1_Transcript_14384/166847_Confidence_1.000_Length_1163_-_ORF_1_(frame_1)_translation NWSPVRTSGDGLLSQTLMMESGEGGCNLHLSSLDSNSATSTGLNPSSASQRTKRMRTSFKHHQLRTMKSYFAINQNPDAKDLEQLAQKTGLSKR VLQVWFQNARAKWRRNNLRQVDLQQGGQNSQNPQPSSQSTHPNSPGATSSFSEPSPCGGGSGMGAELSHASSLPPPAAQPGVMEYEGNSGLTSL VPRHVSPSGEPLPLTSFQELF

$>$ Locus_4700_Transcript_14/16

GCNNNNNNNNNNNNNNNNNNNNNNNNACGCGTACCACACTTTGGGAAATACTGACCAAATGTTTTAATGAAAATTACACCACCCGCTTTATT TGGGCTAATAAAAAAATATTGACTAAGAGCTTTTTATGAAATTTAAAAAAACCATAATATTAACTCCAAAATAATTTCAACATTTAATTTAATT TTTATAAAGATTATAAATAAAGTAAAACTCTTTTAAGTTTTTTTCTTCAAACTTTAATCATTCTAGAAATAAAAAAAAGACATTGCAAAAAATA AATTTTCTTGTGAATACTGGTCTTCCAATACGTCTTACATGTAACAACAGTCATAAATGAAAACACAAAAATAACATTGCGGTGGTCGATATTT GAATACACAATTTGTAGCCAAATAGCAAAAATTACATAAAACGTTAAAATCATATTATGATCAGTCTTTTAATATGTCTTGCAAAAGAACCCCA AAAATATGTAAAATTAAAAATCATAAAGTTAAATAAAAAAGGTAAACCAACGTGTGAAACATGAAAATAATCTTATGTTGATCAGTTCTTTGA AGTCTTATGATTGGACACCTACAAGCGACGTCATCGTGGGTATCGTATCGATCTTGATTGACGATTGGTTTATGTTAGAAACTTTTCTTTCCATT CTATTTCGAATAAGCCTATCCCGTCAAACGTGAATTCGATAAGCGATCTCGATATAGGTTATCGTATCTTGCGATAATTTCGAAACTAATAAATT CTTTTAAATTAATGAAATATGCAATTAATACTTAGAAAAAGAGATAAAATAAGTTATTAATTTTCCTTTCATAACTAAAAGAAGCCTACAATAA AACAAGCCATAATAGTTGTAGCGTTAACACGCCATCTATTTTATAAACATTCAGCTGGTGCTGAGGTTATAGGTCAGGTGGTTGCGTCTCAGCTG TCTGCTTCTCGAGTTGAGAGTATCGCATGGAATAACAGTTAACATTTTTAATATACTTTGCGTAGTCATGGTAACAAAAGTAAATAAAAGGTAG AGGAATCAATTAAAATTCGCTTTACAGTGGAATGTCATTAATAGATGAATATATCCCGTAAATGGGCTATAGTTCACTGAATATGTATAGCCTT GTCAATGGGCTATATCTATGCATGTCCTGTAATCGAGCATAGTACGTCTTGTAATTTCGTAGCGAAATCCATTTAATTGTGGGTTTTAATTAAT ATAAATGCTAATTTTTCATATATGCAATATATTTTTTGTAACATTGAAATGTTTAATTTTATTTCAATTAATTTAATTATTAAGAAATGTAAAT TTTTTAGGCAATATATTTGTTATGAGTACCAATAGCTTTTTTCCCCTTTTTTTCTGTGTGTATGTGATGTTTTCTAAAATAAAATATTTTGAAA ACTGTAATAATAAAATAAGATATCACTTCAGGTATTTAAAAAAAATTTTTTTTGTTGAGGTATTTCTATAATTTAAATATTGTAGAATAATTAC CAAAATAAGCTATACAATATATAGTTTATAAATAATCAATATTATCTAACTTAAAGGTTTTGTAATTCATTAAAAATATATTTAAATGTATCCA ACTTTTATTTAACTTTAACTAGTATTTAAATTTCTTAGATGTTCTTAAAGTTTAGTATTTTCCAGTGATATCAACCTACAAATGAACCATCTTGG TAAACAAAAATACTAGAATTAATAAGAGTGGGGAAAACTCTTTTCTATAATGTTAACTGTTAATTTGTGTTTCCTAAACAGTGATAATGAGGTT TATGCACAGTTTAAGAAAAACCTTAGTACTTATAGACTAACGAAACTTTTTTAAAACTTAATTATCTGATATTAAGTAAATACTATCATATACT ATTTATATATAACGTAAATGTACAATAAAATCGACTGTTTCCTATTCAGTGCTTTTTAAATCTACTTTTTTAAAAGTATTTTTCTTAGTTTTCTT TCTTTTAGTACTTCGTTAACCAAAAAATAATGATAATAATATAAAAAAACAGAATTTGCACTACAACAATAGAGGGAACTCCCACAGAAAGGTT TCCCCATCTCAACTCTAATTTTTATTCTAAATTTAAACAAAGTAAGGATTAAGAAGATTATTCACTATTCCCTAAATAAACAACTAACTAATAA AAATAAAAATAATACTTTATAATAAAAATAATGTATGAAAAAGAAATAAATTTGTTTTGATATCATTAGTATTTGTTTTTATTTTACTTCGCTT 
TTTTTAAAACCTATTTGTTGACCAGTTAGTAAATGAAAACAAAGATTCTGAGATTCAGAGTTTTCCAAAAAGGTTAGGATATATTTTGTGCAAC TTAAACTAAAGAAAAGAAAACGTGAATAATGAGCAAATATTGAACATAAATGGACGTACTATACTTTAAGCTCTATAAACGAAAATCTTTCTCA ATTTTTAAAAGAAAAAAAAATGGAGAGAATTAAGGAAGGTTTTTTTTTTCAACCGAAACTACAATATGTCAATTTCTGTACTTTCATACGTGTT TTATTAATTTTTTTTTTCATTTTATCATAACATTCCTTTTTACATAATTTTATAAGTTTTGATCTATATTTAAATAAGTTAATAAAGGAAATTT TGTGGAAATAAAGGCATTTGTGGACAATGACTGAACACAAAGTTTAAGAAGACTGGCATTCCTTTACTACCGGAGGGTACGAATGATAACGGCG GGTAAAATCATAATTTAATTCAATACGAGAAATCCAGCAGGTCGAACTCAAAATTAAAAGCAATCACTTCCAGACTTTACGAACAACATTTTAT TCCTATGAAACCACGTGTTGCAGCTCCACCAAACGATTTACATGGAATTCAATTCGGAGTTTTAATTATCTGAACCGATAACTTAATTACTTTTA TTTATTAAGTTGGACTTATAGTATTCTTGATGATCCGGAGAGGGTACTGGCCATAAAAAGCCAGTTGCGTTCAATGCTTCTATTAAAGGAAATT GAATTCAGATGATTGTTGAAGTTAAGTCAGAATGCCAGAAGTTCATTGTTAAAATTTGTATTTATTTTCTTAAATTAAATTTTTAAAAAAATTA TCGCGATAGTAAATATGTAAAATGCTACTTTGTGAAAATGCATTTAACAGTTAACAATAATTTATTTTGTTCGCATACTCTATTTGAATCAAAG AAAAAAAAAGTTACTTTTCTTTGAGGTCAATTATTTTAATACACTAATTATGTTTCATCTAGAAATGAATTAATTTGTAATTTATTCTGATATA TTTTTAATTAAAAAAAAACTCAAAAAGCCGTAATCAATTTTTTTTTCCTCATTTGAAGCTACTTTTTGAATGTTCCTTCAGACATTTTTGTATTG CAAAAACTTTTATTTAACTATTATTTGATAAACACTATAGCAAAGGAAGCCACTAAAAATTTTATACATAAAAAATATTAAAACTTTAATAACT TAATACTTTCAAAAAATCCCTCCCTAAATTACAAAATAAACATACTAAATTGGAGAAAAAAAAATTTAAATATAAAACCCCTAAAATATCTCAA CTACTATAAAGGAATTGTGCAGAAATTGGTGAAAATTTAATGTGGTGGGAATTGGCATGCTTTTTAAACCTTTGACGCAGTTGGGAGATATCGC AGTTGGGTGTCCCTTTTATATATTTTCGGGTTGGGTGTACCCACTATTTAAAATACTTAAAACTCAGTATTGTTAAAAAAGTTAAAATCGTTTA AAATTTACATGAGATTCAGAGAGTATATCAAATGGATTAAAGTATTTCCATTAAAAAAAAAATCATTTTACCAGCTCACAATATCATGCTCCGC AATATCGACTCATTTACTAGAACACAGATTGTCCCTACAAAAACAGTGAGCAAATTTTAGAGTAAATTAGTAAAAGTCTGTATGTTCTGTTCCT CTTGTGTTATGGGTGTCGGCTGGACATCGAGGATCGAGGAACCCAGTCGAAATGGATCGCAGAATTGAGGCTGAGACGCCAGTTCAGAGAGAGG TTTGCAGTCCTGTGCGATTTCTGGACCTTGGCTTTGCTGACGGAGCAAATTTCGCCTCCACTTTGCTCTGGCGTTTTGGAACCAAACCTGTAGCA CCCTTTTCGACAAGCCAGTCTTCTGGGAGAGCTGTTTCAGATCTTTGGCATCAGGATTGTGATTAATCGAGAAATATGATTTCATCGCCCTCAGC TGATGATGCTTGAAGGATGTGCGCATGCGCTTTGGACGCGTCAGGTGTTCACTTGAAGTGCTGCTGTCACTTCCCACAGCGGTCAAGCTGTTCGA ATTGTGCTGAAGCGGCGAGTGGTGATCACTTGACAAGGAACAGTGGTCATCGGAGCTGGGACCCCTGTGGGCCAGCAGTAATGAAGAGGAGAGA AAGCTCTTTTGCTTTCTTGGTCTTCCCTTCTGACCAGCATTCTTCCCGATTGCATTCACAGTCCTGATGGGAAGATCTTCCCCACTATTTACTGTC CTCTGCTGCTGTTGGAACAGTTCCATACCGCGGCAGGTTAGGAATCTGTGCAATGTTTTGGCACCTTCCGTTGAAGCACTTGCCGTCTGAATCTT GCTTGCCCTCTTCTTTCAAACACTGCTGGGAACAGAGGTAGGGCCTCTCATATTGCAGAAGCATCTCATAGTGACTCCTGCAATAAATGATGTCC TGCTTCAGTCCGAAGTATTCACCTTTTGTGAGGGGTTTGTTGCAAGTGGCGCAAGTGAAACAGTGGAGGTGGTAGACGTGTTCTCTGGCTCGCAT CACCAGTTCTCTTGGAGAGATGGATAGGTGGCAGCGGGTGCAACGCTTGATAGAAAACAACCTGTAATAATCTTCCTTGCAATAAATATGGCCA TCTCTGGAAAAGCAGGTCAATTCAGAATCCAGTTGCACTTTGCACTGACAGCAGGTCAGGCAAGACATATGCCACTGTCTGTCAACAGCCAACAG GTAGTACCTGTCAACGATGACACCCCCGCATCCAGCACACCTTACAGGTGATGAAGATACAGACGATTTGCCCTCTGCTCCCATCGATCCACCCCT GCTGATCTCTTCCGTGATGCAGGATTCACTTGCGTTGCTGCCACACACCACCAGCATATCGTTCTTTCCCGAAGAATTCTTCGTTCTGGACCAACC TCAGGAGGACGCGCAGCTCAGGAATTCCATATTCTTGGAATAGTTTTTCGTTTTCAGGAAGTGTGGATCCTTCAGACTGTTTCGAATTCTGTGGA ATGACGCTGAGGCTGGCATATCGATGATTTAACAACTCTCAGTTATACTTAGAATCCATTCCGATAAAGAAAAAAGTCGTTTGCAACAAACAGT CAATAAAATTAACTATAAAAAAATAATTATGTAATCACTGTAAAAATTGTTTCTTGCCACCTGATGACTATAGATTATGAACAGAGGGACATTC CACATACTATCTAGTCCTCACTCTTCCATTTGAAGTGTTTGCTGCAAACGACTGTTTCGAATTCTGTGGAATGAC

>Locus_4700_Transcript_14/16_Confidence_0.321_Length_5545_-_ORF_7_(frame_2)_translation MLVVCGSNASESCITEEISRGGSMGAEGKSSVSSSPVRCAGCGGVIVDRYYLLAVDRQWHMSCLTCCQCKVQLDSELTCFSRDGHIYCKEDYYRLFSI KRCTRCHLSISPRELVMRAREHVYHLHCFTCATCNKPLTKGEYFGLKQDIIYCRSHYEMLLQYERPYLCSQQCLKEEGKQDSDGKCFNGRCQNIAQI PNLPRYGTVPTAAEDSK

>Locus_11195_Transcript_3/9

TGCCCAATAAAACTGTACAATAAACAAAAACGATGCATTTCATCGTTTACTGTGTAACCATGAATTTATTACAACCGGCAATTCACCAGAATGCG TATCAGTGAGTATTTATTTCTATATAAAATATGTATGAATGCAATGTGATCAGCAACACAACAAGAGAAAAAAATTATATACACAAAAAACAGA TATGCATTGTCGATGAAAGTGTAGCGTCAGATGTTCTATACAAATAATCGTAAATTCTCTTCGAAAGGAAGAACATTACCGCAAAAACCAAACT TTTTTTTCTTAGTTATTGAACTGGTTTTACATTTATGAATTGCGTCGCTTTGCCCAAACACACTTATTTAGAAGACTCCATCAACTTGTTTAGTT TTTATTTTGCTTTTAGTACCTTACACAAATGTCACGTATGAGTGCCATTAAACGAATGTGAGCTAAGAAAAAAAAATACTGGAGAAACAGTTTC AAAAGACATGATATTTGAAAGAAAGGGAAAAAGCATGCTATACAATTAAATATTTGATCTATCTTGATGTAAAAAATAATAGTTTGCGTAACT TAAAGCTATTTTTGGACAGAATTACTATTTATTCCCAAATAATAAAATTAGAATTTGTATAATTCAAAGAATTAACAATTTGGCTTTATAAAGA ATGATTTAGAAACTGCGCACGGCACTAACATAGTCAAAACACACTAGTATTTTGGGGATTTATTATTATTAATTCTTGACAAATATATAATTTT GGACACAAAGAGAGAATTTTTCATTCATACGAATTCTTTTCATAAGGTACTTCAACATTTAAATTATTTACTTGCTTTTTTTTGAGAATTAATA ATGTTGGTATAATACTTTATAGACCTTTAAGCATTATTCCTTGACTTTAGAAAAACTTGATTTAAAAATTTAAAAAATTTATAAATTCTTAAAA GAGCATAAATTAATTTCTTAAAAGAGCATAAATTTATAGCTCTAGCTGCAGGAGTAAAATAAATAATCAGCAAAGCATTATAAATAAAGATCTT CTTAAAATACTGAAATTTTCCTAAAATCTCACAGAGAATTTTCATGTGATTTAGAAAATAATATCAAATGCAAAAAGTAATTATACAGCACAAC ACAGAAACGGTAAATTATTCTAAATCCGCAAAAAAACAAAAGGAAACGTTTGCAGTTTTAATTAATAAGAGACGTAGGAATAACTGTATGTAA AATTAAAATAAAAATATTTTAAAAATATATATAATTTAGGTTCTTTAAAATAAATTTAAATTTAAACATATGAACGAAGAAGTTTCAAAAAAT ACATTTCTGACACGTGTGTTGACTTTACTTCCTATAGATACTTGACAGCTGCATAAAAATGGTTTAAATTCCACCCGATTTTTCGTGATAATAAG TTTAAAAAAAACTTGTTAAGAATAGATCAAAATAAGCAGCTTTAGATCTTCCGAATGAACAAATTAACTCAACAAATACTTAATCTAATTAGGA TTAACGTATTCACACAAATTGAATTATGCAAAGTAACATTTCTGAATTTGATTTATAACTGTTTAAGATTATTTAATAAACATGGAATCGATTT ATACAAAAATAAATTTTAAAAAGGCTGATAAAATATAAATAAGATTTTAAAAAATTGCTTTTATATTTGGGTTCGTAATAAGTAATAAAATTT ATATGTTTAATCCTCGTTACAATTTGTTTTAAAATCGCATTTATTTAAGCAATGAAATAAGATTAACGAACGCATAAAATAAAGGAAATTTAAG TGATTTGAAAGAAAGTTAATCTTTTAAAATAAAAATCTTAAATTTGATGTGAATAACGTTGTTAGTTCTAAATTAGGAATTTGATTAAAATGG AACGAAAATGAAAGAATAAAAGAATTTATAAGATAAATTAATTTGTTTTAAGAGGAATTTAAAAATTATTTCTTAAAATATTATACAATATTC CAACAAATATTTTAAATCTTCAAATTTATTTCAATAACTGGAATACTGTAATAAAGCTTATCTTATTATAATAAGTTAAAAGTTGTTATAAATG TAAAATAGCTTATGAGATTTATTTTACGCTACGATTTTCATGATTATAAACAATAAAATATCATTTTTAAGAATTGCTGCACTAATATTTTTTC ATAAGACATTGAGTTAGACTACAATTAAAAAATTTTATTTAGATAATAAGCAAATCAAATCTCTGCCTTTTTCTTTCTCATAACTAGTCTTTTT CAGTATTCTTTGCTCGCAAATTCTTTTTCTCAACTTCTCTGAATGATTTTAATGATTTAAATAATTTTAATAATTTAAATATTTTTATCATTTTT TAATTGTTTTTATACAATTCTTACTAAGTATCAAAAAGAAGTAGATTCAGATACTGAATTATAAGCTAAAAAAATATATAAATAAACAACTTCC TTATAGCTATTTAAAAAAAATTAAGTCATTGTACAATTTAGAAGATGACTGCCAGAGATTTTTTTAAAACTAACATTTACTTTTTCTTTGTATT 
ATACTAACAAGTCAAAATTTAGTTAAAATTGACTATTAAAACCATGCTATAAAAAAACTCACGATTGTACAAATAAAGATTTAAAAGCACAAAC ATTGTTGTGTCTAGATTAGAGATATTTTCTTTAAAAATTCTAAACATTAACTAAGTTGATATTCGAATTGAACGTCTCAATTTACACAACTCAA AGTAAATTTTAAGCTTTATCAAAACCATTTAAATTGTTATCTTAGTTAAGAAAGTTAATCGAGTGAAAATGGTTTACATAACCAAAAAAACACG TTTGGTTTAGAGTTAAGCTTCAACAATAAACTTTTTTAAAAAGATTTTTTCTCTCCCGGAAACAAGATAAATTAGATATTCCCATTAAATTTCA ATATATAAAGTTCGAACAAAAAATAATACGATGATAATGGTTATTCTCCACACTCCACTTTTAACAATAATTTTTATTATTAAATAAAATAATT AAATCTGCAATATGGTGTCTCAAATAATTATTATGAGAAAAGGAGAGTTTTGAAACATTTAATTCATACTTATTTTATGAAACCGATAAACGAT TTTAATAATTAATTTTAAAAATCGAGATTTCTTCAACAATTATTAATAAGTGTCAAGTTTCAATGAAAGTTTTCTATTAGATAATATTGATTTT GAGCTAGTAGATTTATTTGTAGTTTAAAAAATATGAGTTTTGCTATCCTTGCTGTTCTTCCATTTAAAAAGTAATTAACTTGTTTTCGGTGTTG AATCAAAAATGCACAGGGTGTTTCGTTATCATAACTCTCAATGCATAATAATATCATGAAACTTTTTCAAAAATAAAGTAAAAATTACAGGCAC AAATGAGGTCAGAAATTAATATTTGAGGAAGAAAAAAGAAACGTTATAAAAATGTAACAAATCATCATTAAGACAAGCGAGAATATTAATGTA TGTCTTCTAATGGACGAAATTTGTCATCTAATATTTTGCACGGATTCTAAAAGTAAATATTAAAGGGGTAATTGAAGAGCACCTTACTCAACAA GAACGATGTTAGAAAACAAACTGTTTATTTTAACAGCAAAATATGACTTCCTTGTTGTATATCTTGTTGATATAAAGGCATGTTTAAAAAAAAA AAAAAAGAAAAAAAACTATATTATTATGCAACGTGTCTCAGCTAAATGTTTCAGAATTTCTAAGAGAGGCAAGGTGCATCTTATCAACTTAAAA TCGCAGAGATGCAAGGTCAGAAAGGCTTTCCTAAAAAGGCAGATGATGTTGCAAGAAAATTTCGATCGTTCCTTCACTGAATCGTCATTTCGCAC AAGGACACAGAATATTCAGGAATTCCATGTTCCGTAGATATGAGATCTGAATTGCAACTTCGAACACTTTCTTTGGTCCGTATTCCTTTCGCGTT TGCACATTTATTATTAAAACAAATATTCAAGTACGGTGATTTTTCCTTTATTCTTATGCACATCACCGCTGCAGGAAAGTATTACCGACCCAGCA TTGCTTGATACTTCGAGTCTTCAGGATACCCTCAACCCCACCTAGAAATTCTGAAACGTTTACCTGAGACCCTTGAATAAGTTTAAATTTCAAAA TAATATTAACCATTTGCTTACGGCTGGTAACACAGGTGGACCAGAATGACGGGCTTCATCATACTAAGCGCCGTAAGCGAAGTGTTAATAATAT AATAGACACATAATATTGTTTATGCAATGCAATATATAATATTAAGATGGTAATATTAATACATTAACCAAAAAAATTACTATTTTGTTCAATT CCAAACTTATACGTGCAATCGAAAGAGTCCAAGATGTTGCTGATGGATCTTTTAAAAAAATGCATGGGCATGATCGACTTTAGGAATATTCATG AGAGTTTTTTGTTGAAATTGTTCTATCAGAACAGTTCATGGAAGGATGTGACATGGAAAGAGACATTATCTCCATTATGGACAGATCCTGGATT AAGTCCTTGGTGATTGACCAGTGGGGTCATAGGATCACTACTGTCAAAGTCCATGGATGCTCCAGGCCCCACGTGGGTGGACAGGGAGCCATCTG AAGCGACACCACCACAGGAATGCACTGGACTGGGCTCGGAATAGGAACTGGTAGCTCCGGGGCTACAGATCCCTCCCCCCATGTGGCCGCCCTGG AGCAACTGAGGGGTGGACGGCTGTTCGCTGGTAATCATACCAGAAACAGAATCTGGTTGTTTGTTGTTGTTTCGTCTCCATTTCGCTCTGGCGTT TTGGAACCATACCTGAAGCACTCTTTTGGAAAGACCTGTTTTCTGGGCTAATTGTTTTAAATCTTTTGCATCTGGATTCTGATTGATGGCAAAAT AGGATTTCATAGTTCTTAATTGATGATGTTTAAAAGATGTGCGCATGCGCTTTGTCCTCTGACTCCCTCCACCACCAGATGATAGTGGGGGTGAA GATTGAGAATTACCAGTACCATCTAAATGGCCTAGGTGTAAATTGCAGGAGGCAGGGTCCAGCGGCGATAAACCTGTATTGTGCCTGGATAGTG GATCTGATCCGGATAGATCCCCATTCTTTTTCTTCCTCGGTCTCCCTTTCCGGCCGGACGCCGGTACTCCGTCTGAGTTATTTACACATCCGGCAG TGTAGGGACCATAGAATCCGGGAGTTGAATCCGGCGTTGGTATTCCGCCGCAGTCAGAAGGTAGACCGGGAGGAGTGAGTAGATGATGCGGAGG ATGAAGGAGGGATGACGGATGATGATCAGGGCTGTAGTAGGAGGTGTCGTCGCGGGAAAACATCTCGTAGTGTGTTCGGCAGTAGACGAGGTTG TCCCGTAGACCGAAGTAATCACCTTGTGTGAGAGCGGTATTGCACCAGGCACATGTAAAACAATGGATGTGGAAGACGAGATCCCGCGCCCGCAT CACCAATTCTTGAGCATGGATACCTTGTTGACATCGGGAACAGCATCTTTTGGATGAGAACAGCCTGTAATAATCTTCTTTACAATAAATATGGC CATCTTTCCAAAAACATGAAAGTTCTTCATCTAGTTGTGCCTTGCACTTATGACACTTTAAACAATAGACATGCCATTGTAAGTCAACAGCATTT AAATAATAACGGTCCACTATATGCCGGCCACAACCCGCACAGTCGTTCGGAGGCGGGGGAGTGTCAAAGTCCGAAGACGATATGACAGGCATGAT CCGACTGTCCGGTGGATGGCTAGTTACCATCTGTGGACCATACGCTTCCGAAGAAAGGGTTCTCTGGTATGGTACCAGGGTGGCCGTTGGTGTGG TTTCGTTAAAAAACAAAACAAAAGTTACAGTAATATTCACCAACGCACCATTAATTTACCAAAGCGCCACTATTTCAGATATTTTGAACTAAAA CATCTATAATTTGTAACATCAAATAATATGGAAATAACTATGCAACACGACTATGCAAATTAATTTGCATGAATTGTATTTCTATAACACGTGC TGTCTAGAACACAACTGAACTTGTACAATGAAAGAATAAGATCGGTCGGCCCCAACCCGCACAGTCGTTCGGAGGCGG

>Locus_11195_Transcript_3/9_Confidence_0.733_Length_6107_-_ORF_7_(frame_2)_translation MVNITVTFVLFFNETTPTATLVPYQRTLSSEAYGPQMVTSHPPDSRIMPVISSSDFDTPPPPNDCAGCGRHIVDRYYLNAVDLQWHVYCLKCHKCK AQLDEELSCFWKDGHIYCKEDYYRLFSSKRCCSRCQQGIHAQELVMRARDLVFHIHCFTCAWCNTALTQGDYFGLRDNLVYCRTHYEMFSRDDTS YYSPDHHPSSLLHPPHHLLTPPGLPSDCGGIPTPDSTPGFYGPYTAGCVNNSDGVPASGRKGRPRKKKNGDLSGSDPLSRHNTGLSPLDPASCNLHL GHLDGTGNSQSSPPLSSGGGGSQRTKRMRTSFKHHQLRTMKSYFAINQNPDAKDLKQLAQKTGLSKRVLQVWFQNARAKWRRNNNKQPDSVSG MITSEQPSTPQLLQGGHMGGGICSPGATSSYSEPSPVHSCGGVASDGSLSTHVGPGASMDFDSSDPMTPLVNHQGLNPGSVHNGDNVSFHVTSFHE LF 


\subsection{4 spineless}

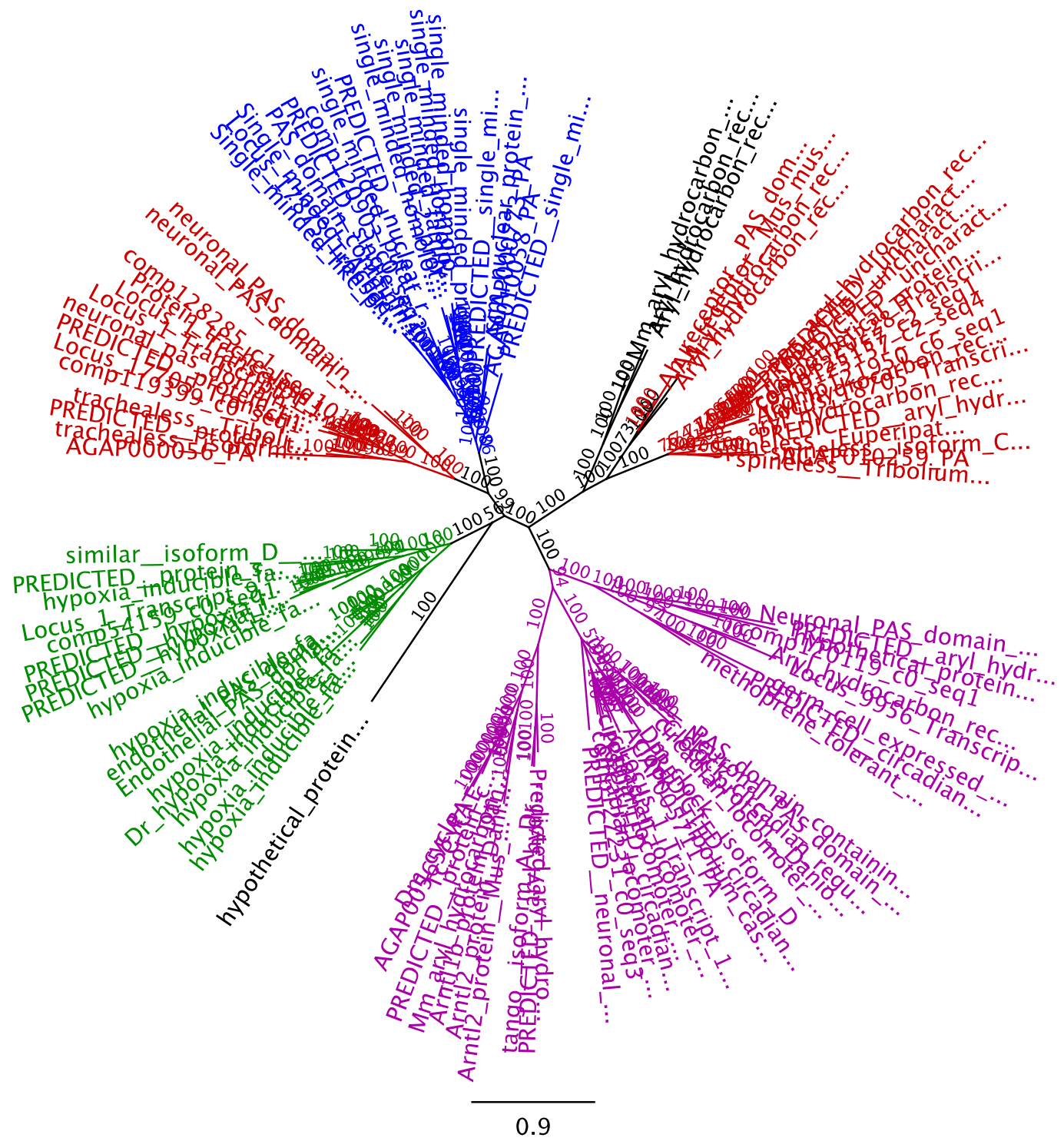

Supplemental Figure 13: Unrooted 50\% majority rule consensus tree to identify true trh and ss homologs.

The Jones amino acid substitution model was chosen after mixed model testing. After reaching $\mathbf{3 0 0 0 0 0 0}$ generations a total number of 12002 trees were written in two files (each file contained 6001 trees of which 4501 were sampled). Colors indicate monophyletic groups in the tree. 


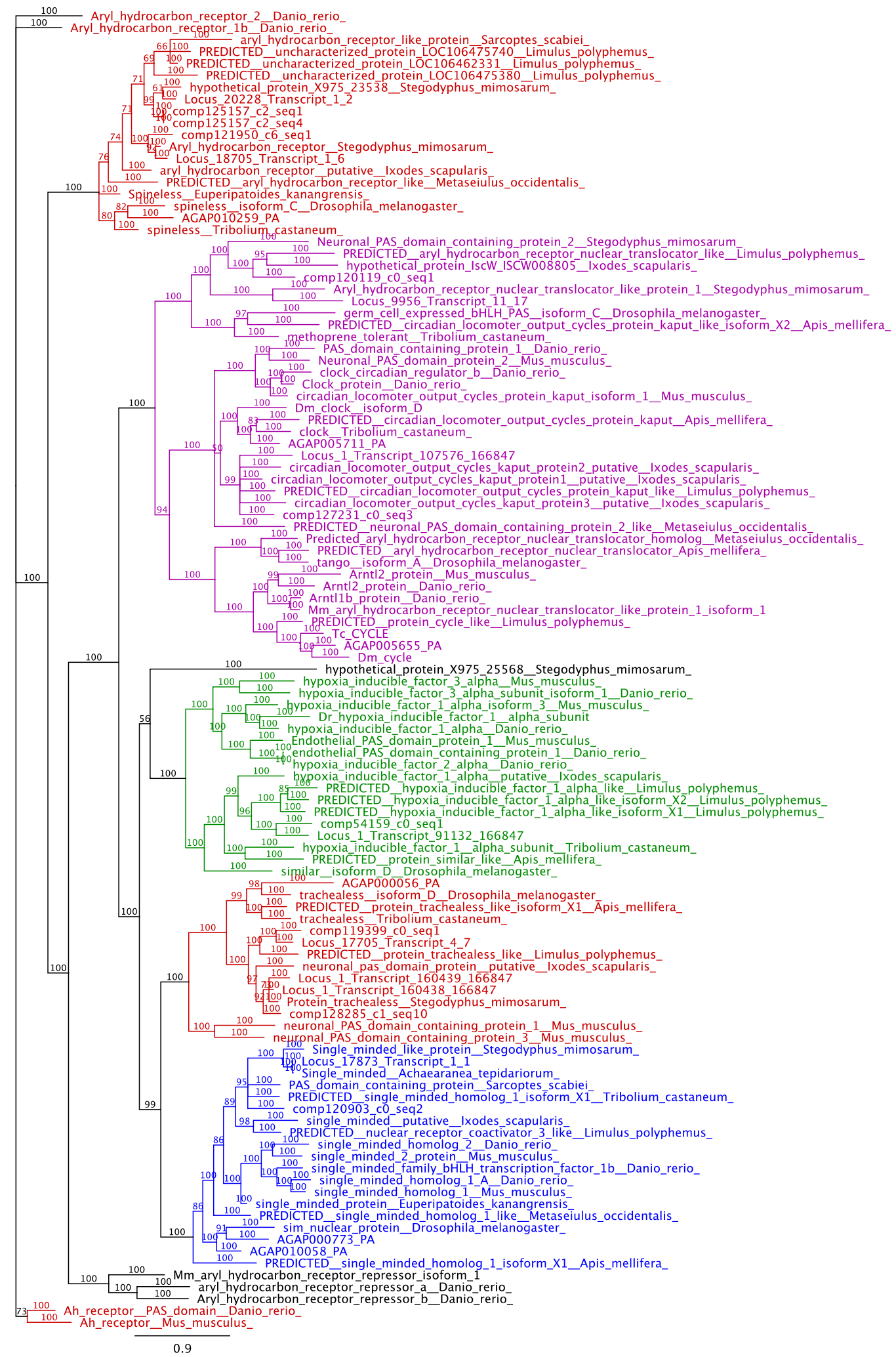

Supplemental Figure 14: Unrooted 50\% majority rule consensus tree to identify true trh and ss homologs.

The same phylogram as in Supplemental Figure 13 in a magnified view to display gene and species names. 
>comp121950_c6_seq2

CGCAAAAATGTGATCGGTGTTCACCCACCTCGAATGAAATGTCCTGTGCAAATGGCGTACTGCTGAAAGTCACTTGAGGAGCCACTTTACCACTT GCAGGACTGGTACTGTTGTGCGGTGAGGCTGATTTTGGAGTTGCTGCGCTACCACTCCATTTGCATGGTGAGCCCATCATATTACCACCACTGAC TTGATCGATTTGCTGGTGCCCGGATTTTGACGACTGCTCCGAGTACCTGCCAGACAATTTCAGGTCATAAGTGTTATTGTTTCCGGGGCTACCGG ACGTCGGGGTTGTCGGGCAACTTCTGTTAGAAACTGGAGACACTCCAGGGAAAGGTCTCCTTTCTCCAGCGTTCGAAGCGTCATTCGTGATGATT CCGTTGTTCCACATGACCACGCTCTGACGATCCGTCTGATAATCCAAAGTCTGTTGCTGATGATGATGATGATGCTGATGAATGATCGCTTTATC CTCTTTCGCAAAAAGAGTCAGATCCCTGGAATTCATCAATACACCGTTCGTCGTCGTACCGTTCATCCCCGGCTTTAGTGTCTTTGCGTTTGTCGT GAGACAATCGTTGGCGAACGTGAAGTTGATGCCACCGTTGTTGCTGCTGCACGTTAGTTCGCGAAATTTGAGATCTCTATCGATCGATGATGGCA AAATAGTCTGAGAGTTGGCCGTGACCACCTGCTGCGGCAAGTTCCTCATCACATCACCATTAGTGGCCACGTAAGGGTCGCAGCAAGAGTTCGAC GATTTAGGTGGAGATTCGGTTCCACCGTTGTATTTCTGGGCCTCCATCTGACACTGGTAGAGCCCTTCGTCTTTACCCAATGGCGAAGTAGCGTT GCTTTCGGCTGTAAATCCAGCCGAAAAACACGTCCTGGCACTTGCCGCTGCAGTCGCCGCAACGTCTAAGAAACCGTTGCTCGGATATTGGGCGA CGGCATAGTCAGTATAGTATGTGCCTATCGGCCTGTAGTGTAGGAAGGCGTTCTCAGCGCTGAGGAACCGGGTGTTGTCTATGGCTGCCGTGAAT AGGTTCTGTTGCATGCTGGCACTGGCGTAAGGTGCAGCGATTCCGTTAACGTTCTCAGCGGCTGATGCGTACGGGTAAACATTATTATAGCCCGC GACTGCAGCCGCTGCCGTTGCTGCCGCTGCCACGGCCGCCGCGTCCTCCATGTACGATGTGTCCGCGAGGGCACCTCCCATCATTGCGGGTGACGT ACCTCCCGAGGAAGAGGACGGACTCTTCCTTTTTCGACAGGTCGAGATCATGTCCCTGATCTGAGATTTGTAGCGGCGCGTTCTCTGACACCTTA GCACGAAGTCACTGTCCGAGAGGAGTCCGCTTCTGTCCGCAAGGGAGTTCAGCCCACCGTCTAGATAAGTCACTTTGAAGTCCATAGTTCTCTTG CCGAGAAGATCTCGCCCTTCTTCTTCCATGAGAGGCCTGTGCGTGCACAAGATGAAATCAGGTTTACTGTTCTTGTAAACGAGCCTGGCACTAGT TTGTAACCACTGCCAGCTGCCGTTCTTTGCGACCAGCCGATAAGCTATGAGACCTGAGGCACCGGTTTTCAAAAGCTCCTGGTGGGCGCTAGCAA CGTAAGCGAGGTCATCGTAATGGACTAGATCGTATCCTCCTTTGCTAGCGAGTTCGGCGTCGGAATAACCCAAAACTTGCTTTGCCCTTTGGTCC AATGAGACTAGAGATAAGTCGAGCTTGTGTTTGCTCTTGAACATGGCGTCCTTTTGGGGCATCTCGAGTAAAGATGGTGGACCGAAAGGGGTGC ACAGAGCGAACAGTGCTAGTGGCGGGTCTTCGGTCTTGTGATTCTGGCCATGCAGAACCTTGATTCTTCCTCGGATGTCCAACCTCAAAAAACCA GATGTGTTATCCAGCAGACACCGAAACCTAACAGTAAAATTCCGCTCCAAGACTTGACTGTTGTCTGACTGTAAAGATTCCCTGAGCGTGAGGGC GCTACGGTCAGCAGGTAACTGGGCATTCCACATCAGGTGGCGTTGTAGCTCTTCCCTATCTTCAGAGTGGACCAGCTCATAGACACTCTGGTGAC AAATATCCGACTGATGGAAACCCAGGTAAGTTTCTACGGTGTGCGAGGTGTAGAAAACTTCCCCTTCACAGGTCAGGATCAACAGAAACCCGTTC AGGGCCTGCAGTATCATGTCACCATCTAACAGATGCTCCTGGTAGAGATCTCTCGCCTTCATCTGACACTGGGATTGCTGCTGCTGCTGCTGTTG CTGTTCTTGGACTCCTTGTCGATCTGAGGTCGGTTTATTGTGCAATGCTGCTTGAAAATAGCTCTTGGTTCGTAGATAGCTCACGGATAACCTGA GAATGGACAGTTTATCCAGTTTAGACAGAACGTTCTGGTCGAAGGGAAGAAGGCTGGCTAAGGTGTCCAATTCGGCGTTGAGTCGCTCTCGATG TCTTTTGCTCGGATTGGATTTGGTTTGGCCTTCTTTTTGCGGGACACGGACGGTTTTGCTGTTCCTCCTTCTCCTCTTAGTAGCGTACATAGTACC AAGATGACTCATCGTTGTCCCGCGAGGACCGTCCATGTAACCAGGACGGCCACTGGAACGGCATGCGCCGGGGCTGGATGTCGTTTCACTGTACA GCAGCACGGTGCAATCTCGATCTTCGAGTCAAATAAGTCGGTAAATCGACCAAATAATTATAAACAAGTAGACAACGTCAACGTGTCGGTCCTTT GAAGAAGGAACCGTTCCATTTTTTCTGTAATCCTGGAGATATAACCAATCACTTCTCGGATTATACGATATCTTTTTCTTTTTTTTTAAGCTAAT GTCATACAGTGCATCAATTTTTTTCCTCTGAAAATTACCTCATTCTTCGAAGTGGAGAAACATCAGTCGATACCTGGAAATCAACGCACTGATCT TCCACTTTCAGTTTTCTCACATTATGAAGAGAAATGATTATTACTTCTGACGCATTTTCCAAGCGTTTTTCTCATGTAATATCGGCTCATAGCGA TTTGTTTATCTTGCGCACAACATGATGCACATTCACGACAGCGCACCTCGGCAACCCGAAAAAATTCACGATTTCCACAAATCCAGCATGCTTAT CAATTTTATC

>comp125157_c2_seq1

CACGAAGGTAAAAAAATCACCAATTGGTTGGTTGTGTTCAGGTGAGAGGCGGGGTTAGTTGAAAGCTGGCCGGCCGCGCACTCCAAGCATCAAT CAAACACTAGCAGCTGGTGCTCGCTCTCACTAGAAAGCCTGGATTTCACTCTTGGACTATTTAATATTATCATCATTTTAATTATTTCTCTCGCA CGTTTATTTCATTTTTTATATAGAAAATATTGAAACGTAAATTTTTATTTTCTGTTCCTCCTCTCGTCGAATGAATCAGCTCAGTCGGATTTCCT CATCATGAATTCAGCTGCTGAATTTATGACTCGCGATCAGTGACAAGGAAAAAGAACAACAAAGTGCTTTAGAATGTCTACGACACGGCAGTGG TGTAAGTGATTACGTCGGTGGCTGCCTTTCTATGTACTGTGCAATAGGTGTGAAATCATACAACCAGAAGATATGAATCATCTGGGTACTATGT ACGCCACTAAAAGGAGGCGTAGGAACAGTAAAAGCGTTCGAGTGCCACCAAAAGACGGCCAAACGAAATCGAATCCAAGCAAGAGGCACAGAGA GAGGCTCAACGCCGAACTGGACACGCTAGCCAGCCTGCTCCCCTTCGAGCAAAGCGTTCTCTCTAAACTCGACAAACTGTCCATACTGAGGCTCA GCGTCAGTTACCTCCGGACTAAAAGTTACTTCCAGGCTGTCATCAGACGGGAAAAGGACATAGAGTTGAAGCCCCGAGAAATGCTTTACCCCGAG 
ATGTTAATGGAAGGTGATCTCATACTTCAAGCTCTGAACGGCTTTCTCATGATATTAACGTGCGACGGAGAAGTATTTTACACATCACACACAGT GGAAACGTATCTAGGCTTCCATCAGAGCGACATCTGCCACCAAAGTGTCTACGAGCTTGTGCATTCGGAAGACAGGGAGGAGCTCCAGAGGCATC TCATGTGGAATTCACACCTCACTTCAGACCGCAGCGATTGCACCATTCACGAGGCTCTTTCTCCAGAAAATGGTCATCTTCTAGAAAGGAATTTC ACTGTGAGGTTTCGGTGTTTATTAGACAACACATCTGGGTTTCTGAGGCTAGACATCAGAGGCAGAATCAAAATACTTCACGGGCAAAATCACA AGACGGAAGAGCCTCCCTTGGCTCTGTTCGCCGTGTGCACTCCTTTCGGCCCACCGTCGCTGCTGGAGATGCCGCAAAAGGATTCGTTGTTCAAG AGCAAGCACAAACTCGACCTATCCCTGGTCTCCATGGACCAGAGGGGTAAGCAGTTACTAGGCTATGCAGACACGGACCTATCAAGCAAAGGAGG TTACGATCTAGTCCATTTCGATGATCTCGCTTATGTAGCAAGCGCCCATCAAGAACTATTGAAGACGGGGGCGTCTGGCCTCATAGCTTACCGAT TATTGACCAAAGATGGAAAATTCCAATGGCTCCAAACAAGCGCAAGATTGGTGTACAAGAACAGCAAACCAGATTTCATCCTCTGCACCCACAGG CCACTCATGGAGGAAGAAGGGCGTGACCTTCTGGGCAAAAGGACGATGGATTTCAAAGTTACGTACCTCGATTCTGGATTGGCCAGCATGAACG ATCGCAGCAGCATTATGTCGGATAGTGACTTTATAACGAGGTGTCAAAGAAACAGGCGATACAAGTCACAAATCCGAGATTTGTTGTCAGCGTC GTGCCGGAAGAGGAAGGTCCCAGGTACCGATCCGTATATCGAGGAGACCACCTATAACAACATGTATCCATACACAACTCCTGAAAATATAAACG GGCTTTCACCTTACGTGGCGACTGCCATGCCCCAGAATATATTCACGGCAGCCATTGACAATTCCAGGTTCCTGTCGGCCGAGAACTTCCTCCATT ATCGTCACCTGGGCAGCTACTACCCGGACTACGCTCACCATCCAGCGGCTCAGTACACGAGCAACGGATTCCTGGACATGACGTCAGCTGCCGCCA GAACAACGTACATCCCGACTGCGTATCCGGATACCAGCCAGTTGGCTAAGGAGGAGAAATACTACCCCTGCCAACTCGAGTCGACGAAGTTCAAC GGCGACGCACAACAGAGACTAGTACAGCAAGTAGGAGTCGCTAACACGAAGCCTTCGTGCTGCGAATCCTACGTGACAGTGAGTTCCGATTCCAG CGCTTCGTTGAGAGGTTGCCTCATGAGCGCTACCAGCGCGGGGATCCAACCCCTGTTGACGACGTTGGACAGAGACCCGAAACTGGGTGAGCTCG GTTGCCACAATCTGAGCTTCACTTTGCCTCCGGTTAGTTCCGAGTGCTTAAACGTGATCAAACCAAAGACTTCACCGCTGCCCACTCATCTCAGG GATTCGCCCCATCAACACCACGTGCCGAAAGACGTCAAAGGTCCCACTACCGCGTCGGACTATGTTTCGGCTTTTTCCAACGACACCAGGCAGAG CGTACTAATGTGGGGTAACTCCTTGAGCAGACCGACCGCAGGTCATCCAATGACCGGCACCATATTCGACAGCAAACTGTGCAAATACAGCGGAC ATTTGGACATGGGAGAAAAATCTCCGCCTTTAGAACCGCTCGGCAGTGGTGTCAGCTCCATATGCAAGTGGAGCAGCAACGCGACCACTCCGAAA TCTTCATCTCCCCACAATAGTACCAGTCCAGCTAGTGGGAAAATTGCACCGGCGGTCAATTACAATGCTACGACCACTTACCATCCGGACATATC TCACGAGGTGTATCTGGCGAGGAACGATCTTGGGCTACAACCTTGGCTGAAGCAAACCGGTAATCCCCTGCTGTCGATATCGGAAGTCACAAATA CACTACTCAATCCAGACTGACATCATTCTACAAAGTCCATTGCAGAGTCTAATGTTCTACTCCGAAATAAAGTAAATTACTCCACAAAAGCTGCA TGTGGAAATAAAGTACAATTGTAATCAGAAGTCAGTCAGACAAAGTGGGGAACAGTGAATATTTGGAAGCAAATCTGGCGAAGAAAAGTCATG ACTCAGAACAAAACTAAAAAAAAGAAAAGTCACATTCCATTGCTCAATTCTGGATGTATGTAATATAATGTATACATATTTTTGAAGCTACGAA AACTATTCTAAAAGCATACGGTAGGGAACTTTAAAATACGCACTGTCCCACAACCTGTTAGAATACTGATAGAAATAGTTTAGGGTTGCCAACC GAAAGTCTGTACCGAAAGTAATACTGCATTTGCTACAACAGTCACGACACGATCACAAATGAATGACTTTTCACACCAGATCGTTCTGCAATGTT TGCTTTGCTGATATGAAGCCCTCGAAAGTTTCAGATAAAAATTACATCGATACAGCACCTGACTTGAAGTTCACAAAATTTTTGTTTTACTCTAT ACAGTATTTAATGCATAAAAATTTAATAGAAGGAATCGTTTAAGTTCCCCACGAGCAATGAAGATCAAATACATTAGTTATGGTAGAAAAAAAA AAAGAAATCCTACCCTCTCTTTTCTCTCACGATCACACACATACACACAATACAGATTAAATAAGTTATAATTTATCCTGAAAGGAATAAAAAT TGTATGTCGTTCTGTTCTCAACTACAATTTGAATTTCAAGCAGAAATTATCCCTGTATCTCTGCTGCAACCGGAACTATCCACTTTGCATCATGA AGAAATGGAATCTGTGAAAACGAGTGGTGTTTTGTGTGACATAATATTATTGAACTATATTTTCAGGAGAGACAAACAAGACTACTGATAATAT ACAGTGATCCCGCTTATAACGTTTTCAAA

>Locus_18705_Transcript_3/6_Confidence_0.762_Length_7353

AATTATGGGGAGAAAGAGAGAGACCGCCGTGCAGGGGGAGGGCCAGCATATGACCCCCCATTTTCGAACGAAAATGAAGAGAACTCCGCGCAGT AGCATGTCAGTTTAGAGTTTGCTGTCCACAGAATAGGAACTATGTGATGTGCGCCCTGAGAGGGGAATTTGTGTAAATAAACGCCCTGGGAAAA GCCCCATCAATTTTTTTGTTGTGATTTTTTTGGATTTGTTTCTTTTTGCGTCCAATTTCACATTATCTATAAAGGATTAATTGTGACACAAGGTG ATGGTTTTGTTTATGCTGAGGCCTATGGTGGCCGGAGTGTCGTAAAGCGGCGGCGGCTAGGCTTGGGCCTGGGGGCCGCCCCTTACTTTAATGTG CAGCCCGTTGGAGAGGCCGCCCCTGTGCATCATGAACCACCTGAGTACTATGTACGCTACCAAAAGAAGAAGACGAAATAGCAAAAGTGTTCGTG TACCACCCAAAGAAGGCCAAACCAAATCAAACCCCAGCAAAAGACATCGTGAACGTCTAAACGCAGAGTTGGACACCTTGGCTAGTCTACTTCCC TTTGACCAAAACGTCCTGTCTAAGCTAGACAAACTCTCCATTCTCCGATTAAGTGTTAGCTATCTCAGGACTAAAAGCTACTTCCAAGCTGCCCT CTATCGGGATCAGGAGAGATCACAGGAGCATAGCATGAAATCTCGAGATCATATGTACAGTGACCTTCCTACAGAAGGAGATCTCATTCTTCAA GCACTCAGCGGGTTTCTTCTGATCTTAACGTGTGATGGAGAAGTGTTCTACACATCGCACACCGTAGAAACTTATTTGGGCTTTCATCAGTCTGA TATTTGTCACCAAAGTGTATACGAGCTTGTCCATTCCGAAGACAGAGAAGAACTGCAGAGGCATCTCATGTGGAATTCCCAGTTGTCACCAGAAA GAAGCAATCTTTCTCTGCAGGAAGCTCTACAGTCGGAAAACAGTCAACTTTTGGAAAGGAATTTTACTGTCCGATTCAGGTGCTTGCTTGACAAC 
ACGTCTGGTTTCTTGAGGTTAGACATTCGAGGTCGCATCAAGGTGCTGCACGGCCAGAATCACAAGACGGAAGACCCTCCTCTTGCCCTCTTCGC TGTGTGCACACCTTTCGGTCCTCCTTCCCTCTTGGAGATGCCCCACAAGGATACCATGTTCAAGAGCAAGCACAAACTTGACCTTTCTCTAGTCTC TTTCGACCAAAGGGCGAAGCAAGTGTTAGGTTACTCTGACACAGAACTCGCCGATAAGGGCTGTTATGACCTAGTCCATTATGATGATCTCGCTT ATGTAGCTAGTGCGCACCAAGAATTACTTAAAACAGGAGCCTCTGGTTTGATAGCATATAGGCTAGCAACAAAAACAGGATCCTGGCAATGGCT TCAAACGAGTGCAAGACTTGTATATAAAAATAGCAAACCAGACTTCATTCTTTGCACACACAGACCTCTCATGGAAGAAGAAGGTCGAGATCTC CTTGGAAAAAGAACAATGGACTTCAAAGTCACCTACTTAGATGGTGGCTTAAATTCCTTGAACGATCGGAGTGGTCTTCTCTCTGACAGTGACTT TGTCCTCCGCTGCCAGAGGACAAAGAGATACAAGTCACAAATTAGAGACATCATTACCACGTGCAGAAAAAGAAAAAGCCCAGTGAATGGACCA CCTTCTACAAGCTCTATTGTAGGAGGCGCATTGCCTGATACATCGACTTATATGGACGATGCTGCTGCAGCTGCAGCTACTGCAGCCGTAGTAGC GGCAGGCTATAACAATGTTTATCCTTATGGCACAACAGACAATATGAATGGTTTAAGTGCTTCTTACGGAGCAACGGCTTCAATGCCACAGAACC TATTCTCAGCTGCCATAGACAATACGAGGTTTATTTCTGCTGAGAACTTTCTTCACTATAGACCTATAGGAACTTATTATCCGGACTATGCAGTC GCGCAGTATCCATCTAATGGTTTTCTGGATGTGGCTGCCTCTGCAACGGCAGCCAGAACGTGTTTCGGCCCGAGCGCTTTTCAAACAGATTCTGC TAGCCTTCAGCTAAGCAACACCAAAGAAGATAAACTGTACCAATGTCAGATTGAAGCGGCAAAGTTTGGCGTCAATTCACCATCTCCTCCTCAGC AACACATAGTGAACAATAAATCCCCACCATCGTCCAACTGCTGCGACAGTGTACCTTCCTACATGAATGGGGTGGATCCCCTAAGGGGGTGTTTG GTGAATAATAATGGAGTGGGTGTCCAATCGTCTTCATCCATCTTACCGGTTACGTTAGATCACCATGATATAAAAGTTCGAGATCTCACATGTAC AGGAAATTTGGCATTCCCTTTACATACCCTCACCACGAACAGCGAGTGTATCAACAATGGACTCAGTGGAACTAAATCCAAAAACACTTTTAATG GAGGTCCGAATAGCATCTTAATGACTATTCGAGATCTACCACATTGGCCTAAAGAAGAAAAGATCTCATCACCTTACGACAGTAGGCATAATAT GCTTGCGTGGAATAGCAGAGATGCACTCTCTGTGGACTGTCCAGCAACAAGCTCCTCCTCTTTCACCACTTATGACATGAAATCGTCCCGATACG GGGACGGTAAGCAAGATGGTGAAATAGGTAATGTGACCAGTTCACCTTGCAAGTGGAGTGGGAGTGCAGCTGCACCCAAATCTGCTTCACCTCAT AACAGTACCAGTCCAGCCAGTGGTAAAGTGGCTCCCCAAGTCACTTACAGCAGCACGCCATATACTCAGGACATGCGGCTAGAGCTTCCACTGGT GAGGTCCGATATCATACGACCATCTTCCGTCAAACAAGAAGGAAACCCACTCTTATCCATATCTGAAGTAACGAACACTTTATTGGACCATCACT AGATGGCGCTCTCTACACCAGTGAAACAGCACCAAAAAAGTGACCTTCATCTACAGTCATTTGGAACTCTTCATCATTTGTAAATTACATTGAAG AATATTATTATAGCAAATGAAAAGTAGTGCAACGGATTGAAAATACTACTACTTTCTCGGTGAAGAAAATACTGAAAAATCAGAAGACCGTATT TCTAAAATAAAAAAAAGTTAACAAATGTAGTTAAGTTTTTAACGTAGTAAACTAAGAAAATTGCAGGCTTTATTGTGCCGTAGAATCTGATTAA TTCTCTTAATTCAGTCAATTATAATAATTTAAAATTTTTATAAAATTTTGTTGAGTTAAAAAATTTATATTGTGTCATGGTGAAATTCAAATTT TTTTTACTAACAAATCTATCAATATATTGTGGTATAAATTAGGTATAAATCATAGAGAACAGAGAAGAATCATAGAGAATCATAGAGTATAAA ACCAGTTCGGAACAAAGCCAGCTTAAATTTAAAATTTGTCTTTTCTTAATTTATATAAAAACTAAAATGTTTTTTCTATAAAAATAGCTTTAAA GCTTTTTTATCCCTTTTAAAAAATTTGCCAAAATTAAATTTAAAATGAAAAAAATCATCGTTTTTGTATAAGTTTTAAAAATGTATTGAGCAAG CATTTAAAGGTACCGTTTGATTCAATACTTTTAACTTATGATGAAACCGTTGTAGCTTATAATGAGAAAGGATCTTTGTGCAATGTCTCTAGTT TTTATGTTTTCTTCTAACAAACGAAATATGGTAAAATGTGTAAATTGAATTTGATAAAAATTATTTTTTTTTGTTCTTTAAAAAAAAAGCTCAA ATTAACTCTGAAGTGTTGTGGTTTGTGTAAAACTTTTAAATATGTTTTGAGCAAGCTATACATCAGCCGTTTCATTCAATACTCTTAAAATAAA ATTAGCTTTTCTTCTTAAAGCCATAAAAAATTAAAATAAATTTTAACATTTTAAATTAGCCTTTCATAACAAAACTATTTTAAGACACTATATC CATATTTTATAATGAATGAAGATATTACCGCTAATCATTACAATGTGTCTATTAATAATAATAGTATATAAAAACACTTTTACAAGTAAAAGCT TTATATAGATATAAAATATTAAGTAAGGGCAAAATATATGTATACATTGAATAAAAATCAAATGTTTTTTTTAATTGCAATTTTTTTAAAACCG TTGTAACCGATAATGAAAAAGGATCGTTGGGCAAGGTCTTTCTTTTCAATATTTTCTTCTAATGGAGAAAATGTGGTAAAATATTTAAAATGAA ATATATAAAAATGGATTTGATAAGAATCAATGAAATCAAAGAAAATATTGAGCAATAGCAACTGTAAAAATTTTTTAAAAAGAAGCGAAAAAC ATTATTATTGTACAGAGAATGTGTTCAAATTGAAACTATAGCATTTTCATGACACAAAACCAGTTTTTTTGTCTGAATTCTCACCCTTCTTCTTT TTTCTATACATATCCTATATGTTATGTTATCGAGTTAGCATTTCTATCCTCTATCACACAGGCACATTTTAAAATCGCTTAGAACTTAACTTGCT TATAAATTCACAAATTATTGAAAAAATTCTGCAAAATTTTAAAAATTGAAAAAATATTTTTTTCCCCAAATATATTTAACTTATACAATAACAT CGCCATTCATGCGTTTATCAAAGACTTGTACAAAAGAATTTTTTGAAACATTACATTTTTAATCTATTTTATCTATTTTGTATTTTTTTAGTTAT ATACATACAGGTAGGTGAAAAATTAAAATATAATAATTAATCTTTGTTTGACATTTGAATTATTGAATATTAATCAGAGCATTTTGAATCAGAT TTATTAACAGTTTAAGTACTAAACAAAACAATGTCTAGGGCAAAATTTTGTACCTTATAAATTGTAAAAATGACTCTTAACTTAGGCTTTCCAG CGCATTAAAAATAAACCATTATAGATTAAGATGAAAAATCGATATAACTGAAAATAAATTTGAAAAATATAAATAGTGCTTTACTGTGTTTGA AATGAATCAATGTAATCTAAATTGTTGTGTAAAGATGAAATTATATGTGAGTACACAAAATATAAGTTTCTGATATTACTCAAATAGATACATT TTGTAACCCGTTATTATAAGTTTTTTGAACATTTTCGTAGAATTTCAATATGGTTTTCTAATGTATTTATAATTTATTTTAAAATAAGGTTTTT TCGTGTAGTTAGTTATTTTCTTAGATTAATATGCAATTCTATTTGCTTGCATTATTTAAAATGANNTAATGATGAAATAATGAATGAATGATGA AATAATTTAAAATAATTTTTAATAAATGATTCATTCATAATGAATGCATTTATTTAAAATAATCAGTCATGATTGATAAAATTAATGAAAATA 
AATAAAAATTTAACTAAGCTCTTAAACCCTACGGTCTACAAGTAGTGTATTATTAATTTGAAAAATCAATCATCTGGTAAATATATGACTCTGA GAACTTAAGACTTATTTTAGATAATCTCATCCTTTAATGTTAGATTTATTTATCATCTTTTCAGTTAATTATTTCATCATTACAAGCCAGGTTTT TTATCAAAAGTTTATTATTTATCAAAAATTAATAATCATATGCTAAGATATTCAAATAACTTGATCCACCAATGTTCAGTTGAGTTAATTATTT TATTATTACAGGTAATTAGTTTCTAATTCAAAGTTAATCATCACATTAAAAAATTAGATCCCTAAAATTTGTGCCTCCTCTGAGTTATCAATTCT TTTAATAACTTAATCAGTTCTTTTAATGGCTTCATTCTTTTAATTAATTGCATAACAGTATAATATTAATACTTCAAAATTAATATTAATATTG GAAAAATAATAATAAATTGTCTACGAAAAAGACGCGACAAACATGTACTTCGATTAATATCGATTAATCGTATATATCGATTAATTAATCTTGA GATTCATTTATAAGGAACTCAGCTTATTGTTTTAATACTACAAGTAATGAGCTATTGTATTCAAAATCAATCATTAGATGTTAAAATTAGACGT GGAATACCTTGAAAAATCGATTCATGAGATTTGAATTCTAAAACATGTACTTCTTTTGTTAAGAAAAAAGCATATACTTTTTTGGTTTAGTTAA ATCCGAGATTCCTTCATGGTAAGTTCAACTAATTATTTTAGTATTATGAATAATGTGACATAGGTTTAAAATTAATCATCATATGCTAGAATTA GACGTGTTAAACTTAGATGTCTGAAAAATTAATTCAATATGAATTTTTTTCATTGTTATAAACATCATATTATTCGGTCAAAACTAATTGTAAT AATTTTTAAAAAAATCATAACTGCTTTGAATAGGAGACGAAAAAAAATTTGACTTCTTCTGTTATTTATCGATTAAAGATGATTTTGATAAATT TTAATGAAGATTTATTGATTAACAATTATTGTTAGCTTTTCTAAGCATTCAAGTAATATATCAGATATAATCATCATATGTTTAGAATAAGACG CGTAAAATAAAGGCATAATATAATCGATTCATTAAATTTATTCATTTCATGCATGGAATTATATCTGATGCTTTTATAAAGAACAACCTTCCTC TAATTATTTTATTCATTTCAGGATACGTGTTATTAATATTAAATATTCAAATATTTGGAATAAGACATAAAAAGACTTTTCCTGATGTCATTAA GTAAATTGTATAAAAATCTATAATGCTAGAGATTAATTTAAAAAGAATCTTAGATTTAAAAAGTTAAAAAAATTTCAACATTCATCACTGCAAA TAAATTGTAAATAAGAAAAAAAAAACATAAATTAACACGTAATTAAAGTCTTTTATATTATCAGGTATTGTCCAAGTAAAAATTACAGAGACT AATCAAGTCGATTGTTACAACGATTGGCATCCGACTTGGCGAGATTTTCGTTTTCTCTGACAGATGAACCTGCTTTATGAATTTGCACATTTTTG AAGTTAATTTTACCAAAGTAGCTCCATCTATCGGAGAAGTATATAACTTGTGAGAAAGTCGAGTCGGCTGCTTCACAAATACAGTCTCTGGTGT GACATAATGCATTTGTAGACTTGTAAAATATTGTTGTTGAATAGTGAAAGTTTTTGTGAATATTATATTTTCTGCTGTAGCTCTTAAAATTTTC ATTTCATGAGATAAATATTTGAGTAAAGACTGGTGAATATTCATTTGTAAATATAAAAATTTGAAGTTTCAACACTTTGCTAATGCAAAACTAG TGTGAAATTGTCACTTATTTCATCGATTTAATTTATTATTTTTGTCATATAAATAAAATTTGCAAATTTTGTTGTAAAAGAAAAAAAA

>Locus_20228_Transcript_1/2_Confidence_0.600_Length_2760

GGATAGTATGAGATAGATAAATATGAATCATTTAGGAACTATGTATGCAACAAAAAGAAGGAGACGGAACAGTAAAAGTGTCCGTGTCCCTCCA AAAGATGGCCAAACTAAATCAAATCCTAGTAAAAGGCATCGAGAAAGATTGAATGCAGAATTGGATACACTCGCAAACCTTCTTCCATTTGAGC AGAGTGTTCTTTCAAAATTAGACAAGTTATCTATACTTCGTCTCAGTGTTAGTTATCTCAGAACAAAAAGTTATTTTCAAGTTGCACTTCGAAG GGAAAAAGACTCAACAGATTTGAAACCCAGAGATTTATTATATTCTGAAATGCTTATGGAAGGTGATCTGATATTGCAGGCATTAAATGGATTC CTAATGATCCTCACCTGTGATGGCGAAGTTTTTTACACATCTCATACAGTGGAGACATATCTGGGATTTCATCAGAGTGATATATGTCATCAGAG TGTTTATGAACTTGTGCATTCAGAAGATAGAGAAGAACTTCAAAGACATTTATTGTGGAATTCTCAATTACCTCCAGAAAGAACAGATCTCAAC TTACAACAAACACTTGAAAACTGTCAATATCTGGAGCGAAATTTCACCGTTCGTTTTCGCTGTCTTTTGGACAATACATCCGGATTTTTGAGATT AGACATTCGAGGGCGAATCAAAATTCTTCATGGGCAAAATCAGAAAGAACCACCTTTAGCATTGTTTGCTGTCTGCACACCGTTTGGTCCTCCAT CCTTACTTGAAATGCCTCAAAAAGATTCCATGTTTAAAAGTAAACATAAGTTGGATTTGTCTCTAGTCTCAATGGACACAAGGGGAAAATGTTG CCAGTGCACATCAGGAATTGCTGAAAACAGGTGCCTCGGGACTTATAGCTTACAGACTTCTTGCAAAGGATCTCAAATATCAATGGTTACAGACT AGTGCTAGACTAGTTTACAAAAACAGCAAACCAGATTTTATACTTTGCACCCACAGACCTTTAATGGAAGAAGAAGGAAGGGATTTGCTTGGTA AGCGTACAATGGATTTCAAAGTAACATATTTAGACAGCGGTTTGGCAACAATGAATGATCGCAGTGGAATGTTATCTGATAGTGACTTTATTAT GCGTTGTCAACGTAACAGACGGTATAAATCTCAAATACGAGACTTGTTGTCATGTCGTAAGAGAAAAGTACCGGATACCTATATCGAAGATACT CCTTATAATAATATGTATACGTACTCTTCAACGGAAAATGGACTTTCACCCTATGCAACGGTACCTCAAAATATATTCACAGCTGCAATAGATAA TAGTAGATTCTTATCTGCTGAAAATTTTTTACATTATAGACATCTGGGTGGATATTATCCTGATTACAGTCATTATGGCTAGGTTTTCTACCTAC CCAGATGGTTCCCAGTTTGCTAAGGAGGAAAAATACTATCCTTGCCAGTTAAATGGAGAACAAAATAATAAACCATCTTGTTGTGAAGATGTAC GCTTAATGACAAGCGCTGATTTAAACTGTTTTCCTTCGACAGATTGTATTAATGTTTTAAAACCACATCTCAGGGATATTTCTAAGGATAATAA ATCANNNNNNNNNNNNNNNNNNNNAAATGCATGTAAATGGAGTGCCGCTACAACGCCAAAATCTTCTTCCCCACAGAATAGTACAAGTCCTG CTAGTGGAAAAGTAGCTCCTTATACGACTACTGCCTATCATCCGGATCCAGAATGGCATGCAGGCAGCCTGGCTACATGGAGACCTCATGTCAGT GAGGAGCGAAGTTGGACACTCCCAGAGAGTCATCGGTAACCCTTTACTCTCCATATCAGAAGTAACAAACACACTTCTTAATCATGACTAAGACT TTATAACTCCTTTAAGAAGCATCTTAAATAGAGTTGGACACTTCCAAGGAGTAACTGTTTGCTCGACACTACATTTTCCTTATTCAGGAGCCTTC AGTTAATTAAACCCTCAGTAACGCTTCCTTGTCAGAAGTAACAAATACCCAGCCAAATTATAACTAAGACTTAGTAAATCCTTTCGGAAATACCT TAAGGAAATAGGACATCATACCACACGTAACAAACACCTTACTCAATCTTAACTGATACTAAGCAAATTCATTAGGAAGAACCTTAAGGGCTCT 
TATAGACAGTCAGCGGTAGCCATTTAATCTCCCCAAAAAAAGTAACAAATACCTCACTCATTCCTAACTAAGGCTGAGTATATTCTTCAAGGAGA ACCTTAAGGATATCGGGATCTCATAGACATTCAGCTCTCCCTATAAGAAACAAAAGACTCAATCCTAACTTGGACTAAGCAAATTCTTTGTACCT CGATATTGGTCTCCTATAGACAATCATCACTTTATCTCTCAAATTGGAAGTAACAACCTTGACTAAGAAAAAACCTTCACCTTCATTAGAAAAAT GATGTCAGAACTTTTAAATTTGATTGCAAAGAGTTAATCAAAAGTAAAATTAAAACGCTGCATTACAGTCTAATAATTTTGAAATAATAAACGT ATTTTTTAAATTGGCTTGTGTATATTTCGACAATTCATAAAATTACTTTTCTTAATAAGATTTATCAGTTGTCTCATTATTTTTCCTTTCGTAAT TGCCAGACACCCAAAAAAAAATTCAAAACTTATTCGCAAATAACCACGTTTCATAAATTCCTAAGTTTTAAATAAAAAATTAAAAAAATGAATG AAAAAAAAAAGAAAAAAGAAAG

>comp121950_c6_seq2_-_ORF_1_(frame_2)_translation

MLYSETTSSPGACRSSGRPGYMDGPRGTTMSHLGTMYATKRRRRNSKTVRVPQKEGQTKSNPSKRHRERLNAELDTLASLLPFDQNVLSKLDKLS ILRLSVSYLRTKSYFQAALHNKPTSDRQGVQEQQQQQQQQSQCQMKARDLYQEHLLDGDMILQALNGFLLILTCEGEVFYTSHTVETYLGFHQSDI CHQSVYELVHSEDREELQRHLMWNAQLPADRSALTLRESLQSDNSQVLERNFTVRFRCLLDNTSGFLRLDIRGRIKVLHGQNHKTEDPPLALFAL CTPFGPPSLLEMPQKDAMFKSKHKLDLSLVSLDQRAKQVLGYSDAELASKGGYDLVHYDDLAYVASAHQELLKTGASGLIAYRLVAKNGSWQWL QTSARLVYKNSKPDFILCTHRPLMEEEGRDLLGKRTMDFKVTYLDGGLNSLADRSGLLSDSDFVLRCQRTRRYKSQIRDMISTCRKRKSPSSSSGGT SPAMMGGALADTSYMEDAAAVAAAATAAAAVAGYNNVYPYASAAENVNGIAAPYASASMQQNLFTAAIDNTRFLSAENAFLHYRPIGTYYTDYA VAQYPSNGFLDVAATAAASARTCFSAGFTAESNATSPLGKDEGLYQCQMEAQKYNGGTESPPKSSNSCCDPYVATNGDVMRNLPQQVVTANSQTI LPSSIDRDLKFRELTCSSNNGGINFTFANDCLTTNAKTLKPGMNGTTTNGVLMNSRDLTLFAKEDKAIIHQHHHHHQQQTLDYQTDRQSVVMWN NGIITNDASNAGERRPFPGVSPVSNRSCPTTPTSGSPGNNNTYDLKLSGRYSEQSSKSGHQQIDQVSGGNMMGSPCKWSGSAATPKSASPHNSTSP ASGKVAPQVTFSSTPFAQDISFEVGEHRSHFC*

$>$ comp125157_c2_seq1_-_ORF_1_(frame_1)_translation

MPFYVLCNRCEIIQPEDMNHLGTMYATKRRRRNSKSVRVPPKDGQTKSNPSKRHRERLNAELDTLASLLPFEQSVLSKLDKLSILRLSVSYLRTKSY FQAVIRREKDIELKPREMLYPEMLMEGDLILQALNGFLMILTCDGEVFYTSHTVETYLGFHQSDICHQSVYELVHSEDREELQRHLMWNSHLTSDR SDCTIHEALSPENGHLLERNFTVRFRCLLDNTSGFLRLDIRGRIKILHGQNHKTEEPPLALFAVCTPFGPPSLLEMPQKDSLFKSKHKLDLSLVSMD QRGKQLLGYADTDLSSKGGYDLVHFDDLAYVASAHQELLKTGASGLIAYRLLTKDGKFQWLQTSARLVYKNSKPDFILCTHRPLMEEEGRDLLGK RTMDFKVTYLDSGLASMNDRSSIMSDSDFITRCQRNRRYKSQIRDLLSASCRKRKVPGTDPYIEETTYNNMYPYTTPENINGLSPYVATAMPQNIF TAAIDNSRFLSAENFLHYRHLGSYYPDYAHHPAAQYTSNGFLDMTSAAARTTYIPTAYPDTSQLAKEEKYYPCQLESTKFNGDAQQRLVQQVGVA NTKPSCCESYVTVSSDSSASLRGCLMSATSAGIQPLLTTLDRDPKLGELGCHNLSFTLPPVSSECLNVIKPKTSPLPTHLRDSPHQHHVPKDVKGPTT ASDYVSAFSNDTRQSVLMWGNSLSRPTAGHPMTGTIFDSKLCKYSGHLDMGEKSPPLEPLGSGVSSICKWSSNATTPKSSSPHNSTSPASGKIAPAV NYNATTTYHPDISHEVYLARNDLGLQPWLKQTGNPLLSISEVTNTLLNPD*

>Locus_18705_Transcript_3/6_Confidence_0.762_Length_7353_-_ORF_1_(frame_2)_-_ORF_1_(frame_2)_translation MCSPLERPPLCIMNHLSTMYATKRRRRNSKSVRVPPKEGQTKSNPSKRHRERLNAELDTLASLLPFDQNVLSKLDKLSILRLSVSYLRTKSYFQAAL YRDQERSQEHSMKSRDHMYSDLPTEGDLILQALSGFLLILTCDGEVFYTSHTVETYLGFHQSDICHQSVYELVHSEDREELQRHLMWNSQLSPERS NLSLQEALQSENSQLLERNFTVRFRCLLDNTSGFLRLDIRGRIKVLHGQNHKTEDPPLALFAVCTPFGPPSLLEMPHKDTMFKSKHKLDLSLVSFD QRAKQVLGYSDTELADKGCYDLVHYDDLAYVASAHQELLKTGASGLIAYRLATKTGSWQWLQTSARLVYKNSKPDFILCTHRPLMEEEGRDLLGK RTMDFKVTYLDGGLNSLNDRSGLLSDSDFVLRCQRTKRYKSQIRDIITTCRKRKSPVNGPPSTSSIVGGALPDTSTYMDDAAAAAATAAVVAAGYN NVYPYGTTDNMNGLSASYGATASMPQNLFSAAIDNTRFISAENFLHYRPIGTYYPDYAVAQYPSNGFLDVAASATAARTCFGPSAFQTDSASLQLS NTKEDKLYQCQIEAAKFGVNSPSPPQQHIVNNKSPPSSNCCDSVPSYMNGVDPLRGCLVNNNGVGVQSSSSILPVTLDHHDIKVRDLTCTGNLAFPL HTLTTNSECINNGLSGTKSKNTFNGGPNSILMTIRDLPHWPKEEKISSPYDSRHNMLAWNSRDALSVDCPATSSSSFTTYDMKSSRYGDGKQDGEI GNVTSSPCKWSGSAAAPKSASPHNSTSPASGKVAPQVTYSSTPYTQDMRLELPLVRSDIIRPSSVKQEGNPLLSISEVTNTLLDHH*

>Locus_20228_Transcript_1/2_Confidence_0.600_Length_2760_-_ORF_1_(frame_2)_translation

MNHLGTMYATKRRRRNSKSVRVPPKDGQTKSNPSKRHRERLNAELDTLANLLPFEQSVLSKLDKLSILRLSVSYLRTKSYFQVALRREKDSTDLKP RDLLYSEMLMEGDLILQALNGFLMILTCDGEVFYTSHTVETYLGFHQSDICHQSVYELVHSEDREELQRHLLWNSQLPPERTDLNLQQTLENCQYL 
ERNFTVRFRCLLDNTSGFLRLDIRGRIKILHGQNQKEPPLALFAVCTPFGPPSLLEMPQKDSMFKSKHKLDLSLVSMDTRGKCCQCTSGIAENRCLG TYSLQTSCKGSQISMVTD*

\subsection{5 trachealess}

>comp119399_c0_seq1 AATAAACACAGTATGGAAAAAAAAAATAATAATAAAAAAAATAAATAATAATAATTCCGTTACAGCACGCCCTCGTTATATACGGACAACCATA ACATGATATCATAATTCGAGGATTTTTCTTACCTCACGCCGGTCGAAAAACAAGGATAAAATCTTCATTCTCGATATCTGATATTGAATAAAAT GTGATCTGAAATTGATCTGATTAATCAATTACCATGGATTATATTCGATGATATTATCAAGATAACTGACTGATAACAGTGGGTTTATATAGGT ATCGAATATCGTCGGGGGAAAAATTCTGGTGACTCTGGATAATGAAAGTGTGACGATGGTGATGGAATGAATGAATACGCCGTTGCGGCTGTGA CTGTCGCTGAATTTCGAGTTGACCGTGCCGACGTCGTTTTCTCGTGCGGATTGACAGGACAAGAGTGGCGATTTTCAGTGCGTAAAAGTGGATAT TGGTACACAAATGTGCTTGACGGTGTAACGTGGTGATTTGTTAAAAAAAAAAAAAAAGAAGAAGAGTCATGCAGCCTGATACTTATGGATCGTT ACCAGTGGATCTACATATACCGCAGCAAGGCTATCCAGCTTATTACAGGAAAGGGCATGACGACAGTCCGGGTCCACCGGTAGTTCAAACACGAA TTTTGGAAATGCGTAAAGAAAAGTCTCGGGACGCGGCAAGATCTCGGCGAGGAAAAGAGAATTATGAGTTCTACGAGTTGGCGAAGATGCTACC ACTGCCAGGGGCGATTACGACGCAGCTTGACAAGGCTTCGATCATTCGACTGACCATAAGCTTTCTCAAGCTGAAGGACTTTGTCGCACATGGAG ATCCTCCGTGGAGGAGAGATGGCCCATCACTGAAAGGAGGGAGCTTACGGTCACGATCGGTGATGATTGATCTTTTCGAGCAGCATCAAGCAACT CACGTATTACAGTCACTTGACGGATTTGCGTTTGCACTTGGAGCTGACGGAAGATTTCTGTACGTATCGGAAACGGTGTCTATCTACCTTGGTCT ATCGCAGGTCGAAATGACTGGTAGTAGCGTTTTCGATTATGTCCATCAGCAGGACCAATCGGAATTGGCCGATATGATTGGCATTAACGTGTGCC ATTCGCCATCCGCGACGTCTCCGGCATCCGTGGCATCGGACGAAGGGTCGTCTTCGACACCGGGGACTTCCACGCCGACGGGATTCGATAGGCCTG CTCCCTTGATGACGCTCAGCAATCAAAGCAACGGCCAAACCTTTCAGAGGTCATTCTGCATTCGCATGAAGTCCACTCTTACAAAGAGAGGCTGC CATTTCAAGTCATCCGGTTACAGAGTTGTATTGATACTTTCCCATCTTAGACCACAGTATACATTTTCATCATCAAAGAAATCTCCTCCAACGAT AATGGGCCTGGTTGGTGTAGCGATTGCACTGCCTCCACCGTCGATGAACGAGCTGAGGTTAGAACAAGACATGTTTGTTACGAGGCTGACGTTCG ATTTCAGGATATCACACTGTGAACCGAAGGTTGCAGATCTCCTCGACTATACAGCTGACGAAATAACTGGCCGCAATATGTACAGTCTTTGCCAC GCACAAGATGCTGAGAATCTTCGAAAGTGCCATATGGATCTCATTCAAAAAGGACAAGTGATGTCTGGTTATTACAGGTTAATGAACAAGACCG GTGGTTACACGTGGGTTCAGAGCTGTGCTACAGTCATATGCAACTCGAAGAACGCGGACGAAGAAACGATCATATGTGTAAATTATGTATTGAG TAACGTTGAATACGAACACTGCATCATGGACTACAGCCAATTACCATCCTCGAGGGAATTGAAAAGTGAGGAGCCTAGTAGTTCGGAAAGGGGT ACGACACCTGATAAGGAAGATGGAAGAGCAGATAGTGGTTCCGCTAGGGATATCCATCCAGATGATCCTCCAGCATCTAGACCAGAGACTGATG AAGGCAAGGAATCCGAACATTACGATCAAGTGACTGAGATCGACGCCAATAAAGGGCACGAACACATTCAAACCTCGGCTTCGCCAACATGTGA GGATTCCAGAGTCAACGAATATCCCAGTGTCAATTACTTTGATGCGAGTGAAATGAACCGCCTTGGACCAACAAATTCGATGGATCAACAGAAC GAAGATCAGCGTTCACCTTCTGTGGTGGCTCACCACAGGAAACGCAAACAAGCCGACTCACTGTCAGTTTCGGCCGAAAGCGAGTCGAAGAGTCC GAGATCCCGTCCAGAAAGTACATCTTCTAGTCTCGAAGTCCTTTCCTTGGATAACGGCCATTACATACAGAGCGAGTCGGACGGGCACGAACAGG CCATACTGACTCCGTCCGTGGACATGTGTCAGCCTCAGAGTCATATTGGAAACAACACCGAAGCCAGTTCACCCGACCGCTCGGACAAAAACTCA GTTTCCAGGCCCTGGACCCGTTCCCCTGTGTCCATCACGAGTCCGGTCGGGTCGTCCTTTCAAGGTGATGGGAACTCGAACAAGGAGACAGCTAG TGCTAGCATGTCCGTCAGAGAACTGGAGGATGCCATGAACAGACATTTGCCCCGCAGCACGTCACCTTCAAACTGCAGTTCTCCGGACTCTCTTA TGCTCAAGACGTACGCTTCGGGTCCCCCGTCTGTACAGCATCCTCACTCGGCGTCGTCCAGGCCCATCCAATGGATAGGTGGGGCACCAGCGACTC TACCAGCTACGACCCTTCTACGGCAGATCTATGTGAGCCGCGAATCAGTGATCCGATCCGGTGCACACGCCAGTAAACACGGCTGCTACGGGGAT GTCCAAGGAACTTTACCGACTCCTCCCGGTGGCCAGAATGAACCCGGTTACGCGGACACGGCACAAATTATGTTACAGTCCAAAATATCGCCAGA CGGTGCGTATGGCGTCATATCCGGGTTCACGTCGGCGCCGGCGTCAGTGGTCAGTGCGGGCGGGTACGCCGATTCGTGCCACGCGATGACCCCGCC GAGCTCGGTATCTCCGAGGGACAAATTCAACACGGAGACGTCAGCCTTCAATGAGGCGGCGGCCGTGGCCGCAGCTGCCGCAATGCCTCACATGA GGCATTACGTCACAGCGGACTCCGGGCCACTGCAGCACCTGCCCCTGAAACCGCAGTTGTTCGTACATCCAGGTAATCTGGACCACGGTGCCTACG GTCATCCCCAACAGACACTCTCGCCCGATCAGCATCAGTTGTATCATCATCCGAGCAGCTTTCATCTATACCATCCTACACCAGGAGGCTCCAAAC CACCATTACATGCTGCTAATGGCACGGCTTCGTGGTTTTGCCAATCTCATTCGTGATCACTCGCGTTGAACTTGATTGAATGTTGTACATTTCGC AGGAGAGAGGAGTCCCTCTTTGTCCCGGAATCTTCTCATAGCCTTCTCAAAGAGGGATTGGTTCATTCGCGGACCGCGTTCTCTTCGAATTTCGT TAAGTGATGATAGGGATATAGAAAAAAATGAAAAGAAAATAATGTGTTTATATATTGTGTGTACATGCACATCGCTCTTAGAACAATAATATC ACGGTGATGATCATTCAGCGCCATCCTGTGTTCATAAAGCAACCTGAAACGCAAGCTAAGTGATCAACTTCATTGTTTACGGAGAGCGATTCGTT TGCGTAAAATATAATGCATATGTTTATGGAAATTTTCTACATGAAATATAACACCTACGTTTTAATTTTATTTTTGTTATTTACCAAAATTTGT TGATTTTCTCTCTCTTTCTCTGTTTTTGCTGTCCGTTTTGAGATATGTCGAAGCTCTAAAACAATGTACAAAATCACAATGTTAGCTATTATTCA GAAATGGCAAAAAAACTACCGACGAAAATGTGATTTTGATGCATTATAAATTTTAATGGGGAAAAAAACATTATTTATTGCACTGACTCTTTCT CTGAATAGTGTTTTTCACACAATTTTTAACAGTCATCATTCAAAGTTTGAATATTTTAGATGTACTTTCACA

>comp128285_c1_seq10

AAATCTCAAAAAAAAAAAAAAACCCGAAAAACAACAGTTTTTTTGTAAGAACGGAATCACAAAATTAATGTTACGAATTTAGTTTTTTGCTAAA AAGTCATATAATATTATCTTATACTTTCTGTCACTCGGAAATAAATACGGTTTTTTTTCAGCGTCAATTGACAATACTTTTGTGACCAGTAGAAT CCAATGATGAGATCATTTGTGTGAGGGATTGATTTTTAGTTTTTCTCGCCATGATTTCTGGGATTAAAATGTTCACTACATGTGCGAAGCGAGA AAACTGACACGAGAACGATGGTATTACTACGACTGTTGTTAATTACTGATCATTTGGTGGATGAACCGGGCAGTGCCCTTTTAATGCATGAAAC CACCCAGCCACGGCTCGAGGAGTCTCAAGAGAAGATGGGCTTCGTAAAAAGGCGATCTGGATGAAATTTTCTACAATTTACCAGGTAGGGTATG CAAAACCACAAGTCCCGGGGCCCCATGATACTTTTGAGACGTCCCTGGAACCAAGATGTCATGATACCACAAATAAGAAACATTTTTATTGGACC AGTACAACAAGGTCAAGCCTAGTAAGGCCAGACTAGCAAATCTGATTGGCGCTTGGGGTATCGCTGAAACTAACATTTCTTTTCTGTTCAAGTGA GTCCCTGTCGTCCATTTCTCTGAAGCATTCATGTACTTTTTTGAAAATTATGTAGTGGGCGGTACGCCTTCACTCATTTCGAAACTCATAACGAG TACCGTAGAATCATTCGGTGAGTTCCAAGCAGAAGCTGAGACTTTTCATTCCCGTTAAAATGCGAAGAGGGCTGAATTTGGCGAGTTCAAGAAC CAACGAGTACTGACCTCATCCAGTAAATTGGCTTTCAGTGGTGAGCAGAGGCATTCTTTATAATTAACAAAGCTCTCAGGGGTGTGCTGTTGTTG TTGTTGTTTTTCAATTGGCTGATTTTAATGGGCGCGTAAAAAGGCTGTGTTGTTTTAATGGTAGAAGAGTTTCGAGCTTGAGTAAAGCAGTTGT GTGTTGTTTTTTCAACACAACTGACAAGTTACGAGCAAGATTTAAAAAAAGTACGGGGTTGTGTTTTTGACTTGGCTGACGAGTTCAGAGCGAA GAAGCTGTGTGTTGCTGTTTTTTTTAGTGGGCAAACGGTTTTCGAGTACCAGTAAAAAAAGTGTGCATGTTGTTTTTCACTGTGCTGACGACTTC CGAGCACGAGTCGAGACTTTTTATTGTTGTCAAAATACGAAAAAGTACTGCGTCCGAGGAGCTTAGACACTAGATTTCTTCCCATCCGGTGAATT 
CGTAAAACTCTGGGTGATAAGTATTGCGCTATTCATAATAAAAATAACAACAACAAAAAAAAACAACATTAAAACAATTTATCCATGCAAATGC CGTCCATCATTACGAAGAAATGTGGAGACCTGTATGAACAAACCAATATATGACTGAAATTACATCATGTAGTATATCGTAATCACCGAAATGA AGCATTGCACTTTAATTTTCTGCATTAAAATCAGTGTCTGACGACTGAATCAGAAGGCTTCAGCGTCGACGGAGCGTCCGTATCGTACGTCCACA CCATGACGTTCATGCATATATTTATATGTACGAGTATATACATGTAAATGGTACGCTTTATTTTTAATTAACCGCAATAAAAACAATCACTGGT TTAATATAATATAGAAAGACTATCTCTTAGAAACAAATTGTTAATGGCTGTGTTGGTCAGTCCGATGCCGAAGTCAGAACAGTTTTACTATTCA GGGAATCATCGCCCTATGATGTTGGAGTCCGTGTTCACGAGAAAGATTAGCGCGTTACGTGTTCGACTGAGGATACCACGACGTCCCGTTCACAG CGTGATGAGATCCTTTACTGGATGCTGAAGGGTGATACAAATGGAAACCGCTAGCGTGGTGAGCGTAGAGTTGCTGCTGCTGCTCCGTGCTCAAA CCCTGCTGGGAGTGAGTATGACTGTACGCTGAAGGATCTAGGGTTCCCGGATGAACAAAGACCTGAGGCTTCAACGGTAGGTGCTGCAGGGCTCC GTCAGAAACGTAGTGGTGCATGTGTGGTATCGCTGCGGCTGCCGCAGCAGCAGCCGCCTCGCTGAAAGCTGCAGTATCCGCGATTCCGGGATGGA ACTTGTCCCTTGGAGACACGGATGCCGGCGGTGTCATAGCAGAATACGTGTCCATGTAGCTCGTGACTGTCACCGGGGTCGTGGAATATCCAGCT ATGACACCGTACGACTCCGCCATCTTGCTTGGCAGCATGAACTGGGCCTGATCTGGATAAGTTTCGGAACTGTTTCCCGGTGGGGTTGGCAGTGT CCCTTGCGCGTCCCCGTAGTACGTTGGTCGACTGACGTGAGATCCAGACCGGATGACTGACTCGCGGTTGACATAGAGTTGCCTCAGAAGCGACG ACGCCGGCAGGGTCGACGTCTGCGGAGCACCGATCCACTGGATAGTGGACCTTTGCTGGTGATTTTTCTCCGCAGAATCCCCGGAGTCCTGAGCA GGCAGATGCTTGCTCATGGCGTCTTCAAGTTCCTTAACCGACATCGACCCACCACCGTTGGTAGGCTCGCTGTAAGACGTCCTAATGGAGCTGCT GGGAGATCGTTTCCAGGGGCGCGTCACCCCGTCTAAGTCCTCGCTGATCACTTCGCTGTTTTGATTAGAACTCGGTGGAGAGGTACAGAGTGGAT CGCTGCCGTTCGTGCTACTTATGGCAGTCCTTTCCGGTGTTATCCTCTCGACGTTGGGCATAGCACCGGTTGTCGAGTCCAGAGGACTCGCGTTCA GACTAGACACCGCACTGTCCGTCAAAGATTTAGGACTCTTCGTGTCCGATTCGAGATGATTACCGTTACTATGGAGTCGCCTTTTACGTATCCTG TGATGGTGCATATCTGACGACCCCGTGACGCATAGGTGCGAGGAATGCTTTGGTTGTGGGTCCTGGTATCTCGACGTATCGACTGTCAAGTCGAG CTGCTTCGGTTCCATGTCGTACGGCGTTACGGTGCCTTCGCGGAGTATACATGGTTCAGCTCTGGATCTCATGGAGTCACCATGCTTTCCAGATG TGAGCTGAACGAGATGCTCACTGTGGTTACTCTCTCCACCCGCTGACGAATGACCCTCTGGAGGTTTCGGTGAACCATTTCTCAAGTCAGTGTCT TTGGATGAAGCATCATCTCGACCCTCATTATCAGGCGTTGATCCACGCTCGGAGTTACTCGGGTCATCAGGTTTCAGATTCGACATCGGCATCTG ACTGCAATCTAATATACAGTGTCCATATTCAGCTCCACTGAGCACGTAATTGACGCAAATGATGCTTTGCTCCTCGGAATTCTTTGTACTGCATA TGACTGTTGCGCAAGTCTGAATCCAAGTGTAACCACCATTTTTATTTATTATTCTGTAATAGCCACTCATGACTTGACCTTTATTCATTAGATCA ACGTGGCATTTTCTCATCTTTTGTACATCTTGCCCGTGACACAGAGTGTACATACTTTTCCCCGTAAACTCCTCTGCGGTATAATCCAGGAGCTCC GATACCCTCGGTTCACAATGAGCTACCCGGAACTCAAAAGTCAGCCTCGTGACGAACATATCGCTCTCCAGTCTGACTTCGTTTATTGAAGGCGG CGGTAACGCTATTGCCAGAGCAACCATACCCATGACTGTCGGTGATTGCTTTCTGTTGTGCGAGAACGAATACTGCGGCCTCAAGTGGCACAGAA CTAGCACTACCCTGTATCCTGATGACTTGAAATGGCAACCTCTCTTTGTAAGAGTCGATTTCATACGAATGCAAAACGATCTCTCCATACCTTTG TAAGGTGTATTGCTATTTAGGGACATCACAGGTACAGGCCTATCAAGACCAGTCGGTGTCGTGGGCGGCCCATGAGCTGACGACGACGATCCATC ATCGGATCCCACAGAGCCAGGTGACGACATGGACTGAGATTGCGATATGTTAATCCCGAGTTGTTCTGCCAATTCAGCTTGATCCTGCTGGTGCG TGTAGTCGAACACACTGCTGCCGGTCATCTCCACCTGTGACAGGCCAAGATATATAGAAACAGTTTCGGATATGTACAAAAACCTGCCGTCAGCG CTTAGGGCGAAAGCGAACCCGTCAAGAGACTGAAGAATGTGAGTCCCTTGATGTTGCTCGAAAATGTCCATGGCTAAACTATTCGCCGTTCTGGA CCTGATAGACCCACCTTTGAGTCCCTTGCTGTTGGTTGGCAAATCCCTGCTCCAAGGTGGATCGCCGTGTGCCGTAAAATCCCGGAGTTTAAGGT AGCTTATGCTGAGCCGAATGATAGAAGCCTTGTCGAGCTGGCTCGTTATGGCCGCCGGTAAAGGCAGCAGCTTGGCCAGTTCGTAGAACTCGTAG TTCTCTTTACCTCGTCTCGAACGTGCCGCGTCTCTCGATTTCTCCTTTCGCATTTCCAATATGCAGTTCTCCCACCCATTGTGCTGGACATCTAGG TTCCCCATCATTTGCAAACATTCCAATTGCCTGTAATAAGGATGATAACCCTGCGGCGGCACGTGCAGGTCCATGGGCAGTGCCCCGTAACTTTC GGGCTGCATGCCGAGGCTCTGTAAGTGTTCCGTCGTCGTGTAACAACACTGGCAGGATGCCGCCGCCGCCGCTGCGGCCGCCACTGCGAGAGCGG AGGACGTCGTCGTATGATGATGTCCGACGAAAACCGATCCTTGATGCTGCTGCTGGTGCTGATGATTCTGGTGTTGGTGATGGTGCTGTTGTTGT TGTTCTTGCTGGTGCAACTGGTGCTCTTGGTGATGATTGTGGTGGCGGGTGAATACGCCATCGCCTCCTCCACCGCCACCACCGACAACAATGGT GCCACCGGCGGGCGGCGTCGGCAACAGCCGGGATAGGCCCGATTCTGCTGCCGCCACGTTTTTATCTTTTAGTAACTTGAAATGTTTCTGTGGCA CACGTGATCACTGTGGTACCATACATATACCAAAAAAAAAAATCCTTTTGTATAAGCCGGAAAAAGAAAATTAATCAATCAAGTCCAAAAAAAT CATTTCAATCACAATCCCATGACCAATACTCAAATCAAAACAGTCCGGTACAAAACAAGTCAAAGACACCGCATAGCCGTAGAATCTATCACTTC AGATATGTACATGACCATAAACTTATGAAATTAATTTTTCTGTAAATTAAAAAAATGGTCATTTAATTTCCTCAAGTTCAAGAAAAATGCACAA CATACTCTCGGTGACGACGGAACCAGGAGTGCCGCGGAAATT

>Locus_1_Transcript_160439/166847_Confidence_1.000_Length_2174

TATCACCCGATGAAGGTGGTGTTGTGGGTCGATGGCATATGCCATCAGAGGAAGTGTTCAGTCCAGTTCGGTCTGGCGTGACCCTATCCACCAAA GTTCCTCCGTTGTCCAGAGGGCTTGTTGCATTAAGACTAGATACCACATTGTCAGGAGGACTCTTACGATCTCCATTACTCATTGAAGTATCACT ATAATTAATTTGATGTCCGTTGCTGATAAGTCGCCTTTTTTTTACTCGCCCATGAGGTTGACTATCAATACATGGGTGATGACTGGAATGTTTTA CTGTTTCTTGATAACGATTTATATCTACTGATAAGTCTAACTGCTTTGGTTCCATATCATATCCATTGCTTGCAACCGAAACATCATCTTGTAAA GAGCATCTCTCCCCTGGAGGTCGCAGAACATTCGAATGTTTCACTAAATGAGATATATGATGATCACTGTCACCGCCTGCAGTTGACGAGGAACA TCGACCTCCATCTGTTTTTGGTGTGCAGCAACCTTCTCTCGAAGATCCTTCCTCTCTAGGGTCGGCTTCTGGAGACGATCCTCTTTCAGAATTGCT GGGATCATCTGGTTTCAGATTTCCAGAATTCGGCATCTGGCTACAATCTAGGATACAATTTTCGTATTCAATCCCACTTATAATATAATTAACAC AGATAATACTCTGCTCATCAGAGTTCTTAGAATTGCAAATGACCGTTGCGCAGGTCTGCACCCAAGTATAGCCGCCATTTTTATTGATGAGTCGA TAGTAGCTGCTCATCACTTGCCCTTTATTGATAAGATCAACATGGCATTTTCTTAGTTTCTGAACATCTTGCCCGTGGCAAAGGGTATACATATT TTTCCCTGTCAACTCATCGGCTGTGTAGTCTAAAAGGTCAGATACCCTCGGTTCGCAATGTGCGATCCTGAATTCGAATGTCAGCCTGGTCACAA ACATATCACTCTCTAGTCTCACCTCGTTGATGGAAGGGGGCGGCAAGGCGATGGCCATGGCCACCATGCCCATAACAGTTTGTGGCCCTGCCCCA GCCCCCTGTTGCTTGCGACCGGAGAAATTGTACTGAGGTCTCATGTGAGACAGGACCAACACTACCCTATAGCCTGAACTTTTAAAATGGCACCC ACGCTTCGTGAGTGTAGACTTCATTCTTATGCAGAAAGATCTGTCCATCCCTTTGTATGGTGTATTGCTGTTCAGTGTCATCACAGGTATTTGTC TGTCCAGGCCCGTTGGAGTGGATGTTCCGCCGGGCGTGGAAGAGGACGAGCCATCATCTGATGCTACAGAACCAGGACTAGAGGCCATTGTTGTC TGTTGCTGCGCCAAGTTGATACCGATCTGCTCCGCCAATTCTGCGTGATCTTGTTGGTGAATATAGTCGAATATACTACTGCCAGTCATTTCAAC CTGAGAAAGGCCTAAGTAAATAGAAACTGTTTCTGATATGTATAAAAACCTTCCATCTGCACTGAGGGCGAAAGCAAAGCCATCTAGTGACTGT AAGATATGTGTTCCTTGGTGCTGCTCGAATAAGTCCATTGCAATAGAAGATCCCGATCTGGAACGGAGTGAACTGCCTTTCATGGCTTTAGAATT GGAGGGAGAATCTCTGTTCCATGGAGGATCTCCGTGAGCTGTGAAATCTCTGAGCTTGAGGTAAGAGATGCTGAGCCTAATGATCGATGCTTTG TCCAGCTGGGTAGTTATGGCAGCGGGCAGGGGTAGCAGCTTGGCTAATTCATAGAACTCATAGTTCTCTTTGCCACGCCGACTCCTGGCCGCGTC CCTTGACTTTTCCTTTCGCATCTCCAAAATGCATGAATGTGTAAAAGGTGGTTGACCAGCAGCAAAAGCAGCATAATGACCGGACAGATCAACAG GGGGTCCGGCCAAGTGATGCGGATCCCCGAGGGTAGGTGCCCCATGTTGGGGGTGATGACGCAACATCAGCATCTCGATCCTCAAACGATAATTT CGACACTACAAACAATTACTTACTTCCTCACAACCAAAACAGAAACTTTTTCTCAACTTTGAAACAAGAGCGGCGCAAAGGGTGGTCGAATGGGC TGCCGCAGACGATCTTTTTGTGGCCGCATTCGATAGGCGATAGGTCTTCTATGTGATGGGGGCCGGTCGCAACGAAATCTCCCCG 
>Locus_17705_Transcript_4/7_Confidence_0.682_Length_6507

ATTTCCCCCTCTTTTTTTTTACATCCACCGATCGCAGTTGCGTCTTCCCGGGCAACGTCACAACTGGACGATCTAGGCCTAACGGGGGACGAAAA AGATTCCAATGCAAAGCCACTAGTCACACTTCTCGGCCGAACGAAGCAGAGAGGTGGTCACCGGCAAAGTCCGACTAGAACTATCCGGCGCGTTA CGCACGCTTTCGCTGTTTTTACGCATTGTTCTTGTTTACATTAAGGCGCAGTAGGGGACATTGCCTGACGTATGTTATTTTGGTCAAGAATATGA TTGTGATATATTGACGAGAAGACTTATGATTATTTTTTTTATTGATTGATAGACTAGAGATTTAAATCACATCTGATTTTGATTTAACTATCGG CTGTCACCGCTGTGCCTAAATTAAAAGTTGCTGAGCTAGTTTTTGTTGTGGATAATTGACGTTAATCAATGGATTTTGAAATAGGTAGTTTACG TAAACAATTGGAGACGTTTTTATCCACGGTAAACAAGTGATTAAAGGAAAAAAATTATACTACAAAAACATTTAATTCTAATAATGACGTAAAC AAATATTTAAATGAGTGAATAGTAAAGAGGAGGTGATTTTTTTTTTCACCCCGAAAAAACAAAGTGGATTTTCATGTTTGTTTATGATTTTGAA TAACAGTTTTTGCAAACGATTTTGGTGAATTTCTCATGACAGAAAGTGGAAGTTAAAGAACACTTTTGGAGGTTATTCCTTTTGTTCTAATAGT GTGTTTTTTCGTTTGGGTTATGTCTTAAAAACAATTGATCTGATGATGATGCAACCTGAAGGATATGGGTCCATACCCATGGACCTGCACATTTC ACAGCAAGGATACCATCCATATTACAGAAAAGGGCATGATGATACACCTGGACCACCAATAGTACAAACGAGAATCCTTGAAATGCGAAAAGAG AAGTCCAGAGATGCTGCTCGTTCAAGGCGAGGCAAAGAAAACTACGAATTTTACGAACTCGCCAAAATGTTGCCTTTACCGGGAGCCATCACAAC TCAGTTAGATAAAGCATCCATCATTAGACTCACCATCAGTTTCCTAAAGCTCAAGGAATTCACGGCACATGGCGATCCTCCTTGGCGAAAAGAAG GTCCATCTCTCAAAAGTGGTAGTCTAAGATCTAGATCGATGACATCGATTGCAATGGATCTCTTTGAGCAGCATCAAGCAACGCACATTCTTCAG TCATTGGACGGTTTTGCTTTTGCCCTTGGTGCAGATGGACGGTTTTTGTATGTCTCAGAAACAGTCTCTATTTATTTAGGTCTTTCACAGGTCGA AATGACAGGAAGTAGTGTTTTCGATTACATCCATCAACAAGACCAATCAGAATTAGCAGATCTAATAGGAATCAACATGTGCCCTTCCCCATCTG CAACATCACCTCCAGCCTCTGTAGCTTCGGATGACGGTTCGTCTTCGAACCCGGGACCATCTACACCCACTGGGTTCGATAGACCCCCTCCAACAA TGAGTATAAGAAGCGAAAGTGCAGGTCAAAGTCTACAAAGATCTTTCTGCATAAGAATGAAATCTACATTGACCAAACGCGGTTGCCATTTTAA ATCCTCTGGCTACAGAGTTGTACTAGTTTTATCACATCTTAGGCCTCAATATAATTTTTCTTCGTCTTCGAGGAAACAGGCACCTACAGTCATGG GACTTGTGGGTTTAGCCATAGCCCTTCCTCCTCCTTCAGTCAACGAGCTACGGCTAGAACCCGATATGTTTGTAACAAGGCTGACATTCGATTTC CGGATATCTCACTGTGAGCCAAGGGTATCTGAACTCTTGGACTACACTGCAGAAGAAATAACCGGAACAAGTATGTACACACTGTGCCACGCACA AGACATCGAAAAACTGCGAAAGTGCCATATAGATTTGATCCAAAAAGGACAAGTAATGAGTGGTTACTATAGGTTAATGAATAAAAATGGCGGT TACACTTGGCTGCAGAGCTGCGCAACAGTCATTTGCAACACCAAGAATGCCGAAGAAGAAACCATTATCTGTGTTAATTACGTCATCAGTCATGT AGAATATCCCCATTGCGTCATGGATTATTGCCAACTACCAGCATCACGTGACTTAAGAAATGAAGATCCCAGCAGTTCTGAGAGGGGTACAAGTC CAGACAAAGAGGGAGGCAGAGACAATACGGCTACAGGGGACGTTGAAGCGGAGCAGAGGTCCACATCGAGGCCAGGAAACGCCGGCGACGTCCA TGACGATGGTCAAGTATTTGAAGGAAGTAGTCATTTGGATATGGAGGGCAACAAAAGAGATTTGCAGGAATTACACAGGACATCGGAGAAACCG GGCAATTCCCCTAAAGAGTTTCCAGGAGTTTCGTTTTACGACCAGTCAAATGCAAGACGTACTCCGACATCCGTTGCCGAGTCTGCCGCGGAAGC AGCCGATAACAGACAACGGAAAAGAAAACTCGCAGATTCCTTATCGGTGATGAATGAAACAAACGACAACATCAAAAGCCCTCGTCCAGGAAGC TCTTCGTCGACTAGTTGAAGTTCTGTCGCTGGACAATGGTACCACGTATAGTGAAGTGACCAACAATGACCTAGTTGTGGACGACATGTCCTGTA GCCAATCCGGTTTAAATTCTTCAAATGGGAGGAGGGAAGGACACTCTGAAAACTCCCCCGACAAAAGTGATAAGGTTACTAGACCATGGACTAG ATCTCCCGCCGCACCGAATGCATACGACACAAACTGTAGTAAAAATTCATCGGCAATGTCTGTTAGAGATTTAGAAGATGTCATGAACAGGCACT TGCCACAGCACAATGGATCTCCTGGCGGAGGCTCGCCAGATGTACTAATGCTCAAATCATTTCCCCCCACATCGGGTTCACCAACACAGCGCCCAA TACAATGGATTGGTGGTCCACCAGCACCCGCATCTTTGCCAGCAACCACACTGTTACGACAAATATACGTGAGTAGAGAGTCTGTCATCCGTTCC GCTGGTGCGCATGCGACGCGTCATGGTTGCTACGGTGATACCATTCAAGGCACACTTCCTACGCCCCCTGGTGGACCTGATCCGCCATACAGTGA TCTCATGTTGCAATCTGCCGCCAAACTTTCAGATTCATCGCCAACTTACGGTTCAACTGGATCATCATATTTGGACAATTGCAATGCCATGACGC CGCCAAGCTCAGTATCTCCGAGAGACAATTTCAACGAGGCAGCAGCTGTCGCCGCTGCTGCGGCAATACCGCACATGAGACATTACGTAACTGCG ACAGACCCGAATTCTCTCCAGCACTTACCGTTAAAACCACATCAATTATTCGTACATCCAAGTAATTTAGACCATCCGGGTGCACCATATGGTCA TCACCACACGCAACAAACACTTTCTCCAGATCAGCATCAATCTTTGTATCATCACCCAAGTAGCTTTCATTTATACCATCCACCGCCAGGTGGCTC TAAAACAGCCCTACATACAACAAACGGCACTTCATGGTTCTGTCAGCCGCATTCATAGATAGTTCATGAATATTTTATTTTGTTTGTACAAAAAA AAAAATATGGTGGAAACTTTTAACGCGTTTTTTTAAACTCTAAAAATTGGCGTCCACCTTTACTTAGCTCGATATGGTTGTGAATTAAGCGCTT GAAACAGCAATGCAAAGTCTAGCTTGCACAACTTTATTGTACATAAAACTTTATATATTAAAAAAAAAAAAAGATTTTAACAAAGAAAGTGAGT GAAAATCAAATTATTGGTGTTATGAAGACAAGAGTTATCGGATTTTTGAAGGAGATGGAAACTATTCATTTCTGATAAATGCAATTTGTGAAAT CAAAATGTTTATTTGTTTATAAAAAAAAATATTTTTATCATTCAAATCAAAATCATGTAATGAAAATTCAAGTTAAAATTGTATTCGCTGCATC AAAATATACACACATGCTTTCATTGTTATTTTTATTACTATAACGTTATGGATGGTACACAAAAATTTATTTATAATGTATTTTTTAGATAATG CAATTATTATTGTTTTTACATAATATGCTTACAACATTGAAATTGGTTTTCAGATAGGTAACAAATATTGCAAAAACGTTTTAAAAATCCCCAA AAACGACATAGATATATTGCCGAAAACATTGAAATATCAACTATTCTACGATCATGAATCAAACGTTCCTGAAATGTTTCTAATATGTTTGAAA TATTTCTGAAACACTCCAGAAATATTTCATTCTTGCCCAAAATGAAACTATTTATCCAAATTTTAATTTATCTGATACGAACTTGCGATGTTTTG AGTTGTATTTGCATAGGAAAAACCTTAAATTGAAAATATCCAACTTAAGTTGAATATTTAAATTAAATATCATTGATTTTAAGTTATTACTTAA TTTATCTGATACATAATTCCGATGTTTTGAGTGGTATAATTGATATAGGAAGAACTTTTCAATTTCTGTTACATTAAATCGAATCTTTTCAAAG AGAATTCGGCTAAATATTTCCTTTTTAGCATTAAACCAATCTCAATGACCAATTAATTTTCAAATTCCCATTTATTGCACAGTTTCAATTATCCA AATTAAATTTTTTTAAAAATCCAAAAATGTTTATTTGAAAAAAATTTGAAACATTTAATAATGACTATAGCCTAAATTTACTAACTTTTTAAAT GTTATGTTTAGTTATAGGCATTTTTTGGTAACGGAATTATAGTTCAAAGCGAATTCGTCACTTTTTTGTTACAAATTCATATTTAATTTTAAGT ATTATTTTCCAATATATTTTTTAAATGCTCGGTAACGGAAATAAGTTTCCTGTAATTCCTTTACCGAACATTTAAAAAACAAATCTTTAAAAAA AATAGTTTGTTTAAATTTGTATATTTGTATAATTGTATAAACAAATTTAAATTTGTATATCTCTGGCTGTATCTAAATGTATATTCAAAAAAAA AAATTAAAATATTAATTATATGAATATTTAACAACATTTTACAAATTTGTTTTGACCTGCAATTCTGTTTTTGTAGAATTGTCTTTATTTGAAT TCACAAAATCTATTCAATGCAAGATAAAGAATATTCACGTGTAAAGAATATTTTTAATGAAAAATTATAATTAAAAAGAAATATATTGATAAT AGAATAAGAACAGCCAAATACAGATTCCATAAAAACAATATTTAAATATCGGAATAATAATTAATGCAAACATTTTACGTAAAAATTGAAATTT AAAACATGAATAATTGGAAATCTTCACAAAAAAAAAAAAATTATGAAAAAATTTAGCACTTTTTTTTTTCTGAAGCCAAGATCAAGTTTTGATT TAAGCTCTTCTTTGCAAGTATACAATCAAAATATTTACAATAAAAATATCATTACAATTTTCACACTTGCACTACTTTTTGCTCAAACAACATTC AAGATAAAAAAAGAAAAAAGGGGGTTAAAAGCACAATGAAAATGATAAGCCACATTTTCATCAAGTTGCACGGAAAGTTATTGATCAAAAACA CCACGATTTTTATGTATAATGTTTGTGTTTATTATACTAATATCAAGTAAGTAAGTCAAGTTTGCGTTTGTGTATGCTAATATCAATCAAAATT TCCGGAAAATTGTAAAGTATGTACCAGAAAACAATACTAAGAAACAGTTTGATCAGAAAATTTTAAAAAAAAAGAGCTTCGATAAAAATGTGG CTTATAATAATTTTATTGGAATAAATGCTTTTCATACATTTATAGATTTACTTCATATTCATAATTTATTATACAACATTTTTCTAATAAAGCA TATATAAAATTAATATACATAATTTTTTTTAACTTTTTTAGCTTAAAAAATTATTCTAGTGTTGTTTTATTAAGTATTTAAATGCTAATTATAA AAATATTCGAATAATTTTTAATCACTATATTTTAAAGACTAGTTTTGATCAAACTAAATTATTTAAGACATTAGAATTAAAGAAAATGCTTTTT ATACATTTACTTTGTTAATTTTACTTCGTTGCTATTTACT ATTATGAAACTGTTTATTGTACTGTATTCTAAATACTGTTTTGAAATTATTTTTT TTTTTACACTTTTGAAATATTTATTCATTTTTAAAAACAGAACTGTGTTATTTTCATCAAATATTTAAATAAAATGAGCCCTTTTTTTGCCCATT TTAAAAGATTTTAAACAAAGTTACATTTAAATCATTCTTAACTGCTAGAAAATAAATGAAAATAAATAAATCATTCCATTACTTTCCCGGTGCT 
ATAATACTCACCCATCATATCATTTCAAATGTGCAAAATACATCTTATTTTATTTTATTTTATGTTTCTTTCAATTTTATTCTGAATCAATTCAA AGAAATTGTGACAAAATACGTTCAATTATGTGATTGATAGAAAAAGAATGAAATGTGAAATAAAGCACTAGGTGAAAAAAAAA

>comp119399_c0_seq1_-_ORF_1_(frame_3)_translation MQPDTYGSLPVDLHIPQQGYPAYYRKGHDDSPGPPVVQTRILEMRKEKSRDAARSRRGKENYEFYELAKMLPLPGAITTQLDKASIIRLTISFLKLK DFVAHGDPPWRRDGPSLKGGSLRSRSVMIDLFEQHQATHVLQSLDGFAFALGADGRFLYVSETVSIYLGLSQVEMTGSSVFDYVHQQDQSELADMI GINVCHSPSATSPASVASDEGSSSTPGTSTPTGFDRPAPLMTLSNQSNGQTFQRSFCIRMKSTLTKRGCHFKSSGYRVVLILSHLRPQYTFSSSKKSPP TIMGLVGVAIALPPPSMNELRLEQDMFVTRLTFDFRISHCEPKVADLLDYTADEITGRNMYSLCHAQDAENLRKCHMDLIQKGQVMSGYYRLMNK TGGYTWVQSCATVICNSKNADEETIICVNYVLSNVEYEHCIMDYSQLPSSRELKSEEPSSSERGTTPDKEDGRADSGSARDIHPDDPPASRPETDEG KESEHYDQVTEIDANKGHEHIQTSASPTCEDSRVNEYPSVNYFDASEMNRLGPTNSMDQQNEDQRSPSVVAHHRKRKQADSLSVSAESESKSPRS RPESTSSSLEVLSLDNGHYIQSESDGHEQAILTPSVDMCQPQSHIGNNTEASSPDRSDKNSVSRPWTRSPVSITSPVGSSFQGDGNSNKETASASMSV RELEDAMNRHLPRSTSPSNCSSPDSLMLKTYASGPPSVQHPHSASSRPIQWIGGAPATLPATTLLRQIYVSRESVIRSGAHASKHGCYGDVQGTLPT PPGGQNEPGYADTAQIMLQSKISPDGAYGVISGFTSAPASVVSAGGYADSCHAMTPPSSVSPRDKFNTETSAFNEAAAVAAAAAMPHMRHYVTAD SGPLQHLPLKPQLFVHPGNLDHGAYGHPQQTLSPDQHQLYHHPSSFHLYHPTPGGSKPPLHAANGTASWFCQSHS

>comp128285_c1_seq10_-_ORF_2_(frame_1)_translation MLPTPPAGGTIVVGGGGGGGDGVFTRHHNHHQEHQLHQQEQQQQHHHQHQNHQHQQQHQGSVFVGHHHTTTSSALAVAAAAAAAASCQCCYT TTEHLQSLGMQPESYGALPMDLHVPPQGYHPYYRQLECLQMMGNLDVQHNGWENCILEMRKEKSRDAARSRRGKENYEFYELAKLLPLPAAITS QLDKASIIRLSISYLKLRDFTAHGDPPWSRDLPTNSKGLKGGSIRSRTANSLAMDIFEQHQGTHILQSLDGFAFALSADGRFLYISETVSIYLGLSQVE MTGSSVFDYTHQQDQAELAEQLGINISQSQSMSSPGSVGSDDGSSSSAHGPPTTPTGLDRPVPVMSLNSNTPYKGMERSFCIRMKSTLTKRGCHFK SSGYRVVLVLCHLRPQYSFSHNRKQSPTVMGMVALAIALPPPSINEVRLESDMFVTRLTFEFRVAHCEPRVSELLDYTAEEFTGKSMYTLCHGQDV QKMRKCHVDLMNKGQVMSGYYRIINKNGGYTWIQTCATVICSTKNSEEQSIICVNYVLSGAEYGHCILDCSQMPMSNLKPDDPSNSERGSTPDNEG RDDASSKDTDLRNGSPKPPEGHSSAGGESNHSEHLVQLTSGKHGDSMRSRAEPCILREGTVTPYDMEPKQLDLTVDTSRYQDPQPKHSSHLCVTG SSDMHHHRIRKRRLHSNGNHLESDTKSPKSLTDSAVSSLNASPLDSTTGAMPNVERITPERTAISSTNGSDPLCTSPPSSNQNSEVISEDLDGVTRP WKRSPSSSIRTSYSEPTNGGGSMSVKELEDAMSKHLPAQDSGDSAEKNHQQRSTIQWIGAPQTSTLPASSLLRQLYVNRESVIRSGSHVSRPTYYGD AQGTLPTPPGNSSETYPDQAQFMLPSKMAESYGVIAGYSTTPVTVTSYMDTYSAMTPPASVSPRDKFHPGIADTAAFSEAAAAAAAAAIPHMHHY VSDGALQHLPLKPQVFVHPGTLDPSAYSHTHSQQGLSTEQQQQLYAHHASGFHLYHPSASSKGSHHAVNGTSWYPQSNT

>Locus_1_Transcript_160439/166847_Confidence_1.000_Length_2174_-_ORF_1_(frame_2)_translation MRIEMLMLRHHPQHGAPTLGDPHHLAGPPVDLSGHYAAFAAGQPPFTHSCILEMRKEKSRDAARSRRGKENYEFYELAKLLPLPAAITTQLDKAS IIRLSISYLKLRDFTAHGDPPWNRDSPSNSKAMKGSSLRSRSGSSIAMDLFEQHQGTHILQSLDGFAFALSADGRFLYISETVSIYLGLSQVEMTGSSIF DYIHQQDHAELAEQIGINLAQQQTTMASSPGSVASDDGSSSSTPGGTSTPTGLDRQIPVMTLNSNTPYKGMDRSFCIRMKSTLTKRGCHFKSSGYR VVLVLSHMRPQYNFSGRKQQGAGAGPQTVMGMVAMAIALPPPSINEVRLESDMFVTRLTFEFRIAHCEPRVSDLLDYTADELTGKNMYTLCHGQ DVQKLRKCHVDLINKGQVMSSYYRLINKNGGYTWVQTCATVICNSKNSDEQSIICVNYIISGIEYENCILDCSQMPNSGNLKPDDPSNSERGSSPEAD PREEGSSREGCCTPKTDGGRCSSSTAGGDSDHHISHLVKHSNVLRPPGERCSLQDDVSVASNGYDMEPKQLDLSVDINRYQETVKHSSHHPCIDSQ PHGRVKKRRLISNGHQINYSDTSMSNGDRKSPPDNVVSSLNATSPLDNGGTLVDRVTPDRTGLNTSSDGICHRPTTPPSSGD

>Locus_17705_Transcript_4/7_Confidence_0.682_Length_6507_-_ORF_1_(frame_3)_translation MGYVLKTIDLMMMQPEGYGSIPMDLHISQQGYHPYYRKGHDDTPGPPIVQTRILEMRKEKSRDAARSRRGKENYEFYELAKMLPLPGAITTQLDK ASIIRLTISFLKLKEFTAHGDPPWRKEGPSLKSGSLRSRSMTSIAMDLFEQHQATHILQSLDGFAFALGADGRFLYVSETVSIYLGLSQVEMTGSSVFD YIHQQDQSELADLIGINMCPSPSATSPPASVASDDGSSSNPGPSTPTGFDRPPPTMSIRSESAGQSLQRSFCIRMKSTLTKRGCHFKSSGYRVVLVLSH LRPQYNFSSSSRKQAPTVMGLVGLAIALPPPSVNELRLEPDMFVTRLTFDFRISHCEPRVSELLDYTAEEITGTSMYTLCHAQDIEKLRKCHIDLIQK GQVMSGYYRLMNKNGGYTWLQSCATVICNTKNAEEETIICVNYVISHVEYPHCVMDYCQLPASRDLRNEDPSSSERGTSPDKEGGRDNTATGDVE AEQRSTSRPGNAGDVHDDGQVFEGSSHLDMEGNKRDLQELHRTSEKPGNSPKEFPGVSFYDQSNARRTPTSVAESAAEAADNRQRKRKLADSLS VMNETNDNIKSPRPGSSSSTS 


\subsection{6 clawless}

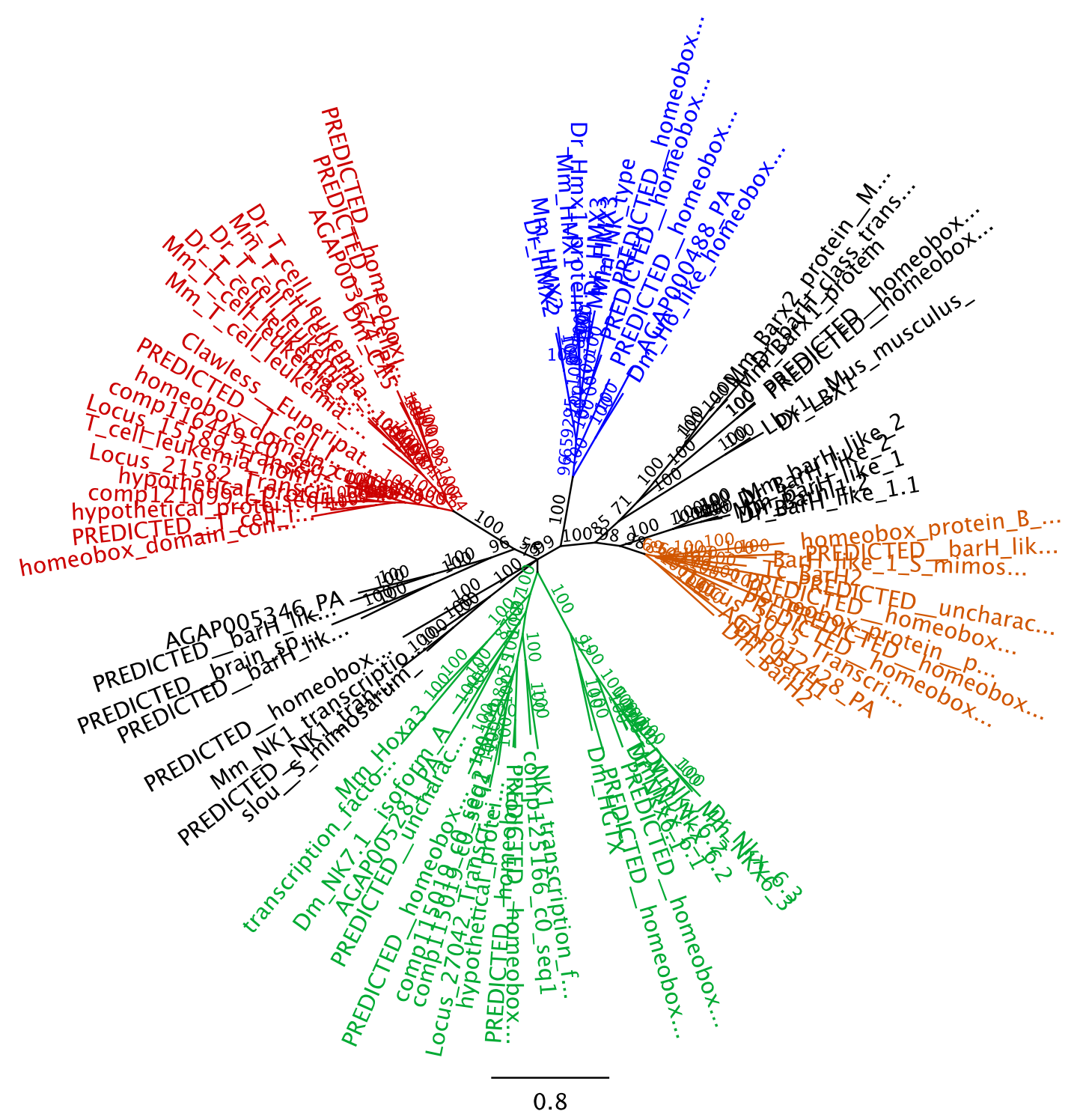

Supplemental Figure 15: Unrooted 50\% majority rule consensus tree to identify true cll homologs. The Jones amino acid substitution model was chosen after mixed model testing. After reaching 3000000 generations a total number of 12002 trees were written in two files (each file contained 6001 trees of which 4501 were sampled). Colors indicate monophyletic groups in the tree. 


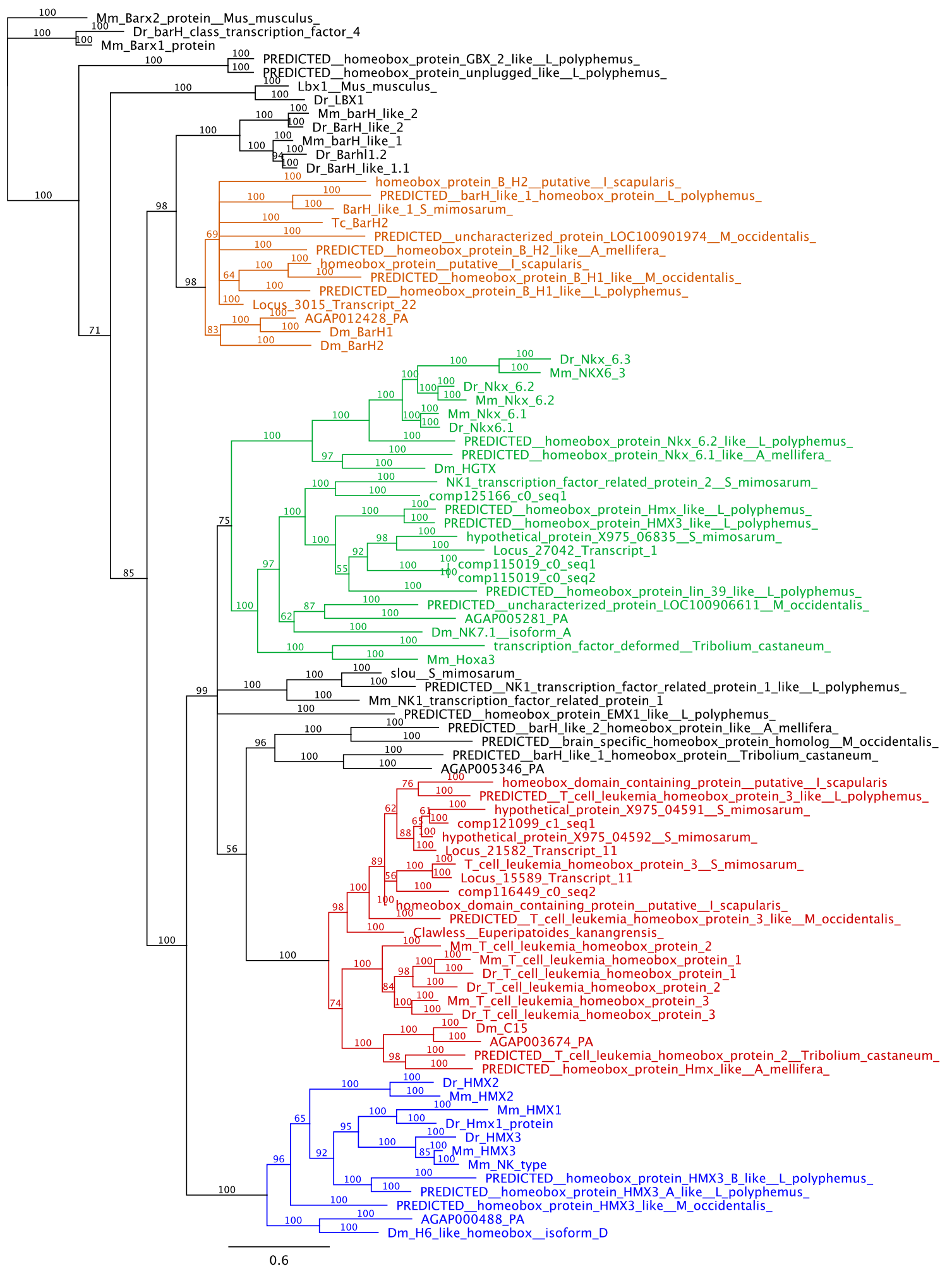

Supplemental Figure 16: Unrooted 50\% majority rule consensus tree to identify true $c l l$ homologs. The same phylogram as in Supplemental Figure 15 in a magnified view to display gene and species names. 
Supplemental Table 10: Accession numbers of cll homologs from different species

\begin{tabular}{|l|l|l|l|}
\hline Name & Accession & Description & Organism \\
\hline Aedes-C15 & EAT43307 & AAEL005250-PA [Aedes aegypti] & Aedes aegypti \\
\hline $\begin{array}{l}\text { Aga- } \\
\text { AGAP003674 }\end{array}$ & EAA08958 & AGAP003674-PA [Anopheles gambiae str. PEST] & $\begin{array}{l}\text { Anopheles gambiae str. } \\
\text { PEST }\end{array}$ \\
\hline Am-hmx-like & $\begin{array}{l}\text { XP_001119 } \\
904\end{array}$ & $\begin{array}{l}\text { PREDICTED: homeobox protein Hmx-like [Apis } \\
\text { mellifera] }\end{array}$ & Apis mellifera \\
\hline Dm-C15 & AAF55898 & C15 [Drosophila melanogaster] & $\begin{array}{l}\text { Drosophila } \\
\text { melanogaster }\end{array}$ \\
\hline Dr-TIx1 & AAI62115 & T-cell leukemia, homeobox 1 [Danio rerio] & Danio rerio \\
\hline Dr-TIx2 & NP_705937 & T-cell leukemia homeobox protein 2 [Danio rerio] & Danio rerio \\
\hline Dr-TIx3 & NP_739572 & T-cell leukemia homeobox protein 3 [Danio rerio] & Danio rerio \\
\hline Ek-Cll & CDK60407 & Clawless [Euperipatoides kanangrensis] & $\begin{array}{l}\text { Euperipatoides } \\
\text { kanangrensis }\end{array}$ \\
\hline Mm-T|x1 & AAH18246 & T-cell leukemia, homeobox 1 [Mus musculus] & Mus musculus \\
\hline Mm-TIx2 & AA138241 & T-cell leukemia, homeobox 2 [Mus musculus] & Mus musculus \\
\hline Mm-TIx3 & AAI45632 & T-cell leukemia, homeobox 3 [Mus musculus] & Mus musculus \\
\hline $\begin{array}{l}\text { STRPU- } \\
\text { W4YBJ6 }\end{array}$ & W4YBJ6 & $\begin{array}{l}\text { Uncharacterized protein OS=Strongylocentrotus } \\
\text { purpuratus PE=4 SV=1 }\end{array}$ & $\begin{array}{l}\text { Strongylocentrotus } \\
\text { purpuratus }\end{array}$ \\
\hline $\begin{array}{l}\text { STRPU- } \\
\text { W4Z6X5 }\end{array}$ & W4Z6X5 & Uncharacterized protein OS= GN=Sp-Tlx3 PE=4 SV=1 & $\begin{array}{l}\text { Strongylocentrotus } \\
\text { purpuratus }\end{array}$ \\
\hline Tc-TC011749 & EFA10692 & hypothetical protein TcasGA2_TC011749 & Tribolium castaneum \\
\hline
\end{tabular}

>comp116449_c0_seq2

GTAGAATTCAGCTCGGAGCGTGTGTCAACCGGTTCAACGTTGTAGGAAAATATCTCCGTGTTTTTATTGAACATCACCAAACATTCGGGAGGTGC GTGAGCACATGCTCGGACGTAAAAAAAATTGAGCTCAGTCGGATGTGTAAAGGATAACGATAATCTCTTTGAAAAATACATGTGCTGAAAATTC GGACTTGAATGCTTAAAAAAGACATTCGTTTTTTCAGCTTCTTCCTGCGAAATTCAAGAATTTCGGACTCTGATAAATTTTTCAGATAGGAATT TGAGTAAGAAGAAAATGGATGTGATTTGGACTAAACGGAACGCGTAGATGTGGCAGAAAAAAAGACATTGGGAAACAGTAAATACACTAGTCT AATAATACTTTGACAGCTGTTGTTTTGTCTCAAGATGTTATGTGATAGGATATCGAGAGTCGACATAGGCTCACCGAGTAGCGTGGTCGACGAC GACGAAGAGCACGTGGACGTCATGGACCACGGTGATACGGACGACGGTGGGATCCATTCCGAGGCGTCCGGACAGTCGCCGTCACCGCTGTCCAC GTCATCGAATTCACCGTGTAACAACAGGTTGCTGCTACCAAACAAACCAAAACTATCTTTCAGCATCAGCCGACTACTCGGAGATGACCATCACA ACCAGCATCAGCAATATCGCCAGTCATCGTCTCCTCACGAGTACCGCGACTCGGAATCGCCACCCGCGAAAGACACCGCCGCCACCAACGTCGAGA ACAGCACGGTCACCGATGACTGCCAGGAGAGTTCGTACAGCTCGGCGTCATCGCCACAGATGTCATCCTCACCGGTGAACTCTGGTACTTTCCCG TCTAGGAGCACCACTAACGGTACGCTGATCAACAGCAGCAGCGGTGCTTGTTGTGCTACTGGACTCGGTACCCACACGTACATTCATTCTGATCT CAAATCTGGTACCATTCCCGTGATGGCTCATTATGATCCTCTACAGAGTGGTGCCAACGGTACCGTGATCAGGGTACCAGCTCACAGGCCGGCAC CGCCTTTACCGTACCATCATCCGGCATCAAGTTTCTCTTGGGTTGGAGCTCAAACCGTAGTCAAAGACCGATTAGCAGTGCCTTTTTCACTCCCAC CCGGTATGCCACCCGTGCCTCCCAGACGTATCGGGCATCCCTACCAGAACCGAACCCCACCAAAGAGGAAGAAACCCCGGACATCCTTCACGAGGA TGCAGATATGTGAACTGGAGAAGAGGTTCCACAAACAGAAATACCTGGCTTCTACCGAGAGGGCCACGTTGGCCAAACAGCTCAAAATGACCGA CGCTCAAGTCAAGACCTGGTTTCAAAACAGGCGGACGAAATGGAGGAGGCAAACAGCCGAAGAAAGGGAAGCAGAACGACAGGCCGCAAACCGA CTGATGATGTCTCTCCAAGCCGAAGTCGTCTCGAAGACGATATACGACCAGAACAGGGACTCGTTATGCATAGGCAACGCGTCGTTGCACGCACT GCAGACTCTGCAACCGTGGGCCGCGGAATCAGCAGCGGCTGCCCACGCGGCCGCGGTGGCCGCTGCCGCAGCCGGACACCACAGGCATCAGGACGG TAGGCCGCAGTTCCTCGGTCCGCCACCTCCACCACCGCCCCCACCACATTCCTTAGCATCACCGATGTGTTGATTTAAGTTATGCGCGTGATTAAC AGACTATAACACCATTATCATCGACTACGAAACCCCCCCCCCTTTCTTTTCAGGACTTGACGAAATATATAGTCTTGTGATATTTCCGATATAAA AATTTCATTTTTGTGACCACCCGTATTATGTTTAGTTTCCCGTGTACAAAGAAAAAATTTGAATAATGTTATATTAAAATATTGAATAGACAGA TAATACAAATTTAGATTTTTTTATTAATTTTTGTTAGAAAGTTCGTGGTGCCTTGACCTATGAGTTTTCCCTATTGCGAGTGATTTCACGCTACA TACGGAAAATATTCGCTGAAAACTGCAGAAATTCATGAAGAATTTTTGATTAATCCTTTGATTGCACCAGTGTCATTTTATGTTGCAACGGCAT CGGAAGCAAACAGCTGAAGAATCGACAGGCCGCAAACCTATAAGATGTAACTTTACAAGATGCGCTCTGGTGTAATGTCTTTTTAGCTTGTAAA AGCTGCTTTCTGGATAACAATTTGAGTTCTGGTTACTTACATTGCGCTCTACTGCAGAGGTCTTCAACCTT

>comp121099_c1_seq1

ATATTTAAAAAAAAAAAATTCACATGTTCTCACACAAAATCATTTCCAATGCAACTGGTATTGATACTTACTTTTATATTAAGAGTAAATGCTA TTTAAGATGTATCCAGCAAGAAAATAGCTTACAATAGTAACATAAAGATGTTCTGATCTCGCTATACATGTCCAAAAAAATAATATTTTTTTTT TACCTTGAATAATATTAGAACTAAGCCAAGACCGGAACAATAAGTTGTTTTCAATCTATGACGGTAATTGAAATGAATGCTGAATCCAGTTTAT AATACTTTTATAAAGTTCATTGTTACTGGAAAAGGAATTGAAAAAATAACACAAAAACATTGATTTCATGGCACGAAACAAAGAATCCATCACT CCATTACGTTTCGAGAAGCAATGAAAACTCGTTACATTTTTCGTGAGTTTCCATCTAGTTATAAAAGAAATGAAAAAAAAGTGCAATTAGTCTT TTTTTTCTTAGTTTTTAATTAACGCGTATATATCACGTCCATCTCACTATAAGTGAAGTTCTAGTTTTGGCACTAATAGGCGAAAATAAAAAAA CAAAAAGGAAACGAGAAGAAACAAGGATTACAAATGCACTTCTAGGCTTTGACGTGTGGACACACATTGATGAAACTAAACGTATTTTCCTATC 
GAACACATGTAACTAATGTTTTATATCTCGCACAATGGCGCGCCAGGAATGTACTCATTAATTGGCACCACTTTGAATACCTTTTGAAGTTGGCT AAAGCGATGAACGTCACTTACTGTGGTTGTCTGTTGATAATAGTTTGAGACATTTATCCATTATGAAGTCTGTTTTATTGAGTCTGTCTGTCTGT TGTGTTCACCGGAATTTGAAAAATCGCATCTCCCGGTTTCCTTATATTTTTTATATAATAGGAAAGACTTACCTCTGTGGAAGTCAGTTCAAAAT CGTCTTTTTAGACTATGAGACTATACAAGGTTCTAATACAAACCTTCGAGATTCCATGAGTACAAAAATACTGCGCAATTTTGTCTTTTCGTAGG AATAGTTAGGATCATAACAAAGCAAGCTCTGGGATTATAGATTTAGGTGAAGATATAAATCGTTATTTTTAGAACTAATTGGCGAGATTTTTCT AAAATATGAACGTCATCTTAAAAAAAATTGTGTGGAATAAAAATTTAAAGAGAATAGATTATAATTAACAAATTTGAAAATGAATTTATGGAT GATTATTTCGTTGCTGATGCGGAAATTTTAAGCCTGCCGATAGTATAATATTTAAATTTTCAACAGTACTATACGCTTTGCGGTTT CTTTCATTG GATTAACTTCAGTTAGTCATCATTTGGCACTGGTTTGCTTTGGCACTATTCCCTATGAGAACTTCGCATCATCTCGTTATCTAAGGGCCAACCTA GGATGAATCTAAGAAGATCTAAACATTTGAAATATTGATGACTAAAAAAAAACACAGGGTGAAATATATTCAGGATTTACCGGGGTTAATTTAG AATGAATTTATCAGATATTAAAGTCGAGGTACTTTCAAGCTATTCTTGGAGACAATAAAGACTAGAGCTCCTATTTATGTGGAGAGAAATAATT GTGTTATTCTCATATTTTTGTAATGAGATCAATATTCCTATTGAAGAAACGATGGGTTATTTTTTTTCACAATGGGCAATTATTTGACGCATTT ATGAAAGACAAGAAATAAGCTCGATCCGTGATCGTTTCCGATTAATTGGTTAATATAAAAGTCCTCCGCCTCGGGTGACATATAGCACAGATTA ATTCCACCGAATTAATTTCATATGGCTGATTTTTATATATAATTTAGAAATGATTTAATAAGTTTTTTAACTGATTTTGAATGTAACTGTACTG AATGTATGTTTTCCAAATAATTTGAATGTATATCGCATTATCAGAACTCATGGAATTCGAGACAGTATGTACTCTTTAACATGAGTCGATGCTT TCAATTAGGATGGTAATGATGACGATATTCTCATGGAATCACATGCAGATAATTGTTTACAGTTGATGTGTATGATGTAACTCATCGTTCGAAA AAAAAAAAAAAACTGATCAGTGTCACGAACATCACTGGACAGACAGTGTTGGTTCGCTGATCTGAGTATCAACGACAATTTATGACATAGACCT ACGCTTCGACAATTTCACTGCTTATGAGTGTTGTCTTTCAGCATATAGGAGCTGTCAGAGATGTCGGTGGTTGCAGGTAAGGAGGTCGATCTGCA GAGTGATGAGATACAGTTGATGATGCGGCGGCTGCAGCGGCCGCAGCTGCAGCGTGTGCAGCAGCTGATGTGCTTTCCGAAGACCATGGTTGTAG GTTCTGGAGGGCGTGCAGAGAAGCGTTGCTTAAGCACAGTGGATCTCTTGTTGGCTCGTACATGGATTTACTGACCACTTCGGCATGTAAGGACA TCATCAAGCGATTCGCGGCCTGCCGCTCCGCTTCTCGTTCTTCTGCCGTTTGACGCCTCCACTTCGTCCGGCGATTTTGGAACCACGTTTTAACCT GGGCATCTGTCATTTTCAGCTGCTTGGCGAGTGCGGCTCTTTCTGCCGAAGCCAAATATTTTTGTTTGTGGAACCTTTTTTCTAATTCACAAATT TGCATCCTTGTGAATGAGGTCCTAGGCTTTTTTCGTTTCGGTGGCGTCCTGTTTTGGTATGGGTGGCCGATCCGACGAGGTGGTACCGGTGGTAC CCCTGGAGGAAGAGAAAAAGGCACATGAAGACGATCTTTGACGAGAGCGGGGCCTGCGGGACCCATCCATGGGAACGACGCAGCGTAGGGAATG GGGGCCGGCCGGTGAGCGGGCACCCTGATCACCGAACCATTAGGCGCTCGTTGAAGCGCGCCAAAAGCGGTCATCATGCTGGCTGGTATAGATGC CTTCATTTCGGCCAGCAAACTAGCCGGGATACCACCCGCAACACACGTGCTGGCCACGGCGGTCATTGCGTCCATGTTGGACACGGATTGAGATG CGTGTCCACTAGCACTCACGTGATTTTGTTCGTCCAGACCGTCTTCGTAAAGCCCACCGTCCGACATCGGCGAAGGTACGTTGCTACCGTTATTGC TATTCTGTCTTTTCTTTTCATCTCCTCCTAATAAACGAGATATGCTGAAAGACAATCTGGGCTTGGAACCTAACGGAGAATGTAACGGAGTCGGC GAAGGATTATTATACAAATGATTCCTGTTCAAATTCGGAGACGAGGAACCCCTTCCGCTGTTAGCAGAACCAGCTCTGCTACTGCTTACAGTCTC AGCACTGTCAGCATCATCCATTTTATCCCAGTTATGTACTGTTTCAGATGCCATGATTAATCCATAGATGACTCCTGGAAAATAATTTCTTGACC AACTCTTGTGGTATTGATAATAATAATACGAAGTTCATTTTTGAATTTGTTGCAAAGTATTTTCAACACTCACAGGTTATTTTCTGGATAAACG ACGCATTTTTTTTTTGCTGTCAACCTATAGAACACTTGATGAAGTCAATGATGGATTTTCCACAAGGACCGCACGTGGGAGAGAATTCAACGACA GGAAATGACGACACACTTGCAGTGTTGTTGTATAAAAAACAAAACAAAACAAACTCGGTATTATTCGCCGAGGAGTTGTGTCCGGTTGCGGGGA ATTGATTCCCTTACACGGCCAAGTTTGTTTTTGCTAATGAGACCGGTGTTTGCTTTTTTCGTCTTCGTTCACCGTCGGGTGGGTTGGG

>Locus_15589_Transcript_1/1_Confidence_1.0_Length_2869

TATCGCTGCTTTTTTTACAGCCGGTTCGGTCTATTGTGTTTGCGTTGTTTACTTCTCGAAAAACTTATCCAATCTGTCACGTGCTTTTTACTTTT GACATTTAATCGGTTTGTGTAGGGTGTGAAAATAAAATAATTCGCTAAATCTGTGATTTGATTTGAAGTGCAAGATGCATTTTTTACTAGTCAT AGATTTTTCGCTGAGTGAAATTGAGCAATGAATGCATTTGAGTTTCTGTCTTGTTTAAATAAACATGCTCTGTGATCGTACTGCTCGCGTCGGGG AGGGCAGGTCCCCTCCCTTAGCTGCCAACGCTATCGATATTGCAAATCCTTCAACAAGGATCGGTGGTGTACTCGATACTGACTACATGGACAAT ATGACCACAAGACACTCTTCCCCACCTACATCGGAAAATAATTCTTCCTCTTCGCCAATCATGCAAGAATCTTCTACGAAACCTAAACTGTCTTT CAGCATCAGCAGGTTGCTCGGTGATGATTCGAAAGTGGACAGTGACAACAACAACAATCATCGAAAAGATGACGGTAGGTCATCAACGGACAAC AACAGCGTGCACGGTTGTGATAGTAGTTCGGCACGAACAACTCCGGCGTCTTCAACCGCAGCATCACCAATGATGGTCATTAGTAGTAGTGGGTG CTGTCCTGGACTTAATAACCAACCACCTTATTTTTCGACCAGTGACCTGAAGATGTCAACTATGTCAGGGATGGTTCCCCATTATGACCCTCTTC AAAGTGGAGCAAATGGAACTGTGATAAGGGTGCCAGCACATAGACCGGCACCTTTATCTTACCATACGACTGTCTCCTTTCCTTGGGTCGGGTCC CAAGGTATAGTCAAAGATAGAATTCCAGTGCCTTTTCCTTTGGCAGCAGGCCTACCTCCGGTTCCTCCTCGACGCATTGGTCATCCTTACCAGAA TCGAACGCCACCAAAAAGGAAAAAACCGAGAACAAGTTTCACGAGGATGCAGATCTGTGAGCTGGAAAAACGATTCCATAAACAGAAGTACTTA GCTTCAGCAGAACGTGCCTCCTTAGCTAAAACTCTTAAAATGACAGACGCTCAAGTTAAAACATGGTTTCAGAACCGAAGGACAAAATGGAGGC GGCAAACAGCAGAGGAGAGAGAAGCTGAACGACAAGCTGCCAATAGACTGATGATGTCTCTACAAGCTGAAGTAGTTTCTAAGTCCATATATGA TCAAAGCAGGGATCCACTCTGTATAAGTAACGCTTCCTTGCACGCGCTTCAAACTTTGCAACCATGGGCTAGTGAGAATGCTGCAGCTGCCCATG CAGCGGCAGCAGCAGCTGCAGCTGTTGGACATCACCAAAGTGGTGTAGAAAGGCCATCTTTCTTAGCACCTCCACACTCTTTATCTTCACCAATT TGTTGAGCTTTCAAATAAATGCAATAAAAACAACATTTCACGTGTTGACTAATGAAATCTAAAGTCATTAAGTCTAATCTAGAGTCGCCGTTGA TCAGATCTTTGTTACAAAGAAGCAAAATGGACAATTTGTTTAAAAAAAAAAGAAGAAAAAGAAGACTTGAAGAGTGATAGCTTATAGTGGGAC TTTTAGCCACCTTACTTCTGATAAAATTATAACCTGAATTTTATAATACATTCTTGCGATAAAAATTGTTGCAATAAATGGTTATAATTAAAAG AGAGATTCGAATTACTATGTAATATAAAATATATGTTGTTCATTGTCAAATTGTTTTTTTTTCTTTTAAAAAACATTGTATTTATTGAAGTTTT GTTCGTTTACTGGCAATAGAATATCATGGTAATCTAATTAAAAGTTGGTTTTATTTAAGAATTAAATTTGTGAAAAAATATAATTCATTTGGTT TGCAGATATTTGAAAATTAATACAAATATTATTATCGTGAATTACTTTTGTAAAAAAAAATATACATATATTTTTATAAAGCTGCTATATCCCA TATTTTCCTTATAGTACAAACTATATAATTTTCTTACTAAGAATAGTAAGATTTTAAATTAAATAACTTTTTACTTTAGAAAACAACAGGAAAT AAAGTATTTTAATTTGAATATTTTGTGTCGATTTTTCCTGTAACAGTGTTAGATAGGATTAACTTTGTTGAGAAACTGAGCTGATTCTTTAAAA TTATTTATAATAAACGATTAAATTTGTTTTATTTTTTAAAGATACCATCTAGATTAATGAAAATGAAAAAAATTAACCAAGCTACTTTGTTTTC CTATTGTTGGAAGAAAATAAAGCGTTTTCAGTTATCATATTATCATTGTAACAATATCTCTGATAACAGTATTGTAAATCAATCTTTTTTTTTT ACATTTATTGGCATTTTTTTAAAGTCTATAACTAATAAACAAATGAAAATGTCAACATGTTTAGCCAAATCGATTAAAAAATATAATACTAACT ATATAATTATTTTTATTTTAGTCATCTAAAATATTAAAAAAGCATTTTGTAATATAACCCATAACACTTTCGATAAATTAAATAAATAATAATT AATTTTAACTACAAGTAGAAATGTGAATAAAAAAACAAATAAAATCATTTCATGTGTAAGAAGAGCAATAATGTACTTAATATTTTTAGCGCAT GTACAGTTTTGCACCGTAAACTAACATAATAAAATAGTCTAATTATTTTACTTTATGATAAACATGTTTTAACTAATTGTTTAACAAAGTAATT ATATAGTTTAGAGCTTATATAGTGTTTATTTATTGCACTTTTTTCTTTTGAAAACCTGATCATTTTTCTTTGTATTTTGTGAGATGAAGTAAGC TCATATTATACTGTTTTAGTTAACTTTATCAAATAGTATT

>Locus_21582_Transcript_1/1_Confidence_1.0_Length_907 
TGAGAATGATAAAAACTAGTCTGAGAAAAATCTTGTAGTTGTCACTTAATATTCTCACTTTTGATCATTTTAATCAATATTGAACGCAAATGGG AACACTTCTTGTGTTCCCATAATCTGTTCATTCAAGTTATTTAAAGTTATAACAAGATGGATGATGCCGATAGTGCGGTCAGCAGCAGAGCAGG ATCGGCTAACAGTGGTCGGGTGTCATCATCCCCTACGCTTAACAACAATCATCTGTACAGTAATAACAATTCGTCGCCGCTAGTTGCGAAACCAA GATTATCTTTCAGTATTTCAAGACTATTAGGTGATGAAAAAAAAAGGATATCAGATGAAATGGCCTGCTGTCCGACTTTGTTAGCCGAAATGCC AGCCTTTGACACCGGAACAGTCATAAGAGTTCCAGCACATAGGCCCTATGCTACACCATTTCCATGGATGGGTCCATTAGTAAAAGACAGACTTC ATGTGCCTTTTTCTCTTCCACCAGGTCTGCCCCCTGTTACACCAAGAAGAATTGGGCATCCATATCAAAATCGAACACCACCAAAAAGAAAAAAA CCAAGAACTTCTTTTACAAGAATGCAAATTTGTGAACTGGAAAAGAGATTTCATAAACAAAAATATTTAGCGTCCGCTGAAAGAGCAGCATTGG CCAAGCAACTCAAAATGACAGATGCACAAGTCAAAACGTGGTTTCAAAATCGAAGAACAAAATGGCGGCGTCAAACTGCGGAAGAAAGAGAAGC GGAACGACAGGCTGCTAACCGACTCATGATGTCTCTTCACGCCGAAGTTGTGAGCAAATCTATGTATGAACCTAGTAGAGACCCTTTATGCTTAA GTAACGCATCATTGCACGCACTTCAAAATCTACAACCATGGTCATCGGAAACCGCTG

>comp116449_c0_seq2_-_ORF_1_(frame_3)_translation MLCDRISRVDIGSPSSVVDDDEEHVDVMDHGDTDDGGIHSEASGQSPSPLSTSSNSPCNNRLLLPNKPKLSFSISRLLGDDHHNQHQQYRQSSSPHE YRDSESPPAKDTAATNVENSTVTDDCQESSYSSASSPQMSSSPVNSGTFPSRSTTNGTLINSSSGACCATGLGTHTYIHSDLKSGTIPVMAHYDPLQS GANGTVIRVPAHRPAPPLPYHHPASSFSWVGAQTVVKDRLAVPFSLPPGMPPVPPRRIGHPYQNRTPPKRKKPRTSFTRMQICELEKRFHKQKYL ASTERATLAKQLKMTDAQVKTWFQNRRTKWRRQTAEEREAERQAANRLMMSLQAEVVSKTIYDQNRDSLCIGNASLHALQTLQPWAAESAAA AHAAAVAAAAAGHHRHQDGRPQFLGPPPPPPPPPHSLASPMC*

>comp121099_c1_seq1_-_ORF_1_(frame_2)_translation

MASETVHNWDKMDDADSAETVSSSRAGSANSGRGSSSPNLNRNHLYNNPSPTPLHSPLGSKPRLSFSISRLLGGDEKKRQNSNNGSNVPSPMSDG GLYEDGLDEQNHVSASGHASQSVSNMDAMTAVASTCVAGGIPASLLAEMKASIPASMMTAFGALQRAPNGSVIRVPAHRPAPIPYAASFPWMGPA GPALVKDRLHVPFSLPPGVPPVPPRRIGHPYQNRTPPKRKKPRTSFTRMQICELEKRFHKQKYLASAERAALAKQLKMTDAQVKTWFQNRRTKW RRQTAEEREAERQAANRLMMSLHAEVVSKSMYEPTRDPLCLSNASLHALQNLQPWSSESTSAAAHAAAAAAAAAASSTVSHHSADRPPYLQPPT SLTAPIC*

>Locus_15589_Transcript_1/1_Confidence_1.0_Length_2869_-_ORF_1_(frame_2)_translation MLCDRTARVGEGRSPPLAANAIDIANPSTRIGGVLDTDYMDNMTTRHSSPPTSENNSSSSPIMQESSTKPKLSFSISRLLGDDSKVDSDNNNNHRK DDGRSSTDNNSVHGCDSSSARTTPASSTAASPMMVISSSGCCPGLNNQPPYFSTSDLKMSTMSGMVPHYDPLQSGANGTVIRVPAHRPAPLSYHTT VSFPWVGSQGIVKDRIPVPFPLAAGLPPVPPRRIGHPYQNRTPPKRKKPRTSFTRMQICELEKRFHKQKYLASAERASLAKTLKMTDAQVKTWFQ NRRTKWRRQTAEEREAERQAANRLMMSLQAEVVSKSIYDQSRDPLCISNASLHALQTLQPWASENAAAAHAAAAAAAAVGHHQSGVERPSFLA PPHSLSSPIC*

>Locus_21582_Transcript_1/1_Confidence_1.0_Length_907_-_ORF_2_(frame_1)_translation MDDADSAVSSRAGSANSGRVSSSPTLNNNHLYSNNNSSPLVAKPRLSFSISRLLGDEKKRISDEMACCPTLLAEMPAFDTGTVIRVPAHRPYATPFP WMGPLVKDRLHVPFSLPPGLPPVTPRRIGHPYQNRTPPKRKKPRTSFTRMQICELEKRFHKQKYLASAERAALAKQLKMTDAQVKTWFQNRRT KWRRQTAEEREAERQAANRLMMSLHAEVVSKSMYEPSRDPLCLSNASLHALQNLQPWSSETA 


\subsubsection{BarH}

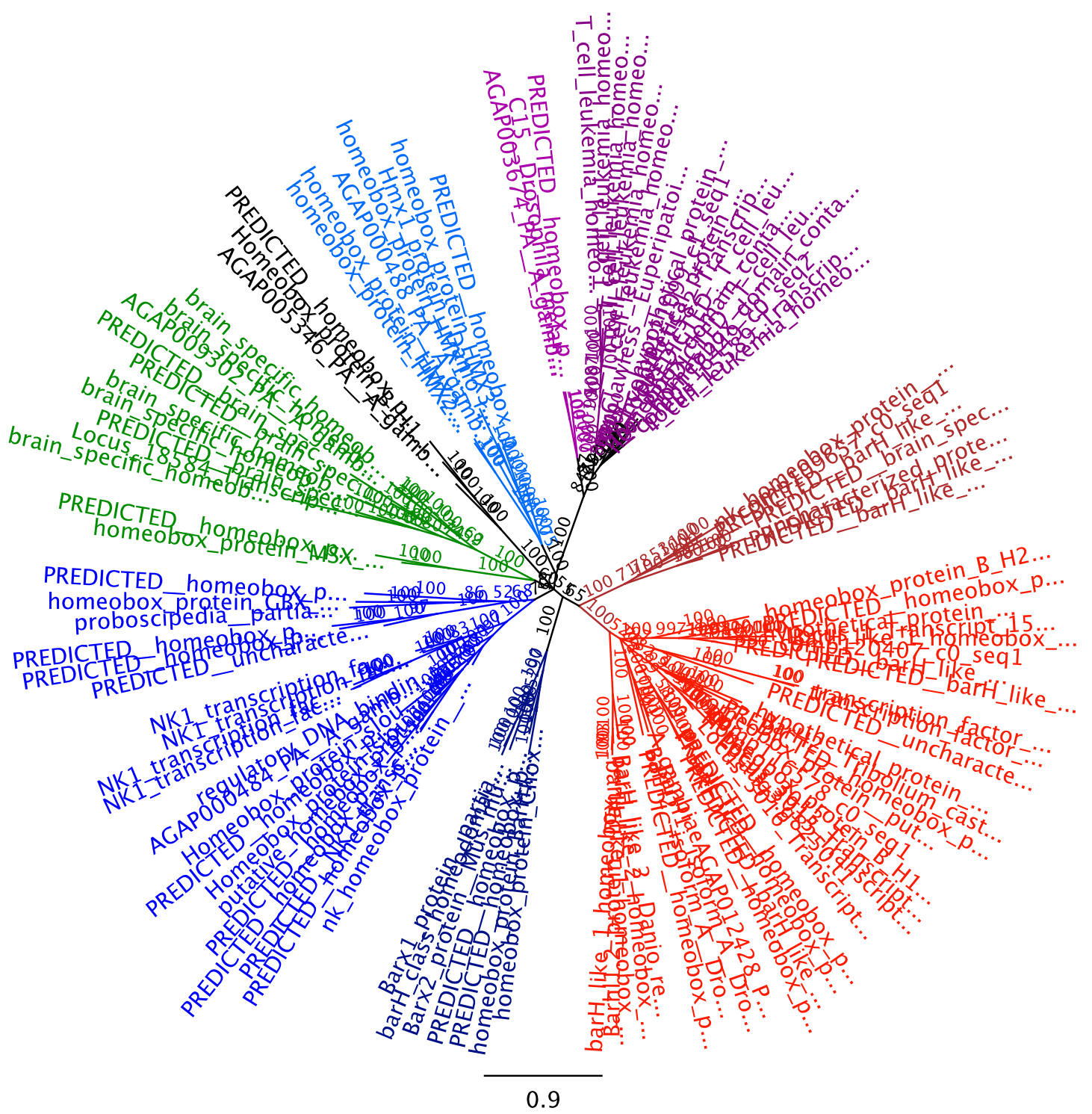

Supplemental Figure 17: Unrooted 50\% majority rule consensus tree to identify true $B a r H$ homologs.

The Jones amino acid substitution model was chosen after mixed model testing. After reaching 3000000 generations a total number of 12002 trees were written in two files (each file contained 6001 trees of which 4501 were sampled). Colors indicate the monophyletic groups in the tree. 


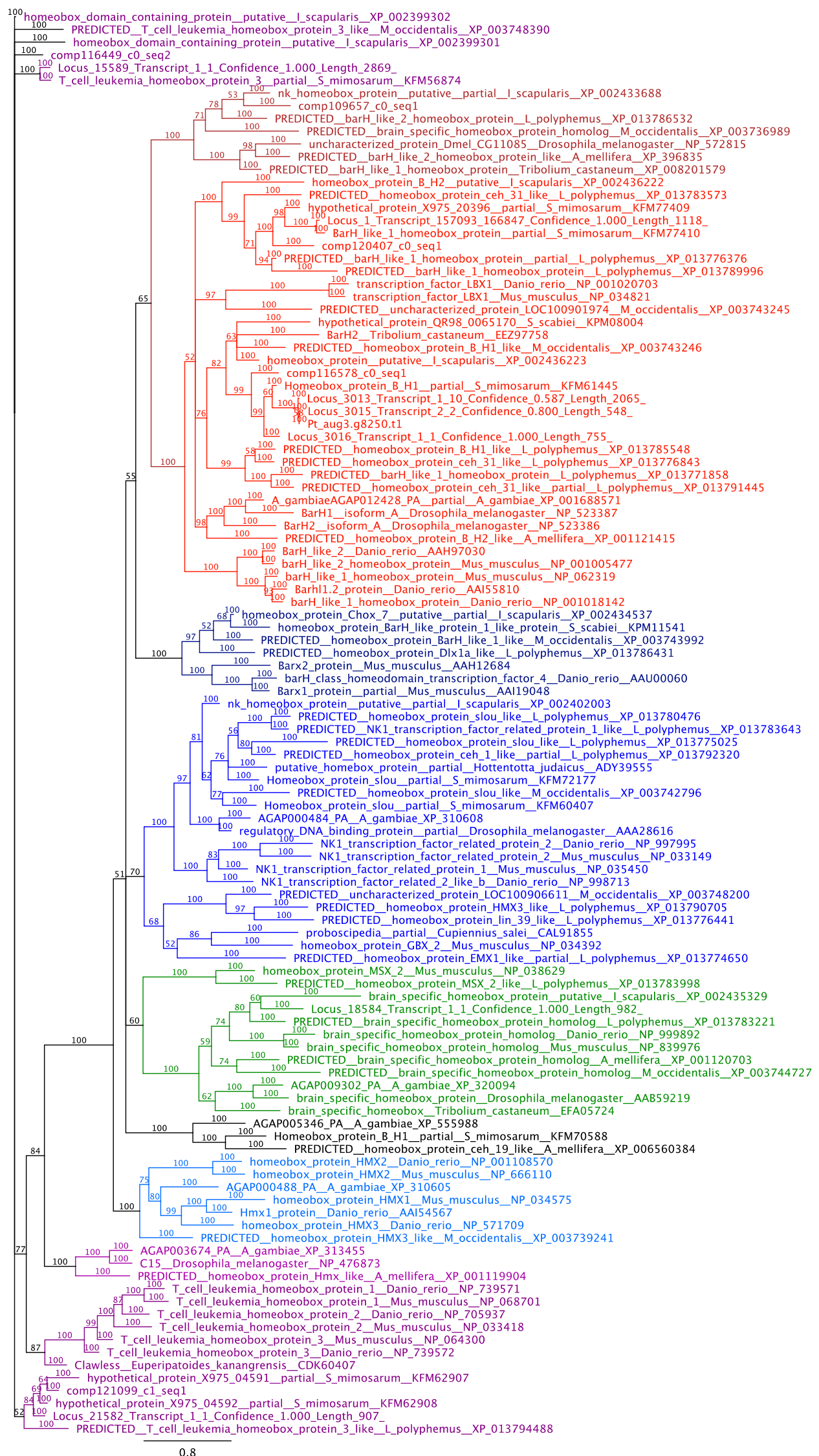


Supplemental Figure 18: Unrooted $50 \%$ majority rule consensus tree to identify true $B a r H$ homologs.

The same phylogram as in Supplemental Figure 17 in a magnified view to display gene and species names.

>comp116578_c0_seq1

CTGGTGTTATCTCGCTACCGGAGGTGCCGGCTGTGATCTCTCTCTCTCTCTTATCTCTTTTTTTTTTTTATTGTAGAAGGAGATAAAAAAAAGAA GGAAAGAAAGAATTAGTTTTTTGGTCGTTTCACCTCCAAGTGAAATTGAGTGTTCACGAATTGATAATATCGTCGGAAAGTGATTTTTTCTCTT CGTTGATTTTTGGAAGACTTTTTTTTTCAAAGAAGAACGATTTGGAACGAAATATACCGACATCACCATTACGGGATCACCTTTTCGTTACGGCC GCCAGGCCATGACTGTTGACCTGCCGCAGAAACCGATGCCGGCCGATCGAGATCTGAGGGTCGTCGTCGATGACGACGGGTCGGTCCGGGACGAC AGTCCGCCACCTCAGTCCACGACCGCTTTCCGAGTCGCGGCGTCGCCTCCGTCGGTGGTCGCCGGCGGTGGGACGACGGCCCCGTCGTCTTTCCTC ATCAGGGACATTCTCAGTGGTACCGAACAGCAGCAACAACAACAAAGACAATCTCACATCCGTGGTTCCGGGTTCAGCCCGGTCGGTTACACGGC ATCGCCGTCGACGACGAATTCCAATCCCACGTCACCGCTGTCCGCCAGCGATCCCGGACACCATCATCATCATCACCCTCCTCCTCCTCCTCACCCG TTGCACATGCACCCGCTCGCTCATCATCATCACGCTCTGCAACATCACCCTGCCATTTCTGGACAGTTTTTCAGTCCCCTGGACATTCGAAGGCCA AACGGTGAAGTTTCGCCTCTCAGAGACGATGAAATCGACGAGGACGGTGTCTCTTCGGACGACGATTGCTCCAGAAGTGGAAAAAACGACGACCC GCAAAAGTTAAATAGTTCGAACAGCCCAGATCCGTCGAAAAGTCCTCCGTACAATTTGAAAGTAAAGAAGCAGCGCAAAGCTCGGACGGCCTTC ACCGACCACCAGCTGCAGACTTTAGAAAAGAGTTTCGAGAGGCAGAAGTACCTCAGCGTCCAGGACAGGATGGAACTCGCCGCTAAACTCAATCT CACGGACACTCAAGTCAAAACTTGGTACCAGAATCGCAGGACGAAATGGAAAAGGCAGACCGCCGTCGGACTAGAACTGCTCGCCGAAGCCGGGA ACTACGCGGCTGTGCAGAGGATGCTGCAGAGCTCCCCTTACTGGCTGAACCACTACGCGGCCAACCTGTCCTTCTCTTCCGTGCCAGGTATGGACA TGTACTACCGGCAGGCGGCAGCGGCCGTCCTGCAGAAGCCGATGGCCTACCGAATGTATCCGACCACGACGGCCACACCGATGGGCATGCCCGTC AACTTCTCGTCGTCCACCGTGCCGCCACCTCCGCCGCCTCCGATGAACTCCAACAACAGTGCACCTTCGCCAACTTCACCGCTGTCTTCTACAACA AGTTCCTCGACGCTGAATTCCTTCTATGGACGAGAGCCTCCACCTTGCTGAGTGTGAGAGACACGCGCGCACACACACACATGCGTTTAGAACTA GACGTATAAGACACAGAGACTCCATTATTTGCCATAAACATTGGGTGTTGAACATTTATTACGTGTAATGTAAATACCGGACAGAGAAATATGT AAATAGTAACGGAATGTGAAGAAAAAATCGTCCGGTTGATTATTATTAATATTATTATCGTACATAAAGCAAAAATGAATGTTGGCCAACCCGT AAGCATGTTGCGTAGCTTCTTCGCCCGTTTTTCTGGAACTTTATTTTGTTTTCCGAGATGTGACACAACACGAGATTCCCAATAGCGTCTGTACT GCATAGAATCGATTCTACTATACAACCATGATCTGCTTCTCAAGAACAAAATCATCTCTCTTCTCATTTATACTTTTGATTATATTTATTTTTCT TCTCCCTTCTTATATTTATTTATTAAAAACAACTGATGTGAAG

$>$ comp120407_c0_seq1

CTAATTTCTTCCTTCTCCCACAAAGGAGAAAAGAAAAAAAAGAAGTAACGAATCCATCAGACCAAATGCTTTTTGAGAGAGGAGAAGAAAAGAA ACACCGGTAGTCCAGTTGTAATGAAGCATGATCACCACCAGTTGGGGGCCCTAATGCGTGTACGGTAGTTAGTTTGGCAGTCGGTGTGTGTGAGT GAGTTGAGTGATGTCACAGCACGCCCTTTTTTTGTTTCAATCCGTGGTGAGGTGCGGTTGAAAGATACCAACGTGTTCCGTTTTTTCCCACACGC ACAGCTGTTGCATGTTCGAGAAATTTACAAATTTTGTGGAATTTGTGACGTGCCTTCGGATATTATAGTTGGGAAAAAAAAAATTGAATGTGAA TGACTATCAACTCGTTGGACACACAACGGATATGACTGTTACGATGTCCGCAGACACTATCAATGGAAGAACGTCCGCGGAGCCGACGGAGGAGC TCGTGGAGCAACCGACTGATCTCAGCACCAAGAAGAAGTCCGAAACGGAATCCGGTTCCGATAACAGCCCGCGGTCTTCCCCGTCGACCCTGGCA GACGTCCGCGATTCGAACCCGGCTCCTACCTCGGGACCGTCGTTCCTGATCAAGAATCTGATCGACCATCACAATAGCCACCATCATCAGCAGAGC CACCAGGACTTTGCGGGTTTGATCCCGTCCACGTTCCACAGGTACCGCCCGTCTTTCGTGCCGTCGCCGAATCCGTATTTCTTGCCGCATTTTGCC GCGAACATTCCTTCCGGGAAGTTTTGTTTCGATCAGGATGGGTCGAATAGGAGGATGGACAGCGACAGTGAAGAGAGTGAGGGCAGGAGACCGT CGCCGGTGCAGTACCAGAGGACTCTCCCTCAGGAACTTGCGGACTACAAGCAGATCTGTGATTCGAGAGACGTTCTCAAGACTAGACCGAACGAT GCTGAGACAGTGTCTTCGTCTTCCAAGGATCGTATATCCTACACTGCGGTTATCGATTCCGACACGGACAGTATCAGCAATCATGATGGCGAAAG TTTCGATGCTCAGCCACCAAGAGAAGACAGCCCGTCTCTCAAAAATAAGAAACCGAGAAAAGCGAGGACAGCCTTTACTGACCATCAGCTAAGAA CACTGGAGAAAAGCTTCGAGAGACAAAAATACCTCAGCGTTCAAGACCGTATGGAATTAGCCGCTAAACTCAATCTGACCGATACTCAAGTCAA AACATGGTATCAGAACAGAAGGACGAAATGGAAGAGGCAAGCAATGATTGGATTCGAGGTTTTTCCACCGGATGCCTGCAGTTTAGCCCGTTTT CCTAGACTCTGGGGACCCGCACAAGCGCCTTACTGGCCTTACAGCTATAGCAGCTACCTTTCCACGATATCGTCTCTGTCGGGATTGAATACAAA CCCTCTGGTGGGACAGACATCATCGTCGCCGGCGTCTCCTCCGTCCTCTCTGGAAGGCTACGGGCGTCAAGCTGGAAGTGCAGTGTCTAATACCGT CAGTCGTCCTCTTTTACCGAGGGCCGTTTATCCGAGTCCGTCCAATTTCTCGTCCGTTTCGCCGTTCTACCAGGGCCAGGCTGGGCCTTGAACAAC AACAAAAAAACAAAGGCCCCAGAATTAACCGCTTGATTTATTTAAAAACCCGAAGATATTTTGCGCTTTTTCTATGGACGGTGGAACGAGGTTT ACTCAGGAAAAAAAAAAAGGCGTGCTTTAGGAACTGATTATAGGAGAGAACTGAGATCATTCTTCCCGTGAAACTGGTGTGTGGTTATGGTCAT TTTCTTAACCGTGGTGAAGAAATTTCATCCAAATGAAAAAAAAAAGGCACGCTTCTAAATAAAATGCCAAAGAGGATTCTTTCTGGACTGAATG CATGTTGCTTGAAGGGAAAAAATAAATAACTGAAATTGCTCTTCTTGTTTTCATCGAGTTACTCATCTTTATTTTTAAGTTATTGTCTTTTCGTA ACATTCCACGTCGAAACCAAATAAGTTATCAGAGTCTAAAGTTTTTAGTTGGAGAGCGGATGAACAGAGATTACGATTTTTTTTTTGTTTTGCT TCAATTTTGAATGCACATGAAATTTGTTGTAATCAAAACACTGATTGCCGTGTGATAATGTTAGTTGTATTGTATATGGAAAAAAAAAATTATC TTCATAAATTATTCTGGTAA

>Locus_1_Transcript_157093/166847_Confidence_1.0_Length_1118

GTAATGACAGATATAGTTCGAGGCAGGTTTCCTCAAGAGATAGTAGCCCTTCACTGAAGAACAAAAAACCTCGAAAAGCAAGAACAGCTTTTAC AGATCATCAATTAAGAACATTAGAGAAAAGCTTTGAGAGGCAAAAATACTTGAGTGTTCAAGACAGAATGGAATTAGCAGCCAAATTAAATTTG ACTGACACACAAGTCAAGACGTGGTACCAAAACCGAAGGACGAAGTGGAAAAGGCAAAATATGACATTCTGTGATTACTACGCTGCTGATAGCG CGACGCTGGCCCGATTCCCGCGTCTCTGGGGACCTAGTCCAGCATCATACTTCCCGTCATACAGCTACAGCAGTTATTTATCCACCATGTCTTCAC TTTCAACCCTTGGAGCAGCCCATTTAGGGACACCGGCTTCTTCTACCTCTGTGCCTACCCTGGACCTTTACCAAAGGCAAAGCCCACCTGTATCAT CAACCATAGGTCGTCCAGTACTACCAAGAGCTGTGTATCCCACCCCAAGTACATATCCATCACTGCAACCGTTTTTCCATGGTAGGCCAACGCAG TGAACTATCGTCAAAAATTGAGTCAGAGAATGAAATAGAATTTTCGTCAAACGTACTGGTTCCGTCAAGATACTGGACAAGTGCTGAGTTTTTA ATTTCGAAAGATTAAGAAGAAACATACTTTGTTTTCTGCTCATTAATGCTTTCGTGTACTGATTATGTTGGAATAGCAACTGGACATTCGTGCG TGTCGGCTGGTCCCGATGTATTACAACAAGTCACAATTGATTCTGTCTTTGGATTGATCTTTTAACTATCGTTTACCCACCCTGATGATAAGAAT CAGATGATTGATTATCTTTGTTACGGGAGAATTAATTGATTGATCCTTTATCATTTAAAAGAACGATTGATTAATCATCTATGCTGTGGAAAAA 
TCAGCGGACTATGCCATGGTAAGATTGACTGGATGATTATACATGCTGTGGTGCAGAAATTAATAAATTGATTTTCTTTAATGTGAAGTGATTT TTTGGTTGAACATCACTGCTATAATTAATTGGCTTAAACAATTGAAATGAATAATAGAAACGAAAAATTAGTACTTAA

>Locus_3013_Transcript_10/10_Confidence_0.587_Length_2075

ACGATCATTCTGGTCTGGAAGAAACAAACAGTAATGACCCATCCAGTAGTCCACCTTACAATCTAAAAGTTAAAAAACAGAGAAAAGCAAGGAC GGCATTTACTGACCATCAGCTTCAAACGCTGGAAAAGAGCTTCGAGCGACAGAAATACCTCAGCGTCCAGGACCGAATGGAGCTAGCGTCCAAAC TCAATCTCACAGATACTCAAGTCAAAACATGGTATCAAAATAGAAGGACGAAATGGAAGCGTCAAACAGCTGTTGGTTTAGAACTTTTAGCCGA AGCCGGTAACTATGCCGCAGTTCAGCGTATGCTTCAGTCTACTCCTTACTGGCTGAATCAATATGCCGCTAATTTACCTTTTACCGCTGGATTAC CAGGAATGGACATGTATTACCGACAAGGACTTGGTCTTCAGAAACCAATGGCTTACAGGATGTATCCATCTGCTCCAACGACAGCCATGGGATTG CCAGTCAATTTCTCTTCATCTACTGTGCCACCAATGGCTGCGGCAAGTGCCACTTCACCCACCTCATCTTCAGCGTCATCTTTACCTTCTTATTTC GCTAGGGAACCCCCTTGCTGAAAATAGATTGATAGCTATTTAAAAAAAATTTAATTTATTATTGTGATCCTCCGTCGAAAAATGACCAGAACTT GTAAATAACTCTTGGACTTTCTCAAACGTTCCAGAACTTGTACATATTTGTATAGAACTCTTGGACTTTCTCTTTGTCAACTATGAAAGAAAAGG TGGCTTCTAATATGAGTTGATGAATTTGGCTGACTACTAGTCTATGGCGCAGGTTTTGGCGAAAAATGATAGAAATGGCGATAAAAATCCAGGA TTGAAGTTAAAAATAGGAGCTGCCTCAGTGATAAGACAAGAGTAAAAGAATGCCTGCGCGCCAGGTTATTCACTTTTTATGGACTGTAACAGAA AATGAAAAACCATTTTGCAATTTTTTTTTTAAATTTCTTTTGATGAAGTTGCATATGTACATTAAAGTGTACATTTTGATGTACATTAAAGTGT ACAATTTCTGTACATTTCATTTATGGCGCGCGTTTATGTAAAAAATAGAAAATTATAGCATCGCTTAGAATGCTGAAAAATGAATATACTTAAA ATGATTTCTAATATTAAATTTTTTTATGAGTGTTTTTGTTAGCTGCATAAAATTTAAAAAAATAATAATAATAATTTTTACATGAAAATATACA TTGTTTTTGAAGACACATTCATTTTAATTTTAAATTCTTAAAACAGATTATGAAGAATTACTTATATTATTACAATGTACTTTTATGCAATTTC AAAAGAATTAGCTTGCATTCTTTTCAATTTTAACTCATATAGTTTTTAATTATATTTTACAGCTAAACTTATTAACAGACCTAATAATTCATGA TTTGATTTTAAAGGGTTGGACGATTAAAGGATTAGTGTAAGTTATAACTTTTCTAAAAGTATTAATTTTCTCTCTAAAATAGTCAATTTTTTTT CATCGAAGAAATAAGTTTTTTTCTGTGATCTCAGTTAATAACTAATTTAATATCGAACAGTGAATTTAATAATCCAATACTACTCGCTAATACG TAGCTATTTCACATCCATGGGCAAGAAACGAGACAGTAGAATTGTCAATTAATCCAGATATGCTTGCAATAACTACTTACCTTTTCGCTGCAAAA GTTTTCAATTGTGCGCACTACCTGTTATTTAATAATTTTTTTCAAATTAGGCATGCACATTTTCTGTTTTCTTTCTTTCCATTTTGATTTATGCA TTTTCATTATAAAATTGCAAATTATTATATTCTCTTTGTGTTTTTCTTTCAATTAGATGCATCGTTTTTATTTATCCATTATGGTGTTAGGTATA GTTTTTATTGTTTTAAAAATTCTTTTGTTACGCTTCCTATACAATTTCGCTTCGGTTTTGGTGCTTTGTATTTCCAATTTTTTTGTGTGCTTCGT CTCTTTTCGTCTCCCTTTATTTTGTGGCGTTTCAGTTTATAGAGTAATGATTAAATTTTCTTTTGTCTTTTAAGTTTAATTTTTGTATTAC

>aug3.g8250.t1

AATCATTCCATGTTCAAAGGTTATTGTTTTATCCCGGCTTGCAGCAATATCTATCCGAGCAAATGTTCGAATTCCGAAAGGGTGGTCCACCACCC ACGCTGCCCTCACAGAATTCACCTCCTTCCCATTCGTGTCAAAAGGTGACCATCCAGAAGACAAATAGTGACGATGATGATTTTTGGATTGACGT CGAAAACGAAACGACAGATGATGAATCAAACGATCATTCTGGTCTGGAAGAAACAAACAGTAATGACCCATCCAGTAGTCCACCTTACAATCTA AAAGTTAAAAAACAGAGAAAAGCAAGGACGGCATTTACTGACCATCAGCTTCAAACGCTGGAAAAGAGCTTCGAGCGACAGAAATACCTCAGCG TCCAGGACCGAATGGAACTAGCGTCCAAACTCAATCTCACAGATACTCAAGTCAAAACATGGTATCAAAATAGAAGGACGAAATGGAAGCGTCA AACAGCTGTTGGTTTAGAACTTTTAGCCGAAGCCGGTAACTATGCTGCAGTTCAGCGTATGCTTCAGTCTACTCCTTACTGGCTGAATCAATATG CCGCTAATTTACCTTTTACCGCTGGATTACCAGGAATGGACATGTATTACCGACAAGGACTTGGTCTTCAGAAACCAATGGCTTACAGGATGTAT CCATCTGCTCCAACGACAGCCATGGGATTGCCAGTCAATTTCTCTTCATCTACTGTGCCACCAATGGCTGCGGCAAGTGCCACTTCACCCACCTCA TCTTCAGCGTCATCTTTACCTTCTTATTTCGCTAGGGAACCCCCTTGCTGAAAATAGATTGATAGCTATTTAAAAAAAATTTAATTTATTATTGT GATCCTCCGTCGAAAAATGACCAGAACTTGTAAATAACTCTTGGACTTTCTCAAACGTTCCAGAACTTGTACATATTTGTATAGAACTCTTGGAC TTTCTCTTTGTCAACTATGAAAGAAAAGGTGGCTTCTAATATGAGTTGATGAATTTGGCTGACTACTAGTCTATGGCGCAGGTTTTGGCGAAAA ATGATAGAAATGGCGATAAAAATCCAGGATTGAAGTTAAAAATAGGAGCTGCCTCAGTGATAAGACAAGAGTAAAAGAATGCCTGCACGCCAGG TTATTCACTTTCTATGGACAGTTACAGAAAATGAAAAACCATTTTGCACTTTTTTTTTTAAATTTCTTTTGATGAAGTTGCATATGTACATTAA AGTGTACATTTTGATGTACATTAAAGTGTACAATTTCTGTACATTTCATTTATGGCGCGCGTTTATGTAAAAAATAGAAAATTATAGCATCGCT TAGAATGCTGAAAAATGAATATACTTAAAATGATTTCTAATATTAAATTTTTTTTATGAGTGTTTTTGTTACGCAGCATAAAATTTAAAAAAAA AATAATAATAATATTTACATGAAAATATACATTGTTTTTGAAGGCACATTCATTTTAATTTTAAATTCTTAAAACAGATTATGAAGAATTACTT ATATTATTACAATGTACTTTTATGCAATTTCAAAAGAATTAGCTTGCATTCTTTTCAATTTTAACTCATATAGTTTTTAATTATATTTTACAGC TAAACTTATTAACAGACCTAATAATTCATGATTTGATTTTAAAGGGTTAGACGATTAAAGGATTAGTGTAAGTTATAACTTTTCTAAAAGTATT AATTTTCTCTCTAAAATAGTCAATTTTTTTTCTTCAAAGAAATAAGTTTTTTTCTGTGATCTCAGTTAATAACTAATTTAATATCGAACAGTGA ATTTAATAATCCAATACTACTCGCTAATACGTAGCTATTTCACATCCATGGGCAAGAAACGAGACAGTAGAATTGTCAATTAATCCAGATATTC TTGCAATAACTACTTACCTTTTCGCTGCAAAAGTTTTCAATTGTGCGCACTACCTGTTATTTAATAATTCTTTTCAAATTAGGCATGCACATTTT CTGTTTTCTTTCTTTCCATTTTGATTTATGCATTTTCATTATAAAATTGCAAATTATTATATTCTCTTTGTGTTTTTCTTTCAATTAGATGCATC GTTTTTATTTATCCATTATGGTGTTAGGTATAGTTTTTATTGTTTTAAAAATTCTTTTGTTACGCTTCCTATACAATTTCGCTTCGGTTTTGGTG CTTTGTATTTCCAATTTTTTTGTGTGCTTCGTCTCTTTTCGTCTCCCTTTATTTTGTGGCGTTTCAGTTTATAGAGTAATGATTAAATTTTCTTT TGTCTTTTAAGTTTAATTTTTGTATTACAATGGAGCGACCGTAGCTGCTCAACAATTTGTAATTCAATACCTAATTTTTGTTTTTTCTTAACGTA AAGCGTAAATATACAGAGCCAGTGAAAGATAAAAAAAATATTTGTGCTTGTATTGACCTTTCCAAGTATTGTTTCGCCATCAAATTTTTTAAAT TTTTTTTTTTTTTTTAATTTATAGAAGCAGCATTGATATTTGCAAAGACCGTTTCAGATACAACTACAAAAGTAGTTGTATTGATCATTTTAAG ATTTATTTATTTATTTATTTTTTTTTTTTGTCGATCGTTTAAAAAAATCAGTTTTATCAAGCATGCACCGTATTGGAATTGTCGCAATGATTCAC TTAGTTTGAACACAAAGAAAAATGAATTTATGCAAAACTGTTTTAACTATATAAATCTTTATTGTTTCTAAACAGTTAGTTACACTTCTTCCAA GAAAGTCAAATCTATTATTTATTTCAAACTTAATTAAGCAAAAAATTGCAAAATGAGTGAATAAACTGTAAAAAATGATTATATATTCAGTAA TTGTACAACTATTATAATTGTACAATTATTGAAGGAATTTTCCTCTTTTAATATGAATAAATAAAAGTGAAGTTACAGTTAACTATGAATTTTT TAGGCTGTAGCTATACAAAAAGTTTAGAAGAAATAAGAATAAAAAGAATGCAACTAATGTAAATTATGAAATTTATTTATCTTTATATATTAG CTCTTTTTTTTTTTTTAATGTTACAGTTTTAAGTTGAATTATTTCAAGAGTCTGCTAAACAAATAATTCAAAATTTTTTTTTTTTTTTTTTTAA ATTTTAATATTGTTACAGTTTTAAGTTGAATTATTTCAAGAGTCTGCTAAACAAATAATTCAAAAATTGCTACATAAAATTTTGACAATTTTAT TGTCATTATTAATATTTTTATTGCTTCAAACTCTAAAAAAGAAAAAATTGCTATATGTGCTATATATTTTCTTTCAGTTTATTAATTTCTCTTC ATGTCCCCGGAATGTAAAAAGTGGTAGCATGTTTTTAATTTGAAAATGTTAGTTTTACAGTAAAACGACTAGCATACGCTTAATGAAAGGATAA CTACTTTTGCGCTTGTATTTTTTAAAAAATAACTGGCTATTTGAATTTATGAAAACATGTATTTATTTGATTTATAATACTATTCTGTCAACCA ATTTTTAATTTTCATAACCATTTGGCGCAGCTGTCACATTCTCACTTATAAATGTGTATTTTAACT

>aug3.g8250.t1 
MFYPGLQQYLSEQMFEFRKGGPPPTLPSQNSPPSHSCQKVTIQKTNSDDDDFWIDVENETTDDESNDHSGLEETNSNDPSSSPPYNLKVKKQRKA RTAFTDHQLQTLEKSFERQKYLSVQDRMELASKLNLTDTQVKTWYQNRRTKWKRQTAVGLELLAEAGNYAAVQRMLQSTPYWLNQYAANLPF TAGLPGMDMYYRQGLGLQKPMAYRMYPSAPTTAMGLPVNFSSSTVPPMAAASATSPTSSSASSLPSYFAREPPC

>comp116578_c0_seq1_-_ORF_1_(frame_2)_translation

MTVDLPQKPMPADRDLRVVVDDDGSVRDDSPPPQSTTAFRVAASPPSVVAGGGTTAPSSFLIRDILSGTEQQQQQQRQSHIRGSGFSPVGYTASPS TTNSNPTSPLSASDPGHHHHHHPPPPPHPLHMHPLAHHHHALQHHPAISGQFFSPLDIRRPNGEVSPLRDDEIDEDGVSSDDDCSRSGKNDDPQK LNSSNSPDPSKSPPYNLKVKKQRKARTAFTDHQLQTLEKSFERQKYLSVQDRMELAAKLNLTDTQVKTWYQNRRTKWKRQTAVGLELLAEAGN YAAVQRMLQSSPYWLNHYAANLSFSSVPGMDMYYRQAAAAVLQKPMAYRMYPTTTATPMGMPVNFSSSTVPPPPPPPMNSNNSAPSPTSPLSST TSSSTLNSFYGREPPPC

>comp120407_c0_seq1_-_ORF_1_(frame_2)_translation

MTVTMSADTINGRTSAEPTEELVEQPTDLSTKKKSETESGSDNSPRSSPSTLADVRDSNPAPTSGPSFLIKNLIDHHNSHHHQQSHQDFAGLIPSTF HRYRPSFVPSPNPYFLPHFAANIPSGKFCFDQDGSNRRMDSDSEESEGRRPSPVQYQRTLPQELADYKQICDSRDVLKTRPNDAETVSSSSKDRISY TAVIDSDTDSISNHDGESFDAQPPREDSPSLKNKKPRKARTAFTDHQLRTLEKSFERQKYLSVQDRMELAAKLNLTDTQVKTWYQNRRTKWKRQ AMIGFEVFPPDACSLARFPRLWGPAQAPYWPYSYSSYLSTISSLSGLNTNPLVGQTSSSPASPPSSLEGYGRQAGSAVSNTVSRPLLPRAVYPSPSNF SSVSPFYQGQAGP

>Locus_1_Transcript_157093/166847_Confidence_1.0_Length_1118_-_ORF_1_(frame_3)_translation MKNKKPRKARTAFTDHQLRTLEKSFERQKYLSVQDRMELAAKLNLTDTQVKTWYQNRRTKWKRQNMTFCDYYAADSATLARFPRLWGPSPAS YFPSYSYSSYLSTMSSLSTLGAAHLGTPASSTSVPTLDLYQRQSPPVSSTIGRPVLPRAVYPTPSTYPSLQPFFHGRPTQ*

>Locus_3013_Transcript_10/10_Confidence_0.587_Length_2075_-_ORF_1_(frame_3)_translation MEETNSNDPSSSPPYNLKVKKQRKARTAFTDHQLQTLEKSFERQKYLSVQDRMELASKLNLTDTQVKTWYQNRRTKWKRQTAVGLELLAEAGN YAAVQRMLQSTPYWLNQYAANLPFTAGLPGMDMYYRQGLGLQKPMAYRMYPSAPTTAMGLPVNFSSSTVPPMAAASATSPTSSSASSLPSYFAR EPPC* 



\section{Curriculum vitae}

\section{Personal Data:}

Name

Date of birth

Place of birth

Home adress

Work adress

Email

\section{Education:}

2011- 2016

$2009-2011$

$2006-2009$
Natascha Zhang neé Turetzek

08th November 1986

Holzminden, Germany

Stumpfe Eiche 12

37077 Göttingen, Germany

Georg-August-Universität Göttingen

Johann-Friedrich-Blumenbach-Institut

für Zoologie und Anthropologie

Abteilung für Entwicklungsbiologie

GZMB Ernst-Caspari-Haus

Justus-von-Liebig-Weg 11

37077 Göttingen, Germany

nturetz@gwdg.de

PhD student of the GGNB (Göttingen Graduate School for Neurosciences and Molecular Biosciences), PhD program: Genes and Development, Georg August University, Department of Developmental Biology

Doctoral Thesis Project "Developmental studies of appendage formation and patterning in spiders"

Master program Developmental, Neural and Behavioral Biology at the Georg-August- University Göttingen, Final grade "very good" 1.2

Master thesis "On the role of hox genes and co-factors in body plan specification in spiders" at the Institute for Zoology and Anthropology, Department of Developmental Biology (Prof. Dr. Wimmer) in the laboratory for Evolutionary Developmental Biology of Arthropods (Dr. Prpic Schäper) Thesis evaluation: "very good" (1.0)

Bachelor of Science in biology at the Georg-August-University of Göttingen. Final grade "good" (1.6). 
Major field molecular biology with 6 focused modules (developmental biology, biochemistry, plant physiology, bioinformatics, genetic, microbiology).

Bachelor Thesis "Function of Mars in Neuroblasts of Drosophila melanogaster and its Interactions with other Mitosis Relevant Proteins" at the GZMB), in the Department of Stem Cell Biology (Prof. Dr. Wodarz) Thesis evaluation: "good" (1.7)

$1999-2006$

$1993-1999$

\section{Stipends and Awards:}

\section{Work Experience:}

Dec. 2010 - Nov. 2011

Aug. 2010

Sep. 2008- Aug. 2009
Secondary school "Campe-Gymnasium-Holzminden"

Elementary school "Grundschule Stadtoldendorf" and orientation stage of the secondary school at the "Orientierungsstufe Stadtoldendorf"
Christiane-Nüsslein-Volhard-Foundation fellowship and a "Women in Science" Award by L'Oréal Deutschland and the Deutsche UNESCO-Kommission.

Student Assistant at the GZMB, department developmental biology (Dr. Prof. Wimmer) in the laboratory for evolutionary developmental biology of arthropods (Dr. Prpic Schäper)

Behavioral Observations of Malagasy verreauxi Sifakas (Propithecus verreauxi verreauxi) in Kirindy Forest, Madagascar, for the German Primate Centre (DPZ), department of Behavior biology and sociobiology/anthropology (Prof. Dr. Kappeler), Student Assistant at the Max Planck Institute for Biophysical

Chemistry Department of Protein crystallization, Structural Biochemistry Group (Prof. Dr. Wahl)

\section{Teaching activities:}


Since 2011

2011

2011

Apr. - Jul. 2008
Teaching assistant of the key competence module evolutionary developmental biology of the Master program Developmental, Neural and Behavior biology

Bachelor thesis supervision of Tobias Klös

Supervision of Tobias Klös (Lab rotation 18.04 - 27.05.2011)

Teaching assistant for the lecture of basic biology

"Ringvorlesung" (for undergraduate students) at the Georg-

August- University Göttingen

\section{Languages:}

German (native)

English (fluent, writing, reading and speaking).

\section{List of Publications:}

Turetzek, N., Prpic, N.-M. Observations on germ band development in the cellar spider Pholcus phalangioides. In preparation.

Turetzek, N., Pechmann, M., Schomburg, C., Schneider, J., and Prpic, N.-M. (2016). Neofunctionalization of a Duplicate dachshund Gene Underlies the Evolution of a Novel Leg Segment in Arachnids. Mol Biol Evol 33, 109-121.

Schomburg, C., Turetzek, N., Schacht, M.I., Schneider, J., Kirfel, P., Prpic, N.-M., and Posnien, N. (2015). Molecular characterization and embryonic origin of the eyes in the common house spider Parasteatoda tepidariorum. EvoDevo 6, 15.

Janssen, R., Schönauer, A., Weber, M., Turetzek, N., Hogvall, M., Goss, G.E., Patel, N.H., McGregor, A.P., and Hilbrant, M. (2015). The evolution and expression of panarthropod frizzled genes. Frizzled 96.

Khadjeh S, Turetzek N, Pechmann M, Prpic NM.

Warum Spinnen (nur) acht Beine haben.

Biologie in unserer Zeit 43, 112-117 (2013).

Khadjeh S, Turetzek N, Pechmann M, Schwager EE, Wimmer EA, Damen WGM, Prpic NM Divergent role of the Hox gene Antennapedia in spiders is responsible for the convergent evolution of abdominal limb repression. 
Proceedings of the National Academy of Sciences of the United States of America 109, 49214926 (2012).

Pechmann M, Khadjeh S, Turetzek N, Prpic NM

Beinentwicklung und Gliedmaßen-Evolution bei Spinnen.

BIOspektrum 18, 365-368 (2012).

Pechmann, M., Khadjeh, S., Turetzek, N., McGregor, A.P., Damen, W.G.M., and Prpic, N.-M. (2011). Novel Function of Distal-less as a Gap Gene during Spider Segmentation. PLoS Genetics 7, e1002342. 